\title{
BOOK OF PROCEEDINGS
}

9th Conference of the European Academy of Occupational Health Psychology

Edited by:

Sergio lavicoli

Aditya Jain

Marta Petyx

Jessica Tang

Nottingham

University Press

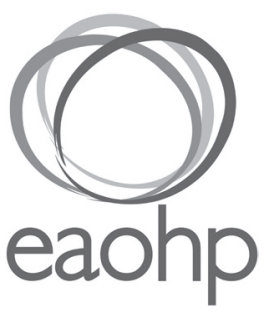

European Academy of

Occupational Health Psychology 
Manor Farm, Main Street, Thrumpton

Nottingham, NG11 0AX, United Kingdom

\section{NOTTINGHAM}

First published 2010

( ) EAOHP

All rights reserved. No part of this publication may be reproduced in any material form (including photocopying or storing in any medium by electronic means and whether or not transiently or incidentally to some other use of this publication) without the written permission of the copyright holder except in accordance with the provisions of the Copyright, Designs and Patents Act 1988. Applications for the copyright holder's written permission to reproduce any part of this publication should be addressed to the publishers.

\section{British Library Cataloguing in Publication Data}

Proceedings of the 9th European Academy of Occupational Health Psychology

Editors: I. lavicoli, S. II. Jain, A. III. Petyx, M. IV. Tang, J.

ISBN: 978-1-907284-46-5

\section{Disclaimer}

Every reasonable effort has been made to ensure that the material in this book is true, correct, complete and appropriate at the time of writing. Nevertheless the publishers, the editors and the authors do not accept responsibility for any omission or error, or for any injury, damage, loss or financial consequences arising from the use of the book. The views expressed by contributors do not necessarily reflect those of the European Academy of Occupational Health Psychology.

Typeset by Nottingham University Press, Nottingham

Printed and bound by Lightning Source, England 


\section{CONTENTS}

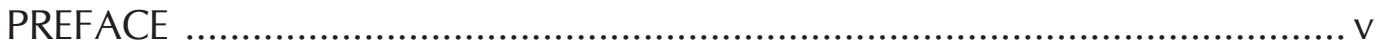

ORGANISING COMMITTEE............................................................... vi

SCIENTIFIC COMMITTEE ................................................................ vii

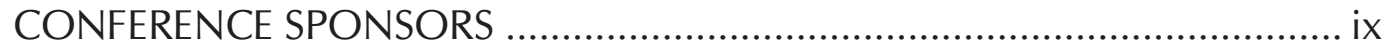

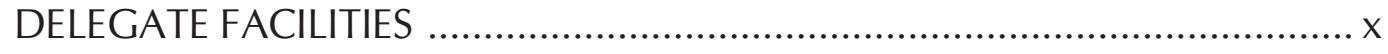

CONFERENCE PROGRAMME ............................................................

\section{ABSTRACTS}

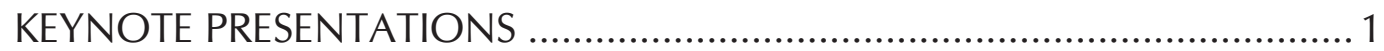

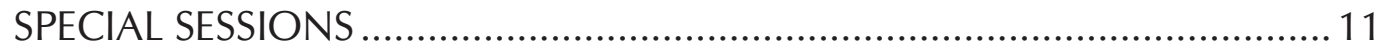

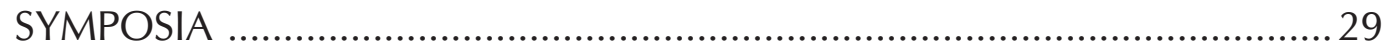

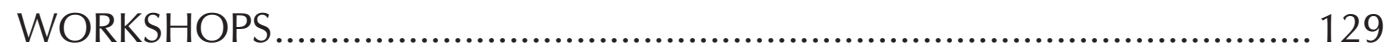

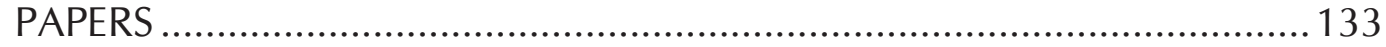

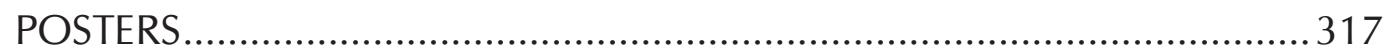

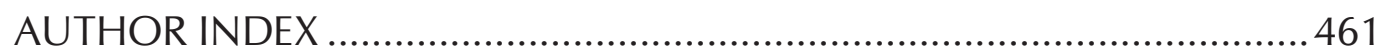





\section{PREFACE}

The European Academy of Occupational Health Psychology in collaboration with the Department of Occupational Medicine of the Italian National Institute of Occupational Safety and Prevention (ISPESL) welcome you to the 'eternal city' of Rome and to the Pontifical Urbaniana University that is playing host to its $9^{\text {th }}$ conference. The Academy's first conference took place in 1999 to provide a new platform to promote the development of research, education and practice in the then emerging field of occupational health psychology. That inaugural event was held in Lund, Sweden. In the intervening years, conferences have been held successfully in Nottingham, England (2000), Barcelona, Spain (2001), Vienna, Austria (2002), Berlin, Germany (2003), Porto, Portugal (2004), Dublin, Ireland (2006) and, most recently, Valencia, Spain (2008). We are delighted to have been able to organise this IX Academy conference in Rome and this has in no small part been possible due to the financial and practical support provided by our co-organiser, ISPESL and by our international sponsors.

The Rome conference promises to be the largest ever organised by the Academy. It has a powerful and engaging schedule of keynote, oral and poster presentations as well as invited special sessions and workshops. The session on 'the management of psychosocial risks: good practices and successful models' organised by ISPESL will showcase examples of the successful experiences in the management of psychosocial risks in Europe. It will discuss experiences from countries like UK, Ireland, Italy and Norway as well as the perspectives offered by the World Health Organisation and the European Agency for Safety and Health at Work (EU-OSHA).

Each year the Academy awards a lifetime Fellowship to an individual who, in the opinion of the Executive Committee, has made an exceptional contribution to the discipline of occupational health psychology. This year we are proud to welcome both, Professor Cary Cooper, University of Lancaster, UK and Professor Wilmar Schaufeli, Utrecht University, the Netherlands into our College of Fellows.

At this juncture, it might also be considered timely to pause to reflect on what has been achieved and what remains to be achieved in respect of research, education and practice in our discipline. The first conference back in 1999 provided a platform for the discussion of how occupational health psychology in Europe might be structured and framed with a view to the creation of a self-sustaining discipline. These achievements will be reviewed following the conference and published in our journal Work \& Stress. We hope that this will stimulate debate on how the discipline might further mature in the coming years.

On behalf of the Organising Committee, we would like to thank you for contributing to this conference. We hope it will meet your expectations and will stoke up your enthusiasm. We continue in our commitment to build a member's Academy and will always welcome those who wish to be actively involved going forward. Finally, we would like to thank all of those who have given so generously of their time in helping to make this event a reality.

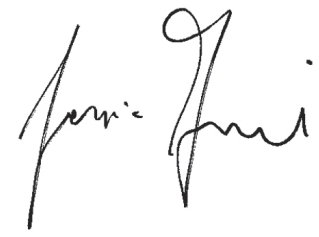

Sergio lavicoli (Conference Chair) Sergio.lavicoli@ispesl.it

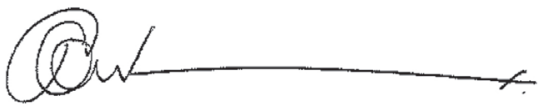

Tom Cox (President, EAOHP)

Tom.Cox@nottingham.ac.uk 


\title{
PROMOTING COMMITTEE
}

\author{
Tom Cox \\ President, EAOHP \\ Sergio lavicoli \\ Conference Chair \\ Antonio Moccaldi
}

President, National Institute of Occupational Safety and Prevention (ISPESL)

Umberto Sacerdote

Director General, National Institute of Occupational Safety and Prevention (ISPESL)

\section{ORGANISING COMMITTEE}

\section{Aditya Jain, Chair of Organising Committee \\ University of Nottingham, UK}

Marina Catelli, National Institute of Occupational Safety and Prevention (ISPESL), Italy

Pasquale Fruscella, National Institute of Occupational Safety and Prevention (ISPESL), Italy

Monica Ghelli, National Institute of Occupational Safety and Prevention (ISPESL), Italy

Juliet Hassard, University of Nottingham, UK

Jonathan Houdmont, University of Nottingham, UK

Stavroula Leka, University of Nottingham, UK

Elena Natali, National Institute of Occupational Safety and Prevention (ISPESL), Italy

Carlo Petyx, National Institute of Occupational Safety and Prevention (ISPESL), Italy

Jessica Tang, University of Nottingham, UK 


\section{SCIENTIFIC COMMITTEE}

\section{Gail Kinman, Chair of Scientific Committee}

University of Bedfordshire, UK

Sergio lavicoli, Conference Chair

National Institute of Occupational Safety and Prevention (ISPESL), Italy

Claudio Barbaranelli, University of Rome "Sapienza", Italy

Julian Barling, Queen's University, Canada

Georg Bauer, University of Zurich, Switzerland

Laura Borgogni, University of Rome "Sapienza", Italy

Victor Catano, St Mary's University, Canada

Tony Cassidy, University of Ulster, UK

Giancarlo Cesana, University of Milan, Italy

Andrew Clements, University of Bedfordshire, UK

Stacey Conchie, Liverpool University, UK

Tom Cox, University of Nottingham, UK

Mark Cropley, University of Surrey, UK

Hans de Witte, Catholic University of Leuven, Belgium

Patrizia Deitinger, National Institute of Occupational Safety and Prevention (ISPESL), Italy

Philip Dewe, Birkbeck College, University of London, UK

Maureen Dollard, University of South Australia, Australia

Julian Edwards, Open University, UK

Paul Flaxman, City University, UK

Roxanne Gervais, Health and Safety Laboratory, UK

Sabir Giga, University of Bradford, UK

Amanda Griffiths, University of Nottingham, UK 
Jonathan Houdmont, University of Nottingham, UK

Michelle Inness, University of Alberta, Canada

Fiona Jones, University of Leeds, UK

Kevin Kelloway, St. Mary's University, Canada

Peter Kelly, Health and Safety Executive, UK

Michiel Kompier, Radboud University Nijmegen, The Netherlands

Stavroula Leka, University of Nottingham, UK

Colin Mackay, Health and Safety Executive, UK

Almuth McDowall, University of Surrey, UK

Elena Natali, National Institute of Occupational Safety and Prevention (ISPESL), Italy

Andrew Noblet, Deakin University, Australia

Mike O'Driscoll, University of Waikato, New Zealand

Nicky Payne, University of Middlesex, UK

José $\mathrm{M}^{\mathrm{a}}$ Peiró, University of Valencia, Spain

Marta Petyx, National Institute of Occupational Safety and Prevention (ISPESL), Italy

Eusebio Rial-Gonzalez, European Agency of Occupational Safety and Health (EU-OSHA)

Per Oystein Saksvik, Norwegian University of Science and Technology, Norway

Steve Sauter, National Institute of Occupational Safety and Health (NIOSH), USA

Norbert Semmer, University of Bern, Switzerland

Arie Shirom, Tel Aviv University, Israel

Johannes Siegrist, University of Dusseldorf, Germany

Noreen Tehrani, Chartered occupational, counselling and health psychologist, UK

Lois Tetrick, George Mason University, USA

Sturle Tvedt, Norwegian University of Science and Technology, Norway

Siobhan Wray, St George's Hospital Trust, UK 


\section{CONFERENCE SPONSORS AND PATRONS}

The following have

generously supported the $9^{\text {th }}$ conference of the

European Academy of Occupational Health Psychology

- Province of Rome

- Municipality of Rome

- European Agency for Safety and Health at Work

- International Commission on Occupational Health (ICOH)

- Italian Society of Occupational Medicine and Industrial Hygiene (SIMLII)
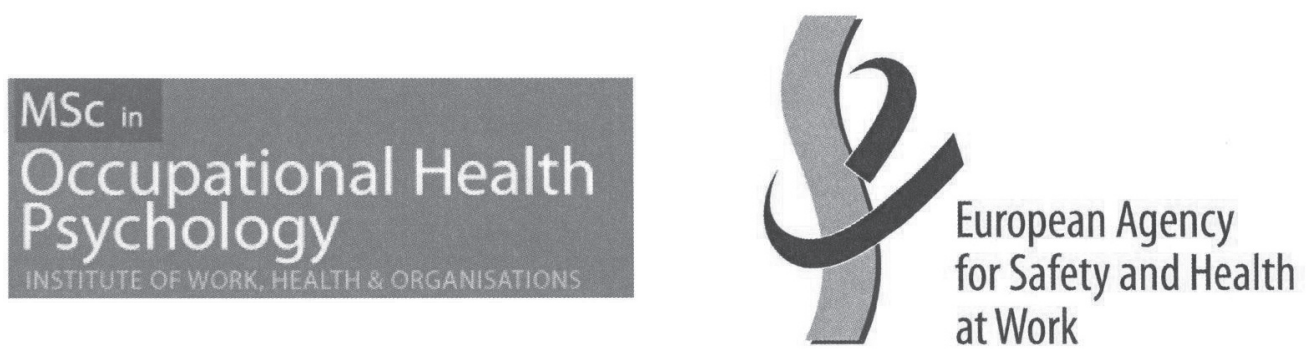

Work \& Stress

A journal of work, health and organisations

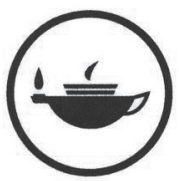

Taylor \& Francis

Taylor \& Francis Group 


\section{DELEGATE FACILITIES}

\section{Venue}

The Conference will be held at the 'Gianicolo Congressi' convention centre, located within the Pontifical Urbaniana University, next to St Peter's Square and with magnificent views of the Gianicolo Promenade and Rome. It is a 10 minute walk from St Peters Square and well connected by public transport.

Address: Pontifical Urbaniana University, Via Urbano VIII, Rome, Italy.

\section{Internet access}

There are no internet facilities at the Venue.

\section{Presentations}

All oral presentations must be supported by a Microsoft PowerPoint presentation. Presentations should be on CD or USB stick and clearly labelled with (i) the name of the lead author, (ii) title of presentation and (iii) day, time and room number of the presentation. Authors for posters presentations should bring their posters (max dimensions $100 \times 70 \mathrm{~cm}$ ) to the conference venue on the day of presentation. Please, note that is not allowed to modify or print the presentation at the venue.

\section{Catering}

Lunch is included in the delegate fee and refreshments will be available at no cost each morning and afternoon of the conference. Refreshments will also be available through out the duration of the conference.

\section{Exhibition stands}

During the conference, you are invited to visit the exhibition stands located in the foyer. Books and journals relevant to occupational health psychology from Wiley-Blackwell, Taylor \& Francis and the EAOHP will be available.

\section{Further assistance}

Should you require any assistance during the conference please don't hesitate to visit the conference registration desk. 


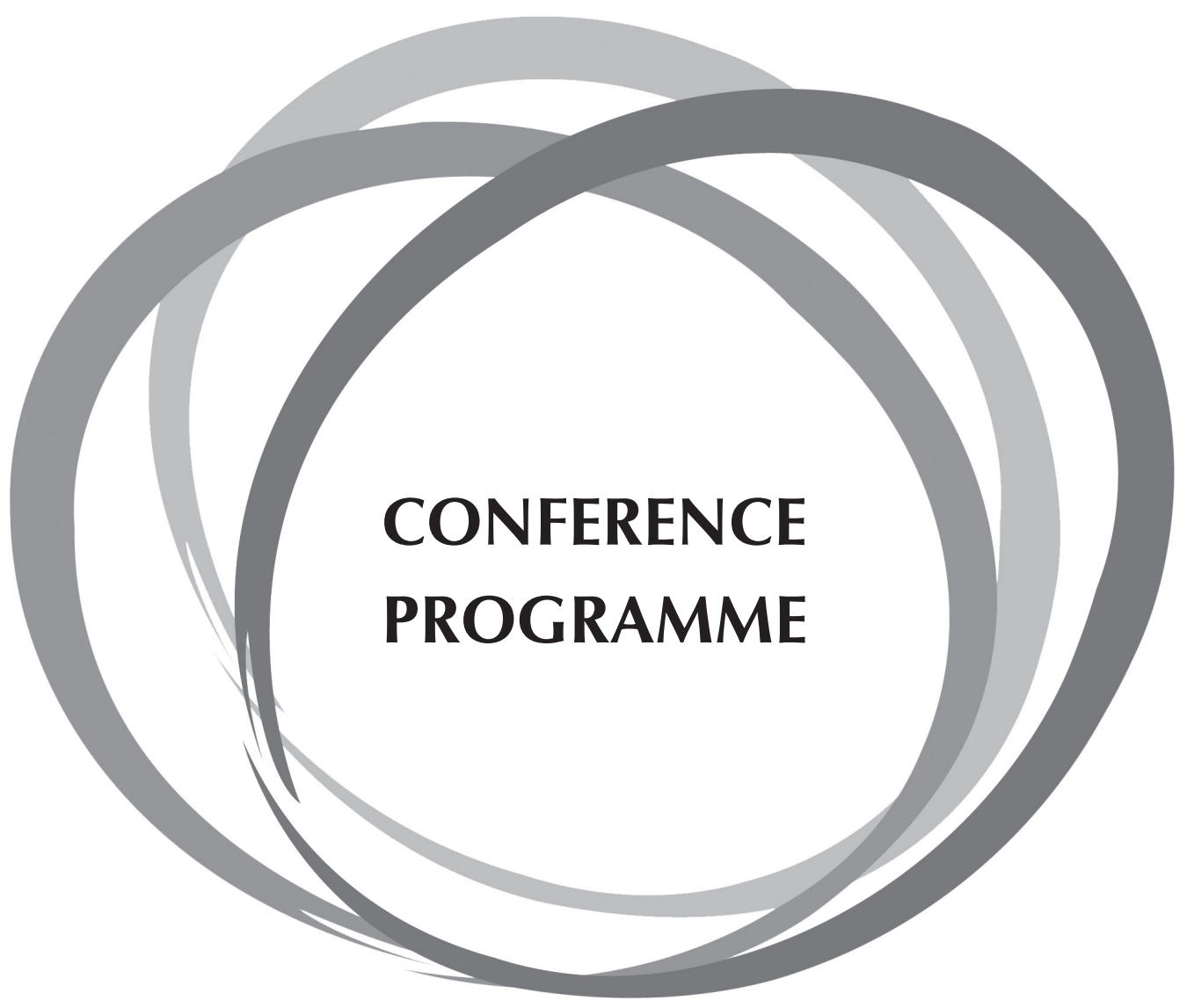




\section{Monday 29 March 2010}

08:00-09:00

Registration

\section{9:00-09:45}

\section{Aula Magna: Opening address}

S. Iavicoli, Conference Chair

S. Leka, EAOHP

A. Moccaldi, President, ISPESL

U. Sacerdote, Director General, ISPESL

F. Fazio, Ministry of Health, Italy

\section{9:45-11:15}

Aula Magna: Special Session "The management of psychosocial risks: good practices and successful models" (S. lavicoli \& S. Leka)

WHO global perspective on psychosocial risk management Kortum, E.

Assessing and preventing psychosocial risks in the EU: An overview of EU-OSHA's work Rial-Gonzalez, E.

UK Management Standards Approach for tackling work-related stress in a global context. Palferman, D.J., Mackay, C. \& Kelly, P.

Republic of Ireland - Work Positive, Risk Assessment method for auditing work-related stress in a global context.

Murray, $P$.

Surveillance of occupational health and safety in Norway: experiences with establishment of a national Surveillance system

Sterud, T.

\section{Aula II: The Changing world of Work and the Aging Workforce}

Age differences in stressors and strain

Rauschenbach, C. \& Hertel, G. 
The Landscape of Psychological Age and Chronological Age at Work

Barnes-Farrell, J., Johnson, N.C., \& Cherniack, M.G.

The effect of Retirement Behaviour on Retirees' Quality of Life

Ceresia, $F$.

The ageing office population: age and the work physical environment

De Been, I. \& De Bruyne, E.

Early retirement: a coping strategy against ageism at work

Desmette, D., Iweins, C.,\& Herman, G.

The aging workforce - more chilled out than burnt out?

Johnson, S., Holdsworth, L., Hoel, H., Cunningham, K.

\section{Aula III: Developing Intervention Process Evaluation (R. Randall)}

Strengthening the evaluation strategy of stress management intervention programs using process evaluation and multilevel methods: The evaluation of a team-based stress management intervention program for low-qualified workers

Busch, C. \& Clasen, J.

Does training managers help? A longitudinal, mixed methods field study of the implementation of team-working

Nielsen, K. \& Randall, R.

Substance over style: The importance of theories-in-use as determinants of intervention outcomes. Randall, R. \& Nielsen, K.

Evaluating stress prevention through work-related interventions: Trade-offs involved, and implementation issues to be considered.

Semmer, N.K.

\section{Aula VII: Bullying in the Workplace}

Monitoring of working and health conditions in victims of bullying at work

Romeo, L., Pelizza, L., Antonini, E., Ballottin, A., Cabianca, M., Tisato, S., \& Perbellini L.

Healthy Change Process Index ( $\mathrm{HCPI})$ as a predictor of workplace bullying during organizational change

Tvedt, S.D., Nielsen, M.B., \& Lau, B.

Workplace Bullying and Staff Well-Being: A Mixed Methodological Approach

Woodrow, C. \& Guest, D.

How bullying changed my life

Cecchini, F. 
Occupational and health conditions among victims of workplace bullying: a prospective study Punzi, S., Pavesi, A., Castellini, G., Conway, P.M., Militello, E., Cassitto, M.G., \& Costa, G.

Bullying among nurses: Extent, context, and consequences

Kaminski, M. \& Sincox, A.K.

\section{1:15-11:30}

Refreshments

\section{1:30-13:00}

\section{Aula Magna: Special Session Part II - The management of psychosocial risks in} Italy (M. Masi \& L. Fantini)

ISPESL's commitment to manage and assess work-related stress lavicoli, $S$.

Inter-regional guidelines for assessment and management of work-related stress

D'Orsi, F.

The slow consolidation of the "right" concept of work-related stress

Frascheri, C.

Work-related stress

Pontrandolfi, F.

\section{Aula II: The Psychosocial Working Environment and Work-related Stress}

Professional competence as an intervening variable in affecting work-related stress: the case of public school teachers in Italy.

Callari, T.C., \& Re, A.

Evaluation of Job Stress Models for Predicting Health at Work

Reineholm, C., Ekberg, K., \& Gustavsson, M.

Social Stress Risk Model

Penati, V., Ferrari, F., Sinibaldi, F., \& Girard, A.

Regulatory focus at Work: the Moderating Role of Regulatory focus in the Job Demands-Resources Model

Brenninkmeijer, $V$.

The success-resource model of job stress

Grebner, S., Elfering, A., \& Semmer, N.K. 


\section{Aula III: Temporary Employment and Well-being: New Avenues for Research (N. De Cuyper \& H. De Witte)}

Temporary versus permanent employment and psychological outcomes: the moderating effect of motives for hiring temporary workers

Bakk, Z., de Jong, J., Cambré, B. \& De Cuyper, N.

Job insecurity in the temporary workforce: A moderated mediation model linking volition, job insecurity and contract expectations to well-being.

Bernhard-Oettel, C., Rigotti, T., \& Clinton, M.

Perceived employability and engagement among temporary versus permanent workers

De Cuyper, N., Van Vuuren, T., Van der Heijden, B., \& Alarco, B.

Core self-evaluations and job satisfaction: Mediation by employability?

Mäkikangas, A., Mauno, S., \& Kinnunen, U.

\section{Aula VII: Burnout Syndrome in Mental Health Professionals: Cross Cultural Perspectives (S.M. Lee)}

Individual and Work-Related Factors influencing Burnout of Mental Health Professionals: A MetaAnalysis.

Lee, S.M. \& Choi, B.Y.

Antecedents and Consequences of Burnout in Mental Health Professionals: A Meta-Analysis

Kim, A. \& Lee, J.

Burnout Syndrome of American Mental Health Professionals

Lee, J. \& Puig, A.

Burnout Syndrome of Korean Mental Health Professionals

Choi, H. \& Yu, K.

Burnout Syndrome of Japanese Mental Health Professionals

Jin, Y.Y. \& Lee, J.

Examining the Burnout Model (Demand-Control vs. Effort-Reward) in Mental Health Professionals Shin, H. \& Lee, J.

\section{Aula VIII: Understanding the Impact of Emotional Labour}

Emotional labour in job-centre workstations and impact on third party violence Manz, $R$.

Emotion work and emotional exhaustion in teachers: What kind of job resources do they need? Naring, G., Vlerick, P., \& Van de Ven, B. 
Resources depletion model of Emotional Labour: Perceived emotional effort as key factor in SpanishEnglish samples

Rodríguez-Carvajal, R., Quiñones-García, C., Clarke, N., Moreno-Jiménez, B., \& De RivasHermosilla, $S$.

Emotion work antecedent or consequence of burnout and work engagement?

Hakanen, J.

A longitudinal investigation into whether emotional labour and other work strains have an impact on the physical and mental health of individuals working in the Service Profession

Al Serkal, A.

How to stand emotionally demanding work settings: workload, emotional regulation and emotional exhaustion among German physicians

Staechele, T., Ensinger, K., \& Schuepbach, $H$.

\section{3:00-13:45}

\section{Aula Magna: EAOHP Fellowship address}

Enhancing Mental Capital and Wellbeing in the Workplace Cooper, C.L.

\section{3:45-15:15}

\section{AULA VIII: EAOHP Research Forum}

Lunch and Poster Session

\section{$\underline{\text { Job Satisfaction }}$}

Job satisfaction and stress in doctors of public tertiary medical care in Mexico.

Colunga Rodriguez, C., Rendon-Manjarrez, J.I., Dominguez-Diaz, R., \& Angel-Gonzalez, M.

Shaping of wellbeing with Job Satisfaction

Molan, M. \& Molan, G.

Quality of Life and Job Satisfaction: Multicultural Perspective

Pereira, J. \& Sousa, A. D.

\section{Mental Health and the Workplace}

Family systems in parents of children with Generalized Anxiety Disorder (GAD) Aleyasin (Al yasin), S.A. \& Salehi, B. 
Psychiatric disabilities and employment discrimination: An empirical analysis of EEOC ADA Title I charges

An, S. \& Roessler, R.

Study of psychological health status in the dental students of the Qazvin University Of Medical Sciences (second semester of the year 2007-2008)

Ansari Ramandi, S., Ramandi, M.M.A., Yazdizadeh, M., \& Sarichlo, M.E.

Explaining an Unexpected Interactive Relationship Between Gender Harassment and Union Tolerance for Harassment in Relation to Mental Health: The Role of Conflict

Golay, L., Mellor, S., \& Magely, V.J.

Shiftwork and mental health - a cross sectional study of shiftworkers in the Norwegian petroleum sector

Ljoså, C.H

The Role of Sense of Coherence as a Mediator between Job Strain and Mental Health in Two Distinct Work Settings

Reeves, D. \& Henning, $R$.

The Effects of Job Demands, Control and Social Support on Exercise

Padover, D., Reeves, D., Henning, R., Faghri, P. \& Warren, N.

Moderated effects of Job Insecurity on Work Engagement and Distress

Lo Presti, A., \& Nonnis, M.

Culture of excellence and mental health of public service workers. A psychodynamic reading of work in Quebec and Switzerland.

Resenterra, $F$.

Predictors of psychological discomfort and well-being in a sample of correctional officers employed in Penitentiary Sector in Italy

Viotti, $S$.

Psychosocial Risk Assessment: a pilot study in an Administration and Coordination Operative Unit Atzori, E. \& Tagliaferri, C.

\section{Burnout}

Prevalence of the burnout syndrome in top managers sent by multinational industrial companies on long-term working stays to foreign culture

Alfoldy, S. \& Gil-Monte, P.

Stress, Burnout and Emotional Disorders in the Emergency Medical Volunteers

Alheiro, A.S. \& Cunha, M. J.

Job burnout and work fatigue in the context of organizational stress among nurses.

Beata, B. \& Wilczek-Ruzyczka, E. 
Investigating the causes of burnout in police officers

Burba, $M$.

Burn out syndrome and contributory work environment factors among psychiatry nurses

Circenis, K., Deklava, L., \& Millere, I

Vulnerability to stress, burnout, emotional disorders and quality life in occupational health care providers in women with breast cancer

Cunha, M.J., Costa, M., Pereira, J.P., \& Gascon, S.

Workload and job burnout among prison educators

Deitinger, P., Nardella, C., Bentivenga, R., \& Ghelli, M.

Burnout and work engagement during the transition from education into work life Hultell, D. \& Gustavsson, J.P.

Burn-out and psychosomatic health complaints in a hospital in Piemonte region

Martini, M. \& Converso, D.

Which is the importance of teachers' competence development programs? The relation between burnout, depression and anxiety in Portuguese teachers

Patrao, I. \& Santos-Rita, J.

School Burnout and Engagement during Educational Transitions

Salmela-Aro, $K$.

How do schools burn-out Portuguese teachers? The role played by age, type of contract and length of experience

Santos-Rita, J. \& Patrao, I.

The effect of perceived support and self efficacy on work related stress and burnout amongst intellectual disability direct care workers in Australia

Walker, A. \& Jackson, B.

The feeling of coherence and burnout syndrome among nurses

Wilczek-Ruzyczka, E.

\section{Work-life balance}

Work-life balance despite or because of volunteering. A comparative, explorative data analysis in Switzerland

Brauchli, R., Bauer, G., Wehner, T., \& Hämmig, O

Vacation Effect: Reduction of Load and Addition of Pleasure?

de Bloom, J., Geurts, S.A.E., Sonnentag, S., \& Kompier, M.A.J.

Till' 5pm Do Us Part: Intimate Relations in the Workplace

Donohoe, $M$. 
Work/family conflict and work stress outcomes in working shifts nurses Iskra-Golec, I.

An investigation of workaholism components in relation to work and life values and satisfaction Kasprzak, M., Tokarz, A., \& Malinowska, D.

To Work or not to Work in an Extended Working Life Nilsson, $K$.

Causes and consequences of the utilization of work-life policies by professionals Peper, B., Dikkers, J., Vinkenburg, C., \& van Engen, M.

A Test of influences of Work-Family Conflict, Job Enrichment and Leader' Member Exchange on Employee Turnover Intentions.

Portoghese, I., Galletta, M., \& Battistelli, A.

Health care managers' boundary approaches to balance their time-commitments at work and in life

Tengelin, E., Wikstrom, E., Arman, R., \& Dellve, L.

Validation Study of a Multidimensional Measure of Work-Family Conflict in Romania Virga, D., Sulea, C., \& Marcutoiu, L.

Work and family support, work to family spillover and job satisfaction: a model Zito, M., Colombo, L., \& Ghislieri, C.

\section{Individual differences: Personality and Coping}

Coping and Quality of Life in Informal Caregivers of Individuals Alves, J. \& Machado, F.

The Relationship between Personality Traits, Job Satisfaction and Job Performance of South African Retail Store Managers

Barkhuizen, N., Ermakova, J. \& van der Walt, R.

The relationship between masculine financial identity, workgroup aggression normalization, and incivility perpetration: a discriminant function analysis.

Bauerle, T., \& Magely, V.J.

Continuance commitment mediating the effect of masculine financial identity on burnout and stress: a case for masculine occupational health.

Bauerle, T. \& Magley, V.J.

Need for achievement as a vulnerability factor in a politicized work setting Berset, M., Henseleit, J., Omlin, F., Willi, S., Semmer, N.K., \& Elfering, A.

Personality factors and professionally relevant behaviour in health care Deklavs, L., Millere, I., \& Circenis, K. 
Conflict Management Styles and Performance Relations Among Voluntary Health Workers Erturk, E.M.

Positive and Negative affect: Diverse pathways on emotion management.

Garrosa. E., Blanco, L. M., Rodriguez-Carvajal, R., Sanz-Vergel, A.I., \& Rodriguez-Muñoz, A.

A Global Look at Workplace Incivility: Does Personality Play a Role Throughout the World? Milam, A., Sulea, C., Hanif, R., Spitzmueller, C.

Coping strategies and positive affect through gender dimension Niculaes, $A$.

Do individual coping strategies help or harm in the work-family conflict situation? Examine coping as a moderator between work-family conflict and well-being.

Rantanen, M., Mauno, S., Rantanen, J. \&Kinnunen, U.

Psychological well-being: levels of vitality and inner resources as relevant components of eudaimonic well-being

Rodriguez-Carvajal, R., de Rivas, S., Moreno-Jiménez, B., Blanco, A., \& Alvarez, A.

Humour Style in Relationship to Stress and Well-being: Four Different Personalities

Stokenberga, I.

Coping and Quality of Life in subjects with Spinal Cord Injury

Teixeira, A. \& Machado, F.

The Impact of Emotional Intelligence on Organizational Citizenship Behaviors

Yildirim, D., Hunerli, S., Atan, O.

\section{5:15-16:45}

\section{Aula Magna: Healthy Organisations and Workers: A Global Perspective}

Witnessing workplace violence, psychological stress and job abandoning: Four case studies from Mexican State universities

Sieglin, V., Coronado, M.Z., \& Ramos, M.E.

Cultural variation and motivation of Indian and Korean employees: A cross-cultural study

Kamalanabhan, T.J. \& Deug Kim, $H$.

Working conditions in a precarious work environment - A global study of aviation workers Greiner, B., Rosskam, E., McCarthy, V., Mateski, M., Zsoldos, L., Marowsky, I., Rodriguez-Muñoz, G., Williamson, E., \& Tyler, J.

Stress and Esfahan steel employees, Iran Lotfizadeh, M. \& Hassim, N. 
Stressed and Fatigued on the Ground and in the Sky: Changes in civil aviation workers conditions of work in the post $9 / 11$ era

Rosskam, E., \& Greiner, $B$.

\section{Aula II: Organizational Health Development (G.F. Bauer)}

Organisational Health Development Model

Bauer, G.F., Hoffmann, S., Müller, F., \& Jenny, G.J.

Organisational Health Capacity: The Role of Capacity Building in the Organisational Health Development Process

Hoffmann, S., Müller, F., Jenny, G.J., \& Bauer, G.F.

Key indicators of organisational health capacities: operationalization, validity and empirical relations with predictors and outcomes

Müller, F., Jenny, G.J., Hoffmann, S., \& Bauer, G.F.

Evaluation of an Organisational Health Development (OHD) Project Jenny, G.J., Müller, F., Inauen, A., \& Bauer, G.F.

\section{Aula III: Employee Empowerment and Self-esteem}

Antecedents and consequence of work empowerment for elementary teachers

Choochom, $\mathrm{O}$.

Wellbeing at work: informing and empowering employees

Hamilton, J.

Influence of empowerment on new graduate nurses' experiences of bullying and burnout in Canadian Hospital settings

Laschinger, $H$.

Parallel Development of Social Stressors, Somatic Complaints and Self-Esteem: A Latent Growth Approach

Gross, S., Semmer, N.K., Meiser, L.L., Kaelin, W., \& Tschan, F.

Does higher professional education increase contingent self-esteem?

Hallsten, L., Rudman, A., \& Gustavsson, $P$.

Exclusion and lack of power at the core of unemployment self-esteem

Herman, G., Bourguignon, D., Desmette, D., Guinote, A., \& Larue, G.

\section{Aula VII: Psychosocial Working Environment, Work Stress, and Worker Wellbeing}

Psychosocial factors promoting well-being in call-center operators

Campanini, P., Conway, P.M., Camerino, D., Punzi, S., Fichera, G.P., \& Costa, G. 
Goal Pursuit Put into Context: Psychosocial Work Environment Associates with Personal Work Goals in a 2-year Follow-up Study

Hyvonen, K., Feldt, T., Kinnunen, U., \& Tolyanen, A.

Effort-Reward Imbalance in combination with overcommitment impairs self-rated health and supervisor-rated job performance

Feuerhahn, N., Kuehnel, J., \& Kudielka, B.M.

Using job strain and organizational justice models to predict the relationship between working conditions and employee performance

Maharee-Lawler, S., Rodwell, J., \& Noblet, A.

Team emotional intelligence as a predictor of employee strain and initiative: A conflict perspective Schraub, E.M. \& Schanz, K.

Switching on and off: the impact of smartphone use on employee well-being

Derks, D., Zecic, D., \& Bakker, A.

\section{Aula VIII: Individual Differences and Personality}

The role of proactive coping in the Job-Demands-Resources Model Ângelo, R., \& Chambel, M.J.

Does successful coping require calming down? Both problem-focused coping and palliative coping are positively associated with well-being, mediated by successful calming down

Kaelin, W., \& Semmer, N.K.

Collective coping within teams of low-qualified workers?!

Requa-Brueckner, G. \& Busch, C.

Effects of Psychological Hardiness on Medically Certified Sickness Absence Hystad, S.W., Eid, J., \& Brevik, J.I.

The Quality of Prior Experiences, Current Job Conditions and Anticipated Stress in a Reorganization Scenario: the Central role of Cognitive Appraisal

Cullinane, S.J., Kirchner, A., Sczesny, S., Meier, L., \& Bosak, J.

Anxiety and Locus of Control in Evaluation of Occupational Stress

Micali, E., \& Bagnato, C.

\section{6:45 -17:00}

Refreshments 


\section{Aula Magna: Workshop}

The Implementation of a New Inspection Tool in the Psychosocial Working Environment: The Experience of the Danish Working Environment Authority

Magwentshu, S.

\section{Aula II: Health-promoting Leadership (S. Vincent \& F. Franke)}

Leadership and job design - Why good leadership is not sufficient for health promotion Wieland, R. \& Krajewski, J.

Health-promoting leadership behaviour: A new measure Vincent, $S$.

Leaders' ability to shape an organisational climate for health: Do personal characteristics matter? Gurt, J.

Health-promoting leadership: A new approach

Franke, F. \& Felfe, J.

What makes supervisors lead in a healthy way? - Organisational and individual antecedents of health-promoting leadership

Wilde, B., Hinrichs, S., Pavez, C.B. \& Schüpbach, H.

\section{Aula III: Improving Worklife: International Perspectives on Workplace Health and Work Engagement (Chair: M. Leiter; Discussant: L. Tetrick)}

How Exposure to Information and Communication Technology is related to Work Engagement: The Role of Positive Appraisal

Salanova, M. \& Llorens, S.

Conflict, Performance And Commitment In Teacher Teams: The Mediating Role Of Team Potency le Blanc, P.M., van Emmerik, H.Y., Euwema, M.C., Salanova, M. \& Vera, M.

Work Positive: An Intervention to Improve Psychosocial Health and Safety in Hospitals

Murray, $P$.

CREW: Improving Worklife Through Collegiality

Leiter, M.P., Laschiner, H.K.S., Day, A. \& Gilin-Oore, D.

Leader-member exchange (LMX) quality and enthusiasm at work: testing a dynamic mediated longitudinal relationship

González-Romá, V. \& le Blanc, P.M. 


\section{Aula VII: Strategies and Practices to Prevent Stress and Promote Employee Health}

Organizations health management systems: Do they improve employee's health? Buech, V., Schraub, E.M.,\& Sonntag, K.

Evaluating occupational health management: Development of the Health Index and its relation to economic outcomes

Schraub, E.M., Buech, V., \& Sonntag, K.

The development of a methodology for developing a healthy organisation culture.

Bos, E., Scheppingen, V.A., Sarren, A., \& Zwetsloot, G.I.J.M.

Effects of a stress management intervention on absenteeism and return to work - results from a randomized wait-list controlled trial

Willert, M.V., Thulstrup, A.M., \& Bonde, J.P.

The Role and importance of goals in output-controlled working systems

Bahamondes Pavez, C., Wilde, B., Hinrichs, S., \& Schuepbach, H.

Relaxed Entrepreneurs: Approaches to Stress Management and Burnout Prevention

Olos, L.

\section{Aula VIII: Workplace Health Promotion}

HRM, psychological employee well-being \& organizational performance van Veldhoven, M., Croon, M., Peccei, R., \& Wood, S.

Wellbeing in a Multinational Company

Gallo, $R$.

Perspectives of workplace health promotion

Noehammer, E., Schusterschitz, C., \& Stummer, H.

Creating and maintaining individual well-being: The potential of worksite health promotion Noehammer, E., Schusterschitz, C., \& Stummer, $H$.

The dark side of high-skilled work. Assessing work-related stress risk in the Italian National Research Council.

Ponzini, G., \& Moccaldi, R.

Recreation on the job: Rest break management among hospital physicians

Ensinger, K., Staechele, T., \& Schuepbach, $H$. 


\section{Tuesday 30 March 2010}

08:00 - 09:15

Aula Magna: Psychologically Healthy Workplaces \& Corporate Social Performance: Applicant, Employee and Organizations Perceptions and Outcomes (A. Day)

Psychologically Healthy Workplaces, Corporate Social Performance, and Applicant Attraction Catano, V. \& Morrow, $H$.

Healthy work as a competitive advantage: What do different generations want from their work? McNally, C. \& Loughlin, C.

Corporate social responsibility through healthy work practices: Implications for employees' work-related attitudes, behaviour and wellbeing

Sverke, M., Näswall, K. \& Göransson, S.

Psychologically Healthy Workplace Practices: Implications for Organisations and Employees Day, A., Randell, K. \& Kelloway, K.

\section{Aula II: Age effects in Work and Organisational Psychology: Young and Older Workers (V. Brenninkmeijer)}

Hindrances, Challenges, and Resources at Work in Relation to Psychological Well-being: Does Age Play a Significant Role?

Van den Broeck, A., Notelaers, G., Van der Heijden, B.I.J.M., De Lange, A.H., \& De Witte, $H$.

The forgotten Potential of Older Employees: Attitudes, Strategies and Well-being of Older Employees in Service Interactions

Machowski, S., Zapf, D., Ohly, S., Bernhardt, L., \& Trumpold, K.

A Self-Regulatory Perspective on Associations between Age Attitudes, Self-Categorization and Emotional Exhaustion

De Lange, A.H., \& Van der Heijden, B.I.J.M.

Fresh and Healthy? Well-being, Health, and Performance of Young Employees with Intermediate Education Akkermans, J., Brenninkmeijer, V., Blonk, R. \& Koppes, L.

\section{Aula III: Psychometrics}

The Development and Validation of the Job Crafting Scale Tims, M., Bakker, A.B., \& Derks, D. 
The development and validation of the Perceived Emotional Effort Scale

Rodríguez-Carvajal, R., Quiñones-García, C., Clarke, N., Moreno-Jiménez, B., De RivasHermosilla, S. \& Alvarez-Bejarano, A.

Measurement and evaluation of perceived stress among students: What supports in an university context?

Tavolacci, M.P., Grigioni, S., Villet, H., Dechelotte, P., \& Ladner, J.

HSE Management Standards and work-related stress: Italian translation and validation of the Indicator Tool

Natali, E., Martini, A., Ronchetti, M., Rondinone, B., \& lavicoli, S.

\section{Aula VII: Occupational Health: Policy and Practice}

Identifying the policy context for psychosocial risk management in Europe Jain, A. \& Leka, S.

EU Policies and Social Dialogue on psychosocial risks- strengths and weaknesses Ertel, M. \& Stilijanow, U.

A Study of Social Capital and Health and Safety Climate-Behaviour-Outcome Relationship in education sector

Tang, J.J., Hunt, N., Leka, S., \& McClellan, S.J.

Work-related stress: guiding employers on the law and good practice Hamilton, J.

Mainstreaming gender in interventions for work-related stress and psychosocial issue: European expert's perceptions and practices.

Hassard, J., Leka, S. \& Cox, T.

\section{Aula VIII: Workshop}

Dealing with the Impact of the "Shadow Side" of Work: An Exploratory Workshop

Gething, N. \& Wren, B

\section{9:15-10:00}

\section{Aula Magna: Keynote Address}

Living Together and well being in organisations and society Avallone, $F$. 


\section{Aula Magna: Promoting Health and Healthy Behaviours}

Now I need a burger! - Job stressors, self-control, and fast food consumption Sonnentag, S. \& Naegel, I.J.

Motives matter - a diary study on the relation between job stressors and sports activity Naegel, I. \& Sonnentag, S.

A qualitative investigation of employees' perceptions of the impact of work on health behaviours.

Payne, N., Jones, F., \& Harris, P.

The Direct and Indirect Antecedents of the Acceptance of Donation After Cardiac Death by Health Care Professionals: Psychological Concerns, Knowledge, and Engagement Peltier, J., \& Alessandro, $A$.

A daily study of effort and recovery: examining the role of time, effort and pleasure Van Hooff, M., Geurts, S., Beckers, D., \& Kompier, M.

\section{Aula II: Is Laughter the Best Medicine? Relations between Humour and Occupational Health (A.H. de Lange \& J.S.E. Dikkers)}

Validation of the 4-dimensional structure of the Humour Styles Questionnaire among a largescale employee sample

Dikkers, J.S.E., de Lange, A.H., de Witte, H., \& den Broeck, A.V.

The associations of humorous coping styles, affective states, job demands and job control with the incidence of upper respiratory tract infection'

Doosje, $S$.

The Associations of Intrinsic and Extrinsic Values with Social Support: Humour as an Explanatory Mechanism

den Broeck, A.V., de Lange, A.H., De Witte, H., Vansteenkiste, M., Dikkers, J.S.E., \& Vander Elst, $T$.

Making jokes at the costs of others? Examining enhancing or buffering effects of humour in relation to workplace bullying

Rodríguez-Muñoz, A., de Lange, A.H., Sanz-Vergel, A.I., Moreno-Jiménez, B., \& Baillien, E.

A self-regulatory perspective on the effects of humour styles in relation to burnout and work engagement

de Lange, A.H., Dikkers, J.S.E. \& Demerouti, E. 


\section{Aula III: Employee Motivation and Performance}

The role of autonomy support and work engagement in job satisfaction and proactivity: A study in the Portuguese financial industry

Cortez, C. \& Passos, A.M.

Does work engagement mediate effects of social and organizational work factors on mental health problems

Hagen, S., Grimsmo, A., Geving, G., Gudbergsson, S., \& Torp, S.

The experience of salesperson engagement: A mixed-methods qualitative investigation of engagement in context

Medhurst, A. \& Albrecht, S.

Exploring the multiple linkages between work engagement and health: An Empirical analysis within the Catalan nursing context.

Moodie, S., Dolan, S., \& Arsenault, A.

The influence of task characteristics and social support on self-rated

employee performance

Blaich, S., Noblet, A., \& Rodwell, J.

Psychophysiological and Personal Characteristics of Marine Cadets: Estimation of Professionally Essential Qualities During Final Examination on the Radar-Tracking Simulator.

Nezavitina, T. \& Vasylyeva, J.

\section{Aula VII: Psychosocial factors, work-related stress and employee wellbeing}

Depressive symptoms and school burnout at middle and late adolescence

Holopainen, L., Salmela-Aro, K., \& Savolainen, $H$.

Interaction Effects of Self-Control Demands and Cognitive Control Deficits on Burnout, Depression, Physical Strain and Daydisfunction

Hupke, M. \& Schmidt, K.H.

The Effects of Job Demand and Job Control on Perceived Stress and Motivators among Information Technology (IT) Consultants

Wallgren, L.G.

How positive psychosocial work factors may promote caring efficacy and mental health among psychiatric nurses

Torp, S., Grimsmo, A., \& Bredrup, O.J.

Perceived work strain: A comparison between models and between occupational groups

Magnavita, N., Garbarino, S., Ciprani, F., Cuomo, G., \& Bergamaschi, A. 


\section{Aula VIII: Changing World of Work: Gender and Diversity}

Does cultural diversity matter for stress of low qualified workers?

Behrendt, K. \& Busch, C.

Diversity management in northern Italy: Value or Bond?

Negri, L. \& Briante, G.

Women who work and OSH: Exploring the limitations

Gervais, R., Schneider, E., Flaspöler, E., Hauke, A., Koppisch, D., Reinert, D., Koukoulaki, T., Vilkevicius, G., Martínez-Casariego, M.A., Martínez, M.B., Lozar, L.G., Martínez, S.V., Carter, L., \& Leah, C.

Effect of Self Managing Life Crisis Programme based on the Oriental Approach towards Midlife Crisis and Well-being of Thai Married Women in Bangkok

Intarakamhang, U. \& Mohan, K.P.

Impact of Gender Identity and Marginality on Occupational Stress

Palermo, J.

Socio-demographic implications of extended working life

Weyman, A.

\section{$11: 30-11: 45$}

Refreshments

\section{1:45- 13:00}

\section{Aula Magna: Current Status and Prospects of Job Stress and Burnout Research in South Korea (K. YU)}

Overview of Job Stress and Burnout Research in South Korea: Current Status and Prospects Lee, S.M. \& Kim, A.

Job Demands and Burnout Syndrome of Private Company Workers

Yu, K. \& Choi, $H$.

Job Ambiguity and Burnout Syndrome of School Personnel

Lee, J. \& Park, Y.M.

Work Demands and Burnout Syndrome of Military Personnel Choi, B.Y. \& Jin, Y.Y.

Academic Demands and Burnout Syndrome of Middle and High School Students Park, Y.M. \& Shin, $H$. 


\section{Aula II: In Search of the X-factor: Within-Person Perspectives on Workaholism and Engagement (I. van Beek \& T. Taris)}

I would if I could and love it too: Self-efficacy and work engagement

Vera, M., le Blanc, P., Salanova, M. \& Taris, T.

Why do workaholics work long hours?

Van den Broeck, A. \& Vansteenkiste, M.

Engaged employees: The role of personal resources in the relationship between job resources and work engagement

Ouweneel, A.P.E., Le Blanc, P.M., \& Schaufeli, W.B.

The motivational bases of job-related well-being: Why do workaholic, engaged and burned-out employees work so hard?

van Beek, I., Hu, Q., Schaufeli, W.B., Taris, T.W., \& Schreurs, B.H.

Under pressure at work: The mediating role of workaholism

van Wijhe, C.I., Peeters, M.C.W., \& Schaufeli, W.B.

\section{Aula III: Promoting Resilience and Developing Wellbeing at Work (B.Wren)}

Building Psychological Resilience in Staff: Individual and Organisational Approaches Jennings, $T$.

Developing Systemic Resilience: Practical Support for Managers Hill-Tout, J.

Building Resilience In Individuals At Work - Coaching To Take Up Role Authority Allen, C.

Developing Resilience At Work - Using A Mindfulness Approach Schwartz, A.

Working with the Shadow Side - Anticipating Obstacles to Resilient Working Gething, N.

\section{Aula VII: How to Predict Work Engagement across Professions and Career Stages (K. Salmela-Aro \& B.S. Wiese)}

Strategies during University Studies predict Work Engagement

Salmela-Aro, $K$.

Work engagement as an indicator of mothers' successful re-entry into working life Wiese, B.S. \& Dalit Jäckel 
How is one partner's career self-efficacy related to the other partner's work engagement?

Neff, A., Niessen, C., Sonnentag, S., \& Unger, D.

When Do Senior Managers Experience Engagement?

Aro, A.

\section{Aula VIII: European Survey of Enterprises on New \& Emerging Risks (ESENER)}

(S. Iavicoli) by invitation only

European Survey of Enterprises on New and Emerging Risks (ESENER): How is occupational safety and health managed in European workplaces?

Rial-Gonzalez, E., Cockburn, W. \& Irastorza, X.

European Survey of Enterprises on New \& Emerging Risks: Key findings on psychosocial risk management at the workplace

Leka, S., Jain, A., Houtman, I., Bakhuys-Roozeboom, M., Ertel, M., Pech, P. \& Riedmann, A.

13:00 - 13:45

\section{Magna Aula: Taylor \& Francis Keynote Address}

Money, Models, and Mental Health: Implications of Economic Stress for Occupational Health Psychology

Sinclair, R.R

\section{3:45-15:15}

\section{AULA VIII: EAOHP Professional Forum}

Lunch \& Poster Session

\section{Diversity and Occupational Health}

Multicentric italian study on gender work related stress among health workers in rehabilitation hospital departments: assessment and management.

d'Ettorre, G., Pati, C., Mazzotta, M. \& Russo, L.

Exploring Racial and Ethnic Differences in Exposure to Work Stressors Roberts, $R$.

Does Diversity Drive Employees Crazy? The Relationship between Organizational Demographics and Employee Mental Health

Waite, E., \& Avery, D.R. 


\section{Physical Health}

Psychological and social factors at work in relation to musculoskeletal disorders among employees in the Norwegian meat process industry

Elka, S., Christensen, J.O., Emberland, J.S., Vindsetmo, K., Pettersen, T., Aune, M., \& Lau, B.

Relationship between cognitive-behavioral treatment and cortisol. A longitudinal study.

Figueiredo-Ferraz, H., Gil-Monte, R., \& Ribeiro do Couto, B.

Job attendance in spite of musculoskeletal pain - a qualitative study on the role of work-related sense of coherence

Geving, G., Torp, S., Hagen, S., \& Vinje, $H$.

Psychogenic mass syndrome in anti-cancer drug exposure

Magnavita, N., lavicoli, I., Leso, V., \& Bergamaschi, A.

The influence of 'significant others' on back pain disability and return-to-work: a qualitative pilot study of illness perceptions.

McCluskey, S., Brooks, J., Burton, K., \& King, N.

Why do some people have no back pain? Subjective characteristics of a middle-aged working population compared to a population-based cohort

Rolli Salathé, C., Melloh, M., Isalue, I., Semmer, N.K., \& Elfering A.

\section{Safety Climate}

Psychological Capital and Safety Climate: A study of Norwegian Air Traffic Controllers Bergheim, K., Hystad, S.W., \& Eid, J.

The Effects of Task Interdependence, Workgroup Efficiency, and Perceived Supervisor Support on Safe Social Status: A Moderated Mediation

Golay, L., Reeves, D.W., Magley, V.J., \& Gilson, L.L.

Promoting Safety Performance

Jones, C., Cox, T., \& Griffiths, A.

Safety Climate as Moderator on the Relationship between Risk Perception and Job-satisfaction Nielsen, M.B., Mearns, K., Matthiesen, S.B., \& Eid, J.

Strategies of moral disengagement and attitude toward risk. Antecedents of the violation of safety rules at work

Palano, F., Cardellicchio, E., \& Ingusci, E.

Correlation between psychosocial factors and work accidents

Picchi, M.P.C. \& Erba, A.M.

Safe workplace design: psychological risk factors

Tint, P., Tkatsova, L., Reinhold, K., \& Tuulik, V. 
Positive Organizational Behaviour and Safety Science: Conceptual Issues and Future Research Questions

Westli, H.K., Eid, J., Mearns, K., Larsson, G., Laberg, J.C., Johnsen, B.H., Nielsen, M.B., Satrevik, B. \& Bergheim, $K$.

\section{Health Promotion in and out of the Workplace}

Quality of Life in subjects with Spinal Cord Injury and their Informal Caregivers Machado, F., Teixeira, A., Alves, J.

Stress, Alcohol and Drug Addiction in workers employed in the building of the great infrastructures

Carpentiero, L., Costa, G., De Luca, G., Piovanelli, S., Vene, D., Righini, S., Marini, M., Baldanzi, S., Selvi, A., Scalas, S., Cassitto, M.G., Sartori, S., Lacangellera, D., \& Bosco, A.

Participation in everyday activities - a comparison of people on sick leave and a reference group from the Swedish population

Eriksson, T., Jonsson, H., Tham, K. \& Eriksson, G.

What is Quality of Work Life? A Research Based on the Workers' Point of View of a Brazilian Public Company

Ferreira, M.C., Ferreira, R.R. \& Seidl, J.

Democratic Participation in NPO as a Factor of Organizational Wellbeing. First Research Results between Italy and Mexico.

Hindrichs, I. \& Converso, D.

Job insecurity, employability and wellbeing: Evidence from an Italian study

Manuti, A., Rosa Pia, F., \& Mariangela, M.

Work-related stress in the Health-Care sector. An evaluation

Raffaele, G., Cataldo, A., Nazzicone, F., \& Di Cecio, M.

Psychological and symptomatic stress-related disorders with Radio Electric Asymmetric Conveyer

Rinaldi, S., Fontani, V., Aravagli , L., Bini, S., Mannu, P., Castagna, A., \& Margotti, M.L.

Complementation and compensation effects of psychological and subjective well-being on their predictive capacity o positive and negative human functioning.

Rodriguez-Carvajal, R., de Rivas, S., Moreno-Jiménez, B., Blanco, A., \& Alvarez, A.

\section{Occupational health interventions}

Worksite health promotion program for physicians: the assessment and management of shortterm stress

Berezovskaya, R. \& Shabelnik, O. 
The Effectiveness of Psychological characteristic and Skill Training on Teacher Supervision for Enhancing Responsibility among Early Adolescent Students

Boonprakob, P., Boonprakob, M., Intarakamhang, U., \& Thongpukdee, T.

Psychopathological screening and assessment of psychological discomfort in a sample of Italian teachers.

Borrelli, I., Bergamaschi, A., di Giannantonio, M., \& Pozzi, G.

Risk stress work-correlated: the results about 6000 Italian workers

Bertana, E., Bisio, C., Menighini, A., Muratore, M., Nocera, A., \& Riva, P.

Good Governance of Thai Small and Medium Sized Enterprises.

Chongvisal, $R$.

Reference regulation and good practices in the assessment of psychosocial risk factors in Italy. Deitinger. P., Nardella, C., Bentivenga, R., Ghelli, M., Persechino, B., \& Iavicoli, S

Work related stress evaluation: two cases studied

Dusi, P. \& Servadio, M.

Stress and coping of unemployed before and after interaction with job coaches Eisele, $P$.

Results of a psycho - educative intervention about the stress symptoms, stressors at work and metabolic indicators in nurses (male and female) of attention of third level

Enriquez Hernandez, C. B., Colunga Rodriguez, C., Preciado Serrano Maria del, L., Angel Gonzalez, M., \& Dominguez Diaz, $R$.

Structure matrix of work related stress based on "Framework agreement on work-related stress" of October 8th 2004.

Erba, A.M. \& Carlo Picchi, M.P.

Some remarks about the function of ergonomics when facing psychosocial risks: an interactive approach aggregating clinical, organizational and activity-centered conceptions of field interventions

Favaro, $M$.

Assessment of Occupational Stress among shopping centre employees.

Fenga, C., Di Nola, C., Cacciola, A., \& Nardella, C.

Well-being Survey in an Italian Research Institute

Ferravante, D.G. \& Gloriani, I.

Quality of Work Life Assessment of a Brazilian Public Company

Ferreira, M.C., Ferreira, R.R. \& Seidl, J.

A quali-quantitative approach for the survey of job stress and the Organizational Development in the organizations

Gattai, A., Scatolini, E., Magnani, M., Mancini, G.A., Majer, V., \& Marocci, G. 
Knowledge Management Systems for promoting Healthy Organizations

Isla-Diaz, R., Hernandez-Fernaud, E., Diaz-Cabrera, D., Ramos-Sapena, Y., \& Casenave, S.

Longitudinal effects in the treatment of occupational stress: Controlled evaluation of an inpatient group-therapy program (7-years follow-up)

Koch, S., Schaaf, V., \& Hillert, A.

Psychosocial risk in finance and banking sector: active participation as a prevention instrument. Application of a multivariate analysis technique (Multiple Correspondence Analysis - MCA).

Macciocu, L., Rossi, I, Nardella, C., Ferrante, P., Buresti, G., \& Iavicoli, S.

Stress prevention for better health, better health to reduce the social costs

Magnani, M., Mancini, G.A., Scatolini, E., Gattai, A., Marocci, G., \& Majer, V.

The assessment of workaholism as a work-life imbalance: "Time Usage Inventory" development and preliminary empirical testing

Malinowska, D., Tokarz, A.\& Jochymek, S.

Preliminary study on the welfare of a community Nosocomial of the province of Rome conversational approach and methodology

Messineo, A., Panunzi, M., Visconti, S., \& Ciconte, A.R.

Stress Management Interventions in Polish Companies

Molek-Winiarska, D.

Non-formal learning: proposal for an occupational health and safety-oriented approach

Nardella, C. Pizzuti, A., \& Deitinger, $P$.

Development of Outstanding Students from the point of view the work of their teachers and companions

Soltero Avelar, R. \& Margarita Torres, T.

Stress management in law enforcement: An integrative perspective

Turc, D. \& TurC, M.

Experimental manipulation of organizational change processes: Effects on Healthy Change Process Index, psychosocial work environment, and stress

Tvedt, S.D.

Experimentally manipulated organizational change: Importance of healthy processes and change resilience for engagement, commitment, stress, and health complaints

Tvedt, S. D.

Flexible Working Time as a Tool to Manage Work-Related Stress and Workplace Bullying for Polish Clerical Workers

Zolnierczyk-Zreda, D. \& Warszewska-Makuch, M. 


\section{5:15-16:45}

\section{Aula Magna: Overtime, Working Hours and Time Pressure}

The long arm of work: Time pressure at work, cognitive failure and commuting accidents Elfering, A., Grebner, S., \& De Tribolet, F.

Darling, it's gonna be late tonight: The relationship of work time on relationship quality Unger, D., Niessen, C., Sonnentag, S., \& Neff, A.

Associations between Overtime and Psychological Well-Being among Chinese Workers Houdmont, J., Hassard, J., \& Zhou, J.

Workplace Conflicts and Employee Well-Being: The Moderating Role of Detachment from Work during Off-job Time

Sonnentag, S., Naegel, I.J. \& Unger, D.

Relaxation or Psychological Detachment? A Diary Study on Recovery among Workaholics Xanthopoulou, D. \& Bakker, A.B.

\section{Aula II: Employee Retention and Sickness Absence}

The Relationship between Skills Training and Retention of Graduate Interns in a South African Information, Communication and Technology Company

Barkhuizen, N. \& Pop, C.

Effectiveness of an empowerment based job retention program for employees with a chronic disease; a randomised controlled trial

Varekamp, I., \& van Dijk, F.

Key Factors for Attracting and Motivating Young People to Work as Employees in Swedish Livestock Farming

Kolstrup, C.

Sustainability among managers in a public health care organisation: a prospective study Skagert, K., Ahlborg, G., \& Dellve, L.

Long sickness absence after depressive disorders - Results from the Finnish Health 2000 Study

Ahola, K., Virtanen, M., Honkonen, T., Isometsa, E.,\& Lonnqvist, J.

\section{Aula III: Traumatic Events and PTSD: impact, Management and Prevention}

Adjustment not PTSD, the real effect of War on Reservist soldiers!

Fairweather, L., Gibbes, C., \& Ridley, D. 
Characteristics of Emergency Nurses Experiencing Traumatic Stress and a Change in Work Productivity

Gillespie, G., Gates, D., \& Succop, P.

Work critical incidents and PTSD: The role of group cohesion

Goncalves, S., Neves, J., \& Morin, E.

The relationship between potentially traumatic experiences, generic workplace stressors and mental health within a large UK police force.

Kerr, R. \& McHugh, M.

The Incidence of Secondary Traumatic Stress in workers dealing with traumatising materials, victims and perpetrators

Tehrani, N.

\section{Aula VII: Psychosocial Risk Management}

Institutional perspectives on psychosocial risk management in Danish elderly care centers

Simonsen, J.S.

Managing Psychosocial Risks for Homeworkers

O'Hara, $R$.

Framing a new proposal for the Psychosocial risk assessment from Europe to Italian context: the Va.RP model

Deitinger, P., Nardella, C., Bonafede, M., \& Aiello, A.

Stress risk assessment in 20 Irish organisations 2008-2009

Murray, $P$.

Assessing occupational hazards in the Ghanaian mining industry and their implications for employee well-being and quality of life

Amponsah-Tawiah, K., Stavroula, L., Cox, T., \& Jain, A.

Utilising the HSE Management Standards approach to predict wellbeing in UK academics: the role of job-specific hazards

Kinman, G.

\section{Aula VIII: Organisational Citizenship, Justice and Culture}

Is Moral Disengagement a moderator within the Stressor-Emotion model of Counterproductive Work Behaviour?

Natali, E., Fida, R., Mamazza, L., \& Barbaranelli, C.

Good citizens are healthy citizens? OCB and health-related attitudes and behaviours

Schusterschitz, C., Stummer, H., Noehammer, E., \& Geser, W. 
Task performance and organizational citizenship behaviour among Chinese nurses: The moderating effects of emotional exhaustion and organizational support.

Tourigny, L., Baba, V.V., Han, J., \& Wang, X.

The work-stress relationship: Examining the independent contribution of organizational justice Noblet, A., Rodwell, J., Demir, D \& Steane, P.

Satisfaction with work-family balance, organizational culture, and stress reactions: Results from an academic institution

Omori, $M$.

\section{6:45-17:00}

Refreshments

\section{7:00-18:30}

\section{Aula Magna: Organisational Development and Change}

The importance of change content: A study of different change contents in the Norwegian food processing industry

Tvedt, S.D.

Workplace changes among Norwegian employees - does information and participation during the change process affect health and well-being?

Sterud, T.

Does the success of change management effect employees' well-being?

Pahkin, K., Leppänen, A., Ala-Laurinaho, A., \& Kajosaari, K.

Chronic Work Conditions as Antecedents of Employees: Anticipated Stress to a Hypothetical Scenario of Organizational Change

Bosak, J., Kirchner, A., Sczesny, S., \& Meiser, L.

Connections of Change Experiences, Work Engagement, and Other Indicators of Work Related Well-Being During Restructuring

Leppänen, A., Pahkin, K., Ala-Laurinaho, A., \& Kajosaari, K.

Qualitative investigation of the relationships between staff health and change management processes in the UK National Health Service

Parkin, T. \& Loretto, W.

\section{Aula II: Understanding Individual Differences and Coping}

Can civil pilots' Personality traits predict their stress, coping types, and job performance? Cherng, C.F.G. 
Are there personal subtypes of company care drivers?

Musicant, $O$.

Adult attachment styles and health-related work attitudes and behaviors

Schusterschitz, C., Stummer, H., Noehammer, E., \& Geser, W.

Humour Style in Relationship to Stress and Well-being: Four Different Personalities

Stokenberga, $L$.

Does starting the day with negative affective appraisal in the morning hinders recovery in the evening? The moderating role of employees perceived job decision latitude at an international airport's hub section

Michel, A., Turgut, S., \& Sonntag, K.

Emotional competencies, resilience and wellbeing in trainee social workers

Kinman, G. \& Grant, L.

\section{Aula III: Employee Engagement, Commitment, and Performance}

The mediating role of work engagement between job characteristics, and positive and negative non-task behaviours

Sulea, C., Maricutoiu, L.P., Virga, D., Schaufeli, W., Dumitru, C.Z.

A dynamic test of job engagement: personal demands, personal resources, and job resources as predictors

Barbier, M., Demerouti, E., \& Hansez, I.

The Relationship between Barriers to Change and Work Engagement of Employees in a South African Property Management Company

Barkhuizen, N. \& Bell, E.

Employee engagement as a mechanism by which organisational climate influences employee job satisfaction and well-being.

Albrecht, $S$.

Committed employees are healthy employees? Employee commitment and health relevant work attitudes and behaviours

Schusterschitz, C., Geser, W., Noehammer, E., \& Stummer, H.

Teachers attributions of the performance appraisal system: implications on well-being Ernesto, F. \& Chambel, M.J.

\section{Aula VII: Wellbeing and Job Satisfaction}

Does Nurse Prescribing Increase Job Satisfaction?

Cousins, R. \& Donnell, C. 
Stress vulnerability, quality of life and well-being in pre-Hospital medical emergency professionals

Cunha, M., Salazar, G., \& Pereira, J.P.

A Hierarchical model of the internal relationship marketing approach to nurse satisfaction and loyalty

Peltier, J, Schibrowsky, J.A., \& Nill, A.

Non- working individuals in Norway, their latest occupation and self- reported health Aagestad, C., Sterud, T., \& Tynes, T.

Return to Work and Workrelated Psychological Complaints

Blonk, R., Oomens, S., \& Huijs, J.

Enterprise bargaining, workers' job satisfaction and union-member relationship: An Australian retail study

Kang, S., Dollard, M.F., Winefield, A.H., Provis C., Black, Q.C., \& Black C.

\section{Aula VIII: Mental Health in the Workplace}

Anxiety and fatigue in on-call workers

Earle, F., Reid, T., \& Clough, P.

Depression at work: The moderating role of depressive symptoms on the association between information flow and work outcomes

ter Hoeven, C., ten Brummelhuis, L.L., \& Peper, B.

Socioeconomic position and common mental disorders in a working age population

Pulkki-Raback, L., Virtanen, M., Ahola, K., Elovainio, M, \& Kivimaki, M.

Job position and mental health: Do Jahodas latent functions matter?

Selenko, E., Batinic, B., Stiglbauer, B., \& Paul, K.

A study of personal and collective efficacy at work in an individualistic culture Torkelson, E.

Predicting Psychological Health at Work among Teachers: Testing a Mediation Model with Social-Organizational, Job-Related, and Individual Predictors

Boudrias, J., Desrumaux, P., Brunet, L., Savoie, A., \& Nelson, $K$.

\section{Secretariat: ICG meeting (closed meeting)}




\section{Wednesday 31 March 2010}

\section{8:00-09:15}

\section{Aula Magna: Occupational Health Climate: Why Does it Matter? (J.A. Lunt)}

Factors influencing choice and implementation of respiratory programmes in the workplace Bell N. \& Vaughan, N.

What influences employers to take noise risks seriously?

Bell N.

The perception of risk, job satisfaction and occupational accidents within a healthy work climate

Gervais, R.L., Weyman, A. \& Williamson, J.

\section{Aula II: How to Find a Balance and Cope with Multiple Roles of Work, Family and Private Life? (S. Mauno \& J. Rantanen)}

Work-family balance and its relations to job and personal characteristics and well-being: A person-oriented approach

Rantanen, J., Kinnunen, U. \& Mauno, S.

Work-Work-Life Balance: Burdens and Chances of Multiple Jobholding and Portfolio Careers

Olos, L.

Work-family conflict: Who is at risk?

Richter, A. \& Näswall, K.

Do work-family coping strategies facilitate successful reconciliation of work and family under high job and family demands?

Mauno, S. Rantanen, M., Rantanen, J., \& Kinnunen, U.

Patterns of coping with multiple roles and their impact on work-family enrichment and conflict Matias, M. \& Fontaine, A.M.

\section{Aula III: Academic Work Demands and Resources and Higher Education Students' Wellbeing (A.M. Pinto)}

The impact of the Bolonha process on student's well-being: A comparison of pre and post Bolonha students on their perceptions of work demands, control, peer support and well-being. Pinto, A.M. 
Volunteering and higher education students' well-being and academic performance Pinto, A.M. \& Figueira, C.

Higher education students' representations of factors associated to burnout and engagement

Figueira, C., Chambel, M.J., Pinto, A.M., Curral, L. \& Castanheira, F.

Academic demands and resources`impact on higher education students ' burnout and engagement: An application of the Job Demands-Resources model

Castanheira, F., Pinto, A.M., Chambel, M.J., Curral, L., \& Figueira, C.

\section{Aula VII: Promoting and Understanding Safety in Organizations}

The Antecedents of safety-related helpful behaviours and its impact on occupational safety performance

Krause-Juettler, G.

Development of a survey for evaluating expert work in safety critical organizations

Pahkin, K., Leppänen, A., Mäki, E., Kuronen-Mattila, T., \& Järvenpää, E.

Risk perception in the health care domain, between clinical risk and practitioners safety: a study among operating theatres practitioners

Bruno, A., Bracco, F., Chiorr, C., Pugliese, F., Sossai, D., \& Palombo, P.

Perceived Risk and Employees' and Supervisors' Participation on Work Safety

Hinrichs, $S$.

\section{Aula VIII: Delivering and Evaluating Training Programmes}

Does training improve participant well-being? The mediated moderation of work environment factors that influence the effects of training

Nielsen, K. \& Daniels, K.

Mindfulness in Dentistry and Dental Hygiene

Durepos, D., Catano, V., \& Lovas, J.G.L.

Developing on-line learning materials to support managers in the prevention and reduction of stress in the workplace

Donaldson-Feilder, E., Lewis, R., \& Yarker, J.

Determinates of Successful, Culturally Competent Disability Service Providers

Dimpfl, S., Taylor-Ritzler, T., Balcazar, F., Oberoi, A., \& Willis, C.

\section{Secretariat: ICG-OHP meeting and EAOHP Education Forum}




\section{9:15-10:45}

\section{Aula Magna: Leadership and OSH}

How is the "Swedish management model" related to organisational effectiveness?

Muhonen, $T$. \& Jonsson, $S$.

Ethical leadership: Authenticity and responsibility

Cherre, $B$.

Leadership and health - Conceptual consideration and empirical evidence

Stummer, H., Noehammer, E., \& Schusterschitz, C.

Do transformational leaders: Enhance their followers daily work engagement

Tims, M., Bakker, A.B., \& Xanthopoulou D.

Work-Family Conflict and Supervisor Support: The Role of Perceived Similarity

Waite, E., Rubino, C., \& Avery, D.R.

\section{Aula II: Recovery processes: Determinants and Effects on Health, Wellbeing, and Workability (J. de Bloom)}

Job characteristics and recovery experiences: testing causal relationships across one year Kinnunen, U. \& Feldt, T

The Moderating Role of Self-Esteem on the Effect of Effort-Reward Imbalance on Recovery - A Diary Study

Meier, L.L. \& Semmer, N.K.

Physiological recovery from stress. Does distraction matter?

Radstaak, M., Geurts, S.A.E., Brosschot, J., Sonnentag, S. \& Kompier, M.

Promoting the prerequisites for recovery through information, counselling and advising activities in the workplace survey process

Kivistö, S., Kallio, E. \& Jalonen, P.

Effects of vacation from work on health and well-being: Lots of fun, quickly gone de Bloom, J., Geurts, S.A.E., Taris, T.W., Sonnentag, S., de Weerth, C. \& Kompier,

Vocationally oriented medical rehabilitation increases well-being at work via increased recovery experiences

Tirkkonen, M. \& Kinnunen, $U$ 


\section{Aula III: Addressing and Understanding Workplace Violence and Bullying: Approaches and Evidence}

Preventing post-traumatic stress following workplace robbery: procedure and preliminary results of a Psychological Debriefing Program for bank employees

Fichera, G.P., Musti, M., Coggiola, M., Russignaga, D., \& Costa, G.

Violence prevention in a psychiatric unit

Magnavita, $\mathrm{N}$.

Are role stressors and workaholism related to abusive behavior at work? The mediating role of workplace bullying

Balducci, C., Schaufeli, W., \& Fraccaroli, F.

An Analysis Of Health Care Employees Experiences Of Mobbing Behaviours At Workplace; A Case Study Of Southern Marmara Region In Turkey

Palaz, S. \& Ozkan, S.

Finish peace keepers psychosocial well being after the home coming

Leskinen, J., Isosomppi, M., \& Nyman, M.

\section{Aula VII: Safety Climate and Behaviour}

The effect of risk perception and optimistic bias on safety climate attitudes and behaviour Walker, A. \& Pearce, M.

The Safe Mindset of Managers, Shift Bosses and Miners of a South African Platinum Mine Barkhuizen, N., Van Rensburg, N.J. \& Stanz, K.

Learning occupational safety and health on the job - how effective is it? Masuhr, K. \& Wetzstein, A.

Presenteeism and occupational accidents: A correlation waiting to happen

Gervais, R., Weyman, A., \& Williamson, J.

Behavioural Safety Approach as a Method of Accident Prevention in Oil and Gas Industry in Nigeria

Kadiri, $S$.

Aula VIII: EU-OSHA: The Way Forward (E. Rial-Gonzalez) by invitation only

10:45-11:00

Refreshments 


\section{1:00-11:45}

\section{Aula Magna: Keynote Address}

Stress and health: the role of working time Costa, G.

\section{1:45-13:15}

\section{Aula Magna: Job Insecurity in Europe: Some New Insights (H. De Witte, \& N. De Cuyper)}

The Job Insecurity Scale: A psychometric evaluation across six European countries Vander Elst, T., De Witte, H. \& De Cuyper, N.

Job Insecurity: Who is at Risk?

Richter, A., Näswall, K. \& Sverke, M.

Job Insecurity and Withdrawal: Different Paths for Different Age Groups?

Stynen, D., Sels, L., Forrier, A., Vander Elst, T., Van den Broeck, A. \& De Witte, H.

Praying for Security: Can Religiosity Offset the Negative Health Effects of Job Insecurity?

Schreurs, B., van den Heuvel, M., van Emmerik, H., De Cuyper, N. \& Demerouti, E.

Associations between Job Insecurity and Workplace Bullying: a two-wave longitudinal test Baillien, E., De Witte, H. \& De Cuyper, N.

\section{Aula II: Worker's Physical Health and Functioning: Causes and Prevention}

The influence of psychosocial workplace factors on the development of musculoskeletal problems: A meta-analysis of longitudinal studies

Lang, J., Lang, P.J.W.B., Kraus, T., \& Ochsmann, E.

The project PAKT - Prevention of Back Pain in German Waste Management Companies Pangert, C., Herold, K., \& Lemke, P.

Psychological Factors, age, and the onset of Cardiovascular Disease De Lange, C.

Job strain and cardiovascular risk factors:- Does social support have an attenuating effect for an ageing Irish workforce

McCarthy, V., Perry, I.V., \& Greiner, B.A.

The interaction between perceived job stress and rumination predicts long-term evening saliva cortisol secretion

Rydstedt, L.W. \& Cropley, M. 
Skin disorders and work strain in Hospital workers

Magnavita, N. \& Bergamaschi, A.

\section{Aula III: Burnout: Antecedents and Consequences}

Is the interpersonal component of burnout still relevant in health care settings?

Consiglio, C., Borgogni, L., \& Ferrillo, M.

Managers' Health - The Case of Person Related Burnout

Hytter, A.

The scientific evidence on occupational health: A meta-analysis on burnout

Palitti, T., Palermo, P., Tomei, G., Cinti, M.E., Rosati, M.V., Scimitto, L., Fiaschetti, M., Schifano, M.P., Panfili, A., Cetica, C., Tomao, E., Fioravanti, M., Tomei, F., \& Sancini, A.

Job dimensions, personal goal facilitation and burnout: a longitudinal analysis.

Pisanti, R., van der Doef, M.P., Maes, S., Violani, C., \& Lazzari, D.

Early-career burnout among nursing professionals: A three-year longitudinal observational study

Rudman, A. \& Gustavsson, P.

Work Stress, Burnout, and Health in Chinese Working men: Mediation Analysis

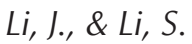

\section{Aula VII: OSH and Leadership}

The Study of Leadership and New Direction for Developing Entrepreneurs in Thai Small and Medium Sized Enterprises

Chongvisal, $R$.

Daily Inspirational Leadership and its Relation to Daily Engagement, Active Learning, and Performance

Hetland, H., Bakker, A., Hetland, J., \& Demerouti, E.

Fulfillment of Psychological Needs as a Mediator in the Relationship between Transformational Leadership and Job Satisfaction

Hetland, J., Hetland, H., Bakker, A.B., Demerouti, E., Andreassen, C.S., \& Pallesen, S.

Nursing leadership style and mental health outcome of nurses in Taiwan Lin, P.Y., Hunt, N., \& MacLennan, S.J.

Exploring the relationship between managers' leadership and their health Lundqvist, D., Eriksson, A.F., \& Ekberg, K. 


\section{Aula VIII: Finding a Balance? The Impact of Work-Life Conflict}

Work-family conflict in the UK offshore oil and gas industry: A qualitative study

Guise, J. \& Cowan, S.

Attitudes to On-call Working and Impact on Family Life

Reid, T., Earle, F., \& Clough, P.

Work place and couple relationship: how are they related?

Vintila, M., Goian, C. \& Cintar, D.

Work-family and family-work conflict and enrichment; relationships with healthy eating in working mothers

Jones, F., \& Grzywacz, J.

Work-life imbalance and effort-reward imbalance as predictors of burnout

Hämmig, O., Brauchli, R., Knecht, M., Bauer, G.

Work-life conflict and health among Swiss physicians - in comparison with other university graduates and with the general Swiss working population

Knecht, M., Bauer, G., Klaghofer, R., Buddeberg-Fischer, B., Samm, M., \& Hämmig, O.

\section{3:15-14:45}

\section{Secretariat: EAOHP Executive Committee Meeting}

Lunch \& Poster Session

\section{Psychometrics}

An evaluation of work-related stress through the integration of objective and subjective measures: the V.I.S. method and the Q-Bo test

Sarto, F., Falco, A., Vianello, L., Corso, L.D., Zanella, D., Magosso, D., Marcuzzo, G., Bartolucci, G.B., De Carol, N.A.

Psychometric Properties of the "Spanish Burnout Inventory" in German Professionals: Preliminary Results

Bosle, A., Gil-Monte, P.R., \& Figueiredo-Ferraz, $H$.

Using the Questionnaire on Experience and Assessment of Work with the Italian population Pace, F., Civilleri, A., Foddai, E., Lo Cascio, V., Passalacqua, C., \& Zanca, M.A.

Confirmatory Factor Analysis of the Toronto Alexithymia Scale among a sample of nurses. Pisanti, R., Violani, C., \& Lazzari, D.

Psychometric analyses of the short-form Utrecht Work Engagement Scale (UWES 9) in Italian Health Care Workers.

Pisanti, R., Violani, C., \& Lazzari, D. 
Psychometric evaluation of radio electric auricular treatment for stress-related disorders: A double-blinded, placebo-controlled controlled pilot study

Rinaldi, S., Fontani, V., Aravagli , L., Bini, S., Mannu, P., Castagna, A., \& Margotti, M.L.

Job Stress Battery: Scales of Job Control and Occupational Outcome Variables

Sertel Berk, H.O., Ozalp Turetgen, I, Unsal, P., \& Basbug, G.

Job Stress Battery: The Job Stress Scale

Unsal, P., Ozalp Turetgen, I, Sertel Berk, H.O., \& Basbug, G.

Antecedents and theory of Organizational Well-being. Variables to measure well-being at work

Trombetta, M. \& Franco, M.

Criterion-related and incremental validity of the Civility Norms Questionnaire - Brief Walsh, B. \& Magley, V.

Exploring nurses' experiences of older adult care: A qualitative pilot study

Watts, J. \& Robertson, N.

Challenge Stressors and work attitudes: Self-esteem tips the scales

Widmer, P., Gertsch, D., \& Semmer, N.

\section{The Psychosocial Working Environment}

Literature Review: Psychosocial Aspects of Injury-Related Occupational Leave and Implications for the Military Context

Lee, J.E.C.

Life After Service Study (LASS): How are Canadian Forces Members doing alter Transition to Civilian Life?

Sudom, K., \& Lee, J.E.C.

A Study Investigating if Emotional Labour and Other work strains impact on wellbeing in Cabin Crew

Al Serkal, A.

The Relationship between Perceived Compensation, Organisational Commitment and Job Satisfaction: The Case of Expatriates in South Africa

Barkhuizen, N. \& Sage, N.

Shift work and health: How personality and circadian typologies affects the relationship between shift work and mental health

Berthelsen, M., Ljoså, C.H., \& Lau, B.

Factors important for a good self-rated health last year in higher education - a nationwide longitudinal study among Swedish nursing students.

Bruce, M., Gustavsson, P.J. \& Omne-Pontén, M. 
Predictors and Mediators of Psychosocial Wellbeing and Discomfort across Different Occupational Sectors. First Research Results.

Converso, D., Gattino, S., Loera, B., Viotti, S., \& Hindrichs, I.

Cultural enablers and barriers for promoting organizational health and safety in aviation companies

Diaz-Cabrera, D., Hernandez-Fernaud, E., Ramos-Sapena, Y., \& Casenave, $S$.

Working in a call centre: the role of personal and organizational resources on positive and negative emotions at work

Emanuel, F., Ricotta, S., Ghislieri, C., Gatti, P., \& Colombo, L.

Job insecurity, job predictability and mental health: a prospective study of Norwegian employees

Finne, L.B. \& Lau, B.

Strike experiences and the post-strike workplace: The strikers' perspective

Francis, L., Kelloway, K., Donohoe, M. Catano, V.M., \& Gilin, D.

Solutions to university faculty work overload: A study of job sharing

Freeman, B. \& Coll, $K$.

Stress factors in the medical profession. Measure of the imbalance in assistance relationships. TEEM questionnaire

Gascon, S., Pereira, J.P., Cunha, M.J., Santed, M.A., \& Martinez-Jarreta, B.

Comparative analysis of stress factors and their influences on employees in a Romanian telecommunication company

Goian, C. \& Vintila, M.

Stress sources in police officers

Goncalves, S., Neves, J., \& Morin, E.

Longitudinal study with Job Demands-Resources Model in police context: Ongoing study Goncalves, S., Neves, J., \& Morin, E.

Analysis of a Subjective Mental Workload Scale and the influence of mental work load in occupational health

Hernandez-Fernaud, E., Diaz-Cabrera, D., \& Rolo-Gonzalez, G.

Does ethical organizational culture associate with managers' occupational well-being?

Huhtala, M., Kaptein, M., Mauno, S., Hyvonen, K., \& Feldt, T.

Nanotechnologies at the Workplaces: Social Impact and Risk Perception

lavicoli, S., Boccuni, F., \& Ronchetti, M.

Assessing impact of work organization on nursing: migration, outsourcing and early retirement lavicoli, S., Boccuni, F., Natali, E., Petyx, C., Ronchetti, M., \& Valenti, A. 
Embitterment at Work and Withdrawal

Jacobshagen, N., Rutz, J., Elfering, A., \& Semmer, N.K.

Risks and hazards affecting Health \& Safety Advisors

Leitão Alexandre, S. \& Greiner, B.A.

The Hazardous Physician

Magnavita, $N$.

Promote organizational wellness through the reduction of constraints and the development of organizational resilience

Maisetti, M., Bonzi, F., Fattori, M., \& Camicia, B.

Assessing the Role of Climate for Mentoring and Distributive Justice in the Relationship Between Perceived Organizational Support and Job Satisfaction: A Moderated Mediation

Marmet, M., Magley, V., \& Gilson, L.

It's good to talk: exploring social support and well-being at work

Parkin, T.

Positive and negative organizational behaviour

Patlan-Perez, J. \& Zorrilla, D.M.N.

Work Contexts and their effects at an individual level

Deitinger, P., Nardella, C., Bentivenga, R., Ghelli, M., \& Bonafede, M.

Evaluation of the work-stress correlation in industrial oil refining operators

Riso, F., De Luca, Annamaria, Barresi, G., Tanzariello, M.G., Velocci, S., Miceli, L., \& Abbate, C.

Influence of workload in emergency on discrimination against patients

Schoenenberger, S., Moulin, P., \& Brangier, E.

Job insecurity, intention to quit, and psychological well-being: Analysing causal relations.

Stiglbauer, B., Selenko, E., \& Batinic, B.

Job Demands-Control-Support Model and Employee Safety Performance

Turner, N., Stride, C. B., Carroll, A. E., McCaughey, D., \& Carter, A. J.

Communication in emergency ambulance setting: observation coding scheme development.

Upenieks, $R$.

Workplace mistreatment and perceived support: Different perpetrators and differential relationships

Walsh, B. \& Magley, V.

Impact of exposure to critical incidents in health and psychological well-being of Emergency Ambulance Personnel

Marcelino, D., Figueiras, J., \& Claudino, A. 
Job-related Stress: a validation study

Tanucci, G., Cristina, E., Palano, F.

\section{Workplace Violence and Bullying}

Moral Agency and Workplace Aggression

Bozeman, J. \& Hershcovis, S.

Organizational justice as predictor of incivility and incivility and verbal abuse at the workplace: The role of recovery experiences.

Diaz-Gracia, L., Garrosa, E., Diaz, L., Moreno- Jiménez, B., \& Rodriguez-Muñoz, A.

Workplace bullying and personal and organizational well-being

Fida, R., Natali, E., Mamazza, L., \& Barbaranelli, C.

Post-traumatic stress symptoms and burnout in doctors and nurses suffering aggressions

Gascon, S., Pereira, J.P., Cunha, M.J., Santed, M.A., \& Martinez-Jarreta, B.

Workplace violence in health care workers

Magnavita, N.

Becoming Person's Values - Protection against violence

Romanyuk, L. \& Surkov, P.

The bullying at work in the public administration

Rossi, De P.

Psychosocial work factors and bullying among employees in Norwegian nursing homes.

Schøning, M., Berthelsen, M., Christensen, J.O., Elka, S., Finne, L.B., Jacobsen, K., Pettersen, T., Utaaker, E., Vindsetmo, K., \& Lau, B.

Verbal Aggression in Workplace Authority Structure: An Experimental Study

Tsang, F. T. \& Chang, Y.W.

Workplace violence against health workers in Turkey

Yildiz, S., Aytac, S., \& Bayram, N.

The experience of work engagement: Examining cross-national differences in hardy personality, optimism and subjective wellbeing among nurses from China and Spain

Carmona, I., Garrosa, E., Ladstätter, F., Moreno- Jiménez, B., \& Gan, E.

\section{4:45-16:15}

\section{Aula Magna: The Healthcare Sector: Identifying Risks and Consequences}

Health and motivation in nurses: A multi-national European study

Schaufeli, W., Torrente, P., van der Heijden, B., \& Hasselhorn, H.M. 
Is primary care physicians' burnout associated with prescribing medications and making referrals to diagnostic tests and to other providers of health services?

Kushnir, T., Bachner, Y., Greenberg, D., Yermiahu, Y., \& Hadari, I.

Stress and Burnout in Canadian Midwives

Schryer LeBel, F. \& Day, A.

Burnout and secondary trauma: a review of the emotional costs of nursing

Watts, J. \& Robertson, N.

Psychological work well-being and occupational accidents in health professionals

Santarém Semedo, C. \& Dominguez, J.L.

An Innovative Approach to Address Workplace Stress in Healthcare

Zogran, C.

\section{Aula II: Promoting Healthy Workplaces and Employees: In and out of the Workplace}

Return to work after a workplace-oriented intervention for patients on sick leave due to burnout

Karlson, B., Jonsson, P., Palsson, B., Abjornsson, G., Malmberg, B., Larsson, B., \& Osterberg, K.

Wellbeing and Contrast of Discomfort in the Health Context: the Institution of Permanent Observatories in the Region Piedmont's Health Units

Converso, D., Hindrichs, I., Mottura, B., \& Viotti, S.

Stress and Burnout Managing Model in Health Care Professionals

Pereira, J., Rodrigues, J., \& Cunha, M.J.

Health status and burnout in physicians: what interventions in a context of decreasing medical demography? A pilot study in France, 2008

Ladner, J., Kuntz, A., Heurtebize, P., Mauviard, E., Manzano, M., \& Caillard, J.F.

The family demands resource model

ten Brummelhuis, L. \& ter Hoeven, C.L.

The impact of subjective occupational success on personal resources

Muehlethaler, C., Jacobshagen, N., Kaelin, W., Grebner, S., \& Semmer, N.K.

\section{Aula III: Organisational Level Interventions}

Organizational-level work stress interventions: The more the better?

Biron, C.

The Three-Level Model of Psychological Stressors - Limitations in Workplace Prevention of Psychological Stress

Windemuth, $D$. 
The implementation of a new inspection tool in the psychosocial working environment : The experience of the Danish Working Environment Authority.

Magwentshu, S.

The working for wellness program: evaluation of an evidence-based employee well-being program using RCT

Page, K. \& Vella-Brodrick, $D$

Work-related stress assessment: an organizational approach

Vecchio, G.M., Fiaschi, S., Pacelli, F., \& Pacelli, E.

Work-related stress assessment: organizational demands and methodological considerations Zuffo, R.G. \& Cortini, M.

\section{Aula VII: Studying Positive Organizational Behaviour using Elaborated Research Designs (E. Demerouti \& M.C.W. Peeters)}

Testing the Job Demands-Resources Model on the Level of Daily Processes: How to Promote Daily Work Engagement

Kühnel, J., Sonnentag, S. \& Bledow, R.

Crafting a job daily: Contextual antecedents and the effect on work engagement

Petrou, P., Demerouti, E., Peeters, M.C.W. \& Schaufeli, W.B.

Daily Detachment from Work and Home: The Moderating Effect of Role salience Sanz-Vergel, A.I., Demerouti, E., Bakker, A.B., \& Moreno-Jiménez, B.

Personality, regulatory focus and the work-family interface: The role of regulatory fit between partners

Demerouti, E., Bakker, A.B. \& Tetrick, L.E.

Work engagement and job performance as correlates of a supportive organisational culture Peeters, M.C.W., Demerouti, E., \& Bakker, A.B.

\section{Aula VIII: The Role and Impact of Contextual Factors on Employee's Health and Performance}

Work and nonwork determinants of Job burnout: a cross validation in time Smulders, P.G.W. \& Houtman, I.L.D.

The role of demographic factors in the experience of the work-home interface MacKinnon, R. \& Gibbes, C.

Family firms Vs non-family firms: The influence of the organizational context on employee behaviour and well-being

Ceja, L., Escartín, J., \& Tàpies , J. 
Organizational justice, stress and commitment: The mediating role of perceived organizational support and work-family conflict

Hansez, I. \& Babic, A.

A Longitudinal Study of Employee Adaptation to Organizational Change: How Information and Individual Adaptability affect Well-being and Satisfaction during Change

Braun, I., Niessen, C., \& Sonnentag, S.

The Interaction of Appreciation and Illegitimate Tasks in the Military.

Jacobshagen, N., Stocker, D., \& Semmer, N.K.

16:15-16:30

Refreshments

16:30-17:00

Aula Magna: Closing Session and Awards Ceremony 


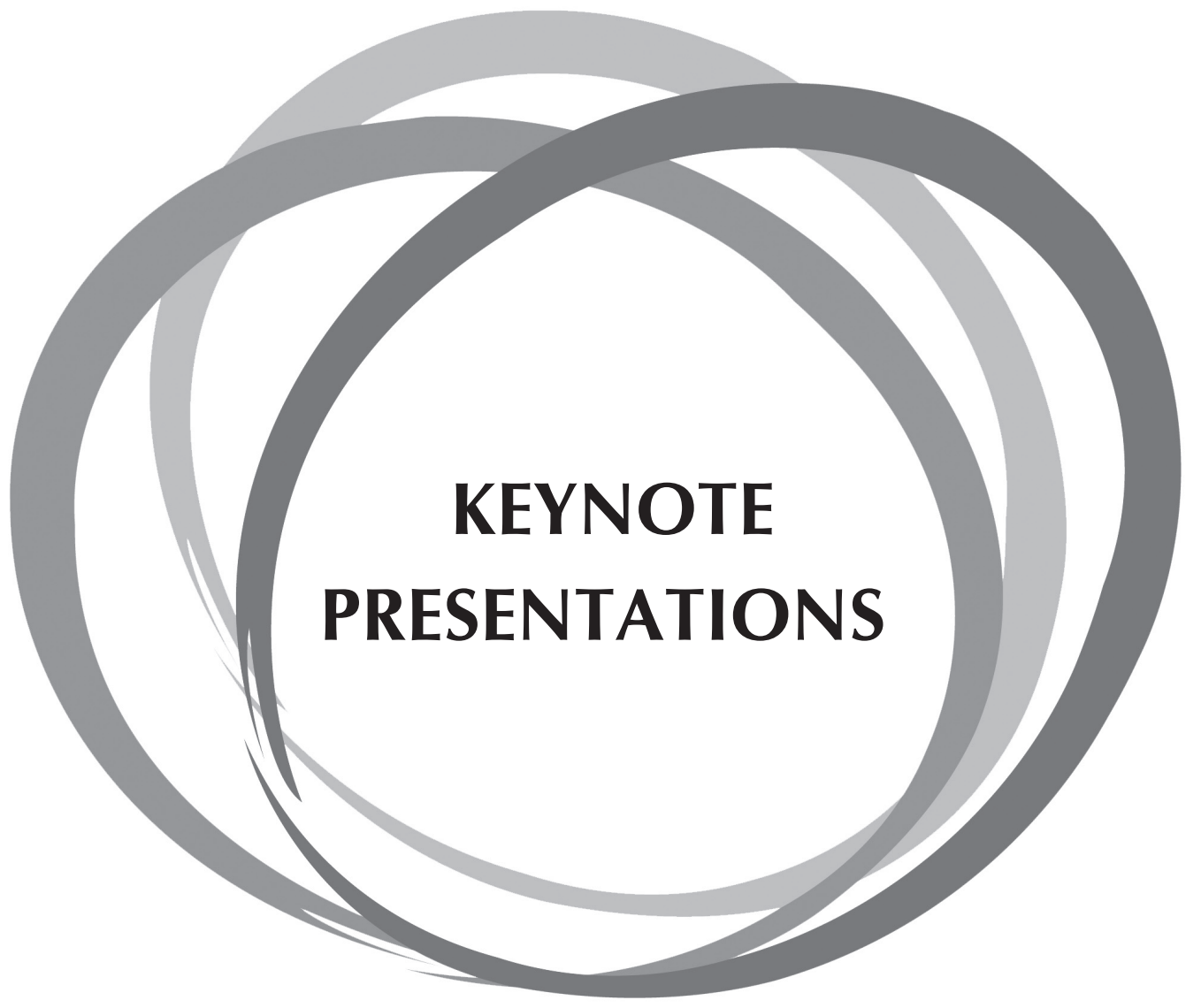





\title{
Living together and well being in organisations and society
}

\author{
Avallone, F. \\ University of Rome "Sapienza", Italy
}

Scientists and politicians agree the main problem of the future will be human living together in a globalized world. A study on more than 5000 individuals tested the 'living together construct in sentimental relationships', in organisations and within a wider social context. Factor analysis confirmed people refer to the following ten dimensions, when describing their living together experiences:

1. Respect of norms and explicit/implicit rules

2. Recognition and respect of the other, diversity tolerance

3. Security and stability

4. Common goals, self and collective efficacy

5. Justice in resource access and in relations management

6. Solidarity, support of the other

7. Trust, hope, confidence

8. Attention to the other and communication

9. Interest and power in relationships, balance between roles

10. Pleasure and involvement in relationships.

Over the past decades, changes in the working environment have made it increasing difficult for people to work together in organisations and in society in general. As a result of this, the literature on work-related stress has been growing rapidly (Cooper and Williams, 1994) highlighting the importance of a proper psychosocial risks assessment in organisational health promotion (Kompier, 2003; Le Blanc et al., 2000). Using this literature as a bsis, researchers have introduced the construct of organisational health (Schabracq, Winnubst, Cooper, 2003),

In order to have a measurement of organisational health, a research instrument: The Multidimensional Organisational Health Questionnaire-MOHQ was developed (Avallone F.\& Paplomatas A., 2005). Based on 14 health dimensions and 3 scales of markers, the MOHQ has been used on 34,000 subjects, within 192 different Italian Public Authorities. The MOHQ uses a Multilevel approach that is able to identify structural, technological, cultural and process variables influencing organisational health and to highlight and compare different organisational health architectures.

Based on to this approach, a new model/instrument is being developed which not only measures an organisation's healthiness but also focuses more on their complexity, and on their processes and practices, rather than on disease itself. This model/instrument will be tested in Italian public and private organisations.

The model identifies several independent variables: physical and technological environment; work content; support and relationship style between managers; support and relationship style between colleagues; security and health, equity, innovation. Through a group/cluster of intermediate variables (efficiency; interpersonal and role conflicts; intrapersonal conflicts; disavowal) and effect of some moderating variables (work control; locus of control; engagement; coping; hope for the future), independent variables directly and indirectly influence 5 dependent variables identified as stress, psycho-somatic disturbances, burnout, uncertain and risk, work dissatisfaction. 
Methodological tests evidence this model/instrument to be a reliable tool in assessing organisational health and identifying higher psychosocial risks, thus enabling planning and implementation of prevention interventions. In the field, it is really useful to put in practice specific actions in psychosocial risks prevention and to define strategies and policies in organisational health promotion, both within single organisation or through overall private and public sector services. 


\title{
Enhancing Mental Capital and Wellbeing in the Workplace
}

\author{
Cooper, C.L. \\ Lancaster University Management School, UK
}

In a recent issue of the European Heart Journal it was estimated that the direct cost of treating depression alone was $€ 44$ billion, but the indirect cost of lost productive value to the European economy was $€ 77$ billion. The UK government were so concerned about the cost of mental ill health in all its' manifestations, with common mental disorders of anxiety, depression and stress affecting one in six of the population, that it initiated a Foresight project in the Government Office of Science on Mental Capital and Wellbeing (Cooper, et al, 2009). This was a two year project which involved over 400 scientists globally, produced over 85 science reviews and established evidence-based policies on how to tackle this costly problem area. As far of this, there was an important stream on work and wellbeing, where the costs to the UK economy were calculated at $£ 25.9$ billion per annum in terms of absenteeism, presenteeism and turnover.

The science reviews established that the main sources of workplace stress and lack of wellbeing were: lack of control and autonomy in the workplace, unmanageable workloads, a long hours culture, lack of work-life balance and above all, an incompetent and inappropriate management style. As a result of these science reviews and discussions with the main stakeholders in the field (i.e. the various senior management teams of the government departments concerned with these issues, the Confederation of British Industry-CBI (business leaders organisation), Trades Union Congress-TUC (trade union organisation), Chartered Institute of Personnel and DevelopmentCIPD (association of personnel managers), etc.), a range of policies were developed. Each policy was then scrutinized in terms of a cost-benefit analysis and those with the most beneficial benefits to costs ratio were highlighted as interventions worthy of strong consideration. These included the following: the right to request flexible working arrangements for all employees and not just those with children; annual or regular stress/wellbeing audits in organisations, followed by appropriate interventions; key performance indicators on wellbeing indicators like job satisfaction levels among employees, stress-related sickness absence, benchmark engagement scores in the annual reports and for line managers; the training of managers on social and interpersonal skills because they are responsible to a great extent for the health and wellbeing of their subordinates. Case studies of some of these will be discussed.

\section{Reference:}

Cooper, C.L., Field, J., Goswami, U., Jenkins, R. \& Sahakian, B. (2009). Mental Capital and Wellbeing. Oxford: Wiley-Blackwell. 


\title{
Stress and health: the role of working time
}

\author{
Costa, G. \\ Università degli Studi di Milano, and IRCCSS Foundation, Italy.
}

Time constraints due to working hours are one of the most relevant psychosocial risk factors at work. Time has become the main dimension of human activities: according to the way and intensity of how we use it, it is possible to do more things in the same or a shorter time, thus enhancing time value. While life expectancy is increasing, time perception/expectancy is increasingly reducing: actually time pressure is one of the main characteristics of our daily life. In the "24-hour Society" the borders between working and social times are no longer fixed and rigidly determined by the normal diurnal working day. Thanks to new technologies, not only is the link between the work place and working hours broken (e.g. telework), but also the value of working time changes according to the economic/productive/social effects produced at different hours/periods (day, week, year, life).

In Europe, according to the most recent Surveys on Working Conditions, only $27 \%$ of employed and $8 \%$ of self-employed workers are engaged in normal daytime work, Monday to Friday, while the majority is engaged on "non-standard" working hours, including shift and night work, overtime, compressed week, Saturday and/or Sunday work, part-time work, irregular or flexible working hours, split shifts, on-call work, etc. What are the advantages and what are the disadvantages for the individual, the social groups, the companies, and society? What is the cost/benefit ratio in terms of psycho-physical health, family life, and social well-being? The knowledge of the past decades shows the negative consequences of non human-centred working time organisation. Irregular working hours and shift work, particularly if including night work, may have a negative impact on health: they perturb the psycho-physical homeostasis (e.g. circadian rhythms, sleep, and performance), hamper family and social relations, and are a documented risk factor for many health disorders, in particular, sleep, digestive, neuropsychological, cardiovascular, and reproductive functions. This may lead to increased morbidity and absenteeism, with high economic and social costs for the individual, the enterprise, and the whole society.

Many factors regarding individual, social and working conditions can interact and influence both short-term adaptation and long-term consequences with age and gender being among the most important ones. Ageing people can encounter greater difficulties in facing irregular work and living patterns due to an increasing rigidity in physical and mental adaptation to a fast changing societal and work organisation (particularly to irregular working hours). Some issues associated with ageing, such as reduced psycho-physical fitness, decreased restorative properties of sleep, proneness to internal de-synchronization of circadian rhythms, increased mental rigidity and resistance to changes, certainly play an unfavourable role. Hence, more age discrimination or unequal treatment at the work place for older workers and consequently more social problems in a progressively aging society.

Also women have to face more specific adverse problems because of their hormonal, family and social conditions. Disorders of menstrual cycle and reproductive function (reduced fertility, higher abortion rates, impaired foetal development) were recorded in many groups of women shift workers. Very recently a probable association between night work and breast cancer has also been claimed. Stress due to time pressure is a constant condition, in particular for those married with small children. They have more difficulties in combining their irregular working 
schedules with the additional domestic duties, that can be more or less demanding according to family composition (e.g. number and age of children, cohabiting persons), personal duties (e.g. school, housework), and availability of social services (e.g. shop opening hours, transportation etc).

Arrangement of working time according to ergonomic criteria, adoption of more flexible working hours, higher autonomy and control of work organisation and conditions, more compensation in terms of social services, job and career opportunities, better information and social support, as well as a more balanced integration among work, environment and society, are the main tools for an appropriate evolution of the homo sapiens in the "24- hour Society". 


\title{
Money, Models, and Mental Health: Implications of Economic Stress for Occupational Health Psychology
}

\author{
Sinclair, R.R. \\ Clemson University, USA
}

The recent world-wide economic downturn has highlighted the fundamental link between economic issues and occupational health. Economic stressors represent a critical concern for workers and require further attention from occupational health psychologists. Some economic issues, such as job insecurity/precarious employment, have been heavily studied and have yielded a large body of research that clearly demonstrates their links to health outcomes. Other economic stressors, such as financial stress, have received disproportionately less attention from OHP researchers. With these concerns in mind, my presentation will address three topics that hopefully will help form a research agenda for OHP scholars interested in economic stressors.

First, the construct of economic stress with particular emphasis on distinguishing two broad forms of economic stress: financial stressors and employment stressors will be discussed. Following which the objective and subjective forms of each of these stressors will be described with particular emphasis on financial stressors. A key theme in the literature on financial stress is the need for more comprehensive and better validated measures of financial stress constructs. Therefore, a multidimensional model of the subjective financial stress construct and some preliminary research findings concerning the structure and correlates of financial stressors will be presented. Some issues that require future research attention will be further discussed.

Second, economic issues reflect interactions among complex systems at multiple levels of analysis. This creates considerable challenges and exciting opportunities for OHP research. Models focusing solely on the individual level of analysis lack the requisite complexity to capture the antecedents and consequences of economic stressors or to fully articulate the mechanisms through which economic stressors affect workers. Therefore, a multilevel framework for economic stress research that illustrates some of the potential influences of the economic context on individual workers will be presented. Recent research efforts to merge publicly available archival economic data with data from a large scale survey study of retail workers will be described in this context, highlighting the tremendous need for further research, particularly research that is sensitive to the unique concerns of different parts of the world and different kinds of occupations. Some research questions and considerations that emerge when considering a multilevel perspective on economic stressors will be proposed to the audience.

Third, despite the importance of interventions to occupational health, relatively little research has focused on economic stress interventions. The first intervention that might come to mind is increasing worker's compensation, which, while normally desirable, may not always be feasible. However, many other potential intervention strategies exist. Therefore, the multilevel perspective on economic stressors will be extended to describe an intervention framework that distinguishes primary, secondary, and tertiary prevention strategies, organisational and governmental intervention agents, and organisational and individual beneficiaries. Hopefully, considering the interrelationships between these intervention strategies will highlight issues that might inform conference attendees' research programs.

Although psychological science must play a central role in understanding how economic stressors affect occupational health, effective economic stress research will require multidisciplinary 
research collaborations. Such collaborations will have to be sensitive to the particular economic concerns of different regions of the world and different groups of workers. The presentation will conclude with a brief discussion on some of these issues. Following which an invitation will be extended to the attendees to attend the next Work Stress and Health conference (in Orlando, May 2011) where economic issues will be the main theme of the conference. 



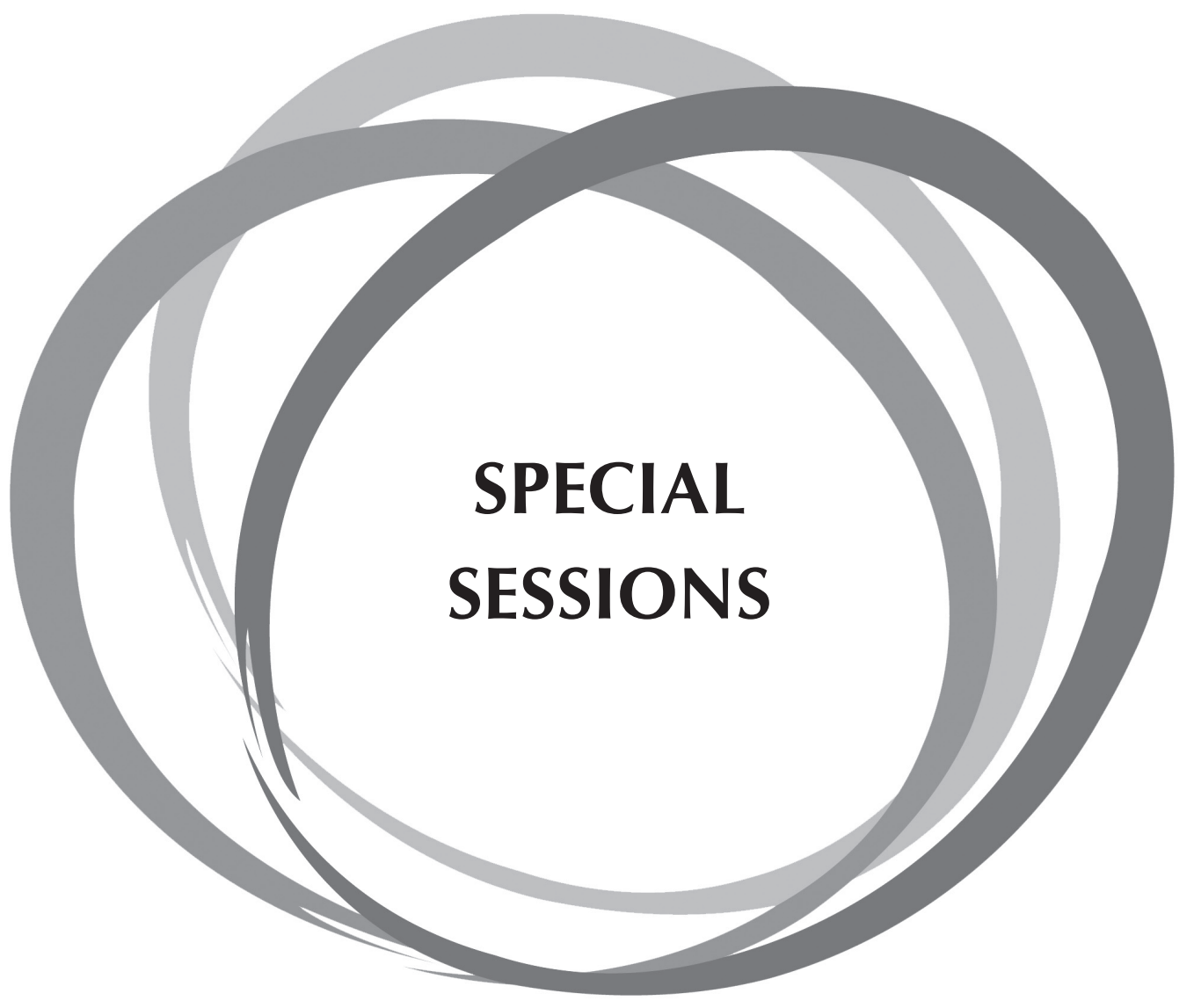




\title{
"The management of psychosocial risks: good practices and successful models"
}

Organised by the Italian National Institute of Occupational Safety and Prevention (ISPESL)

Chairs: lavicoli, S. \& Leka S.

European occupational health and safety policies highlight the importance of work-related stress as well as the need to manage and assess it in the workplace. Both the Council Directive 89/391/ EEC and the European Agreement on work-related stress signed by the social partners in 2004 are key examples of these policies. In compliance with the European regulation, the Italian legislation, as foreseen by the Article 28 of the Italian Lgs. Decree 81/08 on health and safety at work, acknowledges work-related stress as a work-related risk and incisively stresses the duty to enlarge the risk assessment area to all risks arising from work-related stress.

In this context, the Italian National Institute of Occupational Safety and Prevention (ISPESL), the relevant government body on occupational health and safety at national level and the coorganisers of the $9^{\text {th }}$ conference of the European Academy of Occupational Health Psychology have organised a Special Session on 'The management of psychosocial risks: good practices and successful models', which is expected to provide a forum for exchange of views among specialists to address potential strategies for the management and assessment of psychosocial risks at the national level.

In the first part of the session, presents examples of the successful experiences in the management of psychosocial risks of some European countries like UK, Ireland and Norway as well as the perspectives of the World Health Organisation (WHO) and the European Agency for Safety and Health at Work (EU-OSHA), on psychosocial risk management.

In the second part, presentations will focus on cases from Italy. Along with involvement of experts and representatives from the social partners, regions and government institutions the session aims to promote a participative and collaborative approach to addressing the issue. Furthermore, some work-related stress management approaches at European level will be shown, as an example of good practices in the management of this issue in the new occupational scene.

\section{PART I}

\section{WHO global perspective on psychosocial risk management}

\author{
Kortum, E. \\ World Health Organisation
}

The WHO is involved in a number of activities that address the health of workers in an integrated manner. The 60th WHO World Health Assembly, held in May 2007, approved the WHO Global Plan of Action on Workers' Health (2008-2017). This Action Plan (http://www.who. int/gb/ebwha/pdf_files/WHA60/A60_20-en.pdf) has a number of objectives and stipulates that the workplace should not be detrimental to health and well-being and that primary prevention of occupational health hazards should be given priority. It further stresses that the workplace can serve as a setting for delivery of health promotion activities and other essential publichealth interventions, and proposes to improve the assessment and management of health risks at the workplace by defining essential interventions for prevention and control of mechanical, physical, chemical, biological and psychosocial risks in the working environment. 
The WHO Healthy Workplace Global Framework takes a comprehensive approach and includes a multi-stakeholder perspective. WHO defines a healthy workplace as one in which workers and managers collaborate to use a continual improvement process to protect and promote the health, safety and well-being of workers and the sustainability of the workplace by considering the following, based on identified needs:

- health and safety concerns in the physical work environment;

- health, safety and well-being concerns in the psychosocial work environment including organisation of work and workplace culture;

- personal health support/resources in the workplace; and

- ways of participating in the community to improve the health of workers, their families and other members of the community.

The WHO strongly supports the development of all these areas through the harmonization of practical tools for the assessment and management of occupational risks and the collection of tools and programmes to be combined in an inventory. Well advanced is the development and application of the Psychosocial Risk Management toolkit and framework for Europe (PRIMA-EF), which has been developed within the Global Network of Collaborating Centres (CCs) in Occupational Health (http://www.who.int/occupational_health/network/en/) by an international Consortium led by the Institute of Work, Health and Organisations, University of Nottingham, UK. WHO collaborators work with country experts to adapt its application to different contexts, cultures, sectors and companies. In addition, a group of experts assists WHO in addressing the issues of psychosocial risks and work-related stress, their impact on workers' health in their respective countries. Although psychosocial risks are universal, they may differ in various countries due to the situation of the employment market, the availability of legislation and policies at national and company levels, the culture and specific socio-economic situations, or the ethical values applied by employers.

\title{
Assessing and preventing psychosocial risks in the EU: An overview of EU-OSHA's work
}

\author{
Rial-Gonzalez, E.
}

European Agency for Safety and Health at Work (EU-OSHA)

Through the Framework Directive 89/391/EEC and its five individual directives, European Union legislation provides the framework for workers in Europe to enjoy high levels of health and safety at the workplace. Implementation of these provisions differs from one country to another and their practical application varies according to sector, category of worker and size of enterprise. The increasing importance of 'emerging' risks, such as stress, violence and harassment, poses a challenge for policy makers in their development of effective prevention measures.

Since its creation, the European Agency for Safety and Health at Work (EU-OSHA) has paid great attention to these risks. In 2002, the Agency's European Campaign had psychosocial issues as its theme, and included a large number of publications and events. The emphasis of the Agency's work has been on the legal obligation, practical wisdom and economic advantages of assessing and preventing psychosocial risks just like any other workplace risks. 
Putting the management of psychosocial risks in the wider context of occupational safety and health $(\mathrm{OSH})$ management is sensible because experience shows that both domains share some key success factors, such as the importance of management leadership and worker participation, or the need for a thorough assessment of the risks (particularly at the organisational level) followed by carefully planned, implemented and evaluated interventions that address the root causes. Over the years, the Agency has gathered and analysed a wealth of case studies -from enterprises of different sizes, from various sectors in many countries- that illustrate these success factors, and that are transferable across workplaces.

Since the 2002 European Campaign, the Agency's work on psychosocial and organisational risks has grown, including a key report on 'Research on work-related stress' that has become one of its most downloaded and cited publications; a variety of workplace-oriented factsheets in 22 languages giving advice on how to tackle psychosocial risks; an expert forecast report on emerging psychosocial risks, followed up by an international seminar; a statistical overview of stress across the EU; and in-depth reports on issues such as violence and harassment. In 2009, fieldwork was completed on the Agency's largest project to date, the European Survey of Enterprises on New and Emerging Risks (ESENER).

ESENER asks both managers and workers' health and safety representatives about the way health and safety risks are managed at their workplace, with a particular focus on psychosocial risks. A total of 28,649 managers and 7,226 health and safety representatives were interviewed in the 31 countries covered: the EU-27 as well as Croatia, Turkey, Switzerland and Norway. The survey design and its two questionnaires were developed with the support of governments and social partners at European level, as they all share the survey's aim to assist workplaces across Europe to deal more effectively with health and safety and to provide policy makers with cross-nationally comparable information relevant for the design and implementation of new policies.

ESENER explores in detail how both managers and workers are involved in the prevention of stress, violence and harassment. These are some of the key findings on psychosocial issues from the survey:

- Work-related stress is among the top three OSH concerns for European enterprises, together with accidents and musculoskeletal disorders. Violence, and especially harassment, are reported to be a major concern in a fairly large number of enterprises.

- Management of psychosocial risks is more frequent in the health and social work sector and in larger establishments. Southern European countries - except Spain - show less awareness and are less likely to take action to manage psychosocial risks.

- More formalised procedures to manage psychosocial risks appear widespread in only a few countries, such as Ireland, the United Kingdom, the Netherlands and the Scandinavian countries, as well as in large establishments and in the public, financial intermediation, education, and health and social work sectors.

- Establishments generally deal with psychosocial risks by providing training and implementing changes in work organisation. However, only about half of the respondents inform employees about psychosocial risks and their effect on health and safety.

- Fulfilment of legal duties and requests from employees appear to be the main drivers for addressing both $\mathrm{OSH}$ in general and psychosocial risks.

- The most important barriers to addressing psychosocial risks in establishments are the perceived sensitivity of the issue, together with lack of awareness and lack of resources. 
- Managers recognise that employee participation is a key success factor both for OSH and psychosocial risk management and, therefore, the role of the social partners remains crucial for the implementation of effective measures.

\title{
UK Management Standards Approach for tackling work-related stress in a global context
}

\author{
Palferman, D.J., Mackay, C. \& Kelly, P. \\ Health and Safety Executive, UK
}

The Management Standards approach was developed by the United Kingdom Health \& Safety Executive (HSE) to reduce the levels of work-related stress reported/experienced by working people in Britain. The overall aim is to bring about a reduction in the incidence and prevalence of cases and the associated number of employees who are absent from work due to stressrelated sickness or who cannot perform well at work because of their experience of stress. The Management Standards approach was developed to provide managers with the information, procedures and tools needed to achieve these aims. It was intended to demonstrate good practice in the management of health at work through evidence-based, joint problem solving (managers and other employees).

The Management Standards programme has been implemented nationally in three phases:

1. Where 100 volunteer organisations agreed to trial the Management Standards Approach and share their data with the HSE in exchange for help and advice from the regulator (HSE Inspectors). The organisation that participated in this programme have contributed to an evaluation exercise

2. Healthy Workplace Solutions: a series of 64 workshops with approximately 1800 delegates from 900 organisations. Delegates attending the one day events undertook a series of exercises related to the Management Standards and were encouraged to share their good practice examples with fellow delegates. The organisations that sent delegates to these events have had their progress monitored via an inspection programme and have contributed to an evaluation exercise.

3. Wider Implementation Programme (WIP) aims to share the learning from the previous two phases with the all employment sectors within the UK economy. The main delivery mechanism used for dissemination of learning has been via the internet (www.hse.gov.uk/ stress).

A very considerable amount of information has been collected during the various implementation phases of the stress programme, outlined above, The thrust of the second phase was to the integration of our message on 'stress' with that on sickness absence - this was done somewhat in haste and the messages, although palatable, were still somewhat rudimentary. This is partly a reflection of our incomplete understanding of the relationship between the two and, more widely, underdeveloped conceptual models (e.g. linking prevention with the bio-psychosocial approach). 
Additionally, our work on stress has found that messages that are framed positively - playing more to the business case - are likely to be more powerful determinants of decisions to take action and more persuasive than those based solely on harm reduction. It has drawn attention to the contribution of management behaviour and culture on psychological ill-health and more recent work has indicated that other aspects of management, leadership and climate can have separate, but positive effects on well-being. Specifically, the drivers for action in organizations are concerns with impacts on individual and organizational performance.

The intervention programmes were, based on evaluation studies, judged to be a success. As with any intervention programme some participating organisations failed to make progress. In respect to learning, studies of these organisations has provided valuable insights into specific organisation cultures and provided valuable information in respect to the barriers that need to overcome before organisations can make progress. This information has been collated into a "lessons learned" document that has been used to inform the development of additional guidance and to the generation of a new web site (www.hse.gov.uk/stress).

The Management Standards approach was developed within the UK health and safety framework but in themselves they are not legally enforceable, they are guidance. The guidance category means that organisations do not have to follow the Management standards approach. However, an organisation does need to demonstrate whatever approach they choose to take is equivalent, in terms of process and outputs, to the management standards. This is important in a global context as stress is not a problem confined to the UK it is a problem for most of the developed world. Therefore, the Management Standards approach could be accommodated within the regulatory framework of other countries. A number of countries and states including some in continental Europe, Australia, New Zealand and Canada are currently running pilot programmes using the Management Standards approach. The lessons learned from the UK intervention programme should be relevant to other countries considering how to tackle the global epidemic of work-related stress.

\title{
Republic of Ireland - Work Positive, Risk Assessment method for auditing work- related stress in a global context
}

\author{
Murray, P. \\ Health and Safety Authority, Ireland
}

Background: The Work Positive Approach was initially developed by the Health and Safety Authority (HSA) of the Republic of Ireland in partnership with Health Scotland (HS) in 2001/2002 to attempt to tackle the issue of Work-related Stress in a systematic way across various types of organisation in Ireland and Scotland. In 2004, after the Health and Safety Executive in the UK (HSE, UK) had considered and rejected a Code of Practice approach to the issue, alternative ways to promote healthier workplaces were investigated and the Management Standards approach was agreed within the HSE. Thereafter, a further partnership approach was agreed between the HSEUK, HS and HSA. This involved embedding the categories of stressor identified by the HSE in its work on the Management Standards*, into the questionnaire already marketed as 'Work Positive'. Thus, the Work Positive tool had the advantage of having its original questions, with some additions and alterations, arranged in such a way that it could be used as a longer, original 
65 item broader-remit tool, or as a 35 item stress audit tool** measuring the Management Standards alone.

The overall aim is to provide organisations with a user-friendly, cost-efficient measure that allows for in-house organisational profile, benchmark and risk assessment using the tool, relative to other similar organisations and to national and international norms.

*The HSE's Management Standards approach was developed to provide managers with the information, procedures and tools needed to achieve these aims. It was intended to demonstrate good practice in the management of health at work through evidence-based, joint problem solving (managers and other employees).

**The Work Positive tool currently in use is the shorter 35 item tool with results mirroring the Management Standards, using UK norms.

Irish National Project using Work Positive (April 2008 - Sept 2009)

1. 20 organisations across 4 industry areas agreed to implement Work Positive within their organisations and share the results and findings throughout the process with the HSA, in return for support and assistance in the audit and diagnostic phase as well as some training and development inputs. Industry sectors were Health, Education, Public Sector, Semi-state/ local Authority

2. These organisations were profiled as part of the overall larger group and also within smaller industry-specific groups. Profiles of each organisation were taken and compared, as were profiles of each industry area (5 organisations) showing distinct differences between industries across sectors and significant similarities within industries in terms of the relative risk of industry specific stressors.

3. Workshops and presentations as well as training, further auditing using different tools (i.e. Maslach Burnout Inventory - MBI) and stress management sessions for staff were provided by the HSA throughout the process.

4. Seminars on using and promoting the Work Positive Approach - for such seminars were held throughout the process with national and international speakers, encouraging a flexible yet systematic approach to the issue of WRS.

5. 20 case studies were developed by the participant organisations and placed on the HSA website to provide a map for others considering implementing the system. Solutions were identified as were challenges to its success

6. Data was then used to identify possible improvements in the tool - in terms of validity/ reliability and developing Irish norms, as well as improvements in the process postquestionnaire in terms of gaining buy-in and tailoring it to suit different industry cultures and organisational structures and systems.

Brief Summary: This Work Positive project involved national level activities and inputs into prevention of Work-Related Stress. Actions included education and awareness raising, auditing, analysing data, feeding back data, profiling, benchmarking and facilitation of group sessions as well as organisation-wide training sessions on aspects of the Management Standards; Demands, controls, supports, relationships, role and change. On-going further development of the tool and further promotion of risk assessments by organisations is continuing. This paper aims to share the lessons learned from this intervention programme and discuss the findings in a global context. 


\title{
Surveillance of occupational health and safety in Norway: experiences with establishment of a national Surveillance system
}

\author{
Sterud, T. \\ National Institute of Occupational Health (NIOH), Norway
}

The situation in Norway until recently was that information on work-related health outcomes, hazards and exposure factors were fragmented and poorly coordinated, providing no comprehensive information on current status or time trends relevant for decision-makers and other stakeholders. The Department of Occupational Health Surveillance (NOA) was established four years ago to address this concern. The NOA was established by the Norwegian Ministry of Labour and is located at the National Institute of Occupational Health in Oslo. NOA has an advisory board with members from the Ministry of Labour, the Labour Inspection Authority, the Norwegian Labour and Welfare Administration, the Petroleum Safety Authority and Statistics Norway.

At present, NOA has eight employees from various disciplines (psychology, sociology, chemistry, occupational medicine and epidemiology). In addition, NOA has also organized three scientific advisory groups covering different occupational health disciplines ("Psychological, social and organisational work environment", "chemical, biological and physical work environment", and "occupational health"). NOA's main aims:

- To coordinate, systematize and disseminate information on status and time trends on workrelated hazards and occupational health.

- To stimulate our data providers to further develop their data registries to make them more useful for scientific research.

- To establish a common platform for all data collected from different sources, and to make the data easily available to all relevant stakeholders and user groups.

Datasources: NOA's data are provided among others by Statistics Norway, the Norwegian Labour and Welfare Administration, the Labour Inspection Authorities, the Cancer Registry, and the Norwegian Pollution Control Authorities. In the present situation, some suppliers have fairly reliable exposure data, others have none; corresponding situations exist regarding occupational health data. The establishing of NOA has initiated projects to further develop data registries. One example is the plan to make the chemical and biological exposure database at $\mathrm{NIOH}$ nationwide.

NOA has also been involved in the improvement of the Norwegian Survey of Living Conditions - Working Environment, which has been conducted by Statistics Norway every third year since 1989. In 2006, the design of the study was changed to a split panel design, and the sample size was made 4 times larger than in the previous surveys. In addition a large number of new questions were also included, in particular concerning the psychosocial work environment. Every three years, Statistics Norway will provide the surveillance system with both cross sectional and longitudinal data of particular interest for trend analyses.

Examples from recent publications - a focus on psychological, social and organisational factors: Based on the amount of indicators the Norwegian Survey of Living Conditions - Working Environment represents the most important data source of our Surveillance system. In 2008 (NOA) published a report series on recent trends and status of work exposures and health 
among Norwegian employees. This report series gives a comprehensive description of the total exposure to different risk factors (chemical, physical, mechanical, and psychosocial and organisational exposures) at a national level. Moreover, the large sample gave us the possibility to present data on 45 occupational groups, in order to identify occupational groups most at risk, and to describe possibly related ill health and well-being within these groups. We addressed the following aims:

- Describe main trends regarding exposure to occupational stressors during the last decades

- Identify the occupational groups with the highest total level of exposures to stressors, with a special emphasis on organisational and psychosocial stressors

- Identify the occupational groups with the highest total level of work-related health complaints

The results concerning the overall work environment in Norway are positive, but working conditions vary considerably across occupations and sectors. The data shows that several occupations within the health care and service sector have a high exposure to psychosocial stressors. Nurses report a high stressor level on 10 out of 21 psychosocial stressors. Other occupations within the health sector like auxiliary nurse, and social workers also report high levels on several stressors. Applying the terminology from Karasek's demand-control-(support) model an iso-strain group was defined as people who scored in the upper tertile on the dimension high demands, low control and low support. In all, 8 percent were working in an iso-strain situation according to these criteria. Among these 64 percent were women and the largest proportion worked in public sector. The data indicated that the iso-strain group had a higher proportion of sick-leaves and a higher prevalence of musculoskeletal complaints.

Future challenges: The scientific challenge is to provide more reliable data on all aspects of work environment and work related health in Norway. A technological challenge is to establish a common platform for all data collected, opening the possibility to combine data from different sources, and to make the data available to relevant stakeholders and user groups.

\title{
PART II
}

\section{The management of psychosocial risks in Italy}

\author{
Chairs: Masi, M. \& Fantini, L.
}

\section{ISPESL's commitment to manage and assess work-related stress}

\author{
lavicoli, S. \\ National Institute of Occupational Safety and Prevention (ISPESL), Italy
}

Over the last years, stress-related problems have been increasingly affecting the world of work and more attention has been given to psychological distress often caused by poor work organisation. To tackle the issue, in the last few years the Italian National Institute of Occupational Safety and Prevention (ISPESL) tasked to safeguard and promote occupational health and safety, has given a boost to study, research and training within this area. 
In the current Three-Year Activity Plan 2009-2011 more emphasis is given to psychosocial issues and targeted lines of research have been launched to develop practical tools for the assessment, evaluation and management of psychosocial risks with a particular focus on some specific sectors. For the assessment and evaluation of psychosocial risks, a psychosocial and occupational risk preventive tool is being developed and validated and identification of "best practices" is being carried out. Furthermore, giving a key role to the promotion of the corporate wellbeing culture permitted the launch of specific research projects on issues relating to workers' active participation and corporate social responsibility (with a particular focus on credits).

Work-related stress and psychosocial risk factors represent particularly sensitive issues for ISPESL. In 2006, the Institute joined an International Consortium at European level within a project funded by the EC 6th Framework Programme "Psychosocial Risk Management- European Framework PRIMA-EF", which focused on the development of a European framework for psychosocial risk management with a special focus on work-related stress and workplace violence (including harassment, bullying and mobbing). In 2009, as a continuation of PRIMA-EF project and within the "Leonardo da Vinci" European programme, the same consortium launched a new European project titled "Psychosocial Risk Management - Vocational Education and Training PRIMA-eT" aiming at creating an e-learning platform on the basis of the results of PRIMA-EF project.

In addition to the support provided for research activities, investments have also been made on training and networking initiatives. Training initiatives are being promoted by the Institute in order to support and train the professional figures playing a key role in the security system on bullying, work-related stress, psychosocial risk factors and promotion of the corporate wellbeing. In 2007, the launch of a scientific research network applied to psychosocial risks led to the establishment of a "National Network for the Prevention of Psychosocial Distress" involving ISPESL as well as other safety professionals at national level. The Network covers the entire national territory, adapting to local contexts and respecting professional autonomies. A document title, "The assessment of work-related stress: a methodological model" has been produced by the Network.

Finally, considering work-related stress as one of the main causes of occupational ill-health (sickness absences, high turnover, poor performances), ISPESL carried out a study to identify a management approach at organisational level which may be sustained and managed by safety professionals in an autonomous and participative way. In view of the pre-existing European models and the Italian industrial system (mainly made up of SMEs), the study used the UK Health Safety Executive's (HSE) work-related stress management approach as a benchmark and as an example of good practice. The main objective of ISPESL is not only to translate and validate the questionnaire included in the HSE approach (Indicator Tool) with an adequate psychometric validity but also to transfer and adapt the global HSE platform to the Italian context. The Italian validation shall provide organisations with a risk assessment tool included in a multistep process designed to identify work-related stress management standards, taking into account the need to harmonize this process to consolidated risk management frameworks and to the implementation of the Council Directive 89/391/EEC. It shall finally recognize the prominent role of safety professionals also in managing the risk factors for work-related stress. 


\title{
Inter-regional guidelines for assessment and management of work-related stress
}

\author{
D'Orsi, F. \\ Interregional working group coordinator, Italy
}

In order to formulate guidance for work-related stress assessment as provided by the Italian Health and Safety Regulation (D.Lgs. 81/2008), the interregional technical committee has established a working group to define guidelines. The working group activity was based on documents produced by some Italian regions as well as on the methodological proposal issued by the national network for the occupational psychosocial discomfort in coordination with ISPESL. The goal of the guidelines is not to propose a specific assessment method, but rather to indicate criteria and requirements that any assessment method must meet.

The application field is clearly indicated by the European framework agreement on workrelated stress of October $8^{\text {th }}$ 2004; harassment and violence at work (including bullying) are therefore not included in the assessment process as other than indicators of dysfunctions in the work organisation. Besides identifying whether work-related stress is a problem in a specific organisation, the assessment process also helps employers to identify the corrective actions to improve work organisation and, consequently, eliminate or reduce work-related stress. Addressing the issue of work related stress may also help to enhance the organisational efficiency and productivity. For that purpose the assessment process is part of wider activity involving the top management, and requiring appropriate communicative actions, exchange of information, specific training of safety professionals and occupational physicians, promotion of their active participation, training of workers, managers and line management, consultation with workers, risk assessment, adoption of corrective actions, analysis of gained improvements, addressing individual concerns and follow-up activities.

The guidelines show the key elements, criteria and methods of the assessment, the minimum requirements for evaluation tools, and the specific contents of the risk assessment document. The medical surveillance and the role of health and safety local authorities are also considered. The risk assessment must identify the objective indicators of work organisation and the direct or indirect effects of work-related stress, like morbidity, frequent accidents, job change requests, legal arguments, etc. If indicators of work organisation show that work-related stress is a relevant problem for an organisation employing more than 10 workers, the next action to take in the assessment process consists of examining the subjective perception of stress of workers.

\section{The slow consolidation of the "right" concept of work-related stress}

\author{
Frascheri, C. \\ CISL, Italy
}

The concept of work-related stress has officially come into the context of health protection and safety at work, after the implementation of the European Agreement of 2004 (European Social Partners, 2004). This agreement was acknowledged in Italy after four years, exactly on 9 June 2008 by the signatory employers' associations and trade unions which had contributed 
to draw it at European level. Exactly in the same period, after a course of several months, the national legislators had completed the reform procedures carried out in matters of prevention and protection at work (Legislative Decree 81/2008 f.c). Through these procedures the legislator inserted for the first time, in a very clear and precise way, among the duties of the employers, the obligation of evaluating work-related stress.

The sentence passed by the Court of Justice of the European Communities in 2000 (Cfr. C. 49/00 of November 15, 2001) determined an important turning point, because the Court requested Italy to specifically respect the regulations of the European Union on the matters of the employers obligation of evaluating 'all' risks at work (including in this way also risks of work organisation instead of considering only technical risks), but the achievement of the legislators with the recent reform has gone farther. Having in mind the clear objective of improving the text of the law and of the related implementations, both to be faithful to the text of the European Agreement and to confirm the choice made, which is at the basis of the reform, the Italian legislators did not want to confuse the matter or to enlarge the range of action, therefore focused very clearly only on work-related stress.

The two texts, both the European Agreement and the Italian Decree, the Legislative Decree 81/2008 f.c. (Cfr. Legislative Decree n. 81 of 9 April 2008, integrated and modified by the Legislative Decree n.106 of 3 August 2009), were very clear and were able to give a full explanation of the literal meaning of the term. Nevertheless great confusion has been created and spread around regarding the concrete basis of a deep understanding and particularly the problem of tackling the phenomenon, when numerous uncoordinated initiatives were implemented in the past years. Only now, after almost two years of attempts, can the first signs towards the development of coherent and joint coordinated efforts can be seen.

First of all the obstacle of the distinction between the work-related stress and the wider category of the risks of a psychosocial nature had to be overcome. Although in the European Agreement text (art.2 par.3) and, very clearly, in the Legislative Decree 81/2008 f.c. (art.28, par.1) the concept has been well explained, for a very long time, in the months which followed the entrance into force of the decree, a lot of specialised literature continued to insist on the mistake of thinking that inserting the work-related stress in the study and evaluation, as potential risks source, was comprehensive of all phenomena of a psychosocial nature.

The second obstacle, nowadays almost overcome, was the confusion between the concept of stress, in the broader definition of the problems, and the concept of work-related stress. Considering it a very delicate point but at the same time a priority in matters of importance, clarity and distinction, the European Agreement text has continuously tried to give a precise definition of the two concepts and at the same time to clearly separate the two different phenomena and their repercussions at work, starting from the specific criminal liability, in primis, of the employer. However, on this specific point, there have been a lot of "absentminded and inattentive" readers who did not examine carefully and with great attention the text of the Agreement, in particular art.3 of the European text. They did not read carefully the title of art. 3 and they did not examine all the paragraphs of the article (particularly paragraph 4). In this way they thought they could give the definition written in the text to the work-related stress instead of to stress in a general sense, creating confusion and giving wrong information consequently causing inadequate actions and interventions at work.

A third aspect, nowadays consistently reduced, refers to the initial pressure in some of the professional fields to consider the phenomenon from a medical point of view. This attitude has 
caused an exclusive subjective vision of the problem and consequently an inevitable recourse to professional skills outside the companies. Unfortunately they were unfamiliar with the risk evaluating process and with the techniques of tackling specific interventions of prevention and protection to adequately safeguard health and safety at work of the workers involved.

Now, the next important turning point we have to face is the involvement of the Italian Permanent Consultative Committee, by August 2010, in drawing up the 'necessary instructions to evaluate the work-related stress risks' (art.6, par.8, lett.m-quarter). The commitment is high and the challenge is important because the Committee has a serious responsibility. If the Committee does not manage to do it, the burden of evaluation will be on the employers' shoulders. On the other hand, the committee should draw a concrete instrument with helpful and clear instructions to implement the European Agreement on prevention at work in an adequate and simple way to suitably tackle such a delicate topic as work-related stress. These instruments should be effective and realistic in order to improve the organisation of work which is the weakest link in the protection of health and safety at work.

\title{
Work-related stress
}

\author{
Pontrandolfi, F. \\ Confindustria, Italy
}

Risks from work-related stress were officially introduced in the risk assessment process by the Lgs. Decree 81/2008, making reference to the European Agreement on work-related stress signed by social partners in 2004. In line with the subsequent modifications, the Permanent Advisory Commission established within the Ministry of Labour was entrusted with the task to defining new risk assessment criteria. Confindustria - the Confederation of Italian Industriescalled for this indication to be included in the legislative text, thus supplying employers with a reliable risk assessment model instead of uncertain and indefinite predictions that were difficult to interpret and apply.

The issue of psychosocial risks - going beyond the work-related stress that the legislator identified as the specific object of the risk assessment process - has long been studied by specialists; however, fundamental boundaries for a proper work-related stress assessment risk were set only after the European Agreement was signed and implemented in Italy through the Interconfederal Agreement of 2008. In fact, attempts were made to provide a proper definition of stress and stressors and to set out a prevention-oriented policy; yet, the lack of the necessary scientific support caused the hypothesis put forward not to survive the harsh criticism of many. An example of this is provided by the dispute where Confindustria successfully objected (TAR - Regional Administrative Court - Lazio, Section III, 04.07.2005 and Council of State, Section VI, 17.03.2009, n.1576) the guidance note issued by INAIL - the Italian government agency for the insurance against work-related injuries - employing the assumption that the hazardousness of the work organisation is a source of risk itself and thus drawing erroneous and misleading conclusions. This needs to be borne in mind not to make the same mistakes.

The European Agreement of 2004, subsequently transposed into the Interconfederal Agreement of 2008, as well as the future guidelines the Permanent Advisory Commission shall issue remains, 
therefore, the main reference on the work-related stress issue. This needs to be clarified because, even after the legal provision was introduced, a number of different approaches to this issue arose and they were often not coherent with the objective of aiming at supporting an efficient risk assessment in the workplace. Moreover, the legal provisions and the agreement must be applied in full compliance with the objectives identified by the Community Strategy for Health and Safety at Work 2007-2012 defined by the EU Commission.

Simplifying the legal system and adapting it to the evolving world of work by devising simple tools to streamline the risk assessment process, outlining new methods to identify and assess new risks and promoting a change in worker's behaviours are among the most important objectives set out by the EU Commission. The Commission called upon Member States to integrate specific initiatives into their national strategies to strengthen the disease prevention and health promotion, stressing the importance of negotiations among social partners and encouraging them to monitor and report on the implementation of the European Framework Agreement on the work-related stress.

In this context, it appears particularly important to bear in mind the need to simplify the risk assessment process and to integrate it in the organisational context and among the tasks performed by safety professionals (employers, health and safety managers, company physician and workers' safety managers), thus avoiding unnecessary outsourcing and manipulations. 


\title{
European Survey of Enterprises on New \& Emerging Risks (ESENER)
}

\author{
by invitation only \\ Chair: lavicoli, S. \\ National Institute of Occupational Safety and Prevention (ISPESL), Italy
}

\section{European Survey of Enterprises on New and Emerging Risks (ESENER): How is occupational safety and health managed in European workplaces?}

\author{
Rial-Gonzalez, E., Cockburn, W. \& Irastorza, X. \\ European Agency for Safety and Health at Work (EU-OSHA)
}

EU-OSHA's European Survey of Enterprises on New and Emerging Risks (ESENER) asks both managers and workers' health and safety representatives about the way health and safety risks are managed at their workplace, with a particular focus on psychosocial risks, i.e. work-related stress, violence and harassment. In spring 2009 a total of 28,649 managers and 7,226 health and safety representatives were interviewed in the 31 countries covered: the EU-27 as well as Croatia, Turkey, Switzerland and Norway.

Developed with the support of governments and social partners at European level, ESENER aims to assist workplaces across Europe to deal more effectively with health and safety and to provide policy makers with cross-nationally comparable information relevant for the design and implementation of new policies. As well as looking at management practices, ESENER explores in detail how workers are involved in the management of safety and health at work, which is an important factor in the successful implementation of preventive measures at workplace level.

\section{Key findings on OSH management}

As our society evolves under the influence of new technology and of shifting economic and social conditions, so our workplaces, work practices and production processes are constantly changing. These new work situations bring with them new and emerging risks and challenges, which in turn demand political, administrative and technical approaches that ensure high levels of safety and health at work.

The findings from ESENER ${ }^{1}$ suggest that European enterprises use formal policies and procedures to address both general occupational safety and health $(\mathrm{OSH})$ issues and psychosocial risks, but also use more informal ad hoc actions, especially when it comes to psychosocial risks. Some overall conclusions may be drawn on the basis of the survey findings:

- Formal OSH policies are more frequent in larger establishments and, by country, in Ireland, the United Kingdom, the Netherlands and the Scandinavian countries compared to southern

${ }^{1}$ ESENER does not include establishments with fewer than 10 workers, nor does it cover agriculture, forestry and fishing (NACE A), households (NACE T) and extraterritorial organisations (NACE U). 
European countries, the newer Member States and the candidate countries. This might be due to differences in the awareness, knowledge and custom of dealing with OSH in these countries.

- Enterprises that do not have an OSH policy or do not carry out risk assessments or similar measures $^{2}$ cite that these are not necessary or that they lack expertise as the key reasons. This appears to be more frequent among smaller enterprises and in certain countries. Interestingly, legal complexity is not reported to be a main obstacle for the adoption of OSH policies.

- Risk assessments or similar measures are more likely to be carried out by enterprises having a health and safety representative and in larger establishments, as well as in the more hazardous sectors. Employee representation appears to be a key driver for addressing OSH issues.

- More than one third of establishments - particularly the smaller ones- outsource risk assessments to external providers. There are, however, important country differences, with very low outsourcing being reported in Denmark, the United Kingdom, Sweden and Estonia, even among the smaller establishments.

- In about $40 \%$ of establishments OSH issues are regularly raised at high level management meetings, while line manager's involvement in OSH management is reported to be very high or quite high in the majority of establishments (75\%). This is promising as line manager involvement is a key factor in the implementation of good OSH practices.

- Accidents, musculoskeletal disorders (MSDs) and work-related stress are the principal OSH concerns for European enterprises. Violence, and especially harassment, are reported to be a major concern in a fairly large number of enterprises.

- Fulfilment of legal duties and requests from employees appear to be the main drivers for addressing both $\mathrm{OSH}$ in general and psychosocial risks.

- Managers recognise that employee participation is a key success factor both for OSH and psychosocial risk management and, therefore, the role of the social partners remains crucial for the implementation of effective measures.

The survey's specific findings with regard to the management of psychosocial risks are covered in a related session at the conference. The results of the survey will be launched officially in early June, with the full descriptive report, a summary in 22 languages, and an online mapping tool to analyse the results. The full datasets will be available to researchers through the UK Data Archive from mid-June. Secondary analysis reports have already been commissioned to explore four main issues: factors associated with effective management of $\mathrm{OSH}$; factors associated with effective involvement of workers; factors associated with effective management of psychosocial risks; and management of psychosocial risks - drivers, obstacles, needs and measures taken.

More information at: www.ESENER.eu

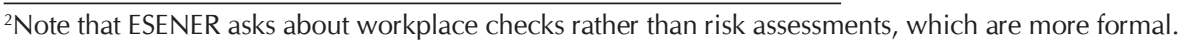




\title{
European Survey of Enterprises on New \& Emerging Risks: Key findings on psychosocial risk management at the workplace
}

\author{
'Leka, S., 'Jain, A., ${ }^{2}$ Houtman, I., ${ }^{2}$ Bakhuys-Roozeboom, M., ${ }^{3}$ Ertel, M., ${ }^{3}$ Pech, P. \& \\ ${ }^{4}$ Riedmann, A.
}

${ }^{1}$ University of Nottingham, UK; ${ }^{2}$ TNO, Work \& Employment, the Netherlands; ${ }^{3}$ Federal Institute for Occupational Safety \& Health, Germany; ${ }^{4}$ TNS Infratest Sozialforschung, Germany

EU-OSHA's Europe-wide establishment survey on new and emerging risks (ESENER) explored the views of responsible actors (managers and workers' health and safety representatives) on how health and safety risks are managed at their workplace. The survey had a particular focus on psychosocial risks. The survey was conducted among establishments with 10 or more employees in the 27 current EU countries plus Croatia, Turkey, Norway and Switzerland. It covers both private and public organisations from all sectors of activity except for agriculture, forestry and fishing, private households and extraterritorial organisations and except for micro-enterprises with less than 10 employees. Data was collected through computer assisted telephone interviewing (CATI). In each establishment surveyed, the highest ranking manager responsible for the coordination of health and safety at work was interviewed. Additionally to the management interview, interviews with the workers' health and safety representative were conducted wherever a formally designated representative with specific responsibility for the safety and health of workers existed.

European enterprises reported using both formal policies and procedures and also informal ad hoc actions to address both OSH issues and psychosocial risks in particular. More ad hoc actions were reported to be used to address the latter. Findings indicate a possible lack of awareness in smaller establishments as concerns OSH in general and psychosocial risks in particular.

Follow-up to risk assessments or similar measures were reported to be centred towards more traditional issues (equipment, work environment and training); however work organisation issues were also reported by a significant percentage of the respondents. This could be indicative of higher concern with new and emerging risks in the modern work environment.

Enterprises make more use of traditional OSH expertise (safety experts and occupational doctors) and less of more modern OSH specialisations such as ergonomists and psychologists. Larger enterprises consistently reported using these experts more. Smaller enterprises have less access to OSH expertise and most enterprises do not have the benefit of multidisciplinary OSH services. Findings also indicated that in some countries a variety of OSH experts are used by enterprises while in others there is a notable minimal use of different types of experts.

Accidents, musculoskeletal disorders and work-related stress are the key OSH concerns for European enterprises. Violence and especially bullying and harassment were reported to be a major concern in a fairly large number of enterprises. Smaller establishments report less often that any risks are a major or some concern which raises questions about the real presence of risks or risk awareness by establishments. The profile of psychosocial risks reported across countries indicates that time pressure, lack of employee control in organising their work and job insecurity are the key psychosocial risk concerns reported by managers. However, less than a third of establishments surveyed had procedures in place to deal with bullying and harassment, work-related violence or work-related stress. These were more frequently found in larger 
establishments, in Ireland and the UK, and in Scandinavian countries and the Netherlands. This finding could be linked to higher awareness in larger companies and the specific countries.

Fulfilment of legal duties and requests from employees were shown to be the main drivers for addressing health and safety (OSH in general as well as psychosocial risks in particular). The most important barriers to address psychosocial risks in establishments relate to the perceived sensitivity of the issue, together with lack of awareness and lack of resources. Employee participation is a key success factor both for OSH and for psychosocial risk management. 


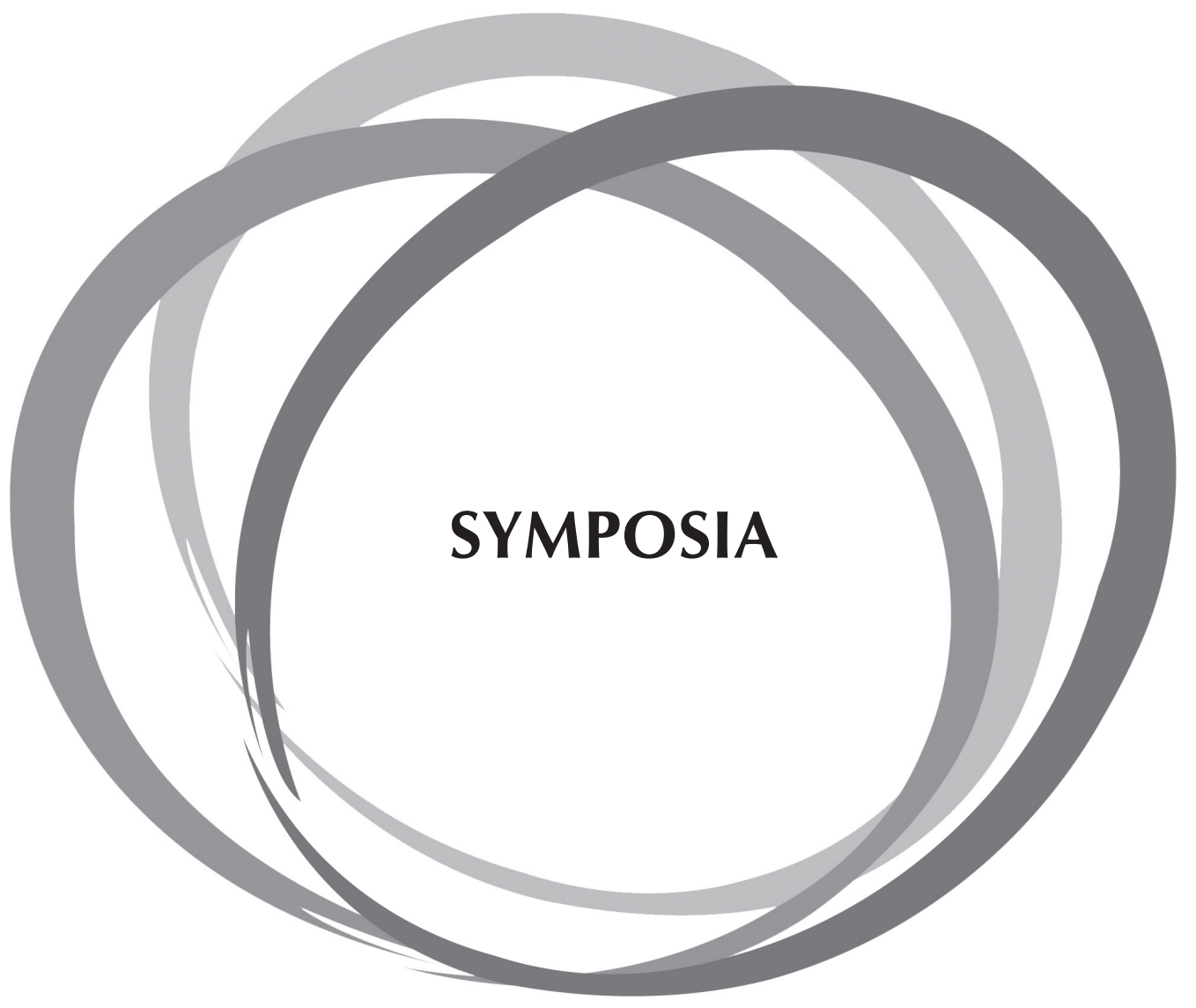




\title{
Organisational Health Development
}

\author{
Chair: Bauer, G.F. \\ University of Zurich \& ETH Zurich, Switzerland
}

Objectives: In our complex, fast changing knowledge society, health is strongly influenced by the interaction of individuals with organisations. Thus, disciplinary, expert driven approaches to occupational health have limitations. Organisations themselves need to know and improve their continuous influence on health of their employees. To shift the focus of attention from individual employees, workplaces and occupations to the entire organisation, our symposium proposes the approach of "Organisational Health Development" (OHD) as a re-framing of occupational health interventions - advancing our earlier proposal called "Occupational Health Management" (Bauer \& Jenny, 2007).

Methods: The OHD approach has been developed based on the collaboration between the organisational health research group and the consulting centre of our division. It is based on both literature reviews and the overall empirical findings of several large-scale organisational health intervention studies conducted by this research-practice partnership. The symposium covers both the conceptual basis and implications for appropriate indicator development and evaluation research approaches.

\section{Results - content of the symposium}

1. OHD model: This paper provides a common group action theory for organisations, researchers and practitioners in regard to OHD. Based on models of general health development, systemic management and organisational change, it shows how health is created by continuous interactions between persons and organisations - and how OHD can improve these.

2. Organisational health capacity: In order to achieve sustainable OHD, building organisational health capacity is needed. This paper discusses how to conceptualize capacity building in an OHD context. For now, we suggest organisational skills, volition, opportunities and norms as key dimensions of organisational health capacity to be considered in planning and evaluating OHD interventions.

3. Key indicators of organisational health capacity: This paper presents health-oriented readiness for change as a key aspect of organisational health capacity. We distinguish this readiness on an individual and organisational level in order to identify where it needs to be increased as a first step of OHD. Validation of the indicator and relation to important antecedents and outcomes are presented.

4. Evaluation of OHD: To be effective, OHD needs to be tailored to the complex situation of organisations. Based on a large-scale intervention study, the paper presents how such context-specific interventions can be evaluated based on a generic OHD outcome model and related indicators.

Implications: Implications of the OHD approach for future organisational health research and practice will be discussed with the audience. 


\title{
Paper 1:
}

\section{Organisational Health Development Model}

\author{
Bauer, G.F., Hoffmann, S., Müller, F., \& Jenny, G.J. \\ University of Zurich \& ETH Zurich, Switzerland
}

Objectives: Based on earlier conceptualizations, we define Organisational Health Development $(\mathrm{OHD})$ as the continuous improvement of organisational structures and processes aiming simultaneously at the health of employees and of the organisation. Supporting such an OHD process first of all requires a common group action theory where organisations, practitioners and researchers can equally relate to.

Methods: The OHD model builds on a generic health development model, organisational change models and the generic New Management Model of St. Gallen (NMSG) (Rüegg-Stürm, 2003), which is committed to structuration theory, a systemic viewpoint, social constructivism and organisational ethics. Based on these models, our OHD model focuses on those elements particularly relevant to health development of employees and of the organisation.

Results: The OHD model shows the organisational system as organisational structure, strategy and culture in interaction with individual competence, identity and behaviour, contributing to individual health, job quality and organisational sustainability as key success criteria for OHD. The interaction encompasses both factual business-/management-/support processes as well as social processes between managers, employees and customers. These interactions are influenced by and in turn shape both individuals and the organisation. Further the model shows customers, society, financers and private life as relevant environment of the organisation. Finally, the model contains the OHD process in the centre, which aims to improve the interactions within the organisation and with its environment.

Implications and Conclusions: The OHD model is expected to focus attention on key aspects to be improved and key outcomes to be expected by systematic OHD. As the OHD model links better to the logic of organisations it should particularly facilitate to consider key aspects of OHD during routine management and job tasks. For practitioners and researchers, the model is expected to facilitate hypothesis building and testing about possible interactions as well as positive and negative (side-) effects of OHD interventions. The practical application and usefulness of the OHD model will be tested in a currently planned intervention research project.

\section{Paper 2:}

\section{Organisational Health Capacity: The Role of Capacity Building in the Organisational Health Development Process}

\author{
Hoffmann, S., Müller, F., Jenny, G.J., \& Bauer, G.F. \\ University of Zurich \& ETH Zurich, Switzerland
}

Objectives: Sustainable Organisational Health Development is a process that has to be maintained over time. Therefore we identified organisational capacity as a precondition. While 
the concept of "capacity building" has emerged in the field of health promotion, a linkage to the organisational logic, the organisational stage of development and well-based indicators are still required. Capacity building is defined as the development of knowledge, skills, commitment, structures, systems and leadership to enable effective health promotion (Eriksson, 2007). We will show, how to integrate and measure capacity building in an Organisational Health Development Process.

Methods: We reviewed several established capacity building concepts and determined the relation to our Occupational Health Model (Bauer \& Jenny, 2007). Additionally, we integrated the main findings of four intervention research projects. By focusing on applied implementation strategies and dissemination at company and intercompany level, we identified key aspects of health capacity on the organisational level. We will point out differences between capacity building and related concepts, such as competence building, resource allocation, option for action, achievement and functioning. Finally we will integrate capacity building in our Organisational Health Development Model.

Results: For now, according to Pelikan (2007), we suggest four dimensions of organisational health capacity $(\mathrm{OHC})$ : skills, volition, opportunities and norms. Indicators and criteria for measuring and developing $\mathrm{OHC}$ can be deduced from it, for example health orientation, readiness for change and employee orientation.

Implications and Conclusions: We benefit from the concept of $\mathrm{OHC}$ as we distinguish between individual and organisational level and elaborate interaction as a main focus in Organisational Health Development Projects. We discuss how OHC stabilizes and critically removes barriers between individual and organisational learning. The role of $\mathrm{OHC}$ in the Organisational Health Development Process is both initial position as well as an achieved outcome. OHC enfolds both processes of learning, adoption and processes of change. OHC strengthens our organisational health model, emphasizing the focus on interaction.

\title{
Paper 3:
}

\section{Key indicators of organisational health capacities: operationalization, validity and empirical relations with predictors and outcomes}

\author{
Müller, F., Jenny, G.J., Hoffmann, S., \& Bauer, G.F. \\ University of Zurich \& ETH Zurich, Switzerland
}

Many variables contribute to the effectiveness of the implementation of organisational changes. For the success of organisational health development it is important that an organisation possesses a certain health-oriented readiness for change on an individual and organisational level. Understanding how employees perceive their own health-oriented readiness for change and the readiness for change of their organisations is essential in order to comprehend the change process and the subsequent effects on health outcomes in the course of organisational health development interventions. Thus, we will present our conceptualization, operationalization and validation of these constructs within the framework of a large-scale organisational stress management intervention in Switzerland. 
Methods: A longitudinal stress management intervention study in 9 medium- and large-scale enterprises in Switzerland will be the basis for these analyses. Overall, in 2008/09 3703 employees completed a comprehensive baseline questionnaire, covering a variety of work and health-related topics with validated instruments. This questionnaire included our newly developed measure of individual and organisational health-oriented readiness for change for which validity and reliability analyses were conducted.

Results: Readiness for change is a valid indicator for change initiatives on the individual and organisational level. Factor analyses resulted in two factors, depicting an individual and organisational dimension of health-oriented readiness for change. The importance of the construct is highlighted as we found crucial relationships with antecedents (e.g. self-efficacy) and outcomes (e.g. stress and health).

Implications: Individual and organisational health-oriented readiness for change is a promising indicator in assessing, developing and evaluating organisational health capacity in the context of organisational health development projects. It permits to identify groups and sub-units within companies in which specific organisational health capacity building is needed.

Conclusions: Employees' readiness for change is an important factor in understanding sources of resistance to health-oriented change and it is expected to be important to tailor interventions to increase individual and organisational readiness for change. In the future, longitudinal analyses will be applied to study a potential causal impact of these indicators within organisational health development processes.

\title{
Paper 4:
}

\section{Evaluation of an Organisational Health Development (OHD) Project}

\author{
Jenny, G.J., Müller, F., Inauen, A., \& Bauer, G.F. \\ University of Zurich \& ETH Zurich, Switzerland
}

Objectives: The project 'SWiNG' was initiated by Health Promotion Switzerland and the Swiss Insurance Association (SVV), to assess the effectiveness and benefits of workplace health promotion, with particular emphasis on reducing stress levels in the organisation. From mid 2008 to end of 2010, an intervention program aimed at developing organisational health is being implemented and evaluated in 9 pilot companies with a total of 5'500 employees. The evaluation of the project SWiNG assesses the effectiveness of the implemented project architecture regarding individual and organisational health determinants as well as health status. Further, the financial benefit (ROI) is estimated and procedural knowledge on health-oriented organisational change is generated.

Methods: To structure the great amount of information generated in nine company-specific development processes led by three consultancy agencies, the evaluation grounds its work on a generic OHD outcome-model. It further considers established evidence on work and health, optimal stakeholder involvement, criteria for assessing the potential public-health impact 
(RE-AIM-criteria; Glasgow et. al, 2003), relevant constructs depicting change in the working environment, including work-related sense of coherence (Work-SoC; Bauer \& Jenny, 2007), health orientation and readiness to change, and contextual factors. Longitudinal data is collected through employee surveys on three occasions, complemented with qualitative data from interviews, group discussions and observations.

Results: Preliminary analysis using structural equation modeling is currently being conducted with baseline data from the employee surveys. For the first project year, the evaluation focuses on the organisational conditions in which the project develops, the range of organisational health demands and resources, and the company-specific implementation of the common project architecture. Here, qualitative data show how in each company the initially devised project architecture has been adapted by the consultants to mobilize energy for the change process and lead the project to success.

Conclusions: The experiences and findings from the first year of SWiNG advance the field of organisational health development (OHD): Under the roof of a common project architecture innumerous interactions between a dozen consultants and a multiple of company members have taken place. These interactions gradually shaped the company maps for OHD and led to more adequate and specific ways to walk these paths. OHD can learn from this self-organisation between consultants and clients. For OHD evaluation research, we learn how company-specific, adaptive processes can be structured and assessed with a common OHD model and OHD relevant criteria. 


\title{
Age effects in Work \& Organisational Psychology: Young and older workers
}

\author{
Chair: Brenninkmeijer, V. \\ Utrecht University, the Netherlands
}

\section{Paper 1:}

\section{Hindrances, Challenges, and Resources at Work in Relation to Psychological Well- being: Does Age Play a Significant Role?}

\author{
${ }^{1}$ Van den Broeck, A., ${ }^{2}$ Notelaers, G., ${ }^{3,4,5}$ Van der Heijden, B.I.J.M., ${ }^{6}$ De Lange, A.H., \\ \& ${ }^{1}$ De Witte, $H$. \\ ${ }^{1}$ K.U. Leuven, Belgium; ${ }^{2}$ University of Bergen, Norway; ${ }^{3}$ Maastricht School of Management, \\ the Netherlands; ${ }^{4}$ Open University of the Netherlands, the Netherlands; ${ }^{5}$ University of \\ Twente, the Netherlands; ${ }^{6}$ University of Groningen, the Netherlands
}

Introduction: Despite a growing managerial and scholarly awareness of an increasing age diversity in the workforce (De Lange, Taris, Jansen, Kompier, Houtman \& Bongers, in press), few studies have examined whether inter-individual age differences may alter how workers perceive and respond to their work environment. For example, within the job characteristics literature, age has seldom been taken into consideration (Griffiths, 1997), and has often been treated as a control variable (De Lange, Taris, Kompier, Houtman, \& Bongeres, 2003). The aim of the current study was therefore to investigate potential age differences in the realm of the Job Demands-Resources model in relation to worker well-being (Bakker \& Demerouti, 2007;De Lange et al., in press; Matthews, Bulger, \& Branes-Farell, in press) In particular, we build upon a recent expansion of this well-established model (Van den Broeck, Vansteenkiste, De Cuyper, \& De Witte, in press) wherein job hindrances (e.g,. role conflict and emotional demands), job challenges (e.g., workload and cognitive demands), and job resources (e.g., task autonomy and social support) yield health-impairing, mixed and health-enhancing relationships, respectively with workers' well-being in terms of exhaustion and vigour as outcomes.

As it is important to take a life-stage perspective in examining workers' age (Levinson, 1978) we tested this model in three separate age groups: (1) workers aged between 18-35 years (younger), (2) workers between 36-45 years (mid-career), and (3) workers of 46 years and older (older) (Schultz \& Adams, 2007). We expected to find the three factor structure among the job characteristics in each of the age groups, which would attest to the validity of the model. However, based on life-span theory (Baltes, Staudinger, \& Lindenberger, 1999), differences in mean levels and strengths of the structural relationships between the job characteristics' categories and workers' functioning were hypothesized.

Method: To test our hypothesis, we applied Multiple-Group analysis (Lisrel 8.80) using a sample of police officers, a vital group of employees in society which may represent the growing group of service workers $(\mathrm{N}=3446)$.

Results: CFAs confirmed the three-factor structure of the distinguished job characteristics in each of the three age groups. MANOVAs indicated that older workers experienced more job 
hindrances compared with mid-career and younger workers, whereas the younger workers experienced less job challenges and exhaustion compared to the other age groups. Finally, multiple group SEM analysis indicated that a variant model showed the best fit to the data, which points out important age differences.

Conclusions: Our results confirm the validity of the extended JD-R model for different age groups. They also highlight particular age differences in the experience of job characteristics and the importance of using a life span perspective in studying and applying job design.

\title{
Paper 2:
}

\section{The forgotten Potential of Older Employees: Attitudes, Strategies and Well-being of Older Employees in Service Interactions}

\author{
Machowski, S., Zapf, D., Ohly, S., Bernhardt, L., \& Trumpold, K. \\ Goethe-University Frankfurt, Germany
}

In the past, research on the elderly has focused on their increasing deficiencies in basic cognitive and sensori-motor skills. In contrast, only limited research exists on the development of socioemotional competences of the elderly despite these competences being required in every workplace, particularly in the service sector. In the ongoing study our central question being: compared to younger employees, which advantages and disadvantages do older employees have in service interactions regarding social and emotional work demands?

Based on theoretical concepts and empirical findings from areas such as stress research, emotion regulation and the psychology of wisdom we hypothesise that older employees are better at the intra-psychological regulation of emotions and use conflict avoidance and cognitive change strategies such as deep acting more often than younger employees. Therefore they can master social and emotional demands in service interactions as well as, or even better than, their younger colleagues. In addition, we expect that older employees show higher job satisfaction, higher affective commitment and higher customer orientation, which supposedly has positive effects on service work. The better regulation strategies should lead to better well-being of older employees compared to younger.

The hypotheses will be tested in a sample of 300 service employees from different service occupations using self-ratings. Antecedents of the organisation, antecedents of the individual, attitudes and strategies of the individual, and well-being are assessed using established instruments.

Preliminary results based on a sample of 82 service employees reveal that older employees report higher affective commitment and less role-conflict. They more often use anticipative deep acting, a subscale of deep acting, and more often avoid conflicts than the younger. Older employees also report less emotional exhaustion. These results so far support our hypotheses. We conclude that older employees show the same or better social and emotional competences in service interactions, especially with regard to emotion regulation.

Limitations of the study and implications for research and practice will be discussed. 


\title{
Paper 3:
}

\section{A Self-Regulatory Perspective on Associations between Age Attitudes, Self- Categorization and Emotional Exhaustion}

\author{
${ }^{1}$ De Lange, A.H., \& ${ }^{2.3 .4}$ Van der Heijden, B.I.J.M. \\ ${ }^{1}$ University of Groningen, the Netherlands; ${ }^{2}$ Maastricht School of Management, the \\ Netherlands; ${ }^{3}$ Open University of the Netherlands, the Netherlands; ${ }^{4}$ University of Twente, \\ the Netherlands
}

Introduction: In view of the world-wide graying of the workforce (De Lange, Taris, Jansen, Kompier, Houtman, \& Bongers, 2010), we are facing a scenario that presents critical challenges for numerous stakeholders (e.g., politicians, employers, managers, career counselors, and employees) across countries and industries (Alley \& Crimmins, 2007). One of these challenges is preventing possible mental health effects of negative age attitudes held by managers and employees themselves (Van der Heijden, De Lange, Demerouti, \& Van der Heijde, 2009). Based on earlier social psychological theories [e.g., self-categorization theory of Tajfel (1978)], and life span developmental theory [e.g., Selection Optimization with Compensation (SOC) model of Baltes, Staudinger and Lindenberger (1999)], we want to address the effects of different agerelated variables (age attitudes, self-categorization, and regulatory focus) in relation to emotional exhaustion. More specifically, we want to test whether:

1a. Negative age attitudes, self-categorization with older workers, and a prevention focus are positively related to emotional exhaustion; and $1 \mathrm{~b}$. A prevention focus plays a moderating role in the aforementioned associations, such that the negative impact of age attitudes and selfcategorization is reduced in case of a higher prevention focus.

Method: 217 Dutch blue- and white collar employees participated in a study on the attitudes of older workers. 67.3 percent was male, and their calendar age ranged from 21 to 70 years $(M=53.6$ years, $S D=9.8)$. All included scales revealed good psychometric qualities. In the preliminary analyses we controlled for age, gender, level of education and job tenure.

Results: The results of multiple hierarchical regression analyses revealed positive significant main effects of negative age attitudes and prevention focus in relation to emotional exhaustion, but no significant effects of self-categorization were found. Furthermore, prevention focus appeared to play a moderating role in the relationship between age attitudes and emotional exhaustion (reducing the negative impact of age attitudes), yet did not have an effect in case self-categorization was included as the predictor variable.

Discussion: Our results underline the need to incorporate age attitudes, and to add self-regulatory processes into occupational health models. Given the moderating effect of prevention focus, a factor that, in general, appears to increase with age (Baltes, Staudinger, \& Lindenberger, 1999), it is important to hypothesize relationships for distinguished age groups. During the presentation we will further discuss theoretical as well as practical implications of the results found. 


\title{
Paper 4:
}

\section{Fresh and Healthy? Well-being, Health, and Performance of Young Employees with Intermediate Education}

\author{
${ }^{1}$ Akkermans, J., ${ }^{2}$ Brenninkmeijer, V., ${ }^{1,2}$ Blonk, R. \& ${ }^{2}$ Koppes, L. \\ ${ }^{1}$ Utrecht University, the Netherlands ; ${ }^{2} \mathrm{TNO}$ Work \& Employment, the Netherlands
}

This study was conducted to gain more insight into the well-being, health and performance of young intermediate educated employees. First, we compared employees with low education (9 years or less), intermediate education (10-14 years of education), and high education (15 years or more) on a number of factors related to well-being, health, and performance at work. Second, determinants of well-being, health and performance were examined for the intermediate educated group, based on Demerouti's Job Demands-Resources model.

Data from the Netherlands Working Conditions Survey 2007 were used: the largest working conditions survey in the Netherlands. ANOVAs with post-hoc Bonferroni correction and linear regression analyses were used for the analyses.

Young intermediate educated employees differed from young high educated employees with regard to job demands, job resources and health. They reported less demands, but these demands were nevertheless associated with well-being and performance. They also reported less resources, while these resources were strongly associated with their health and performance: both directly and indirectly via job satisfaction and emotional exhaustion.

Limitations of the study are that cross-sectional data were used and the theoretical model was tested using regression analyses. In a follow-up study, longitudinal data and structural equation modelling will be used. The study adds to the limited knowledge on young employees with intermediate education and gives insight into the processes that are important for their wellbeing, health, and performance. The study shows that this group deserves the attention of both researchers and professionals. 


\title{
Psychologically Healthy Workplaces \& Corporate Social Performance: Applicant, Employee, and Organisations Perceptions and Outcomes
}

\author{
Chair: Day, A. \\ Saint Mary's University, Canada
}

There has been an increased interest in developing workplaces that are psychologically healthy and that engage in corporate social responsibility. This interest is likely fuelled by a better understanding that employees who suffer from poor well-being tend to cost the organisation in terms of absenteeism, turnover, and decreased performance (e.g., Casio, 1991; Kuoppala, Lamminpaa \& Husman,2008), and that aspects of the work environment are able to enhance the well-being of employees (e.g., Kelloway \& Barling, 1991; Grawitch, Trares, \& Kohler, 2007; Quick, Quick, Nelson \& Hurrell, 1997). A healthy workplace incorporates practices and policies that ensure a safe and healthy physical environment, help promote healthy employee behaviours and lifestyles, and facilitate a positive psychosocial environment (e.g., Burton, 2004; Kelloway, Teed \& Prosser, 2008). Corporate social performance incorporates corporate social responsibility and responsiveness, and it involves activities and practices that benefit the community and society in general Carroll, 1991). It is directed at positive behaviours toward the environment as well as toward employee and community relations.

This symposium involves different perspectives on the impact of workplace practices on applicant reactions, employee well-being, and organisational performance. The first presentation involves a lab-based study assessing the impact of healthy workplace practices and corporate social policies on applicant perceptions. The second presentation also has implications for recruitment: It examines the extent to which different applicant characteristics may impact one's view of healthy practices. The third presentation examines the impact of employees' perspectives of their organisation's healthy and socially responsible practices on individual health and satisfaction. Finally, the last presentation examines the link between healthy workplace practices and organisational performance and employee functioning.

\section{Paper 1:}

\section{Psychologically Healthy Workplaces, Corporate Social Performance, and Applicant Attraction}

\author{
Catano, V. \& Morrow, H. \\ Saint Mary's University, Canada
}

Objectives: Organisational recruitment campaigns are geared to attracting high quality applicants (Carless \& Imber, 2007; Grawitch, Tares \& Kohler 2007). Applicant attraction is defined as an individual's perceptions of or interest in an organisation; it is a desire to work for an organisation (Allen, Mahto, \& Otondo, 2007). Individuals who perceive congruence of their values and an organisation's policies are more likely to be attracted to that organisation. Corporate social policies (CSP) on the environment, employee relations, community relations and diversity influence applicant attraction. The American Psychological Association's psychologically 
healthy workplace (PHW) awards recognize organisations that excel in one of the following areas: Employee recognition, work and family-life balance, employee involvement, employee growth and development, and health and safety (APA, 1999). The current study investigated whether incorporating information on a PHW into recruiting material would lead to greater attraction than material on CSP or just on salary and benefits. We predicted that organisations whose recruiting material contained information on both CSP and PHW policies would be more attractive than organisations that presented only one or none of these types of information.

Method: Four groups of job seekers $(N=116)$ were given recruitment advertisements that contained information on the organisation as follows: Group1. Salary and benefits; Group2. PHW policies plus salary and benefits; Group3. CSP, plus salary and benefits; and Group4. PHW and CSP, plus salary and benefits. Each group saw four vignettes containing recruiting information specific to the group, and then rated the attractiveness of each organisation as a potential employer using a scale developed by Highhouse (2003). The scale had two factors: Good Workplace and Reputation.

Results: Both outcome measures were analyzed using a repeated measures MANOVA. For Good Workplace, our prediction was fully supported. Group4, which saw both CSP and PHW information had significantly higher ratings than when the other types of information were presented alone. For Reputation, Group4 ratings were significantly higher than those for Group 2 (CSP alone) and Group1 (Control) but not higher than Group3, which contained only PHW data.

Implications \& Conclusion: Organisations that seek to attract the best job seekers should emphasize their policies on being a psychologically healthy workplace. Together with information on CSP, PHW information presents the organisation as a good place to work. PHW information, either alone or together with CSP data, enhances the reputation of the organisation and makes it more attractive as a place to work.

\title{
Paper 2:
}

\section{Healthy work as a competitive advantage: What do different generations want from their work?}

\author{
McNally, C. \& Loughlin, C. \\ Saint Mary's University, Canada
}

Objectives: More educated employees expect more from their employers, and companies are seeking a competitive edge in recruiting the best people. Some employers have turned to generational profiles in determining what benefits will best attract and retain employees. Growing interest from HR specialists, managers and researchers is based on the assumption that employees from different generations will differ in their work values, expectations and what they believe comprises a 'good' job. By tailoring rewards and benefits to each generation, some organisations believe they can attain a competitive advantage (seen as critical in these tough financial times). Generational profiles are widely cited in the popular press; however, some suggest that they may be backed by little empirical evidence (Cennamo \& Gardner, 2008). The purpose of the present study was to empirically study any generational differences in the healthy 
work practices expected, valued and used by employees in each of the generations. Our results offer insight into what each generation wants from their work; Information that can contribute not only to recruitment and retention efforts, but help create healthy workplaces in general.

Method: 159 Canadian employees from three generations typically profiled in the popular press (Baby Boomers (1846-1964), Generation X (1965-1980), and Generation Y (1981-2000)) completed a questionnaire based on the American Psychological Association's Psychologically Healthy Workplace Program.

Results: One-way ANOVAS examined group differences. Results suggest that there are differences in the extent to which some healthy workplace practises are valued by each cohort (e.g., educational opportunities). However, overall, there were also many similarities in the extent to which each generation valued, benefitted from, or used healthy workplace practices (e.g., Flexible Work Arrangements). Two of the most valued offerings among all cohorts were Adequate Health Insurance and Compensation, and the practices used most by employees were Self Managed Work teams and Flexible Work Arrangements.

Implications and Conclusion: The results of the present study suggest that while there are some clear differences based on demographic profiles, there are also many healthy work practises that are valued and utilized by all generations in the workplace. Given these and others findings, mangers should practice caution in moving forward based on the profiles championed in the popular press, and psychologists should look beyond these profiles to create healthy workplaces for the most employees possible. Our paper offers some practical suggestions in this regard.

\title{
Paper 3:
}

\section{Corporate social responsibility through healthy work practices: Implications for employees' work-related attitudes, behaviour and wellbeing}

\author{
Sverke, M., Näswall, K. \& Göransson, S. \\ Stockholm University, Sweden
}

Objectives: Extensive changes are taking place in working life as organisations adjust to an increasingly competitive climate. The fact that work intensification and job stress may not only affect employee health and well-being but may also have detrimental consequences for the organisation has attracted growing recognition in recent years. As a result, many organisations are actively implementing healthy work practices and engage in activities that may make them more socially responsible. Corporate social responsibility activities that are directed at employees often involve issues of personal fulfilment and work-related development. The aim of the present study was to evaluate how employees' perceptions of such practices relate to their work-related attitudes, behaviour, and well-being.

Methods: Data are being collected among all 600 employees of an accountant firm that has placed great emphasis on implementing healthy work practices. The data collection is currently being completed and postal questionnaires have been returned by approximately 70 percent of the employees. The survey includes measures on various factors reflecting employees' evaluations of the organisation's work practices, in domains such as opportunities for participation, organisational justice evaluations, supportive leadership, employee recognition, and prospects 
of growth and development at work. The ambition is to supplement the range of self-reported outcome measures (work-related attitudes, behaviour, and well-being) with performance and sickness absence data from company records.

Results: Preliminary results of multiple regression analysis indicate that employee evaluations of healthy work practices may have important implications for their levels of job satisfaction and commitment to the organisation. In addition, the preliminary analyses found healthy work practices to be positively related to work-related behaviour (self-reported performance and intention to remain in the organisation) as well as well-being (mental and physical).

Implications \& Conclusion: Engagement in corporate social responsibility related activities can make an organisation more attractive to both current and prospective employees. Employees would rather choose a company that, for example, has good personnel policies and protects employees' health in comparison with a company that does not. These practices also have a positive impact on employee performance and well-being.

\title{
Paper 4:
}

\section{Psychologically Healthy Workplace Practices: Implications for Organisations and Employees}

\author{
Day, A., Randell, K. \& Kelloway, K. \\ Saint Mary's University, Canada
}

Objectives: Despite the increased interest in psychologically healthy workplaces (PHW), little is known on the extent to which organisations implement initiatives to increase the 'healthiness' of their members. Some PHW literature has emerged on the hypothesized antecedents and consequences of healthy and unhealthy workplaces (e.g., APA, 2009; Grawitch et al., 2007; Kelloway \& Day, 2005). Researchers have called for studies assessing a broader array of practices (e.g., Parker \& Wall, 1998). We must examine organisational factors that enhance the well-being of employees (e.g., Kelloway \& Day, 2005a; Luthans \&Youssef, 2007; Parker et al., 2003). The present study goals are to: (1) examine the structure of PHW measure; (2) identify the usage of PHW initiatives, and (3) link PHW components to organisational outcomes. Method: Organisational representatives from 118 organisations completed an online survey assessing: Organisational Descriptors; Specific Healthy Workplace Practices; General PHW, Financial/ Operational Performance; Employee Attitudes, and Employee Withdrawal Behaviours. Based on healthy workplaces models (e.g., APA, 2009; Kelloway \& Day, 2005; Randell \& Tatarkiewicz, 2008), we assessed several broad PHW factors: employee involvement, work-life balance, employee growth and development, employee safety, employee health, employee recognition, and a culture of respect, communication, and fairness.

Results: A PCA identified three PHW components accounting for 55.7\% of the variance: Positive Communication; Control; \& Health \& Safety. Organisations reported that $45 \%$ of the PHW initiatives listed were utilized in their workplaces. As expected, high-risk industry organisations offered more health practices and more safety practices than low-risk industry organisations, and larger organisations reported to offer more formal PHW practices than smaller organisations. 
Interestingly, size of organisation was negatively related to communication and control.

All PHW components were correlated with financial/operational performance. Both communication and control were associated with employee attitudes, but only control was related to withdrawal behaviours. When controlling for organisational characteristics, the healthy workplace components accounted for incremental variance in financial/operational performance $(16 \%, p<.01)$, employee attitudes $(25 \%, p<.001)$, and withdrawal behaviours $(14 \%, p<.01)$. Communication was uniquely associated with financial/operational performance $(ß=.36, p<.01)$; Control was uniquely associated with attitudes $(B=.41, p<.01)$ and withdrawal behaviours $(B=.47, p<.001)$. Attitudes mediated some of the relationships between healthy components and financial/operational performance.

Implications \& Conclusion: This study provides evidence for the 'business-case' of developing psychologically healthy workplaces, in that all PHW components were associated not only with positive employee attitudes, but also with decreased turnover, and improved financial/ operational performance. 


\title{
Recovery processes: Determinants and Effects on Health, Well-being and Workability
}

\author{
Chair: de Bloom, J. \\ Radboud University Nijmegen, the Netherlands
}

Research in the field of occupational health has consistently demonstrated the adverse impact of stress in the workplace on individuals' health and well-being (e.g., Belkic, Landbergis, Schnall, \& Baker, 2004; Ferrie, Westerlund, Virtanen, Vahtera, \& Kivimaki, 2008; De Lange, Taris, Kompier, Houtman, \& Bongers, 2003). Recovery during nonwork time plays a crucial role in protecting employees against the adverse effects of exposure to job stressors. The essence of recovery is that psychophysiological systems that were activated during work will return to and stabilize at a baseline level, that is, a level that appears in a situation in which no special demands are made on the individual (Geurts, \& Sonnentag, 2006). Recovery after work may occur regularly in-between workdays (e.g., during evening hours and during weekends) and during longer periods of off-job time like vacations, constituting meta- and macro-recovery, respectively (Sluiter, Frings-Dresen, Meijman, \& Van der Beek, 2000).

Although the body of research on recovery is growing, research in this area could be improved by providing more insight in the underlying processes of recovery and the effects of different types of recovery on health, well-being and working ability, and by methodologically stronger designs and measurements. The primary aim of this symposium is to contribute to our understanding of recovery processes by presenting six studies that contribute to recovery research in several ways.

This symposium has a two-fold emphasis: (i) it will focus on processes and factors that may impede or improve recovery processes, and (ii) it will center on effects of different types of recovery on health, well-being and working ability. By using a diversity of research methods (longitudinal field studies, diary studies, experiments), we hope to contribute to our understanding of recovery and its underlying processes.

In the first contribution, Ulla Kinnunen will present the results of a one-year longitudinal field study on 274 Finnish employees. She investigated the effects of job demands and job resources on recovery experiences. The results showed that especially job demands were detrimental to recovery and that in order to promote recovery, work demands should be decreased.

In line with these results, Laurenz Meier will present his findings from a diary study on the short-term effect of effort-reward imbalance at work on recovery processes (psychological detachment and sleep quality in particular), health and well-being. He will also focus on the moderating role of self-esteem within this relationship. His results demonstrated that effortreward imbalance negatively affected particularly psychological well-being in the short run.

The experimental study of Mirjam Radstaak will supplement these findings by focusing on the role of distraction in the process of psychological (thoughts and affect) and physiological recovery (blood pressure and heart rate) from stress.

In the fourth contribution, Sirkku Kivisto will present the results of her project "The Healthy Mind at Work" that seeks to promote effort-recovery balance at work by counseling employees 
and managers on how to enhance control, increase mental and social resilience at work and recovery capacity. In this project, she also identified working conditions that impede recovery processes and that help employees recover more efficiently.

In the fifth contribution, Jessica de Bloom will present her results on vacation, a relatively long and uninterrupted period of absence from work. On the basis of her longitudinal study on wintersports, she explains why vacation, as a naturally occurring event, has recovery-potential and what its short- and long-term effects are on health and well-being.

Finally, Maija Tirkkonen will speak about her study on Vocationally Oriented Medical Rehabilitation as a unique, exemplary form of an intervention for enhancing well-being at work in Finland. It is aimed at employees with signs of fatigue or other risks to their working ability. It intends to maintain and improve working ability and well-being at work on the individual level and reduce early retirement on a societal level.

\title{
Paper 1:
}

\section{Job characteristics and recovery experiences: testing causal relationships across one year}

\author{
${ }^{1}$ Kinnunen, U. \& ${ }^{2}$ Feldt, T \\ ${ }^{1}$ University of Tampere, Finland; ${ }^{2}$ University of Jyväskylä, Finland
}

Objectives: The aim of the study was to shed new light on the relationships between job characteristics and recovery experiences using one-year longitudinal data. Previous cross-sectional studies suggest that job demands prevent and job resources facilitate recovery from job strain. However, there is a lack of longitudinal studies; therefore the relationship is not fully established. In addition, recovery experiences - mechanisms helping to restore energy resources - have not been used as indicators of recovery in the previous studies. Thus, our study contributes to the literature by investigating normal causal (job characteristics $\rightarrow$ recovery), reversed causal (recovery $\rightarrow$ job characteristics) and reciprocal (job characteristics $\rightarrow$ recovery) relations between job demands (time demands, demands in decision making, weekly working hours), job resources (job control, social support from coworkers, justice of the supervisor) and four recovery experiences (psychological detachment, relaxation, mastery and control during non-work time).

Methods: The data were collected among 274 Finnish employees from a variety of different jobs in two phases; first, in spring 2007 (Time 1) and, second, in spring 2008 (Time 2) using questionnaires. Of the participants, $57 \%$ were women, the average age was 45.9 years (SD $=10.9$ ), and $60 \%$ had an academic degree. Job characteristics were measured using the QPSNordic Questionnaire (Lindström et al., 2000) and recovery experiences using the Recovery Experience Questionnaire (REQ, Sonnentag \& Fritz, 2007). The main statistical method used was SEM based on the Mplus statistical program.

Results: The results showed that both job characteristics and recovery experiences were moderately stable across one year (stability coefficients ranging from 0.61 (control during non-work time) to 0.85 (job demands). Of the models tested (stability model, normal causal model, reversed causal model, and reciprocal model), the normal causal model turned out to be the best fitting model. 
According to this model, high job demands at Time 1 were linked to low levels of psychological detachment $(ß=-.0 .24)$ and relaxation $(ß=-.0 .18)$ at Time 2 .

Conclusions and implications: The relationships between job characteristics and recovery experiences seem to follow the normal causality. Of the job characteristics, job demands seem to be more important than job resources; they had lagged relationships to recovery experiences over one year. Thus, in order to promote recovery it is crucial to decrease demands at work.

\title{
Paper 2:
}

\section{The Moderating Role of Self-Esteem on the Effect of Effort-Reward Imbalance on Recovery - A Diary Study}

\author{
Meier, L.L. \& Semmer, N.K. \\ University of Bern, Switzerland
}

Objectives: It is well-established that a lack of recovery after work has negative effects on health (e.g., Geurts \& Sonnentag, 2005) and that work stressors may impede the recovery process (e.g., Sonnentag \& Bayer, 2005). An impressive body of research shows that effort-reward imbalance (ERI) is related to an impaired recovery process and well-being on a chronic level (see Siegrist, 2002). However, little is known about short-term effects of ERI on recovery. Furthermore, little is known about individual differences with regard to vulnerability to the experience of ERI. As ERI signals a lack of respect and because people with a low self-esteem react particularly strongly to signs of other's devaluation (e.g., Nezlek, Kowalski, Leary, Blevins, \& Holgate, 1997), we assumed that ERI is more strongly related to impaired psychological detachment and sleep among individuals with low self-esteem than among individuals with high self-esteem.

Methods: 122 participants filled in a general survey and completed daily surveys over 10 working days. Before starting work, participants reported sleep quality of the last night (Buysse, Reynolds, Monk, Berman, \& Kupfer, 1989), and psychological detachment at the previous evening (Sonnentag \& Fritz, 2007). In the afternoon, they reported their ERI during the work day (Van Yperen, 1996).

Results: We used multilevel random coefficient models to regress psychological detachment and sleep quality on ERI. We controlled for sex, age, education, job tenure, supervisory function, and trait self-esteem (Level 2 variables), and for the baseline of the outcomes (Level 1 variables). In accordance with our hypothesis, ERI was negatively related to psychological detachment. In contrast, there was no main effect on sleep quality. However, both relationships were qualified by a significant cross-level interaction (ERI (Level 1) x Self-Esteem (Level 2)). Contrary to the assumption, ERI was particularly strongly related to psychological detachment and sleep quality among individuals with high self-esteem.

Implications and conclusions: The experience of an ERI during work time has negative effects of the recovery process, especially for people with a high self-esteem. ERI was only related to sleep quality for people with high self-esteem and it was more strongly related to psychological detachment for people with high rather than low self-esteem. This pattern was unexpected and needs further replication. Nevertheless, the result suggests that self-esteem plays an important role in the recovery process and future studies may not only look at the level but also the stability of the self-esteem (see Kernis, 2005). 


\title{
Paper 3:
}

\section{Physiological recovery from stress. Does distraction matter?}

\author{
${ }^{1}$ Radstaak, M., ${ }^{1}$ Geurts, S.A.E., ${ }^{2}$ Brosschot, J., ${ }^{3}$ Sonnentag, S. \& ${ }^{1}$ Kompier, M. \\ ${ }^{1}$ Radboud University Nijmegen, the Netherlands; '2Leiden University, the Netherlands; \\ ${ }^{3}$ University of Konstanz, Germany
}

Objectives: This experiment explored whether preventing stressful thoughts by providing distracting stimuli could improve cardiovascular (CV) recovery. Hereby, the quality of distraction was taken into account.

Methods: Resting baseline systolic blood pressure (SBP), diastolic blood pressure (DBP) and heart rate (HR) were assessed among 110 participants. After baseline measurement, participants were exposed to a stressful task, i.e., a mental arithmetic task with harassment. Next, participants were randomized to a positive, a negative, a neutral, or no distraction condition, followed by a 10 minute recovery period. Blood pressure and heart rate were continuously measured.

Results: At first, paired t-tests revealed that all CV recovery indicators (SBP, DBP, HR) increased during the stressful task. In addition, a 3(Time: stressful task, distraction period and recovery period) x 4 (Distraction: positive, negative, neutral or no distraction) repeated measures analysis of variance yielded (i) a main effect for Time: a reduction in CV arousal after having conducted the stressful task, and (ii) an interaction effect for SBP: this was hampered for participants in the negative distraction condition during the distraction period. Negative affect hampered the recovery process.

Implications: The results of our research suggest that negative affect is an important obstructive factor in the recovery process.

Conclusions: After exposure to a stressful task, the body seems to recover no matter the quality of the distraction. Negative affect seems to play a more crucial role. Future research in this area needs to take negative affect into account.

\section{Paper 4:}

\section{Promoting the prerequisites for recovery through information, counselling and advising activities in the workplace survey process}

\author{
Kivistö, S., Kallio, E. \& Jalonen, P. \\ Finnish Institute of Occupational Health, Finland
}

Introduction: Working conditions can either improve or hinder mental recovery capacity. "The Healthy Mind at Work" project adapted the Mental Well-Being Impact Assessment (MWIA) procedure for the workplace survey conducted by the Occupational Health Services (OHS) in Finland (MIVA). An essential part of the workplace survey procedure consisted of informing, counselling and advising (ICA) employees, managers, supervisors, and occupational safety representatives on how to promote mental well-being at work. We wanted to improve the ICA procedures in 
workplace surveys by using the MIVA approach in order to 1) enhance control, 2) increase mental resilience and recovery capacity, 3) increase social resilience and recovery capacity, and 4) facilitate participation and promote inclusion.

Objectives: The purpose of our presentation is to discuss the contribution of ICA carried out by $\mathrm{OHS}$ to the learning of health issues, focusing particularly on the promotion of effort-recovery balance at work.

Methods: Together with representatives of four workplaces and the serving $\mathrm{OH}$ units, we sorted out the workplace survey material and assessed the situation using the MIVA frame. When working conditions hindering the recovery process were identified, we helped the workplace to solve the problem using various ICA means.

Results: Our ICA feedback in official reports, workplace meetings and in "occupational health letters" to the workplace was focused on MIVA areas. To enhance shared understanding of stress, each workplace formulated its own definition of harmful stress. To support mental resilience and recovery capacity, we formed a graphic representation of the restorative aspects of the physical work environment survey. To strengthen social resilience and recovery capacity in one workplace, we wrote an $\mathrm{OH}$ letter entitled "Frequent interruptions and intervening tasks at work - what is the background?" Another workplace received an $\mathrm{OH}$ letter about irrelevant and meaningless tasks (illegitimate stressors) or stress as offence to self, in order to overcome problems of participation.

Implications: The ICA approach in OHS is based on the Occupational Health Care Act of Finland, but is unfortunately underused in daily practice. It focuses more on individual counselling, but is scarcely explored on the work place level. Without active ICA activity, the benefits of the workplace survey remain incomplete.

Conclusions: ICA activities are the main vehicle in the workplace survey process to help the workplace to gain health literacy in effort-recovery.

\title{
Paper 5:
}

\section{Effects of vacation from work on health and well-being: Lots of fun, quickly gone}

\author{
${ }^{1}$ de Bloom, J., ${ }^{1}$ Geurts, S.A.E., Taris, T.W., ${ }^{3}$ Sonnentag, S., ${ }^{1}$ de Weerth, C. \& ${ }^{1}$ Kompier, \\ ${ }^{1}$ Radboud University Nijmegen, the Netherlands; ${ }^{2}$ Utrecht University, the Netherlands; \\ ${ }^{3}$ University of Konstanz, Germany
}

Objectives: Our study on vacation (after-) effects focused on two related questions: 1) Do health and well-being of working individuals improve during a vacation?, and 2) How long does a vacation effect last after work resumption?

Methods: In a longitudinal field study covering 7 weeks, 96 workers reported their health and well-being levels 2 weeks before a winter sports vacation, during vacation, and 1 week, 2 weeks and 4 weeks after vacation on 7 indicators. 
Results: Participants' health and well-being improved during vacation on 5 indicators: health status, mood, tension, energy level and satisfaction (average $d=0.54$ ). However, during the first week of work resumption, health and well-being had generally returned to pre-vacation levels.

Conclusions and implications: A winter sports vacation is associated with improvements in self-reported health and well-being among working individuals. However, these effects fade out rapidly after work resumption. We propose a framework for future vacation research and suggest investigating the role of vacation type, duration, and means to prolong vacation relief.

\title{
Paper 6:
}

\section{Vocationally oriented medical rehabilitation increases well-being at work via increased recovery experiences}

\author{
Tirkkonen, M. \& Kinnunen, $U$ \\ University of Tampere, Finland
}

Objectives: This study examined the effects of an early rehabilitation intervention on perceived job demands and resources, individual resources and well-being among Finnish employees. In addition, we looked at whether recovery experiences might mediate the possible improvement in well-being. The intervention aims at maintaining and improving employees' work ability and ensuring a supply of skilled labour force in society.

Methods: The study was conducted among the participants $(n=148)$ of the vocationally oriented medical rehabilitation (VOMR) in one rehabilitation centre during 2007-2009. These participants were managers and experts from different fields. Of the participants, 56\% were women, and the average age was 49 years. The intervention lasted three weeks (three one-week periods) during one year. The intervention consisted of sessions covering work and vocational topics, psychological well-being, and physical activity and ergonomics. The participants answered to a questionnaire every intervention week (i.e., three times) and still 5 months after the intervention. The questionnaire included questions about job demands (i.e., work load, demands for decision making), job resources (i.e., control, climate, social support), personality resources and wellbeing (need for recovery, stress, work engagement, positive mood). Managers and experts that did not participate in any rehabilitation intervention but who could have applied for one were invited to the control group ( $n=34$ ). They answered to the questionnaires three times at the same rhythm as the participants. ANOVA for repeated measures, intervention/control group as a between-groups variable and time as a repeated measure, was used as the statistical method.

Results: Well-being at work was improved among the rehabilitation participants although job demands remained at the same level and job resources increased only marginally. The participants' stress and need for recovery decreased and perceived work ability, health and positive mood increased, when compared to the control group. Recovery experiences (psychological detachment from work, relaxation and mastery) seemed to have a mediating role in the process, for example, the decrease in need for recovery seemed to occur via increased recovery experiences. However, positive changes in personality resources, vitality and life satisfaction occurred in the control group, too. 
Implications: Recovery experiences play an important role in improving well-being.

Conclusions: To conclude, early rehabilitation seems to improve participants' well-being at work. The improvement seems to be linked with the enhancement of recovery experiences. To have more influence on job conditions, too, more co-operation between rehabilitation centers, occupational health care and work organisations is needed. 


\title{
Temporary employment and well-being: New avenues for research
}

\author{
Chairs: De Cuyper, N. \& De Witte, N. \\ K.U.Leuven, Belgium
}

This symposium brings together four studies on the subject of temporary employment; i.e. dependent employment of limited duration. The common stand is that the studies seek to relate temporary employment and well-being, however taking two different approaches.

First, the symposium addresses how contract type relates to well-being, including aspects of job (in)security; a topic that is covered in the contributions by Zsusza Bakk, Jeroen de Jong, Bart Cambré and Nele De Cuyper (Study 1), and Claudia Bernhard-Oettel, Thomas Rigotti, and Michael Clinton (Study 2). The first study demonstrates that the relationship between temporary versus permanent employment, and job security and well-being is conditional upon specific motives for hiring temporary workers. Innovative to this study is the idea that the relationship between contract type and well-being is moderated by factors at the level of the organization. The second study focuses upon the heterogeneity that exists in the temporary workforce, notably in terms of volition. The authors argue that volition associates negatively with job insecurity, and through job insecurity, to well-being. They then go one step further by including also contract prospects as a possible buffer for vis-à-vis job insecurity. This so-called hypothesis of moderated mediation is a particular strength, as is the large sample $(N=1909)$ of temporary workers from six countries.

Second, two other papers portray temporary employment as a moderator of the relationship between perceived employability and engagement (Study 3), or between core self-evaluations and job satisfaction (Study 4). This is addressed in the studies by Nele De Cuyper, Tinka Van Vuuren, Beatrice Van der Heijden, and Barbara Alarco, and by Anne Mäkikangas, Saija Mauno, and Ulla Kinnunen, respectively. In Study 3, the argument is that perceived employability is a personal resource with particular resonance to temporary workers, implying that the relationship between perceived employability and engagement is stronger among temporary compared with permanent workers. The study is based on a sample of 643 Peruvian workers; this context being an added value. The fourth study takes a dispositional approach vis-à-vis well-being: it relates core self-evaluations to job satisfaction, accounting also for employability as a possible mediator. The aim is to investigate whether these relationships are similar or instead different for temporary and permanent workers. This study is based on three cross-sectional samples, thereby presenting possibilities for generalization.

Altogether, we believe that this symposium highlights possible new avenues in the realm of temporary work research. A further strength is that we arrived at an international, global view on temporary employment with data from, in the order of the papers presented in this abstract, Dutch and Belgian workers (Study 1), European workers from six countries (Study 2), Peruvian workers (Study 3) and Finnish workers (Study 4). 


\title{
Paper 1:
}

\section{Temporary versus permanent employment and psychological outcomes: the moderating effect of motives for hiring temporary workers}

\author{
${ }^{1}$ Bakk, Z., ${ }^{1}$ de Jong, J., ${ }^{1}$ Cambré, B. \& ${ }^{2}$ De Cuyper, H. \\ ${ }^{1}$ Tilburg University, the Netherlands; ${ }^{2}$ K.U.Leuven, Belgium
}

Objectives: The relationship between contract type (temporary versus permanent) and wellbeing, and the organisation's motives for hiring temporary workers have attracted much scholarly attention. However, the individual and organisational factors have not yet been studied simultaneously. In response, our aim is to explain the relationship between contract type and well-being, accounting for organisational motives as a possible moderator. By including permanent workers in our analyses, we show (1) that the difference in individual level outcomes (i.e., job insecurity and well-being) can be explained by the organisational strategies for hiring temporary workers, and (2) that organisational strategies targeting temporary workers may affect permanent workers as well.

Methods: The main motives for hiring temporary workers are: labour cost reduction, labour flexibility, hiring specialized skills and replacing core workers. A model for the four motives was fitted using confirmatory factor analysis. In a next step, multilevel analysis was applied using a mixed model. We sampled Belgian and Dutch workers from 60 organisations. Of respondents, 601 were temporarily and 841 permanently employed.

Results: We hypothesized that temporary employment relates negatively to job security and wellbeing when motives related to labour cost reduction and labour flexibility dominate (hypothesis 1). In contrast, we expected that temporary employment relates positively to job security and well-being in organisations where motives related to hiring specialized skills and replacing core workers dominate (hypothesis 2). We found support for hypothesis 1, but not hypothesis 2 .

Implications: Our results have implications for studies about individual level differences between temporary and permanent workers, in the sense that they highlight the importance of organisational level factors. We also see implications for research about externalization strategies and performance: this link can be better understood by analyzing employees' psychological outcomes.

Conclusions: We found evidence that the organisation's motives for hiring temporary workers affect well-being outcomes of both temporary and permanent workers. Well-being and job security of both permanent and temporary workers are higher when the central motive relates to hiring specialized skills. In organisations that predominantly use cost reduction and flexibility motives, well-being is lower among temporary compared with permanent workers. 


\title{
Paper 2:
}

\section{Job insecurity in the temporary workforce: A moderated mediation model linking volition, job insecurity and contract expectations to well-being.}

\author{
${ }^{1}$ Bernhard-Oettel, C., ${ }^{2}$ Rigotti, T., \& ${ }^{3}$ Clinton, $M$. \\ ${ }^{1}$ Stockholm University, Sweden; ${ }^{2}$ University Leipzig, Germany; ${ }^{3}$ Kings College London, UK
}

Objectives: Although temporary work almost inevitably involves job insecurity, recent studies show that not all temporary workers feel job insecure, and also, that job insecurity does not necessarily have negative effects on occupational health and well-being in temporary as compared to permanent workers. The present study probes two possible explanation for this finding related to (a) contract volition and (b) contract prospects, that is, expected prolonged employment after the end of the temporary contract. More specifically, we tested whether high levels of voluntary contract choice associated with lower values of job insecurity feelings, which in turn may explain more positive outcomes (mediation hypothesis). Furthermore, we tested whether contract prospects played an additional role, possibly buffering the proposed negative effects of job insecurity (moderated mediation hypothesis).

Methods: Questionnaire data for this study has been gathered as part of a European project across three sectors (retail, food industry, education) in six European countries (Sweden, Germany, Belgium, the Netherlands, Spain, UK) and Israel. In a total, the sample for this study consists of 1909 temporary workers. The hypotheses of this study were tested with regression analyses applying bootstrapping techniques for more adequate tests of the proposed indirect effects. In these analyses we controlled for country, sector, age, gender and type of temporary contract.

Results: We first tested a simple mediation model, which supported the hypothesis that the association between contract volition and positive outcomes for occupational health and wellbeing was mediated by (lower levels of) job insecurity. Thereafter, contract expectations were added to this simple mediation model in order to test the moderated mediation hypothesis. This hypothesis was also supported, showing that the association between job insecurity and occupational health as well as work attitudes was affected by high levels of contract expectations. However, the direction of this moderation effect was opposite to the hypothesis: High levels of contract expectations strengthened the positive associations between low levels of job insecurity and occupational health and work attitudes.

Implications and conclusions: Theresults supported the idea that volition and contractexpectations are important factors enhancing our understanding of the development and consequences of job insecurity feelings in the temporary workforce. Also, by testing a parsimonious model, this study offers valuable new insights into the mechanisms that explain associations between job insecurity and well-being for temporary workers. 


\title{
Paper 3:
}

\section{Perceived employability and engagement among temporary versus permanent workers}

${ }^{1}$ De Cuyper, N., ${ }^{2}$ Van Vuuren, T., $3,4,5$ Van der Heijden, B., \& ${ }^{1}$ Alarco, B.

\author{
${ }^{1}$ K.U.Leuven, Belgium; ${ }^{2}$ Loyalis Consult, the Netherlands; ${ }^{3}$ Maastricht School of Management, \\ the Netherlands; ${ }^{4}$ Open University, the Netherlands; ${ }^{5}$ University Twente, the Netherlands
}

Objectives: Our main objectives are to investigate (1) perceived employability in relation to engagement, and (2) to account for contract type as a possible moderator. Perceived employability (PE) concerns the individual's perceived chance to get a job in the internal (i.e., internal PE) or the external labour market (i.e., external PE). PE is sometimes portrayed as a resource. In this respect, some authors have demonstrated a positive relationship between PE and well-being. However, few studies have distinguished between internal and external PE. Furthermore, PE has particular resonance in the context of temporary work research: temporary workers have to be employable in order to secure their labour market position, unlike permanent workers who can still rely on job security offered by the employer. This hints at the idea that employability is perhaps more predictive for temporary than for permanent workers' well-being. Accordingly, we test the hypothesis that the positive relationship between both internal and external PE is stronger among temporary compared with permanent workers.

Methods: We tested the hypothesis among a sample of 642 Peruvian workers from eight organisations and using moderated hierarchical regression analysis.

Results: The interaction term between internal PE and contract type contributed in explaining variance in engagement: internal PE was positively related to engagement, and this relationship was stronger among temporary compared with permanent workers. However, the interaction between external PE and contract type was not significant. Moreover, external PE related negatively to engagement.

Implications and conclusions: From this study, two implications stand out. First, internal PE may be a resource that is associated with higher engagement, particularly among temporary workers. The reason is that temporary workers may see internal PE as a stepping stone towards permanent employment in their current organisation. For many temporary workers, stepping stone motives are the most important consideration in accepting a temporary assignment. Second, external PE related negatively to engagement, meaning that external may not be a resource, rather to the contrary: when workers perceive other employment opportunities with another employer, they feel they can easily replicate the deal with the current employer elsewhere. This may have unfavourable consequences. 


\title{
Paper 4:
}

\section{Core self-evaluations and job satisfaction: Mediation by employability?}

\author{
${ }^{1}$ Mäkikangas, A., ${ }^{1}$ Mauno, S., \& ${ }^{2}$ Kinnunen, U. \\ ${ }^{1}$ University of Jyväskylä, Finland; ${ }^{2}$ University of Tampere, Finland
}

Background: Dispositional approach to job satisfaction research suggests that relative stable characteristics of a person determine the level of job satisfaction, regardless of one's job. Core self-evaluations indicated by self-esteem, locus of control, generalized self-efficacy, and high emotional stability is such a personality construct that strongly associates with job satisfaction. Typically the mechanisms linking core self-evaluations and job satisfaction are sought in appraisal processes, therefore the actions employees take as a result of the personality (i.e., qualifications, social capital) have remained under-investigated. Against this background, employability can be seen as a potential factor that mediates the link between personality and job satisfaction.

Aim: The main aim of this study was to investigate the relationships between core self-evaluations, employability, and job satisfaction. The special target was to test whether employability mediates the relationship between core self-evaluations and job satisfaction. These relationships were tested between permanent and temporary employees and between different samples studied.

Methods: The study was based on three cross-sectional samples of employees. Two of these samples were derived from university staff ( $n=1197$ for University 1 and $n=940$ for University $2)$, and the third one from hospitals $(n=916)$. The main statistical method of analysis used was Structural Equation Modelling computed by Mplus statistical package. In addition, the main and interaction effects of the job contract type and the sample studied were examined.

Results: Core self-evaluations showed strong positive relationships with employability and job satisfaction. Employability associated positively with job satisfaction when these two constructs were analyzed separately, but this connection no longer existed in the final model which contained also core self-evaluations. Thus, no evidence was found that employability would mediate the relationship between core self-evaluations and job satisfaction. The associations were mainly similar across the samples. The only difference was that the association between core self-evaluations and employability was weaker in hospital than in universities. No differences were found in the relationships between permanent and temporary workers.

Conclusion: Altogether, the findings suggest that personality measured in terms of core selfevaluations is a strong predictor of both employability and job satisfaction. These associations remained mainly constant across comparison groups, although there was some evidence suggesting that core self-evaluations are a stronger predictor of employability in the university than in hospital context. 


\title{
Is Laughter the Best Medicine? Relations between humour and occupational health
}

\author{
Chairs: ${ }^{1}$ de Lange, A.H. \& ${ }^{2}$ Dikkers, J.S.E. \\ ${ }^{1}$ University of Groningen, the Netherlands; ${ }^{2} \mathrm{VU}$ University Amsterdam, the Netherlands
}

A popular view is that a greater sense of humour enhances psychological well-being and physical health (Kuiper and Nicholl, 2004). Recently, several studies have begun to examine the role of humour in the workplace finding positive relationships with job performance (Avolio, Howell, \& Sosik, 1999), increased employee morale (Gruner, 1997), leadership effectiveness (Priest \& Swain, 2002), group effectiveness (Romero \& Pescoslido, 2008), and even productivity (Clouse \& Spurgeon, 1995). However, these new studies have some limitations. First, there is a paucity of studies that examines the relations between humour and occupational health, and most studies failed to define or examine humour in a multi-faceted manner. Humour can be a double-edged sword in the sense that it can be either harmful or beneficial for the actor and/ or the target. It is, therefore, crucial to distinguish between different types of humour. Martin, Puhlik-Doris, Larsen, Gray, \& Weir (2003), for example, have specified four humour styles (i.e., affiliative, self-enhancing, aggressive, and self-defeating humour). Finally, previous research on humour in the workplace does not test theory-driven mechanisms underlying the associations of humour (styles) with occupational health.

This symposium aims to present relevant insights of new research that: i) examines relationships of (multi-faceted perceptions of) humour with occupational health and well-being in working samples, and ii) tests theory-based mechanisms underlying these associations.

In a first contribution, Dikkers, De Lange, De Witte, and Van den Broeck will present their psychometric test of the previously mentioned four-factor structure of humour styles developed by Martin et al (2003) among a large-scale Belgian employee sample. Secondly, Doosje presents the findings of his study examining the contribution of job demands, job resources and humorous coping styles to the risk of upper respiratory tract infections in a sample of 2094 Dutch employees. Thirdly, Van den Broeck, De Lange, De Witte, Vansteenkiste, Dikkers, and Vander Elst will present their study in which employees' intrinsic and extrinsic values are related to the availability of social support, and in which the possible explanatory mechanism of humour is examined in a sample of 3308 Belgian employees. In the fourth presentation, RodríguezMuñoz, De Lange, Sanz-Vergel, Moreno-Jiménez, and Baillien examine the moderating effects of humour styles in relation to workplace bullying in a sample of Spanish employees. Finally, De Lange, Dikkers, and Demerouti present a study, among 409 undergraduate Dutch psychology students and 70 Dutch police officers, on the associations of the four humour styles of Martin et al. (2003) with work engagement and burnout. Furthermore, they explore the influence of selfregulatory focus as possible underlying mechanism in these relations. 


\title{
Paper 1:
}

\section{Validation of the 4-dimensional structure of the Humour Styles Questionnaire among a large-scale employee sample}

\author{
${ }^{1}$ Dikkers, J.S.E., ${ }^{2}$ de Lange, A.H., ${ }^{3}$ de Witte, H., \& ${ }^{3}$ den Broeck, A.V. \\ ${ }^{1} \mathrm{VU}$ University Amsterdam, the Netherlands; ${ }^{2}$ University of Groningen, the Netherlands; ${ }^{3} \mathrm{~K} . U$. \\ Leuven, Belgium
}

Introduction: Most definitions of humour encompass an actor (sender) and a target (receiver), and the qualitative aspect of humour as something 'amusing' with positive consequences for the actor's environment (e.g., Cooper, 2005; Romero and Cruthirds, 2006). Empirical studies have shown that sense of humour can indeed enhance psychological well-being and physical health (Kuiper and Nicholl, 2004; Szabo, 2003). More recently, studies have begun to examine the role of humour in the workplace, with positive relationships found with factors such as job performance (Avolio, Howell, \& Sosik, 1999), increased employee morale (Gruner, 1997), leadership effectiveness (Priest \& Swain, 2002), group effectiveness (Romero \& Pescoslido, 2008), creativity (O'Quin \& Derks, 1997), a more positive organisational culture (Clouse \& Spurgeon, 1995) and even productivity (Clouse \& Spurgeon, 1995).

Most previous studies, however, failed to define or examine humour in a multi-faceted manner. Humour can be a double-edged sword in the sense that it can be either harmful or beneficial for the actor and/or the target. It is, therefore, crucial to distinguish between different types of humour. Martin, Puhlik-Doris, Larsen, Gray, \& Weir (2003) recently developed a theory in which they specify four humour styles: i) affiliative humour, which is used as a social lubricant in order to amuse others and facilitate one's social relationships; ii) self-enhancing humour, which is a more self-centered style used to cope with stressful situations and have a more positive view of life; iii) aggressive humour, which includes the use of sarcasm, threats, and ridicule in order to manipulate and hurt others; and iv) self-defeating humour, through which individuals will amuse others by putting themselves down in an effort to hide their true feelings, or to avoid dealing with failure or losses in terms of one's self image. There is still a paucity of research examining the aforementioned multi-faceted perception of humour styles among worker samples. Therefore, the current study aims to test this four-factor structure of humour styles among a large-scale employee sample.

Method: This study tests the psychometric quality of the Humour Styles Questionnaire (HSQ) developed by Martin et al. (2003) among $\mathrm{N}=3,308$ Belgian employees from various sectors. With regard to the factor structure of the HSQ, we expect that it will reflect the four humour styles. We will test the psychometric qualities of the HSQ and its factor structure by means of reliability analyses, a Confirmatory Factor Analysis in LISREL (8.5), and an examination of its (convergent) associations with well-being.

Results and discussion: Preliminary results in SPSS suggest that a four-factor model would fit the data well, and that the four humour styles have acceptable to good Cronbach's alphas (ranging from $\alpha=.65$ for the aggressive humour style to $\alpha=.81$ for the affiliative and self-enhancing humour styles). Theoretical and practical implications of the results will be discussed. 


\title{
Paper 2:
}

\section{The associations of humorous coping styles, affective states, job demands and job control with the incidence of upper respiratory tract infection}

\author{
Doosje, S. \\ Utrecht University, the Netherlands
}

Introduction: There is some evidence that job demands, job resources and humorous coping contribute to the risk of upper respiratory tract infections. Research purpose: The purpose of this study was to test a model including these variables as well as job-related affect and humorous coping styles, in order to explore their role in the incidence of upper respiratory tract infection.

Method: A sample of 2094 employees filled out questionnaires assessing job demands, job control, generic (MSHS-C), antecedent-focused and response-focused humorous coping $(\mathrm{QOHC})$, and job-related affect (JAWS).

Results: It was shown that job demands were indirectly related to the incidence of upper respiratory tract infections, mediated by their relationships with job control and negative jobrelated affect, both of which influenced the outcomes. Generic and response-focused humorous coping were less relevant for the explanation of the incidence of upper respiratory tract infections than the presumably 'healthy' antecedent-focused humorous coping style, mainly because of its favourable, negative association with negative job-related affect. However, the incidence of upper respiratory tract infections was predicted better by job control and negative job-related affect than by humorous coping, in the expected directions.

Conclusions: These findings may have practical relevance for the improvement of stress management interventions in organisations. Contribution/value-add: Although it was shown that healthy humorous coping does contribute to decreases in upper respiratory tract infection, job demands, job resources and negative affective state seem more important predictors.

\section{Paper 3:}

\section{The Associations of Intrinsic and Extrinsic Values with Social Support: Humour as an Explanatory Mechanism}

\author{
${ }^{1}$ den Broeck, A.V., ${ }^{2}$ de Lange, A.H., ${ }^{1}$ De Witte, H., ${ }^{4}$ Vansteenkiste, M., ${ }^{3}$ Dikkers, J.S.E., \& \\ VVander Elst, T. \\ ${ }^{1}$ K.U. Leuven, Belgium; ${ }^{2}$ University of Groningen, the Netherlands; ${ }^{3} \mathrm{VU}$ University \\ Amsterdam, the Netherlands; ${ }^{4}$ Ghent University, Belgium
}

Introduction: Employees' intrinsic (e.g. valuing community contribution and personal growth) and extrinsic values (e.g. financial success and power; Deci \& Ryan, 2000) may guide their job selection (Van den Broeck, et al., in press) and preparedness to act upon particular job characteristics (Van den Broeck, et al., 2009). The first aim of this study was to examine whether these values may also assist in jobcrafting (Wrezesniewski \& Dutton, 2001) and may 
in particular influence the availability of social support. Employees' values may indeed effect social relationships: Extrinsic values have, for instance, been shown to increase individuals' tendency to strive to outperform others (Vansteenkiste, et al., 2004), to consider themselves as superior (Duriez, et al., 2008), and to 'use' others to advance in life (Sheldon, et al., 2000). Approaching social relationships from the positive side and contextualizing these findings to the work context, we hypothesize that intrinsic values relate positively (Hypothesis $1 \mathrm{~b}$ ) and extrinsic values relate negatively (Hypothesis $1 \mathrm{~b}$ ) to social support.

Second, we examine humour as a possible explanatory mechanism of these relations. Humour may indeed play a crucial role in social bounding, but may also be harmful, depending on the humour style used. Intrinsic values likely relate positively to both personal and social constructive (i.e., self-enhancing and affiliative) styles of humour (Martin, et al., 2003) which reflect an open, responsive behavioural style typical for intrinsically oriented individuals (Hodgins \& Knee, 2002; Sheldon \& Kasser, 2001) and likely reinforce social relationships (Cooper, 2008). Extrinsic values, in contrast, likely relate positively to destructive (i.e. self-defeating and aggressive) styles of humour, which are characterized as manipulative and defensive and would therefore fit with the behavioural style of extrinsically oriented employees and forestall social support. Accordingly, we hypothesize that intrinsic values relate positively to social support via affiliative and self-enhancing humour (Hypothesis 2a), whereas extrinsic values associate negatively with social support through aggressive and self-defeating humour (Hypothesis 2b).

Method: The hypothesis will be examined in a heterogeneous sample of Belgian employees ( $\mathrm{N}$ =3308) using structural equation modelling in Lisrel (8.54).

Results: Preliminary results using hierarchical regression analysis confirmed Hypothesis 1a that intrinsic values were positively related to social support. This relationship was (partially) mediated by self-enhancing and affilitative humour, supporting Hypothesis 2a. Unexpectedly also aggressive humour intervened, such that intrinsic values related to more social support via self-enhancing and affiliative humour, as well as a lack of aggressive humour. Extrinsic values were unrelated to social support, rejecting Hypothesis $1 \mathrm{~b}$ and $2 \mathrm{~b}$.

Conclusions: The discussion centres on the importance of work values as personal resources fuelling job crafting and the particularities of the different humour types in this process.

\section{Paper 4:}

\section{Making jokes at the costs of others? Examining enhancing or buffering effects of humour in relation to workplace bullying}

${ }^{1,2}$ Rodríguez-Muñoz, A., ${ }^{3}$ de Lange, A.H., ${ }^{1}$ Sanz-Vergel, A.I., ${ }^{1}$ Moreno-Jiménez, B., \& ${ }^{4}$ Baillien, E.

${ }^{1}$ Autonoma University of Madrid, Spain; ${ }^{2}$ IE University, Spain; ${ }^{3}$ University of Groningen, the Netherlands; ${ }^{4}$ K.U. Leuven, Belgium

Introduction: Research has shown that the experience of workplace bullying is followed by a number of dysfunctional outcomes. During the last few decades, several studies have shown that bullying is associated with severe health problems, such as anxiety, depression, 
and psychosomatic symptoms (Einarsen \& Mikkelsen, 2003). In this field, researchers have directed their attention to variables that may mitigate these negative effects. For instance, Moreno-Jiménez et al. (2008) showed that psychological detachment buffered the relationship between bullying and sleep problems. Similarly, Mikkelsen et al (2002) found that self-efficacy attenuated the effects of bullying on mental health. In contrast to the vast amount of studies about the consequences of workplace bullying, research regarding the question on how individual variables may influence bullying-strain relationships is limited. In order to completely understand the bullying phenomena, we should explore how individuals differentially react to stressful situations rather than simply relating stressors to strains (Rodriguez-Muñoz, 2009).

Following this suggestion, the goal of this study is to examine the moderating role of humour styles on the relationship between workplace bullying and several strain indicators (e.g., somatic symptoms, social dysfunction, anxiety and depression). Sense of humour has frequently been regarded as an effective resource to alleviate work stressors (e.g., Avolio, Howell, \& Sosik, 1999). Recently, Martin et al. (2003) have distinguished between four different types of humour styles. On the one hand, positive humour styles are directed to enhance the self (Self-enhancing) and to enhance one's relationships with others (Affiliative). It has been pointed out that these styles are coping mechanisms for dealing with stress, diminishing interpersonal tensions and aid in relationship building (Romero \& Cruthirds, 2006). On the other hand, two other negative humour styles have been described; to enhance the self at the expense of others (Aggressive), and to enhance relationships at the expense of the self (Self-defeating). For example, a manager tells an aggressive joke in order to distance herself from her subordinates (Clouse \& Spurgeon, 1995).

In this line, Malone (1980) advanced that humour at work may act as a double-edged sword, because humour styles may at times exacerbate (rather than attenuate) the impact of stressors on strains. According to the Conservation of Resources Theory of Hobfoll (1989), workers will strive to obtain or retain their job-related as well as personal resources at work. From this point of view, self-enhancing and affiliative humour can be seen as a way to restore or gain resources. For instance, humour may increase one's level of social support. On the contrary, aggressive and self-defeating humour may exacerbate the negative effect of bullying on health, by increasing the loss of resources. In these situations, the use of this kind of humour would be like "adding fuel to fire".

In line with the aforementioned line of reasoning we will test the following two hypotheses: Hypothesis 1. The positive humour styles (self-enhancing and affiliative) will buffer the relationship between bullying and psychological strain indicators, such that bullying will be less strongly associated with strain for employees with high level of these styles.

Hypothesis 2. The negative humour styles (aggressive and self-defeating) will enhance the relationship between bullying and psychological strain indicators, such that bullying will be more strongly associated with strain for employees with high level of these styles.

Method: The present cross-sectional survey study was part of a broader project on health and employee well-being in Spain. The sample will consist of 300 Spanish employees, from the security sector. Participants were recruited from 58 organisations located in 11 different communities. Hierarchical moderated multiple regression analyses will be used to test the hypotheses. 
Results and discussion: Preliminary results revealed that both negative and positive humour styles moderated the relationship between workplace bullying and strain. In line with our expectations, the effect of bullying on strain indicators was stronger for employees with high levels of negative humour styles, and weaker for employees with high levels of positive humour styles.

Discussion: Our results highlight the importance of humour in the context of workplace bullying, suggesting that in some cases, humour is effective as a stress-relieving method. Possible underlying mechanisms and practical implications will be discussed.

\title{
Paper 5:
}

\section{A self-regulatory perspective on the effects of humour styles in relation to burnout and work engagement}

\author{
${ }^{1}$ de Lange, A.H., ${ }^{2}$ Dikkers, J.S.E. \& ${ }^{3}$ Demerouti, E. \\ ${ }^{1}$ University of Groningen, the Netherlands; ${ }^{2} \mathrm{VU}$ University Amsterdam, the Netherlands; \\ ${ }^{3}$ Technical University Eindhoven, the Netherlands
}

Introduction: A popular view is that a greater sense of humour enhances psychological wellbeing and physical health (Kuiper and Nicholl, 2004). Martin, Puhlik-Doris, Larsen, Gray, \& Weir (2003; Martin, 2004) recently developed a more extensive theory specifying four 'humour styles' varying in their association with well-being. Aggressive and self-defeating humour are potentially detrimental to psychological well-being, whereas self-enhancing and affiliative humour can promote psychological well-being (Martin et al., 2003). Tümkaya (2007) found in a cross-sectional study among university lecturers negative relations between affiliative and selfenhancing humour and burnout, wheras positive relations were found between self-defeating and aggressive humour and burnout. However, the influence of these different humour styles have not been tested in relation to work engagement, and no study to date has examined possible underlying self-regulatory processes to further explain the effects found (Higgins, 2000). It is interesting to include both work engagement and burnout as these outcomes have been assumed to be polar opposites of each other (Demerouti, Bakker, Nachreiner, \& Schaufeli, 2001).

Using a sample of undergraduate psychology students as well as a worker sample, we will test the following Hypotheses:

Hypotheses $1 \mathrm{a}$, b: The positive humour styles self-enhancing and affiliative humour will be positively related to work engagement (1a), and negatively related to burnout (1b).

Hypotheses 2 a, b: The negative humour styles aggressive and self-defeating humour will be negatively related to work engagement (2a), and positively related to burnout (2b).

Regulatory fit. To further understand the motives of people to use these different humour styles, we will include the influence of self-regulatory focus. We want to examine whether workers use certain humour styles to prevent further loss (in terms of burnout), or increase further gains (in terms of work engagement). According to Higgins (2000), regulatory fit occurs when a worker 
uses strategic means congruent with his or her regulatory orientation. Strategic means or (e.g. humour) behaviours that fit one's regulatory focus will result in improved work outcomes. We therefore exploratory want to examine whether a regulatory fit between humour style and regulatory focus predicts work engagement and burnout.

Method: $N=409$ (81\%) undergraduate psychology students were included in our analyses. Of these students, 71.4 percent was female, and the students' age ranged from 17 to 50 years (M $=20.22$ years, $\mathrm{SD}=3.34$ ). Furthermore, a new sample of $\mathrm{N}=70$ Dutch police officers will be included.

Results: The preliminary results of multiple hierarchical regression analyses using data of the undergraduate students revealed support for our hypotheses $1 \mathrm{a}, \mathrm{b}$ and $2 \mathrm{a}, \mathrm{b}$. Furthermore, significant interaction effects were found between the humourstyles and regulatory focus in explaining work engagement and burnout.

Discussion: Our results underline the importance of adding personal resources and self-regulatory processes into occupational health models. During the presentation we will further discuss the theoretical as well as practical implications of the results found. 


\title{
Job Insecurity in Europe: some new insights
}

\author{
Chairs: De Witte, N. \& De Cuyper, H. \\ K.U.Leuven, Belgium
}

Job insecurity is commonly defined as perceived uncertainty about the future existence of one's job. Job insecurity research has been increasing during the last decades. The growing interest in this topic is not surprising, given the large scale changes our economies are going through at the moment (e.g. restructuring, downsizing, mergers etc.). Research thus far has documented the detrimental effects of job insecurity for individuals and organisations. Many research issues still remain unanswered, however, and some recent insights on this topic will be presented and discussed in this symposium.

First, Vander Elst et al. discuss the measurement of job insecurity. Many instruments have been used to measure this concept, but their psychometric characteristics are often unclear. In an attempt to develop a generally agreed-on measurement of job insecurity, paper 1 analyse the Job Insecurity Scale (JIS; 4 items). They evaluate its validity and reliability across six European countries (i.e., Sweden, Germany, the Netherlands, Belgium, the UK and Spain), using the data of the Psycones project. Richter et al. concentrate on the issue of the antecedents of job insecurity by focussing on risk groups. They use a Swedish dataset to identify employees who are more vulnerable to experience job insecurity. Their longitudinal design allows them to investigate how different groups of employees feel depending on their pattern of job insecurity over time. In addition, they also test the moderating effect of two sorts of social support: support from supervisor and co-workers.

The next two papers continue examining moderators of the job insecurity-strain relationship. The contribution of Stynen et al. focuses on the associations of two kinds of job insecurity (quantitative and qualitative) with work engagement, OCB and organisational commitment. They analyse two intervening mechanisms of these relationships: psychological contract breach and the frustration of three basic needs, as conceptualised in Self Determination Theory. Their main focus, however, is on age differences. They expect that older workers may experience more contract breach when facing job insecurity. They additionally expect basic needs to be thwarted by job insecurity for all age groups.

Schreurs et al. focus on a forgotten moderator: religious attitudes. They start by analysing both quantitative and qualitative job insecurity and their relationship with the core components of burnout: emotional exhaustion and cynicism. Religiosity is expected to buffer this relationship: the negative relationship between both kinds of insecurity is supposed to be weaker with increasing levels of religiosity. Using cross-sectional data from the Netherlands, they however find the opposite pattern: religiosity was found to exacerbate the negative effects of job insecurity.

In the last paper, Baillien et al. focus on a rather new outcome variable in job insecurity research: workplace bullying. In a longitudinal, two-wave study they analyse the relationships between both targets and offenders of workplace bullying and job insecurity over time. They test three causal models: normal causation (job insecurity causes bullying), reversed causation (bullying causes insecurity) and reciprocal causation (in which job insecurity and bullying are each other's antecedents and consequences over time). 


\title{
Paper 1:
}

\section{The Job Insecurity Scale: A psychometric evaluation across six European countries}

\author{
Vander Elst, T., De Witte, H. \& De Cuyper, N. \\ K.U.Leuven, Belgium
}

Objectives: Job insecurity is defined as the perceived uncertainty about the future existence of one's job. Multiple instruments have been developed and used to measure this concept, often without proper evaluation of their psychometric characteristics, and this may hamper comparisons of the results across studies and countries. A commonly used measurement in this respect is the Job Insecurity Scale (JIS), a scale that consists of four items derived from De Witte (2000). The purpose of the current study was to evaluate the validity and the reliability of the JIS across six European countries (i.e., Sweden, Germany, the Netherlands, Belgium, the UK and Spain), in an attempt to offer a generally agreed-on measurement of job insecurity.

Methods: Data were collected in 2005 within the PSYCONES project; a European project that aimed to investigate the effects of the changing nature of employment contracts on employees' job security, well-being and health in different European countries. The total sample consisted of 2268 respondents $(\mathrm{nSweden}=310$; $\mathrm{nGermany}=302$; $\mathrm{nNetherlands}=394 ; \mathrm{nBelgium}=$ $377 ; \mathrm{nUK}=369 ; \mathrm{nSpain}=516$ ).

First, the validity of the JIS was investigated in terms of construct validity, concurrent validity and discriminant validity, by performing multi-group confirmatory factor analyses (CFA). The construct validity was evaluated by testing whether the same one-dimensional structure was found across all countries. In order to evaluate the concurrent validity, we examined whether job insecurity was negatively related to job satisfaction, organisational commitment, general health and performance in every country and whether these associations were invariant across countries. The discriminant validity of the JIS was tested with respect to employability. In particular, we investigated whether the model with the dimensions job insecurity and employability fitted the data better than the alternative one-factor model and whether the associations with the four outcome variables differed for job insecurity and employability in every country. Second, the reliability of the JIS was evaluated by calculating the Chronbach's alpha coefficient. The results and its implications will be presented during the symposium.

\section{Paper 2:}

\section{Job Insecurity: Who is at Risk?}

\author{
Richter, A., Näswall, K. \& Sverke, M. \\ Stockholm University, Sweden
}

Objectives: Job insecurity is a work stressor which has become increasingly relevant in the last decades due to major changes such as globalization and flexibility demands, which have been followed by organisational restructuring. This leaves a lot of employees worried about the future 
of their job. Research has contributed to a great body of knowledge around this work stressor regarding antecedents as well as consequences and moderators. Two recent meta-analyses have summarized the major consequences associated with job insecurity, such as decreased mental as well as physical health and impaired organisational attitudes such as job satisfaction, organisational commitment and trust. However, there is a need for a closer focus on identifying risk groups, that is, employees who are more vulnerable to experiencing job insecurity in order to apply knowledge in organisations and focus primarily on these employees to build up future preventions. The major aim of this study is to investigate how different groups of employees feel depending on their pattern of job insecurity over time. This is done by studying those who feels most threatened by job insecurity and who have responded the most negatively in terms of decreased work attitudes as well as decreased mental health. In addition we test who benefit the most from supervisor and co-worker support.

Methods: A sample of 567 Swedish accountants has been examined longitudinally, where the majority of the sample had a university background and $60 \%$ was female. The hypothesis was tested whether there are differences in relations between groups of different job insecurity patterns and the known outcomes of job insecurity such as mental health, job satisfaction, commitment as well as work-family conflict.

Results: Results show a difference between groups of insecure employees. Implications and conclusion: As this study tests a new approach on how to view job insecurity, with following change over time, it is first of all important to evaluate if previous results which did not differentiate between those groups hold also for in this study. Moreover, it has major implications for potential interventions to identify groups of employees that are suffering the most.

\title{
Paper 3:
}

\section{Job Insecurity and Withdrawal: Different Paths for Different Age Groups?}

\author{
${ }^{1}$ Stynen, D., ${ }^{1}$ Sels, L., ${ }^{2}$ Forrier, A., ${ }^{1}$ Vander Elst, T., ${ }^{1}$ Van den Broeck, A. \& ${ }^{1}$ De Witte, H. \\ ${ }^{1}$ K.U.Leuven, Belgium; ${ }^{2}$ Lessius University College, Belgium
}

Objectives: Besides its adverse effects on well-being (Ashford, Lee \& Bobko, 1989), job insecurity also leads to withdrawal behaviours (e.g. less OCB) (Bultena, 1998) that may set off a downward spiral (eventually enhancing one's objective chance on lay off). Withdrawal in response to both quantitative and qualitative insecurity may be of particular interest with regard to older workers. With fewer perceived external opportunities (Berntson \& Marklund,

2007), it may hamper their active participation to the workforce. We therefore focus on the effects of both types of insecurity on work engagement, OCB and organisational commitment and address whether age matters.

We expect to find some age-related differences because the intervening mechanisms may function differently. A first mechanism that is often suggested is psychological contract breach (Sverke, Hellgren, Näswall, Chirumbolo, De Witte \& Goslinga, 2004). Although older workers may be less prone to contract breach in general (Bal, De Lange, Jansen \& Van Der Velde, 2008) 
we expect that older workers may experience more contract breach when facing quantitative than qualitative job insecurity, since they may hold more traditional psychological contracts (Granrose \& Baccili, 2006). We expect the opposite pattern within younger age groups, assuming they hold a more transactional, short term psychological contract. In addition to psychological contract breach we also take into account basic need frustration as a mediator. Latter concept stems from Self-determination theory (Ryan \& Deci, 1985) and regards the need for autonomy, competence and belongingness as nutrients for well-being. Since basic needs are innate and individuals do not differ in need strength, we expect that basic needs will be thwarted by both types of job insecurity for all age groups (direct effect). However, age differences are expected since need frustration may be strengthened in response to psychological contract breach (indirect effect).

Methods: To test our hypothesis, we apply Multiple-Group analysis (M-plus) using a sample of Flemish workers $(\mathrm{N}=3239)$. The analyses are carried out on three different age groups: (1) workers aged 18-35 years (younger), (2) workers between 36-45 years (mid-career), and (3) workers of 46 years and older (older).

Results: Preliminary results of regression analyses point to some interesting age differences. Effects will have to be confirmed using the Multiple-Group procedure described above.

Implications and conclusions: The results may contribute to a better understanding of how job insecurity is related to withdrawal and how both differences and similarities may occur between workers from different age groups.

\title{
Paper 4:
}

\section{Praying for Security: Can Religiosity Offset the Negative Health Effects of Job Insecurity?}

\author{
${ }^{1}$ Schreurs, B., ${ }^{2}$ van den Heuvel, M., ${ }^{3}$ van Emmerik, H., ${ }^{4}$ De Cuyper, N. \& ${ }^{5}$ Demerouti, E. \\ ${ }^{1}$ European University College Brussels, Belgium; ${ }^{2}$ Utrecht University, the Netherlands; \\ ${ }^{3}$ Maastricht University, the Netherlands; ${ }^{4}$ K.U.Leuven, Belgium; ${ }^{5}$ Eindhoven University of \\ Technology, the Netherlands
}

Objectives: Job insecurity has been identified as a major source of job stress, leading to a laundry list of negative outcomes, including impaired employee health. The job insecurity literature has successfully tested the stress-buffering role of various personality dispositions and environmental variables, such as locus of control, social support, and fairness. In the present study, we examined the stress-moderating effect of yet another variable: religiosity. Previous research has shown that religiosity can mitigate the negative health consequences of severe life crises, but it has been largely ignored in research on coping with occupational stress. Hence, our goal was to extend the job insecurity literature by investigating the stress buffering effect of religiosity in the relationship between job insecurity and employee health. This buffering effect was examined for two types of job insecurity: quantitative (the perceived threat of job loss) and qualitative job insecurity (the perceived threat of loss of values of aspects of the job). Our hypothesis was that quantitative and qualitative job insecurity and religiosity interact so that the 
negative relationship between both types of job insecurity and health is weaker with increasing levels of religiosity.

Methods: To test this hypothesis, cross-sectional survey data were collected from 238 Dutch workers employed in a variety of public and private organisations undergoing change. 44 percent of the respondents was not affiliated with any religion; 33 percent was Catholic; 16 percent Protestant; 15 employees reported other affiliations (e.g., Muslim, Hindu). The two core components of burnout, emotional exhaustion and cynicism, were used as indicators of impaired health. Religiosity was measured with a ten-item scale that assessed importance of religion, strength in the belief that religion give one's life meaning, and religion as part of one's identity.

Results: Hierarchical moderated regression analyses (separate for quantitative and qualitative job insecurity) showed that both types of job insecurity were positively associated with emotional exhaustion and cynicism. Significant interactions were found between quantitative and qualitative job insecurity and religiosity for emotional exhaustion as well as for cynicism. However, the direction of the interactions was opposite to the ones predicted: religiosity did not buffer but rather exacerbate the negative effects of job insecurity on employee health while for those low in religiosity there was no effect of job insecurity on both burnout dimensions. These results might be explained by the fact that almost $50 \%$ of the sample was not affiliated to any religion while the other $50 \%$ was affiliated to different religions.

Implications and conclusions: Results indicate that religiosity is not a protective factor against the detrimental effects of job insecurity. Rather it makes them more vulnerable to job insecurity, perhaps because highly religious employees view their insecure job situation as a punishment from God.

\title{
Paper 5:
}

\section{Associations between Job Insecurity and Workplace Bullying: a two-wave longitudinal test}

\author{
Baillien, E., De Witte, H. \& De Cuyper, N. \\ K.U.Leuven, Belgium
}

Objectives: Recently, a range of studies have revealed significant associations between job insecurity and workplace bullying, both for targets and offenders (De Cuyper, Baillien, \& De Witte, 2009). Most empirical evidence to date has however been based on cross-sectional designs. Accordingly, little is known about how job insecurity and targets'/offenders' reports of bullying influence each other over time; a void we wish to address in the current study. Specifically, we aim to test various causal models that link job insecurity to targets'/offenders' reports of bullying over time. First, job insecurity and bullying may be linked through normal causation: Job insecurity may be seen as a stressor, hence causing strain, which employees may try to reduce in ways that promote workplace bullying (Baillien, Neyens, De Witte, \& De Cuyper, 2009). From the target's perspective, employees may adopt a rather passive coping style when confronted with job insecurity, which may be reflected in withdrawal behaviour 
(Sverke, Hellgren, \& Näswall, 2002) and non-compliant job behaviour (Lim, 1996). This, in turn, may lead these employees to become a 'safe' or 'legitimate' target (Felson \& Tedeschi, 1993). Alternatively, job insecurity may wear out the employees' resources; and insecure employees may become 'easy' targets that offer little resistance against bullying (Hoel \& Salin, 2003). From the offender's perspective, employees may vent negative emotions associated with job insecurity on others through a process of projection (Hinduja, 2007). Second, they may be linked through reversed causation: From the target's perspective, bullying may be seen as a social stressor that triggers a range of negative consequences such as severe health problems, burnout and decreased job satisfaction (Mikkelsen \& Einarsen, 2002; Rodriguez-Muños, Baillien, De Witte, Moreno-Jiménez, \& Pastor, 2009); which may eventually lead to resignation or dismissal (Leymann, 1996). From the offender's perspective, bullying may be picked up by the organisation's policy officers, who may choose to fire the offender. Third, they may be linked through reciprocal causation, in which job insecurity and bullying appear to be each other's antecedents and consequences on time.

Methods: We collected a complete panel two-wave heterogeneous sample of Flemish employees (six months time lag). SEM (Amos 7.0) will be used to test and compare the competing models reflecting the relationships between job insecurity and bullying, i.e. the baseline or stability model (M1), the normal causation model (M2), the reversed causation model (M3) and the reciprocal causation model (M4).

Results and discussion: Preliminary results in SPSS revealed significant correlations between (a) targets' reports of bullying Time 1 and job insecurity Time 1 and job insecurity Time 2, (b) targets' reports of bullying Time 2 and job insecurity Time 1 and job insecurity Time 2, and (c) offenders' reports of bullying Time 1 and job insecurity Time 1. Lagged Hierarchical Regression analyses only revealed a lagged effect of job insecurity Time 1 on targets' reports of bullying Time 2; suggesting normal causation between job insecurity and target's reports of bullying. These results will be analyzed in more detail using SEM. Theoretical and practical implications of the results will be discussed. 


\title{
Studying positive organisational behaviour using elaborated research designs
}

\author{
Chairs: ${ }^{1}$ Demerouti, E \& ${ }^{2}$ Peeters, M.C.W. \\ ${ }^{1}$ Eindhoven University of Technology, the Netherlands; ${ }^{2}$ Utrecht University, the Netherlands
}

This symposium aims to uncover the processes in the work and non-work domain through which positive organisational behaviour can be enhanced. The symposium integrates different indicators of positive organisational behaviour, namely: (1) work engagement (2) cognitive liveliness and (3) family-work-facilitation. Next to uncovering the processes leading to positive organisational behaviour, the symposium has several other aims and innovative aspects. First and foremost the symposium consists of studies using elaborated research designs: three of the contributions are daily diary studies, one study is conducted among couples and yet another contribution uses other-ratings of performance. Second, the symposium sheds light on both work and non-work processes leading to positive organisational behaviour. Some studies focus particularly on work-related processes leading to positive organisational behaviour, whereas other contributions integrate also non-work processes. Third, the symposium integrates the role of some new constructs in predicting positive organisational behaviour, such as job crafting, role salience, diversity and work-home culture and regulatory focus.

Specifically, Kühnel et al. found that daily resources promoted daily work engagement. Daily job control qualified the relationship between daily time pressure and daily work engagement: On days with higher job control, daily time pressure was beneficial for work engagement. On days with lower job control, daily time pressure was detrimental for work engagement.

Similarly, Petrou et al. found that the daily work pressure was stronger related to daily job crafting (positively to resources seeking and negatively to demands reducing) when daily autonomy was high vs. low. Work engagement was predicted positively by resources and challenges seeking and negatively by demands reducing.

Furthermore, Sanz-Vergel et al. suggested that work role salience moderated the relationship between home detachment and work outcomes while home role salience moderated the relationship between work detachment and outcomes in the home-setting. The overall results suggested that job performance and well-being can be achieved when people detach from home and the work role is more salient to the individual.

Demerouti et al. found that the interaction between own and partner's promotion focus predicted the levels of developmental possibilities at home and family-work facilitation and the interaction between own and partner's prevention focus predicted work pressure at home. Overall, it was beneficial for family life and family-work-facilitation when both partners use the same self-regulation systems.

Finally, Peeters et al. found that work engagement fully mediates the relationship between organisational culture (work-home and diversity culture) and objective performance and partially mediates the relationship between organisational culture and subjective performance.

The symposium emphasizes the multifaceted character of positive organisational behaviour. 


\title{
Paper 1:
}

\section{Testing the Job Demands-Resources Model on the Level of Daily Processes: How to Promote Daily Work Engagement}

\author{
${ }^{1}$ Kühnel, J., ${ }^{1}$ Sonnentag, S. \& ${ }^{2}$ Bledow, R. \\ ${ }^{1}$ University of Konstanz, Germany; ${ }^{2}$ Justus-Liebig University, Germany
}

This diary study adds to research on the Job Demands-Resources (JD-R) model (Demerouti et al, 2001). We test main propositions of this model on the level of daily processes and extend the model by including daily recovery. More precisely, we test additive and interactive effects of job demands and job as well as personal resources on daily work engagement. Daily team climate, daily job control, and being recovered in the morning are hypothesized to be positively related to daily work engagement. According to the JD-R model and the transactional stress model (Lazarus \& Folkman, 1984), daily time pressure and daily resources should jointly predict daily work engagement.

Method and Results: One hundred and fourteen employees from German companies in diverse industries completed a general questionnaire once and electronic questionnaires three times a day over the course of one working week. Each day, we assessed participants' state of being recovered at the beginning of the working day, daily team climate, daily job control, and daily time pressure at noon, and daily work engagement at the end of the working day. Results from hierarchical linear models indicated that daily resources (team climate, job control, and being recovered in the morning) promoted daily work engagement. As predicted, daily job control qualified the relationship between daily time pressure and daily work engagement: On days with higher job control, daily time pressure was beneficial for work engagement. On days with lower job control, daily time pressure was detrimental for work engagement. Contrary to expectations, being recovered in the morning failed to qualify the relationship between daily time pressure and daily work engagement.

Practical Implications: To ensure that employees' work engagement is not negatively affected by high time pressure, employees should be provided with control over their tasks especially when they face high time pressure. Moreover, organisations can indirectly foster employees' work engagement by ensuring that employees have enough daily recovery time at their disposal.

Conclusion: To sum up, our study pointed out that work engagement substantially varies over time and that these variations can be explained by daily job and personal characteristics. Thus, we have shown that it is necessary studying the processes related to daily work engagement to gain a valid understanding of work engagement as a vivid experience that takes place day for day at work. 


\title{
Paper 2:
}

\section{Crafting a job daily: Contextual antecedents and the effect on work engagement}

\author{
${ }^{1}$ Petrou, P., ${ }^{2}$ Demerouti, E., ${ }^{1}$ Peeters, M.C.W. \& ${ }^{1}$ Schaufeli, W.B. \\ ${ }^{1}$ Utrecht University, the Netherlands; ${ }^{2}$ Eindhoven University of Technology, the Netherlands
}

Objectives: Recent perspectives on job design integrate dynamic views of organisational behaviour (i.e. job crafting) with classic job design theories. Job crafting behaviours involve shaping the task or the relational boundaries of the job or both. In the present paper, our aim is to expand literature on job crafting, investigate the conditions that promote crafting and the outcomes of crafting behaviours. To do so, we used the Job Demands-Resources model. Job crafting was conceptualized in terms of "resources seeking", "challenges seeking" and "demands reducing". We expected that the combination of high daily work pressure and high daily job autonomy ("active jobs") will be associated with higher daily resources seeking, higher daily challenges seeking and lower daily demands seeking. Furthermore, we hypothesized that daily work engagement will be positively predicted by daily resources seeking and challenges seeking, and negatively predicted by daily demands reducing. We used a diary methodology to shed light on the dynamic nature of job crafting.

Methods: The participants of the study, 95 employees working in several Dutch organisations that had undergone some kind of organisational change, filled in a questionnaire providing information on the general level (trait) variables and then, for five consecutive working days, they filled in a diary, providing information on the day-level (state) variables.

Results: Multilevel analyses were conducted to analyze data. Findings provided full support to three out of four hypotheses: The relationship between work pressure and resources seeking was positive for high job autonomy whereas the relationship between work pressure and reducing demands was negative for high job autonomy. No support was found for the interaction hypothesis about challenges seeking. Work engagement was predicted positively by resources seeking and challenges seeking, and negatively by demands reducing. Additional analyses provided some support for the link between job crafting and ongoing organisational changes that employees are exposed to.

Implications: Our findings have implications for management and practice and propose new research avenues towards integrative job characteristics frameworks. Employers should create conditions that promote job crafting and consequently work engagement.

Conclusion: The study suggests that job crafting does occur on a daily basis, it is predicted, at some extent, by the interaction between work pressure and autonomy and has a favourable effect on work engagement. Future research should conceptualize more comprehensively organisational change and use longitudinal methodologies to test if job crafting has long lasting, reversed and reciprocal effects. 


\title{
Paper 3:
}

\section{Daily Detachment from Work and Home: The Moderating Effect of Role salience}

\author{
${ }^{1}$ Sanz-Vergel, A.I., ${ }^{2}$ Demerouti, E., ${ }^{3}$ Bakker, A.B., \& ${ }^{1}$ Moreno-Jiménez, B. \\ ${ }^{1}$ Autonoma University of Madrid, Spain; ${ }^{2}$ Eindhoven University of Technology, the \\ Netherlands; ${ }^{3}$ Erasmus University Rotterdam, the Netherlands
}

Researchers generally agree that psychological detachment from work has beneficial effects for well-being, and empirical findings from cross-sectional and diary studies have confirmed the recovery value of detachment. However, studies focusing on the importance of detaching from the home domain are still lacking (Moreno-Jiménez et al., 2009). Taking home detachment into account is especially important when studying the links between work and non-work domains. Detachment both from work and from home can help the individual to reduce conflict between work and family as well as to improve performance and well-being in the other domain.

In the present study, we contribute to research on the link between work and non-work by examining detaching from work and non-work domains, as well as by assessing the importance of trait role salience for the relationship between daily detachment and several outcomes at the day-level. Based on role theory (Kahn et al., 1964), we hypothesized that:

$\mathrm{H} 1$ : Work role salience will moderate the relationship between (1) daily home detachment and

(2) work performance and home-work interference.

$\mathrm{H}$ 2: Home role salience will moderate the relationship between (1) daily work detachment and

(2) cognitive liveliness and work-home interference.

Method/ Procedure: Employees from different organisations filled out a general questionnaire and after that, they completed daily questionnaires three times a day, during five consecutive working days (Monday-Friday).

Results: Multilevel analyses provided evidence for a moderating effect of work role salience on the relationship between home detachment and outcomes in the work-setting. Specifically, home detachment had a negative effect on home-work interference and was particularly beneficial for work performance among employees with low levels of work role salience. In addition, we found a moderating effect of home role salience on the relationship between work detachment and outcomes in the home-setting. Specifically, work detachment had a negative relationship with work-home interference and resulted in more cognitive liveliness at bedtime for employees who scored high on home role salience.

Conclusion: The overall pattern of these results suggests that performance and well-being in the work domain can be achieved when people detach from home and the work role is more salient to the individual. The same pattern is observed in the case of the home domain. Our findings point out that difference in trait role salience may affect the beneficial impact of detachment from work and non-work domains. 


\title{
Paper 4:
}

\section{Personality, regulatory focus and the work-family interface: The role of regulatory fit between partners}

\author{
${ }^{1}$ Demerouti, E., ${ }^{2}$ Bakker, A.B. \& ${ }^{3}$ Tetrick, L.E. \\ ${ }^{1}$ Eindhoven University of Technology, the Netherlands; ${ }^{2}$ Erasmus University Rotterdam, the \\ Netherlands; ${ }^{3}$ George Mason University, USA
}

Objective: The aim of the present study was to examine the relationship between personality, regulatory focus, regulatory fit between marital partners and work-family balance. According to regulatory focus theory, individuals can be promotion focused - driven by challenges; or prevention focused - driven by obligations. Moreover, the fit between individual and situational focus is expected to lead to positive emotions and better performance. Because neuroticism is related to avoidance behaviour it was expected to be related to prevention focus, while openness to experience is related to developmental outcomes and therefore presumably also to promotion focus. Because promotion-focused individuals are driven by challenges, families where both partners are high on promotion focus (fit) should offer to both partners possibilities for development and an increased likelihood of family-work facilitation (FWF). On the contrary, when both partners are prevention focused (fit) they should experience less work pressure at home and less family-work conflict (FWC) than when one partner is low and the other high on prevention focus.

Methods: In order to test these assumptions we conducted a study among 131 couples, where each partner provided information about their own personality, regulatory focus, perceptions of work pressure, and developmental possibilities at home as well as FWC and FWF.

Results: Results largely supported our hypotheses since, for both partners, neuroticism was positively related to prevention focus, and openness was positively related to promotion focus. Furthermore, the interaction between own and partner's promotion focus predicted the levels of developmental possibilities at home and FWF such that there was a positive relationship between promotion focus and developmental possibilities as well as FWF when also the partner was high on promotion focus. Additionally, the interaction between own and partner's prevention focus significantly predicted work pressure at home such that there was a negative relationship between prevention focus and work pressure at home when partner's prevention focus was high.

Implications: This study is, to the best of our knowledge, the first to confirm the assumptions of the regulatory focus theory in the family domain by showing how regulatory focus is predicted by personality and consequently is related to perceived home demands, home resources and FWF. Family counselors should enlighten partners about the consequences of regulatory fit for home life.

Conclusion: The regulatory focus of individuals in the family system can help to uncover the way through which the personality of each partner influences family life and consequently work. 


\title{
Paper 5:
}

\section{Work engagement and job performance as correlates of a supportive organisational culture}

\author{
${ }^{1}$ Peeters, M.C.W., ${ }^{2}$ Demerouti, E., \& ${ }^{3}$ Bakker, A.B. \\ ${ }^{1}$ Utrecht University, the Netherlands; ${ }^{2}$ Eindhoven University of Technology, the Netherlands; \\ ${ }^{3}$ Erasmus University Rotterdam, the Netherlands
}

Objectives: Over the past decades, the labor force of many Western countries has witnessed two important changes, being an increase in the number of employees who are part of dual-earner families and an increase of ethnic minority employees. A relevant question is how organisations can adopt to these changes in the labor force. Organisations can be supportive in this respect by creating and stimulating an organisational culture that supports workers' diversity. Diversity refers to all of the characteristics that make individuals differ from each other. It is the aim of the present study to examine whether a positive organisational culture relates to increased work engagement and consequently to improved employee performance. In this study, organisational culture is considered regarding two issues: (1) with respect to work-life balance and (2) with respect to cultural diversity. Work engagement is characterized by vigor, dedication and absorption. It is a work-related positive persistent and pervasive state of mind, which is present over a longer period of time.

Method: Data were collected among 1296 employees of an organisation in consultancy, technology and outsourcing. Organisational culture and work engagement were assessed by means of self-reports. Employee's performance was assessed by both objective and subjective indicators. Subjective performance referred to in-role performance and extra-role performance. Objective performance was measured by a 'banding' (evaluation) grade which employees received from their employer. Promotions and incentives depend on the received banding grade. Banding grades varied from (1) Requires improvement to (6) At the very top of peer group.

Results: With regard to the work-home culture, results of SEM analyses provide evidence that work engagement fully mediates the relationship between work-home culture and objective performance and partially mediates the relationship between work-home culture and subjective performance. With regard to the diversity culture the results showed the same pattern: work engagement fully mediates the relationship between diversity culture and objective performance, and partially mediates the relationship between diversity culture and subjective performance. Surprisingly, the structural relationships were statistically identical for relevant socio-demographic subgroups (parents - non-parents; Dutch - non-Dutch).

Implications: These results show that it pays of to invest in a supportive organisational culture. HR-tools and -instruments that may stimulate such a culture will be discussed. 


\title{
Burnout Syndrome in Mental Health Professionals: Cross-Cultural Perspectives
}

\author{
Chair: Lee, S.M. \\ Korea University, South Korea
}

\begin{abstract}
A panel of six experienced presenters will address their research findings related to burnout characteristics (e.g., job environments, job stress and demands, reward systems. ) of three populations (i.e., Japanese, Korean, and American mental health professionals) to gain knowledge, awareness, and skills in working with these populations.
\end{abstract}

The goal of this symposium is to give counselors, educators, researchers, supervisors, and administrators a comprehensive overview of the burnout syndrome of mental health professionals from three countries. Participants will learn about burnout syndrome as defined by past literature and research. Additionally, participants will gain an understanding of the similarity and dissimilarity of this syndrome's manifestations among mental health professionals in three countries. Additionally, in this session, through exploring mental health professionals' burnout patterns and typologies, we compare the roles of psychological factors influencing these burnout types. The audience will have an opportunity to identify their own burnout levels and types using a newly developed Counselor Burnout Inventory (CBI).

Presenters will provide handouts on materials presented in the symposium. Media may be used in the delivery of the materials.

\section{Paper 1:}

\section{Individual and Work-Related Factors influencing Burnout of Mental Health Professionals: A Meta-Analysis.}

\author{
Lee, S.M. \& Choi, B.Y. \\ Korea University, South Korea
}

Burnout is a specific kind of occupational stress that results from demanding and emotionally charged relationships between caregivers and their recipients (Maslach \& Schaufeli, 1993). Burnout accompanies various symptoms of physical, emotional, behavioural, interpersonal, and attitudinal problems (Kahill, 1988). These negative consequences have drawn particular attention of researchers studying burnout of mental health professionals (Rosenberg \& Pace, 2006). The original research of burnout centered on healthcare workers in general (Caldwell, 1984; Savicki \& Cooley, 1981). However, more recent studies found burnout of mental health professionals a more compelling subject (Lee et al., 2007). The work of mental health professionals such as psychologists, therapists, and counselors consists of emotionally demanding relationships with their clients (Bakker et al., 2006; Renzi et al., 2005), often resulting in physical and emotional manifestations of burnout symptoms (Pines \& Aronson, 1981). The present study aims to identify and assess individual and work-related factors as correlates of burnout among mental health professionals using meta-analysis approach, which has been characterized as “a statistical analysis of statistical analyses. Only articles from 1988 to 2008 (20-year period) 
were considered. Initially, we obtained 67 articles. We included articles that are available for meta-analysis containing the statistical data such as mean, standard deviation, t-statistic, or correlation coefficients. Research that only showed multiple regression results have been excluded. We selected articles that include individual or work-related variables: age, gender, educational level, work hours, work experience, and work setting. Based on these criteria, the analysis included a total of 3,613 participants from 15 studies. Results of meta-analysis indicate that age and work setting variables are the most significant indicators of emotional exhaustion and depersonalization. In terms of personal accomplishment, age and work-hours variables are the most significant indicators with positive correlations. Interestingly, while individuals with higher degrees experience emotional exhaustion and depersonalization, they also feel more accomplished. This can complicate the manifestation of burnout. These mental health professionals experience burnout in some dimensions, but not in others. Moreover, the pursuit of a sense of personal accomplishment through demanding work and long hours may exacerbate emotional exhaustion and depersonalization. This underscores the importance of considering burnout as multidimensional. Based on these results, we seek to determine resources that can assist with developing programs for preventing and treating burnout syndromes of mental health professionals.

\title{
Paper 2:
}

\section{Antecedents and Consequences of Burnout in Mental Health Professionals: A Meta-Analysis}

\author{
Kim, A. \& Lee, J. \\ Korea University, South Korea
}

Burnout is conceptualized as a psychological syndrome in response to chronic emotional and interpersonal stress on the job, and is most widely defined by the dimensions of exhaustion, depersonalization, and inefficacy (Maslach, Schaufeli, \& Leiter, 2001). It is related to not only negative occupational indicators including poor job performance, absenteeism, and turnover (Kahill, 1988) but also a variety of mental and physical health problems such as depression, insomnia, and gastrointestinal disturbances (Burke \& Deszca, 1986; Lee \& Ashforth, 1990). Therefore, it is important to find intervention strategies by understanding the relationships between antecedent and consequent variables of burnout. The purpose of the present study was to identify antecedents and consequences of burnout among mental health professionals. A total of 4,303 participants from 17 studies were included in this study. All of them are mental health professionals and all studies used the Maslach Burnout Inventory. Five antecedents (i.e., job stress, self-control, over-involvement, job support, and professional identity), and two consequences (i.e., job satisfaction and turnover intention) were included. The results of the meta-analysis revealed over-involvement, control, and professional identity were significantly related to all dimensions of burnout. However, job support was related only to personal accomplishment of burnout. In addition, job stress and over-involvement were more closely related to emotional exhaustion, while the magnitude of association of control and professional identity were similar across three dimensions of burnout. On the other hand, two consequent variables, turnover intention and job satisfaction, were closely related to all three dimensions of burnout. The correlates of job satisfaction were greater than those of turnover 
intention with all three dimensions. The findings of the association patterns of antecedents and consequences are partially consistent with the conservation of resources theory. Within the framework of the conservation of resources, job stress and over-involvement are categorized into job demands, while control, job support, and professional identity represent the resources. As proposed by the conservation of resources theory, job demands, or job stress and over-involvement, have stronger direct relationships with emotional exhaustion than with depersonalization or personal accomplishment. In summary, burnout is a multifaceted experience developed through the interaction of various demands and resources and resulting in costly consequences. The process of burnout may be more complicated with mental health professionals because the nature of their work challenges them to deal with great distress and high expectations.

\title{
Paper 3:
}

\section{Burnout Syndrome of American Mental Health Professionals}

\author{
Lee, J. \& Puig, A. \\ University of Florida, United States of America
}

Therapeutic relationships regularly challenge the professional counselor's ability to recognize and attend to an array of physically and emotionally taxing experiences (Kesler, 1990; O'Halloran \& Linton, 2000). Maintaining a sense of equilibrium while still providing quality therapeutic services in the wake of managed care constraints, increased budget cuts, and burgeoning caseloads illustrate only a small sampling of issues that could strain a counselor's physical and psychological resources. If not closely monitored, counselor burnout, conceptualized as a combination of multiple emotional and physical ailments manifesting cognitively or within the workplace, could ensue and jeopardize both the counselor's well being and treatment efficacy. While most burnout studies have focused primarily on the correlates of burnout, including counselors' characteristics, work environments, and client attributes (Vredenburgh, Carlozzi, \& Stein, 1999), research examining specific burnout typologies experienced among professional counselors remains limited. The majority of the current research on burnout utilizes the Maslach Burnout Inventory - Human Services Survey (MBI-HSS; Maslach \& Jackson, 1984). While the $\mathrm{MBI}-\mathrm{HSS}$ provides some insight regarding counselor burnout, it falls short in accurately assessing burnout specifically related to counselors. Therefore, the present study assesses American counselor burnout with the newly developed Counselor Burnout Inventory (CBI; Lee et al., 2006). In this study, MBI-HSS was used to compare the patterns derived from the CBI. The CBI provides norm-referenced measures of a counselor's burnout syndrome on five factorially derived burnout dimensions: Exhaustion, Incompetence, Negative Work Environment, Devaluing Client, and Deterioration of Personal Life. Unique to this inventory is its focus on the counselor's work environment. This unique component corresponds with recent counseling burnout literature that accentuates the role one's workplace environment plays in promoting burnout (Azar, 2000; Maslach, 2005; Osborn, 2004; Savicki \& Cooley, 1981; Thompson, 1999). Using our survey data as well as reviewing the existing relevant studies, first, we will explore the multiple-cluster structure of counselor burnout. Second, because there are several known demographic variables linked to burnout, we categorize demographic variables that best appear to discriminate between the burnout clusters. Third, we explore which psychological variables (e.g., job satisfaction, self- 
esteem, and locus of control) best contributed to the cluster differences. Finally, the audience will get the opportunity to identify their level and type of burnout with standardized counselor burnout inventory $(\mathrm{CBI})$.

\title{
Paper 4:
}

\section{Burnout Syndrome of Korean Mental Health Professionals}

\author{
Choi, H. \& Yu, K. \\ The Catholic University of Korea, South Korea
}

Current Korean mental health professionals (e.g. counselors and school counselors) are faced with many challenges because of the nature of the work and the role expectations of the profession (Evans \& Villavisanis, 1997). In addition, many Korean mental health professionals are expected to have numerous non-counseling responsibilities. These challenges which may lead to burnout, affect counselors' work performance both as it pertains to their work with clients and in their personal lives (Yu, 2007). For mental health professionals, heavy workloads, conflicts with staff or co-workers, and a lack of social or network support are the major stressors which may lead to burnout (Choi, Yang, \& Lee, 2002; Park, 2006; Yoo \& Park, 2002). Most of studies related to burnout in the mental health profession have been focused on the dimensions of individual sources including ego-resilience, perfectionism, self-soothing ability, and spiritual well-being (Choi \& Chung, 2003; Choi, Yang, \& Lee, 2002; Kwon, 2004; Park, 2006; Yeo, 2005; Yoo \& Park, 2002). Recently, focus has been placed on the organisational sources of burnout and researchers have investigated these sources, including poor interaction among organisations, unstable working condition, additional expenses for continuing education, and other occupational stressors such as heavy workloads and a lack of network support (Park, 2006; Yoo \& Park, 2002). The comprehensive understanding of burnout patterns and related variables would be critical to help them more effectively. Thus, the study aimed to 1) review mental health professionals' burnout patterns and related variables shown in the literature, 2) introduce recently conducted studies by using the recently developed Counselor Burnout Inventory (CBI, Lee et al., 2007) in the profession, and 3) propose prevention and intervention strategies for mental health professionals. The data for mental health professionals were collected from mental health counselors and school counselors $(n=217)$. This study also investigated the relationship among job dissatisfaction, professional identity, and burnout for Korean mental health counselors. The results indicated that professional identity mediated between job dissatisfaction and burnout. In addition, this study was conducted for Korean school counselors to examine the job demands control model of school counselor burnout and to investigate the relationship between role conflicts and burnout. The results of this study will be presented in the program in detail. Based on the studies, the implications to mental health professionals will be discussed. 


\title{
Paper 5:
}

\section{Burnout Syndrome of Japanese Mental Health Professionals}

\author{
Jin, Y.Y. \& Lee, J. \\ Korea University, South Korea
}

For the past 25 years, counseling and psychology professional literature has examined the issues of stress on the job. Research and discussion initially identified burnout resulting from job stress as an important area for treatment and prevention. In the last decade, the focus has shifted from burnout to secondary traumatic stress due to the recognition of the specific challenges of working with traumatized individuals. Whether mental health professionals are addressing the impact of working with others in general or those who have been traumatized, research agrees that mental health professionals have a responsibility to maintain their own health and wellness (Iliffe \& Steed, 2000; Miller, 1998; Savicki \& Cooley, 1982; Sexton, 1999; Sherman, 1996). Mental health professionals who are trained to care for others often overlook the need for personal self-care and do not apply to themselves the techniques prescribed for their clients. Burnout of health professionals is becoming a serious social problem in maintaining stable and sufficient staffing. One recent study (Narumoto, 2008) reported that the unemployment rate for Japanese health professionals (e.g., nurses, occupational therapists, and social workers) was $22.6 \%$ in a year and higher than other occupations, and burnout affected caregiver's intention to continue their work. For Japanese mental health professionals, we assume that they also express a similar degree of burnout syndrome as other health professionals. Thus, the study aimed to: 1) review health professionals' and mental health professionals' burnout syndromes in Japan and related variables shown in the literature, 2) conduct studies by using the recently developed Japanese Version of the Counselor Burnout Inventory to explore the characteristic of Japanese mental health professionals' burnout, and 3) develop prevention and intervention strategies for mental health professionals. The data for mental health professionals were collected from 258 Japanese professional counselors and psychotherapists $(n=258)$. The result indicates that the Japanese mental health professionals $(M=3.01, S D=.64)$ have higher scores on incompetence than their counterparts, American mental health professionals $(M=2.30, \mathrm{SD}=.56)$ and Korean mental health professionals $(M=2.65, S D=.61)$. Namely, Japanese mental health professionals felt less competence on their job than American and Korean mental health professionals. Based on these results, we discussed resources that can assist with developing programs for preventing and treating burnout syndromes of mental health professionals in Japan.

\section{Paper 6:}

\section{Examining the Burnout Model (Demand-Control vs. Effort-Reward) in Mental Health Professionals}

\author{
Shin, H. \& Lee, J. \\ Korea University, South Korea
}

Several previous studies indicated that exposure to adverse psychosocial work environments is a major hazard for the mental health professionals. Much of this evidence, accumulated over the 
past two decades, is based on the demand-control model (DCM; Karasek, 1979). According to the DCM paradigm, task-level work conditions can be characterized by a combination of job demand and job control. Combining high demand and low control is the worst situation for burnout of mental health professionals whereas combining high demand as well as high control provided well-being, learning, and personal growth (Theorell \& Karasek, 1996). However, one of the criticisms of this DCM paradigm is its reliance on "objective" measures of the work environment alone (Ostry et al., 2003). As a result, many studies failed to examine the interaction effects of job demand and job control on burnout syndrome (De Rijk, et al., 1998; Jimmieson, 2000). In the early 1990s, as an alternative model, the effort-reward imbalance model (ERI; Siegrist et al., 1990) was developed. The ERI model hypothesizes that jobs characterized by a perceived imbalance between high efforts and low rewards are stressful and will lead to negative health outcomes. Many studies point out high effort and low reward were a stronger predictor of poor health (Siegrist \& Johannes, 1996; Jonge et al., 2000; Vegchel et al., 2005) The purpose of this study was to compare the DCM and ERI models to explain the burnout syndrome of mental health professionals. A total of 198 mental health professionals participated in this study. The results showed that a combination model of both DCM and ERI was more predictive than the demandcontrol and effort-reward imbalance models alone. That is, the independent cumulative effects of both the DCM and the ERI Models were observed when examining the burnout syndrome of mental health professionals. The results of this study provide mental health professionals with a better understanding of burnout as it relates specifically to work environments. Both DCM and ERI models have a great deal of potential to assist the mental health profession in many ways including: providing a clearer picture of burnout syndrome, assisting employers with assessing work environment, and meeting the requirements laid out in the new Code of Ethics for mental health professionals. Continued research on the burnout models should provide a clearer understanding of burnout in the field of mental health professionals. 


\title{
Improving Worklife: International Perspectives on Workplace Health and Work Engagement
}

\author{
Chair: Leiter, $M$. \\ Acadia University, Canada \\ Discussant: Tetrick, L., \\ George Mason University, USA
}

Employees experience the quality of their work environments through their interactions with people and systems within the workplace. Contemporary research on occupational health and work engagement have identified points of interaction between employees and their work environments that are especially consequential for employees' experience of work.

This symposium considers two points on which employees interact with their work environments: learning systems and collegial relationships. The paper, "How Exposure to Information and Communication Technology is related to Work Engagement: The Role of Positive Appraisal" considers the connection of critical, work-relevant information with work engagement. Greater confidence in their knowledge base enhances employees' energetic involvement with work. The paper, "Conflict, Performance And Commitment In Teacher Teams: The Mediating Role Of Team Potency," shifts the focus to collegiality, especially the intensity of collegial teamwork as a function of team conflict and productivity.

"Work Positive: An Intervention to Improve Psychosocial Health and Safety in Hospitals," used information from surveys across a variety of organisations to help leaders guide their organisational health initiatives. The information supported a safer, healthier worklife.

The fourth paper, "CREW: Improving Worklife Through Collegiality," presents the results of an intervention study designed to improve collegial relationships as a means of enhancing workplace health and wellbeing. Building on a long tradition of research emphasizing the importance of workplace relationships, the study investigated whether these relationships could be deliberately improved and the impact of improvements on employees' experience.

The fifth paper, "Leader-member exchange (LMX) quality and enthusiasm at work: testing a dynamic mediated longitudinal relationship" tests longitudinal research which shown that leader-member exchange (LMX) quality is related to affective well-being at work.

Together, these papers provide an international perspective (Spain, The Netherlands, Ireland, Canada) on workplace health and work engagement. The presentations provide an active approach to worklife research with implications for both theory and practice. 


\title{
Paper 1:
}

\section{How Exposure to Information and Communication Technology is related to Work Engagement: The Role of Positive Appraisal}

\author{
Salanova, M. \& Llorens, S. \\ Universitat Jaume I. Castellón, Spain
}

Information and Communication Technology (ICT) plays a pivotal role as a key advantage in our current labor market and occidental societies, with an important impact on employee well-being as well. However, different scholars have shown the negative effects of technology on users, and others have demonstrated that its impact differs depending on the type of exposure and the mediating variables, like appraisal of exposure.

Based on the Positive Occupational Health Psychology, the present study focuses on the positive impact of types of technology exposure and cognitive positive appraisal of past experience with ICT on work engagement. Specifically, this paper tests the hypothesis that the positive appraisal of ICT exposure mediates the impact of technology exposure (i.e., frequency of use and technology training) on work engagement (i.e., vigor, dedication and absorption) in a sample of 645 workers from Spanish private and public companies (337 males, 52.8\%) who used technology as an inherent part of their jobs.

Results using SEM analyses revealed that it is not the technology per se, but the types of technology used in terms of the frequency of use and technology training, that (directly and indirectly) relate to work engagement. In light of this, the results show that, as expected, the effects of technology exposure (i.e., frequency of use and technology training) on work engagement are not direct, but mediated by positive cognitive appraisal. That is, the more experience with technology, the more positive the technology appraisal and, consequently, the higher the levels of work engagement (i.e., more vigor, dedication and absorption). This result is also in line with the non-deterministic approach where technology exposure does not determine the consequences of technology on users; rather, it depends on other factors, such as the employee's cognitive appraisal of technology.

However, we obtained a result that we did not expect as a direct and negative relationship was found from one of the two types of technology exposure and work engagement. That is, the more technology exposure in terms of frequency of use, the lower the work engagement (i.e., less vigor, less dedication and less absorption). Study limitations and practical implications are discussed.

\section{Paper 2:}

\section{Conflict, Performance And Commitment In Teacher Teams: The Mediating Role Of Team Potency}

${ }^{1}$ le Blanc, P.M., ${ }^{2}$ van Emmerik, H.Y., ${ }^{3}$ Euwema, M.C., ${ }^{4}$ Salanova, M. \& ${ }^{4}$ Vera, M.

${ }^{1}$ Utrecht University, the Netherlands; ${ }^{2}$ Maastricht University School of Business and Economics, the Netherlands; ${ }^{3} \mathrm{KU}$ Leuven, the Netherlands; ${ }^{4}$ Universitat Jaume I. Castellón, Spain

Objectives: Interpersonal conflict is inherent to the life and dynamics of work teams, and is 
related to important outcomes such as team performance as well as team member satisfaction and commitment. However, still little is known about the mediating mechanism by which intrateam conflict affects outcomes. According to Antoni and Hertel (2009), we need to know more about the psychological mechanisms of teamwork in order to explain and predict why certain teams are functioning successfully while others don't. The current paper tries to fill this void by proposing a model in which team task conflict and team relationship conflict are indirectly related to team performance and team commitment through team potency. Moreover, the model proposes that both types of conflict make an independent contribution to team potency.

Methods: Data were collected as part of a larger study on the functioning of interdisciplinary teacher teams in Dutch secondary education. Teachers from 38 teams received a structured questionnaire on team(work) characteristics and work-related well-being. Three weeks after the start of the study, a $65 \%$ response rate was obtained. We used a minimum within-group response rate of $60 \%$ as criterion for inclusion of teams in the data analyses. As a result of this criterion, the number of teams that was eligible for inclusion was 33, consisting of 269 teachers.

For each of the participating teams, teachers provided questionnaire data on team conflict, team potency, and team commitment, whereas the performance of each team was rated by the respective school principals. Analyses were performed at the team level. In order to test our hypothesized model, we performed structural equation modelling with AMOS using the bootstrapping resampling method (Preacher \& Hayes, 2008).

Results: Both types of team conflict were negatively related to team potency, which in turn was positively related to both outcomes. Moreover, the results of the SEM-analyses demonstrated that team potency fully mediated the relationships between each of the two types of team conflict and team performance and team commitment, respectively.

Implications: In order to prevent high levels of intra-team conflict from negatively affecting team outcomes, teams could be trained in conflict management strategies which strengthen - instead of weaken - collective feelings of potency .

Conclusions: Team potency can be considered a key explanatory variable linking team conflict to team outcomes.

\title{
Paper 3:
}

\section{Work Positive: An Intervention to Improve Psychosocial Health and Safety in Hospitals}

\author{
Murray, P. \\ Health and Safety Authority, Ireland
}

\begin{abstract}
A busy theatre in the main Dublin teaching hospital, ANMCH (Tallaght Hospital) was the site of this project led by the Stat agency for implementation the European Directives on health and Safety at Work. The Health and Safety Authority, as part of its prevention strategy, tackled Psychosocial Risk through promotion, product development and in this case, assistance of a practical nature to at risk groups.
\end{abstract}


This intervention (from Sept 2008 - Sept 2009) which used both the Management Standards (UK) the Health and Safety (Ireland) survey tool, Work Positive, and the Burnout Inventory (Maslach \& Leiter). The theatre is a busy unit where surgeries are on-going; it is the main and largest surgical theatre unit in the Republic with a wide catchment area. As a workplace it's a meeting point for four main roles - nursing, medical doctors in training, medics out of training and other service and admin personnel. ON the basis that it is often our role within our work unit which predisposes us to stress, a survey was carried out on 250 workers across these four main areas. The results of the Work Positive survey showed widely different hazards for each role. Non Consultant Hospital Doctors (NCHDs) showed the highest reports of stress-related hazards. This sub group was then further surveyed using the Burnout inventory across the 100 NCHDs across 4 different specialisms. Differences were found across specialisms. Correlations were found between the Burnout and Work Positive findings within groups.

Suggestions are made for the findings, for the hazards and recommendations made for improvements.

\title{
Paper 4:
}

\section{CREW: Improving Worklife Through Collegiality}

\author{
${ }^{1}$ Leiter, M.P., ${ }^{2}$ Laschiner, H.K.S., ${ }^{3}$ Day, A. \& ${ }^{3}$ Gilin-Oore, D. \\ ${ }^{1}$ Acadia University, Canada; ${ }^{2}$ University of Western Ontario, Canada; ${ }^{3}$ St Mary's University, \\ Canada
}

Objective: The research question was determining the impact of changing workplace civility on the three aspects of burnout and work engagement.

Method: The presentation tests these concepts in a survey of health care providers before and after an intervention to enhance workplace civility. The initial survey included 1130 providers working in 40 units within 5 hospitals across Nova Scotia and Ontario, Canada. Participants from eight units completed the first wave of an intervention known as CREW: Civility, Respect, and Engagement at Work. A central quality of this intervention is a series of regular meetings among members of workgroups who are committed to enhancing the quality of their workplace community. The research team guides this process in various ways, including didactic training sessions, ongoing mentoring sessions throughout the six-month process, and community gatherings that bring together leaders from all of the participating sites.

Results: The post-intervention survey assessed differences in the social qualities of the worksetting, including civility, incivility (Work Incivility Scale (Cortina, et al, 2001), and trust. It also assessed participants' perception of core areas of worklife, using the Areas of Worklife Scale (Leiter \& Maslach, 2004), as well as burnout and work engagement using the Maslach Burnout Inventory-General Scale (Schaufeli, Leiter, Maslach \& Jackson, 1996) and a short version of the Utrecht Work Engagement Scale (Schaufeli \& Bakker, 2003). Overall, these measures indicated an improvement in civility within the intervention units in contrast with the waiting list control units. The presentation will describe the specific areas of improvement. 
Structural equation analyses determined that incivility from both colleagues and supervisors serve as workplace demands, contributing along with work overload to predicting exhaustion, a core aspect of job burnout. Further, the cynicism aspect of burnout was closely related to instigated incivility, or the frequency with which respondents acknowledge behaving incivility towards other people at work. The model builds on these relationships to depict the qualities underlying burnout and work engagement as mediating the relationship of experienced incivility to instigated incivility.

Conclusions: The session concludes with discussing the practical implications of this model. It also considers the potential application of the intervention approach to settings in health care and in other sectors.

\title{
Paper 5:
}

\section{Leader-member exchange (LMX) quality and enthusiasm at work: testing a dynamic mediated longitudinal relationship}

\author{
${ }^{1}$ González-Romá, V. \& ${ }^{2}$ le Blanc, P.M. \\ ${ }^{1}$ University of Valencia, Spain; ${ }^{2}$ Utrecht University, the Netherlands
}

Objectives: Recent longitudinal research has shown that leader-member exchange (LMX) quality is related to affective well-being at work (Atwater \& Carmeli, 2009; Sparr \& Sonnentag, 2008). However, these studies did not model the dynamic relationship between the aforementioned variables over time, and did not investigate the role of hypothetical mediating variables. Considering that LMX quality is related to psychological climate (Kozlowski \& Doherty, 1989), and that the latter variable is related to affective well-being (Carr et al. 2003), the objective of this study is to test a dynamic mediated relationship that involves the following hypotheses: 1 . change in leader-member exchange (LMX) quality over time is positively related to change in enthusiasm at work over time, and 2. the aforementioned relationship is mediated by change in psychological climate of innovation over time.

Methods: The study sample was composed of 711 employees of 33 health care centers. Data were gathered at 4 time points, with a 1-year lag between successive points. LMX quality was measured by means of Scandura and Graen's (1984) 7-item LMX scale. Innovation climate was measured by using the innovation scale of the FOCUS questionnaire (Van Muijen et al., 1999). Enthusiasm was measured by means of Warr's (1990) enthusiasm-depression scale. The data were analyzed by means of hierarchical linear modeling. The study hierarchical models were estimated by using the MULTILEVEL module of PRELIS/LISREL 8.80.

Results: The estimated coefficients yielded support for the study hypotheses. Change in LMX quality over time was positively related to change in enthusiasm at work over time $(.15, p<.01)$ and change in psychological climate of innovation over time $(.25, p<.01)$. Moreover, after controlling for change in LMX, change in innovation climate was positively related to change in enthusiasm at work over time $(.21, \mathrm{p}<.01)$. The Sobel test associated with the indirect effect (LMX-innovation climate-enthusiasm) was statistically significant $(2.59, p<.01)$.

Implications: The results obtained showed that the three study variables did not remain 
stable over the period of time considered but changed according to growth trajectories. More importantly, these results support the idea that the addressed relationships are dynamic and develop over time. Future investigations should take into account these findings and implement research designs that allow researchers to estimate relationships that are dynamic in nature.

Conclusions: Change in LMX quality is positively related to change in enthusiasm at work over time. Moreover, this dynamic relationship is mediated by change in psychological climate of innovation. 


\title{
Occupational Health Climate: Why Does it Matter?
}

\author{
Chair: Lunt, J.A. \\ Health and Safety Laboratory, United Kingdom
}

The contemporary evidence-base on the importance of organisational culture and climate variables on health and safety outcomes is compelling. The root causes of high profile accidents can typically be traced back to variables such as senior management commitment and the prioritisation given to health and safety. The method of choice for assessing the significance of these variables has, however, focussed on safety climate (e.g. Flin, 2007). Even where measures ask about both health and safety, both terms are treated as if one of the same entity. For three key reasons the case is building for separating occupational health from safety when defining culture and climate and gauging the role of organisational variables. Firstly, the characteristics of exposure to safety hazards can differ substantially from occupational health hazards. For the latter, harm can be latent and occur up to 40 years after initial exposure as in the case of asbestosis. For safety, harm can be instantaneous. Similarly, exposure to occupational health hazards is often insidious and cumulative in nature, which can make it difficult to attribute cause to work or non-work sources. For safety, the cause is usually more obvious. Collectively, these key differences in hazard characteristics means that safety risks will be perceived differently from health risks, and responded to in different ways. Secondly, wellbeing is now high on the UK government's agenda (Working for Health, 2008). This includes calls for assessment of attitudes in relation to occupational health. Finally, the constructs and behaviours that help shape occupational health climate may differ from those that affect safety climate. For example, the intangible nature of occupational health hazards undermines the 'face validity' of safety climate indicators such as near miss reporting.

This symposium presents a recent body of research undertaken by the Health and Safety Laboratory that sheds further light on the meaning of occupational health climate, its antecedents and consequences. It commences with two papers assessing the motivators that influence occupational health management in respect to respiratory protective equipment and noise control, and their knock on effect for occupational health climate. The first paper elucidates these motivators; the second indicates their relative strength. The final paper highlights some of the consequences of a 'healthy' organisational climate, in terms of job satisfaction and risk perceptions. By the end of the symposium a robust justification for developing and measuring occupational health climate as a distinct construct should have been provided.

\section{Paper 1:}

\section{Factors influencing choice and implementation of respiratory programmes in the workplace}

\author{
Bell N. \& Vaughan, N. \\ Health and Safety Laboratory, United Kingdom
}

Objectives: Little is known about the influences on managers' choice and implementation of respiratory controls. This paper presents findings from a cross-sector study carried out to address these gaps in current understanding. 
Methods: The research was qualitative involving a purposive sample of 20 organisations (mostly small-medium sized). Semi-structured interviews were carried out with 40 managers responsible for health and safety (on average two per company). The interview transcripts were analysed using a systematic approach, widely used in policy research, incorporating 'within' and 'between' case analysis that is not possible with a purely 'theme' based approach.

Results: Discrete factors were found to influence the choice of respiratory controls and implementation of these. For the former, legislation and external audits by the Health and Safety Executive, insurers and industry groups, motivated managers to consider appropriate controls. Managers sought advice on which controls to select from a range of external contacts; small companies looked to their suppliers. With regards to actual practice, regular supervision of operatives using respiratory protective equipment (RPE) and maintenance of kit and systems (e.g. refresher training) were often neglected. This was partly as organisations at early stages of implementation were constrained by economic issues. It was also apparent that managers often lacked knowledge of how to sustain good practice and, for some, knowledge of respiratory risks and RPE generally.

Implications: Training is an obvious mechanism to improve RPE practice e.g. communication campaigns on respiratory risks, developing simple RPE guidance for managers, including materials to use with their workforce. Existing communication channels could also be exploited e.g. cascading knowledge to managers through insurers and RPE manufacturers/suppliers. Of most importance, managers need to be convinced of the need to secure sustained resources for to combat adverse health, such as respiratory disease, by demonstrating the benefits of an ongoing commitment to health promotion.

Conclusions: This research raises the need for HSE, industry and the occupational health community to better communicate respiratory risks and good RPE practice to organisations. It is vital that managers better understand the necessary controls, including the importance of a maintenance regime. Creating a climate where respiratory risks are taken seriously is reliant upon managers: communicating health risks, possible consequences, and the required protective behaviour(s). Managers must also maintain momentum through regular training and updating of controls.

\title{
Paper 2:
}

\section{What influences employers to take noise risks seriously?}

\author{
Bell N. \\ Health and Safety Laboratory, United Kingdom
}

Objectives: The Health and Safety Laboratory is conductig research to determine which factors influence employers' noise management, inclusive of strength of effect, to suggest appropriate behaviour change interventions. This paper presents findings from an initial fact-finding stage that explored potential salient factors.

Methods: Fact-finding involved a literature review following systematic methodology and semistructured interviews with six Health and Safety Executive Inspectors. Interviews were used 
due to the scarcity of research on noise management; these were transcribed and thematically analysed. Factors extracted from both sources were grouped according to the PRECEDE model (Green \& Kreuter, 1991), useful for understanding possible interactions between factors and hypothesising their potential effect on employers' noise reduction behaviour. An objective assessment (strong, moderate or weak) was made for each factor extracted from the literature according to the quality of the underlying evidence. A simple distinction between influential, having some influence and not being influential was made for interview findings.

Results: A diverse range of individual and organisational factors influenced whether employers take steps to reduce noise. Overall, 15 factors emerged as pertinent for noise reduction, 14 from both sources (e.g. health and safety attitudes, climate, values, managerial knowledge and competence, financial drivers), and an additional factor from the literature, 'control, to accommodate employers who delegate noise decisions to lower-level managers. Some differences were noted in their apparent strength of influence. For instance, 'compliance' was considered a key driver in the literature, yet Inspectors considered 'fear of civil claims' to exert greater influence.

Implications: It is widely accepted that employers who set a good example in their attitudes and actions towards health and safety promote similar attitudes and behaviour in their workforce. As such, workers will only take noise seriously if a strong message of the necessity to do so comes from their leaders. Understanding what will motivate employers to reduce noise, ideally going beyond simply issuing hearing protection, is therefore vital.

Conclusions: This research will develop an understanding of which of the 15 factors identified to date are potentially more effective at motivating employers to reduce noise or indeed, whether there are leverages that are currently unknown. In doing so, it should help inform some of the 'transferable' constructs that make up occupational health climate, and isolation of the drivers affecting the priority an organisation attaches to occupational health.

\title{
Paper 3:
}

\section{The perception of risk, job satisfaction and occupational accidents within a healthy work climate}

\author{
${ }^{1}$ Gervais, R.L., ${ }^{2}$ Weyman, A. \& ${ }^{3}$ Williamson, J. \\ ${ }^{1}$ Health and Safety Laboratory, United Kingdom; ${ }^{2}$ University of Bath, United Kingdom; \\ ${ }^{3}$ Department for Work and Pensions, United Kingdom
}

Objectives: To explore the relationships between the belief in the risk of having an accident at work, the recording of accidents, the reporting of accidents to the Health \& Safety Executive (HSE), experiencing occupational accidents and job satisfaction within a healthy work climate.

Methods: The HSE's Survey of Workplace Absence Sickness and (ill) Health (SWASH) 2006/06 was used to generate a sample of the working population $(N=10,193)$. This survey provides comparison of data by age, gender, role, sector (Standard Industrial Classification) and occupation. Household telephone interviews were used to collect the data, with items on: the risk of experiencing an occupational accident, actual experience of accidents, the recording and 
reporting of accidents, with the mean measure of the following items used to create a healthy work climate index (i.e. support from manager, control of work, consultation on organisational changes, health and safety officers on site, workplace safety representative on site, trade union at workplace, employers' interest in employee health, safety or well-being, employer invests in prevention). ANCOVA and t-tests tested the relationships, inclusive of exploring these by sector and size of organisation.

Results: Individuals who felt that they worked in a healthy work climate were more likely to believe that they were not at risk of being involved in an accident at work, were more satisfied with their jobs, were less stressed and less likely to be involved in accidents. Sector or size of organisation influenced job satisfaction (size), experiencing an accident (size) and stressfulness of job (sector, size). Interestingly, the ANOVA analyses showed that employers sending a record of accidents to HSE positively influenced individuals' perceptions of whether or not they would experience an accident at work over the next 12 months.

Implications: Previous research shows individuals who perceive that they work within a healthy and safe environment are less likely to engage in unsafe or dangerous working practices. Similarly, workers are motivated and influenced to engage in safe practices by wider organisational actions, such as the provision of support and involvement in changes.

Conclusions: The safety element within organisations is progressing from one that is purely 'safety' and 'behaviour' specific to include other organisational factors, such as management practices and principles that are known to impact on outcomes e.g. performance and productivity. This research adds to the literature in this area, especially in understanding how the climate of organisations is essential to promote safe and healthy outcomes. 


\title{
Academic work demands and resources and higher education students' well-being
}

\author{
Chair: Pinto, A.M. \\ University of Lisbon, Portugal
}

Higher education institutions can be thought-out as specific occupational contexts in which students face academic work demands, are offered resources (Chambel \& Curral, 2005) and experience different levels of well-being (Schaufeli, Martinez, Marques Pinto, Salanova and Bakker, 2002). Accordingly, some previous research on higher education students' well-being (e.g. Chambel and Curral, 2005; Schaufeli et al, 2002) borrowed models such as Karasek and Theorell's (1990) Job Demands-Control-Support (JD-C-S) model, and concepts like burnout and engagement from the occupational health psychology domain.

In this symposium we draw on these previous works and introduce some innovative perspectives to study higher education students' well-being. Four presentations are included.

The first presentation, addresses the impact of the changes implied by the Bolonha process on the well-being of higher education students; based upon Karasek and Theorell's (1990) JD-C-S model they compared pre and post Bolonha students, from the same university, and found significant differences on their perceptions of work demands, control, peer support and academic well-being, that give support to the use of the JD-C-S model. In the second presentation, the authors use Keyes` (Keyes and Magyar-Moe, 2003) multidimensional perspective on context free well-being to explore differences in perceived well-being levels between higher education students who do volunteer work and those who do not; results supported the usefulness of a multidimensional perspective on students' well-being. Next, the authors present a qualitative, exploratory work on higher education students' representations of factors associated to burnout and engagement; (Demerouti, et al, 2001) as a heuristic model of academic work demands and resources related to higher education students burnout and engagement. In the final presentation, the authors use the Demerouti, et al, (2001) model to explore, with a longitudinal design, the main effects of academic demands and resources on higher education students ` burnout and engagement; once again results supported the usefulness of the to study the role of academic work characteristics on higher education students ' well-being.

\section{Paper 1:}

\section{The impact of the Bolonha process on student's well-being: A comparison of pre and post Bolonha students on their perceptions of work demands, control, peer support and well-being.}

\author{
Pinto, A.M. \\ University of Lisbon, Portugal
}

The Bologna Declaration has put in motion a series of reforms to the Portuguese Higher Education system. In this study we assessed the impact of the changes implied by Bolonha process on the well-being of higher education students. These changes were mostly structural, specifically with the introduction of a tutorial organisation of the courses. Thus, based upon Karasek and Theorell's (1990) Job Demands-Control-Support model, we compared two 
samples of psychology students from the same university with a gap of five years. In the first moment 348 students from a 5-year undergraduate psychology course answered a questionnaire comprising measures of work demands and control, peer support and anxiety/depression. In the second moment 283 students from a 3-year undergraduate course and a 2-year master course in psychology answered a questionnaire comprising measures of work demands and control, peer support and emotional exhaustion.

Results showed that post-Bolonha students perceived higher levels of demands, higher levels of control and lower levels of peer support than their counterparts in the pre-Bolonha organisation. The post-Bolonha students also showed lower levels of well-being than the preBolonha students. A tutorial arrangement with smaller groups, more contact hours and more peer interaction seemed to increase the demands and the perception of control, but do not contribute to increase the perception of peer support. We argue that this lower perception of support might account for the lower levels of well-being reported by post-Bolonha students. Results give support to the heuristic value of Karasek and Theorell's (1990) model for the study academic work characteristics associated to higher education students' well-being.

\title{
Paper 2:
}

\section{Volunteering and higher education students' well-being and academic performance}

\author{
Pinto, A.M. \& Figueira, C. \\ University of Lisbon, Portugal
}

Volunteering for community service is increasingly frequent among higher education students, thus becoming a phenomenon of growing interest for the study of educational practices and to knowledge building about positive psychological processes (Martínez-Odría, 2002). According to some authors the practice of volunteering can have positive impacts on the lives of students, namely on students ` well-being (e.g. Davila De Leon, et. al., 2005). This study aimed to test differences in perceived well-being levels and academic performance between higher education students who do volunteer work and those who do not. For this purpose we drew on Keyes ` (Keyes and Magyar-Moe, 2003) integrative perspective of well-being that conceptualizes wellbeing along three dimensions - emotional well-being, psychological well-being and social wellbeing. Using a cross-sectional design we collected data on higher education students $(\mathrm{N}=323)$ and found out that $18 \%$ of participants were doing volunteering work at that time while $82 \%$ were not. All the participants answered the Mental Health Continuum, long form (Keyes, 2005) a measure of emotional $(\alpha=.84)$, psychological $(\alpha=.80)$ and social $(\alpha=.75)$ well-being. Academic performance was assessed through previous term grades and also by computing the ratio of the number of passed exams in the previous term relative to the total number of exams during that same period; the resulting ratio was multiplied by 100 so that the final index indicates the proportion of passed exams. We performed ANOVAs to test for mean differences in well-being and academic performance across volunteer and no-volunteer groups.

Results showed that higher education students who do volunteer work have significantly higher levels of perceived psychological and social well-being and had significantly higher grades on 
the previous term. No significant differences were found on emotional well-being levels or on the ratio of passed exams. Our research design does not allow us to conclude whether the differences found in higher education students well-being levels and academic performance are due to their volunteer work practices or are prior differences to volunteer work (Thoits and Hewitt, 2001). Nevertheless, results support the usefulness of a multidimensional perspective on well-being and encourage further research on the potential role of volunteering as a valuable academic resource for the promotion of higher education students' well-being.

\title{
Paper 3:
}

\section{Higher education students' representations of factors associated to burnout and engagement}

\author{
Figueira, C., Chambel, M.J., Pinto, A.M., Curral, L. \& Castanheira, F. \\ University of Lisbon, Portugal
}

Some previous research on higher education students' well-being borrowed burnout and engagement concepts from the occupational health domain (Schaufeli et al, 2002). Students' burnout refers to feelings of exhaustion associated to academic demands, cynical attitudes toward one's study, and feelings of low self-efficacy; conversely students' engagement is defined as a positive, fulfilling, and academic work related state of mind, characterized by vigor, dedication, and absorption (Schaufeli et al, 2002).

The Job Demands and Resources model (JD-R) (Demerouti, et al., 2001) has been extensively used to explain burnout and engagement reactions at work, but as far as we know no studies have been carried out on student burnout and engagement using this model. The present study aimed to explore higher education students' representations of factors associated to burnout and engagement. To accomplish this goal we used the JD-R model (Demerouti, Bakker, Nachreiner, Schaufeli 2001) as a conceptual frame of reference and developed a qualitative exploratory study through focus group interviews, following Krueger and Casey (2000) guidelines. Focus groups $(\mathrm{N}=8)$ were conducted with students from a 3-year undergraduate course and a 2-year master course in psychology $(\mathrm{N}=47)$ from a Portuguese University, until theoretic saturation was attained about academic demands and resources perceived as contributing to their own and their colleges burnout and engagement levels.

Participants expressed satisfaction with academic resources such as role clarity and peer social support. High emotional academic-work demands and low institutional support on areas like personal and career development or employability skills training were perceived as major determinants of high burnout and low engagement levels. Results give preliminary support to the use of the JD-R model ((Demerouti, et al., 2001) as a heuristic model of academic work demands and resources associated to higher education students' burnout and engagement. Additionally, results offer some clues on relevant areas for the promotion of students' well-being. Of course, given the exploratory nature of our study and the limited number of participants, further research is recommended. 


\title{
Paper 4:
}

\section{Academic demands and resources `impact on higher education students` burnout and engagement: An application of the Job Demands-Resources model}

\author{
Castanheira, F., Pinto, A.M., Chambel, M.J., Curral, L., \& Figueira, C. \\ University of Lisbon, Portugal
}

According to the Job Demands- Resources Model (JD-R) (Demerouti et al, 2001) employees ` work conditions can be categorized into demands and resources. Job demands and resources are typical of specific occupations and relate in different ways to employees ' well-being, namely to professional burnout and engagement (Hakanen et al, 2008). Higher education institutions can be thought-out as specific occupational contexts in which students face demands and are offered resources (Chambel \& Curral, 2005), develop burnout and engagement reactions and do their academic work with varying degrees of success (Schaufeli et al 2002). To our knowledge the JDR model hasn 't still been used to study higher education students' well-being. Therefore, the present study aimed to test, with a longitudinal design, the main effects of academic demands and resources on higher education students ` burnout and engagement $(N=73)$ during an examination period (T1) and 4 months later (T2), as proposed in the JD-R model.

Job demands $(\alpha \mathrm{T} 1=.85 ; \alpha \mathrm{T} 2=.85$ ) were assessed with the workload scale developed by Chambel and Curral (2005). The three job resources included in this study were work conditions $(\alpha \mathrm{T} 1=.79 ; \alpha \mathrm{T} 2=.77)$, role clarity $(\alpha \mathrm{T} 1=.91 ; \alpha \mathrm{T} 2=.87)$ and peers social support $(\alpha \mathrm{T} 1=.91$ ; $\alpha \mathrm{T} 2=.89$ ) and were also assessed with Chambel and Curral `s (2005) work characteristics scales. Work engagement was assessed with vigour $(\alpha \mathrm{T} 1=.72 ; \alpha \mathrm{T} 2=.73)$ and dedication $(\alpha \mathrm{T} 1=.70 ; \alpha \mathrm{T} 2=.71)$ scales from the UWES (Schaufeli et al., 2002). Burnout was measured with exhaustion $(\alpha \mathrm{T} 1=.73 ; \alpha \mathrm{T} 2=.79)$ and cynicism $(\alpha \mathrm{T} 1=.84 ; \alpha \mathrm{T} 2=.84)$ scales from the MBI-SS (Schaufeli et al., 2002).

The hypotheses were tested with cross-lagged regression analysis based on two waves over the 4 month period. Auto-regression effects were included in order to control for baseline levels for each dependent variable. Although we hypothesized causal paths, we also tested synchronous relationships between the study variables. Academic demands and resources influenced synchronously students' burnout and engagement, as hypothesized, the only unexpected result being the positive effect of role clarity on cynicism, both at T1 and T2. Furthermore, role clarity had a negative impact on exhaustion and a positive impact on vigour and dedication, and again on cynicism, over time.

Results give additional support to the usefulness of JD-R model, as they confirmed the role of academic work characteristics on higher education students' well-being explanation. A deeper analysis of role clarity - cynicism relationships in this particular academic context is recommended. 


\title{
How to find a balance and cope with the multiple roles of work, family and private life?
}

\author{
Chairs: ${ }^{1}$ Mauno, S. \& ${ }^{2}$ Rantanen, J \\ ${ }^{1}$ University of Jyväskylä, Finland; ${ }^{2}$ University of Tampere, Finland
}

Background \& Objective: The difficulty of balancing work, family and private life domains has received much more attention than the positive interplay between these domains. In addition, much is known about the situational and psychosocial (e.g., working hours, parenting) antecedents of work-family interface but less about the various organisational and individual strategies which might alleviate negative and enhance positive interplay between work, family and private lives. Therefore, this symposium of five presentations aims to answer the question: How to find a balance and cope with work and non-work roles?

In the first two presentations the essence and possible multiple forms of work-nonwork balance as well as factors connected to these different forms of balance are considered. The following presentation will investigate co-worker and supervisor support as moderators between job demands/resources and work-family conflict. Finally, the last two presentations will clarify what kinds of coping strategies employees use in balancing between work and family demands, and which coping strategies work best in this regard.

Methods: All the presentations will be based on a quantitative design and the first two presentations will be based on a person-oriented approach, whereas the last three presentations will utilize a variable-oriented analysing strategy. Specifically, in the first presentation, the Finnish university and hospital employees ( $\mathrm{n}=2718$ ) will be analyzed through latent profile analysis. In the second presentation, the occupational trajectories of German psychologists with 15 years work experience $(n=585)$ are identified and then examined in more detail based on sub-sample ( $n$ $=20$ ) interviews. The third presentation is based on a longitudinal sample of Swedish service employees, whereas the fourth presentation is based on a large cross-sectional sample of Finnish nurses and service workers $(n=2764)$. In the fifth presentation about 200 hundred employees from Portugal will be studied.

Results \& conclusions: Altogether, the presentations will show what sort of resources (e.g. autonomy and control, social support, and coping skills) are beneficial in searching a better balance between work, family and personal lives. Furthermore, they will also show that work-family interface can well be approached also from a positive perspective, that is, from facilitation/enrichment. Finally, from a methodological point of view, the presentations will portray interesting results complementing each other because both variable- and person-oriented approaches to work-life balance are included. 


\title{
Paper 1:
}

\section{Work-family balance and its relations to job and personal characteristics and well- being: A person-oriented approach}

\author{
${ }^{1}$ Rantanen, J., ${ }^{1}$ Kinnunen, U. \& ${ }^{2}$ Mauno, S. \\ ${ }^{1}$ University of Tampere, Finland; ${ }^{2}$ University of Jyväskylä, Finland
}

Objective: Work-family balance is a multifaceted construct which according to the fourfold taxonomy by Frone (2003) consists of low work-family conflict and high work-family enrichment in two directions: from work-to-family (WF) and from family-to-work (FW). The problem with this variable-oriented approach is, however, that the numerous possibilities how the four dimensions of work-family balance (i.e., WF-conflict and -enrichment, FWconflict and enrichment) may be combined complicate the precise specification of the workfamily balance (Grzywacz \& Bass, 2003). Therefore, in this presentation a person-oriented approach to work-family balance is taken and empirically examined in order to get a better understanding of the different combinations of the work-family conflict and enrichment experiences as well as their implications for well-being.

Method: The data to be analysed consists of 2,718 participants representing two occupational groups that will also be compared: 1,888 university employees (mostly upper white-collar workers) and 830 nurses (lower white-collar workers). Through latent profile analyses we investigate what kind of latent groups can be identified when the experiences of WF-conflict and -enrichment as well as FW-conflict and -enrichment are considered simultaneous instead of separate constructs. For example, one interesting question is do we find a 'fully balanced' group who is both high in WF- and FW-enrichment and low in WF- and FW-conflict? And what kind and how many other work-family balance groups can be identified via latent profile analysis? We also validate the identified latent groups by examining whether they differ from each other in terms of job conditions (time pressures, insecurity, control, support), core-self evaluations (i.e., emotional stability, self-efficacy, self-esteem, and locus of control), and well-being (vigor and exhaustion at work, self-rated health and psychosomatic symptoms, life satisfaction).

Conclusions and implications: The presented results will answer the question of what kind of combinations of work-family conflict and enrichment are typical, and what kind of workfamily balance they reflect. In addition, the results will help scholars and practitioners to direct work-family initiatives and assistance policies towards those actions that seem most relevant. For example, it is not yet known whether actions directed towards increasing work-family enrichment are sufficient to overcome experiencing work-family conflict and negative well-being consequences related to it. The other possibility might be that it is better to concentrate on reducing work-family conflict. 


\title{
Paper 2:
}

\section{Work-Work-Life Balance: Burdens and Chances of Multiple Jobholding and Portfolio Careers}

\author{
Olos, L. \\ Free University Berlin, Germany
}

Objective: The initial hypothesis was that multiple jobholding would exacerbate the difficulties of combining work and private life; multiple jobholders have to reconcile not only "work" and "life," but also "work" and "work."

Methods: In a study on the occupational trajectories of German psychologists with 15 years' work experience ( $\mathrm{N}=585 ; 68 \%$ women), long-term multiple jobholding $(\mathrm{N}=121 ; 64.5 \%$ women) emerged as the most prevalent career pattern. Multiple jobholders hold two or more jobs in parallel for years; these jobs may or may not be in similar areas and/or forms of employment. Some of the respondents with such portfolio careers $(N=20)$ were interviewed in detail on their occupational trajectories and the relationship between work and private life.

Results: A first group of respondents—the "conflicted" - indeed has great difficulty coordinating the spheres of life. These respondents are primarily mothers who, finding only "minijobs," are forced to do more than one job for financial reasons. Their lifestyle is characterized by conflicts of interest and multiple commitments. The diverse occupational commitments of a second group of "work-centered self-actualizers" can even cause personal relationships to fail. The men and women in this group themselves use the term "workaholic" to describe their strong focus on working life, and they tend to remain childless. "Work-centered breadwinners," on the other hand, are men who have found wives with a traditional view of gender roles and who delegate family responsibilities to them. Finally, a fourth group of "balanced" respondents chose multiple jobholding precisely because it offers the opportunity to integrate the work and private spheres of life, and use it as an optimal form of work-life balance. For these men and women, integrity, autonomy, temporal flexibility, and the support of the spouse/partner play a key role. They see work and family as a double benefit rather than as a double burden.

Conclusion: As the structural conditions of work change, multiple jobholding is likely to increase, opening up new opportunities and creating new risks for the individual work-life balance.

\section{Paper 3:}

\section{Work-family conflict: Who is at risk?}

\author{
Richter, A. \& Näswall, K. \\ Stockholm University, Stockholm
}

Background: Work-family conflict has increased relevance in today's work environment, as both men and women are working full time to a greater extent, due to increased gender 
equality. Work-family conflict has become one of the 10 most common stressors experienced by employees. An increasing proportion of individuals are managing both the family and the work role simultaneously which might lead to an inter-role conflict where the work role inhibits the proper fulfillment of the family role and therefore is experienced as a stressor. Previous research has shown that this domain-crossing stressor is associated with negative consequences such as burnout, marital and life dissatisfaction as well as other health issues. This implies that it is a serious stressor as it affects both the work as well as the family domain. Therefore, research on work-family conflict should receive high priority, and investigate factors that can help identify employees who are more at risk than others, as well as study factors which might work as buffers to decrease the experience of work-family conflict, which is going to be addressed in this study. Primary antecedents of work-family conflict have been structural factors in the work domain, such as work overload, distress, as well as work-role ambiguity.

Aims: The present study aims at integrating a stress perspective with work-family conflict research and investigates a model where work-family conflict is predicted by certain demands such as role conflict, powerlessness, job insecurity and carry over between work and family. Potential resources such as the perception of control and goal clarity, as well as trust in the organisation are also included in the model, in order to investigate whether they lead to decreased experiences of work-family conflict. In addition, social support from the supervisor as well as colleagues is introduced as a potential moderator of the relation between demands and the experience of work-family conflict. Such support can be influenced by the organisation and therefore might be a first step towards potential interventions with the aim to decrease work-family conflict.

Methods \& Results: This study was conducted with a longitudinal Swedish samples from the service sector. The aim of the current study is to test a model including variables which can be modified by organisations as well as employees themselves, so that it is applicable in practice.

\title{
Paper 4:
}

\section{Do work-family coping strategies facilitate successful reconciliation of work and family under high job and family demands?}

\author{
${ }^{1}$ Mauno, S. ${ }^{2}$ Rantanen, M., ${ }^{2}$ Rantanen, J., \& ${ }^{2}$ Kinnunen, U. \\ ${ }^{1}$ University of Jyväskylä, Finland; ${ }^{2}$ University of Tampere, Finland
}

Background: Coping strategies have evoked less interest among work-family researchers than other kinds of "family-friendly" resources, for example, organisational work-family benefits. However, over decades, coping strategies have shown to be important factors in job stress and health research (e.g., Folkman \& Moscowitz, 2004; Lazarus \& Folkman, 1984; Penley et al., 2002). Thus, it is well arguable that coping strategies deserve more attention also in the workfamily context as a reconciliation of work and family is often experienced stressful. Despite the importance of coping strategies in stress adaptation, it has been argued that they are hard to measure via overall scales. Consequently, context-specific coping scales have been called for also in the arena of work-family interface. Namely, Somech and Drach-Zahavy (2007) suggested that general measures of coping are inadequate in assessing coping in work-family research. In response to this, they developed a context-specific coping scale: Work-Family Coping Strategies $(=$ WFCS $)$. 
Aims: The aim of our study is to examine the structure of WFCS among Finnish employees and to clarify how its different eight dimensions (i.e., super at home/work, good enough at home/work, priorities at home/work, and delegation at home/work) relate to negative and positive workfamily experiences: work-to-family and family-to-work conflict and enrichment. Furthermore, we will also examine whether, and if so, which dimensions of WFCS buffer against the negative effects of job and home demands (e.g., perceived workload at job and home, parenting stress) on conflict and enrichment. Testing these buffering effects has theoretical foundations in stress theory and coping research.

Methods \& Results: This study will be based on data collected in ongoing research project "Work-family coping strategies and their role in the work-family interface". Data collection from 1712 nurses and 1106 service employees has been just finalized and the full data will be ready for analyses in December 2009. Data will be analyzed via Confirmatory Factor Analysis (psychometrical validation) and hierarchical moderated regression analysis (buffering effects).

Conclusions: The findings will show how important are individual work-family coping strategies in alleviating work-family/family-work conflict and promoting work-family/familywork enrichment. Moreover, the results will also reveal do certain work-family coping strategies ameliorate the negative effects of job and home demands on conflict and enrichment, illuminating potential buffering effects.

\title{
Paper 5:
}

\section{Patterns of coping with multiple roles and their impact on work-family enrichment and conflict}

\author{
Matias, M. \& Fontaine, A.M. \\ University of Porto, Portugal
}

Background: Managing work and family responsibilities has become an increasing concern in today's societies due to part to the changes in the roles of men and women in both workplace and at home. Dual-earner families are increasing and the way individuals and couples can balance family and work is now, more than ever, a challenge. Traditionally, research has focused on the conflict and strain that dual-earner couples experience. More recently, the interest has shifted towards the analysis of the adaptive strategies they use. Furthermore, since work and family are highly gendered domains, the study takes into account gender by analysing men and women separately. Preliminary qualitative studies have shown that both men and women tend to rely on familial coping strategies and on resources and support in order to manage their multiple responsibilities, nevertheless some gender differences have also been found. Women tend to rely more on reconciliation strategies than men; men emphasize more individual characteristics than women do, and women emphasize more daily planning than men do. Thus, gender differences are likely to be found in the ways how work-family coping behaviours are associated with workfamily conflict and enrichment.

Aims: One of the purposes of this study is therefore to characterize the work-family interface by describing the strategies used by dual-earners to manage work, family and personal roles, that is, work-family coping patterns. A second goal of this study is to analyse the impact of distinct work-family coping patterns on work-family conflict and enrichment. 
Methods \& Results: Two hundred individuals, part of dual-earner families responded to a paper and pencil survey. In order to determine work-family coping patterns, data reduction techniques will be performed and distinct work-family coping behaviours will be identified. To examine the second research question, multiple regression analyses were performed to clarify the relationship between work-family conflict/enrichment and work-family coping patterns. Gender differences will also be analysed.

Conclusions: The findings to be obtained may help us explain why individuals in the same work and family environment have different work-family coping behaviours. 


\title{
Developing Intervention Process Evaluation
}

\author{
Chair: Randall, R. \\ University of Leicester, United Kingdom
}

In this symposium we present three empirical papers that show how the use of new methods of process evaluation can help to further our understanding of the effectiveness of organisationallevel interventions. The fourth paper draws together the key findings from contemporary intervention research to set out an agenda for future research and practice. The symposium has been designed to present a detailed examination of the importance of process evaluation in intervention research.

\section{Paper 1:}

\section{Strengthening the evaluation strategy of stress management intervention programs using process evaluation and multilevel methods: The evaluation of a team-based stress management intervention program for low-qualified workers}

\author{
Busch, C. \& Clasen, J. \\ University of Hamburg, Germany
}

Objectives. The evaluation of occupational stress management intervention programs has proved challenging for researchers and practitioners alike. This applies in particular for interventions with low-qualified workers and for interventions, which should prove external validity, i.e. intervention programs should be effective in various work organisations. In this contribution, we present the evaluation of a team-based stress management intervention program for lowqualified workers using process evaluation and multilevel methods.

Methods. Although low-qualified workers are at particular risk in terms of their health, intervention programs for this target group are lacking. Therefore, a manualized intervention program was developed and evaluated. The project was funded by the German Federal Ministry of Education and Research (2006-2009). The intervention program is team-based and combines four training sessions for team workers with two training sessions for the direct supervisor. The training sessions for team workers deal mainly with physical exercise, resources and collective coping in team work, like social support among team members and problem-solving activities. The intervention covers a training of the direct supervisors to ensure their support for the program and to enhance participation and appreciation.

The evaluation was split into two phases, the pilot and the evaluation phase. In the pilot phase the program was carried out in six German companies of different industries with an extensive process evaluation $(n=73)$. The process evaluation showed e.g. the differential team quality and the effects of trainers' adherence to the manual on the session and program evaluation by the participants.

On the basis of the results of the pilot phase the whole program was revised and carried out nine times in eight companies. The quasi-experimental control-group design in this phase comprised 
pre-, posttest, and follow-up measurements among 268 employees from 34 teams. Hierarchical linear modeling was used to handle the hierarchical data structure. The main analysis model was a three-level model, with repeated measurements nested within individuals, who are nested within teams.

Results. The intervention program affected job strain in the full model as hypothesized. The process evaluation showed session specific outcomes to be effective, like exercise, social support among team members, if team meetings are held regularly, if the trainers' adherence to the manual is moderate and if the cultural diversity in the teams is low. The number of sessions attended by the participants did not affect the outcome.

Implications. Using process evaluation and multilevel methods, we could strengthen the evaluation strategy and mechanisms of change became visible.

\title{
Paper 2:
}

\section{Does training managers help? A longitudinal, mixed methods field study of the implementation of teamworking}

\author{
${ }^{1}$ Nielsen, K. \& ${ }^{2}$ Randall, R. \\ ${ }^{1}$ National Research Centre for the Working Environment, Denmark; ${ }^{2}$ University of Leicester, \\ United Kingdom
}

Objectives: The introduction of teamworking sometimes fails to bring about positive effects. This may be due to poor implementation practices and negative concurrent events. Unfortunately, little is known about how the process of implementing teamwork should be conducted in order to achieve the best results. In particular it is unclear whether its impact is enhanced by giving training to team managers to help them respond to the demands of the changes in their role that occur with team implementation. This paper presents a longitudinal field study of the impact of manager training that was delivered during the implementation of teamwork. The study explored both the processes and outcomes of the training intervention within the four levels of Kirkpatrick's model of training evaluation. As such we aimed to explore at which level training had exerted an effect: 1) The participating managers' perceived usability of the course, 2) changes in values and attitudes, 3) changes in behaviours and finally, 4) changes in followers' perceptions of teamwork, involvement and job satisfaction. Finally, team implementation and the transferability of training within the organisational context (barriers and facilitators were explored).

Methods: A longitudinal intervention design was applied in a part of the Danish the elderly care sector that was implementing teamwork. 12 managers received training and 18 managers functioned as a comparison group. A combination of qualitative and quantitative methods was employed: The training course was evaluated using observations during the training course, module evaluation and internal reference evaluation. Action plans developed at the training course were also collated. Questionnaires from followers, interviews were conducted with all managers and employee representatives in both the intervention and the comparison group. 
Results: At level 1, managers participating in training felt the training course had been useful. At level 2, there were some indications that attitudes had changed but not for all managers. It appeared that only some managers had changed behaviours (level 3). Followers whose managers had been on training reported significantly higher involvement and job satisfaction (level 4). Context analysis revealed that concurrent changes had hindered team implementation.

Implications: This study has three important implications. First, manager training may help ensure successful team implementation. Second, the Kirkpatrick model allowed change to be examined at several levels and was a useful way of separately identifying theory failure and process failure. Third, it shows the importance of careful documentation of the implementation processes.

\title{
Paper 3:
}

\section{Substance over style: The importance of theories-in-use as determinants of intervention outcomes}

\author{
${ }^{1}$ Randall, R. \& ${ }^{2}$ Nielsen, K. \\ ${ }^{1}$ University of Leicester, United Kingdom; ${ }^{2}$ National Research Centre for the Working \\ Environment, Denmark
}

Objectives: Research has shown that a variety of organisational change interventions can be effective but that problems with implementation and a difficult intervention context often undermine their impact. Intervention plans are not always translated into intervention activities or theories-in-use. There is a growing body of research that shows middle managers often play a crucial role in shaping employees' experiences of interventions, and hence intervention outcomes (Nielsen \& Randall, 2009). It also appears that transformational leadership behaviour can have a strong positive impact on employees. In this study we examined the extent to which middle mangers' transformational leadership behaviours were important in determining exposure to a teamwork intervention. We then examined the extent to which intervention-related activities determined the impact of a teamwork intervention on employee working conditions, well-being and job satisfaction.

Methods: A longitudinal intervention study was carried out in the elder care sector in a large Danish local government organisation with 539 participants (as reported in Nielsen \& Randall (2009), paper 2 in this symposium). Before the intervention, poor role clarity, and low influence and few opportunities for development were found to be a problem. The introduction of teamworking was designed to address these problems.

Results: Structural equation modelling showed that transformational leadership behaviour was significantly linked to employees' self-reported exposure to teamwork intervention activities. This exposure to intervention theories-in-use - and not transformational leadership behaviour - was directly linked to post-intervention working conditions. This two-stage mechanism fully mediated the impact of the intervention on various outcome measures.

Implications: These results suggest that the transformational leadership behaviour is important during interventions because it is linked to employee exposure to intervention activities. Our 
findings also indicate that transformational leadership is behaviour is not necessarily a sufficient substitute for well-designed and properly implemented intervention activities.

\title{
Paper 4:
}

\section{Evaluating stress prevention through work-related interventions: Trade-offs involved, and implementation issues to be considered.}

\author{
Semmer, N.K. \\ University of Bern, Switzerland
}

There is now ample evidence that work characteristics and working conditions are related to well-being and health. Interventions aimed at work-related changes therefore seem a "natural priority". They can take variety of forms, changing (1) tasks itself and the way work is organized (e.g., by increasing autonomy), (2) physical working conditions (time, ergonomics); and / or (3) the social work environment, e.g., by training supervisors to be more supportive. Many interventions combine several measures, often based on a participatory approach. Furthermore, many interventions combine work-related and person-related (e.g., cognitive-behavioural stress management) approaches.

Altogether, however, person-related interventions are dominant. Their effectiveness has been shown empirically in quite a convincing manner. Regarding work-related changes, the evidence is more mixed. There are many examples of successful interventions, but there still is a paucity of strong evaluation designs. Overall, results tend to be positive, but inconsistent. Often, there are improvements in some outcome variables but not in others; improvements in variables that were immediately targeted (e.g., job autonomy) do not necessarily "translate" into improved health and well-being. Often, effects are confined to subgroups of participants. Negative effects, although rare, do occur, often due to poor implementation, and to increased time pressure following the introduction of teamwork. Recent meta-analyses that included only strong designs have yielded rather disappointing results. I will argue that there are substantive reasons that the results are mixed, since work-related interventions intervene in highly complex social systems. Furthermore, I will argue that we have to think more in terms of "trade-offs" with regard to what can reasonably be expected (e.g., changes in only some variables, for subgroups of participants).

I will also argue that more studies with better designs are needed, but that studies with less than optimal designs still can yield important information if investigators really go to the limits of what is possible. This involves, for instance, adapted designs, and nonequivalent dependent variables. Furthermore, more precise and clear documentation of processes and events is needed, preferably in a way that allows that information to be included in quantitative analyses. Recent developments are in this respect are encouraging. Still, more careful process analysis is needed in order to disentangle the effects of implementation from conceptual aspects. 


\title{
How to predict work engagement across professions and career stages
}

\author{
Chairs: ${ }^{1}$ Salmela-Aro, K. \& ${ }^{2}$ Wiese, B.S. \\ ${ }^{1}$ University of Helsinki, Finland; ${ }^{2}$ University of Basel, Switzerland
}

The four papers of the symposium approach work engagement (i.e., being happy to work and willing to invest effort) during different phases of the career by using sophisticated longitudinal approaches. Whereas the first two papers analyze the long-term development of work-related engagement, the last two papers examine how work engagement evolves on a daily basis.

The first paper focuses on the role of achievement and social strategies during university to early career work engagement and burnout. Using 18 year longitudinal study she shows how the level and increase of social and achievement-related optimism during university studies predict high level of work engagement and low level of burnout ten to fifteen year later. Second paper focuses on women's work engagement while re-entering into working life after maternity leave. Based on longitudinal data, she shows that work engagement is most likely to be high among those who appreciate work-related learning opportunities and who are willing to affectively commit themselves to the employing organisation. The third paper focuses on the role of social context to engagement. Here, examines how one partner's career self-efficacy is related to the other partner's work engagement using standardized diary data from couples. Final paper examines senior managers daily critical decisions, related affects as well as work engagement using diary approach.

In sum, the present papers point to the need for macro- and micro-longitudinal data as well as for a stronger social contextualization of individual work behaviour and decisions to deepen our understanding of work engagement as a crucial facet of positive experiences and well-being at work.

\section{Paper 1:}

\section{Strategies during University Studies predict Work Engagement}

\author{
Salmela-Aro, K. \\ University of Helsinki, Finland
}

The transition from university to work life presents a substantial challenge for youth in modern societies. The present longitudinal study examines the role of strategies young people apply during their studies to their early career adaptation. Some students deliberately seek to avoid challenging situations rather than make an active effort to deal with them. In contrast, others use more active, task-focused strategies when they face challenging situation, which are characterized by optimism, high effort and intensive problem solving. Achievement strategies refer to ways of approaching and responding to academic situations and individual differences in such patterns were operationalized by two constructs: task-optimism and -avoidance. Social strategies refer to ways of approaching and responding to social situations and individual differences in such patterns were operationalized as social-optimism and -avoidance. Early career adaptation was 
operationilized by work burnout and engagement. Work engagement refers to a positive, full-filling, work-related state of mind that is characterized by vigour, dedication and absorption, while work burnout is characterized by exhaustion, cynicism and inadequacy at work. The aim of this six-wave longitudinal study was to examine whether student achievement and social strategies measured during university studies would impact on work burnout and work engagement measured 10 to 17 years later. 292 university students completed the SAQ strategy questionnaire three times while at university, and the work burnout inventory three times and the work engagement inventory twice during their early career. The results were analysed using Latent Growth Curve Modelling. The results for strategies showed that both achievement and social optimism increased during the studies. Next, optimism both at achievement and social domains predicted a high level of work engagement and low level of work burnout 10, 14 and 17 year later. Moreover, increase of optimism during the studies predicted a high level of work engagement and low level of work burnout during early career. By contrast, a high level of avoidance predicted a low level of work engagement and high level of work burnout during early career. The results of our longitudinal study that spanned over 17 years showed that the achievement and social strategies young people evidenced when they were at university predicted their work engagement and burnout during their early career. Functional strategies and increase of them predicted successful career adaptation, while dysfunctional strategies predicted burning out.

\title{
Paper 2:
}

\section{Work engagement as an indicator of mothers' successful re-entry into working life}

\author{
Wiese, B.S. \& Dalit Jäckel \\ University of Basel, Switzerland
}

Despite being a common transition, mothers' return into working life after a maternity leave has been largely overlooked in psychology so far. It is particularly important to understand what predicts whether this transition is successful or not. In the present paper, we use work engagement as success criterion. Work engagement has been defined as a positive state characterized by enthusiasm for one's work, a happy engrossment in one's work, and a willingness to invest effort even when difficulties arise (see Schaufeli, Bakker, \& Salanova, 2006). Because engagement is inherently motivational and action-oriented it is not only desirable from the employee's point of view but also from the employer's perspective. We are testing the influence of work-related goal orientations (i.e., having a focus on learning, on proving competencies, or on avoiding competence evaluations, see VandeWalle, 2001) as well as of individual's organisational commitment on the development of work engagement. Our data stem from a four-wave longitudinal research project with 300 women re-entering the workforce. First measurement took place immediately before re-entry, the following three measurement points are scheduled about one, two and six months after re-entry. About half of them returned to the organisation at which they were previously employed, the other half of the women were organisational newcomers.

Our analyses revealed that work engagement is most likely to flourish among those employees who display high learning orientation and who are willing to affectively commit themselves to the organisation. Note, however, that already during the first few months of organisational (re) entry, work engagement turned out to be a relatively stable component of an employee's work motivation and attitude. 


\title{
Paper 3:
}

\section{How is one partner's career self-efficacy related to the other partner's work engagement?}

\author{
Neff, A., Niessen, C., Sonnentag, S., \& Unger, D. \\ University of Konstanz, Germany
}

During the last decades the percentage of dual-career couples has rapidly increased. Within these couples, partners often hold similar roles as they both seek competence and expertise at work. The effects of this similarity on positive health-related constructs are largely unexplored. If a successful career is important to both partners, do high career self-evaluations of one partner have salutary effects on the other partner in terms of improved work engagement or are they rather detrimental to the other partner? To investigate this question we examined the relation between one partner's career self-evaluations and the other partner's work engagement. More specifically, we focused on career self-efficacy as the cognitive component of career self-evaluations. In a diary study over five consecutive working days among 72 dualcareer couples $(\mathrm{N}=144)$, we examined how one partner's perceived career self-efficacy in the evening might be related to the other partner's work engagement the next morning. We wanted to identify specific moderators that influence the strength and direction of this relation.

Multilevel analyses using an actor-partner interdependence model revealed two crosslevel interactions for the relation between one partner's perceived career self-efficacy in the evening and the other partner's work engagement the next morning. First, the trait level of career self-efficacy played an important role. For participants high in trait career self-efficacy, high perceived career self-efficacy of their partners in the evening was associated with next day's higher work engagement. On the contrary, participants low in trait career self-efficacy showed the opposite pattern. Second, participants' general construal style moderated the relationship. We found that participants who construed themselves rather as part of their relationships than as independent individuals, showed higher work engagement the next morning when their partners had shown high career self-efficacy the evening before. In contrast, for participants construing themselves rather independently from their partners, the partners' high career self-efficacy evaluations was negatively related to their work engagement the following day. Both interactions stayed stable after controlling for age, gender, parenthood, and length of relationship.

In conclusion, high career self-efficacy of one partner seems neither to merely foster the work engagement of the other partner, nor just to impair it. The effects rather seem to depend on personal variables. Future research is needed to help dual-career couples to both establish successful careers and personal well-being. 


\title{
Paper 4:
}

\section{When Do Senior Managers Experience Engagement?}

\author{
Aro, A. \\ Diacor, Finland
}

Engagement experience can be defined as a situation in which high challenges are matched wit high competence. However, there is lack of studies conducted among senior managers' daily engagement experiences. Consequently, the aim of the present intensive longitudinal study was to examine the daily dynamics of senior managers' affects, personal decisions and related appraisals. To examine this, 30 senior managers' appraised their daily experiences of their decisions, related competence and challenge, and their positive and negative affects during a twoweek period. In addition, before and after the two week period they filled in Work Engagement (Schaufeli et al, 2002) and Bergen Burnout (Näätänen et. al., 2002) inventories and the Recovery Experiences Questionnaire (Sonnentag \& Fritz, 2007) and the Need for Recovery questionnaire (Veldhoeven \& Broersen, 2003). The participants belong to the senior management of a global ICT company. As a part of a broader coaching intervention project aiming to increase their abilities of self-leadership and competence in leading their own well-being at work, they participated in a two-week daily data collection period. Altogether 196 observations were collected on their experience, positive and negative moods, challenge, competence, their goals, situation and interaction. The preliminary results showed that high levels of both competence and challenge contributed to particularly high level of positive affects, such as flow, whereas a combination of a high level of challenge and a low level of competence contributed to a particularly high level of negative affects, such as anxiety. It is highly important to provide senior managers reflective tools which they can use to enhance their well-being and work-related engagement as well as their subordinates. Highlighting the individual decision-making process in the everyday work life context can provide these kinds of means of self-reflection and self-direction. 


\title{
Promoting Resilience and Developing Wellbeing at Work
}

\author{
Chair: Wren, B. \\ Royal Free Hampstead NHS Trust, United Kingdom
}

\begin{abstract}
Resilience is increasingly recognised as a key aspect of psychological health. Resilience is our ability to withstand stress and continue successfully in times of adversity - a key skill for the modern turbulent world of work! This workshop provides an overview of a range of approaches to developing resilience at work. Leading contributors in the field will describe cutting edge interventions at individual, management, team and organisational level designed to promote resilience. We also aim to provoke new thinking about resilience by considering how it needs to be grounded in both the lighter and darker side of organisational life The symposium will be of interest to all those with responsibility for staff health, relationship dynamics at work and organisational effectiveness. The speakers are all experts in their field and combine an evidence-based approach with cutting edge, successful and practical interventions in public and private sector organisations.
\end{abstract}

\section{Paper 1:}

\section{Building Psychological Resilience in Staff: Individual and Organisational Approaches}

\author{
Jennings, $\mathrm{T}$.
}

Northumbria Healthcare NHS Foundation Trust, United Kingdom

Healthcare workers experience relatively high levels of psychological morbidity compared with other workers in other sectors. The nature of healthcare work is inherently stressful and high levels of organisational change and uncertainty add to the risk factors. Developing high psychological resilience and positive coping in healthcare staff is critical in helping to help protect against the impact of stress and adverse events. This in turn reduces the likelihood of more serious mental health problems developing.

This paper outlines some of the individual and organisational approaches to improving psychological resilience of employees in a large acute healthcare trust: Firstly individual approaches to improving resilience will be described. This includes the provision of a psychological therapies service to staff experiencing mental health or stress related problems in the workplace. Some of the psychological strategies used in promoting positive coping with staff will be described using case study examples. However, as we know, managers can also have an effect on employees' psychological resilience in terms of their own behaviour and skills in promoting psychological health in their teams. This second part of this paper will describe some organisational approaches to improving resilience including the provision of psychological skills training for managers. The training covers areas such as preventing stress, improving wellbeing and dealing with interpersonal problems in teams such as conflict, bullying or harassment. Some of the outcome measures to date have been positive. Clinical outcome measures show a significant drop in psychological morbidity following psychological intervention. Training provided for managers has received positive evaluation with managers reporting greater levels of awareness 
and confidence in dealing with issues relating to wellbeing in their teams. Implications for future strategies for improving resilience are discussed.

\title{
Paper 2:
}

\section{Developing Systemic Resilience: Practical Support for Managers}

\author{
Hill-Tout, J. \\ Aneurin Bevan Health Board, United Kingdom
}

Work is known to be a protector for our mental health but some work environments are better for us than others. Workplaces that are good for us are likely to offer employment security, appropriate autonomy, a balance between efforts and rewards, procedural justice and strong workplace relationships. The quality of relationships at work is important not only personally but it has also been demonstrated that cohesion and friendship are related to organisational functioning and increased job satisfaction. Middle managers have challenging and sometimes isolated roles and are often exposed to high workplace stressors and so this group of staff in particular merit special attention when considering resilience at work. In addition manager behaviour is known to have a significant influence on staff stress and so ensuring managers are well supported is important for staff and organisational performance.

The initiatives described in this paper set out to enhance the quality of workplace relationships for middle managers, improve their support and thereby enhance their resilience when coping with workplace pressures. The first initiative was a facilitated network for managers who met regularly over a 12-month period and undertook a range of problem-solving discussions and exercises. The second is a series of Psychology@Work seminars where larger numbers of managers came together with the dual aim of learning about how psychological models and theories might be applied to management, and also to network with each other and discuss common problems. The paper will describe in more detail the content of these initiatives and some quantitative and qualitative outcome measures for the managers involved. Overall the outcome data show that participants greatly valued the opportunity to share problems, normalise their feelings and meet others dealing with similar situations, and the Psychology@Work events in particular were consistently oversubscribed.

\section{Paper 3:}

\section{Building Resilience In Individuals At Work - Coaching To Take Up Role Authority}

Allen, C.

\author{
Central Manchester NHS Foundation Trust, United Kingdom
}

Occupational Health Psychology practitioners have been engaged for some time in building capacity and capability in people at work, particularly in the context of stress and wellbeing. Theoretical models and frameworks informing the practice of OHP draw on cognitive, emotional and behavioural elements of experience at work, that impact on resilience (Padesky, 2009). 
This paper will argue, with illustrative case examples, that many management development approaches are insufficient to foster the internal changes individuals must make to manage themselves in roles in work environments that are increasingly turbulent and demanding, that is, to build resilience.

Three individual coaching case studies are used to illustrate the use of cognitive, emotional and behavioural elements of building resilience, focussed on taking up authority within a professional role. Cognitive frameworks include Implicit Leadership Theories, the 'Organisation-in-the-Mind' (Armstrong, 2006) and organisational role analysis (Reed, 2001). Emotional dimensions focus on specific aspects of the underlying motivations and related affective states of professional carers, here, healthcare professionals. Behavioural facets of the coaching interventions include practising self-observation, linked to Mindfulness Training (Kabat-Zinn, 1990). Finally, relevant outcome measures of these developmental interventions are discussed, with particular focus on resilience and wellbeing.

\title{
Paper 4:
}

\section{Developing Resilience At Work - Using A Mindfulness Approach}

\author{
Schwartz, A. \\ Staffordshire University, United Kingdom
}

Developing resilience to help manage complexity, challenge and commitment at work is central to the role of Occupational Health Psychologist practitioners working with people in organisations. This has always been applicable when considering stress at work, conflict, or change, and has become even more critical under economic constraints posed by the global recession.

Maintaining productivity and enhancing efficiency despite 'leaner working' is promoted at national, regional and local levels. In the United Kingdom a recent report (McKinsey, 2009) advised a cut in the National Health Service workforce by $10 \%$ over the next five years, in order to save $£ 20$ bn by 2014. At the same time the CIPD suggests that line managers need to have more training and to spend time coaching and reinforcing the skills of their team members (CIPD Employee Outlook Report, Summer 2009). Employees are reported as being worried about job security, with morale being damaged due to redundancies and the manner in which these were handled, and having excessive pressure at work. Yet, Taylor et al., (2003) contend that organisations are becoming more punitive in their attempts to reduce sickness absence. More recently they suggest that people are too scared to go sick, due to fear of being discriminated against in appraisal, promotion or selection for redundancy (Preparatory work for the International Labour Process Conference, 2010). Financial imperatives affect the context within which organisations and individuals operate, and there is increasingly little room for manoeuvre; hence the challenge for OHP practitioners to provide practical tools to promote wellbeing and alleviate distress.

The need for OHP Practitioners to engage with the concept of resilience will be examined, taking into account cognitive behavioural approaches (Neenan, 2009), the notion of hardiness (Kobasa, 1979) and current work in this area. Key aspects of resilience will be highlighted, such as the cognitive, physical, emotional, social-relational, environmental, spiritual and moral 
dimensions (Padesky, 2009). These impact on an individual's ability to address problems and to develop optimism, based on a more flexible adaptive disposition. Resilience will be linked to developing a range of possible actions, including the use of Mindfulness Training (Kabat-Zinn, 1990), which is considered under the umbrella of cognitive and behavioural therapies (Baer, 2003; Hayes \& Smith, 2005). Like any other endeavour, improving resilience skills involves a change process. This paper will, therefore, also present a number of frameworks to explore the application of mindfulness training in order to build resilience in individuals at work (e.g. the Change Model (Prochaska et al., 1992), Person-Environment Fit (Lewin, 1951), and the Developmental Pipeline Model (Peterson, 2006).

\title{
Paper 5:
}

\section{Working with the Shadow Side - Anticipating Obstacles to Resilient Working}

\author{
Gething, N. \\ Westminster Primary Care Trust, United Kingdom
}

Carl Jung postulated the notion that every person contains within his or her psyche a repository of undesirable thoughts, feelings, images, and behaviours. This aspect of the psyche is called the Shadow. It contains all those aspects of ourselves that, for whatever social, personal, or traumatic reason, have been relegated to a position in the mind where it is hoped they will remain out of conscious life and, most importantly, away from everyday interactions in the workplace. This Shadow repository can contain our worst fears; it harbours rage and can precipitate cruel and uncharacteristic behaviour. It contains those aspects of ourselves we deem disgusting; it contains our stereotypes and prejudices. Yet it can, if dealt with properly, prompt change, growth, and development. In a manner of speaking the Shadow can be described as the inner personality; a psychological twin of the conscious mind, who observes everything we experience and who makes comments on everything we do.

These concepts have particular relevance to the field of resilience, and for those who manage, supervise, or advise others. Being unaware of the thoughts, prejudices and (hidden) agendas of the Shadow leads to those moments where we succumb to uncharacteristic behaviour, slips of the tongue, regrettable outbursts, and behaviour that leaves us feeling like we're not in control. Unsupervised, the Shadow can leave us feeling depleted and traumatised. Supervised, the Shadow enriches our work and adds to our wisdom. This paper describes the development of a model of coaching with managers to help them conceptualise themselves using these ideas. It suggests that every experience in work is processed through two, and often opposing, 'minds'.

This model is used to highlight the following aspects of work life: [a] dealing with painful and difficult management decisions, [b] dealing with challenging and thought-provoking staff members, and [c] dealing with the after-effects of containing and managing emotionally charged work situations. This model demonstrates how it is possible to form an internal working alliance and in so doing enrich work relationships. As such, it also proposes that to remain fully resilient at work we need to have integrated both these parts of ourselves. 


\title{
In search of the X-factor: Within-person perspectives on workaholism and engagement
}

\author{
Chairs: van Beek, I. \& Taris, T. \\ Utrecht University, the Netherlands
}

During the last decade a body of research has focused on concepts that are associated with high levels of effort expenditure at work. E.g., several publications have addressed the workrelated antecedents of work engagement, an affective-motivational state of mind consisting of high levels of energy, dedication and absorption. Other research has considered the antecedents and consequences of workaholism, referring to the combination of a compulsion to work hard with working excessively long hours. Interestingly, in both lines of research the role of personal resources (such as personality characteristics and individual differences in motivation) is largely neglected. This is unfortunate, in that examining these resources in relation to workaholism and/ or engagement could contribute to our understanding of the etiology and development of these concepts, as well as of the differences between workaholism and engagement.

The five contributions to the present symposium focus on the personal resources relating to engagement and workaholism. They have in common that they search for the X-factor (with " $\mathrm{X}$ " denoting a range of personal characteristics and motivations) that triggers and stimulates engagement and workaholism. The first two contributions examine the person-bound antecedents of engagement. In a controlled experiment, Vera et al. show that high levels of selfefficacy prevent the deterioration of engagement during task execution. Ouweneel et al. report that personal resources such as hope and optimism foster high engagement.

The third and fourth contribution focus on the motivational antecedents of workaholism and engagement. Using self-determination theory, Van Beek et al. show that engaged and workaholic workers differ in their underlying work motivations. Similarly, Van den Broeck and Vansteenkiste show that whereas workaholics worked long hours because they felt they had to (controlled motivation) whereas engaged workers did so because they wanted to do so (autonomous motivation). As these two contributions examine both engagement and workaholism, they also contribute to our understanding of the similarities and differences between these concepts.

Finally, Van Wijhe et al. consider the psychological mechanisms that makes workaholics work long hours: when do workers feel that they can stop working? Interestingly, workaholics are more likely to feel that they can stop working only after the work is done than others.

Together, the findings reported in this symposium show that (a) personal resources play an important role in the etiology and development of both workaholism and engagement; and

(b) that workaholism and engagement are characterized by different patterns of underlying motivations. 


\title{
Paper 1:
}

\section{I would if I could and love it too: Self-efficacy and work engagement}

\author{
${ }^{1}$ Vera, M., ${ }^{2}$ le Blanc, P., ${ }^{1}$ Salanova, M. \& ${ }^{2}$ Taris, T. \\ ${ }^{1}$ Universitat Jaume I, Spain; 2 Utrecht University, The Netherlands
}

Objectives: Work engagement is a key concept within Positive Occupational Psychology. Halbesleben's (2009) meta-analysis of the antecedents and consequences of work engagement not only demonstrated that self-efficacy was positively associated with work engagement, but also that self-efficacy had the strongest relationship with work engagement compared to other job and personal resources. However, at present it is still unclear how exactly self-efficacy beliefs affect levels of engagement during the execution of tasks. The present study tests whether differences in initial levels of efficacy beliefs are related to differences in (the development of) work engagement levels over time at both the individual and the group level.

Methods: In order to test whether different initial levels of efficacy beliefs lead to different courses of work engagement over time, growth curve modeling using LISREL 8 was performed on data from 372 psychology students from a Spanish University randomly assigned to one of 79 working groups of four or five members each. The groups carried out three tasks, collecting data directly after the completion of each tasks, in a laboratory setting with an intranet connection and five work stations, on which the Moodle online collaboration software system was installed.

Results: Results demonstrated that whereas for people with high initial levels of self efficacy the level of work engagement remained high and stable over time, for people with low initial levels of self efficacy levels of work engagement were lower and decreasing over time. The same pattern of results was found at the group level, using measures of efficacy and work engagement in which the work group (instead of the individual worker) was the referent.

Implications: The available literature on work engagement has focused almost exclusively on the way in which efficacy beliefs may enhance (work) motivation, but has not yet paid attention to their buffering effect in preventing a decline in motivation. So, in order to prevent a possible process of demotivation in organisations, efficacy beliefs levels must be always optimal.

Conclusions: The most important conclusion of this study is that efficacy beliefs should not only be considered as a personal characteristic that enhances (work) motivation, but also one that buffers a decline in (work) motivation. This applies to both the individual and the group level.

\section{Paper 2:}

\section{Why do workaholics work long hours?}

\author{
${ }^{1}$ Van den Broeck, A. \& ${ }^{2}$ Vansteenkiste, $M$. \\ ${ }^{1}$ Catholic University of Leuven, Belgium; ${ }^{2}$ Chent University, Belgium
}

Objectives: Workaholism refers to the compulsion or uncontrollable need to work incessantly. Workaholism thus includes a behavioural component: working excessively and an attitudinal 
component: compulsiveness. Although from a theoretical point of view both workaholism components are closely intertwined, they are generally measured in juxtaposition. As such, compulsive work is generally indicated as the most detrimental component. Excessive work yields mixed results, which is in line with the research on overtime (e.g., Beckers et al., 2008).

In order to further clarify the concept of workaholism, the current study aims to examine whether workaholics indeed work excessively, operationalized as overtime (Hypothesis 1a) because they feel they 'have to' (i.e. controlled motivation; Deci \& Ryan, 2000; Hypothesis 1b), and whether this further associates with negative outcomes such as negative work-life interference (Hypothesis 1c). We further aim to contrast these findings with similar analysis regarding a closely related, yet different concept: work engagement. Although work engaged employees may work overtime as well (Hypothesis 2a), their motives likely differ. Specifically, we expect work engagement to associates with working overtime because one freely 'wants to' work long hours (autonomous motivation; Gagné \& Deci, 2003; Hypothesis 2b), which might even associate with positive work life interference (Hypothesis 2c).

Methods: Currently data is being collected in a heterogeneous sample of Belgian employees. The hypothesis will be examined using structural equation modelling.

Results: Results in the yet available data $(N=108)$ indicated that both workaholism $(\beta=.45, p$ $<.001)$ and work engagement $(B=.38, \mathrm{p}<.001)$ associated positively with working overtime, thereby confirming Hypotheses $1 \mathrm{a}$ and 2a respectively. Furthermore, workaholism associated positively with working overtime out of controlled reasons $(B=.34, p<.001$ ), which is in line with Hypothesis 1b, but unexpectedly also with working overtime out of autonomous reasons $(ß=.27, \mathrm{p}<.001)$. The motivations to work overtime could however not explain the positive association between workaholism and negative work life interference; only a direct relationship emerged $(~ B=.69, \mathrm{p}<.001$; Hypothesis 1 not supported). In line with Hypothesis $2 \mathrm{~b}$, work engagement associated positively with working overtime out of autonomous reasons, which - in line with Hypothesis 2c - partially explained the positive association between work engagement and positive work life balance $(z=.04, p<.10)$.

Conclusion: The discussion centres on the importance of overwork in the concept of workaholism and the underlying motivation.

\title{
Paper 3:
}

\section{Engaged employees: The role of personal resources in the relationship between job resources and work engagement}

\author{
Ouweneel, A.P.E., Le Blanc, P.M., \& Schaufeli, W.B. \\ Utrecht University, the Netherlands
}

Objectives: Work engagement can be considered one of the key concepts in Positive Occupational Psychology that focuses on optimal functioning of individuals and groups in organisations. According to the Job Demands-Resources (JD-R) model, job resources are positively related to work engagement through a motivational process. Consequently, the majority of studies have focused on the role of job resources in predicting engagement. Recently however, personal resources were found to contribute to engagement as well. In this study, we propose that the 
positive relationship between job resources and work engagement is mediated by personal resources. More specifically, it was hypothesized that the positive relationship between collegial support and learning opportunities and work engagement is mediated by hope and optimism.

Method: Our hypotheses were tested on data from 350 employees of a Dutch University who filled out an online survey on their work and well-being. The research population consisted of $34 \%$ men and the average age was $38.4(\mathrm{SD}=11.8)$.

Results: Regression analyses revealed a mediating role of hope as well as optimism in the relationship between collegial support and learning opportunities on the one hand and work engagement on the other hand. Sobel tests confirmed the mediating effects of both hope and optimism. In the coming months, follow-up data will be collected, so that the results of a longitudinal test of our mediation model will be reported at the conference.

Implications: This study supports the theoretical notion that personal resources could be part of a so-called gain cycle, as it was found that job resources (learning opportunities and collegial support) enhance the experience of personal resources (hope and optimism), which in turn predicted work engagement. Other studies already demonstrated that work engagement has a positive impact on (the perception of) job resources, thereby closing the cycle. Moreover, individual interventions to enhance work engagement could be targeted to increase for example hope and optimism by means of for example goal setting.

Conclusions: Taken together, the research findings provide support for our hypothesis that personal resources mediate the relationship between job resources and work engagement. Hence, the inclusion of personal resources as mediators in the JD-R model should be considered a useful refinement.

\title{
Paper 4:
}

\section{The motivational bases of job-related well-being: Why do workaholic, engaged and burned-out employees work so hard?}

\author{
${ }^{1}$ van Beek, I., ${ }^{2} \mathrm{Hu}, \mathrm{Q} .,{ }^{1}$ Schaufeli, W.B., ${ }^{1}$ Taris, T.W., \& ${ }^{3}$ Schreurs, B.H. \\ ${ }^{1}$ Utrecht University, the Netherlands; ${ }^{2}$ Yongkang Nursing School, Zhejiang Province, China; \\ ${ }^{3}$ European University College Brussels, Belgium
}

Objectives: The world of work is rapidly changing and people work harder than ever before. Working hard involves spending more hours on work than formally agreed upon, and being absorbed while working or thinking about work when not working. Two types of working hard can be distinguished: workaholism, a "bad" type of working hard, and work engagement, a "good" type of working hard. Previous research has focused on conceptualizing and operationalizing these two constructs and on identifying their consequences. However, their antecedents are still largely unknown. The current study was designed to fill in this gap by examining the motivation underlying workaholism, work engagement, and burnout, the antipode of work engagement. The Self-Determination Theory of Ryan and Deci (1985; Ryan \& Deci, 2000) constituted the theoretical framework for our study. 
Methods: Data were collected through a survey held among 544 nurses and 216 doctors in China. Hypotheses were tested using structural equation modelling.

Results: Results showed that workaholism was positively related with introjected regulation and identified regulation, while it was negatively related with intrinsic regulation. Work engagement was positively related with introjected regulation, identified regulation and intrinsic regulation. The relationships were stronger the more autonomous the motivation. Furthermore, burnout was negatively related with identified regulation and intrinsic regulation. These results hold for nurses and doctors. In addition, burnout was positively related with introjected regulation, but only for doctors.

Implications and conclusions: In conclusion, self-determination theory provides a valuable theoretical framework that helps to explain the antecedents of workaholism, work engagement and burnout. Introjected regulation and identified regulation foster workaholism, while intrinsic regulation is negatively associated with workaholism. This suggests that experiencing an activity by itself as inherently enjoyable and satisfying is a way to discourage workaholism. Furthermore, as motivation becomes more autonomous employees become more work engaged and less burned-out. This suggests that enhancing feelings of relatedness, feelings of competence, and feelings of autonomy stimulate the development of work engagement and discourages the development of burnout.

\title{
Paper 5:
}

\section{Under pressure at work: The mediating role of workaholism}

\author{
van Wijhe, C.I., Peeters, M.C.W., \& Schaufeli, W.B. \\ Utrecht University, the Netherlands
}

Objectives: Due to the increasing flexibility in many work situations, more and more employees can decide themselves when they want to stop working on a workday. The Mood as Input model assumes that individuals use certain subjective mental rules (so-called persistence rules) when they have to make such decisions. One basic mental rule that workers may use is the rationale that they have to continue working until enough work has been done. Assuming that using this so-called 'Enough rule' may put pressure on people, we expect that this rule may induce negative emotions in workers. Furthermore, it is already well-known that experiencing high workload is also related to negative emotions. It is the aim of the present study to examine if workaholism, characterized by working excessively and working compulsively, mediates the relationship between the Enough rule and workload on the one hand and negative emotions on the other hand. We expect that employees will experience negative emotions if they respond to these internal (enough rule) and external pressures (workload) in a workaholic manner, especially by working compulsively.

Methods: A survey study was conducted among a sample of 340 employees of a Dutch University. The questionnaire contained measures of workload, Enough rules, workaholism and negative affect. Structural Equation Modelling was used to test the hypothesized model.

Results: The results revealed that both the Enough rule and workload were significantly related to negative emotions. Working compulsively (fully) mediated the relationship between the Enough 
rule and negative emotions, and between workload and negative emotions. No mediation was found for working excessively.

Implications and conclusions: Using an Enough rule and having a high workload may lead people to work excessively and compulsively, but only in the latter case it results in experiencing negative emotions. In conclusion, workaholics don't feel unhappy because they respond to the Enough rule and workload by working exceptionally hard, but by approaching their work in a compulsive manner. These findings support the assumption that working compulsively is the more detrimental and central component of workaholism. 


\author{
Health-promoting Leadership \\ Chairs: ${ }^{1}$ Vincent, S. \& ${ }^{2}$ Franke, F. \\ ${ }^{1}$ University of Hamburg, Germany; ${ }^{2}$ University of Siegen, Germany
}

For decades of leadership research, subordinates' health has not been frequently mentioned as a relevant factor for leadership effectiveness. Factors like productivity, employee job motivation, and job satisfaction appear as definitions of successful leadership. However, in recent years we observe a growing interest in analysing the impact of leadership behaviour on employees' health and well-being.

Leadership theories like the consideration and initiating structure approach and transactional and transformational leadership have been found to dominate leadership research. In a number of studies relationships between these leadership concepts and indicators of employees' wellbeing were postulated, but the magnitude of the relations varies across studies. The effects of leadership behaviour on employees' health have been gradually more focused. However, there is still a relative lack of well-founded prospective studies targeting the association between leadership and employee health.

There is consensus in the research literature that leaders directly or indirectly influence employees' well-being at the workplace, but it remains unclear to what extent and in what ways leaders influence their subordinates' health. It is therefore of interest to clarify the characteristics of health-promoting leadership. In order to understand to what extent leaders show healthpromoting behaviour, it is also important to examine which prerequisites and conditions encourage supervisors to lead in a health-promoting way.

Within this symposium new concepts of health-promoting leadership are illustrated. We present empirical findings on the characteristics of health-promoting leadership and the results of a systematic investigation of antecedents of health-promoting leadership. We draw conclusions from our findings and discuss prospects for future work on the topic.

\title{
Paper 1:
}

\section{Leadership and job design - Why good leadership is not sufficient for health promotion}

\author{
Wieland, R. \& Krajewski, J. \\ University of Wuppertal, Germany
}

Recently, growing attention has been paid on the effects of leadership on employees' health. The results of empirical studies show that inadequate leadership behaviour has negative effects on subjective well-being during work, motivation, physical disorders, health, and absenteeism. In business reality, however, managers too often failed to recognise health promotion and healthrelated work-design as a leadership task and accordingly fail to put it into action. Based on a comprehensive model of stress at work, this paper investigated the combined effects of work design (i.e. regulation obstacles) and leadership behaviour (i.e. employee oriented leadership 
behaviour) on functional and dysfunctional strain and feelings of control during work, physical disorders, absenteeism, and presenteeism.

Three cross-sectional empirical studies $(\mathrm{N}=877, \mathrm{~N}=300, \mathrm{~N}=570$ ) were conducted. Data were collected from white- and blue collar employees in a range of manufacturing companies and an insurance company. Data analysis was based on a four-fields pattern combining a high vs. low employee oriented leadership style (median split) and a workplace with a low vs. high amount of regulation obstacles.

The results show that the combination of a low employee oriented leadership style and a high amount of regulation obstacles has strong negative effects on the "strain-balance" (difference between functional and dysfunctional strain), feelings of control during work, physical disorders as well as absenteeism and presenteeism. In contrast, an employee oriented leadership style and a low amount of regulation obstacles, has positive effects with regard to these employee health variables. Good leadership is not solely responsible for healthiness. Psychological workstrain, physical disorders, absence and presenteeism attribute to a strong interaction effect of leadership behaviour and job design on strain. The implications deriving from these data for health promotion and job design as a leadership task are discussed.

\title{
Paper 2:
}

\section{Health-promoting leadership behaviour: A new measure}

\author{
Vincent, S. \\ University of Hamburg, Germany
}

In the field of occupational stress research a wide variety of work characteristics that affect employee well-being has been identified. They can be categorised as stressors, task requirements, and resources at the workplace. In recent research regarding the relationship between transformational leadership and subordinates' well-being, different work characteristics - e.g. meaningfulness, role clarity, opportunities for development - were found to mediate this relationship. Furthermore, the direct relationships between leadership and health outcomes show an inconsistent pattern. These findings suggest that further research should focus on the indirect relationship between leadership and employee health by analysing supervisors' influence on different work characteristics.

Therefore, the aim of the present study is to integrate occupational stress research and leadership research to help explain the mechanisms behind the relationship between leaders' behaviour and followers' health. In order to realise this integrative approach, an instrument has been developed measuring the direct influence of the supervisors' behaviour on the stressors, task requirements, and resources of the employees. In this contribution, the results from a validation study will be presented. 822 employees from multiple organisations were asked to assess their direct supervisors' behaviour. In addition, data on their well-being was collected.

The leadership scales show good psychometric properties and substantial correlations with emotional exhaustion, psychosomatic complaints, irritation, and occupational self-efficacy of the employees. The examination of the factor structure confirmed three underlying factors according to the findings of occupational stress research: demanding leadership, development-oriented 
leadership and supportoriented leadership. Regression analyses show that these factors explain $33 \%$ variance of employees' emotional exhaustion. Compared to individualized consideration, a subscale of transformational leadership, the derived factors explain an incremental amount of $18 \%$ variance. The test of two and three way interactions reveals moderating effects and stresses the importance of the interplay of the three leadership factors. The results indicate that these factors constitute relevant characteristics of health-promoting leadership behaviour. On the basis of these findings a model for health-promoting leadership behaviour has been developed.

Concluding, a stress-theoretical foundation of assessing leadership behaviour offers multiple implications for research and practise. On the one hand, this integrative approach gives detailed insights regarding the interaction of the different factors of leadership behaviour and the employee well-being. On the other hand it provides new opportunities for interventions by imparting knowledge to supervisors on how to influence their subordinates' work characteristics in a healthpromoting way.

\title{
Paper 3:
}

\section{Leaders' ability to shape an organisational climate for health: Do personal characteristics matter?}

Gurt, J.

\author{
Ruhr-University Bochum, Germany
}

Shared norms, values and basic assumptions are at the core of organisational culture, facilitate and motivate employee behaviour giving orientation and linking everyday work to long-term organisational goals. Therefore, for organisational health promotion to be successful and sustainable, the development of a corresponding organisational culture is considered indispensable. Following the corporate culture approach organisational culture is at least to some degree considered an organisational domain that can be intentionally shaped and designed. It is widely agreed among researchers within health promotion that leader support and engagement for health promotion is essential for the emergence of a climate for health (which is regarded to be the 'surface manifestation' of culture) and a health promoting culture. Drawing on social cognitive learning theory, leaders serve as role models and reflect the organisation's attitude towards the importance of employee health.

While climate research is well advanced within other contexts, e.g. services or safety, empirical studies on the leadership - climate link concerning health are rare. The present study examines the influence of (1) leadership behaviour and (2) leader characteristics, such as gender and age, on the emergence of an organisational climate for health applying hierarchical linear modeling (HLM). Data was collected using the FAGS-BGF questionnaire, which covers health domain-specific leadership next to general leadership. Sampling took place in 2005 in nine local tax offices in North RhineWestphalia, Germany, who agreed to participate in a health promotion project for the upcoming three years. The overall response rate amounted to $72.3 \%$ comprising 1,454 employees and 110 supervisors. Results indicate that health domain-specific leader behaviour has a beneficial effect on climate for health. Additionally, leader characteristics (e.g. gender) influence employee perception of health domain-specific leadership. Theoretical considerations concerning future research and practical implications for organisational health promotion professionals are discussed. 


\title{
Paper 4:
}

\section{Health-promoting leadership: A new approach}

\author{
Franke, F. \& Felfe, J. \\ University of Siegen, Germany
}

Research has shown that leadership is an important factor for employees' health and well-being. Previous leadership approaches provide rather surface information about relevant leadership aspects in this regard. Hence, it is insufficently explained what constitutes health-promoting leadership and which aspects of leadership are particularly relevant. Therefore, the instrument health-oriented leadership ( $\mathrm{HoL})$ was developed. It considers two important conditions of health-promoting leadership. First, the role of self-leadership is emphasised. The underlying assumption is that leaders who take care of their own health are more promoting for employees' health than leaders who do not mind their health. Second, cognitive and motivational aspects which influence health behaviour (e.g., health awareness, value of health) are considered. Here it is assumed that being aware of health and signs of stress and dealing cautiously with these aspects are important determinants of health-promoting behaviour. Aim of the study was to test these assumptions.

More than 500 leaders and employees from different branches were asked for rating health-oriented leadership with the HoL instrument. Additionally, different health indicators were assessed (e.g., irritation, work-family-conflict). In terms of reliability and validity the HoL instrument showed sufficient psychometric properties. As expected, results support the assumption that leaders who care about their own health lead their employees more health-promoting than leaders who do not care about health. Moreover, findings indicate that also cognitive and motivational aspects are important predictors of health. For example, leaders that are perceived to be aware of employees' health situation and signals of stress can mitigate employees' irritation. Although health-oriented leadership is related to transformational leadership, it explained unique variance in different health indicators.

The findings imply that health-oriented self-leadership is an important condition for healthpromoting leadership of employees. This is of particular importance when the meaning of leaders as role models in health management is regarded. Thus, considering health-oriented self-leadership in stress interventions might foster both, leaders own health and their dealing with employees' health.

Furthermore, adding cognitive and motivational aspects allows a clearer examination of the influences of leadership on health. Thus, for leadership trainings we would recommend to focus on sensitisation and training of the perceptions of signs of stress. The study indicates that it is important to extend previous leadership approaches to understand better how leadership and health are related. Furthermore, a more comprehensive assessment of health-promoting leadership facilitates clearer instructions for practical fields. 


\title{
Paper 5:
}

\section{What makes supervisors lead in a healthy way? - Organisational and individual antecedents of health-promoting leadership}

\author{
Wilde, B., Hinrichs, S., Pavez, C.B. \& Schüpbach, H. \\ University of Freiburg, Germany
}

Supervisors have a twofold role in workplace health promotion. On the one hand, they have a stressing job themselves and are therefore a target group of workplace health promotion. On the other hand, they have an influence on their employees' health and are therefore considered as supporters and "realisers" of workplace health promotion as well. Concerning the last mentioned point, supervisors have different opportunities to influence their employees' health. On the basis of former research, the following factors can be subsumed under health-promoting leadership (HPL): considerate and supportive leadership, the design of healthy working conditions and engagement in workplace health promotion. One of the goals of workplace health promotion is to strengthen HPL. For this purpose, it is necessary to know which conditions on the level of the individual and on the organisational level encourage supervisors to show HPL.

Thus, it is the goal of our first study to derive and test organisational and individual antecedents of HPL. Based on the Theory of Planned Behaviour (Ajzen, 1991) the following factors are considered to influence the extent to which supervisors lead in a health promoting way: culture of HPL, supervisors' attitudes concerning HPL, supervisors' personal skills and the possibilities on the company level to lead in a healthy way. To test the empirical relevance of the derived factors, data were collected from 221 lower- and middle-level supervisors in several German companies using a questionnaire.

The findings reveal that all factors are significantly correlated with HPL. Regression analysis show, that the derived factors can explain 35\% of HPL, thereby each factor has an independent effect. As different authors assume that the stress and strain of supervisors themselves influence how they lead, we focused on the associations between supervisors' own working conditions (demands, autonomy, social support) and HPL as well as the mentioned antecedents in a second study. Analysing the same data set we found the expected correlations between working conditions and HPL as well as the former named antecedents. Associations between working conditions and organisational antecedents are higher than those with individual antecedents.

The results show that the derived factors are relevant antecedents of HPL. Thus, the combination of organisational and individual antecedents seems to be the best setting for HPL; interventions should take both levels into account. Further analysis should have a closer look on the interplay between supervisors' working conditions and HPL and its antecedents. 


\title{
Current Status and Prospects of Job Stress and Burnout Research in South Korea
}

\author{
Chair: Yu, K. \\ The Catholic University of Korea, South Korea
}

A panel of five experienced presenters will address the current status and prospects of job stress and burnout research in South Korea. The goal of this symposium is to share the Korean research findings with international scholars who are interested in job stress and burnout syndrome. Participants will learn about the characteristics and environments of workplaces in South Korea (e.g., School, Military, and Private Company). Additionally, participants will gain an understanding of the similarity and dissimilarity of these job environments and stress and burnout syndromes for potential application to their own countries' situations. Presenters will provide handouts on materials presented in the symposium. Media may be used in the delivery of these materials.

\section{Paper 1:}

\section{Overview of Job Stress and Burnout Research in South Korea: Current Status and Prospects}

\author{
Lee, S.M. \& Kim, A. \\ Korea University, South Korea
}

Job stress in modern times has been reported to be associated with many diseases such as musculoskeletal disorder, hypertension, coronary heart disease, mental stress, and burnout. South Korea has experienced rapid social and economic changes since the 1997 economic crisis. Structural changes in the form of organisational downsizing and restructuring, as well as instability in the labor market, have increased job insecurity and created both physical and psychological stress among Korean employees (Khang et al. 2005; Kim et al. 2006). Along with these changes, compensation claims for work-related cardiovascular diseases, musculoskeletal diseases, and mental illness sharply increased (Korean Ministry of Labor 2005) and this has been attributed to job stress resulting from the unstable labor market. Furthermore, Korean traditional cultural values and practices favoring authoritarianism, collectivism, regionalism, and kinship ties are starkly divergent from the horizontal organisation and rationalism of Western societies and exert a critical influence on the workplace infrastructure. The increasing influence of Western cultures, and the more widespread adoption of individualism, has placed these traditional values at the center of organisational and interpersonal conflicts at places of work. As a result, diseases resulting from job stress have been rapidly increasing. Therefore, it is important to scrutinize the current status of job stress and burnout research in South Korea and discuss the future agendas and prospects by examining the factors related to job stress and burnout. This effort is needed to comprehensively discuss those concerns, agendas, and issues that may need to be taken into consideration for the future. Specifically, this research has investigated several objectives. First, it presented the historical development and current trends of job stress and burnout research and discussed the troubles that have limited the impact of burnout research throughout its history. Second, this research described the factors to have impacted the development of the job stress and burnout research and presented the issues that have been raised in the Korean burnout research field. Lastly, the prospects for job stress and burnout research, for example, societal and cultural issues that are important to consider when studying job stress and burnout models, were discussed. 


\title{
Paper 2:
}

\section{Job Demands and Burnout Syndrome of Private Company Workers}

\author{
Yu, K. \& Choi, H. \\ The Catholic University of Korea, South Korea
}

Ninety five percents of Korean workers reported that they experience occupational stress. This is significantly higher than that of counterparts such as $61 \%$ of Japan or $20 \%$ of U.S. workers (Korean Minstry of Labor, 2006; Korean Occupational Stress Association, 2001). Work related environment variables (e.g. occupation, level of job demands, or rewards) or personal characteristics (e.g., coping styles, personality, or gender) have been reported as major stressors for Korean workers (Kim \& Lee, 2009; Standfield, 1998). Role ambiguity or role conflicts could also be other stressors for workers (Lee \& Kim, 2001). Particularly, when people have higher level of job demands, lower level of control over work, and lower level of social support, they experience higher stress (Human Resources Development Services of Korea, 2001). These stressors may cause various mental health problems (e.g., depression, anxiety disorders, substance abuse and sleep disorders) and somatic symptoms (e.g., cronic headache and stomachache) (Public Health Service, 1991; Korean Academy of Family Medicine, 1996). Thus, it is imperative to understand Korean workers' major stressors and burnout syndrome to develop effective prevention and intervention strategies for Korean workers. The objectives of the study were to 1) review literature related to occupational stress/burnout of private company workers, 2) investigate specific burnout patterns and related variables for private company workers, and 3) examine the demand-control model (DCM: Karasek \& Theorell, 1990) of occupational burnout. Participants of this study were recruited from several private companies in South Korea. Based on the study, the researcher examined the relationship among stressors and burnout patterns and how the DCM model explained the burnout syndrome of private company workers. Using a cluster analysis procedure, participants' burnout patterns were identified. In addition, using structural equation modeling to examine the demand-control model, the results revealed that "control variables" play an important role in explaining the relationship between job demands and burnout syndromes. Finally, prevention and intervention strategies for individual workers who might experience burnout were discussed. Being aware of burnout and providing appropriate interventions for burnout could assist company stakeholders to prevent potential future problems.

\section{Paper 3:}

\section{Job Ambiguity and Burnout Syndrome of School Personnel}

\author{
Lee, J. \& Park, Y.M. \\ Korea University, South Korea
}

School personnel play a significant role in controlling the quality of education. Each school staff such as the principal, teachers, school counselors, and school nutritionists, share in leading the school system and have well defined roles. The role of school counselors, however, is not as clearly defined. Since school counseling programs began in 2005 in South Korea, these school personnel still cannot clearly identify their role. There is no regulation governing the role of 
school counselors, therefore, their role ambiguity has been worse than that of other school personnel. School counselors' role expectations are defined by the counselors themselves as well as by others within the school (e.g., school boards, teachers, parents, and students) and outside of the school system (e.g., counselor educators, state and national legislators) (Culbreth et al. 2005). Another stressor is role conflict. Role conflict occurs when a person is confronted by incongruent role demands from two or more role definers or when a person has different expectations than those associated with his or her position (Coll \& Freeman, 1997). Given the inconsistency of role expectations, there is a potential for school counselors to experience role stress. Prolonged periods of stress, in turn, can generate burnout, leading to deterioration in the quality of service yielded (Lambie, 2006). Even though school counselors' burnout could be major issue, little attention has been given to school counselors' burnout in South Korea. Therefore, the purposes of this study were (1) to explain the relationships among job stressors, burnout, and depression of school counselors and teachers, and (2) to explore the level of stressors, burnout, and depression of school counselors and teachers. Using structural equation modeling (SEM), relationships among variables (e.g., stressors, burnout, and depression) were examined. In addition, using latent mean analysis, mean differences between school counselors and teachers on level of job stressors, burnout, and depression were examined. The findings indicated that burnout plays as a mediator between job stressors and depression for both school counselors and teachers. In addition, school counselors have higher scores on job stressors and burnout than classroom teachers. Specifically, school counselors perceived job ambiguity and role conflict were the major stressors among job stressors. No significant difference was found on depression. Results of this study could begin to draw attention to the problem of counselor burnout in South Korea. Results could also inform the development of treatment or psychoeducation interventions for reducing school counselors' burnout.

\title{
Paper 4:
}

\section{Work Demands and Burnout Syndrome of Military Personnel}

\author{
Choi, B.Y. \& Jin, Y.Y. \\ Korea University, South Korea
}

In Korea, there are a total of 690,000 military personnel, including approximately 160,000 professional military officers and 530,000 soldiers. Korea, a divided country, is under unique national defense circumstances. The Korean Armed Forces have sustained a draft system since the Korean War of the 1950s. The Korean Armed Forces is the organisation that safeguards the country and protects its citizens. The majority of studies about the military have focused on officers' leadership, soldiers' adaptation to the military environment, and the factors influencing physical and psychological health (Kim, 2007; Son, 2001; Vinokur, 2009; Yun, 2004). Recently, suicide in the barracks has developed into a big problem. According to Jeong (2008), 60-70 military personnel commit suicide every year, it take $50 \%$ of reported deaths in the military. In addition, it was reported that $8.8 \%$ of military personnel feel suicidal impulses (Yoon, 2008). Suicide attempts impact other military personnel negatively and bring losses in military power. As a result, recently, Korean military has developed the professional military counseling system to prevent suicidal behaviour among troops (Kim, 2008). The main causes of suicide are stress and maladjustment resulting from the controlled and hierarchical military environment (Lee, 2006). In addition, some studies showed that an individual's depression and anxiety are related suicide attempts or suicidal impulses in military personnel (Gu, 2009; Lee \& Jung, 2001). However, there 
are few studies that examine the burnout of military personnel, although burnout is a significant factor associated with both job-related stress (environmental factor) and psychological instability (individual factor) (Hanye \& Long, 1989; Lee \& Lee, 2009; Thomas, 1982). The purpose of this study was to find out the causes of job stress and investigate the relationship between job burnout, depression and suicidal impulse as a consequence of job stress. Results of the study were as follows: first, the interpersonal relationship factor showed the highest score among the work demands. Second, work demands, especially the interpersonal relationship factor, are related significantly with burnout, depression, and suicidal impulse respectively. Finally, burnout partially mediated between work demands and depression, and fully mediated between work demands and suicidal impulse. These results indicate that counselors should evaluate and understand the degree of burnout when they counsel military officers and soldiers who feel depression or show suicidal impulse.

\title{
Paper 5:
}

\section{Academic Demands and Burnout Syndrome of Middle and High School Students}

\author{
Park, Y.M. \& Shin, H. \\ Korea University, South Korea
}

There are numerous studies indicating that academic stress is generally a main concern of adolescents around the world and the pressure to perform in school-work is more acute in Asian countries such as Korea (Friedman, 1991; Huan, Yeo, \& Chong, 2006; Isralowitz \& Ong, 1990). Korean adolescents experience great stress with regard to general academic demands or preparation for college (Juon, Shin, \& Nam, 1995) Thus, Korean students ranked academic stress as the most stressful aspect of their lives (Lee \& Kim, 1996; Hwang, 2006). There is evidence that these extreme academic stressors cause negative consequences such as mental health problems (Misra \& Mckean, 2000; Shek, 1995). It appears that there is also a strong relationship between academic stressors and mental health problems such as the suicidal ideation (Toero et al., 2001). Therefore, it is important to explore academic burnout of Korean students to prevent more severe problems. The objectives of this study were to: 1) validate a Korean version of the Maslach Burnout Inventory-Student Survey (MBI-SS), originally developed by Schaufeli et al (2002), 2) identify specific burnout patterns among Korean students, and 3) examine the demand-control model (DCM; Karasek \& Theorell, 1990) of academic burnout. We used confirmatory factor analysis (CFA) to test the goodness of fit of the three factor MBI-SS on Korean middle and high school students. The goodness of fit index indicated the satisfactory construct validity of the CFA. Using a cluster analysis procedure, participants were classified into four identifiable groups: (1) distressed group, (2) laissez-faire group, (3) persevering group, and (4) well-functioning group. In addition, using structural equation modeling we examined the demand-control model; results revealed that "control variables" play the role of suppressors between academic demands and academic burnout. The present study yielded important information about academic burnout for Korean adolescent students with several implications for educators including teachers and school counselors. Individualized strategies could target problem areas (e.g., emotional exhaustion, cynicism, academic efficacy). Awareness and understanding of a student's burnout type could assist teachers and counselors to identify at-risk students and develop targeted prevention programs to address potential problems before they emerge. Specific intervention programs can also be devised to help students already exhibiting academic burnout and related issues. 



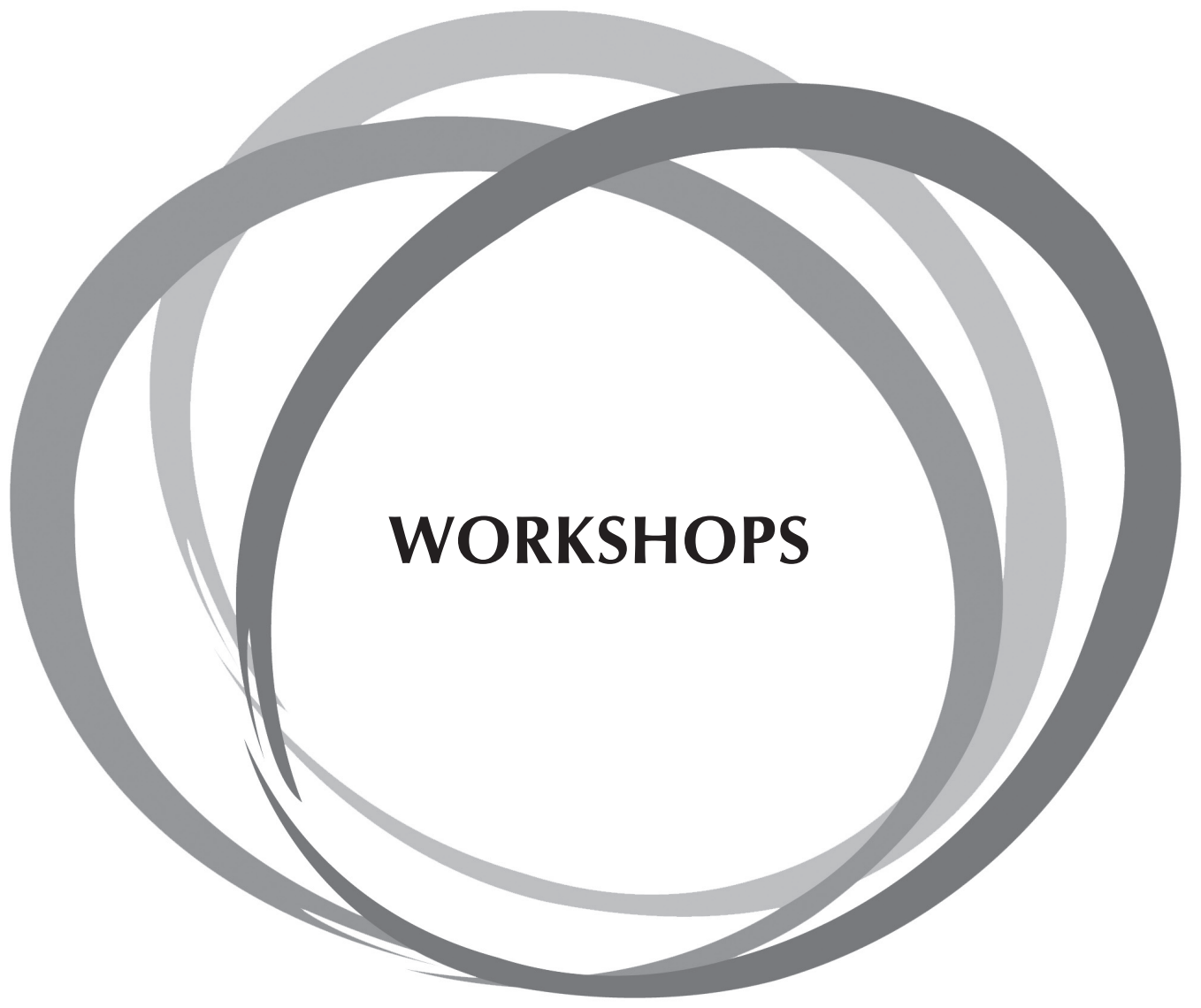




\title{
Dealing with the Impact of the "Shadow Side" at Work: An Exploratory Workshop
}

\author{
${ }^{1}$ Gething, N. \& ${ }^{2}$ Wren, B. \\ ${ }^{1}$ Westminster Primary Care Trust, United Kingdom; ${ }^{2}$ Royal Free Hampstead NHS Trust, \\ United Kingdom
}

"There is a deep gulf between what a man is and what he represents, between what he is as an individual and what he is as a collective being".

Psychological Types (1921). CW 6: P.III

Much theory and practice in occupational health psychology is informed by rational approaches to human behaviour at work. Cognitive Behavioural and other scientific frameworks aim to provide interventions to improve the experience of work, and enhance performance at work. But all practitioners know that there are aspects of working life that demand a greater understanding of complexity, irrationality, and other interactions that defy logical intervention and thought. If these dynamics are approached creatively, managers and practitioners can avoid dead-end or one-dimensional solutions to workplace challenges.

Carl Jung postulated the notion that every person contains within his or her psyche a repository of undesirable thoughts, feelings, images, and behaviours. This aspect of the psyche is called the Shadow and contains all those aspects of ourselves that, for whatever social, personal, or traumatic reason, have been relegated to a position in the mind where it is hoped they will remain away from conscious life and, most importantly, away from everyday interactions in the workplace. Moreover, commissioners often demand time limited straightforward interventions that minimise funding and time loss. However we neglect complexity at our peril; conversely, embracing illogical complexity can lead to the disruption of outdated habits resulting in change, growth, and development.

Jung's Shadow can be described as the inner personality; a psychological twin who observes everything we experience and who makes comment on everything we do. Being unaware of the thoughts, prejudices and agendas of the Shadow leads to those moments where we succumb to uncharacteristic behaviour, slips of the tongue, regrettable outbursts, and behaviour that leaves us feeling like we're not in control. Unsupervised, the Shadow can leave us feeling depleted and traumatised. Supervised, the Shadow enriches our work and adds to our wisdom.

This model will be used to describe and highlight the following aspects of work life:

- Making decisions using both the logical mind and the Shadow mind thereby acknowledging complexity in work interactions.

- Dealing with painful and difficult encounters that arise because of Shadow influences.

- Using the Shadow effectively [in self and other] thereby promoting change and creativity. In other words, the Shadow becomes a change agent rather than a disruptive force. 


\title{
The implementation of a new inspection tool in the psychosocial working environment: The experience of the Danish Working Environment Authority
}

\author{
Magwentshu, S. \\ The Danish Working Environment Authority, Denmark
}

Objective: The Danish Working Environment Authority (DWEA) launched a new strategy in 2007 to strengthen and qualify primary prevention of work related stress. Part of the strategy consists of increased inspections of the psychosocial working environment as well as the development and implementation of a new inspection tool, namely the "guidance tool".

Method: Twenty four sector specific guidance tools have been developed to assist DWEA inspectors in assessing psychosocial working environment problems in all Danish enterprises. The guidance tools assists the DWEA inspectors in their assessment of the three to five most important risk factors in the specific sectors, (for example quantitative demands, emotional demands, and work related violence) as well as the most important preventive factors, e.g. quality of management, influence/control, and training. The guidance tools are based on recent research.

Results: The guidance tools enables all DWEA inspectors to make an assessment of psychosocial working environment problems, and thus ensures that both DWEA inspectors with and without specialist background are able to assess the psychosocial working environment. During the first years after implementing the strategy, an increasing number of inspectors have issued improvement notices regarding work related stress and violence, and there has been an increasing number of notices issued on several risk factors.

Implications: Overall, the tools have contributed to an increase in the clarity and comprehensibility of the improvement notices and have reduced the DWEA's time consumption per enterprise, both during the inspection and in the production of notices.

Conclusion: The guidance tools seem to be a suitable and efficient way of assessing several health and safety risks concerning work related stress and violence thereby allowing the DWEA to detect more of the existing problems. Future challenges projected for the strategy include continual training of all DWEA inspectors in the proper use of the tools and continuously refining the tools - or developing new tools - based on further research and experiences from practice.

The Workshop:

As follows the workshop will consist of:

- A presentation of the Danish Working Environments' way of inspecting psychosocial work environment.

- Discussion on the risk-factor approach of inspecting psychosocial work environment

- Discussion on the Theoretical and empirical background for the guidance tool

- Discussion of the use of DWEA inspectors without specialist background in the assessment of psychosocial work environment. 



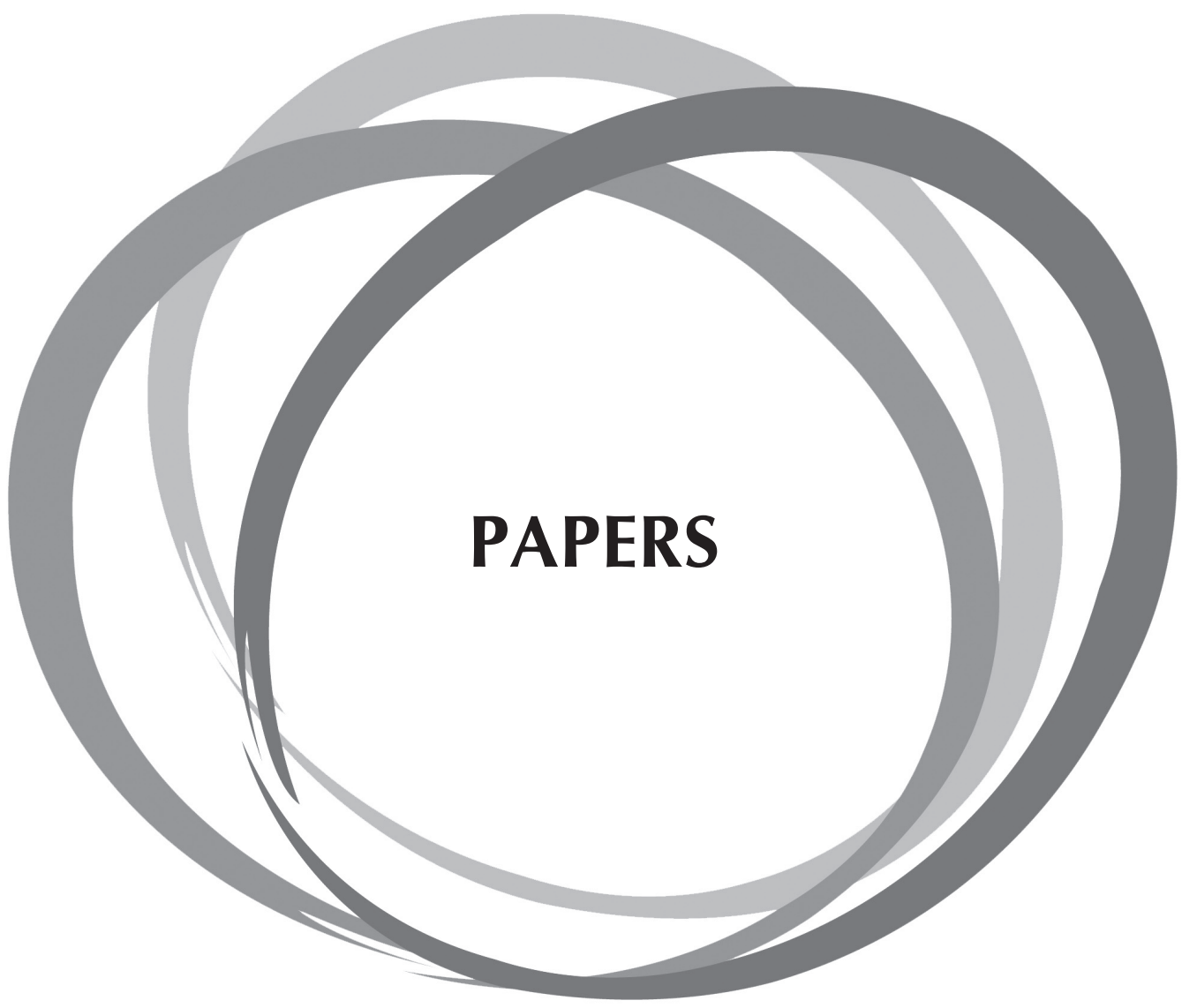




\title{
The Role of Proactive Coping in the Job Demands-Resources Model
}

\author{
Ângelo, R. \& Chambel, M.J. \\ University of Lisbon, Portugal
}

The present study research has positive occupational psychology as a paradigm, so it consists in the study of success, of individuals and productive work groups, instead of studying the flaws or the problems of the individuals and organisations. The Job Demands-Resources (JD-R) Model (Demerouti, Bakker, Nachreiner, \& Schaufeli, 2001; Schaufeli \& Bakker, 2004), is the research theoretical framework.

The main goal of this study consists in analyzing the expansion of the JD-R Model with the introduction of the coping strategies as a moderation variable, in both health impairment and motivational processes.

Participants are fire fighters and the sample is representative of the Portuguese reality since 1,487 rescue mission firefighters from the 18 districts of Portugal participated in the research. The methodology is quantitative. The questionnaire integrates the following scales: professional demands in the fire fighters rescue mission context (developed by the authors), Job Content Questionnaire (Karasek, R., 1985), Proactive Coping Scale (Greenglass, E.; Schwarzer, R. \& Taubert, S., 1999), Engagement Inventory (Schaufeli, W.; MartÃnez, I.; Pinto, A.; Salanova, M. \& Bakker, A., 2002), and Maslach Burnout Inventory (Schaufeli, W.; Leiter, M.; Maslach, C. \& Jackson S., 1996). The factor structures of all scales included in the questionnaire were tested using a confirmatory factor analysis with AMOS software package (Arbuckle, 2005). Afterwards, the measurement model examined whether indicators suitably measured their designated construct and how constructs in the model were related, also with SEM techniques. The hypothesized moderation effect of proactive coping in the health impairment and motivational processes were then examined through hierarchical regression analyses.

Results revealed that proactive coping moderated the relationship between job resources (supervisor social support) and engagement. In a context of low supervisor social support, firefighters with a high usage level of proactive coping strategies show similar engagement values in comparison to firefighters with high supervisor social support and low proactive coping. Proactive coping did not moderate the relationship between job demands and burnout.

The results highlight the importance to invest in the promotion of coping strategies adapted to the work context and roles, such as proactive coping in the case of rescue mission firefighters. These findings shed light on the JD-R model by suggesting that a personal characteristic has a moderating role within its framework. Furthermore, the results suggest that proactive coping has a more powerful effect on the promotion of positive occupational well-being indicators, than on the mitigation of negative well-being indicators. 


\title{
Non- working individuals in Norway, their latest occupation and self- reported health
}

\author{
Aagestad, C., Sterud, T., \& Tynes, T. \\ National institute of occupational health, Norway
}

Objectives: In 2006, almost 25\% of the Norwegian population between 16-66 years was regarded to belong to the group of non-working individuals. Reasons for not working was defined into 6 categories; disabled, unemployed, old-age/ early retirement pensioner, homemaker, student or military service. While students constitute the largest share of the non-working group with $8.4 \%$, disabled persons and unemployed constitutes the second and third largest share with $8.2 \%$ and $3 \%$ respectively. Knowing there are differences between occupational groups in exposure to health damaging factors, it is crucial to identify occupations most at risk for disablement.

Methods: The study is cross - sectional, is based on two representative population surveys conducted by Statistics Norway; The Survey of living Conditions - Working environment 2006 with a sample size of 10,000 workers and 2589 non- working individuals, age 18-66 years and The Labour Force Surveys consisted of about 21090 persons, 14872 workers and 6218 nonworking individuals in the age group $16-74$ years.

Results: In occupations with no requirement for education almost 30 percent are none - working individuals and more than 20 percent define themselves as disabled. More than 30 percent say that they quit working because of health problems and the share reporting this is largest in occupations with limited or no requirement for education.

The level of several self-reported afflictions was also studied, and for all afflictions the incidence was found to be higher amongst non-working people compared to workers. However, distinct differences within the group were also seen like disabled persons differing from the rest of the group by having significantly higher occurrence of self- reported health afflictions, and in especial muscle and skeletal pains.

The occurrence of psychological afflictions is also seen to be higher among disabled individuals being without employment for 2-3 years compared to those being without employment for 0-1 year, but the occurrence of musculoskeletal afflictions is lower among disabled individuals being without employment for 2-3 years compared to those being without employment for 0-1 year.

Implications: Our analyses, based on cross- sectional data, implicate further studies on the health effects of being non-working, as a function of time for disabled persons to be needed. For this work, both prospective and register based studies should be used.

Conclusions: The findings in these surveys show large differences between occupational groups. As non- working individuals in general report more health problems than the working population, the surveys also seem to support the importance of work on health. 


\title{
Long sickness absence after depressive disorders - Results from the Finnish Health 2000 Study
}

\author{
${ }^{1}$ Ahola, K., ${ }^{1}$ Virtanen, M., ${ }^{2}$ Honkonen, T., ${ }^{3}$ Isometsa, E., \& ${ }^{4}$ Lonnqvist, J. \\ ${ }^{1}$ Finnish Institute of Occupational Health, Finland; ${ }^{2}$ Ministry of Social Affairs and Health, \\ Finland; ${ }^{3}$ University of Helsinki, Finland; ${ }^{4}$ National Institute for Health and Welfare, Finland
}

Objectives: Work disability due to mental disorders has increased during the past ten years. We found that 29 per cent of employees with a depressive disorder or minor depression were absent from work for a more than 9 working day period during two years around our representative study. The aim of the present study was to investigate how common similar absences are in 12 months' follow-up after the study period and which factors predict these absences.

Methods: An epidemiological Finnish Health 2000 study was conducted during August 2000 and June 2001. The data collection included a CIDI mental health interview, a clinical health examination, an interview, and questionnaires. The participants of the present study $(\mathrm{n}=409)$ were those who had fulfilled the DSM-IV criteria of major depressive disorder, dysthymic disorder, or minor depression. Compensated sickness absence days for all causes in 2002 were extracted from a national register. The association between baseline socio-demographic, clinical, work-related, and treatment factors and subsequent sickness absence were examined with logistic regression models.

Results: In 2002, 18\% of those employees who had a depressive disorder during 2000 and 2001 had at least one sickness absence lasting more than 9 working days. Sickness absence during the follow-up was more common among the employees who at baseline were over 35 years old, had low basic or vocational education, had already been on a long absence, had negative expectations regarding their future work ability, used antidepressants, and had participated in vocational rehabilitation. Instead, future long absences were rarer among those in temporary employment, experiencing uncertainty, and receiving moderate support from their supervisor at baseline. In a multivariate model, the effects of baseline absence, experienced uncertainty, and participation in vocational rehabilitation remained statistically significant. In addition, a coexisting alcohol use disorder predicted subsequent sickness absence and low physical work strain and the anticipation of better work ability in the future protected against it.

Implications: Work places should be encouraged to develop models to prevent sickness absence in co-operation with occupational health services. Special attention should be targeted to coordinate the treatment among the employees with comorbid mental disorders and physical illnesses. Knowledge on the ways to strengthen the individual expectations regarding future work ability should be further studied.

Conclusions: Depressive disorders predict long sickness absences but the majority of the depressed employees do not have such absences. Both clinical and work-related factors predict the subsequent absences among the depressed employees. 


\title{
A Longitudinal Investigation Into Whether Emotional Labour and Other Work Strains Have an Impact on the Physical and Mental Health of Individuals Working in the Service Profession
}

\author{
Al Serkal, A. \\ Emirates Integrated Telecommunications Company (PJSC), United Arab Emirates
}

Objective: Emotional Labour is a term coined by Hochschild (1983). Since then, numerous studies have been conducted, but very few are longitudinal in nature. This study has attempted to investigate into whether Emotional Labour and other work strains have a long term impact on physical and mental health of individuals working in the service industry.

Method: A questionnaire which included measures of Emotional Labour (FEWS), organisational factors (Job Satisfaction, Decision Making, RoleClarity, RoleConflict, Autonomy and Control and Peer Support), the experience of physical symptoms, and the General Health Questionnaire (GHQ) was administered to a convenience sample of new cabin crew $(\mathrm{N}=299)$ based in an airline in the Middle East (Time 1). Fifteen to eighteen months later (Time 2), 35 cabin crew were traced, and in addition to the above mentioned questionnaire, the Maslach Burnout Inventory $(\mathrm{MBI})$ was administered. The response rate was $13 \%$. The average age was 24 years old (SD 2.37), and 20\% of the participants were male. Participants from 18 different nationalities participated. There are 2 waves of data.

Results: The results indicated that expecting to experience emotional dissonance as well as less job satisfaction were predictors for experiencing emotional dissonance at a later stage. While the expectation of having less autonomy and control, and the expectation of not having role clarity were predictors of Depersonalisation. Expecting job satisfaction as well as displaying positive emotions were predictors for Personal Accomplishment, Also, experiencing less Job Satisfaction at time 2 was a predictor for Emotional Exhaustion, even though expecting Emotional Dissonance at time 1 was a predictor for Emotional Exhaustion. It was observed that expecting to receive less Autonomy and Control at time 1 was a predictor for Depersonalisation, and no other variables were present. While expecting to Display Positive Emotions (time 1), was a predictor for Personal Accomplishment. At present, no other literature supports these findings, as no longitudinal research has been conducted in the field of Emotional Labour.

Conclusion and Implications: It was observed that cabin crew had realistic expectations concerning the Emotional Labour variables when they first started their role, and that in the long term, Emotional Labour did impact on the wellbeing of the individuals. This research could aide Employee Assistance Programmes in order to help service industry employees deal better with their health issues, therefore minimising sick leave or retaining them as they may not be able to cope with job demands.

\section{Employee engagement as a mechanism by which organisational climate influences employee job satisfaction and well-being}

\author{
Albrecht, S. \\ Monash University, Australia
}

Objectives: The relationship between organisational climate and important employee health outcome variables has been hampered by a lack of validated and theoretically derived climate 
measures and by an absence of research into potentially helpful explanatory mechanisms. Moreover the study set out to test whether or not employee engagement fully or partially mediates the effects of the six Human Relations dimensions of the Organisational Climate Measure (Patterson et al., 2005) on employee job satisfaction and job-related well-being.

Methods: The sample on which conclusions are based consisted of 306 full time employees occupying a diversity of roles within a diverse range of organisational settings. Confirmatory factor analysis (CFA) and structural equations modelling (SEM) were used to test the measurement and structural models proposed. Results CFA showed good fit indices for the six factor climate measurement model after specifying a reduced number of items per climate factor. Structural equations modelling of the proposed model yielded acceptable fit indices and showed that four of the six climate measures influenced employee engagement, and that employee engagement influenced both outcome variables.

Implications: Results are discussed in terms of the importance of engagement as a motivational explanatory mechanism by which organisational climate influences important employee health outcome variables. The results suggest organisational climate interventions and engagement strategies to influence employee satisfaction and well-being. The results will be of interest to practitioners and researchers.

\title{
Assessing occupational hazards in the Ghanaian mining industry and their implications for employee well-being and quality of life
}

\author{
Amponsah-Tawiah, K., Stavroula, L., Cox, T., \& Jain, A. \\ University of Nottingham, United Kingdom
}

While in recent years mining companies have become more aware of the need to address environmental issues and to incorporate environmental management systems into their overall policy, there are still issues to be addressed in terms of the social and health impact a mine may have on its employees.

A cross sectional survey was administered to 330 employees of five large scale mining companies producing three different mineral products (Gold, Manganese and Bauxite) to examine physical and psycho-social hazards in the Ghanaian mining industry and their consequences for the quality of life (QOL) and general well-being (GWB).

Response from 307 participants showed mining equipment (ME), ambient conditions (AC), and work demands-control (WDC) as being significant predictors of QOL and GWB after controlling for demographics. Age and type of mine within which participants operated had important implications for their well-being, and quality of life, with older workers and workers from nongold mines experiencing better well-being and quality of life. 


\title{
Organisations' health management systems: Do they improve employees' health?
}

\author{
Buech, V., Schraub, E.M.,\& Sonntag, K. \\ Ruprecht-Karls-University Heidelberg, Germany
}

In times of demographic change, financial crisis, and altering markets, employees' health plays an important role because only healthy employees are able to adapt to new circumstances. Therefore, organisations' health management systems become more important. The current study aimed to evaluate organisations' health management systems. Drawing from health research, we suggested positive relations between health management, a positive supervisor style, and employees performance and health. Moreover, we assumed the supervisor style to partially mediate the positive relation between health management and employee health.

The study was part of a greater research project (BiG) 'Benchmarking in a Health Network' funded by the German Federal Ministry of Education and Research (BMBF) and supported by the German Aerospace Center's Project Management Agency (PT-DLR)). About 1.800 employees of five German companies were asked to fill out questionnaires concerning their perceptions of health management, supervisors, performance, and health. Moreover, we collected objective measures, such as absenteeism and number of accidents.

Results: revealed a positive relation between health management and both employees' performance and health. As hypothesized, the supervisor style mediated the relation between health management and employees' health. Furthermore, our results were supported by the objective measures: a positive health management was negatively related to both absenteeism and number of accidents.

Implications: for future research concern further examination of health management's effects. Such evaluation studies 'showing positive effects of health management' would lead to a better recognition of these systems. However, our results indicate the roles of health management and of supervisor style to be important for employees' health. The indirect effect shows that health management affects other organisational factors such as supervisor style. This leads to the conclusion that health management creates a kind of health-supportive environment. It is therefore worth for companies to invest in adequate health management interventions.

\section{The Role and importance of goals in output-controlled working systems}

\author{
Bahamondes Pavez, C., Wilde, B., Hinrichs, S., \& Schuepbach, H. \\ University of Freiburg, Germany
}

Several changes have taken place in the world of work in the last decades. The main change concerning the control of work is related to the increasing output-orientation of working processes: The assigning of tasks is highly output-oriented, employees are more and more responsible for both the working process itself and the achievement of accorded or assigned goals, and the importance of goal attainment for the assessment of employee performance has increased. Consequently, we assume that the quality of the goals which employees have to 
achieve might be critical for characteristics of the working situation (such as the amount of stressors and the available social and organisational resources).

Objectives: This study examines how goal characteristics in output-controlled working systems are associated with well-being on the one hand and with characteristics of the working situation (e.g. stressors and resources) on the other hand.

Methods: Data from 170 employees of two German companies from the financial and industrial sector were obtained by means of a questionnaire. The questionnaire assessed perceived goal characteristics and working conditions (stressors and resources) which were considered to be especially relevant in output-controlled working systems. As an indicator of impairment of well-being, we included the Irritation scale from Mohr, Rigotti and MÃ $1 / 4$ ller (2005).

Results: Preliminary findings in output-controlled working systems show that goal characteristics (e.g. goal attainability as well as goal and resource adaptability) are positively linked to wellbeing. However, the effect of these goal characteristics on well-being is completely mediated by stressors. Further analysis will also shed some more light on the role of resources (e.g. social support, job control) and the interplay of these factors.

Conclusions: Goal characteristics influence other aspects of the working situation (e.g. stressors) in output-controlled working systems. If we intend to achieve healthy working conditions, therefore, special attention has to be paid to the setting of goals. These results and their implications will be presented and discussed at the conference.

\title{
Are role stressors and workaholism related to abusive behaviour at work? The mediating role of workplace bullying
}

\author{
${ }^{1}$ Balducci, C., ${ }^{2}$ Schaufeli, W., \& ${ }^{3}$ Fraccaroli, F. \\ ${ }^{1}$ University of Bologna, Italy; ${ }^{2}$ University of Utrecht, The Netherlands; ${ }^{3}$ University of Trento, \\ Italy
}

Research on workplace aggression has been carried out either from the perspective of the victim or from the perspective of the perpetrator. Considered separately, however, these two approaches may yield only a partial picture of the aggression phenomenon at the workplace. Building upon the concept of an incivility spiral (Anderson \& Pearson, 1999), the analysis reported in the present study tested a model of abusive behaviour at work in which work-environmental factors (i.e. role stressors) and personal factors (i.e. workaholism) trigger abuse and being the target of bullying acts as a mediating process. The participants were 462 employees of the health sector in Italy. The results of structural equation modelling showed that role stressors and workaholism were independently related to abusive behaviour, and that undergoing bullying acted as a mediating process. Furthermore, an interaction between role stressors and workaholism had added value in explaining the hypothesised path to abusive behaviour: at higher levels of workaholism the path leading from role stressors to abuse was significantly stronger. 


\title{
A dynamic test of job engagement: personal demands, personal resources, and job resources as predictors
}

\author{
${ }^{1}$ Barbier, M., ${ }^{2}$ Demerouti, E., \& ${ }^{3}$ Hansez, I. \\ ${ }^{1}$ National Fund for Scientific Research, Belgium; ${ }^{2}$ Eindhoven University of Technology, the \\ Netherlands; ${ }^{3}$ University of Liege, Belgium
}

Objective: With the emergence of positive psychology, work engagement has gained research interest. The application of longitudinal studies has enabled a deeper understanding of its causes and consequences. The role of job resources as initiators of work engagement has particularly been emphasized. Recent studies started to uncover the importance of personal resources for the experience of work engagement. The role of personal demands, like workers expectancies, in predicting work engagement has not been studied in the past. The goal of this study is twofold: (1) longitudinally testing causal and reversed causal relationships between personal demands, personal resources and job resources on the one hand, and work engagement on the other; (2) longitudinally testing the relationships between personal demands and both personal and job resources.

Methods: Personal demands were operationalized as workers' expectancies regarding their own performance. Personal resources were operationalized as organisation-based self-esteem (OBSE) and optimism. Job resources were operationalized as development opportunities at work. A total of 373 employees working in a Belgian public administration participated in this threewave longitudinal study. Data were collected with eight months interval and were analysed using structural equation modeling analyses.

Results: Three models were tested. The model testing direct effects of personal demands, personal resources and job resources on engagement showed good fit to the data. All paths were significant and positive, excepted for personal resources whose impact on engagement was not significant. The model including reciprocal effects fits badly to the data; therefore reciprocal links were not included further. The model that includes relationships between personal demands and job and personal resources showed good fit to the data. In addition to significant links found in the first model, changes in job resources had a positive impact upon further changes in personal demands. Changes in OBSE also had a positive impact on further changes in job resources.

Implications and Conclusion: This study makes several contributions. It adds to the understanding of work engagement by showing that not only job factors but also personal factors have an impact upon its development. Moreover, not only resources but also personal demands play a role in the experience of engagement. The study fails to replicate the previously found reciprocal link between job and personal resources. Finally, the study adds to the understanding of the dynamic nature of work experiences in explaining work engagement. 


\title{
The Relationship between Skills Training and Retention of Graduate Interns in a South African Information, Communication and Technology Company
}

\author{
Barkhuizen, N. \& Pop, C. \\ University of Pretoria, South Africa
}

Objective: The war for graduate talent necessitates the development of very specific skills required by the workplace in the 21 ste century. In reality, companies are not just assessing their current staff and future recruits on their business/hard/technical skills but also on their soft skills (Clymer, Roberts \& Strawn, 2001; Collective Resources, 2008). The main objective of the research was to determine whether soft skills and technical skills training, as part of a graduate internship programme, contributed to the retention of graduate interns in a South African Information, Communication and Technology (ICT) company.

Methods: An exploratory, ex post facto research design was followed using a combination of quantitative and qualitative data gathering techniques. A soft skills and technical skills survey were administered among a purposive selected sample of graduate interns $(N=79)$ and mentors $(\mathrm{N}=39)$ in a South African ICT company. Open ended questions were included to allow for the triangulation of results.

Results: The overall results of the soft skills training indicated that the internship programme contributed to a large extent to the soft skills of the graduate interns. On average, the graduate interns indicated that the programme training contributed most to business etiquette, conflict management, problem-solving, self-management and goal-directedness soft skills. Both groups of participants indicated that the soft skills presented in the training are important. Combined, the participants identified verbal communication, self-motivation, teamwork and goal directedness as the most important for graduate employability. Results further showed that the technical skills training contributed to a large extent to the employability of the graduate intern. Technical skills training were also significantly related to the graduate intern's intention to quit the internship programme.

Implications: The research confirmed that skills and capacity building is a significant factor in employee retention. Soft skills training are as imperative to the preparation for employability and must not be deemed optional or regarded as secondary to the technical skills training. The relevant technical training requirements should be identified for the specific position the intern is placed in. This requires a more rigorous conceptualisation and planning of the graduate internship programme to ensure that the outcomes are achieved (Metcalfe, 2001).

Conclusion: In conclusion, the current graduate internship programme focuses on the majority of employability soft skills required for the acquisition and retention of a job. Practical training is therefore important for the application of knowledge and to enhance graduate employability. 


\title{
The Safe Mindset of Managers, Shift Bosses and Miners of a South African Platinum Mine
}

\author{
${ }^{1}$ Barkhuizen, N., ${ }^{2}$ Van Rensburg, N.J. \& 'Stanz, K. \\ ${ }^{1}$ University of Pretoria, South Africa; ${ }^{2}$ University of Johannesburg, South Africa
}

Objective: The mining industry in South Africa is in a process of transformation. Central to the transformation process is maintaining and improving production output in a safe manner. Leadership is one of the most crucial aspects in organisational performance and safety efforts (Hidley, 2004). The aim of this research was to determine the safety mindset of Managers, Shift bosses and miners in a South African Platinum mine.

Method: The research applied a quantitative descriptive research approach. The Safe Human Mindset Measuring Instrument (SHMI) was administered among Managers $(\mathrm{N} 1=18)$, Shift bosses $(\mathrm{N} 2=28)$ and Miners $(\mathrm{N} 3=60)$ of a South African platinum mine. The SHMI measures the constructs relationship credibility, workforce satisfaction, work environment and supportive relationships (climate) and safety culture.

Results: The results showed statistical significant differences between Managers, Shift bosses and Miners in their respective safe mindsets. More specifically, results showed with the exception of Culture (where no significant differences exist between Managers, Shift bosses and Miners), that differences in Climate and Trust originated at Managerial level. Relationship credibility, Caring support, Ownership, Physical environment and Safety intent originated at Shift boss level. Results further showed that Shift boss level acts as a filter between Management and the lower levels (Miners) in the organisation. The result is disconnect to the organisation and has dire consequences for communication lower down the hierarchy, teambuilding and participation in health and safety matters.

Implications: The development of credible relationships comprising of trust, caring support, respect and ownership, influencing of attitudes towards the job and organisation and cultivating a values and belief system falls within the ambit of leadership development that is required at the identified organisational levels. It has been recommended that a behaviour - based approach to safety improvement be introduced that will include employee involvement. In terms of management (leadership) practice the identified areas for development include trust, respect, ownership and caring support.

Conclusion: In conclusion, the study elicits the factors that influence and contribute to the safe mindset of the Manager, Shift boss and Miner leader levels in the organisation. The Safe Human (Mindset) Model provides a sound theoretical and management model which, when applied, suggests focus areas from which interventions can be planned and executed. The model is a diagnostic instrument that assists in managing safety either re-actively or pro-actively in eliminating incidents and fatalities. 


\title{
The Relationship between Barriers to Change and Work Engagement of Employees in a South African Property Management Company
}

\author{
${ }^{1}$ Barkhuizen, N. \& ${ }^{2}$ Bell, E. \\ ${ }^{1}$ University of Pretoria, South Africa; ${ }^{2}$ University of Johannesburg, South Africa
}

Objective: Resistance to change has long been recognized as a critically important factor that can influence the success or failure of any organisational change effort. For any merger / acquisition to achieve its goals, each individual in that organisation needs to contribute and be committed to the process involved. However, the expense, time and energy invested to take staff through these changes are challenging. The objective of this research was therefore to determine the relationship between barriers to change and the work engagement of employees in a South African property management company.

Methods: A cross-sectional survey design was used with a convenience sample taken from employees $(\mathrm{N}=234)$ in a South African property management company. The Barriers-to-Change Questionnaire (BCQ) and the Utrecht Work Engagement Scale (UWES) were administered.

Results: Confirmatory factor analysis resulted in a one factor-solution for both the BCQ (labelled barriers-to-change) and the UWES (labelled work engagement). Both scales showed acceptable internal consistencies. Practically significant differences were found between barriers-to-change and job level of employees. Practically significant differences were found between work engagement, age of employees and entitlement to annual leave. Results showed a practically significant relationship (medium effect) between barriers-to-change and the work engagement of employees. Results further showed practically significant relationships between the dimensions of barriers to change (i.e. change, project, people and environment) and work engagement (i.e. vigour, dedication and absorption).

Implications: The results highlighted the need for future organisations to spend more time focusing on organisational barriers-to-change and the impact thereof on work engagement of employees. Apart from the time that organisations need to spend on interventions, organisations also need to acquire tools and the "know how" to keep employees engaged during change interventions.

Conclusion: In view of organisations experiencing continuous change, as well as the fact that people in these organisations will want to resist these changes, more research into the matter of barriers-to-change can prove to be beneficial to both industry as well as the academic domain. Nicholas (2000) writes that "it is people who make the transition from the old "as-is" to the new "to-be". If barriers-to-change can be correctly identified and addressed, change initiative will have more of a chance to succeed. 


\title{
The Landscape of Psychological Age and Chronological Age at Work
}

\author{
'Barnes-Farrell, J., 'Johnson, N.C., \& ${ }^{2}$ Cherniack, M.G. \\ ${ }^{1}$ University of Connecticut, USA; ${ }^{2}$ University of Connecticut Health Center, USA
}

Objectives: Work is an arena where aging processes have the potential to be reflected in a variety of outcomes that matter to workers and employers alike. Nonetheless, studies of aging and work phenomena suggest that chronological age is of limited utility as an indicator of work well-being or as a predictor of work performance. Other aspects of age, such as age self-construals, appear to have more value than chronological age for understanding work and aging. A primary goal of this study is to continue building our understanding of the landscape of psychological age and chronological age at work. We focused on the feature of subjective age known as Felt Age (FA), which refers to how old one "feels." Our objectives were to examine drivers of FA and relationships of FA with employee reactions to work demands in two distinctly different work environments.

Methods: Employees of two organisations completed a survey that assessed aspects of their physical and mental health (SF-12 Physical and SF-12 Mental scales), personal and work functioning (mastery, sense of coherence, workability), reactions to work (job satisfaction, burnout-disengagement, burnout-exhaustion, emotional labor, experienced stress), and working conditions (physical demands, psychosocial demands, social supports), as well as FA and chronological age. Participants included correctional officers from a state correctional system $(n=277,73 \%$ males, mean age $=$ 42 years) and employees of a large manufacturing organisation ( $n=285,80 \%$ males, mean age $=48$ years). Hierarchical multiple regression analyses were conducted, first regressing FA on measures of health and personal functioning, then regressing work reactions on FA (statistically controlling for chronological age in all analyses). Finally, work design and work environment were considered as potential moderators of relationships between FA and work reactions.

Results: Physical and mental health and assessments of personal functioning accounted for significant variance in FA in both work environments. FA, in turn, accounted for significant incremental variance in work reactions, showing its primary impact on stress reactions such as work disengagement and work exhaustion. Features of job design were generally unrelated to chronological age or age selfconstruals, nor did they moderate relationships between FA and personal/work reactions.

Implications and conclusions: Our discussion focuses on notable similarities and differences in the psychological age landscape in the interpersonally demanding context of a correctional institution and the physically demanding context of a manufacturing operation, and implications for supporting the well-being of an aging workforce in such environments.

\section{Does cultural diversity matter for stress of low qualified workers?}

\author{
Behrendt, K. \& Busch, C. \\ University of Hamburg, Germany
}

Low-qualified workforces have been mainly neglected in occupational stress research. They are often multicultural. In this contribution, we present the results of a study, which investigated social stressors, social resources and psychological well-being of multicultural workforces in comparison 
to mono-cultural personnel. Respective samples consisted of low-qualified employees working in teams in different industries in Germany, like manufacturing, kitchen work or waste disposal.

A preliminary study (Study 1) investigated potential differences of culturally diverse and culturally homogenous (German) workforces in relation to their social resources and well-being. Results revealed that members of multicultural workforces $(n=31)$ reported a significantly higher degree of social resources than members of mono-cultural workforces $(n=33)$. Despite this difference, both groups did not significantly vary in their psychological well-being.

Based on these results, Study 2 examined 120 members of multicultural work teams and 145 members of mono-cultural teams. Structural Equation Modeling (SEM) was applied to compare the respective direct effects of social stressors and social resources on employees' well-being. Twogroup comparisons of hierarchical structural equation models analysed whether cultural diversity has a moderating effect on the postulated relations between social stressors and well-being, and social resources and well-being. Model A (social stressors and well-being) was proven to be largely valid for members of mono-cultural low qualified teams. As expected, this model did not apply to members of multicultural low qualified teams. A moderating effect of cultural diversity was confirmed. Validity of Model B (social resources and well-being) was verified for members of monocultural teams. Contrary to expectations, it was valid for members of multicultural work teams, too. Social resources were determined to be an even better predictor for well-being of the multicultural sample. The hypothesis of a moderating effect of cultural diversity had to be rejected. However, an inhomogeneous picture of fit indices suggests to carefully handle results concerning Model B. However, the results of this study show the requirements of culturally sensitive approaches within occupational stress research, when it comes to low-qualified workforces. Culturally sensitive measures and instruments are strongly needed for this target group.

\title{
Organisational-level work stress interventions: The more the better?
}

\author{
Biron, C.
}

\section{Lancaster University Management School, United Kingdom}

This 2-wave longitudinal study evaluated (a) whether work-oriented stress interventions improved the psychosocial work environment (i.e. job demands, job control, reward, participation in decision, support, and relationship with manager), job satisfaction, and psychological distress, and b) whether a linear relationship existed between exposure to interventions and improvements in outcomes. To evaluate the effects and level of exposure to interventions, a prospective quasi-experimental design with measures before $(\mathrm{N}=301)$ and 18 months $(\mathrm{N}=285)$ after the beginning of the intervention was used to study three intervention groups and two comparison groups. To inventory interventions implemented and evaluate the process, group and individual interviews with a total of 73 participants were conducted, in addition to 20 months of observation of every meetings related to the intervention. This data was used to collect a list of interventions implemented in each unit, and to create a set of items measuring the exposure to interventions for the follow-up questionnaire. Interventions aimed at modifying relations with line manager, team processes, tasks, or work conditions.

Results showed significant positive improvements in 75\% of outcomes measured in the intervention group compared to $0 \%$ in the comparison groups. Significant dose-response effects were found for 
job control, participation in decisions, reward, and relationship with manager. Participants who reported a high level of exposure to the overall intervention program improved significantly on all outcomes compared to employees who reported a lower level of exposure to the program. This study adds to a scarce literature considering process in organisational-level stress interventions. The results support the relevance of organisational-level interventions to manage psychosocial risks and improve wellbeing.

\title{
The influence of task characteristics and social support on self-rated employee performance
}

\author{
Blaich, S., Noblet, A., \& Rodwell, J. \\ Deakin University, Australia
}

Objectives: The primary objective of the current study is to examine the relationship between job stressors as defined by the demand-control-support (DCS) model and both in-role and extrarole measures of employee performance behaviour. The extra-role behaviours will take into account organisational citizenship behaviours (OCB) and counter-productive work behaviour (CWB). A further objective of the current study is to test for the linear, non-linear and interactive effects associated with the independent variables. Of particular interest is the extent to which the relationship between the DCS and self-rated employee performance is mediated by health and attitudinal variables, including job satisfaction, affective commitment, well-being and psychological distress.

Methods: The sample comprised of public servants from a state-based law enforcement organisation. Six hundred and sixty two public servants employed in a range of departments within the organisation participated in a survey via a self-completed questionnaire. An initial factor analysis of the three-factor OCB scale (Williams \& Anderson, 1991) produced a fourth factor containing negatively-oriented behaviours, termed CWB. Participant responses were subsequently analysed using hierarchical multiple regression analyses. The main effect variables were "centred" prior to undertaking these analyses in order to reduce multicollinearity when incorporating the interaction terms and squared variables.

Results: Although the DCS variables accounted for relatively small proportions of explained variance in the outcome measures, their collective R-squared contributions were nevertheless significant. Results indicated the individual components of the DCS, particularly workload, nonwork support and job control, predicted all four outcome variables. There was support for nonlinear effects, with support-at-work squared predicting all four outcome variables. There was also evidence of mediation with job satisfaction fully mediating the relationship between support-atwork and IRB. CWB was negatively related to both forms of support, affective commitment and workload, but positively related to psychological distress.

Implications: The present study provided only limited support for the interactive components of the DCS, but strong support for non-linear effects in relation to support-at-work. Resources, such as job control and both forms of support (work and non-work), were important predictors of employee performance outcomes.

Conclusion: Both managers and researchers should be aware of the importance of resources in relation to employee performance, but not assume that relationships are linear, especially in 
regard to support. It is important to delineate CWB when investigating discretionary behaviour in the workplace considering it is associated with a lack of support, lower commitment and higher psychological distress.

\title{
Return to Work and Work-related Psychological Complaints
}

\author{
Blonk, R., Oomens, S., \& Huijs, J. \\ TNO Quality of Life, the Netherlands
}

The present study was conducted to gain new insights concerning factors that contribute to recovery of work related psychological complaints. Recovery is defined here not only as recovery from complaints but also as return to work. 155 participants on sick leave due to psychological complaints were recruited via psychologists.

The study design was a longitudinal cohort design with 4 waves and measurements every three months. Measurements included work aspects, complaints, return to work and personal factors such as self-efficacy, coping, work-life balance. Data of 155 participants were obtained from 71 psychologists who participated in this study. Data collection has just been finished.

Preliminary analyses revealed that eighty percent of the participants fully returned to work within one year. Concerning psychological complaints a significant decrease was find overtime, with a largest decrease in the first three months. Analyses of predictors of these changes indicated that reduction of complaints predicted the duration until full return to work. However when work related self-efficacy was entered into the analyses reduction in complaints did not remain significant. This indicates that the process of recovery concerning work functioning is partly independent from the recovery of complaints.

This finding concurs with the results of other recent studies. Further analyses on the process of change in recovery of both work functioning and psychological complaints and changes in selfefficacy will be presented.

\section{The development of a methodology for developing a healthy organisation culture}

\author{
${ }^{1}$ Bos, E., ${ }^{1,2}$ Scheppingen, V.A., Sarren, A., \& ${ }^{1,3}$ Zwetsloot, G.I.J.M. \\ ${ }^{1} T N O$ Quality of Life, The Netherlands; ${ }^{2} \mathrm{VU}$ University Amsterdam, the Netherlands; \\ ${ }^{3}$ University of Nottingham, United Kingdom
}

Introduction: The social environment is a major health influencing factor in organisations, besides other factors such as workplace health promotion, improving the quality of work and design for human factors. In literature the issue of a healthy culture is barely explored, while the concept of a safety culture is widely acknowledged. Methods and tools for developing a healthy organisation environment are spare up till now. That is why we developed the Health and Safety $(\mathrm{H} \& S)$ toolkit consisting of a survey with 2 questionnaires and 2-3 workshops. The GVV toolkit is based on a set of core values underlying healthy and safety behaviour. The incorporation of a set of core 
values related to health in organisational culture results in a framework for developing a healthy organisational culture, and opens up new perspectives in psychosocial risk research as well as for workplace health promotion.

Objective: Aim of this study is the development of the H\&S toolkit for the improvement of health and safety culture in organisations. The H\&S toolkit consists of 2 parts. One part includes two questionnaires on two organisational levels: employers and employees. The other part includes the intervention process.

\title{
Methods:
}

1. We used a variety of methods: literature search, multidisciplinary discussions with experts and stakeholders and an evaluation of 10 frontrunner OHS companies to identify 7 core values which together form a framework for a healthy organisation culture

2. Development questionnaires: for each of the 7 core values we composed behaviour expressions theorems for employees and managers/ staff as well. We evaluated some psychometric qualities of the questionnaires.

3. Intervention: 2-3 workshops with management, employees and staff based on large scale intervention techniques. The outcomes of the survey are input for those workshops.

Results: Corporate values in companies are a framework for human behaviour and social interactions in organisations. Seven core values relevant for the management of health, safety and well-being at work were identified: sustainability, justice, growth and development, social involvement, participation, trust, and resilience. These were made operational and measurable in two questionnaires. The content validity was good. On this moment a survey and dialogues are carried out.

Conclusion: The incorporation of health as related to a set of core values in organisational culture results in an interesting framework for developing a healthy organisational culture including social capital, opening up new perspectives in psychosocial risk research as well as for health promotion. Practical and research implications will be discussed.

\section{Chronic Work Conditions as Antecedents of Employees' Anticipated Stress to a Hypothetical Scenario of Organisational Change}

\author{
${ }^{1}$ Bosak, J., ${ }^{2}$ Kirchner, A., ${ }^{2}$ Sczesny, S., \& ${ }^{2}$ Meiser, L. \\ ${ }^{1}$ Dublin City University, Ireland; ${ }^{2}$ University of Bern, Switzerland
}

Previous research has repeatedly demonstrated the negative effects of organisational change on employees. For example, research findings indicate that employees mostly are left uninformed about what is expected of them (Shaw, 1993) which causes adverse feelings of uncertainty (Kivimäki, et al., 2000) and fears about loss of employment (Fugate, Kinicki, \& Scheck, 2002). Nevertheless, little research has investigated employees' reactions to the anticipation of organisational change, even though researchers have proposed that the anticipation of a stressful event is at least as likely to produce various types of negative outcomes as the event itself (Lazarus, 1966, Perrez \& Reicherts, 1992). 
Therefore, the purpose of this study is to examine anticipated stress in a reorganisation scenario presented to 654 employees of a German telecommunication company. Specifically, the employees were asked to put themselves into the situation of involuntary job change due to workplace reorganisation and to indicate their perceptions of job insecurity, job control, and job rewards in a questionnaire. These current, chronic occupational conditions were regarded as potential antecedents of anticipated stress using the Job Demand Control Model (Karasek, 1979) and the Effort Rewards Imbalance Model (Siegrist, 1996) as theoretical frameworks. Moreover, the questionnaire assessed employees' anticipated emotional reactions to this potentially stressful situation of organisational change. Moderated multiple regression analyses tested the hypothesis that chronic resources (that is job control and job rewards) can buffer the negative effects of chronic stressors (that is job insecurity) on anticipated stress.

As expected, both job control and job rewards separately attenuated the relationship between job insecurity and anticipated stress, accounting for considerable variance over and above the additive effects of each individual factor. Job insecurity predicted anticipated stress at all levels of the moderator job control, yet the relationship was less pronounced for high levels of job control than for low levels. Similarly, job security predicted anticipated stress at all levels of the moderator job rewards, yet the relationship was less pronounced for high levels of job rewards than for low levels.

In sum, the results have supported the idea that anticipated stress might be a function of particular combinations of chronic work conditions. Job insecurity increased anticipated stress for employees but job control and job rewards were able to attenuate this relationship to some extent. The results have implications for both researchers and practitioners who would benefit from considering chronic work conditions as grounds upon which organisational change is built upon.

\title{
Predicting Psychological Health at Work among Teachers: Testing a Mediation Model with Social-Organisational, Job-Related, and Individual Predictors
}

\author{
${ }^{1}$ Boudrias, J., ${ }^{2}$ Desrumaux, P., ${ }^{1}$ Brunet, L., ${ }^{1}$ Savoie, A., \& ${ }^{1}$ Nelson, K. \\ ${ }^{1}$ University of Montreal, Canada; ${ }^{2}$ University of Lille3, Canada
}

Among the numerous research on stress conducted over the past 20 years, one published by the International Labor Organisation (2003) confirms that 25\% to $33 \%$ of teachers in most OECD countries suffer elevated levels of work-related stress. However, testable multidimensional models are still lacking to predict and prevent psychological health problems among populations at risk.

This study proposes to test a predictive model of psychological health at work among teachers. In this model, psychological health is conceptualized as an integrative second-order variable covering both positive (well-being) and negative (distress) health symptoms. Predictors of psychological health, selected in diverse theoretical perspectives, were integrated in a framework distinguishing personal resources (optimism, resiliency), social-organisational resources (supportive climate, procedural and distributive justice), and job demands. The model hypothesized that satisfaction of intrinsic needs (competence, autonomy, affiliation) mediates the influence of organisational and personal resources on psychological health. Structural equation modeling performed on cross-sectional data collected from 391 teachers supported the mediating effects expected (Ïキ2(311) $=616, \mathrm{CFI}=.97$, $\mathrm{TLI}=.96, \mathrm{RMSEA}=.05, \mathrm{SRMR}=.07$ ). 
Results indicated that satisfaction of needs completely mediated the influence of social-organisational resources on psychological health. The mediation was partial for personal resources, which still exerted a strong direct effect on psychological health. Perceptions of job demands were influenced by personal resources and were also directly related to psychological health. The importance of different categories of psychological health predictors will be discussed based on our findings.

\title{
A Longitudinal Study of Employee Adaptation to Organisational Change: How Information and Individual Adaptability affect Well-being and Satisfaction during Change
}

\author{
Braun, I., Niessen, C., \& Sonnentag, S. \\ University of Konstanz, Germany
}

Today's work environments are characterized by a variety of change processes. Mergers and acquisitions, technological innovations and new organisational concepts are some of the reasons for change in the workplace. When employees are faced with organisational change, uncertainty appears to be one of the most critical aspects (e.g. Jimmieson, Terry, \& Callan, 2004). Therefore, individual factors as well as environmental variables that relate to uncertainty should affect individual outcomes during organisational change. In this regard, this study examined the role of information about change and the Individual Adaptability dimension 'Uncertainty' in predicting job satisfaction and psychological well-being during organisational change. Ployhart and Bliese (2008) define the 'Uncertainty' dimension as an ability to handle unsecure and unpredictable situations.

Based on the work of Fugate, Kinicki and Prussia (2008) this study further investigates appraisal, coping and emotion as mediating variables to explain the effects as mentioned above.

A total of 118 logistics employees of a large German multinational company participated in this study. This longitudinal study collected data before and after a broad organisational change process, which incorporated a relocation to a new building, restructuring of work processes and technological innovation.

Multiple regression results showed that information about change is indirectly related to emotion focused coping via its negative relationship to threat appraisal. Results also indicated that emotion focused coping is indirectly related to job satisfaction as well as psychological well-being via its positive relationship to negative emotions. Thus, people who received more information about change appraised the situation as less threatening, thereby attenuating the use of emotion focused coping. Reduced use of emotion focused coping itself lessened negative emotions and thereby increased job satisfaction and psychological well-being during change. The same positive effects have been found for the Individual Adaptability dimension 'Uncertainty'.

In terms of practical implications, these findings emphasize the importance of providing employees with adequate information about change. Furthermore, the results suggest the importance of acknowledging the Individual Adaptability dimension 'Uncertainty' when hiring employees in highly dynamic work environments. 


\title{
Regulatory focus at Work: the Moderating Role of Regulatory focus in the Job Demands-Resources Model
}

\author{
Brenninkmeijer, $\mathrm{V}$.
}

Utrecht University, the Netherlands

\begin{abstract}
Objectives: The Job Demands-Resources Model (Demerouti, Bakker, Nachreiner, \& Schaufeli, 2001) addresses the influence of job demands and resources on the well-being and performance of employees. Individuals may differ, however, in their sensitivity to the harmful effects of demands and the beneficial effects of resources. An important individual difference variable that could influence the effects of demands and resources is regulatory focus. Regulatory focus refers to the way in which individuals approach situations and try to reach their goal. Regulatory focus theory (Higgins, 1997) distinguishes between a promotion focus (aimed at growth and development) and a prevention focus (aimed at safety and security). This study examined the influence of regulatory focus in the Job Demands-Resources model.
\end{abstract}

Methods: A survey was conducted among 146 teachers in secondary education.

Results: As expected, effects of job demands (i.e., conflict and workload) were more pronounced among individuals high in prevention focus. Unlike expected, effects of job resources (i.e., support from colleagues and autonomy) were more pronounced among individuals high in promotion focus. Effects of resources were also moderated by prevention focus.

Implications \& Conclusion: It is concluded that regulatory focus may be an important determinant in individual responses to demands and resources in the work situation.

\section{Risk perception in the health care domain, between clinical risk and practitioners' safety: a study among operating theatre practitioners}

\author{
${ }^{1}$ Bruno, A., 'Bracco, F., 'Chiorr, C., ${ }^{1}$ Pugliese, F., ${ }^{2}$ Sossai, D., \& ${ }^{3}$ Palombo, P. \\ ${ }^{1}$ Università di Genova, Italy; ${ }^{2}$ AUSL Piacenza, Italy; ${ }^{3}$ A.O.U. San Martino Genova, Italy
}

Objectives: Risk perception among operating theatres' practitioners is investigated concerning both the relationship between personal safety and patients' safety and the perception of causes of accidents.

Methods: A quantitative checklist of Incident Reporting has been used. It consisted in 13 items about clinical risks and 8 items about practitioners' risks, considering injury frequency and seriousness, its causes and near misses. Participants were 139 and were recruited among physicians, surgeons, anesthetists, nurses of 14 operating theaters of a north Italian wide hospital.

Results: Spearman's Rho index reveals mild positive correlations between injuries to operators and injures to patients concerning frequency (means $=0,10$ ) and gravity (means $=0,22$ ). The same trend is found for near misses involving operators and patients $(r=0,26)$; the perception of the frequency and severity of injuries (means $=0,54$ ), and the frequency of accidents 
and near misses (means $=0,66$ ). We notice also that injuries are positively associated with communication problems and the lack of personal protection devices. The personal and professional characteristics have a positive correlation with accident frequency in case of missed control of devices (correlation with age: $r=0,29$; with job experience: $r=0,24$ ). Age and expertise negatively correlate with the frequency of accidents in case of patient injury due to devices failures ( $r=0,30$ and 0,27 respectively).

Implications: Frequency and seriousness of the practitioners' and the patients' risks are positively correlated, and this relationship strengthens when also considering near misses. Communication problems and the absence of devices for personal protection seem to be the most probable causes of accidents, the first if injuries are very frequent, the second if injuries are serious. Professional experience seems to be linked with perceptions of accidents due to behavioural routines, while low surgical skills are correlated with perceptions of accidents due to lack of technical expertise or a scarce sensitivity to dangerous conditions.

Conclusions: These results suggest that the promotion of safety in operating theatres could concern both operators and patients, thanks to an organisational commitment investing in technical matters like personal protection devices to reduce the most frequent accidents (technical approach to safety), but to reducing severe accidents needs making actions at the level of staff management (social approach to safety), also considering age and expertise differences. This tool could be used as a link between organisational practices and the health and safety promotion culture.

\title{
Professional competence as an intervening variable in affecting work-related stress: the case of public school teachers in Italy
}

\author{
Callari, T.C., \& Re, A. \\ University of Turin, Italy
}

Objective of the research was to study work-related stress among school teachers in a primary public school in Northern Italy. Work-related stress was studied not only as the process arising when work demands of various types and combinations exceed the person's capacity and capability to cope (Lazarus \& Folkman, 1984), but where the organisation, and in particular the school system, is not able to respond to the daily workers' needs and demands (Clot, 2006). Under this framework and in line with studies on moderating effects of commitment on job satisfaction (Begley \& Czajka, 1993), the research studied whether in school teachers the professional competence might play a mediator role in affecting work-related stress.

The study is nowadays, more than ever, of interest: after the structural reforms carried out by the Italian Minister of Education Gelmini, affecting the entire educational system and directly the school teachers (2009); in compliance with the Italian legislative decree 81/2008 (art. 28) and the European autonomous framework agreement on work-related stress (2004); taking into account few studies investigating the specific subject (Bhagat \& Allie, 1989; Favretto \& Comucci Tajoli, 1988; Pedditzi, 2005).

The project researched the hypothesis that "competent" school teachers might perceive an added stress-strain source from a school environment unable to valorise their capabilities and involve them as resources of value for a school of quality; on the contrary, "less-competent" teachers, who 
deliver their activities without enthusiasm and dynamism, might be less affected by frustration and dissatisfaction. The identified school teachers sample was classified in "competent" and "less-competent" based on the colleagues' assessment according to the social validation of professional competence (Re, 1990). To both "competent" and "less-competent" school teachers sub-samples was distributed a questionnaire (Favretto, 1994; Favretto \& Comucci Tajoli, 1988) consisting of four sections: personal data; comments on own workplace; self-assessment on own competence; perceived state of health and wellbeing.

The results confirmed that professional competence is an intervening factor able to affect the relation with stressors present in the work place and the perceived strain. The research provides interesting elements to plan preventive and ameliorative interventions in complex organisations. A study considering a wider sample to prove the research results is in progress.

\title{
Psychosocial factors promoting well-being in call-center operators
}

\author{
Campanini, P., Conway, P.M., Camerino, D., Punzi, S., Fichera, G.P., \& Costa, G. \\ Università Degli Studi di Milano, Italy
}

Objective: The association between an adverse psychosocial work environment and poor psycho-physical health is well-known. Few studies considered the association between psychosocial work characteristics and the opposite health condition (well-being). Call-centre operators may be under risk for job stress due to several work characteristics; some of these aspects lie at the core of the Effort/Reward Imbalance model (ERI). Study aim is to evaluate the relationship between ERI, its components taken separately, and well-being among call-centre operators.

Methods: The present study was conducted on Italian call-centre operators. At the presence of a researcher, subjects filled in a questionnaire following routine worksite health surveillance. In addition to the ERI Questionnaire, we investigated psychological and physical health outcomes using different scales (the General Health Questionnaire, the Inventory of Subjective Health and the Health Survey). The direction of the effort scale was inverted (higher values mean less effort). All health measure were rescaled to the same range and categorized into tertiles. Psychological well-being was defined as having a GHQ score equal to 0 (no anxiety and depressive symptoms), while physical health as having a score on the Inventory of Subjective Health and the Health Survey equal to 0 (no digestive and cardiovascular symptoms).

Results: Of participants $(n=1106$, response rate $=99,9 \%), 77.4 \%$ women, mean age was 33.4 years (s.d. 3.9) and mean job seniority 8.0 (s.d. 2.2). Most worked parttime $(75.8 \%)$ and performed in-bound call handling $(58.6 \%)$. In all, $72.3 \%$ reported a balanced condition between ERI; $36.6 \%$ of the participants had a GHQ score equal to $0 ; 19 \%$ and $25 \%$ did not report any digestive and cardiovascular symptoms respectively. After adjustment for sex, age, marital status, number of children (under 7 years), education, job seniority, commuting time, overcommitment, job activity (inbound/outbound), working time (part time/full time; shift type), a balanced situation between effort and reward was associated with higher well-being (ORs from 2.16 to 2.25). Compared to high effort, low effort was more strongly associated with psycho-physical well-being (ORs from 1.72 to 
2.33); the same was for high reward compared to low reward (ORs from 1.80 to 3.46). With regard to the reward components, only high esteem reward, compared to low, was associated with higher psychological well-being (OR 2.91) and absence of cardiovascular symptoms (OR 2.54).

Conclusions: Different factors of the ERI model (mostly reward and in particular its esteem component) may be important for maintaining and promoting psychological well-being and reducing work-related stress among operators.

\title{
How Bullying Change My Life
}

\author{
Cecchini, F. \\ Patronato INAS CISL, Italy
}

This study illustrates a research conducted on a group of workers, mainly from central Italy, exposed to harassment on the job. The research was inspired by the experiences gathered by the anti-mobbing counselor's office of INAS-CISL. These testimonies show that the harassments do not only cause damage at the moment in which they do happen, but most of the times, they change radically the aspects of the life of the workers; they ultimately lose their loved ones, health and economic means. Out of these considerations came the need to plan a research to verify le impressions gathered so far, to make public the fact that mobbing is not just a transitory phenomenon, but it brings about radical negative and dramatic changes in the life of a person.

To carry out this research, the operators INAS-CISL have interviewed about two hundred workers who have been victims of moral harassment. Two different questionnaires have been used: one for the workers who have left the company and the other for the ones who remained employed. The questionnaire contains 50 questions, based on the most frequent statements of the workers. The questions regard: relationships in the company, aspects tied to the kind of harassment suffered, social status in the personal and family relationships; economic difficulties generated by the situation, psychophysical conditions and a comment of the worker about his/her experience. The information gathered have given semi-quantitative. This study shows interesting data, which confirms previous results, but also gives new surprising elements. Among the most significant data showed: 39\% of the interviewed workers changed their job, 28\% separated/divorced from their spouse, 54\% isolated themselves from friends, 33\% faced economic problems, 49\% suffered from various medical pathologies, 75\% professionally demotion, $40 \%$ stopped working, 53\% turn to the courts and one interviewed has committed suicide. From the comments it is clear that the harassment has a higher effect on the workers who ignore their union rights, that the irreversible pathologies of a psychophysical nature mine is health, which increase the possibility of accidents on the job; that the phenomenon causes social damage to the worker, the same company where the mobbing is perpetrated and the entire national community due to the economic, social and sanitary support. All this information shows the necessity of a national legislation dedicated to the mobbing phenomenon, which will make light on all its aspects and be a deterrent to the perpetrators. 


\title{
Family firms Vs non-family firms: The influence of the organisational context on employee behaviour and well-being
}

\author{
${ }^{1}$ Ceja, L., ${ }^{2}$ Escartín, J., \& ${ }^{3}$ Tàpies , J. \\ ${ }^{1}$ IESE Business School, Spain; ${ }^{2}$ University of Barcelona, Spain
}

Objectives: The literature has often been highly critical of family-owned firms as an organisational form. Family businesses are frequently viewed as suffering from a lack of professional management, destructive nepotism, and appear to be short-lived. Although this is true for some family businesses, recent research has begun to show that family firms often outperform non-family businesses along various dimensions. One example is that family firms generally act with altruism for the benefit of the organisation and the community they operate in. This can be associated to the fact that family firm leaders are either family members or emotionally linked to the family. Such executives often commit deeply to the values, mission and vision of the business, treasure their employees and feel motivated to do their best for the owning family and the organisational collective. Building on the stewardship theory (e.g. Davis, Schoorman, \& Donaldson, 1997; Miller \& Le Breton-miller, 2005, 2006) and following the three way model of workplace bullying (Baillien et al., 2009), the present study seeks to explore how family business and non-family business contexts affect employee behaviour (i.e. altruism, workplace bullying and job performance) and employee well-being (i.e. work engagement and job satisfaction).

Method: The sample was composed of 287 employees (135 from family firms and 152 from non-family corporations). Every employee gave free descriptions of their own organisation and filled out a self-administered questionnaire. In order to asses the organisational variables associated with different levels of the outcome variables, the Multiple Correspondence analysis (MCA) was used.

Results: The study results revealed the following: 1) family firms are associated with more positive perceptions of the organisational context than non-family businesses; 2) family firms are perceived as having a balanced task/employee oriented focus, a positive work atmosphere and balanced power relationships/hierarchy within the organisation; 3) family firms are associated with higher levels of workplace engagement, job satisfaction, altruistic behaviours and lower levels of workplace bullying than their non-family business counterparts. Discussion: This results support the assumption that the organisational context has an important impact on employee behaviour and well-being. More specifically, our findings support previous theoretical and empirical research, which view family firms, as an organisational form that shapes its strategy around a set of family values that emphasize continuity, integrity and trust among stakeholders. In this sense, family firms can have distinctive capabilities that may be difficult for non-family firms to match. Also discussed are the implications of the study, its limitations and areas for future research. 


\title{
The effect of Retirement Behaviour on Retirees' Quality of Life
}

\author{
Ceresia, F. \\ University of Palermo, Italy
}

Studies of retirement behaviour have mainly followed two substantially different approaches: a) identity-based theories, emphasize on the individual's coping and adjusting behaviour; b) multidimensional theories, consider systemically the social, organisational and political factors as well as individual factor. Systemic theories have developed numerous models, yet the limits reported are such that only parts of the models are applied in the study of retirement.

This paper has two main aims. The first one is underline that a specific methodology called System Dynamics allows the building of a general framework in which psychological, economic, social, legal and organisational variables converge to describe the retirement behaviour and its main unintended consequences on retirees' quality of life. The second one is emphasized that organisational commitment and psychological contract breach play a considerable role in the dynamics of retirement behaviour, affecting retirees' quality of life.

A total of 916 retired individuals ( men $=62 \%$; women $=38 \%$ ) living in Italy participated in this study. The participants worked and lived in the North $(n=398)$, Centre $(n=264)$ and Sud $(n=254)$ part of Italy, and they were fully retired and receiving a retirement pension. The data collection procedure has been supported by a Retirees Association of a Labor Union (FNP-CISL).

Meyer and Allen's (1991) three-component Organisational Commitment Questionnaire modified by Clugston et al. (2000), Dorfman et al. (1988) Organisational Cultural Dimensions Inventory, Floyd et al. (1992) Retirement Satisfaction Inventory and Kickul et al. (2001) Psychological Contract Breach Inventory have been given to the participants.

To develop the System Dynamic Model, a causal loop diagram has been provided to show the causal relationship between the main variables of the considered system. Then a stock and flow structure was built to explore the behaviour of the system dynamics model. Finally, alternative scenarios and results from simulation runs have been provided.

The effects of retirement behaviour on retirees' quality of life have been outlined. Retirement phenomenon, dynamics of the key-variables, problem issues, feedback analysis of adopted public policies, unintended consequences of public policies and policy design to remove these consequences are discussed. The methodological implication of this paper refers to the usefulness of adopting the System Dynamics approach to explore the consequences of retirement behaviour on retirees' quality of life. The results show that Human Resources Management policies, that are effective in enhancing organisational commitment, can produce delayed positive effects on retirees' quality of life. 


\title{
Can civil pilots' Personality traits predict their stress, coping types, and job performance?
}

\author{
Cherng, C.F.G. \\ Chang Jung Christian University, Taiwan, R.O.C.
}

Introduction: Pilots' work is categorized as one of the high risk jobs and is expected to be sensitive to stress. It was of importance to understand civil pilots' baseline stress level, sources of stress, as well as their reactions and habitual coping behaviours. The current study was to explore the constellation of civilian pilots' stress, coping strategies and their flight performance in terms of their national cultures and their personality traits.

Methods: 721 civil aviators were recruited in this study. Among them, 82.7\% are Taiwanese and $17.3 \%$ are foreigners. A researcher-made questionnaire, using a 5-point scale, consisting of 131 close-end items related to work stress, reactions under stress, personality, present work, and stress coping behaviours, was used in this study. In terms of the coping types, Havlovic \& Keenan's (1995) categorizations--positive thinking, direct action, help-seeking, avoidance/ resignation, and alcohol were applied. Moreover, three personality factors, namely, the hardy personality, the fragile trait, and the other-orientation trait were assessed.

Results: The current results had been shown that there are significant differences between Taiwanese pilots' and foreign pilots' perceptions on stress, stress reactions, coping types, and their job performances. The two groups of pilots' personality traits were also different significantly. Although pilots' personality traits cannot predict their flight accidents directly, pilots with different personality traits did show react differently on their stress, coping types, and job performance which were related to flight accidents.

Discussion: The current results suggest that three personality traits could predict specific stresses and their coping types. The results support that pilots' personality traits play the main role in understanding of pilots' stress and coping strategies which closely related to the pilots' flight safety. It is of importance to make sure that pilots' perceptions on their work situations, such as operation rules, work climate, and promotion are suitable to their flight performance as well as personal needs. It is beneficial to reducing the human errors and guarantee for flight safety as well as pilots' mental health With respect to their different stressors and coping types, designing suitable intervention training programs to increase the use of adaptive or effective coping were suggested. It is not only important for individual needs and health, but also is good for establishing well organisational culture.

\section{Ethical Leadership: authenticity and responsibility}

\author{
Cherre, B.
}

Group sup de co La Rochelle Business School, France

Objectives: The crucial characteristic of a leader, by opposition to a manager, is the ability to manage subordinates in periods of chaos (Zalesnick, 1977). In order to decide through chaos and ambiguity, the leader must have values grounded on ethical principles (Llewellyn 2007). 
Ethical Leadership can have positive effects for both Leaders and followers and generate good outcomes for organisations especially in ambiguous times. In this research, we revisit the philosophical principles for Ethical Leadership through the scope of Sartre's conception of Authenticity and Responsibility (Sartre 1996; Cherre 2007).

Methods: We conducted interviews with top managers and explored the way they solved ambiguous issues resulting from a moral dilemma. Afterwards, we tried to understand the approach they were promoting during the resolution of those moral impasses and the impact it had on their subordinates. We interviewed fifteen managers from private and public sectors located in the City of Montreal, Canada. The methodology of this investigation is based on the qualitative method. Their discourses were analyzed and coded with the Atlas TI software.

Results: Leaders' faced a dilemma and experienced tension when they were confronted to choose between their organisation's obligations and their personal convictions. In each dilemma, the stake was to keep their personal reputation and professional obligations. To solve this matter, these managers relied on authenticity by aligning their actions with their personal values. At the same time, they considered the rules and the respect of social norms during their decision making process. When they had to settle the dilemma, Leaders decided to exercise their freedom of choice and take into consideration the specific context of the situation. By acting this way, they were able to maintain their leadership towards subordinates and clients, and preserve their psychological well being.

Implications and Conclusions: These results confirm that Sartrian Philosophy is present in Leader's discourses. In order to be ethical, Leaders must act with authenticity, freedom and self-awareness. Furthermore, Leaders have to take responsibility for their actions. According to them, taking responsibility means primarily to be responsible to others, especially to subordinates. This response is a definition closer to Levinas definition of responsibility (1982) than Sartre's. These two findings allow us to put forward a conceptual framework on ethical leadership based on Sartrian and Levinasian Ethics. These findings can be a platform for discussion and reflexion on how to train managers to lead in difficult and ambiguous situations.

\title{
The Study of Leadership and New Direction for Developing Entrepreneurs in Thai Small and Medium Sized Enterprises
}

\author{
Chongvisal, R. \\ Kasetsart University, Thailand
}

This research comprised of 2 phases. The first phase was designed to study the leadership of Thai SMEs entrepreneurs and the second phase was to develop new leadership through training course. For the first phase, there were three sample sets. In the first sample set, the data were collected from 30 successful SMEs entrepreneurs by in-depth interview and critical incident technique (CIT).

The data were analyzed to generate items of leadership scale for Thai SMEs entrepreneurs. The pilot 120-item leadership scale was tried out on the second sample set consisting of 544 SMEs entrepreneurs. Exploratory factor analysis (EFA) was used to explore the dimensions 
underlying the leadership scale. Confirmatory factor analysis (CFA) was used as a follow-up to determine the definite number of factors. Six factors resulting from CFA were 1) encouragement of subordinates' inspirational motivation, 2) consideration and well wishing toward others, 3) morality in business, 4) capability of strategic thinking, 5) encourage social consciousness, and 6) openness. The final 30-item leadership scale was validated with the third sample set composing of 1,064 SMEs entrepreneurs. The known-group technique was used to test the construct validity using the success of the entrepreneurs as the criterion.

The results indicated that the leadership scores of all 6 factors from the highly successful SMEs entrepreneurs were statistically higher than the scores from the low successful SMEs entrepreneurs. In the second phase, new direction for developing leadership of Thai SMEs entrepreneurs was proposed through the training course developed from Phase 1. The Training Workshop for Developing SME Entrepreneurs Leadership was developed and assessed its quality by the experts. There were 3 stages in the training course; assessment before training, leadership development by training, and evaluation after training. The course was organized into 9 modules. After the training, two meetings were held to monitor and evaluate the progress of leadership development plan, to let them share their experiences with each other, to advise them on how to solve the problems during implementing, and to adapt their plan accordingly.

\title{
Antecedents and consequence of work empowerment for elementary teachers
}

\author{
Choochom, O. \\ Behavioural Science Research Institute, Thailand
}

The purpose of this study was to propose and test a causal relationship of work empowerment model for elementary teachers. The model posits that psychosocial factors influence work empowerment, which in turn predict work satisfaction and effectiveness.

The sample consisted of 572 elementary teachers under the Bangkok Metropolis, Thailand. Self- report inventories with 5 rating scales ranging from true to untrue designed to measure antecedents and outcomes of work empowerment were administered to collect data. The selfreport inventories were acceptable in terms of discrimination power, validity, and reliability. The hierarchical set multiple regression analysis was conducted to determine the relative strength of different sets of independent variables. The structural equation model was also employed to examine the causal relationship model of work empowerment.

Results using structural equation modeling suggested that the model fit the data. That is, selfesteem, optimism, policy and administration, and collegial relationship positively affected work empowerment, whereas withdrawn coping style negatively affected work empowerment. In addition, work empowerment had impact on work satisfaction and effectiveness. Specifically, work empowerment played a mediating role between psychosocial factors and work satisfaction and effectiveness. The findings also indicated that the psychological characteristics factor contributed to work empowerment the most and the work environment factor was seconds in terms of strength of contribution. 
In conclusions, the study provides evidence in support of the work empowerment model. The data also suggest that the enhancement of work empowerment for elementary teachers needs to take into consideration both teachers' psychological characteristics and work environment.

\title{
Is the interpersonal component of burnout still relevant in health care settings?
}

\author{
Consiglio, C., Borgogni, L., \& Ferrillo, M. \\ University of Rome, Sapienza, Italy
}

Objectives: The present study intends to deepen the role of emotional and interpersonal dimensions in predicting health symptoms among hospital workers.

The first aim is to contribute to the investigation of burnout syndrome introducing a new facet, named interpersonal strain (Borgogni, Armandi, Amaducci, Consiglio, 2007), specifically referred to the psychological reaction to exceeding relational pressure and requests. If cynicism implies a distant and detached attitude towards one's work in general, interpersonal strain recaptures in a new light the original conceptualization of depersonalization, representing a distant and detached attitude towards other people. Thus, the study intends to test the discriminant validity of interpersonal strain and cynicism scales.

The second aim of the study is to examine the relationship between interpersonal strain, emotional dissonance (Zapf, 2002) and health symptoms. Given the mediating role of burnout between stressors and physical and psychological well being (Maslach \& Leiter, 2008), similarly we assume that interpersonal strain and exhaustion mediate the relationship between emotional dissonance and health symptoms.

Methods: A sample of 347 subjects working in an Italian hospital (56\% nurses, 16\% physicians and $28 \%$ other staff) filled a self-report questionnaire measuring exhaustion, cynicism, interpersonal strain, emotional dissonance and health symptoms scales. Confirmatory factor analysis was performed to test the discriminant validity of interpersonal strain and cynicism. Structural equation modelling were performed to test the mediation model hypothesized.

Results: Confirmatory factor analysis as certains the discriminant validity of interpersonal strain and cynicism. Structural equation modeling revealed the mediating role of exhaustion and interpersonal strain in the relationship between emotional dissonance and health symptoms. In particular emotional dissonance displayed the stronger relationship with interpersonal strain that in turn had a stronger relationship with health symptoms than did emotional exhaustion and cynicism.

Implications: Implications with regard to the emotional and interpersonal aspects of health care work are discussed, specifically related to training and people management interventions.

Conclusions: The study attested the distinction between cynicism and interpersonal strain, underlining the added value of interpersonal strain facet in predicting health symptoms in hospital settings. The study also corroborated the role of emotional dissonance as a key burnout predictor in health-care professionals. 


\title{
Wellbeing and Contrast of Discomfort in the Health Context: the Institution of Permanent Observatories-in the Region Piedmont's Health Units
}

\author{
Converso, D., Hindrichs, I., Mottura, B., \& Viotti, S. \\ University of Turin, Italy
}

Objectives: In 2007, the Health Councillorship of the Piedmont region decreed, in convention with the Psychology Department of the University of Turin, the institution of the 'Workgroup for Safeguard of Organisational Wellbeing' composed by psychologists, occupational doctors, and superintendents for Prevention and Protection services. This group is aimed at promoting pilot experiences in the Health Units in order to prevent and contrast psychosocial discomfort and to sustain workers' wellbeing.

Methods: A complex intervention started in January 2009: it begun with the formation of multi-professional project-groups in all of the 23 regional health units, who then became generally permanent workgroups, recognised by their General Directors. By the regional group's process-accompaniment, these groups grounded their projects among five different subjects: evaluation of psychosocial risks, opening of listening and support spaces for workers, redefinition of bonus-system, training interventions for the promotion of wellbeing, and the formation of 'observatories' aimed at monitoring critical areas indicators linked to organisational health.

Results: The first data and the development prospective of this last subject are presented here. The groups, belonging to five health units, were working on a parallel way in identifying objective indicators for the indirect observation of organisational discomfort and wellbeing: starting with the guidelines from the PRIMA-EF (Psychosocial RIsk MAnagement - European Framework) and $\mathrm{NIOSH}$ projects, a broad bibliography was set up, alliances with the organisational key-structures for database-communication (human resources and personnel, prevention and protection services, training, equality-committees) were created, and the experimentation of indicators began.

Subsequently, groups began to work together at regional pilot-project. They defined the purpose of an observatory such as a strategic function and organisational process that allows to collect, in a recursive way, data useful for the identification of organisational health maps, from which the widening and designing of interventions could be drawn, in order to promote organisational wellbeing.

Implications and Conclusions: The project contemplates the sharing of the following indicators, recognised both as relevant in literature and as empirically detectable in the specific health units: turnover, transfer requests and voluntary resignation, demands for parttime, extraordinary medical check on worker's demand, absence, surplus of working hours, customer reports, legal cases, and compensation requests. Inter- and intra-organisational networking will allow the comparison between permanent and recursive observation of organisational health, as well as the activation of jointed interventions aimed at promoting the wellbeing in the health units. 


\title{
The role of autonomy support and work engagement in job satisfaction and proactivity: A study in the Portuguese financial industry
}

\author{
${ }^{1}$ Cortez, C. \& ${ }^{2}$ Passos, A.M. \\ ${ }^{1}$ Departament of Social and Organisational Psychology, ISCTE-IUL, Portugal; ${ }^{2}$ MRC - \\ Management Research Center, ISCTE-IUL, Portugal
}

In a highly competitive and continuously changing environment, financial organisations need to promote the active performance of their employees. As work activities are playing an increasing importance in people's lives, it seems that employees are expecting higher levels of job satisfaction. Work engagement appears to simultaneously promote the active performance and the well-being of the employees.

The purpose of this study is to investigate the impact of the perceived autonomy support given by the supervisors on the work engagement and to investigate the mediating role of work engagement among autonomy support, job satisfaction and proactivity in the Portuguese financial industry. The present study also aims to present some suggestions to the managers on how to improve both the individual well-being, measured by job satisfaction and the individual active performance measured by the personal initiative of the employees.

A self report questionnaire was completed by a group of individuals working in the major Portuguese financial institutions with executive functions in commercial and business support areas. The questionnaire was sent directly to the participants and included measures of perceived autonomy support from their supervisors; work engagement (using the Utrecht Work Engagement Scale); job satisfaction; and personal initiative as an indicator of proactivity. A principal component analysis and some regression analysis were used in this study.

Results show a strong positive relationship between autonomy support and work engagement, a partial mediation effect of work engagement in the relationship between autonomy support and job satisfaction and a full mediation effect in the relationship between autonomy support and proactivity.

This study gives an empirical background for the managers of financial institutions to understand the importance of the supervisors' autonomy support for the individual well-being and the organisational competitiveness. In addition, this study gives some clues to the management on how to simultaneously improve the job satisfaction and the proactivity of their employees.

This study gives a contribution to the academic research as it is the first one to address work engagement in the Portuguese financial industry as well as the impact of the perceived supervisor's autonomy support as a job resource on the work engagement. 


\title{
Does Nurse Prescribing Increase Job Satisfaction?
}

\author{
${ }^{1}$ Cousins, R. \& ${ }^{2}$ Donnell, C. \\ ${ }^{1}$ Liverpool Hope University, United Kingdom; ${ }^{2}$ Alder Hey Hospital, Liverpool, United Kingdom
}

Objectives: Nurse prescribing was introduced in the UK October 1994 in a limited capacity. Following recommendations for further expansion, which took off in 2002, it is estimated that 14,000 nurses now have almost the same prescribing rights as doctors (Courtenay \& Carey, 2009). Beyond the central driver of improvements to clinical services, a discrete assertion of this development is that "nurse prescribing increases job satisfaction". Research to support this hypothesis is scarce and under-developed. Some support comes from previous research using relatively new nurse prescribers (e.g. Bradley \& Nolan, 2004, 2007; Lewis, Ellis \& Jester, 2004). This study sought to test this hypothesis using a sample of highly qualified and experienced nurse prescribers, and to examine the context in which job satisfaction is increased.

Methods: A qualitative methodology was used. Six nurse prescribers working in a community setting, in an inner city general practice were interviewed in depth. All had held the qualification V300 - Independent and Supplementary Nurse Prescribing for at least three years, and were required to issue prescriptions on a regular basis.

Results: The interviews were analyzed using a thematic approach. The main themes identified were increased role satisfaction and increased work-related stress. Sub themes included financial reward, job demands and job support. All participants reported increased role satisfaction, perceiving prescribing to be a way of providing a more holistic and complete type of care. However, the nurse prescribers also reported that increased demands, noting the management of more complex patients, the need for more knowledge, increased responsibility and the pressure to prescribe, alongside a lack of sufficient consultation time and insufficient training support. Rewards issues were important - no financial reward is attached to the extended role - confirmed by reference to the Government's Agenda for Change documentation (2004).

Implications: The findings will be discussed in terms of theories of Job Satisfaction and EffortReward Imbalance, and we will also consider the value of qualitative methodology towards extending models of occupational health and providing interventions.

Conclusions: The hypothesis was only partially supported. Whilst highly experienced nurse prescribers reported increased role satisfaction and autonomy, and assert an unwillingness to return to non-prescribing nursing, there were nevertheless reports of occupational stress, and dissatisfaction with the rewards of the job, especially pay.

\section{The Quality of Prior Experiences, Current Job Conditions and Anticipated Stress in a Reorganisation Scenario: the Central role of Cognitive Appraisal}

${ }^{1}$ Cullinane, S.J., ${ }^{2}$ Kirchner, A., ${ }^{2}$ Sczesny, S., ${ }^{2}$ Meier, L., \& ${ }^{1}$ Bosak, J.

${ }^{1}$ Dublin City University, Ireland; ${ }^{2}$ University of Bern, Switzerland

The effects of previous exposure to change on employees have been targeted in stress literature 
in terms of both positive effects such as familiarity (Axtell et al, 2002) and negative effects such as uncertainty (Rafferty \& Griffin, 2006). It may seem self-evident that current work conditions and prior efficacy in dealing with a similar stressor would influence anticipated stress in a new stressful situation by influencing cognitive appraisal. However, until now, this idea has been neglected by researchers in favour of more dispositional approaches to anticipated stress (Peacock \& Wong, 1996). Therefore the central aim of this study was to investigate the process through which external job resources (job security, job control and job rewards) and personal coping efficacy in dealing with a similar situation are related to anticipated stress to a hypothetical scenario describing workplace reorganisation. Job insecurity and the specific resource job control were selected due to their prominent status in the work stress literature (Bordia et al., 2004; Hartley et al., 1991; Karasek, 1979; Spector, 1986). A reward composite similar to the one proposed by Siegrist (Siegrist \& Peter, 1996; Siegrist, Siegrist \& Weber, 1986) including social support, career, and financial outlooks, was chosen to support a more general approach. Finally, coping efficacy in a similar situation was considered relevant due to the frequent reoccurrences of various types of organisational changes in the company of interest. In accordance to the Transactional Model of Stress (Lazarus and Folkman, 1984) cognitive appraisal was hypothesized and confirmed to function as a mediator for the relationship between these resources and anticipated stress.

Participants ( $\mathrm{N}=654$ ) were employed by a German telecommunication company with a history of rapid organisational change. The employees were asked to put themselves into the situation of involuntary job change due to workplace reorganisation and to indicate their perceptions of job insecurity, job control, job rewards, coping efficacy and change appraisal in a questionnaire adapted from the Stress and Coping Process Questionnaire (SCPQ) by Reicherts and Perrez (1991). In addition the questionnaire assessed employees' anticipated emotional responses to this situation.

The hypothesis was tested using hierarchical regression analysis which yielded the expected outcome that cognitive appraisal mediates the effects of all three indicators of chronic work conditions and prior coping efficacy on anticipated stress. This research contributes to both research and practice in terms of identifying factors that contribute to how employees perceive organisational change.

\title{
Stress Vulnerability, Quality of Life and Well-Being in Pre-Hospital Medical Emergency Professionals
}

\author{
Cunha, M., Salazar, G., \& Pereira, J.P. \\ Instituto Superior da Maia, Portugal
}

The authors present a study which aims to characterise the population of Emergency Telecommunications Operators (TOTE), Emergency Ambulance Technicians (TAE) and Immediate Life Support Ambulance Nurses (SIV) from the National Medical Emergency Institute (Instituto Nacional de Emergência Médica - INEM). The dichotomy between health and sickness, its relationship to stress, quality of life and well-being and the arising emotional consequences were the main focus of the study.

Purposes: to characterise the population of pre-hospital medical emergency professionals in terms of stress vulnerability, quality of life and well-being, emotional disturbs and burnout; to characterise 
the relationship between those variables; to investigate if the role they perform will influence the levels of stress vulnerability, quality of life and well-being, emotional disturbs and burnout.

Results: the 183 professionals participating in the study are vulnerable to stress, and the majority evaluates as good their Quality of Life, being the Physical and Environmental domains evaluated as less positive. On average, these emergency professionals are not emotionally disturbed, nor suffer from burnout. However, and curiously, stress vulnerability is positively related to these professionals' quality of life and well-being, and negatively related to emotional disturbs and burnout manifestations. To enhance that, probably due to the environment, individual space, impotence feeling, difficulty to manage the contacts' emotions or even the obligatoriness to adopt a physically passive role, TOTE is the group presenting more problems, which may compromise their health.

\title{
The ageing office population: age and the work physical environment
}

\author{
De Been, I. \& De Bruyne, E. \\ Delft University of Technology, the Netherlands
}

Objectives: This study investigates the hypothesis that age has a significant influence on the satisfaction about the work environment, the perceived productivity and the importance of different work environment aspects.

Methods: A survey was carried out with the Work Environment Diagnosis Instrument (WODI) in 18 organisations (51 office buildings) resulting in 7084 respondents. The respondents were asked to rate their level of satisfaction on 19 different aspects of the work environment and the extent in which the work environment supports their (perceived) productivity (3 items). These items were rated on a five-point Likert scale. Besides this, respondents selected the 3 most important aspects of the work environment out of 17 aspects. To compare age groups five age intervals were determined. ANOVA and Post Hoc tests were used for analysis.

Results: Statistical significant differences were found between the age groups regarding satisfaction, perceived productivity and relative importance of the different aspects. Overall, the respondents younger than 31 years old and the respondents older than 60 years are relatively more satisfied about the aspects of the work environment in comparison with the respondents from 31 to 60 years. Exceptionally, some aspects showed a different tendency and were rated lower by the youngest and oldest employees. Both the youngest and oldest groups ( $<31$ years and $>60$ years) find the work environment significantly more supportive for their productivity than the other age groups. There are some significant differences found between the age groups regarding the relative importance of the different aspects.

Conclusions: Although the data was gathered over several years and in different organisations, and differentiation might be influenced by the variation in buildings, a large number of responses were collected to give founded insights into how different generations evaluate the physical workplace. The youngest and oldest workers are overall most satisfied about their physical work environment and find the work environment significantly more supportive for their productivity than the other age groups. 
Implications: To keep all office workers feeling satisfied with their work environment, attention needs to be given to the different standards generations seem to have in relation to their workplace. Since most research concludes that extra attention should be given to the eldest workers, we would like to point out that one should also consider the middle age groups, since they seem to feel least satisfied and productive. Further research is needed to pinpoint the determinants of satisfaction related to age.

\title{
Psychological Factors, age, and the onset of Cardiovascular Disease
}

\author{
De Lange, C. \\ University of Groningen, the Netherlands
}

Introduction: Cardiovascular disease was the second overall cause of death in the Netherlands for the year 2008, as well as the main cause of death amongst the female Dutch population. Only by relentlessly investigating the myriad causes of this devastating disease can we ever hope to eradicate it as one of the main killers in the Western World. Previous research has shown that people with certain psychological factors can be more susceptible to cardiovascular disease. Moreover, in recent studies consistent evidence has been found that with the increase of age, the chances of falling victim to cardiovascular disease will subsequently increase. In this study the relationship between psychological factors and cardiovascular disease as a function of age was investigated. The psychological factors of interest were: "discrepancy" between self set high standards and actual performance, "high standards", and "negative coping styles" (in particular "avoidant coping").

Method and results: The results of this Dutch cross-sectional study, amongst a group of cardiovascular disease patients $(n=23)$ and a healthy control group $(n=25)$ provide support for the hypothesis that psychological factors only influence the late onset of cardiovascular disease, and not the early onset of cardiovascular disease.

Discussion: We can conclude that support for the proposed model was found in the sample of participants studied in this study. Moreover, this study will hopefully provide food for thought on the inclusion of age as a moderator in future research on the contributing factors to the onset of cardiovascular disease.

\section{Framing a new proposal for the Psychosocial risk assessment from Europe to Italian context: the Va.RP model}

\author{
${ }^{1}$ Deitinger, P., ${ }^{1}$ Nardella, C., ${ }^{2}$ Bonafede, M., \& ${ }^{2}$ Aiello, A. \\ ${ }^{1}$ National Institute for Occupational Safety and Prevention-I.S.P.E.S.L., Italy; ${ }^{2}$ University of \\ Cagliari, Italy
}

Introduction: On the basis of the findings of the project entitled Psychosocial RIsk MAnagementEuropean Framework PRIMA-EF (Leka and Cox, 2008), designed to develop a European model for the management of psychosocial risk, a specific model has been developed: the Va.R.P. Model 
(Deitinger, et al, 2008) starting from the specificity of the Italian context. Italian Law on occupational health and safety (D.Lgs 81/08) requires employers to make regular assessments of the risk of work-related stress so as to identify all risks that could affect workers' physical or mental health. Despite the importance of these problems and their impact on health and productivity, so far only a few Countries have defined standards directly aimed at managing psychosocial risks, with specific reference to work-related stress, thus raising the importance of focusing on the Italian context.

The Va.RP model is divided into five different stages: 1) identification of hazards; 2) risk assessment; 3) intervention strategies; 4) monitoring the efficacy of these strategies; 5) reassessment of the risk.

Objective: The main objective of this work is to present the Va.RP model and the development of the associated psychometric tool, within the multidimensional operational frame as provided by the PRIMA-EF project, which promotes the use of an integrated, multifaceted model for monitoring psychosocial risks.

Methods: Focusing mainly on documentary researches, we deeply reviewed the main areas investigated, according to the international literature, to define the main categories on which the Va.RP model shaped a multidimensional, measurement and rating questionnaire. To ensure a common approach for managing psychosocial risk in the workplace, we also interviewed stakeholders such as occupational health and safety experts, employers, union officials and workers.

Results: The scope of this assessment tool in Italy was clearly outlined. We then generated the items for the questionnaire, which most be modular and flexible, reflecting the various ATECO macro-sectors.

Implications: For Italian companies, including the many small and medium-sized ones throughout the Country, this tool should prove reliable and easy to use, fostering improvements and the adoption of good management practice.

Conclusions: The questionnaire is now undergoing a validation procedure on extended and varied typology of samples to verify its psychometric characteristics in order to approach the final validation of the proposed model and the associated tool.

\title{
Switching on and off: the impact of smartphone use on employee well-being
}

\author{
Derks, D., Zecic, D., \& Bakker, A. \\ Erasmus University Rotterdam, the Netherlands
}

This paper focuses on the impact of the increasing use of smartphones, mobile devices with the opportunity to send and receive emails regardless of office hours and location, on employee well-being. Research has indicated that smartphones can lead to increased productivity and more flexibility in work schedules. This flexibility gives individual workers the opportunity to a better work-life balance. Paradoxically, a major drawback of the same smartphone is that it is associated with difficulties in managing a work-home balance. Employees high on work-home 
interference put effort in their jobs during the time that might be used to recover from load effects that were build up during regular work time.

We propose that the extensive use of smartphones might inhibit the process of daily recovery and may lead to a decrease in well-being on the long run. To our knowledge there are no empirical studies on the effects of $24 / 7$ availability facilitated by smartphones on well-being. This paper aims to fill this gap. There are four indicators of well-being included in this study; experienced work-home balance, burnout, engagement and recovery style. We hypothesized that smartphone users experience more problems in work-home balance than the control group. Furthermore we hypothesized that smartphone users experience more burnout and less work engagement than the control group. And finally we explored whether smartphone users engage in different recovery styles than the control group. A total of 80 employees (40 smartphone users, 40 control group) completed a questionnaire on a daily survey over a period of 6 work days over 2 weeks.

Results showed that there was no difference in experienced work-home balance between groups. Smartphone users experienced more work engagement and less burnout than the control group. The more engaged smartphone group made more use of mastery experiences in the evening hours in order to recover. Apparently the smartphone users in our group experienced the smartphone more as a resource than as an increasing demand. They found a way to overcome the potential pitfalls of smartphone use and to make most of the advantages. Theoretical and practical implications are discussed.

\title{
Early retirement: a coping strategy against ageism at work
}

\author{
Desmette, D., Iweins, C.,\& Herman, G. \\ Université catholique de Louvain, Belgium
}

Objectives and hypotheses: Because the workforce is growing old, more and more studies are concerned with aging at work in connection with issues such as the longer working life. Until now, research has mainly investigated the influence of individual (e.g., gender) and organisational (e.g., working conditions) variables on older workers' performance and attitudes (see Faurie, Fraccaroli, \& Le Blanc, 2008) and, more recently, wellbeing at work (e.g. Elovanio et al., 2005). However, most of these studies have neglected the influence of psychosocial dimension of age, i.e. the social meaning of aging (Sterns \& Miklos, 1995) on older workers' attitudes. Numerous data have shown that older workers are stigmatised and discriminated at work (e.g., Finkelstein \& Farrell, 2007). We supposed that, like other stigmatised groups (e.g. Miller \& Major, 2001), when their age group experiments discrimination in the workplace, older workers may try to selfprotect by disengaging from the work domain. In other words, withdrawal attitudes such as early retirement might be conceived as coping strategies against age-related threat in the workplace. The study we will present examined, on the basis of the JDR Model (Schaufeli \& Bakker, 2004) how ageism (i.e. the social devaluation of individuals because they are old, Butler, 1969) influences the motivational and energetic processes which are likely to lead to early retirement.

Methods: Three hundred fifty-two Belgian workers aged from 50 to 59 years old answered questionnaires in various private organisations. The sample was counterbalanced on the basis of gender and professional status. Multiple hierarchical regressions were computed to test the 
psychosocial hypothesis controlling for individual (gender, health, wealth) and job variables (physical strain, autonomy in the task).

Results and implication: Results supported that early retirement was a self-protecting strategy against ageism: the more participants perceived that older workers were likely to experience stigmatisation and discrimination in the workplace, the more they were stressed and psychologically disengaged from work and the more they were likely to retire early. Moreover, findings underlined the importance to distinguish between complete early retirement and bridge employment (i.e., partial retirement): whereas complete early retirement intentions were mainly explained by the motivational process, bridge employment intentions were mainly predicted by the energetic process.

Conclusion: To conclude, we will discuss new research perspectives regarding analyse of aging at work just as propositions for age management in organisations.

\title{
Determinates of Successful, Culturally Competent Disability Service Providers
}

\author{
Dimpfl, S., Taylor-Ritzler, T., Balcazar, F., Oberoi, A., \& Willis, C. \\ University of Illinois at Chicago, United States of America
}

Objectives: According to the 2000 U.S. Census approximately $80 \%$ of the disabled population in the United States were considered to be non-white. So it is vital that disability service providers serve their clients in a culturally competent manner. The overall objective of the current study is to investigate what makes a culturally competent and successful disability provider, i.e. an individual that is successful at outreaching and retaining clients from diverse ethic backgrounds.

Methods: 249 disability service providers participated in the study as part of an 8 or 16 hour cultural competence training. Prior to the start of the training participants were asked to fill out the Cultural Competence Assessment Instrument (CCAI). The CCAI consists of Demographic questions, previous cultural competence training, questions about their position in their organisation, success in outreach and retention of different ethnic/racial groups, and a 24-item survey assessing participants' cultural awareness, skills, and level of organisational support.

Results: Paired t-test showed that disability service counselors have higher outreach and retention success rates with clients of the same race as themselves than with clients of another race. ANOVA's showed that there were significant differences in outreach and retention of clients from a different race for depending on the counselor's race. ANOVA's also showed significant differences on the three subscales (skills, awareness, and organisational support) of the CCAI depending on participants position in the organisation and educational attainment. Multiple linear regression showed that cultural skills were the only successful predictor of both retention and outreach.

Implications: The results of the study implicate that the best strategy to ensure successful outcomes for clients is to match them with a counselor of the same race as themselves. Additionally, it seems that many counselors are lacking the cultural skills necessary to successfully outreach and retain clients of a race different from their own. 
Conclusions: This study showed that disability counselors have greater success rates with clients of the same race versus those of a race different from their own. Secondly, Cultural skills are the most important predictor of outreach and retention success of a disability service counsellor. Cultural competence training is a means to increase the cultural skills of counsellors and could help overcome the "same race" success bias. Future studies need to take a post assessment of counsellors cultural skills after cultural competence training to see if this is in fact the case.

\title{
Developing on-line learning materials to support managers in the prevention and reduction of stress in the workplace
}

\author{
${ }^{1}$ Donaldson-Feilder, E., ${ }^{2}$ Lewis, R., \& ${ }^{1}$ Yarker, J. \\ Affinity Health at Work, United Kingdom; City University, United Kingdom
}

Objectives: The purpose of this project is to develop on-line learning materials to support managers in managing stress in their teams. The materials are based on a three-phase research programme that developed a framework of line management behaviours identified as important for preventing and reducing stress at work.

Methods: In the initial two phases of the research, a combined qualitative and quantitative methodology was used to develop, refine and validate a four-factor framework identifying the management behaviours that are important for preventing and reducing stress in those being managed (see Yarker et al, 2007, and Yarker et al, 2008). In the third phase, a longitudinal research design was used to evaluate a learning and development intervention, which aimed to help managers show the management behaviours identified in phases one and two (see Donaldson-Feilder et al, 2009). This showed that it is possible to help managers develop the relevant behaviours, particularly if they have development needs in this area.

The current project took the elements of the learning and development intervention from phase three of the research and converted them into on-line materials for use by two different audiences: - Materials for line managers to learn directly about how to prevent and reduce stress in their staff. - Materials for practitioners supporting line managers, for use as part of training, coaching, consultancy and other learning and development interventions.

A prototype version of the materials will be made available to 300 managers and 100 practitioners (trainers, consultants, coaches etc). Initial feedback data will be sought through a questionnaire immediately after usage. Further data will be sought through a further questionnaire three months later, to understand the effectiveness of the materials in achieving manager behaviour change. This data will be analysed and used to refine the materials prior to launch.

Results: Examples of the on-line materials will be shown during the presentation and early results from the feedback data discussed.

Implications: The final version of the materials, which will have been refined and improved as a result of feedback and evaluation of the prototype, will be freely available via the UK Health and Safety Executive website in late 2010. 
Conclusions: Research has identified the manager behaviours that are important for preventing stress in those being managed and shown that it is possible to help managers show the relevant behaviours. This new project takes the research a step further by designing and testing on-line materials based on these findings.

\title{
Mindfulness in Dentistry and Dental Hygiene
}

\author{
${ }^{1}$ Durepos, D., ${ }^{1}$ Catano, V., \& ${ }^{2}$ Lovas, J.G.L. \\ ${ }^{1}$ Faculty of Psychology, Saint Mary's University, Canada; ${ }^{2}$ Faculty of Dentistry, Dalhousie \\ University, Canada
}

Objectives: There is considerable evidence that the stress inherent in health care professions negatively affects health care professionals, and may reduce professional effectiveness (Shapiro, Astin, Bishop, \& Cordova, 2005). Prior research has demonstrated that mindfulness interventions may be used to reduce stress, anxiety, and depression in both clinical and nonclinical populations (Miller, Fletcher, \& Kabat-Zinn, 1995; Shapiro, Schwartz, \& Bonner, 1998). Mindfulness practice is a novel way of improving the teaching of professionalism, and has had success within the medical and nursing fields (Kinghorn, McEvoy, Michel \& Balboni, 2007; Kirk, 2007; Louie, Roberts \& Coverdale, 2007). To date, only one study examined the impact of mindfulness training on dental students (Raymond, Kinnunen, Clark, Sherman, \& Strafford, 2008). The present pilot study sought to address the gap within the mindfulness literature by examining the effects of mindfulness training among dental and dental hygiene students at a Canadian university.

Methods: All first year dental and dental hygiene students were required to attend a lecture that was followed approximately four weeks later by an optional 2-hour small-group Seminar focusing on mindfulness training. Subsequently, all students were provided with the opportunity to complete an 8-10 week Mindfulness Elective. Time 1 data was collected following the completion of the Lectures and Seminars. Time 2 data was collected upon completion of the elective. Measurements of self-compassion, personality, empathy, mindfulness, professionalism and career-related values were obtained.

Results: A total of 34 participants completed Time 1, and 9 participants completed Time 2. Outcome measures were analyzed using ANOVA to determine if there were significant differences between participants who had: 1) not attended the lecture or seminar; 2) had attended the lecture only; 3) had attended both the lecture and the seminar; and 4) who had attended the elective. Preliminary results indicate there were significant effects for the Selfcompassion Mindfulness scale, the Neuroticism scale, the Openness to Experience scale, the Conscientiousness scale, and the Career Related Values scale.

Implications: The present study served to expand the existing mindfulness literature by further examining the impact of mindfulness training on dental and dental hygiene students. The results of the present study indicate that participating in mindfulness training lead to increases in conscientiousness and career related values, and suggests a relationship between specific personality dimensions and mindfulness training. 


\title{
Anxiety and fatigue in on-call workers
}

\author{
Earle, F., Reid, T., \& Clough, P. \\ University of Hull, United Kingdom
}

Objectives: A diary study was carried out to investigate the impact of on-call working on two occupational groups of physiotherapists and fire officers. Subjective anxiety and fatigue were assessed following three types of shift, including normal working, on-call working and rest days.

Method: The study used a diary methodology to provide multiple ratings of subjective anxiety and fatigue following different shifts. Prior to analysis, the on-call shift was subdivided into two categories; those in which participants were called out and those in which they were not called out. This resulted in four shift categories of 'normal working', 'rest', 'on-call called out' and 'on-call not called out'. The two IVs for the study were the shift category and the occupation group and the DVs were the mean ratings of anxiety and fatigue. The participants consisted of 11 Physiotherapists (10 females and 1 male; aged between 20 and 49) and 11 Fire Officers (all male; aged between 30 and 65).

Results: The data were analysed using $2 \times 4$ mixed design ANOVAs. The analysis found a significant effect of shift type on anxiety, with post hoc tests revealing that both categories of on-call working resulted in significantly higher anxiety than rest days. There was no effect of occupational group and no interaction. A similar pattern of results was found for the fatigue ratings, with higher levels of fatigue following on-call shifts (although the difference between 'rest days' and 'on-call not called out' did not reach significance at the 5\% level). There was again no significant effect of occupational group and no interaction.

Implications: On-call working is a regular feature in the working lives of individuals across a broad range of occupational groups. Although operational working practices differ greatly across occupational categories, it is not uncommon to consider that being on-call, but not called out is the same as being at rest. The research presented here suggests that being on-call has an impact on the subjective state of individuals, even when they are not called out. A greater understanding of the psychological impact of this type of work scheduling could inform and improve operational practices, limiting the impact on the individuals working in key occupational groups.

Conclusion: This study highlights that working on-call can have an impact on the psychological well-being of workers, even when not called out.

\section{The long arm of work: Time pressure at work, cognitive failure and commuting accidents}

\author{
${ }^{1}$ Elfering, A., ${ }^{1}$ Grebner, S., \& ${ }^{2}$ De Tribolet, F. \\ ${ }^{1}$ University of Bern, Switzerland; ' University of Applied Sciences Northwestern Switzerland, \\ Switzerland
}

Objectives: This study investigates the association between time pressure at work, time control at work, cognitive failures, i.e. cognitive-based errors on everyday tasks, commuting accidents 
and nearby commuting accidents. Time pressure at work puts high demands on cognitive control and therefore increases the risk of cognitive failures during work and commuting between workers regular place of residence and place of work. We expected cognitive failures to mediate the influence of time pressure and time control at work on commuting accidents and nearby commuting accidents.

Methods: The sample consisted of 83 employees (56 \% female) who mostly worked in service sector (54\%) and health care system (31\%) and who individually commuted between the regular place of residence and place of work using vehicles. Participation rate in this questionnaire study was $71 \%$ (83 returned questionnaires out of 117 that were sent). The mean age was 43 years (SD = 12 years). The Workplace Cognitive Failure Scale (WCFS) consisted of 15 items with a five point Likert response format, asking for the frequency of failure in memory function, failure in attention regulation, and failure in action excretion. Time pressure and time control at work were assessed by the Instrument for Stress-Oriented Task Analysis (ISTA). Outcome variables were commuting accidents in last 12 months (yes/no), and nearby commuting accidents in last 4 weeks (yes/no). The mediation hypothesis was tested using a multiple mediator model and software of Preacher and Hayes (2008) including a bootstrap test of the indirect effect. Age, gender, neuroticism, conscientiousness, and time pressure during commuting were controlled in mediator analyses.

Results: Commuting accidents in last 12 months were reported by $10 \%$ of participants, while half of the sample reported nearby commuting accidents in last 4 weeks. Time pressure at work was positively associated with cognitive failure and nearby commuting accidents. Cognitive failure significantly mediated the influence of time pressure on nearby accidents even when age, gender, neuroticism, consciousness, and time pressure during commuting were controlled for. Time control was negatively related to cognitive failure, but no association with commuting accidents or nearby commuting accidents was found.

Implications: Time pressure at work is likely to increase cognitive load and thereby boost cognitive failure at work and during commuting enhancing the risk of accidents.

Conclusion: The results show that job redesign that reduces time pressure is crucial to improve road safety.

\title{
Recreation on the job: Rest break management among hospital physicians
}

\author{
${ }^{1}$ Ensinger, K., ${ }^{1}$ Staechele, T., \& ${ }^{2}$ Schuepbach, H. \\ ${ }^{1}$ University of Freiburg, Germany; ${ }^{2}$ University of Applied Sciences Northwestern Switzerland, \\ Switzerland
}

Objectives: To determine correlations between job conditions and contemporary recreation among hospital physicians.

Medical activity in the hospital would be associated with high job demands: The workload is frequently unforeseeable and can suddenly change. Long work hours are rather the rule than the exception. Research in the area of occupational health psychology has shown that long work hours can lead to increased fatigue, with unfavourable conditions as well as to an increase 
of stress. Thus the need for sufficient and good recovery periods during work hours (internal recovery) is increasingly needed to reduce or at least control fatigue. The question now is how physicians can deliver the high job demands and long work hours, without risking negative consequences for themselves and others.

Quantitative data were collected from about 398 physicians in a large hospital in Germany using self-reporting questionnaire. Preliminary findings reveal that internal recovery (e.g. regular short breaks) was not possible for the majority of physicians considering the intensity of medical activity as well as the overlap of partial activities: For three out of four physicians the opportunity to take a break is hardly given - once a workday at best. Time pressure is significantly related to the perceived lack of regeneration mediated by the recovery strategies.

In this lecture, the coherency between the requirements at work, contemporary recreation and the physician's state of health will be presented.

Conclusions: The findings provide information for tailored interventions. The question is, hence, which conditions on the level of the individual and on the hospital level encourage physicians to enhance their rest break management?

\title{
Teachers' attributions of the performance appraisal system: implications on well-being
}

\author{
${ }^{1}$ Ernesto, F. \& ${ }^{2}$ Chambel, M.J. \\ ${ }^{1}$ FPCE-UL, Portugal; ${ }^{2}$ Universidade de Lisboa, Portugal
}

The Holistic Model of Stress (Nelson \& Simmons, 2003) incorporates both negative impacts that stressors have on individuals, distress, and the existence of positive responses that provide eustress. Nishii, Lepak \& Schneider (2008) stress the importance of the attributions that employees make about the reasons why management adopts the Human Resources practices and their consequences for attitudes and behaviours.

This study aims to examine to what extent the Performance Appraisal system recently implemented in Portugal can be interpreted by teachers in a positive or in a negative way, and check to what extent these different interpretations are related to distress and eustress responses.

Participants in the study were 218 Portuguese secondary school teachers. Results revealed the existence of two different interpretations of the Performance Appraisal system: a Positive Attribution (in order to improve the quality of education and to enhance the teaching profession); a Negative Attribution (in order to reduce costs and to get the most work out of teachers). These different attributions are related to eustress and distress responses, respectively. In addition, the negative interpretation of this system is related to the desire to leave the teaching profession, but this relationship occurs because this negative interpretation triggers distress in teachers.

In summary, this study showed that the same practice of Human Resources can have both positive and negative interpretations and that these different interpretations are related differently to teachers' well-being. We argue that when implementing organisational practices it is necessary to develop positive arguments rather than negative ones. The suggested focus on eustress is in tune with the new development of the field of positive psychology and the emergence of positive 
organisational behaviour. Positive feelings at work possibly allow employees to effectively cope with work-related demands and, more importantly, are likely to have a positive impact on their well-being. Therefore, there exists a need for future research on positive states at work.

\title{
EU Policies and Social Dialogue on psychosocial risks- strengths and weaknesses
}

\author{
Ertel, M. \& Stilijanow, U. \\ Federal Institute of Occupational Health (BAuA), Germany
}

Background and Objective: Over the last years, significant progress has been achieved in the $\mathrm{EU}$ as regards the management of psychosocial risks at work. This includes, in particular, the common regulatory framework on $\mathrm{OSH}$ and European Social Dialogue. However, in spite of these promising developments, there are rising levels of stress at work in many EU countries, which raises doubts as to whether the application of instruments and approaches has kept pace with emerging problems. In the context of the project PRIMA-EF (www.prima-ef.org), this presentation will focus on strengths and weaknesses of the way psychosocial risks are regulated and managed in EU, considering also different perspectives and interests of relevant stakeholder groups and the challenges of EU enlargement in this context.

Methods: To capture relevant issues and trends concerning regulations and Social Dialogue in the area of psychosocial risks, we conducted an in- depth literature review and also involved important stakeholders in the research through focus groups and expert interviews. The focus groups were recorded, transcribed and analysed using thematic analysis. Additionally, some quantitative results from a stakeholder survey $(\mathrm{N}=75)$ conducted by the Italian project partner ISPESL on the perception of work-related stress were integrated.

Results: In general, problem awareness toward psychosocial risks is higher in the EU 15 than in the new EU countries (those having joined the EU in 2004 and in 2007). In terms of stakeholder perceptions, representatives from employers' associations tend to favour voluntary agreements to manage work-related stress, violence and harassment, whereas union representations are sceptical in this respect and prefer binding regulations. The discussion in the focus groups showed agreement among all stakeholders that there is a need for better guidance, tools and training on psychosocial risks.

Implications and Conclusions: Although work-related stress gradually has been established on the OSH agenda, it remains an issue that is the subject of contested claims. The results of PRIMAEF suggest that to successfully manage psychosocial risks, social dialogue and enforceable regulations should act in a complementary way, depending also on the overall context (e.g. the state of industrial relations in a specific branch, country, etc.). Particularly in new EU countries with only weak $\mathrm{OSH}$ capacities and short on experience in social dialogue, regulations and focused activities of the labour inspectorate will be needed to bring about improvements in psychosocial risk management. All in all, the political will to address psychosocial risks and to allocate sufficient resources to this end remains critical. 


\title{
Adjustment not PTSD, the real effect of War on Reservist soldiers!
}

\author{
Fairweather, L., Gibbes, C., \& Ridley, D. \\ University of East London, United Kingdom
}

Objectives: This research has studied the experience of being a Reservist soldier in the present situation of increased military tempo; Iraq, Afghanistan and the Global War on Terrorism. It focuses on exploring the lived experiences of UK Volunteer Reservists returning to civilian life, following a prolonged period of military mobilisation (active duty). The main objective of this research is to find out if there is a shared experience of mobilisation for Volunteer Reserves that is different from that of other soldiers and enduring beyond their mobilisation.

Methods: A qualitative study has been conducted. This consisted of semi-structured interviews with 12 members of the Volunteer Reserve, who have been on operational deployment. The aim of the interviews has been to describe and interpret the "lived experience" of retuning to civilian life. The interviews have been recorded and transcribed verbatim, before being analysed in line with the general phenomenological method of epoche, phenomenological reduction and imaginative variation in order to come up with the "essence" of what is it to come home after operational service (Interpretative Phenomenological Analysis).

Results: It has been found that mobilisation has long term effects on Volunteer Reservists, whether or not they see combat, that is different from post-traumatic stress disorder (PTSD). Also although a less acute condition, this adjustment has a chronic effect much greater in terms of numbers than the medical condition PTSD. In particular, what causes the returning Reservist the most issues is not work related; rather the general case is that it is home life and family issues that are the hardest to adjust back in to.

Implications: The implications for finding a generally shared phenomenon are that such experiences have to be catered for in government policy, as part of the military's duty of care towards its part-time employees (Reservists). In the same way that in theory, Reservists require more pre-deployment training than their regular counter-parts, they and their families may also require more explicit post-deployment assistance to readjust back into civilian life, if the duty of care is to be maintained.

Conclusions: A part of a mixed methods research project, the next stage will be to conduct quantitative research in order to assess the depth and consistency of the qualitative findings. It will be important for policy formulation to find out whether these same clusters are experienced throughout the wider body of mobilised Volunteer Reserves in the UK at this time.

\section{Effort-Reward Imbalance in combination with overcommitment impairs self-rated health and supervisor-rated job performance}

\author{
${ }^{1}$ Feuerhahn, N., ${ }^{2}$ Kuehnel, J., \& ${ }^{1}$ Kudielka, B.M. \\ 1Jacobs University Bremen, Germany; ${ }^{2}$ University of Konstanz, Germany
}

Objectives: The two central aims of this study are (1) to investigate the relationship between Effort- 
Reward Imbalance and employee job performance and (2) to examine the interaction between EffortReward Imbalance and overcommitment on health and performance. The three main assumptions of the Effort-Reward Imbalance (ERI) Model by Siegrist and colleagues (2002) postulate that (A) high effort in combination with low rewards (ERI) impairs health, (B) intrinsic overcommitment (OC) impairs health, and (C) the ERIxOC interaction synergistically impacts on health. The latter assumption has received inconsistent findings so far. We hypothesize that overcommitment aggravates the detrimental relationships between ERI and health, and between ERI and burnout. The present study extends the ERI Model by investigating associations with job performance. Therefore, we hypothesize that ERI is negatively related to performance and that overcommitment aggravates this negative relationship.

Methods: Employee and supervisor data was collected from 140 employees in 22 teams of a mechatronic company in Germany. Individuals were nested within teams. The central measures in this study were ERI/OC (Siegrist et al., 2009), general subjective health (one self-report item), work-related burnout (CBI; Kristensen et al., 2005), individual task performance and contextual performance (three supervisor-rated items each). We controlled for gender, age, education, type of job, and job autonomy.

Results: Multilevel analyses showed thatERI was negatively related to subjective health and positively related to burnout. In contrast to the significant positive relationship between overcommitment and burnout, the negative relationship between overcommitment and subjective health was only marginally significant. In line with the interaction hypothesis, these harmful relationships were aggravated for individuals scoring moderate or high on overcommitment. Furthermore, ERI was negatively related to task performance as well as contextual performance. The result of the interaction hypothesis showed that overcommitment moderated the relationship between ERI and job performance, such that ERI impaired job performance only for individuals reporting moderate or high overcommitment.

Implications: In order to support employees' health and performance, organisations should seek to foster a balance between employees' efforts and rewards, even more so for overcommitted employees.

Conclusion: Our study contributes to research on the effects of working conditions on health and supervisor-rated performance. We extend the Effort-Reward Imbalance Model by linking ERI to job performance. Furthermore, we found evidence that supports the postulated (but inconsistently reported) interaction effect of theERI Model on health. More specifically, overcommitment aggravates the detrimental relationship between ERI and health, and between ERI and performance.

\title{
Preventing post-traumatic stress following workplace robbery: procedure and preliminary results of a Psychological Debriefing Program for bank employees
}

\author{
${ }^{1}$ Fichera, G.P., ${ }^{2}$ Musti, M., ${ }^{3}$ Coggiola, M., ${ }^{4}$ Russignaga, D., \& ${ }^{5}$ Costa, G. \\ ${ }^{1}$ University of Milan, Italy; ${ }^{2}$ University of Bari, Italy; ${ }^{3}$ University of Turin, Italy; ${ }^{4}$ Intesa San \\ Paolo Bank, Milan, Italy; ${ }^{5}$ University of Milan, Italy
}

Objectives: The present study aims at reporting preliminary results of a project intended to provide Psychological Debriefing (PD) for bank employees' victims of robbery in order to reduce post-traumatic stress. 
Methods: Within 7 days after robbery, a collective interview was provided to employees by the occupational physician. The interview aims were to collect information about robbery and employees' reactions, and to provide PD. After 45 days, follow-up interviews were administered individually by occupational physician to assess employees' psychological health, and their perception about PD efficacy. The project comprised several preliminary activities, including creation and testing of semi-structured interviews for physicians, and training programmes for all company staff involved in the project. Only for physicians, a specific training course on conducting interviews and listening to the person victim of robbery were designed and performed. Finally, all physicians have been supported by psychologists for at least one interview each.

Results: Sixty-nine collective interviews - related to 69 different robberies - and 166 followup individual interviews were analyzed. The most frequent post-robbery reactions reported during PD were 'hypervigilance' (54 interviews), sleep disturbances (28), startle responses (17), avoidance symptoms (9). Psychosomatic reactions, 'intrusive' symptoms and lack of concentration at work were reported less frequently. In 5 interviews, at least one employee was symptom-free. At follow-up, 97 employees (59\%) reported complete well-being; 57 (34\%) partial recovery; 8 (5\%) persistency of symptomatology; 4 (2\%) worsening. One hundred fortyeight employees expressed their opinion about the efficacy of PD in providing support: $76 \%$ perceived it as 'effective', 16\% 'ineffective' , 8\% were not able to provide any opinion. We also asked which aspect of PD was particularly useful to subjects. The main feature - expressed by 60 employees - was " sharing with my colleagues the same feelings and reactions following robbery"; for 58 was "possibility of expressing my mood with someone who encouraged me to do it"; for 19 was "feeling social support by the company" ; for 10 was "learning from the doctor that emotional reactions were considered physiologic after robbery, and not pathological."

Implications: At follow-up interviews a complete or partial remission of symptoms occurred after the robbery was reported by the major part of employees. The PD was perceived as effective in high proportions and the reasons expressed by subjects are consistent with PD theoretical assumptions.

Conclusions: PD may play an important role in mitigating post-traumatic reactions; further studies are needed to assess its efficacy quantitatively.

\title{
Wellbeing in a Multinational Company
}

\author{
Gallo, R. \\ Watson Wyatt, Italy
}

This research was carried out in response to the 2002-2006 European Directive for Member States regarding psychophysical health in the workplace (COM/2002/0118 def.). It promotes a preventive approach to emerging psychosocial risks in order to define systematic intervention in favour of well-being at work. The aim of this research was to identify indicators of organisational well-being so as to reveal the conditions and the characteristics of the well-being of human resources and strategies to promote an intervention model.

Objectives: -identify psychophysical indicators of discomfort to prevent uneasiness, accidents and individual and group pathologies (for example: stress, burn-out) 
- identify indicators of psychophysical wellbeing to give value to personal, professional and organisational resources

- identify personal and group strategies to eradicate uneasiness and produce well-being present best practices to prevent organisational uneasiness and promote well-being

Methods: This research was done in a Multinational Company and involved an Italian headquarters that leads European branches in Austria, the UK, France and Germany. One hundred and sixteen people were involved in qualitative and quantitative investigations. We did 15 focus-groups and 30 interviews, and we used a questionnaire called "Investigation of psychosocial risks", an adaptation of the Job Content Questionnaire of R.A. Karasek (the European model). This basic text was modified and integrated to be more focused on needs and characteristics of the Company's people. The sample included more males $(76.4 \%)$ with a lifetime contract $(91.3 \%)$. The sample was clustered by blue collars $(45.7 \%)$, white collars $(42.2 \%)$ and executives $(12.1 \%)$. The majority $(62.6 \%)$ had been working in the same area of activity for over 6 years and $53 \%$ had been working for over 11 years.

Results: are related to recurring elements in the groups interviewed, stress and emotions at work and indicators. The indicators are prevalently identified as:

-Indicators belonging to the personal sphere and indicators belonging to the organisational sphere; -Indicators of well-being at work and indicators of uneasiness at work;

-Relationships with supervisors/managers, relationships with clients, relationships with colleagues; -Strategies for dealing with uneasiness and strategies for promoting well-being.

\title{
Presenteeism and occupational accidents: A correlation waiting to happen
}

\author{
${ }^{1}$ Gervais, R., ${ }^{2}$ Weyman, A., \& ${ }^{3}$ Williamson, J. \\ ${ }^{1}$ Health \& Safety Laboratory, United Kingdom; ${ }^{2}$ University of Bath, United Kingdom; \\ ${ }^{3}$ Department for Work and Pensions, United Kingdom
}

Objectives: This paper will examine the effect that working when ill i.e. presenteeism, has on the occurrence of occupational accidents. It is known that emotional and mental stressors could influence adversely the sustaining of work-related accidents and injuries. As illness is both a mental and emotional stressor it is useful to assess how great its impact, as a risk factor at the workplace.

Methods: A cross-sectional design using the Health \& Safety Executive's Survey of Workplace Absence Sickness and (ill) Health (SWASH) - 2005/06 of working individuals $(N=10,193)$ was used to generate data on sickness and work-related incidents, e.g. the number of accidents experienced at work, working when ill and sector and size of organisation. SWASH was a random stratified household telephone survey conducted amongst the working population in Great Britain and permits comparisons by age, gender, job role, sector (Standard Industrial Classification), and occupation.

Results: Correlation and ANOVA analyses showed individuals were more likely to experience occupational accidents if they went to work when ill. This was regardless of sector or size of organisation. In addition, the results showed that those who were involved in occupational 
accidents also worked longer hours and experienced higher job strain (i.e. lower job satisfaction, higher stress levels, lower control, lower coping and higher depression).

Implications: These results highlight that working when fitness level is low may have more negative consequences on individuals and ultimately on the working environment. Presenteeism may contribute to unsafe practices and may lead to negative work patterns, such as occupational accident outcomes in some individuals. Moreover, as individuals are less effective when ill, and may experience occupational accidents, this may have a consequential effect of a reduction in performance and thereby productivity.

Conclusions: This research supports previous research in this area showing that presenteeism as work behaviour may generate negative outcomes. Further, the results link the connection between stressors and strains on the job and occupational accidents, a linkage that is said to require further exploration. The perceived pressures on individuals to work when ill cannot be healthy for them or for their jobs. These pressures will need to be explored in further research, as the human factor element of understanding occupational accidents is one area that needs more investigation.

\section{Women who work and OSH: Exploring the limitations}

${ }^{1}$ Gervais, R., ${ }^{2}$ Schneider, E., ${ }^{3}$ Flaspöler, E., ${ }^{3}$ Hauke, A., ${ }^{3}$ Koppisch, D., ${ }^{3}$ Reinert, D., ${ }^{4}$ Koukoulaki, T., Vilkevicius, G., ${ }^{5}$ Martínez-Casariego, M.A., ${ }^{5}$ Martínez, M.B., ${ }^{5}$ Lozar, L.G., ${ }^{5}$ Martínez, S.V., ${ }^{1}$ Carter, L., \& ${ }^{1}$ Leah, C.

${ }^{1}$ Health \& Safety Laboratory, United Kingdom; ${ }^{2}$ European Agency for Safety and Health at Work; ${ }^{3} B G I A$ - Institut für Arbeitsschutz der Deutschen Gesetzlichen Unfallversicherung, Germany; ${ }^{4}$ ELINYAE, Greece ; ${ }^{5}$ NSHT - Instituto Nacional de Seguridad E Higiene en el Trabajo

Objectives: To highlight the issues which limit the occupational safety and health (OSH) standards for women within the European Union (EU), in line with previous research of EUOSHA. Women are a significant component of the employed, but are still not largely considered when defining and implementing effective OSH standards.

Methods: Desk-based research and analysis of online statistical data were used to review and assess work-related safety and health issues associated with women who work.

Results: The percentage of women who work continues to increase across the EU-27 countries, rising to $49 \%$ in the Baltic States. Despite this, women remain concentrated in certain sectors e.g. health, social and informal, with a limited choice of 'professions'. Of the 110 major occupation groups defined by the International Labour Organisation, half of all women work in eleven or fewer. The women employed in these 'specific' sectors/jobs are less likely to be given adequate training e.g. on chemicals or hazards.

Women are in marginalised jobs; they are at a higher poverty risk; work longer hours than men; are mainly responsible for family care and household work; have jobs with less control over decision making; and more likely to experience sexual harassment and discrimination; the number and rate of occupational injuries and illness as well as fatal incidents they experience 
are increasing, especially as they move into more hazardous jobs; the majority of equipment is designed for the average male worker, so women using it are at higher risk.

Although some progress was made, policy decision makers and associated bodies responsible for recommending and implementing policies for better working practices still do not focus enough on gender, e.g. EU documents and guidelines developed to determine the causes of and measures to address informal work do not focus on gender.

Implications: Women's work contribution is vital to the economy, and if they are unhealthy and unsafe, this lessens.

Conclusions: This research updates previous EU-OSHA research and proposes a more consistent and comprehensive approach to assessing women who work. Women are a significant aspect of the workforce; they make large economic contributions and are working increasingly in more dangerous and hazardous jobs, thereby increasing the likelihood of being injured or becoming ill. The prevalence of women in specific sectors, and ones seen as not generally hazardous or high risk has restricted their 'power' to act on their OSH. Change is required to acknowledge and address their OSH needs.

\title{
Characteristics of Emergency Nurses Experiencing Traumatic Stress and a Change in Work Productivity
}

\author{
Gillespie, G., Gates, D., \& Succop, P. \\ University of Cincinnati, United States of America
}

Objectives: Emergency nurses are exposed to 30 million traumatically injured patients annually suffering injuries such as burns, falls, motor vehicle crashes, and violence that may lead to traumatic stress symptomatology (TSS) and decreased work productivity. The objective of this study is to compare worker and workplace characteristics for emergency nurses who experienced TSS and a change in work productivity.

Methods: Surveys were mailed to a randomized sample of U.S. emergency nurses. Surveys included the Impact of Events Scale-Revised (IES-R) as a measure of TSS and the Healthcare Productivity Survey (HPS) as a measure of work productivity change. Data were received by 264 participants. Sixty four (24.4\%) participants met criteria for TSS (IES-R score 24-88) and a change in work productivity (decreased or increased). Emergency nurses with TSS and decreased work productivity (HPS score -58 to -1 ) were compared to emergency nurses with TSS and increased work productivity (HPS score 1 to 58). Groups were compared using Chi-square for categorical variables and a t-test for scaled variables.

Results: There were 27 emergency nurses with TSS (mean 40.67) and decreased work productivity (mean -14.74). There were 37 emergency nurses with TSS (mean 40.00) and increased work productivity (mean 18.95). Emergency nurses who received training for critical incident stress debriefing (CISD) were significantly more likely to report increased work productivity following trauma patient care compared to nurses with no training $(X(1)=5.247, p=.022)$. No significant differences were seen for the emergency nurse characteristics of TSS $(p=.816)$, age $(p=.072)$, race $(p=.550)$, gender $(p=.610)$, educational attainment $(p=.870)$, or shift worked $(p=.553)$. In 
addition, no differences were seen for the workplace characteristics of employer offered stress debriefing following trauma patient care $(p=.109)$ or patient population $(p=.677)$.

Implications: CISD training needs to be offered to all emergency nurses upon hiring. Employers may also consider providing annual training updates to protect the emergency nurses from concurrent TSS and decreased work productivity.

Conclusion: Work productivity change in emergency nurses experiencing TSS is largely unpredictable. Only CISD training was identified as a variable to explain the resilience seen for some emergency nurses experiencing TSS following trauma patient care. Further research is needed to determine why some emergency nurses are resilient for work productivity while experiencing TSS.

\title{
Work critical incidents and PTSD: The role of group cohesion
}

\author{
Goncalves, S., Neves, J., \& Morin, E. \\ CIS/ISCTE-IUL, Porugal
}

This research work has as central goals to determine the incidence of Post-Traumatic Stress Disorder (PTSD) and co-morbid disorders (depression and anxiety) in victims and witnesses of work critical incidents, and to understand if group cohesion moderate the relation between the evaluation of the degree of trauma associated with the work incident and the severity of symptoms of PTSD. To conduct this work we used an empirical methodology based in the quantitative method, through the application of questionnaire, in a Portuguese Police Institution. The sample was composed of 267 respondents. According to the results we believe that the study of psychological consequences associated to work incidents is worthy of attention from research and intervention perspective, since $14,3 \%$ and $15,2 \%$ of the victims and witnesses, respectively, exhibit a diagnosis consistent with PTSD. Moreover, $80 \%$ and $48 \%$ of the individuals with a clinical diagnosis of PTSD also show anxiety and depression diagnosis symptoms, respectively. The moderation results will be discusses in the oral presentation.

\section{The success-resource model of job stress}

\author{
${ }^{1}$ Grebner, S., ${ }^{2}$ Elfering, A., \& ${ }^{2}$ Semmer, N.K. \\ ${ }^{1}$ University of Applied Sciences Northwestern Switzerland, Switzerland; ${ }^{2}$ University of Bern, \\ Switzerland
}

Theoretical approaches to stress should include all factors which promote human functioning, well-being, and health. Achievements define human functioning. However, their role in the stress process is hardly understood. The success-resource model (Grebner, Elfering, \& Semmer, 2008, 2010) defines subjective success as positive and meaningful events that are related to work goals and behaviour and which are salient for the individual like reaching goals and milestones, outcomes of work processes (e.g., service or product), short-term consequences of work behaviour (e.g., positive feedback), and long-term outcomes (e.g., promotion). 
The model assumes that subjective success is a resource because it is valued in it's own right (e.g., Hobfoll, 2001), triggers positive emotions (e.g., Weiss \& Cropanzano, 1996), has positive effects on well-being (e.g., Locke \& Latham, 1990) and health (e.g., Carver \& Scheier, 1999), helps protecting and gaining other resources (e.g., Fredrickson, 2001), facilitates learning (e.g., Frese \& Zapf, 1994), ameliorates detrimental effects of job stressors, and has energizing (e.g., Locke \& Latham, 2002) and attention directing effects (Carver, 2003), which can promote recovery.

Based on qualitative (critical incidents) and quantitative evidence (PCA, CFA), the model defines 4 dimensions of subjective success: Goal attainment, pro-social success, positive feedback, and career success. The model posits reciprocal direct effects of success on well-being, health, and recovery, a buffer effect of success on the stressor-strain relationship, positive effects of resources like job control and problem-focused coping and negative effects of job stressors (e.g., role ambiguity, conflicts) on success.

Evidence for several aspects of the concept is presented. For instance, research among Swiss service employees and metal industry workers showed that success is positively related to situation- and person-related resources (e.g., job control, social support, self-efficacy, problemfocused coping) and different types of well-being including job satisfaction, work engagement, affective commitment, and exhaustion. Moreover, job stressors were found to impede subjective success. For instance, conflicts were negatively associated with subjective goal attainment. Moreover, subjective success mediated the coping-well-being relationship. Little evidence is available on the proposed buffer effect of subjective success on the stressor-strain-relationship and on beneficial effects of success on health including psychological and physiological recovery.

The success-resource model provides a useful framework for understanding the role of selfevaluated achievements in the job stress process. The value of the model for person- and organisation-targeted job stress interventions is outlined. Research requirements, possible costs of success and methodological concerns are debated.

\title{
Working conditions in a precarious work environment - A global study of aviation workers
}

\author{
${ }^{1}$ Greiner, B., ${ }^{2}$ Rosskam, E., ${ }^{3}$ McCarthy, V., ${ }^{4}$ Mateski, M., ${ }^{5}$ Zsoldos, L., ${ }^{6}$ Marowsky, I., \\ ${ }^{6}$ Rodriguez- Muñoz, G., ${ }^{6}$ Williamson, E., \& ${ }^{6}$ Tyler, J. \\ ${ }^{1}$ University of Cork, Ireland; ${ }^{2}$ Graduate Institute of International and Development Studies, \\ Geneva, Switzerland ; ${ }^{3}$ University of Cork, Ireland; ${ }^{4} \mathrm{MPP}$, Washington, DC, USA; ${ }^{5}$ Data \\ Analyst and Statistician, Budapest, Hungary; ${ }^{6}$ Civil Aviation Section, ITF
}

Objectives: Civil aviation is highly globalised industry that has been experiencing major restructuring due to increased competition, liberalisation policies and 9/11 worldwide. These changes presumably led to an increase of precarious work and changes in the temporal structure of jobs potentially associated with burnout. Although associations between job characteristics and burnout are well established, less is known about how the context of economic and social insecurity affects these associations.

Methods: Using a participative Action Research approach a questionnaire was developed together with union affiliates and sent to all International Transport Workers' Federation global 
affiliates in 116 countries. Key informants completed the questionnaires on behalf of the group (cabin crew, ground workers and air service workers) they represented, including questions on burnout, changes in precarious work since the year 2000 (e.g. outsourcing, contract work), temporal structure (e.g. fixed versus rotating shift work, working hours, rest breaks, turn around time between flights) demands, control and social support (JCQ). Participation rate was $67 \%$.

Results: Some regions showed a clear increase in insecure and unprotected working conditions since the year 2000 whereas other regions were not affected by these changes and working conditions even improved. The increases in precarious work was mostly noticeable in ground staff such as check-in workers and baggage handlers. An increase in precarious working conditions was also associated with a poorer temporal structure of the job such as longer working hours, less protected rest breaks and shorter turn-around time. Reported changes in working conditions work varied systematically whether a country had an expanding or downsizing airline industry. Stratified multiple regression models showed stronger associations between demands, control, social support, temporal aspects and precarious work environment as predictors and burnout as outcome in countries with a downsizing aviation industry (as compared to countries with an expanding industry).

Implications and conclusions: Although causality cannot be stated based on these crosssectional results, an environment with increasing non standard employment globally may give raise to unhealthy working conditions. As aviation industry expands in some but decreases in other regions leading to differential outcome for workers a global strategy is required to protect workers health that takes regional concerns into account.

\title{
Parallel Development of Social Stressors, Somatic Complaints and Self-Esteem: A Latent Growth Approach
}

\author{
${ }^{1}$ Gross, S., ${ }^{1}$ Semmer, N.K., ${ }^{1}$ Meiser, L.L., ${ }^{1}$ Kaelin, W., \& ${ }^{2}$ Tschan, F. \\ ${ }^{1}$ Departement of Psychology, University of Bern, Switzerland; ${ }^{2}$ Departement of Work and \\ Organisational Psychology, University of NeuchÃषtel, Switzerland
}

Objectives: Occupational stress research has rarely examined developmental processes, despite theoretical frameworks suggesting growth processes of stressors and well-being (cf., Sonnentag \& Frese, 2003). For the analysis of development processes standard statistical approaches (e.g., regression analysis) are not appropriate, as they rely on interindividual variations to obtain model estimates. The study of development processes needs to focus on intraindividual variation (e.g. latent growth models, LGM) to obtain valid results (Molenaar \& Campbell, 2009). Drawing from Mohrâ's (1991) theory of stress reactions and Sociometer theory (Leary \& Baumeister, 2000), two parallel growth processes were hypothesized: (a) Increases in somatic complaints and (b) decreases in self-esteem were proposed to develop in parallel to increases in social stressors at work.

Methods: Data from a longitudinal study (four waves: 1998, 1999, 2001, 2007) of young adults entering the workforce were analyzed. The sample consists of 675 employees ( $45 \%$ men). Social stressors were measured with regard to social animosities and conflicts at work (Frese \& Zapf, 1987), somatic complaints were measured with an adapted version of a scale by Mohr (1986) and self-esteem was measured with the Rosenberg Self-Esteem Scale (Rosenberg, 1965). 
Results: The data was analyzed in a structural equation framework of intraindividual change across measurements (LGM) with the program Mplus, Version 5.2 (Muth $\tilde{A}^{\odot} \mathrm{n}$ \& Muth $\tilde{A}^{\odot} \mathrm{n}$, 2007). To investigate the hypothesized parallel growth processes across the first nine years of professional life two bivariate LGMs were specified: The latent growth curve of social stressors was related in the first model to the latent growth curve of somatic complaints and in the second model to the latent growth curve of self-esteem. Results indicate that increases in social stressors were closely related to increases in somatic complaints $(r=.42, p<.01)$ and decreases in selfesteem $(r=-.39, p<.01)$ supporting our propositions.

Implications and Conclusions: These results suggest that social stressors and somatic complaints on the one hand, and social stressors and self-esteem on the other hand, demonstrate a close parallel growth process. Thus, social stressors at work constitute an essential stress factor that is related to the long-term development of one's physical and psychological well-being, which is in accordance with the stress reactions model of Mohr (1991), the Sociometer theory of selfesteem (Leary \& Baumeister, 2000) and the Stress-as-Offense-to-Self perspective (Semmer et al., 2007). Moreover, this study demonstrates the utility of bivariate LGMs for detecting parallel growth processes in stressors and strain variables and indicates that occupational stress research may gain new insights by focusing more attention to the issues of development.

\title{
Work-family conflict in the UK offshore oil and gas industry: A qualitative study
}

\author{
Guise, J. \& Cowan, S. \\ University of Abertay Dundee, Scotland
}

Objectives: The North Sea oil and gas industry plays a vital role in the European economy, in terms of the financial benefits deriving from the extraction of oil and gas reserves, and the substantial workforce employed by the operations that support the industry. However, the UK sector of this industry currently faces serious staff recruitment and retention problems, for reasons that have to date been unclear. The objectives of our study were, therefore, to explore the views and experiences of UK sector employees, in order to provide insights into how employers might address these problems.

Methods: The study took a qualitative approach to ensure that data collected were grounded in the experiences and views of employees themselves. Twenty-one, semi-structured focus groups were conducted with 86 employees representing a range of occupations, working on five offshore installations. The initial topic guide was based on issues that emerged from exploratory work with offshore employees using a modified nominal group technique, and key issues relating to occupational demand identified in the literature. Data were recorded verbatim, transcribed and analysed using framework analysis (Ritchie and Spencer, 1994).

Results: Several themes emerged from the data analysis. This paper focuses on those factors that relate to work-family conflict. Offshore work is physically isolated. The limited opportunity for communication with home while at work, and in particular face-to-face contact, was especially felt when there were home-related problems. In addition, offshore workers often missed significant social events, and their regular and lengthy absences from home had an impact on their role in the family. Participants also described the need for a period of re-adjustment to 
being at home, and then to leaving again for their next trip. Many of the problems related to family life can be exacerbated by the fact that partners may not understand the pressures of offshore working, and that workers are often disinclined to discuss them in home time.

Implications: The issues identified in relation to work-family conflict are of significant importance to employees and, in the main, could be relatively easily addressed at low cost by employers. The findings of the study also have potential implications for other areas of employment involving extended periods of absence from family.

Conclusions: In conclusion, recommendations are made for employers in relation to strategies that might reduce work-family conflict.

\title{
Work-life imbalance and effort-reward imbalance as predictors of burnout
}

\author{
Hämmig, O., Brauchli, R., Knecht, M., Bauer, G. \\ University of Zurich and ETH Zurich, Switzerland
}

Objectives: Burnout as a state of long-term exhaustion and diminished interest and a prolonged response to chronic stressors at work by now is well explored. In this context, it is well known that work stress can lead to burnout syndrome or at least to several burnout symptoms. It is also evident and has been found that negative spillover or role conflict between work and family indicating work-family or work-life imbalance and producing stress as well is positively associated with burnout. However, much less is known about the relationship between effortreward imbalance at work and burnout. Only very few studies have focused on that. And there is no single study published that is looking at differential and independent effects of both stressors work-life imbalance and effort-reward imbalance on burnout at the same time. The aim of this cross-sectional study, therefore, is to explore to what extent these stressors (work-life imbalance and effort-reward imbalance) are associated with burnout and which one of these two stress concepts is more strongly related to burnout than the other.

Methods: The study is based on survey data that were collected in 2007 within four large and well-known companies in Switzerland of different industrial sectors covering a sample of 6,091 employees in total across all hierarchical positions and all occupational fields. By using well established, validated, and for the purpose of our study adapted measures to assess concepts such as work-life imbalance, effort-reward imbalance and burnout we investigated our research questions.

Results: Preliminary results from first bi- and multivariate statistical analyses of the pooled data indicate that work-life imbalance, effort-reward imbalance and burnout are strongly associated (correlation coefficients) among each other. And independent of each other, the two stress concepts both show quite strong effects (regression coefficients) on burnout. Work-life imbalance or rather work-life conflict turned out to be more predictive for burnout than effortreward imbalance as the more commonly used work-related stressor.

Implications and conclusion: Both work-life imbalance and effort-reward imbalance seem to play an important and complementary role for burnout and that independent of one another. 
Thus, companies should take this into account when planning intervention strategies in order to meliorate conditions at work and especially to prevent burnout.

\title{
Does work engagement mediate effects of social and organisational work factors on mental health problems
}

\author{
${ }^{1}$ Hagen, S., ${ }^{2}$ Grimsmo, A., ${ }^{3}$ Geving, G., ${ }^{4}$ Gudbergsson, S., \& ${ }^{1}$ Torp, S. \\ ${ }^{1}$ Vestfold University College, Norway; ${ }^{2}$ Work Research Institute, Norway; ${ }^{3}$ Hjelp24 \\ Occupational Health Service, Norway; ${ }^{4}$ University of Oslo, Norway
}

Objective: The objective of this study was two-fold: Firstly, to investigate whether demands and resources affect a negative mental health measure differently than a positive measure such as work engagement; Secondly, to investigate if work engagement mediates effects of work-related demands and resources on mental health problems. Methods: A cross-sectional questionnaire study was performed among a general population in Norway $(n=700)$. The Demands-Control-Support Questionnaire (DCSQ), the Utrecht Work Engagement Scale (UWES) and the depression part of the Hospital Anxiety and Depression Scale (HADS) were used to measure the different independent and dependent variables.

Results: Bivariately, workers with high personal control and social support reported significantly less mental health problems and were more engaged at work compared with workers low in control and social support. High demands correlated positively with mental health problems while there was no significant bivariate correlation between demands and engagement. All these significant bivariate correlations were retained in multivariate regression analyses including the three work factors. In addition, the non-significant negative correlation between demands and engagement was changed to a positive relationship. When engagement was included in the multivariate analysis as an independent variable together with demands, control and social support, the positive correlation between demands and depression was retained, while the significant correlations between depressive symptoms and control and support were reduced to non-significance. This indicates that engagement mediates the effects of control and social support on depressive symptoms, but does not mediate the effect of demands.

Conclusion and implications: These results are in line with the demands-control-support model (Karasek \& Theorell 1990) and also the demands-resources model (Bakker \& Demerouti 2007). As engagement seems to mediate the effects of work environment on mental health problems, it may be worthwhile to encourage enterprises to improve engagement instead of focusing on preventing mental health problems as the main aim of the enterprise's health promoting activities. A focus on engagement may have more positive organisational effects as compared to the more traditional negative preventive focus, since engagement is contagious and closely related to, for instance, work performance and motivation. 


\title{
Emotion work - antecedent or consequence of burnout and work engagement?
}

Hakanen, J.

\author{
Finnish Institute of Occupational Health, Finland
}

Objectives: The aim of this longitudinal study was to investigate causal and reversed crosslagged relationships between emotional demands (five dimensions of emotion work), emotional dissonance, burnout, and work engagement in a large sample of Finnish dentists.

Method: I used a full panel design on a representative sample of Finnish dentists. The study was based on two-wave three-year follow-up data. At T1, the response rate was $71 \%$ of the whole profession in Finland, and at T2 three years later, the response rate was $84 \%(\mathrm{~N}=2$ 555). The Finnish version of the Frankfurt Emotion Work Scales (Zapf et al., 1999) was used to measure six dimensions of emotion work: positive emotions, negative emotions, neutral emotions, emotional sympathy, sensitivity requirements, and emotional dissonance.

Results: The best-fitting model indicated reciprocal relationships between emotion work and employee well-being over time. More specifically, emotional demands had a cross-lagged impact on emotional dissonance, which in turn predicted both future burnout and (decreased) work engagement. Interestingly, emotional demands and engagement positively influenced each other over time, and in addition, burnout had positive cross-lagged effects on both emotional demands and emotional dissonance. Investigating the relationships between the dimensions of emotional demands, burnout, and engagement more closely revealed that burnout impacted on the display of both positive and neutral emotions, whereas engagement predicted a display of neutral emotions.

Implications: Emotion work is a multi-dimensional salient work characteristic for employees carrying out client work. Theoretically, emotion work, i.e., the emotional regulation required of employees to display certain emotions, stems from the requirements of the organisation (or e.g. professional ethics). This study showed that the relationships between emotion work, burnout, and work engagement may be more complex than theoretically expected. More longitudinal studies on emotion work in different service sectors are needed. In practice, employees carrying out client work could benefit from emotion work training, thus becoming more aware and capable of meeting the specific requirements of their jobs.

Conclusions: This was among the first longitudinal studies on emotion work and employee well-being. The findings showed that emotion work may have both negative and positive effects on employee well-being. In addition, burnout and engagement may also influence emotion work. For example, high levels of burnout may further affect emotional dissonance, but also lead to increased efforts to regulate emotions in client work, and to the display of both positive and neutral emotions. 


\title{
Does higher professional education increase contingent self-esteem?
}

\author{
Hallsten, L., Rudman, A., \& Gustavsson, P. \\ Karolinska Institutet, Sweden
}

Contingent self-esteem is a vulnerable orientation that entails self-validation strivings and long-term costs such as depression, burnout and long-term sickness absence. An extensively used instrument in Sweden for assessing global contingent self-esteem is a scale for performance-based self-esteem (PBSE) that has shown higher PBSE scores for young and well-educated individuals. Whilst showing high rank-order stability, contingent self-esteem has been assumed to rise during socialization processes. Hitherto, however, no studies have been carried out in relation to natural social contexts for young adults such as higher education that exerts considerable socialization pressures.

Objectives: To examine the mean-level changes of PBSE for a national cohort of nursing students during their professional education from the first to the second and third years of education. Another objective was to compare the mean-level PBSE change for the students between the first two years with a corresponding PBSE change from a matched group of work employees.

Methods: PBSE data were obtained annually from a national cohort study of 1,220 Swedish nursing students during their 3-year nursing education programme. In addition, two-wave PBSE data one year apart were collected from a matched group of 115 work employees, derived from a nationally representative sample. Data were analyzed by repeated-measures analysis of variance and by paired samples t-tests.

Results: The mean-level of PBSE increased from the first to the second and third year, and the increase was greater for younger than for older students. Furthermore, the mean-level increase over one year was greater for the nursing students than for the matched group of work employees.

Conclusions: Participation in higher education increased global contingent self-esteem for the nursing student group, thereby lending support for the socialization assumption and indicating that global contingent self-esteem is a developmental construct. Considering the negative consequences of contingent self-esteem and self-validation strivings to ill-health, the present outcomes make a case for giving special attention to unintended socialization effects of higher education. The succeeding occupational years may become particularly demanding and strenuous for individuals experiencing elevated contingent self-esteem.

\section{Wellbeing at work: informing and empowering employees}

\author{
Hamilton, J. \\ Leeds Metropolitan University, United Kingdom
}

Objectives: Much of the research and practitioner focus on establishing healthy and safe working environments has been on the role of the line managers, particularly in the identification and control of workplace hazards. However employees themselves have an important role in their own health and wellbeing through the way they carry out their work and how they live their life away 
from work. This project aimed to provide an online wellbeing resource for Leeds Metropolitan University's staff and students that was (i) user friendly, (ii) accessible, (iii) provided a broad range of wellbeing related advice, (iv) aligned to the university's occupational health and student support measures, and (v) the basis for the development of a universal tool for the public sector.

Methods: The project combined the University's academic wellbeing-related expertise across a range of subjects including relaxation (Thew, 2008), work-life balance (McKenna, 2008), diet and exercise (Sutton, 2009); practitioner guidance on subjects such as stress (HSE, 2009) and posture (Bell, 2007); guidance on study, revision, and examinations; work-related subjects such as using email, report writing and managing meetings; and life issues such as money worries and coping with loss. The user interface, designed by the University's own web and multimedia team, avoids presenting large quantities of information that users don't have time to read. Whilst there are 65 wellbeing-related topics consisting of 25,000 words, no more than five pieces of information are presented at any one time. Guidance is accessed in one of three main ways; firstly based on the user's current feelings or what is on their mind at the time. Secondly task-based advice is built around every-day work activities, with related health advice offered alongside hints and tips. Finally, the site home page offers some time-based guidance which is updated throughout the day. The website can be viewed at www.leedsmet.ac.uk/wellbeing

Results: The website has received significant positive feedback from users and has resulted in a significant increase in usage when compared with its predecessor. The website has received external recognition, and a number of other public sector organisations are working with the University to develop a similar resource for their employees.

Conclusions: This project has shown the benefit of an organisation taking a broad view of the wellbeing of its employees, and pooling its various resources to provide an accessible, informative source of guidance and support for its employees.

\title{
Work-related stress: guiding employers on the law and good practice
}

\author{
Hamilton, J. \\ Leeds Metropolitan University, United Kingdom
}

Objectives: Work-related stress is a major cause of absence for British employers yet their legal obligations for managing it are complex and multi-faceted, supported by guidance that is fragmented. Reforming both the statutory and common law is a complex and lengthy process, as a result this study aimed to produced a guide to the present law for employers that would be (i) simple and accessible, (ii) a concise summary of all aspects of the law, (iii) referenced to good management practice and other research, (iv) developed, owned and promoted by national stakeholders.

Methods: The study resulted from previous research on the current British legal framework regarding work-related stress (Hamilton, 2008). In addition to developing a model for reform, it identified a number of barriers to employers understanding of their duties to their employees; including the lack of a single concise form of the law, and the nature of the ownership and promotion of policy at a national level. In recognition of this, and pending legal reform, the 
study has developed an employer's guide to the law that embraces all elements of the current statutory and common law obligations in this area. The legal principles were mapped on to four simple steps for employers derived from the reform model; identifying a problem, preventing harm, protecting individuals, and managing the workplace. The guide also incorporates related good practice (Acas, 2006) and management competencies (CIPD, 2009). A synopsis and initial draft were completed in conjunction with the UK's Health and Safety Executive. Development of the guide was then undertaken in coordination with the other main stakeholders; (i) government agencies, (ii) employer representative groups, (iii) academics, (iv) legal profession, (v) health, safety and HR professionals, and (vi) trade union representatives. The published version of guide will be co-branded and co-promoted by these stakeholder organisations in Spring 2010.

Results: The study has produced an employer's guide to the law regarding work-related stress that provides a single, concise authoritative source, developed and promoted in a collaborative way.

Conclusions: The production of an employers guide will provides employers with simple concise information on their statutory and common law duties to employees regarding work-related stress. Presenting these duties alongside suggested good practice should ensure employers are better informed on how to prevent stress-related ill-health amongst their employees.

\title{
Organisational justice, stress and commitment: The mediating role of perceived organisational support and work-family conflict
}

\author{
Hansez, I. \& Babic, A. \\ University of Liège, Belgium
}

Objectives: Judge and Colquitt (2004) examined the relationship between organisational justice (OJ) and stress and the mediating role of work-family conflict (WFC). The presence of justice seemed to allow participants to better manage the interface of their work and family lives, which was associated with lower stress. The present study proposes to extend this idea to additional outcomes: intent to quit, job satisfaction, involvement and commitment. Second, the mediating role of perceived organisational support (POS) is examined in addition to that of WFC, assuming a double mediation effect between OJ and stress and commitment outcomes.

Methods: A questionnaire was used to assess distributive, procedural (active and passive) and informational justice, POS, WFC, job strain and job engagement, work commitment, job satisfaction, job involvement and intent to quit. Structural equation modelling was performed using Lisrel 8.80 on 535 data collected amongst health care personnel in a Belgian hospital. The basic model (model 1) includes that the four dimensions of OJ have a direct impact on POS, which in turn impacts WFC. Work-family conflict impacts on job strain, job satisfaction, intent to leave and involvement. A second model (model 2) was tested with multiple-foci commitment as outcomes.

Results: The two models fit adequately the data $(\mathrm{X} 2=1808.15, \mathrm{df}=386, \mathrm{RMSEA}=.04$ and $\mathrm{X} 2=2276.87, \mathrm{df}=573, \mathrm{RMSEA}=.04$ respectively). In both models, only distributive and informational justice are significant in explaining POS $(\gamma=.24, t=3.05$ and $\gamma=.34, t=4.59$ 
respectively). The coefficient path from distributive justice to WFC is also significant $(\gamma=-.30$, $t=-3.9$ ), giving a status of partial mediator to POS. The coefficient path from informational justice to WFC is not significant $(\gamma=-.09, \mathrm{t}=-1.04)$, giving a status of total mediator to POS. The path coefficient from active procedural justice to WFC is significant $(\gamma=.15, t=2.46)$. POS is significant in explaining WFC $(\gamma=-.24, \mathrm{t}=-3.46)$. Specifically in model 1, WFC has a positive impact on job strain $(\gamma=.79, t=17.05)$, involvement $(\gamma=.13, t=2.44)$ and intent to quit $(\gamma=.51, t=11.5)$ and a negative impact on satisfaction $(\gamma=-.63, \mathrm{t}=-14.28)$. In model 2 , WFC has a negative impact on affective $(\gamma=-.22, \mathrm{t}=-4.08)$ and normative $(\gamma=-.20, \mathrm{t}=-3.61)$ commitment to the organisation, affective commitment to the occupation $(\gamma=-.16, \mathrm{t}=-2.8)$ and affective commitment to the work group $(\gamma=-.17, \mathrm{t}=-3.04)$.

Implications: WFCs have a negative impact for workers but also for companies. Our results support that POS and OJ decrease work-family conflict. This study suggests developing workfamily policies which are clearly and formally established, communicated and applied fairly.

\title{
Mainstreaming gender in interventions for work-related stress and psychosocial issue: European expert's perceptions and practices
}

\author{
Hassard, J., Leka, S. \& Cox, T. \\ University of Nottingham, United Kingdom
}

Strong gender segregation within the labour market can be observed; men and women tend to work in very different jobs and in different occupational sectors, resulting in differential exposure to workplace hazards and impacts on occupational health and wellbeing (Messing et al., 2003). The European Commission (EC; 2002) in the 'Community Strategy on Health and Safety at Work 2002-2006' included the integration of gender (i.e. gender mainstreaming) into occupational health and safety activities as a key objective and priority for future action. The European Agency of Health and Safety (2002) further emphasis the need to mainstream gender into risk assessment for occupational health and safety; reinforcing this issue as a key priority for future action. Currently, within the context of the EU, gender issues and differences have been ignored in policy, strategies, and actions (European Agency, 2002).

Objective. The objective of the current research endeavour is threefold: namely, to examine the degree and extent to which gender is addressed in strategies to manage and prevent psychosocial issues and work-related stress; yield an assessment of intervention expert's perceptions surrounding issues of gender and their ascribed importance; and the identification of key gender differences needing to be addressed in psychosocial risk management as indicated by experts.

Method. A systematic review of best-practice interventions (including primary, secondary and tertiary) from across Europe was conducted. Authors of identified interventions were recruited via email to participate in a semi-structured interview. Experts identified had a concentrated expertise and knowledge in the design, implementation and evulation of interventions. Experts were asked "Has the intervention [you have designed, implemented, and/or evaluated] been useful in addressing gender issues? If so how?", "Do you think that gender differences are important to address in work-related stress interventions? If so, how?", and "What gender issues do you think are important to address in work-related stress interventions?". Telephone interviews were conducted, recorded, and subsequently transcribed. 
Results. A total of 32 interviews were conducted ( $47 \%$ female; $n-10$ primary -, $n=12$ secondary -, and $10=$ tertiary -level intervention experts) from the 15 European countries: U.K., Germany, Netherlands, Denmark, Norway, Sweden, Poland, Italy, Portugal, International, Finland, Ireland, Switzerland, Spain, and Belgium. Thematic analysis was used to analyze the collected data. The findings will be presented.

\title{
Exclusion and lack of power at the core of unemployment self-esteem
}

\author{
${ }^{1}$ Herman, G., 'Bourguignon, D., 'Desmette, D., ${ }^{1}$ Guinote, A., \& ${ }^{2}$ Larue, G. \\ ${ }^{1}$ Université catholique de Louvain, Belgium; ${ }^{2}$ University of Liège, Belgium
}

The effect of unemployment on mental health has been under scrutiny since many decades. Recent meta-analyses (McKee-Ryan, Song, Wanberg, \& Kinicki, 2005; Paul \& Moser, 2009) have shown that the negative effect of unemployment on mental health is a rather broad one: it can be detected on a large range of mental health indicators (mixed symptoms of distress, depression, anxiety, psychosomatic symptoms, subjective well-being, and self-esteem). Furthermore this effect has been stable throughout the last 30 years: in other words, societies do not "habituate" to high unemployment rates.

How to explain the deleterious effect of unemployment? At the core of a jobless experience lays processes such as lack of power and perceived exclusion. As exclusion has been often applied to unemployment context (Castel, 2009), lack of power which could be related to exclusion is less documented. Yet we know that both these variables negatively impact on selfesteem (Wojciszke \& Struzynska-Kujalowicz, 2007; Leary, Cottrell, \& Phillips, 2001). They also negatively affect projection into the future: lack of power thwarts goal setting (Guinote, 2007) and exclusion impedes future time orientation ((Twenge, Catanese \& Baumeister, 2003).

This presentation aims to clarify the relations between exclusion, power, time orientation and self-esteem, and particularly to propose a general model that predicts the latest variable. 197 jobless people attending a public work integration agency have answered a questionnaire. Data have been analysed through structural equation modeling (LISREL). Partly according to our predictions, power and time orientation mediate the relationship between exclusion and self-esteem. The results are discussed in line with a general model about power and raise some implications on the wellbeing of unemployed people and work integration.

\section{Daily Inspirational Leadership and its Relation to Daily Engagement, Active Learning, and Performance}

\author{
Hetland, H., Bakker, A., Hetland, J., \& Demerouti, E. \\ Bergen University, Norway
}

Introduction: The aim of this study is to investigate how daily inspirational leadership (e.g., intellectual stimulation and inspiration) is related to daily employee engagement and daily employee active learning. Our central hypothesis is that the more employees perceive their 
leader as being inspirational, the more they will be engaged in their work on a daily basis. In addition, we predict that daily employee engagement, in turn, is positively linked to daily active learning and performance. On the basis of theory, we further hypothesize that employees' goal-orientation (learning goal orientation vs. performance goal orientation) and psychological reactance (the tendency to react negatively toward restrictions of one's freedom to choose freely among possible behaviours), measured on the person level, will moderate the relationship between work engagement on the one hand and active learning and performance on the other hand.

Methods: In total, 50 employees participated in this study. They initially filled out a background survey, and then completed a daily questionnaire during five consecutive days. Daily Inspirational leadership was based on the MLQ (Bass and Avolio, 1995); Daily Work Engagement (vigor, dedication and absorption) was measured with the state version of the Utrecht Work Engagement Scale (UWES; Bakker, 2008); Active learning was assessed with the 7-item scale developed by Bakker and Demerouti (2009); Performance was measured with the state version of Goodman and Svyantek's (1999) performance scale; and Psychological Reactance was measured by the a recently developed 4-item scale assessing psychological reactance (Wiium, Aarø, Hetland, 2008).

Results: The data will be analyzed using multilevel analyses.

Conclusion: The findings can shed light on how intra-individual variability in employees' experience of leadership and inter-individual differences in goal orientation and psychological reactance are related to daily engagement, active learning, and performance. Moreover, findings can be used for leadership and employee training interventions aimed at enhancing leadership and employee effectiveness and motivation.

\title{
Fulfilment of Psychological Needs as a Mediator in the Relationship between Transformational Leadership and Job Satisfaction
}

\author{
${ }^{1}$ Hetland, J., ${ }^{1}$ Hetland, H., ${ }^{2}$ Bakker, A.B., ${ }^{3}$ Demerouti, E., ${ }^{4}$ Andreassen, C.S., \& ${ }^{1}$ Pallesen, S. \\ ${ }^{1}$ University of Bergen, Norway; ${ }^{2}$ Erasmus University Rotterdam, the Netherlands \\ ${ }^{3}$ Utrecht University, the Netherlands; ${ }^{4}$ The Bergen Clinics Foundation, Norway
}

Objective: The link between transformational leadership and job satisfaction is well established (Alvolio, Bass et al., 2004), and it has been suggested that transformational leaders enhance followers' effectiveness, confidence and motivation by meeting followers' needs (Bass, 1998). The aim of the present study is to examine this potential mediating role of psychological needs fulfilment in the relationship between transformational leadership and job satisfaction.

Methods: The sample includes 661employees working in several occupations: a major pharmaceutical company $(n=127)$, a regional health sector company $(n=96)$, a national TV station ( $n=172)$, two different HR-sector consultancy companies $(n=80)$, and two university faculties $(n=186)$. In the total sample, 301 respondents $(46 \%)$ were female, and the mean age was 42.6 years. In total 1300 employees were invited to participate yielding a response rate of $51 \%$. Transformational leadership was measured by The Multifactor Leadership Questionnaire (MLQform 5X). Fulfilment of basic needs (autonomy, relatedness, and competence) was measured 
by the Basic Needs Satisfaction at Work (BNSW) scale (Deci et. al., 2001), and job satisfaction was measured by 5 items from the original Brayfield and Rothe's index for job satisfaction suggested in Judge et al. (2001). Structural equation modeling analysis was performed using AMOS 16.0.1.

Results: Preliminary confirmatory analysis revealed that the three sub dimensions of the basic need scale, autonomy, relatedness, and competence, were highly inter-correlated (.83 to .94). Consequently, the composite scores of the three psychological needs were used as indicators of a general "need satisfaction" factor in the subsequent analysis. In the structural model total, direct, and indirect effects (through fulfilment of psychological needs) from transformational leadership to job satisfaction were estimated. Initially a substantial direct effect $(.46, p<.001)$ between transformational leadership and job satisfaction was found. In line with the mediation hypothesis, this effect was no longer significant (.04, n.s.) when the indirect path (.43) was included in the model. The final model without the direct path showed an acceptable fit to the data (chi-square $=229.12, \mathrm{df}=63, \mathrm{CFI}=.97, \mathrm{TLI}=.96$ ). The significance of the indirect effect was formally tested by performing a Sobel test ( $z$-value $=9.28, p<.001$ ).

Conclusion: The results from the present study support the assumption that fulfilment of psychological needs has an important mediating role in the relationship between transformational leadership and job satisfaction. Implications for research and practice will be discussed.

\title{
Perceived Risk and Employees' and Supervisors' Participation on Work Safety
}

\author{
Hinrichs, S. \\ University of Freiburg, Germany
}

Departments of work safety traditionally prefer technical remedies and establish rules for safety problems in the workplace. The increasing percentage rate of so called "behaviour based" accidents opens the door for psychological interventions. Behaviour in the workplace is learned, and this applies for safe as well as for unsafe behaviour. As accidents are seldom events, it is very likely that unsafe behaviour by means of "negative reinforcement" is being learned (i.e. the expected aversive stimulus does not occur). This leads to most accidents happening in connection with activities which the workers consider to be unrealistically safe-they underestimate the danger of their own activities. The detection of these misjudgements in risk perceptions is the first step in participating employees and supervisors working on safety and health.

Methods: The first step in each company (three steel companies in Germany) was to analyse the accident data by activities for a sufficient period of time. The second step was to assess the employees' subjective accident frequency for those activities.

Results: The comparison of objective accident data and subjective estimation of frequency resulted in the operational definition of "overestimated", "realistically estimated" and "underestimated" activities. Our studies show that about $15 \%$ of the single activities at a workplace are underestimated, $44 \%$ of all accidents happening with these few underestimated activities. 
Intervention: The results were reported in subsequent workshops for supervisors. The psychological background, implications of unrecognised near-accidents for the process of learning unsafe behaviour and the necessity of safety circles were extensively discussed. Additional training enabled the supervisors to initiate safety work in small groups.

Evaluation: The Evaluation shows high activity in the small groups. After the intervention the underestimated activities were estimated more realistically and the accident rate decreased.

Discussion: Continuous work on safety has to be well organised to reach sustainable effects. This has to be done by means of a high degree of employee involvement. The starting point on this way is the correction of underestimated activities. Workers and supervisors have to work on these activities with the aim of uncovering unrecognised nearaccidents. Successful efforts to correct individual risk perception result in a decrease of accidents. The described method was successfully transferred to health and quality. The implication for practice and further research will be discussed.

\title{
Depressive symptoms and school burnout at middle and late adolescence
}

\author{
${ }^{1}$ Holopainen, L., ${ }^{2}$ Salmela-Aro, K., \& ${ }^{1}$ Savolainen, H. \\ ${ }^{1}$ University of Joensuu, Finland; ${ }^{2}$ University of of Jyväskylä, Finland
}

Objectives: The main purpose of this study was to examine the extent to which middle and late adolescents' depressive symptoms predict their later school burnout and, in turn, the extent to which school burnout predicts depressive symptoms. The aim was also to examine the extent to which cross-lagged paths between school burnout and depressive symptoms vary by school track. We hypothesized that both school burnout and depressive symptoms would show some stability during middle and late adolescence. On the basis of the literature on burnout in the work context we assumed that school burnout would more strongly predict depressive symptoms later on than vice versa and that to some extent school burnout would predict depressive symptoms, and, in turn, depressive symptoms would influence school burnout. We assumed that crosslagged effects between school burnout and depressive symptoms would be found particularly among adolescents who study in upper secondary education.

Methods: This study is part of the Staying in Track of Learning research (Holopainen \& Savolainen, 2005). In this study one age-cohort $(N=517)$ of young people at comprehensive school from a middle-sized town in eastern Finland (population about 50 000) are being followed through post-comprehensive education. More than half of the students (58\%) were studying at upper secondary high schools (academic track) and the rest students at vocational schools (vocational track). The median age of the participants during the first measurement was $16.45(\mathrm{M}=16.51$; $\mathrm{SD}=.37$ ). Drawing on data gathered in three-wave longitudinal study, cross-lagged paths between school burnout and depressive symptoms were investigated. School burnout and depressive symptoms were measured three times annually (Time 1: $N=474$, Time 2: $N=412$, Time 3: $\mathrm{N}=414$ ) during students' three years of secondary education.

Results: Results revealed moderate stability for both school burnout and depressive symptoms. They also showed that school burnout more strongly predicted subsequent depressive 
symptoms later on than vice versa. Lastly, they revealed cumulative cycles between school burnout and depressive symptoms. The cross-lagged effects between school burnout and depressive symptoms were somewhat stronger among the vocational school students, although the same effect was also found among the upper secondary high school students.

Implications and Conclusions: Our findings increase understanding the well-being of adolescent's development in different school contexts. School burnout both during middle and late adolescence should be taken seriously as it can lead to depressive symptoms later on.

\title{
Associations between Overtime and Psychological Well-Being among Chinese Workers
}

\author{
Houdmont, J., Hassard, J., \& Zhou, J. \\ University of Nottingham, United Kingdom
}

Objectives: The relationship between overtime and psychological well-being has been studied exclusively in the North-West European individualist cultural context through the lens of EffortRecovery theory. No studies have been conducted to assess whether extant theory and evidence can be generalized to the collectivist cultural setting and used therein to inform organisational human resource and occupational health policy and practice. This study aimed to address this shortcoming by investigating the overtime/well-being relationship, and the moderating influence of four reasons for working overtime, in China.

Methods: Data were collected for 130 employees (65\% response rate) who worked in two Chinese branches of an international information technology company. All participants were full time employees contracted to work a minimum of 40 hours per week and had been in the company's employ for at least 12 months. Self-reported data were collected via a questionnaire on overtime hours worked, psychological well-being, job demands, intrinsic motivation, anticipated rewards, and overtime work culture.

Results: All participants had worked overtime in the previous six month period $(M=14.2$ hours per week; SD = 7.8). Of these, $23 \%(n=30)$ were classified as working an average of $\leq 7$ hours overtime per week, 46\% $(n=60) 8-19$ hours, and 31\% $(n=40) \geq 20$ hours per week. There was a significant positive correlation between overtime hours and (impaired) well-being $(r=0.22, p<0.05)$. ANOVA analysis revealed a significant difference between each of the three overtime-hours groups in terms of their well-being. Intrinsic motivation, job demands, and overtime work culture were found to be significant moderators of the overtime/psychological well-being relationship.

Conclusions: The findings of this exploratory study suggest that extant theory and evidence on the overtime/well-being relationship can be generalised with caution to the Chinese work context. The findings may usefully inform the organisation of work for the improvement of occupational health, and may facilitate the implementation, integration, and adaptation of multinational operations in collectivist cultures. 


\title{
Interaction Effects of Self-Control Demands and Cognitive Control Deficits on Burnout, Depression, Physical Strain and Daydisfunction
}

\author{
Hupke, M. \& Schmidt, K.H. \\ Leibniz Research Centre for Working Environment and Human Factors, Germany
}

Objectives: The concept of self-control has recently gained increasing attention in occupational health research as today's work is more and more requiring employees to flexibly self-control their behaviour on the job. This means that people have to consciously regulate and control themselves by showing only organisationally desired emotional reactions, overcoming inner resistance or resisting distractions. Self-control demands have been found to be related to job strain and further research indicated that this relationship is moderated by individual cognitive control deficits. The aim of this study was to further investigate this interaction effect and provide wider evidence by placing it into a larger methodical framework.

Methods: 257 elderly care nurses, hospital nurses and nurses caring for disabled people in 16 different small organisations completed a questionnaire during normal working hours, that contained scales measuring self-control demands, cognitive control deficits and five job strain dimensions (burnout dimensions: emotional exhaustion and depersonalisation, depression, physical strain, daydisfunction) that were expected to be closely related to self-control demands. 107 of these nurses also participated in a second part of the study three to six months later. This part contained computer-based cognitive control tasks on response inhibition (Stroop) and working memory (N-Back).

Results: Cognitive control deficits and self-control demands showed significant interaction effects on the burnout dimensions emotional exhaustion and depersonalisation as well as on depression, physical strain and daydisfunction. Multiple regression results indicated a buffering effect of a low level of cognitive control deficits on the effects of self-control demands. Participants showing high emotional exhaustion performed weaker in the cognitive control task then did those with low emotional exhaustion.

Implications: Participants with a high level of cognitive control deficits show significantly higher job strain when self-control demands are high compared to people with a low level of cognitive control deficits. These findings implicate that cognitive control deficits increase the risk to suffer from burnout or other forms of job strain in the world of work today. While organisations should be aware of the self-control demands they expect their employees to cope with, training programs might also be developed to strengthen the individual self-control resource. Conclusions: Interaction effects of cognitive control-deficits and self-control demands on burnout, depression, physical strain and daydisfunction could be replicated and further evidence was found by using multiple measuring methods. 


\title{
Effects of Psychological Hardiness on Medically Certified Sickness Absence
}

\author{
${ }^{1}$ Hystad, S.W., ${ }^{1}$ Eid, J., \& ${ }^{2}$ Brevik, J.I. \\ ${ }^{1}$ University of Bergen, Norway; ${ }^{2}$ Norwegian Armed Forces Medical Services, Norway
}

Objectives: Considerable research has demonstrated that psychological hardiness protects against stress and predicts healthy functioning. The aim of the present study was to prospectively explore the relationship between hardiness and medically certified sickness absence.

Methods: Psychological hardiness was measured in a sample of employees from the Norwegian Armed Forces $(\mathrm{N}=7254)$ in 2007. Total number of sickness absence spells was subsequently retrieved for the period January 1st to December 31st 2008. To predict the effect of hardiness on absence spells, we used negative binomial and hurdle regression models that take into consideration the distributional nature of sickness absence data (typically positively skewed and with excess zeroes). All regressions were computed using the $\mathrm{R}$ system for statistical computing.

Results: After controlling for age and absence at baseline, hardiness significantly predicted the likelihood of having at least one absence spell, as well as the number of subsequent spells.

Conclusions: The present study extends previous research on hardiness and health by demonstrating a prospective link between hardiness and an objective measure of health outcome (medically certified sickness).

Implications: Employee absenteeism can have dire individual, organisational, and societal consequences. An explicit focus on developing and fostering the individual resource factor of hardiness could thus yield huge benefits, both in terms of individual health and in terms of economical savings related to reduced absenteeism.

\section{Managers' Health - The Case of Person Related Burnout}

\author{
Hytter, A. \\ Linnaeus University, Sweden
}

Objectives: Work related health is receiving a lot of attention in terms of the number of scientific studies being carried out. The vast majority of those studies however look at employee health. Surprisingly few studies report on manager's health. The objective of this study is to explain symptoms of person related burnout among managers.

Methods: A cross sectional study was carried out within four Swedish public organisations. One hundred and thirty eight managers, equal to a response rate of eighty five percent, responded to one survey on work environment and health and to one survey on managerial behaviour. Person related burnout symptoms were measured with items from the COPSOQ.

Results: Regression analysis revealed that forty one percent of the variance of self reported person related burnout symptoms among managers was explained by "being tired and listless 
within the last three months" together with "having job related sleep problems within the last three months".

A SEM analysis showed that work family balance was the factor impacting managers' symptoms of person related burnout the most. The influence from WFB was direct as well as mediated through "being tired and listless within the last three months", and "having job related sleep problems within the last three months". Furthermore influence from WFB was mediated through "coping strategies". "Coping strategies" also had a mediating role for work demands, while "work satisfaction" was mediated through "having job related sleep problems within the last three months".

The model also contained aspects of the own managers' behaviour. The own managers' "power orientation" was mediated through "having job related sleep problems within the last three months" while the own managers' "charisma" had a small but direct influence on the person related burnout symptoms.

Conclusions: Findings showed that person related burnout symptoms among managers is a complex construct, and that sources of burnout can be found within the psycho social dimensions of work, in the interface between work and family life, as well as within the own managers' behaviour.

Implications: One important implication of the study is a need to understand what influences managers' health more in depth. Furthermore that this is done from multitude of perspectives.

\title{
Goal Pursuit Put into Context: Psychosocial Work Environment Associates with Personal Work Goals in a 2-year Follow-up Study
}

\author{
${ }^{1}$ Hyvonen, K., ${ }^{1}$ Feldt, T., ${ }^{2}$ Kinnunen, U., \& ${ }^{1}$ Tolyanen, A. \\ ${ }^{1}$ University of Jyväskylä, Finland; ${ }^{2}$ University of Tampere, Finland
}

The relation of the components of the Effort-Reward Imbalance model (ERI; Siegrist, 1996) to goal pursuit was investigated over a 2-year period. Goal pursuit was studied through categories of goal contents - competency, progression, well-being, job change, job security, organisation, and finance - based on the personal work goals of managers (Hyvönen, Feldt, SalmelaAro, Kinnunen, \& Mäkikangas, 2009). The associations of the levels and changes in the ERI components (effort, reward) with the stability of goal contents were investigated through data collected in two measurement points (2006 and 2008).

The participants consisted of 433 young Finnish managers (age range 24-35 years in 2006). Repeated measures MANCOVAs (significant background variables controlled for) showed that the levels and/or the changes in the level of rewards were associated with the stability of goals related to competency, progression, job change, job security, organisation, and finance. In terms of effort, only changes in the level of effort were associated with the stability of job change goals. Taken together, this study contributes to the understanding of the relationship between psychosocial work environment and work goal pursuit indicating that rewards - and changes in rewards - play an important role in the work orientation of young managers. 


\title{
Effect of Self Managing Life Crisis Programme based on the Oriental Approach towards Midlife Crisis and Well-being of Thai Married Women in Bangkok
}

\author{
Intarakamhang, U. \& Mohan, K.P. \\ Srinakharinwirot University, Thailand
}

Women between 35-55 years old play several roles in the Thai society. They continue to be principally responsible for childcare and housework. In addition, women working outside the homes tend to have the same responsibilities as men in their work contexts. Moreover, the degenerative changes in the body, and declined sex hormone can cause both internal and external stress. This group is therefore at risk to become stressed, which is considered as a life crisis. The researchers were therefore interested in training the women in order to build up self protection to cope with the problems. The life crisis management according to the doctrine of Buddhism means adapting through the principles of meditation and body exercise of Taoism. The midlife women would be able to apply this knowledge and skill to their daily life, to reduce perception of life crisis and enhance their own well-being, and become good quality assets of Thai society.

The main objectives were: 1) to study the efficiency of Managing Life Crisis Program (MLCP) based on the oriental approach towards the self-perception of life crisis among Thai married women, 2) to investigate an interaction between psycho-social characteristics (of neuroticism, self control and social support) and MLCP, and 3) to study the variables predicting the well-being. Subjects, who scored high on self perception of life crisis, were mothers of sixth graders, studying in schools under the Bangkok Metropolis. Using simple random sampling, 32 participants were selected as the experimental group and 40 as the control group. The data was collected in three phases - before training, at the end of training, and three months after training. Data was analyzed by MANOVA statistics and multiple regression.

The results indicated that after training the experimental group who trained with MLCP, scored lower on the total perception of life crisis, emotion oriented coping, and adapting. These scores were statistically significant different at 0.05 level from the control group. No interaction was observed among psycho-social characteristics and training program towards the self- perception of life crisis. Finally adapting, emotion oriented coping, and stress predicted the well-being of participants at $41.1 \%$ and adaptation had greatest influence over well-being in opposite direction $(\beta=0.741)$. Second was emotion oriented coping $(\beta=0.185)$ and last was stress $(B$ $=0.021$ ).

\section{The Interaction of Appreciation and Illegitimate Tasks in the Military}

\author{
Jacobshagen, N., Stocker, D., \& Semmer, N.K. \\ University of Bern, Switzerland
}

Objectives: Appreciation at work has seldom been researched in detail, even though it sounds plausible that appreciation might be an important resource. Illegitimate tasks are a new stressor concept, which refer to tasks that violate norms about what can reasonably be expected from a person in a given job (Semmer, Jacobshagen, Meier, \& Elfering, 2007). 
They consistently predict different parameters of well-being, which was demonstrated in several cross-sectional as well as longitudinal studies (Jacobshagen, 2006). Our goal was to explore whether appreciation should be considered a resource on its own right and whether the relationship of illegitimate tasks and well-being would be mediated by appreciation.

Methods: 228 officers of the Swiss Military filled in an online- questionnaire. Illegitimate tasks were assessed with the Bern Illegitimate Tasks Scale (eight items; Semmer et al., 2007), which asks to what extent participants have to carry out tasks they consider unreasonable or unnecessary. Appreciation was assessed wit a self-developed measurement containing ten items, which measures appreciation by supervisors and colleagues (Stocker, Jacobshagen, Annen, \& Semmer, in press). Well-being indicators were feelings of resentment towards the organisation (Geurts, Schaufeli, \& Rutte, 1999) and job satisfaction (Baillod \& Semmer, 1994). Data were analyzed with multiple regression analyses, controlling for age, gender, and the three important resources job control (Semmer, Zapf, \& Dunkel, 1995), social support (Frese, 1989), and interactional justice (Colquitt, 2001).

Results: Illegitimate tasks were associated with feelings of resentment and job satisfaction. Also, appreciation predicted both outcomes over and above all the control variables. Finally, the relationships between illegitimate tasks and both outcomes were mediated by appreciation.

Conclusions and Implications: Illegitimate tasks constitute a threat to one's professional identity, and, therefore, the self. The results imply that the mechanisms connecting illegitimate tasks with well-being should be the experience of not being acknowledged and appreciated. This is the mechanism suggested by our mediation analysis. It is noteworthy that the importance of appreciation could be confirmed in a sample of professional military officers, as the stereotype of the military entails command and obedience and depicts them as tough. Our results show that they appreciate appreciation no less than other people.

\title{
Identifying the policy context for psychosocial risk management in Europe
}

\author{
Jain, A. \& Leka, S. \\ University of Nottingham, United Kingdom
}

A number of significant developments towards the management of psychosocial risks have been achieved at the policy level in the EU since the introduction of the 1989 EC Council Framework Directive 89/391/EEC on Safety and Health of Workers at Work on which a new EU risk prevention culture has since been established, combining legislation, social dialogue, best practices, CSR, and building partnerships. Important documents in this context include: the European Commission's Guidance on Work-Related Stress (2002); the European Commission's Green Paper on Promoting a European Framework for Corporate Social Responsibility (2001); the European Framework Agreement on Work-Related Stress (2004); the European Framework Agreement on Harassment and Violence at Work (2007). Examples of these policy-level interventions can also be found at the national level: the Management Standards approach (HSE, 2005) to work-related stress in the UK, the Health Covenants in the Netherlands, the 'Victimisation at work' ordinance in Sweden, specific anti-bullying legislation recently introduced in some countries, for example in France, Finland, Belgium and the Netherlands, are just few of the many key initiatives taken at the national level across many EU member states. 
However, it has been widely acknowledged that initiatives aiming to promote workers' health have not had the impact anticipated both by experts and policy makers and the main reason for this has been the gap that exists between policy and practice (Levi, 2005). There are a number of reasons for this gap. One is a lack of awareness across the enlarged EU that is often associated with lack of expertise, research and appropriate infrastructure. At the same time, the responsibility for understanding and managing the interface between work, employment and mental health varies greatly across countries (Cox, Leka, Ivanov, \& Kortum 2004). It is therefore essential to understand the 'policy context' within which policy initiatives for psychosocial risks are developed and implemented. In this context, this paper presents the findings of two studies that aim to explore the 'European policy context' and views of stakeholders and policy experts in relation to the current state of the art in the area of policy-level interventions in relation to psychosocial risk management.

This paper presents the findings of sixteen interviews conducted with key organizational stakeholders in occupational health (International Organizations, NGOs, Trade Unions and Employer organizations, such as the ILO, WHO, ISSA, EU-OSHA, IALI, ITUC, IOE) to assess their role in promoting occupational health policy, especially policies relating to psychosocial risk management. The findings highlighted a number of important issues in relation to psychosocial risk management at the policy level. These findings will be further discussed in the presentation.

\title{
The aging workforce - more chilled out than burnt out?
}

\author{
Johnson, S., Holdsworth, L., Hoel, H., Cunningham, K. \\ Manchester Business School, United Kingdom
}

Objectives: It is widely accepted that prolonged or intense stress can have a negative influence on well-being. A topical area is the role of emotion work and its effect on work stress and health. However, despite interest in the stress and emotion work relationship there is no clear understanding about the role age plays. The objective of this funded European research project is to investigate the relationship between stress and emotion work with a focus on the influence of age, as experienced by both employees and customers. As a high risk area in terms of emotion work demands, stress and burnout, the research focuses on the service sector.

Methods: The research utilises a mixed methodology. Semi-structured interviews and focus groups in customer service departments of a UK University $(n=20)$ explored attitudes, perceptions, and beliefs about emotion work demands, work pressures, coping strategies and age. Forthcoming interviews and focus groups will further explore these issues and investigate industry differences.

An employee questionnaire will gather information on emotion work and regulation strategies, stressors, burnout, health, and age orientation. A customer questionnaire will look at the interaction experience, service quality and the role of age.

The main study data are currently being gathered in Germany and the UK. Cross-cultural comparison will be made. 
Results: Early findings from the qualitative data suggest age affects emotion work in several ways. From the evolution of coping strategies: "when I was a lot younger I used to take it quite personally"; to regulating emotions to fit the customer's age: "I definitely deal with older people very differently to ... younger people"; and employee coping differences: "older people.... are generally calmer".

A shortened version of the main study questionnaire was used in a related study of UK teachers $(n=137)$. Findings revealed that emotion work, i.e. the requirement to display negative emotions and emotional sympathy, and emotional dissonance, negatively affected psychological wellbeing. Emotional sensitivity was linked to physical health. Furthermore, the experience of emotion work varied significantly with age.

Implications: Shifting European demographics will result in an ageing workforce. Understanding the impending impact of age on employee and customer behaviour has implications for service organisations with regards to training needs, employee well-being and customer satisfaction.

Conclusions: Preliminary findings suggest that awareness of the role age plays in service interactions can help to manage both expectations of employees and customers, with subsequent implications for stress management.

\title{
Work-family and family-work conflict and enrichment; relationships with healthy eating in working mothers
}

\author{
${ }^{1}$ Jones, F., \& ${ }^{2}$ Grzywacz, J. \\ ${ }^{1}$ University of Leeds, United Kingdom; ${ }^{2}$ Wake Forest University, United States of America
}

Objectives: This study investigated the relationship between work-family (and family-work) conflict and enrichment and healthy eating in working women with small children. It further aimed to explore the role of time-based or strain-based mechanisms. Specifically, it aimed to test the role of work hours and emotional-eating (i.e, the tendency to eat more when anxious and stressed) in the relationship between work-family variables and eating behaviours.

Methods: The data were extracted from a large study of non-Hispanic white and black working mothers in Forsyth County, North Carolina, U.S. A sample of 183 women who had returned to work after childbirth completed measures during an interview conducted 16 months after childbirth. Measures included work-family conflict (WFC) and family-work conflict (FWC), work-family and family-work enrichment, work hours, emotional eating and measures of eating behaviour (snacking, eating out and eating take-out food, and eating convenience food at home). Preacher and Hayes (2002) bootstrapping techniques were used to test mediated relationships.

Results: Relationships between WFC and eating behaviours were stronger than those for FWC. Greater WFC was associated with more snacking, eating out/take-out and eating convenience food at home. FWC was only significantly related to snacking and eating convenience food at home. There were few relationships between enrichment variables and eating. Results suggested investigation of mediated relationships was only appropriate for the conflict variables. The relationship between work hours and eating out and fast food was partially mediated by WFC. While there were no direct relationships between work hours and the 
other eating variables, results did suggest that there was an indirect relationship between work hours and snacking via WFC. In relation to emotional eating, the relationship between WFC and both snacking and eating out/take-out was partially mediated by emotional eating. Neither work hours, nor emotional eating were related to eating convenience food at home.

Implications: This study suggests that WFC has a stronger relationship with eating behaviours than FWC. Both work hours and emotional eating are implicated in this relationship. Further research is needed to investigate whether these impacts on women's health behaviours also extend to affect the food they provide for other family members including children.

Conclusion: The study suggests that working women with children reporting higher levels of WFC have poorer eating behaviours than those who report less conflict. Both time-based and strain-based factors are implicated in the relationship.

\title{
Does successful coping require calming down? Both problem-focused coping and palliative coping are positively associated with well-being, mediated by successful calming down
}

\author{
Kaelin, W. \& Semmer, N.K. \\ University of Bern, Switzerland
}

Objectives: Classical emotion-focused coping scales have been criticized for not really measuring coping but distress (cf. Semmer, 1996; Stanton et al., 2000). We developed a new instrument measuring 'true' emotion-focused coping, which we call palliative coping. In contrast to classical emotion-focused coping, which typically shows negative associations with well-being, we hypothesized palliative coping to correlate positively with well-being (Hypothesis 1). If this is the case, and if our assumptions are correct, this relationship should be mediated by successfully calming down (i.e. palliative coping success; Hypothesis 2). In addition we assumed that the association between a problem-focused coping style and well-being would also be mediated by successful palliative coping.

Methods: 685 persons from several Swiss organisations filled in an online questionnaire. Mean age was 38.93 years ( $\mathrm{sd}=12.18), 51.8 \%$ were women. Palliative coping was measured using a new instrument by KÃalin and Semmer (2007) referring to attempts to calm down and relaxation (8 items) as well as to palliative coping success (4 items). Problem-focused and classical emotionfocused coping were measured with the Coping Inventory for Stressful Situations (CISS; German version by KÃalin \& Semmer, 1997). Well-being was measured using the positive attitudes towards life scale (Grob, 1995) and a 7-point Kunin-faces item assessing job satisfaction (cf. Wanous, Reichers \& Hudy, 1997). Mediation was tested using regression analyses (Baron \& Kenny, 1986) and Sobel tests.

Results: Confirming Hypothesis 1, palliative coping was positively associated with well-being, whereas classical emotion-focused coping was negatively associated with well-being. The relationship between palliative coping and well-being was fully mediated by palliative coping success, confirming Hypothesis 2. The relationship between task-oriented coping and positive attitudes towards life was partially mediated by palliative coping success, while the association 
between task-oriented coping and job satisfaction remained statistically significant with $\mathrm{p}=.08$ when controlling for palliative coping success.

Conclusion: In contrast to classical measures of emotion-oriented coping, our measure of palliative coping, which is not confounded with distress, seems to be beneficial for well-being. It is associated with calming down successfully, which in turn is associated with better wellbeing. At the same time, the well-known positive effects of problem-focused coping in terms of good strategies and plans seem to be due not only to successful problem solving but also to successfully calming down. If replicated, our results imply that the role of emotional coping has to be reconsidered.

\title{
Behavioural Safety Approach as a Method of Accident Prevention in Oil and Gas Industry in Nigeria
}

\author{
Kadiri, S. \\ Gateway Industrial and Petrogas Institute, Nigeria
}

Safe behaviour programs are currently a popular strategy for improving safety in large organisations. Behaviour modification programs are now widely advocated as a means of reducing injuries at work. Given that it is the behaviour of management which is most critical in creating a culture of safety in any organisation, behavioural safety observations are likely to have their greatest impact if directed upwards, at managers. Behavioural safety focuses on the interaction between people and their working environment, not just employee's behaviour. Designed to dictate people's daily work-related behaviour, an important feature of the working environment are organisations management systems. The presence, quality and functioning of various management systems (safety and non-safety), the quality of leadership, the amount of resources available (financial and non- financial) and the overall safety culture are all vital factors exerting an influence on results. These aspects are also important to other safety systems (e.g. Training, Permits to Work, Risk Assessments, Safety Audits, etc.) and other business improvement initiatives. This paper provides a critical look at the assumptions which underlie such programs and identifies some of their limitations. It suggests one of the behavioural approach concept Xstrata Coal's S.L.A.M, which stands for Stop, Look, Assess, Manage for adoption by oil and gas industry.

\section{Bullying among nurses: Extent, context, and consequences}

\author{
${ }^{1}$ Kaminski, M. \& ${ }^{2}$ Sincox, A.K. \\ ${ }^{1}$ Michigan State University, United States of America; ${ }^{2}$ Michigan Nurses Association, United \\ States of America
}

Objectives: Workplace bullying is a recognized problem among nurses, where nurse-on-nurse bullying is often referred to as "lateral violence". We collected data in four unionized hospitals to assess the extent of workplace bullying, work environment, impact on nurses, and responses to bullying. 
Methods: Data were collected via an on-line survey of nurses in four hospitals in the Midwest region of the United States. Response rates varied from 25 percent to 75 percent within each hospital. Analysis is based on data from 485 nurses.

Results: About 25 percent of nurses reported being the target of bullying behaviour on a regular basis. Nurses were bullied by other nurses significantly more often than they were bullied by doctors or charge nurses. Nurses who were the target of bullying were significantly more likely to report lower job satisfaction, higher intent to turnover, and more symptoms of post traumatic stress disorder. Demographic differences in who was the target of bullying were inconsistent.

In spite of a new requirement that certain hospitals must develop and implement a code of conduct that addresses a range of behaviours including bullying, most nurses said their hospital does not have a system to deal with bullying. Among those that do have a policy, many believe that the policy is not implemented fairly and is not applied equally to all.

The most common response nurses had to bullying was to ignore it. Some also sought social support from friends, family members, and other nurses. About 40 percent said they confronted the bully. Confronting the bully resulted in marginally better treatment than ignoring the bully.

Implications: In the U.S., most work organisations have not yet developed effective means to deal with bullying. Some have policies, but those policies are not consistently implemented. Unions are just beginning to take on the issue of bullying. Currently, there is no anti-bullying legislation in the U.S. (although there is in other countries). Thus a U.S. worker who is bullied finds that there is virtually no institutional mechanism for fighting this problem.

Conclusion: Bullying and particularly bullying by peers - is a significant problem for nurses. Negative consequences for nurses include decreased job satisfaction and possible health consequences such as post traumatic stress disorder. Negative consequences for work organisations include possible turnover. Given the current lack of nurses who are willing to work in the hospital setting, the consequences of turnover are significant for U.S. hospitals.

\title{
Enterprise bargaining, workers' job satisfaction and union-member relationship: An Australian retail study
}

\author{
Kang, S., Dollard, M.F., Winefield, A.H., Provis C., Black, Q.C., \& Black C. \\ University of South Australia, Australia
}

The Australian system of labour relations had a long historical preference to a centralised wage fixation system. Although after the 1980s the shift has been moving toward an enterprise-based approach (e.g. enterprise bargaining agreements), large employers in Australia, large retail employers in particular have maintained their industrial relations preference for collective negotiation with the workers' union(s). At the time of its introduction, EB was regarded as a new form of negotiation approach that could improve productivity, the living standards of workers and the relationship of all negotiating parties (including employers, employees and the unions).

While the nature and effects of different forms of negotiation agreements, such as the enterprise 
bargaining agreements, on the economy, legal and industrial relations systems have drawn plenty of attention (Dowrick, 1993), the extent of success of these agreements, especially from the employee's point of view has been neglected in the industrial relations literature. However, there has been little or no systematic research in Australia addressing employee satisfaction with the bargaining process since 1991 (Black, 2004). In addition, the retail sector has been underrepresented in the Australian literature, despite the fact that its employees make up a significant portion $(20 \%)$ of the Australian labour market, of them $66 \%$ are women, and $30 \%$ are young (under the age of 18 years) and inexperienced who are also under-represented in the industrial relations system (Bahrami, 1996; O’Brien et al., 2006).

Thus, this study focused on employees' satisfaction with the bargaining process and its relationships with employees' satisfaction with their union(s) and satisfaction with their job, we also looked at psychological job demands and decision latitude at work. 256 employees from the retail sector (large fast food restaurants and discount stores) participated in this survey study. We found a significant relationship between employees' satisfaction with the bargaining process and their satisfaction with the union; we also found a positive relationship between employees' satisfaction with the union and their job satisfaction. These results support the view that wellconducted bargaining process could improve union-member relationship and satisfaction at workplace.

\title{
Return to work after a workplace-oriented intervention for patients on sick leave due to burnout
}

\author{
${ }^{1}$ Karlson, B., ${ }^{1}$ Jonsson, P., ${ }^{2}$ Palsson, B., ${ }^{2}$ Abjornsson, G., ${ }^{3}$ Malmberg, B., ${ }^{2}$ Larsson, B., \& \\ Osterberg, K. \\ ${ }^{1}$ Lund University Hospital, Sweden; ${ }^{2}$ Lund University Hospital, Sweden; ${ }^{2}$ Linköping University, \\ Sweden
}

Objectives: Previous studies have shown that individual oriented treatment is insufficient to improve return-to-work (RTW) after burnout. The objective of the present study was to evaluate the effect of a work-place oriented intervention for persons long-term sick-leave due to burnout, aimed at facilitating return to work (RTW) by job-person match through patient-supervisor communication. We hypothesized that the intervention group would show a more favourable rate of RTW than a control group.

Methods: In collaboration with regional social insurance offices (RSIO) all subjects with at least 50\% sick-listing with (ICD-10) F.43 diagnoses, pre-dominantly due to work stress, were consecutively identified 2-6 months after the first day on sick leave, and invited. After acceptance a thorough clinical examination was carried out including the patient's view of the changes needed to facilitate RTW. Next, the patient's supervisor was interviewed at the workplace, responding to the same questions about needed changes. Then followed the core of the intervention - a convergence dialogue meeting (CDM). The purpose was to initiate a dialogue between the patient and the supervisor to find solutions to facilitate RTW. Out of 92 clients with work related burnout, as validated after the clinical examination, 76 participated in CDM (78\% women, mean age 47 years). As our primary control group we used 178 persons who were sent an information letter about the study but were uninterested in 
participating without giving any specific reason for that. From them 76 persons (74\% women, mean age 44 years) were matched for length of sick-listing before CDM as final controls. The control group was only followed by sick listing register data.

Results: There was an almost linear increase in the RTW rate in the intervention group during the 1,5 year follow-up period after the CDM, and $88 \%$ of the subjects had returned to work to some extent at the end of the follow-up period. The increase in RTW rate in the control group came to a halt after six months, and only $71 \%$ had returned to work to some extent at the end of the 1,5 follow-up.

Implications: A practice implication is that, to improve RTW, workplace-oriented interventions should be added to individual treatment for patients with clinical burnout.

Conclusion: We conclude that the present study demonstrated an improvement of longterm RTW after a workplace-oriented intervention for patients on long-term sick leave due to burnout.

\title{
The relationship between potentially traumatic experiences, generic workplace stressors and mental health within a large UK police force.
}

\author{
Kerr, R. \& McHugh, M. \\ Ulster Business School, University of Ulster, Northern Ireland
}

Objectives: To investigate the relationship between generic workplace stressors, potentially traumatic experiences and mental health within a large UK police force.

Methods: An anonymous cross-sectional questionnaire was distributed by e-mail to all employees within the Police Service Northern Ireland. Generic workplace stressors were assessed using the UK Health and Safety Executive's Management Standards Indicator Tool (IT). A six item scale was created to assess the respondents frequency of exposure to potentially traumatic events while on duty. An example event would be 'at the scene of a traumatic death'. Individual item responses were aggregated to create a 'traumatic exposure' score. Mental health was assessed by the 12-item General Health Questionnaire (GHQ-12), as well as Breslau's 7-item screen for posttraumatic stress disorder (PTSD). Single item measures investigating 'stress at home' and 'stress at work' were also included. Controlling for age, gender, years of service and rank, associations were investigated using regression statistics.

Results: A total of 2,715 employees completed the questionnaire, representing a response rate of $29 \%$. The Indicator Tool (IT) scores explained 34\% of variance in GHQ-12, $41 \%$ of the variance in 'Stress at work', $14 \%$ of the variance in PTSD responses, and less than $1 \%$ of the variance of 'stress at home' responses. All of the Indicator Tool (IT) subscales displayed a strong negative association with both GHQ-12 and 'stress at work' scores. The IT demands, peer support, and relationships subscales were negatively associated with Breslau's PTSD screen scores. Only the IT relationships subscale displayed a weak negative relationship with the 'stress at home' scores.

Only $29 \%$ ( $n=793$ ) of respondents had no exposure to potentially traumatic events. As expected, 'traumatic exposure' scores were positively associated with GHQ-12, PTSD, and 'stress at work' 
scores. However the scale of these associations was lower than expected. The 'traumatic exposure' scores explained $4 \%$ of the variance in PTSD scores and less than $1 \%$ of the variance in the other dependent variables. The 'traumatic exposure' scores were not associated with 'stress at home' scores.

Implications: This study provides empirical evidence to support the use of the Management Standards Indicator Tool and the Management Standards approach in tackling workplace stress.

Conclusions: The HSE Management Standards Indicator Tool is a valid and reliable measure of workplace stressors. These workplace stressors are associated with employee mental health. Within police organisations generic workplace stressors may have a greater overall impact on employee mental health than exposure to potentially traumatic events.

\title{
Emotional competencies, resilience and wellbeing in trainee social workers
}

\author{
Kinman, G. \& Grant, L. \\ University of Bedfordshire, United Kingdom
}

Objectives: Emotional resilience is defined as "the general capacity for flexible and resourceful adaptation to external and internal stressors" (Klohen, 1996, p. 1068). It is thought to be a key attribute for social workers as it may protect against the high levels of distress and burnout endemic to this profession. In order to guide the development of interventions designed to enhance wellbeing in this group, this study tested several inter- and intra-personal emotional competencies as predictors of resilience in trainee social workers. The contributions of emotional intelligence, reflective ability (encompassing self reflection, empathetic reflection and reflective communication), empathy and social competence were assessed. Resilience was also tested as a potential mediator of the relationship between emotional competencies and psychological wellbeing.

Methods: a cross-sectional correlational design was utilised. Two hundred and forty trainee social workers ( $82 \%$ female) completed a range of validated questionnaires.

Results: Significant positive relationships were found between all emotional competencies and both stress resilience and psychological wellbeing. Together, the emotional competencies accounted for $47 \%$ of variance in resilience. Stress resilience was found to fully mediate the relationship between emotional intelligence and psychological distress. Forty-three percent of the sample achieved scores indicative of "caseness" levels of psychological distress, where some intervention is deemed appropriate.

Conclusions: The results of this study indicate that trainee social workers who are more emotionally intelligent and socially competent are more resilient than those whose emotional competencies are less well developed. Evidence was also found that the capacity for self reflection, empathetic reflection and reflective communication may be protective qualities in trainee social workers. Empathy, operationalised in this study as the ability to take the perspective of other people and feelings of warmth, compassion and concern for others, was also significantly related to resilience. The comparatively high levels of psychological distress found in this study 
suggest that some intervention to enhance resilience is required in this occupational group. The findings of this study suggest that interventions designed to enhance inter-personal and intrapersonal emotional competencies are likely to enhance resilience which, in turn, will protect the wellbeing of an occupational group that is highly vulnerable to work-related stress.

\title{
Utilising the HSE Management Standards approach to predict wellbeing in UK academics: the role of job-specific hazards.
}

\author{
Kinman, G. \\ University of Bedfordshire, United Kingdom
}

Objectives: Research conducted in several countries suggests that academic employees experience high levels of work-related stressors and strains. This study examines the workrelated stressors that predict strain in academic employees working in UK universities using the framework for the measurement of job stressors (or psychosocial hazards) developed by the UK Health and Safety Executive (HSE). The HSE framework encompasses a series of workplace psychosocial hazards: demands, control, support from peers and managers, relationships, role and involvement in change. These hazards are considered relevant to the majority of employees and, it is argued, have a strong evidence base as the "most critical predictors" of employee wellbeing. There is also evidence, however, that job specific hazards can also be considerable sources of strain. This study also investigates whether hazards that are specific to the academic job role account for additional variance in strain to that explained by the global hazards in the HSE framework.

Methods: Seven thousand, one hundred and ninety-seven academic employees (50\% female) participated in the study. The HSE Management Standards Indicator Tool was used to assess employees' levels of exposure to key psychosocial hazards. Stressors related to teaching and research and dealing with students were also measured.

Results: Significant relationships were observed between all categories of hazard (both global and job-specific) and perceived stress. Hierarchical multiple regression found that hazards that were specific to academic work accounted for variance (9\%) beyond that explained by global hazards $(48 \%)$.

Conclusions: The findings of this study indicate that a global measure of job-related stressors is a valid predictor of perceived stress in a sample of academic employees working in UK universities. The utility of the HSE management standards approach is therefore supported in this occupational context. Nonetheless, the importance of recognising job-specific stressors has also been highlighted Findings suggest that interventions to enhance wellbeing in university settings should address psychosocial hazards that are specific to the academic working context, such as those relating to teaching and research, as well as those that are more global. 


\title{
Work-life conflict and health among Swiss physicians - in comparison with other university graduates and with the general Swiss working population
}

\author{
${ }^{1}$ Knecht, M., 'Bauer, G., ${ }^{1}$ Klaghofer, R., ${ }^{2}$ Buddeberg-Fischer, B., ${ }^{2}$ Samm, M., \& ${ }^{1}$ Hämmig, O. \\ ${ }^{1}$ University of Zurich and ETH Zurich, Switzerland; '2Zurich University Hospital, Switzerland
}

Objectives: An increased number of employees face substantial domestic duties as well as work obligations. These general social changes apply in particular for physicians, where a strong "feminization" takes place in Switzerland. The competing demands from different life domains can generate work-life conflict, which may be time-based as well as strain-based. As international research has shown, work-life conflict is associated with poor mental and physical health. The present study aims to compare the prevalence of work-life conflict of physicians with a representative sample of university graduates and with a representative sample of the general working population of Switzerland. Furthermore it aims to compare the health status of these samples and wants to analyze whether work-life conflict is a correlate of health for physicians as it is in the general working population.

Methods: To compare physicians concerning work-life conflict and health with university graduates and the general working population, the present cross-sectional study analyses data from the year 2007 of the SwissMedCareer Study (a prospective cohort study of physicians who graduated in 2001; $n=543$ ) and the Swiss Household Panel (a representative survey in Switzerland covering a broad range of topics on living and working conditions; university graduates of the same age range: $n=172$, general working population of the same age range: $n=670$ ).

Results: Physiciansreportsignificantly stronger time-basedas well as strain-based work-lifeconflictthan university graduates and than the general working population. Significantly more physicians report moderatetovery poor health than theother two samples. Strongassociations between work-lifeconflict and self-rated healthand differenthealthcomplaintswerefound for physicians. Physiciansexperiencing strong strain-based work-life conflict are 15 times more likely to report moderate to very poor health. Implications and Conclusions: Work-life conflict is strongly related with health problems. Since physicians report work-life conflict above average, it is a substantial problem for this profession and furthermore a health risk. The high prevalence of work-life conflict and its strong association with poor health may be an explanation for the high prevalence of poor self-rated health in the physicians' sample. Additionally, strong work-life conflict generally is a predictor of lower commitment, lower job satisfaction and higher turnover rates. To provide good health care services, work-life conflict of physicians should be minimized.

\section{Key Factors for Attracting and Motivating Young People to Work as Employees in Swedish Livestock Farming}

Kolstrup, C.

\author{
Swedish University of Agricultural Sciences, Sweden
}

Introduction: Swedish livestock farming has undergone considerable structural changes and this has lead to fewer but larger farms with employed workers - and in Sweden it is difficult to recruit 
qualified workers. If agriculture should be an attractive occupation for young people in the future, it is important to know what attracts and motivates them to work and stay in the profession.

Objectives: The aim of the study is to identify key factors which are important when young people choose to work with livestock.

Methods: The study was based on a questionnaire to elucidate young people's attitudes towards working in agriculture e.g. What will attract you to choose livestock farming as a profession? What will motivate you to stay as employee in livestock farming? Furthermore, in order to elucidate the farm employer's view, they were asked what they believed were important as key factors to attract and motivate young people to choose livestock farming as a profession'? The study was conducted 2008 and comprised 183 agricultural students 197 employed livestock workers and 146 farm employers.

Results: The preliminary results showed that the five most important key factors for agricultural students to be attracted and motivated to work with livestock farming were: Having fun at work, good leadership, feeling proud of their work, living in the country and safe employment. The least important factors were: Regular working hours and working overtime. Having a high wage was low prioritised. Among employed livestock workers the five most important key factors were: Good leadership, working with animals, having fun at work, living in the country and feeling proud of their work. The employers believed that safe employment, having fun at work, good team work, good leadership and feeling proud of the work were the most important factors for employed workers. The least important factors among employees and employers were the same as for the agricultural students.

Implications: The preliminary results of this study have enlarged the knowledge about how farmers can increase the interest to the occupation by identifying the most important key factors that workers appreciate when they are choosing to work with livestock farming.

Conclusions: In general, the results indicated that the most important key factors that attract and motivate presumptive and existing employees to a large degree corresponded with the employer's perception. The results will be further analysed to study the effect of gender, age, geographical location or level of education.

\title{
The Antecedents of safety-related helpful behaviours and its impact on occupational safety performance
}

\author{
Krause-Juettler, G. \\ University of Technology, Dresden, Germany
}

Objectives: Research results show that helpful behaviours of employees contribute to the performance of organisations (e.g. OCB). Until now it is relatively unexplored to what extent safety-related helpful behaviours improve the occupational safety performance. The present study deals with that research question. By using the Theory of planned behaviour (TopB) the preconditions of safety-related helpful behaviours are investigated on the individual level. On group level it is surveyed whether safety-related helpful behaviours among co-workers are able 
to reduce unsafe behaviours indicated by violations of job-related safety rules, near misses, occupational injuries and accidents.

Methods: The collected data derive from a "self-report" - questionnaire which is completed by employees working in the producing divisions in three Saxon enterprises belonging to the metal respectively electrical industry $(\mathrm{N}=232 ; 21$ work groups). On individual level the explanatory components of the TopB "attitude toward behaviour" and "perceived behaviour control" are recorded by existing validated scales that have been already used in safety-related research studies. The measurements of the safety-related "subjective norm" (TopB) and safety-related helpful behaviour are based on self-developed scales. Theoretically helpful behaviours are constructed as a two-factor-concept separated into "advising co-workers of potential safety hazards" and "removing potential safety hazards". In order to test whether individual helpful behaviours benefit the reduction of unsafe behavioural acts in teams data are aggregated and analysed on group level.

Results: Factor analysis provides evidence that safety-related helpful behaviour can be separated into the two assumed factors. "Advising co-workers of potential safety hazards" and "removing potential safety hazards" are predicted by the independent variables postulated by the TopB whereas the "subjective norm" contributes most to its explanation. On group level it is shown that widespread safety-related helpful behaviours among employees in terms of "advising co-workers", support the improvement of safety indicators, whereas violation of safety rules and near misses are direct antecendents of real injuries and accidents.

Implications: Safety-related helpful behaviours provide high potential to improve organisational safety performance. Enterprises have the ability to enhance safety-related behaviours and finally its occupational safety by shaping the "subjective norm", employee's "attitude toward safety-related behaviour" and their "perceived behaviour control" concerning safety issues by means of knowledge transfer and constant practical trainings.

Conclusions: The study provides another evidence for the predictive power of the explanatory variables of TopB. Furthermore it shows that safety-related helpful behaviours consist of two factors whereof the factor "advising co-workers" has a significant impact on safety indicators.

\title{
Is primary care physicians' burnout associated with prescribing medications and making referrals to diagnostic tests and to other providers of health services?
}

\author{
${ }^{1}$ Kushnir, T., ${ }^{1}$ Bachner, Y., ${ }^{1}$ Greenberg, D., ${ }^{1}$ Yermiahu, Y., \& ${ }^{2}$ Hadari, I. \\ ${ }^{1}$ Ben-Gurion University of the Negev, Israel; ${ }^{2}$ Leumit HMO, Beer-Sheva, Israel
}

Background: Physicians in general and primary care physicians in particular, are vulnerable to develop burnout due to chronic stresses in the medical environment. Burnout, as defined in most current studies in the medical literature, is a syndrome of emotional exhaustion, depersonalization and low personal accomplishments. It is commonly assumed that burnout has significant negative consequences on job performance, yet there is little research testing this assumption in healthcare settings. Moreover, there have been no empirical studies on burnout and physicians' referral behaviour. 
Objective: To assess the associations between primary care physicians' burnout levels and the rates of prescribing medications and referring patients to diagnostic tests (imaging and laboratory tests), and to other providers of health services (specialists, emergency room, nurses). Methods: 136 primary care physicians in community clinics in one $\mathrm{HMO}$ in Israel responded to a structured questionnaire in a personal interview. Burnout levels were assessed by the Maslach Burnout Inventory $(\mathrm{MBI})$. The rates of prescriptions and referrals were obtained from the HMO's data bases.

Results: $56 \%$ of the physicians had high burnout levels. In bivariate correlations, emotional exhaustion was associated positively with prescriptions and referrals to both inexpensive (e.g. X-ray) and expensive imaging tests (e.g. MRI); depersonalization was associated with expensive imaging and laboratory tests; and reduced personal accomplishments were associated with referrals to laboratory tests. In multiple regression analyses, after controlling for physician characteristics, prescription rates and referrals to imaging tests were associated with increased emotional exhaustion, but not with other components of burnout.

Conclusions: The hypothesis of significant associations between burnout and objective measures of physician behaviour in the medical encounter has been upheld for prescribing medications and referrals to imaging tests, both in univariate and multivariate analyses. It appears that in this rather small sample of primary care physicians, burnout level had some effect on the objective parameters of patient care examined and therefore may have some economic implications to the $\mathrm{HMO}$. Future analyses should also control for patient characteristics and disease severity.

Implications: The alarming high burnout rate replicates similar findings in other countries. Organisational-level interventions for reducing burnout among physicians should be devised: 1. Because of established associations between burnout and physical and emotional health; 2 . To prevent physicians from searching for alternative professional and occupational alternatives, thereby increasing the existing shortage of physicians that exists locally.

3. To improve patient care and contain $\mathrm{HMO}$ costs.

\title{
Health status and burnout in physicians: what interventions in a context of decreasing medical demography? A pilot study in France, 2008
}

\author{
${ }^{1}$ Ladner, J., ${ }^{1}$ Kuntz, A., ${ }^{2}$ Heurtebize, P., ${ }^{2}$ Mauviard, E., ${ }^{1}$ Manzano, M., \& ${ }^{1}$ Caillard, J.F. \\ ${ }^{1}$ Rouen University Hospital, France; ${ }^{2}$ French Medical Association of Seine-Maritime, France
}

Objective: To study physicians health status and burn-out characteristics in physicians

Methods: Following a psychological study including 20 physicians, a cross-sectional study was conducted in 1,235 primary care practitioners (PCP) and specialist practitioners (SP) randomised, within a total of 2,819 ambulatory practitioners working in Upper Normandy region in 2008. The postal anonymous questionnaire contained 200 items collecting data about work (environment, content, schedules and ability), personal situation, burnout, health status, self-care and difficulties with their professional practice. 
Results: A total of 543 physicians were included (354 PCP, 189 SP), with an overall response rate was $44.7 \%$. The mean age was 50.9 in PCP and 52.7 SP $(p=0.02)$. PCP weekly work hours were higher than SP (51.0h vs. 46.5, p<10-5); PCP were more unsatisfied with their work situation $(42.1 \%$ vs. $25.6 \%, p=0.001)$, especially because of the administrative work part $(60.1 \%$ vs. $41.0 \%, p=0.01)$. Exhaustion periods (18.6\% vs. $12.4 \%, p=0.008)$ and psychiatric diseases $(32.1 \%$ vs. $18.0 \%, \mathrm{p}<10-4)$ were more prevalent in PCP; they also took more antidepressant drugs $(10.6 \%$ vs. $4.3 \%, \mathrm{p}=0.01)$. PCP were more affected by osteoarticular diseases $(66.6 \%$ vs. $58.3 \%, p=0.06)$, overweight $(34.9 \%$ vs. $22.8 \%, p=0.02)$ and addictions $(17.2 \%$ vs. $11.1 \%, p=0.06$, essentially eating disorders). Prevalence of high burnout was $16.3 \%(95 \%$ $\mathrm{Cl}=14.7-17.9)$. Burn-out was significantly associated to primary care practice, high workload, psychotropic drugs consumption and sleep disorders. Physicians with burnout symptoms presented impacts on professional activity: insufficient time pour discuss with the patient, low motivation to practice, accumulation of delay, fear of making mistakes in professional exercise, absence of work control.

Implications: There is a significant degree of burnout among PCP in Upper Normandy region. It is time to physicians, public health managers and politicians to develop specific services for primary care practitioners. It would be preferable to develop a policy based on healthy approaches and prevention of impairment. We need to implement prevention (including screening strategies) and curative programs specifically dedicated to PCP. Evaluation of these experimental programs are also needed.

Conclusion: Physicians increasing workload and health status are emergent public health issues while French medical demography has reached a critical threshold, with serious difficulties expected in the next years. Furthermore, medical physician population is progressively aged. Some worrying aspects of physician health status support the fact that they should benefit from a specific health service as a public health priority, with a particular attention for PCP, in order to maintain "the delights of working in health" and to ensure a care physician workforce in a next future in France.

\title{
The influence of psychosocial workplace factors on the development of musculoskeletal problems: A meta-analysis of longitudinal studies
}

\author{
${ }^{1}$ Lang, J., ${ }^{2}$ Lang, P.J.W.B., ${ }^{1}$ Kraus, T., \& ${ }^{1}$ Ochsmann, E. \\ ${ }^{1}$ RWTH Aachen University, Germany; ${ }^{2}$ Maastricht University, the Netherlands
}

Objectives: The purpose of the present meta-analysis was to conduct a systematic review of baseline-adjusted prospective longitudinal studies estimating the lagged effect of psychosocial workplace factors on musculoskeletal problems.

Methods: Literature review was conducted by searching the MEDLINE (1966 to August 2009) and PsychINFO (1872 to 2009) databases. The authors independently classified studies into categories of psychological work stressors (e.g., job demands), and musculoskeletal problems. Available effect sizes were converted to Odds ratios (OR). ORs were then pooled for each stressorproblem relationship using a random-effects model. Additionally, the possibility of publication bias was assessed with the Duval and Tweedie nonparametric -trim and fill- procedure. 
Results: At least five effect sizes were available for 16 of the 44 possible psychosocial work stress - musculoskeletal problems relationships. For 12 of these stressor' problem relationships, pooled OR estimates were positive and significant. Pooled ORs ranged from 1.154 to 1.623 with the largest pooled OR estimating the relationship between highly monotonous work and lower back pain. The lagged effect of low social support on lower back problems was the only effect size for which the statistical test for bias was significant. However, the corrected effect size remained significant. The limited number of longitudinal primary studies did not allow further moderator analyses on the nature of the stressor-musculoskeletal problems relationship. Additionally, the large majority of the primary studies in our meta-analysis did not report the reliability of the measures and dichotomized continuous independent and outcome variables. Thus the estimates, likely seriously underestimate the true lagged effects.

Implications: Under the assumption that the lagged effects in our study did not originate from an unstable unmeasured background variable, our review suggests that psychosocial work stressors are antecedents of subsequent musculoskeletal problems. From an applied perspective, some of the psychosocial work stressors in this investigation are inherent in a variety of jobs and cannot always easily be altered (e.g., high job demands). Still changes to job conditions (e.g., through job redesign) and specific interventions (e.g., stress prevention trainings) could be implemented to minimize psychosocial workplace stressors. From a methodological perspective future research should use structural equation modeling techniques and include tests of the lagged effect of musculoskeletal problems on the development of psychosocial work stressors.

Conclusions: Most psychosocial stressor had small but significant lagged effects on the development of musculoskeletal problems. Thus, organisational interventions to minimize these stressors may be promising to prevent musculoskeletal problems at the workplace.

\title{
Influence of Empowerment on New Graduate Nurses' Experiences of Bullying and Burnout in Canadian Hospital Settings
}

\author{
Laschinger, $\mathrm{H}$. \\ University of Western Ontario, Canada
}

The purpose of this study was to test a model linking new graduate nurses' perceptions of structural empowerment to their experiences of workplace bullying and burnout in Canadian hospital work settings using Kanter's (1993) work empowerment theory. New graduate nurses are a precious health human resource in today's worsening workforce shortage and every effort is required to ensure that their transition to the full professional role is supported through positive work environments that promote their development. Yet, there are numerous anecdotal reports of bullying of new graduates in current health care settings (Duchscher and Cowin, 2004) and Cho, et al (2006) found that $66 \%$ of new graduates experience severe burnout, related to negative workplace conditions. Workplace bullying threatens new graduates' adjustment to their roles, possibly contributing to their leaving the profession. Bullying has been linked to employee burnout, absenteeism, and turnover in other occupational groups, but there is little research on this phenomenon in nursing, particularly with new graduate nurses, who represent the future of the profession. Kanter's structural empowerment theory suggests actionable approaches to creating conditions that foster optimal performance in work settings. 
We tested the model in the first wave of a longitudinal study of 300 newly graduated nurses in acute care hospitals across Ontario. The sample was drawn from the registry list of practising nurses in Ontario. Variables were measured using the Conditions of Work Effectiveness Questionnaire (Laschinger et al 2001); the Negative Acts Questionnaire-Revised (Einersen and Hoel, 2001), and the Maslach Burnout Inventory-General Survey (Schaufeli et al., 1996).

The final model fit statistics revealed a reasonably adequate fit $\left(\chi^{2}=27.1, \mathrm{df}=7, \mathrm{IFI}=0.91\right.$, $\mathrm{CFI}=0.91, \mathrm{RMSEA}=.10)$. Structural empowerment was significantly related to workplace bullying exposure $(B=-.30)$, which in turn, was significantly related to all 3 components of burnout (Emotional exhaustion: $B=.39$, Cynicism: $B=.18$; Efficacy: $B=-.17$ ). The burnout variables followed the theorized pattern of relationships in that emotional exhaustion had a direct effect on cynicism $(B=.51)$, which in turn, had a direct effect on efficacy $(-.34)$. The results suggest that new graduate nurses' exposure to bullying may be lessened when their work environments provide access to empowering work structures, such as information, support, resources, and opportunities to learn and grow, and that these conditions promote nurses health and well being. The results suggest managerial strategies to create work environments that are likely to improve retention of new graduates.

\title{
Connections of Change Experiences, Work Engagement, and Other Indicators of Work Related Well-Being During Restructuring
}

\author{
Leppänen, A., Pahkin, K., Ala-Laurinaho, A., \& Kajosaari, K. \\ Finnish Institute of Occupational Health, Finland
}

Objectives: At the beginning of 2008, the Finnish Institute of Occupational Health (FIOH) launched a two-year study to examine the current state of occupational well-being among paper industry workers and to follow-up the changes in well-being during great reorganisations on an important industrial sector. The aim of the study is also to find good practices to master changes, and methods to be used in the evaluation of changes and their effects. In this paper we study the relations of change experiences, work engagement, and other indicators of work related well-being.

Methods: Eight production facilities participated in the study. In the spring of 2008, a questionnaire was sent to all blue-collar employees (response rate was $52 \%, \mathrm{n}=1$ 955) and in autumn 2009, a follow-up was carried out (response rate $48 \%, n=1518$ ). During the study period, all the participating units had faced organisational restructuring. In the first measurement both the previous experiences of the mastery of change processes in the organisation and the meaning of changes were studied with three questions. Work engagement was studied with the Utrecht work engagement scale (Schaufeli et al. 2002). Experienced stress, job satisfaction and experience of future were measured with single questions. The relations of change experiences, work engagement and the other indicator of work related well-being in the first measurement were studied with Pearson Correlations.

Results: Positive change experiences were positively correlated with all three dimensions of work engagement, job satisfaction, positive attitude to future, and fewer experiences of stress with two exceptions. Experienced significance of the changes was not related with any of the 
dimensions of work engagement. But the more significant the changes had been, the higher was the level of stress, and the fewer were the experiences of job satisfaction and positive future. Neither was the amount of participation in planning and realization of the changes related to experienced stress.

Conclusions: The cross-sectional study on the relations of experiences revealed a connection between the workers' change experiences, and their level of well-being. However, follow-up data and further analyses are required to be able to determine the directions of the relationships. Also knowledge of objective changes and their implications e.g. to work and employment of the respondents must be added into the analyses.

\title{
Finish peace keepers psychosocial well being after the home coming
}

\author{
Leskinen, J., Isosomppi, M., \& Nyman, M. \\ National Defence University, Finland
}

Objectives: 1.To study the possible post traumatic experiences and related post operational psychological problems after the different peace keeping missions (Kosovo and Afghanistan) 2. To study the intra personal and the mission focused factors related to the home coming experiences.

Methods: survey questionnaire, logistic regression analysis

Results: The peace keepers in Kosovo, compared to the peace keepers in Afghanistan after the mission (2007-2008) had:

- less mission related psychological burden, traumatic experiences and stress reactions

- more alcohol consumption

- no difference in depression, general health or post traumatic stress reactions

The peace keepers in Afghanistan experienced more psychologically burdening situations and potentially traumatic events in spite of the basically similar work content. The results of the logistic regression analysis highlight the contribution of the sense of coherence as a mediating and stress protecting personality orientation factor.

Conclusions: The Finish peace keepers, mostly in reconstruction work and disarming mines, didn't usually have any severe mental symptoms after their home coming. But if they had difficult combat experiences, the strong sense of coherence protected them from the long term stress reactions.

\section{Work Stress, Burnout, and Health in Chinese Working men: Mediation Analysis}

\author{
${ }^{1} \mathrm{Li}, \mathrm{J} . \&{ }^{2} \mathrm{Li}, \mathrm{S}$. \\ ${ }^{1}$ University of Wuppertal, Germany; ${ }^{2}$ Kunming Medical University, Kunming, China
}

Objective: The mechanisms linking work stress with health are still uncertain. This study was 
to examine the impact of work stress on health in Chinese working men, and to explore the mediating effect of burnout between them.

Methods: A survey was conducted in a sample of 1133 male subjects at age of 30-65 years, working in Kunming City, China. Work stress, burnout, and health were measured by Copenhagen Psychosocial Questionnaire (COPSOQ), Copenhagen Burnout Inventory (CBI), and Medical Outcomes Study 8-Item Short Form (SF-8), respectively. The mediation analysis was used for testing the associations among work stress, burnout and health.

Results: It was found that the increase of work stress was associated with the decline of both physical health and mental health $(\mathrm{P}<0.001)$, and dose-response relationships could be observed obviously. As a mediator, burnout contributed $15.08 \%$ mediating effect on poor physical health and $23.84 \%$ mediating effect on poor mental health.

Conclusion: The psychosocial stress at work had adverse effects on health in Chinese working men, mediated partly by burnout.

\title{
Nursing leadership style and mental health outcome of nurses in Taiwan
}

\author{
${ }^{1}$ Lin, P.Y., ${ }^{1}$ Hunt, N., \& ${ }^{2}$ MacLennan, S.J. \\ ${ }^{1}$ University of Nottingham, United Kingdom; ${ }^{2}$ University of Aberdeen, Scotland
}

Objectives: The aim of the research was to understand nursing leadership style in three types of ownership of hospitals in Taiwan. It also investigated the relationship between leadership style and the mental health of nurses, organisational commitment and job satisfaction. The hypothesis of this research was that different leadership styles of nursing would have an influence on the quality of nurses' working life.

Methods: This was a cross-sectional quantitative study. Samples from each type of hospital ownership (private, public and religious hospital) were recruited. Participation was voluntary and signed informed consent was obtained. The inclusion criteria were nurses graded from N1 to N4 with at least had one year's work experience in the hospitals. Self-administrated questionnaires were conducted within each hospital.

Results: A total of 807 participants were obtained. The overall response rate was around $85 \%$, with 651 questionnaires fully completed. The mean age was $30.53 \pm 6.18$ (SD) years. The majority of the participants were female and more than half of them were single. In items of grade of nursing and educational level, there were significant differences $(p<0.01)$ between three hospitals. General health status was significantly different $(p<0.01)$ in the three hospitals. The degree of organisational commitment scores were the highest in the religious hospital compared with the others. In term of job satisfaction, although the participants in public hospital scored the highest job satisfaction (mean $\pm \mathrm{SD} ; 46.51 \pm 7.74)$, this was not significant.

Implications: The results provided information about current nursing leadership styles in different hospital settings as well as identifying organisational factors that could improve the quality of nurses' working life. These findings increase our understanding of the relationship between nursing leadership and nurses' mental health outcomes. The findings could also be used as a reference to improve leadership styles and further promote organisational health. 


\title{
Stress and Esfahan steel employees, Iran
}

\author{
Lotfizadeh, M. \& Hassim, N. \\ National University of Malaysia (UKM), Malaysia
}

\begin{abstract}
Nowadays, stress is a common feature of everyday life. Stress is defined as a state of tension that is created when a person responds to the demands and pressures that come from work, family and other external sources, as well as those that are internally generated from self-imposed demands, obligations and self-criticism. Since 1960s, occupational stress has been associated with worker alienation, job dissatisfaction, mental health problems, concerns originating from the Industrial Revolution, mass production technologies, and the re-design of jobs. NIOSH (2008) defined work stress as "the harmful physical and emotional responses that occur when the requirements of the job do not match the capabilities, resources, or needs of the worker." However, this is one of premier studies on occupational stress with such a great distribution in Iran. Esfahan steel company organisation was established in 1959; diverse range of ESCO products in Iran and Middle East is unique.
\end{abstract}

Objectives: To determine the factors causing occupational stress among Esfahan steel company employees (Iran).

Methods: In Iran, workers are highly vulnerable to the risk of occupational stress. The big manufacturing part of steel industry in Iran is ESCO that is located somewhere near Esfahan and has approximately 6000 formal employees working in different unit. The design used in this study is Cross-sectional carried out in 2008 on 200 ESCO employees. For this research, the Depression Anxiety and Stress Scales (DASS) were selected since it is reasonably short to be easily answered by respondents.

Results: The distribution shows that the age of the respondents ranged between 20 and 58, the mean age of respondents was $35.3 \pm 7.2$ years. Majority of respondents, $167(83.5 \%)$ participants reported being married, 33(16.5\%) single or never married. The average number of years an individual employed was $11.8 \pm 7.4$ years. The result shows there is no significant relationship between stress and age, marital status, experience, literacy and shift work, but economy subject has a high significant relationship with stress. The result also illustrated there is significant relationship between work position and environmental of workplace $(P<0.05)$.

Discussion: On the basis of the present study results, it can be said that around half of the respondents suffer from stress. This result concurs with the findings in other similar studies in Iran but internationally and in comparison with industrial countries, Iranian people have a much higher level of stress.

\section{Exploring the relationship between managers' leadership and their health}

\author{
Lundqvist, D., Eriksson, A.F., \& Ekberg, K. \\ Linköping University, Sweden
}

Objectives: The relationship between managers' leadership and their health has been studied in 
two ways. Both emphasise psychosocial conditions at the workplace as important, but while one suggests that managers' health is a result of a match between conditions and their leadership, the other suggests that managers' leadership is a result of how conditions affect their health. The purpose of this paper is to deepen the understanding of how leadership and health is related by combining the two perspectives. What psychosocial conditions at work affect managers' health, and how does managers' health influence their leadership?

Methods: Semi-structured interviews were conducted with forty-two male and female managers at different managerial levels in a large Swedish industrial production company. All interviews were transcribed verbatim and analysed with qualitative content analysis.

Results: Managers' health is closely related to their own and their employees' performance. They stress the importance for their health of achieving the results that are expected of them. It is also of importance that their achievement is acknowledged and rewarded in the organisation. Performance and ability to achieve results are reported to be dependent of how favourable their psychosocial work conditions are. Unfavourable psychosocial conditions, such as low degree of decision latitude or control and/or bad social climate and uncooperativeness in the workplace, make the managers' work more difficult and prevent them from reaching results as expected, which in turn affects their health. The managers' health has a large influence on their leadership, mainly affecting the quality of their work and the quality of their relationship with subordinates. When the managers do not feel well they withdraw themselves, their work performance deteriorates, e.g. in decision making and problem solving, and they provide the subordinates with less support and guidance, affecting both production and health in the workplace. Thus, the managers are dependent of psychosocial work conditions to produce results, and the psychosocial work conditions are partly shaped by their health and leadership.

Conclusions: Managers' leadership, their health and their psychosocial work conditions are reciprocally related to each other.

Implications: To facilitate leadership that is productive and healthy for the workplace, improvement strategies should focus on the managers' psychosocial work conditions and organisational possibilities for fulfilling production goals.

\title{
The impact of subjective occupational success on personal resources
}

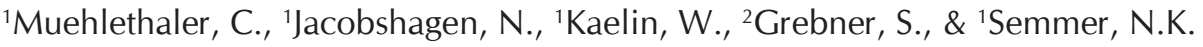 \\ ${ }^{1}$ University of Bern, Switzerland; ${ }^{2}$ University of Applied Sciences, Olten
}

Objectives: Self-esteem, self-efficacy and internal locus of control are among the most important personal resources (Hobfoll, 2001; Semmer, 2003). According to Fredrickson's broaden-and-build theory (1998, 2001) positive affective experiences build personal resources. Subjective occupational success (e.g., goal attainment, prosocial success, positive feedback) is such a positive affective experience. Occupational success has, however, not been studied widely in the context of occupational stress and resources. Since success experience affirm the self (Grebner et al., 2008), we expect that subjective occupational success will predict self-esteem, self-efficacy and internal locus of control over time. 
Methods: Data are from a longitudinal study in Switzerland, containing three waves, with half a year time lag. The sample consisted of 164 employees (60\% men). Subjective occupational success was measured with a short version of the SUCCESS-scale (Grebner, Elfering, Achermann, Knecht, \& Semmer, 2008) self-esteem with Rosenberg-scale (1965), selfefficacy with a scale by Krampen (1991), and locus of control with a scale by Sapp (1991).

Results: Regression analyses were run referring to different time lags. Sex, age and baseline values of the resources were used as control variables. Supporting our proposition self-esteem and internal locus of control were predicted by subjective occupational success over a time-lag of one year. The same results were obtained when using a time-lag of six months, the prediction of self-esteem from t2 to $\mathrm{t} 3$ being the only exception. All relationships were in the expected direction.

Implications and conclusions: We proposed that experiencing success at work builds personal resources over time. In this longitudinal study subjective occupational success predicted selfesteem, self-efficacy and internal locus of control. Therefore we conclude that occupational success experiences can help to generate important personal resources, which are essential for a better well-being and coping (Grebner et al., 2008). This has important practical implications: Organisations should provide a work environment where the experience of success is promoted.

\title{
The role of demographic factors in the experience of the work-home interface
}

\author{
MacKinnon, R. \& Gibbes, C. \\ Talent Q, University of East London, United Kingdom
}

Casper et al (2007) provide an excellent critique of existing research into the work-life interface (to which they refer as 'Work-Family research'). In their methodological review, they identify a number of weaknesses in the research base of this area, including: an over-reliance on quantitative methodology, the use of poor measures, a preponderance of unidirectional (work to home) investigation, and the inclusion of unrepresentative participant groups and thus ungeneralisable results.

This study aimed to address some of these weaknesses, specifically examining the work-life interface from a bi-directional perspective (work to home interference and home to work interference). Utilising an online survey, data were collected about employees' work-life interface, self-reported health status, locus of control and coping strategies when experiencing imbalance between work and personal domains. Additional demographic information including gender, sexual orientation and relationship status were also collected.

Analysis indicated that significant gender differences in the experience of positive work-home interference, full-time employees were significantly more likely to experience work to home interference and gay/lesbian employees were significantly less likely to report positive bidirectional emotional overspill between domains. Unsurprisingly, both work to home and home to work interference predicted higher scores on the GHQ12 health measure. 


\title{
Perceived Work Strain: A Comparison Between Models and Between Occupational Groups
}

\author{
${ }^{1}$ Magnavita, N., ${ }^{2}$ Garbarino, S., ${ }^{2}$ Ciprani, F., ${ }^{2}$ Cuomo, G., ${ }^{1}$ Bergamaschi, A. \\ ${ }^{1}$ Catholic University, Italy; ${ }^{2}$ Health Service of State Police Department of the Interior, Italy
}

Background: The social epidemiological approach for explaining the causes of work-related stress, that is commonly adopted in occupational medicine, suggests that certain work characteristics increase the susceptibility of the worker to the risk of job strain, with negative consequences for mental and physical health. The two leading models, that are claimed to have the most explanatory power are: the Job Demand/Control (JDC) model, developed by Karasek, and the Effort/Reward Imbalance (ERI) model, developed by Siegrist.

Objectives: The aim of this study was to explore the explanatory value of these two models in different groups of workers, addressing the relationship between stress at work and health outcomes, in terms of physical or psychological problems.

Methods: To compare the demand control model (DCM) and the effort/reward imbalance model (ERI), self-reports for psychological work conditions were obtained by 1183 workers belonging to 6 occupational groups: radiologists $(N=314$ workers), health care workers from a general hospital $(N=162)$ and from a specialized Infectious Diseases hospital $(N=217)$; clerks $(N=51)$; aged workers $(\mathrm{N}=197)$; policemen $(\mathrm{n}=292)$.

Results: Measured stress levels were significantly different between groups (ANOVA $p$ $<0.0001)$. Aged workers showed the higher levels of self-perceived work strain using the JDC model, while with the ERI model radiologist had the higher stress level. These two groups showed the lower levels of social support at work. Radiologists scored higher than other groups in overcommitment. Policemen committed in public safety actions during the G8 meeting in Italy surprisingly scored low, when measured by the ERI method.

Conclusions: The models, or dimensions of the models, made distinct contributions to explaining perceived work stress in different types of occupations.

\section{Violence Prevention in a Psychiatric Unit}

\author{
Magnavita, N. \\ Catholic University, Italy
}

Background: Aggression against nurses and ancillary personnel is a major, albeit underreported, occupational problem in long-term psychiatric health-care. Assaults may represent a problem also in social-sanitary facilities for psychiatric and demented patients. In such residences, staff is mainly constituted by unskilled workers.

Objectives: This study indicates strategies which have been adopted by a health-care facility to address violence, and evaluates the output of the preventive action. 
Methods: The frequency of violent incidents against health care workers in a residential rehabilitation unit has been assessed during medical visits at the workplace, in the 1996-2009 period. A violence prevention program, including educational, organisational, and medical measures, was developed in 2002. Interrupted time series analysis was used to determine the impact of the intervention.

Results: The majority of the workers had been subjected to physical aggression over time. Violent incidents were severely underreported and only 2 cases have had formal injury report. At the completion of the aggression minimisation program assault rate was significantly reduced.

Implications and Conclusions: The program proved to contribute to reducing violence at the workplace, and improved the quality of health care.

\title{
Skin Disorders and Work Strain in Hospital Workers
}

\author{
Magnavita, N. \& Bergamaschi, A. \\ Catholic University, Italy
}

Background: Eczematous hand dermatitis, a common skin disorder among hospital workers, is classified among psychophysiologic disorders, i.e., conditions that involve an interaction between the mind and the skin.

Objectives: The aim of this study was to evaluate the association of occupational stress with skin disorders in hospital workers.

Methods: 1744 hospital workers (767 male, 977 female workers) were invited to compile a questionnaire concerning the occurrence of skin disorders. Perceived work strain was assessed by the Demand/Control/Support model (Karasek). Anxiety and depression were assessed by the Goldberg's scales.

Results: 382 workers (21.9\%) reported work-related skin disorders at fingers and hands, that had occurred in the 12-months period before the examination; 138 of these workers $(7.9 \%$ of the exposed cohort) showed actual skin lesions. Workers with skin disorder showed significantly higher levels of job strain, and lower levels of social support than control workers $(p<0.001)$. Workers with eczema showed higher levels of anxiety and depression than other workers $(p<0.001)$.

Implications: Literature shows that there is a relationship between psychiatric and dermatological diseases. The incidence of psychiatric disorders among dermatological patients is estimated at about 30 to $60 \%$. Effective management of at least one third of the patients attending the skin department depends, to some extent, upon the recognition of emotional and psychological factors. Psychological and stress-reduction interventions were recently shown to improve patient well-being, and to significantly improve cutaneous manifestations. According to these data, hand skin disorders in hospital workers appear to be related to emotional states and stress. 
Conclusions: In an holistic perspective, the so-called 'glove dermatitis' is not merely an allergic disease, but a more complex condition, involving individual and organisational factors. The prevention of hand dermatitis in hospital workers should be based upon a multi-level approach.

\title{
The implementation of a new inspection tool in the psychosocial working environment: The experience of the Danish Working Environment Authority
}

\author{
Magwentshu, S. \& Gyldholm Møller, K. \\ The Danish Working Environment Authority, Denmark
}

Objective: The Danish Working Environment Authority (DWEA) launched a new strategy in 2007 to strengthen and qualify primary prevention of work related stress. Part of the strategy consists of increased inspections of the psychosocial working environment as well as the development and implementation of a new inspection tool, namely the 'guidance tool'.

Methods: Twenty four sector specific guidance tools have been developed to assist DWEA inspectors in assessing psychosocial working environment problems in all Danish enterprises. The guidance tools assists the DWEA inspectors in their assessment of the three to five most important risk factors in the specific sectors, (for example quantitative demands, emotional demands, and work related violence) as well as the most important preventive factors, e.g. quality of management, influence/control, and training. The guidance tools are based on recent research.

Results: The guidance tools enables all DWEA inspectors to make an assessment of psychosocial working environment problems, and thus ensures that both DWEA inspectors with and without specialist background are able to assess the psychosocial working environment. During the first years after implementing the strategy, an increasing number of inspectors have issued improvement notices regarding work related stress and violence, and there has been an increasing number of notices issued on several risk factors.

Implications: Overall, the tools have contributed to an increase in the clarity and comprehensibility of the improvement notices and have reduced the DWEA's time consumption per enterprise, both during the inspection and in the production of notices.

Conclusion: The guidance tools seem to be a suitable and efficient way of assessing several health and safety risks concerning work related stress and violence thereby allowing the DWEA to detect more of the existing problems. Future challenges projected for the strategy include continual training of all DWEA inspectors in the proper use of the tools and continuously refining the tools or developing new tools based on further research and experiences from practice. 


\title{
Using job strain and organisational justice models to predict the relationship between working conditions and employee performance
}

\author{
Maharee-Lawler, S., Rodwell, J., \& Noblet, A. \\ Deakin University, Australia
}

Objectives: Job stress research has tended to focus on the health and attitudinal outcomes associated with adverse psychosocial working conditions and, by comparison, much less is known about the relationship between job stressors and employee performance, particularly specific forms of performance. We aimed to address this gap in the literature by, first, assessing the extent to which working conditions accounted for explained variance in three forms of employee performance - namely in-role behaviour (IRB), organisational citizenship behaviour directed at the organisation (OCB-O) and at the individual (OCB-I) - and, second, by clarifying the nature of the relationships between these conditions and job performance through tests for direct, interaction and non-linear effects. The working conditions examined in the current investigation were based on the Job Strain Model (JSM)- incorporating additive and interactive demand, control, support models, as well as organisational justice theory.

Methods: A sample of 640 operational employees from an Australian police force took part in the current study via a mail-out survey. The questionnaire included the JSM variables, four organisational justice dimensions, and the three performance measures. Participant responses were analyzed using hierarchical multiple regression and these analyses included tests for direct, interaction and curvilinear effects.

Results: The results of the regression analyses indicated that a significant proportion of explained variance in all three performance measures was attributed to the additive JSM model. While the level of variance associated with the organisational justice dimensions was relatively small, there were indications that specific forms of justice may provide unique insights into the stressor-performance relationship. There was also some support for curvilinearity, particularly in relation to procedural justice items and the two OCB measures.

Implications: The results suggest that stressful working conditions may not only impact on employee well-being but may also influence employees' ability to perform important work roles. The findings also indicate that work-based resources, especially job control, social support and specific forms of justice (interpersonal and procedural), may offer useful avenues for preventing/ reducing stress-impaired performance.

Conclusion: Care needs to be taken when interpreting the above results, particularly given the design limitations (cross-sectional, self-report data). Nevertheless, there are signs that the same conditions that impact on employee well-being may also be associated with fluctuations in both in-role and extra-role performance. While the prominence of the JSM suggests that future stress-performance research should focus on job design features, there is some support for also including the more resource-oriented justice dimensions. 


\title{
Emotional labour in job-centre workstations and impact on third party violence
}

\author{
Manz, R. \\ German Stutatory Accident Insurance, Germany
}

Emotion work can been defined as the management of one's own feelings. It is work done in a conscious effort in order to maintain a good relationship (Hochschild, 1979). Emotional labor refers to the emotional work done in a paid work setting, while emotion work relates to any unpaid emotional work that a person undertakes. Fore example in their relationships with family and friends. Examples of emotion work include showing affection, apologizing after an argument, bringing up problems that need to be addressed in an intimate relationship or any kind of interpersonal relationship, and making sure the household runs smoothly. Emotional labour is often studied in the context of service work. Several types of emotion regulation are can be used in order to modify ones emotion within a service task.

Methods: Working in public unemployment administration offices (Job-centres) differs in certain ways from usual services. It is characterized by high demand of emotion control. Violence and harassment are typical occupational hazards in job-centres. Within a large-scale study on third party vio-lence in job-centres we try to explain employee's health complaints and violence by customers as a function of the type of emotion regulation used. We report survey data from 1581 employ-ees out of 12 job-centres. Emotion regulation is conceptualized within a model that includes the demand of emotion control and the experience of emotion within the service process.

Results: The model predicts 4 types of emotion management which are compared with respect to their health complaints and their risk for employees of being exposed to third party violence. Emotion control is not merely a necessary precondition for service, it also serves as a protection against third party violence. Insufficient emotion control is frequent and it turns out to be a high risk for third party violence.

\section{Learning occupational safety and health on the job - how effective is it?}

\author{
Masuhr, K. \& Wetzstein, A. \\ Institute Work and Health of the German Social Accidence Insurance, Germany
}

Objectives: In Germany the instruction of the employee in occupational safety and health by the entrepreneur is statutory and one of the basic measures for the prevention of accidents and occupational diseases. What is special in comparison to other learning settings is, that trainer and supervisor are the same. Furthermore the learning content is company- and workplacerelated. The practical realisation of this statutory demand as well as its impact on a safe and healthy behaviour of the employees still remains unknown. At the same time there is a growing market of providers for electronic instruction-software. These two facts lead to an increasing uncertainty of companies and Statutory Accident Insurances if these new materials are adequate for instruction. To give answers the recent study concentrates on two central questions: 
1. Which impact can be expected from instruction at the workplace?

2. Is the impact that can be expected from electronic instruction equal to that of personal instruction?

Instruction can be understood as short trainings for company-specific health and safety issues. The impact of training measures is described by Kirkpatrick (1998) on four levels. According to him instruction should

1. be accepted by the employers as a necessary prevention measure in the field of OSH,

2. lead to an enhancement of behavioural safety knowledge and it should influence the attitudes of the employees positively, 3. increase safe behaviour and, 4. lead to changes in relevant key data in $\mathrm{OSH}$ (management outcomes)

Methods: The sample consists of employees of the industrial department on one location of the enterprise. The 34 employees were randomly subdivided into three groups of different interventions. The first group received personal instruction. The second group was instructed electronically and the third group received no intervention so far. A pre-post experimental control group design was used. Questionnaires were used to measure the satisfaction with the instruction, the attitude towards occupational safety and health, the safety climate, behavioural competence to react properly in case of emergency. The company related specific knowledge in occupational safety and health was measured by a multiple choice test.

Results: To answer the question, whether instructions have an influence at all, the two groups which had been instructed (either personally or electronically) were compared with the group without instruction. The results show that these groups do not differentiate regarding the attitude towards occupational safety and health and the behavioural competence to react properly in case of emergency. The same could be observed for the comparison between electronic and personal instruction. However, instructed and non-instructed groups show certain differences in the results to the company-internal knowledge in occupational safety and health. While the noninstructed group shows a decrease, the instructed groups show improvement in the knowledge test. In comparison, the personally instructed group shows little advantage in the improvement of the test results. Due to the small sample the differences between the two groups as well as the little advantage of the personal instruction can not be confirmed by statistical significance.

Implications: The results reveal that instructions conducted personally or electronically lead to consolidation of relevant knowledge of occupational safety and health. This study shows that with instruction as a measure of prevention a basis for health and safety behaviour is established. Recommendations and statements for the use of electronic instruction should be made however only with consideration of the background of company-internal structures and processes.

Conclusions: The aim of instruction beyond the knowledge transfer is the promotion of safe and healthy behaviour. Therefore it is not sufficient to examine only the effectiveness of the media used for instruction. That means that it will be even more necessary in future investigations to ask how instructions can be embedded into the structure of a company and which processes e.g. during the preparation, execution and post-processing are of importance. 


\title{
Job strain and cardiovascular risk factors: Does social support have an attenuating effect for an ageing Irish workforce
}

\author{
Mc Carthy, V., Perry, I.V., \& Greiner, B.A. \\ University College Cork, Ireland
}

Objectives: To establish if there is an association between job characteristics as defined by the demand/control model (high-strain, active, low-strain and passive jobs) and cardiovascular risk factors. To establish if supervisor and co-worker support attenuates the association between job characteristics and risk factors for both paid and unpaid workers.

Background: Studies demonstrate a dose response relationship between exposure to job strain/ low control and Cardiovascular Disease and cardiovascular disease risk factors. Social support is recognised as an important component for the reduction of workplace stress. Work stress can be experienced in a paid or unpaid role (housewives or helping in family business). Ireland still has a high proportion of people working in farming with family members helping out in the family farming business. The latter are rarely recognised as 'workers'.

Materials and Methods: A 10-year follow up study was conducted on a cohort of the general population (59-80 year olds) in the Republic of Ireland. Anthropometric measurements, lipoprotein profile and data on medical history were collected on 179 study participants who were classified as having paid $(n=80)$, and unpaid work (housewives or helping in the family business), $(n=99)$. Almost a quarter $(n=18)$ of the paid workers were farmers and $18 \%(n=17)$ of the unpaid workers were farmers' wives. The job content questionnaire was used to assess job strain and support at follow-up. Cardiovascular risk factors were defined as: a prior doctor diagnosis of hypertension, a total cholesterol levels $>5$ mmols and waist circumference $>$ half their recorded height.

Results: Twenty-four percent of the sample had all three CVD risk factors. Using linear regression and the passive-job as reference, paid workers in the high-strain job have significantly less cardiovascular risk factors $(B=-0.46, S E=0.22, p=0.04)$. When stratified by gender, the findings for female paid workers were similar $(B=-0.78, S E=0.32, p=0.02)$. These associations remain significant when adjusted for co-worker support $(p=0.03, p=0.02$ respectively) but nonsignificant when adjusted for supervisor-support $(p=0.10)$. There was no significant association found when looking at housewives.

Conclusion: The effect of support at work appears to be dependant on whether it is from supervisors or co-workers. The Irish culture and the environment we are exposed to may have led to these unexpected results with regard to job strain and cardiovascular risk factors. However, this is a unique sample of ageing paid and unpaid workers, which warrant further investigation. 


\title{
The experience of salesperson engagement: A mixed-methods qualitative investigation of engagement in context
}

\author{
Medhurst, A. \& Albrecht, S. \\ Monash University, Australia
}

Context: Engagement has been highlighted as a key research area within occupational health psychology (OHP). Engaged employees demonstrate more functional work attitudes and behaviours and experience greater well-being. Although much quantitative research has explored this positive performance state, there is a lack of consensus regarding its theoretical base, nature, form, dimensionality and measurement, as well as the conditions under which it manifests, We argue that qualitative methods that explore employees' experiences of engagement may provide important insight into these areas.

Objectives: The purpose of the study was to qualitatively examine the conditions and antecedents that facilitate engagement; the characteristics or dimensions of the experience itself; stages in its development; and the outcomes of such experiences within the 'lived' experience of sales employees.

Method: A mixed-methods qualitative investigation was conducted with a sample of 14 salespeople from a large consumer goods enterprise. Ethnography was used to focus on participant descriptions of engagement within their daily work routines. The aim was to delineate patterns of action within the sales function and explore the conditions under which work engagement manifests. Interpretive phenomenological analysis was used to interpret the psychological and experiential nature, behaviour, and dimensionality of each individual's experiences by uncovering and interpreting the meaning behind participant narratives.

Results: Results revealed five core contexts related to salesperson work engagement. These encompassed a variety of engagement-related routine roles, situations, activities and tasks, namely: Job, Organisation, Team, Leader, and Client. Dominant cultural features were identified along with a variety of key antecedents and the necessary conditions for engagement. In line with definitions of engagement as a state-like psychological experience, results suggested that affective, cognitive, conative, and behavioural mechanisms are involved. For each of these mechanisms, a variety of characteristics emerged. A detailed interpretive account of the psychological experience of engagement at work as a positive phenomenon of human performance is posited, along with a conceptual process by which engagement manifests.

Implications and Conclusions: The results present a useful extension to the current literature by providing both a comprehensive description of engagement, and a deeper interpretation of sales peoples's lived-experiences of engagement. It is hoped that this study will further the emerging positive research agenda with OHP. In line with the definition of OHP as an applied science, the paper will conclude with implications for both researchers and practitioners, highlighting future research areas as well as potential areas for intervention. 


\title{
Anxiety and Locus of Control in Evaluation of Occupational Stress
}

\author{
Micali, E. \& Bagnato, C. \\ University of Messina, Italy
}

\begin{abstract}
Objective: Hypothesis of a relationship between occupational stress and mental suffering must be sought to document a causal link between environmental factors and damage-biopsychic in mensuretion and evaluation of occupational stress. This survey aims to assess a sample consisting of social health assistant working for Messina's University Hospital (Italy) the impact of levels of' anxiety (manifest and latent) on internal locus of control to highlight impact measurement and assessment of occupational stress sources.
\end{abstract}

Methods: Study sample consisting of 30 social-health assistants (22 females and 8 males) with chronological age between 35 and 50 years and average working seniority of 17 years, providing the setting in a maximum time of $45 \mathrm{~m}^{\prime}$ without interference and interruptions, was given a psycho-diagnostic protocol consisting of: Occupational Stress Indicated (OSI) and Anxiety Scale IPAT-ASQ. Entry criteria for subjects recruited implied: average level of education, dwelling the same geographical area and absence of organic nervous diseases that might interfere with the purpose of the study.

Results: With grids of correction for the 7 OSI variables was performed as the scoring detail, as mode of overall scoring and for Anxiety Scale IPAT-ASQ were recorded levels of anxiety: total, latent and manifest. The raw scores were converted into sten points. In the study sample results showed that: $40 \%$ recorded with anxiety sten middle level, locus of control and sources of stress for middle level.35\% had elevated sten for anxiety, sten for low and high perceived locus of control of sources of stress. $25 \%$ showed low levels of anxiety, adequate capacity locus of control and medium-low perception of the sources of stress

Implications: The confounding factors and variables that come into play in the measurement and evaluation of occupational stress are difficult to control so it is inevitable impact of "bias."

Conclusion: The levels of anxiety disorders and psycho-emotional impact on people's abilities to internal locus of control in various situations and relationships determined work with the mindset which believes that it can assess and control specific actions and circumstances beyond Our investigation has shown that high levels of anxiety and excessive anxiety specifically affect the latent capacity of the internal locus of control resulting in an 'excessive and distorted perception of the sources of occupational stress.

\section{Does starting the day with negative affective appraisal in the morning hinders recovery in the evening? The moderating role of employees' perceived job decision latitude at an international airport's hub section}

\author{
Michel, A., Turgut, S., \& Sonntag, K. \\ University of Heidelberg, Germany
}

Aim of this study was to examine how negative affective appraisal in the morning and perceived 
job decision latitude at work effect recovery in the evening among blue-collar workers at an international airport's hub section.

Based on the demand-control model (Karasek, 1979) and the effort-recovery model (Meijman \& Mulder, 1998), we tested the following assumptions in a diary study: firstly, it was assumed that negative affective appraisal in the morning is negatively related to recovery after work. Secondly, it was postulated that this negative relationship is moderated by job decision latitude at work. More specifically, we assume that the negative relation between negative affective appraisal and recovery is stronger for employees perceiving low levels of job decision latitude at work than for those perceiving high levels.

To test our assumptions, we firstly assessed employees' perceived job decision latitude at work and the control variables age and shift system at the person level. Secondly, we applied a daylevel measurement design to specify effects of negative affective appraisal on recovery processes. We tested our hypotheses by using multilevel analyses (HLM 6, Raudenbush, Bry, Cheong, Fgdon \& Du Toit, 2004) that fit the hierarchical data structure.

By using the described cross-level measurement design, the assumption that job decision latitude at work moderates the negative effect of negative affective appraisal in the morning on recovery in the evening could be confirmed.

Our results underline the importance of employees' job decision latitude at work with reference to their recovery after work. This is of great interest because research has shown that successful recovery after work plays a pivotal role with reference to both employees' wellbeing and work engagement (Sonnentag, 2003; Sonnentag \& Ziljlstra, 2006; Sonnentag \& Fritz, 2007). Thus, organisations should contribute to employees' recovery processes by offering high job decision latitude at work.

\title{
Exploring the multiple linkages between work engagement and health: An Empirical analysis within the Catalan nursing context
}

\author{
${ }^{1}$ Moodie, S., 'Dolan, S., \& ${ }^{2}$ Arsenault, A. \\ ${ }^{1}$ ESADE Business School, Spain; ${ }^{2}$ Montreal Institute of Cardiology, Canada
}

Objectives: This paper set out to explore the multiple linkages between facets of work engagement (using combined elements of Schaufeli and Bakker, (2003) and Shirom, (2003) and dimensions of both physical health (Cardiovascular risk) and mental health (depression, anxiety and burnout).

Methods: An empirical study was designed to test possible linkages between work engagement and certain personal health factors. Regression analysis was used to identify the contributing influences linking health and engagement. An online questionnaire completed by 1709 nurses working in the Catalunya region of Spain was used for the analysis. The selection of nurses as the object of study was intentional so that reliable metrics of personal health data could be obtained. Engagement was measured through the UWES scale and health related constructs included body-mass-index, obesity via waist circumference, self-rated overall health, depression and 
anxiety, and a range of somatic health measures assessing digestive, cardiac, neurological, and muscular complaints. A combination of ANOVAs and multiple linear regression was employed to test the relationships and identify quasi-causal profiles.

Results: Preliminary analysis confirms that the factors of depression, neurological somatic complaints, obesity, and self-rated health significantly are significantly linked to an employee's level of work engagement. By separating in-role and external health factors, we were able to identify multiple linkages between health and engagement.

Implications: While much of the research in work engagement focuses on work-related influences, it is important to identify both personal health factors that can impact employee work engagement and those that can be affected by low levels of engagement and burnout. By addressing the in-role and extra-role health factors of employees, managers can design intervention strategies to improve the overall quality of life of employees as well as levels of work-related engagement connected to productivity and health.

\title{
How is the "Swedish management model" related to organisational effectiveness?
}

\author{
Muhonen, T. \& Jonsson, S. \\ Malmö University, Sweden
}

A current overview of Swedish and international management research (Backström, Granberg \& Wilhelmson, 2008) maintains that the Swedish management tradition has special features. The Swedish way of managing and organising work is claimed to be characterised by teamwork, trust, cooperation, participation, consensus, independent workers and innovation. On the other hand, it is also regarded as being characterised by vagueness, a fear of making decisions and time-consuming alignment (consultative) processes (Backström, Granberg \& Wilhelmson, 2008; Gullers grupp, 2007). However, there is no agreement amongst researchers as to what a Swedish management tradition means, or if it is competitive and efficient in an era of rapid technological development and globalisation. IKEA is often mentioned as a successful example of the Swedish management tradition, with 128000 co-workers and 253 stores in 24 countries.

This study is part of a recently launched research programme called A global perspective on the Swedish management model, conditions and challenges. The overall aim of the programme is to examine different aspects of the Swedish management model and how it functions in a global context as represented by the IKEA-group. The specific aim of the present study is to examine how different aspects of leadership: change, production and employee oriented style (Ekvall \& Arvonen, 1991), empowering and fair leadership (Dallner et al., 2000) are related to effectiveness, here defined by both more subjective measures like employee well-being and more objective measures like sales figures. Psychological well-being is measured by the short version of General Health Questionnaire (Goldberg, 1972; Sconfienza, 1998).

Questionnaires are being distributed in three IKEA stores in Sweden and all the employees (around 300 in each store) have been requested to participate. The design of the study enables us to make comparisons between managers and co-workers, women and men and between the stores. In the next stage of the project a study with the same questionnaire will be conducted 
in two IKEA stores in China during April 2010. As IKEA has developed into a global megacompany purporting to apply the same management model all over the world, comparing the Swedish management model at IKEA in Sweden and in China is pertinent, especially as China is associated with other management and leadership traditions. Since the questionnaire study is being conducted during October-November 2009 we do not yet have any results at the time of this abstract submission.

\title{
Stress risk assessment in 20 Irish organisations 2008-2009
}

\author{
Murray, P. \\ Health \& Safety Authority, Ireland
}

Objectives: The state agency charged with promotion and enforcement of health at work undertook this project in partnership with private and public sector enterprises. 20 organisations across 4 industry sectors' education, health, public sector agency and semi state bodies were participants. Work related stress was the target and risk assessment/control measures were the interventions.

Its aims were:

1. to provide expertise to industry through its in-house Org Psychologist and to streamline its practical application

2. to develop out of the project case studies, results data, process information and improvement metrics for publication on the HAS website to guide non-users and to act as marketing tool for the use of the audit instrument, Work Positive

3. to provide evidence based findings of the differences in stressors for difference types of organisation and across different functional roles within and across organisation types. Its method included presentations, survey data, data analysis, cross-analysis, feedback, focus groups, targeted intervention, recording and on-going supports.

Findings from the groups ( 4 X 5 design) were compared and different primary strssors were identified in each of the 4 areas. Differences in stressors for sub categories within each group were also identified.

Conclusions: 1) the practical application of the tool brings its own stresses which should be pre empted so the process isn't in itself an added stressor; suggestions are made. 2) Case studies and supports were developed and are now on the HSA website for use generally and the tool is downloadable with no charge. Backup support can be availed of through the 20 organisations who participated and through their experiences 3) What stresses teachers is not what stresses nurses or office workers. Tips for each sector were developed in terms of likely stressors and likely interventions. Each job-role embedded in the organisational culture has to be taken into account for optimum intervention. 


\title{
Are There Personal Subtypes of Company Car Drivers?
}

\author{
Musicant, $\mathrm{O}$. \\ Ben-Gurion University of the Negev, Israel
}

Objectives: In many countries, work related crashes are a leading cause of death in the workplace. It was suggested that in addition to their personal dispositions regarding road safety, company car drivers are also exposed to work related factors such as the organisation safety climate. The purpose of this study was to identify subtypes of company car drivers based on their reported driving behaviours, road safety attitudes and safety climate perceptions.

Methods: 110 employees from a large organisation responded to a specialized questionnaire that was developed for this study. An exploratory factor analysis implemented on 34 questionnaire items yielded six factors: tendency for violations, attitudes toward overspeeding and alcohol use, opinion on law enforcement, driver ability to bend the rules when the situation allows, and also two factors describing drivers' subjective opinion about safety climate - work pressure, and management commitment to safety. The k-means procedure was undertaken to identify subtypes of company car drivers based on their score patterns. The unique properties of each subtype were studied by a series of ANOVA tests followed by Tukey post- hoc analysis.

Results: 3 subtypes (A, B, C) of company car drivers were identified; the "safe drivers" (subtype A) reported positive attitudes across all factors while the other 2 subtypes ( $B$ and $C$ ) reported higher involvement in traffic violations and "riskier" attitudes toward overspeeding and alcohol use. These 2 subtypes differ in their road safety attitudes as well as in their safety climate perceptions. Subtype B expressed positive opinion about law enforcement and disapproved of "bending the rules" even if the situation allows it, but they also expressed less approbation for management commitment to safety and to the way work pressure is being managed. Subtype C expressed the opposite pattern in their views. We propose that these subtypes' tendency for road violations and riskier attitudes toward overspeeding and use of alcohol stems from different reasons.

Implications: Understanding the attitudinal properties of company car drivers different subtypes can help create more effective road safety countermeasures. Moreover, the questionnaire we developed is relatively short but consists of multiple factors. Thus, it may serve as a practical tool for researchers and safety officers within organisations.

\section{Motives matter - a diary study on the relation between job stressors and sports activity}

\author{
Naegel, I. \& Sonnentag, S. \\ University of Konstanz, Germany
}

Objectives: High occupational stress levels contribute to the development of health problems and changes in health behaviours. In our study, we focused on the relation between job stressors and health behaviours. Particularly, we examined sports activity because studies have demonstrated that individuals tend to engage less in sports activity after a stressful day at work. The motives for 
engagement in sports activity have been addressed in many studies. However, no study has examined the role of motives on the relation between job stressors and sports activity. To reveal processes within persons' daily work lives it is crucial to analyze relationships on a day-level. Therefore, we conducted a diary study and investigated the moderating role of motives on the relation between daily job stressors and daily sports activity after work. In particular, we examined the relation between daily situational constraints and social stressors at work and the motive for revitalisation on sports activity. Revitalisation means recharging ones batteries during sports activity.

Methods: We conducted our diary study with 105 employees over five consecutive working days from Monday to Friday. We collected our data with a general paper-based questionnaire and daily surveys on pocket computers. Thus, for each participant, we gathered data at the person level (e.g., motives) and at the day level (e.g., daily job stressors). To analyze our data we used multilevel random coefficient modelling.

Results: Interestingly, we did not find a main effect of daily job stressors on daily sports activity. However, the motive for revitalisation moderated the relationship between daily job stressors and daily sports activity. On days individuals experienced a high level of job stressors they tended to engage in sports activity when they indicated a high motive for revitalisation. In contrast, on days individuals experienced a high level of job stressors they did not tend to engage in sports activity when they indicated a low motive for revitalisation.

Implications: After a stressful day at work individuals are indeed able to engage in sports activity depending on the individual motives they have. We identified the motive for revitalisation as an important factor to engage in sports activity after a stressful day at work.

Conclusions: When investigating relationships between daily job stressors and daily sports activity it is crucial to consider individuals' motives.

\title{
Emotion work and emotional exhaustion in teachers: What kind of job resources do they need?
}

\author{
${ }^{1}$ Naring, G., ${ }^{2}$ Vlerick, P., \& ${ }^{2}$ Van de Ven, B. \\ ${ }^{1}$ Radboud University Nijmegen \& Open University, the Netherlands; ${ }^{2}$ Chent University, \\ Belgium
}

Objectives: Many professions require that workers regulate their own and others' emotions. A relation of such emotional demands with negative outcomes is often, but not always found. In order to clarify this inconsistency in findings we investigated whether emotional and cognitive job resources moderate this relationship. We hypothesized that the positive relation between emotion work and emotional exhaustion would be weakened by the availability of emotional or cognitive job resources. Emotion work was not only operationalised as a job demand, but also as the self regulation characteristic emotional labor.

Methods: In a survey study 219 Flemish teachers participated. Emotional labor was measured with the Dutch Questionnaire on Emotional Labor (D-QEL) (Näring, Briët, \& Brouwers, 2007), which measures surface acting, deep acting, and suppression of emotions. Emotional job demands and emotional and cognitive job resources were measured with the DISQ 2.0 (de 
Jonge et al., 2007). Emotional exhaustion was measured with the Dutch version of the MBI (Schaufeli \& van Horn, 1995).

Results: In line with previous findings from other studies both emotional demands and emotional labor were related to emotional exhaustion. Regression analyses showed that the relationship between emotion work and emotional exhaustion was only moderated by cognitive job resources. Furthermore, in all analyses, there was a strong direct beneficial influence of cognitive resources on emotional exhaustion.

Implications: This study replicates several well-established findings. As in previous studies, emotional demands (Ybema \& Smulders, 2002) and surface acting (Näring, Briët, \& Brouwers, 2006) were related to emotional exhaustion. In teachers, emotional job resources are apparently less important as moderators of the relationship between emotional demands and emotional exhaustion than cognitive resources.

Conclusions: Even when facing emotional demands, cognitive resources are very important resources. The relatively unimportant role of emotional resources may be due to the solitary nature of teaching. The important role of cognitive resources can be a signal to schools to think about ways how to provide these resources on an institutional level.

\title{
Is Moral Disengagement a moderator within the Stressor-Emotion model of Counterproductive Work Behaviour?
}

\author{
${ }^{1}$ Natali, E., ${ }^{2}$ Fida, R., ${ }^{2}$ Mamazza, L., \& ${ }^{2}$ Barbaranelli, C. \\ ${ }^{1}$ National Institute for Occupational Safety and Prevention (ISPESL), Italy; ${ }^{2}$ University of Rome \\ 'Sapienza', Italy
}

Objectives: This study is aimed at investigating the role of moral disengagement with respect to the relation among organisational frustrations and counterproductive work behaviours. The theoretical frames of this study are: a) stressor-emotion model of Counterproductive Work Behaviour (Spector \& Fox, 2004); b) social cognitive theory of moral reasoning (Bandura, 1986).

Counterproductive work behaviours are volitional acts aimed at harming organisations or people in organisations. These include aggression, hostility, sabotage, theft, and withdrawal. According to Stressor-Emotion model, these behaviours arise from a number different personal as well as situational variables. Situational conditions are mainly stressful job conditions. Personal variables are related to emotional and cognitive processes that mediate or moderate the counterproductive response. Among these different processes it seems to be particularly relevant to consider the mechanisms of moral disengagement through the use of which regulatory self-sanctions can be selectively disengaged from the effects of a detrimental conduct (by converting harmful acts to moral ones, obscuring personal causal agency, misrepresenting or disregarding the injurious effects inflicted on others, vilifying the recipients of maltreatment).

In this study we considered stressful job conditions such as the Interpersonal Conflicts at Work, Organisational Constraints, Quantitative Workload, Physical Symptoms, and Negative Acts directed to the subject. These conditions were considered as independent variables with 
respect to counterproductive work behaviours. Within this nomological network, the role of mechanisms of moral disengagement related to organisational transgressions was investigated. In particular, following previous experimental research on the role of individual differences in frustration-aggression hypothesis (Caprara, Perugini \& Barbaranelli, 1994), we hypothesized that moral disengagement acts as a moderator, so the more people use mechanisms of moral disengagement, the stronger is the relation among stressful job conditions and counterproductive behaviours.

Methods: A specific questionnaire has been developed included measures of Interpersonal Conflicts at Work, Organisational Constraints, Quantitative Workload, Physical Symptoms, and Negative Acts.

Results: Results of structural equation models on a sample of about 700 Italian adults supported the evidence of the moderating action of moral disengagement in the causal network linking stressful job condition to counterproductive work behaviours. Alternative models where a mediational role of moral disengagement was posited were not consistent with empirical data.

Implications and conclusions: On the basis of the present study, further research should be finalized to study the relationship among moral disengagement construct and other aspects at work as work-related stress or counterproductive work behaviours.

\title{
HSE Management Standards and work-related stress: Italian translation and validation of the Indicator Tool
}

\author{
Natali, E., Martini, A., Ronchetti, M., Rondinone, B., \& lavicoli, S. \\ National Institute for Occupational Safety and Prevention (ISPESL), Italy
}

Objectives: Over the last years, stress has been recognized as a potential work-related risk factor. Unfortunately, work-related stress is a very delicate subject, especially because it is difficult to assess it objectively and in broadly acceptable terms. In fact, work-related stress is a subjective, personal response to a specific work environment, and is of a multifactorial origin. Nowadays work-related stress affects $22 \%$ of all workers in the EU and it accounts for more than $50 \%$ of lost working days (European Agency, 2009). In the past years, a specific European regulation was issued (i.e. European Directive 89/391) and an important European Framework Agreement was signed in 2004 by the European social partners on work-related stress.

In view of that, a number of European institutions suggested specific models in order to assess and manage the causes of work-related stress. Among these models there are the six Health and Safety Executive (HSE) Management Standards. HSE suggests a global approach including a questionnaire validated in the UK called the Indicator Tool (Mackay et al., 2004; Edwards et al., 2008, Cousins et al., 2004). The Indicator Tool assesses the six key dimensions associated with levels of health and wellbeing at workplace, known as the six Management Standards. These are: Demands, Control, Support, Relationships, Role and Change. The questionnaire, already translated in many languages like Arabic, Chinese, Russian and many more, consists of 35 items using a 5-point Likert scale. The aim of this work is to translate and validate the Indicator Tool in Italy, as the first step towards the implementation of the global HSE framework as an important European model in compliance with relevant national and international regulations. 
Methods and results: A preliminary version of the questionnaire is completed and the pre-testing phase took place from October to December 2009. The following step is the dissemination of the final version of the Indicator Tool at national level. The public and private organisations are covered, including a large part of the productive sector. The results are expected to confirm the English factorial structure in the Italian version.

Implications and conclusions: The aim of this work is to provide a practical tool for the assessment of work-related stress. This tool is expected to be a cross-culture validation and the results provided might confirm that hypothesis. Finally, among long-term objectives is the implementation of the global and participative HSE model for managing work-related stress in the Italian context.

\title{
Diversity management in northern Italy: Value or Bond?
}

\author{
Negri, L. \& Briante, G. \\ University of Turin, Italy
}

Organisations, groups and society as a whole are affected by global and local changes. The future will see a diversification of cultures, practices and way of doing business across the countries but also inside them because of the growing diversity of the workforce. Diversity emerged as an autonomous research domain in the 1990s in the USA (Johnston \& Packer, 1987), highlighting the increasing shift of the workforce from white and male to black and female. In most recent literature on workplace diversity, a wider definition of diversity has been given, considering not only "classical" distinction criteria (gender, race, ethnicity or functional background), but defining diversity as "any attribute that may lead to the perception that another person is different from self" (Van Knippenberg, De Dreu \& Homan, 2004, p. 1008). Thinking at diversity as a value inside the organisations instead as a bond can represent a challenge for organisations in the new economy, shifting the attention from norms that force organisations to include diversity to a way of considering diversity in thinking, acting and behaving inside companies, as a way to better face complexity and uncertainness.

The present study investigates how Diversity Management (DM) is perceived in a sample of companies in the north of Italy.

The HR managers of 13 companies have been interviewed on their representation of DM in Italy and about their experiences inside their companies, comparing them with equal opportunity norms adoption. As Stakeholders they have been requested to express their opinion on how to facilitate the diffusion of DM in our Country. Data were content analysed and show main differences between companies on their knowledge about DM and how to make profitable the growing diversity of the workforce (immigrants). However most of the companies share the view of DM as a way to enhance open-mindness through problem solution, showing positive effects on team performance. Data will be commented on the light of the two main theories related to DM (Social categorization and Information-Decision making, Cfr. Van Knippenberg \& Schippers, 2007). 


\title{
Psychophysiological and Personal Characteristics of Marine Cadets: Estimation of Professionally Essential Qualities During Final Examination on the Radar-Tracking Simulator
}

\author{
${ }^{1}$ Nezavitina, T. \& ${ }^{2}$ Vasylyeva, J. \\ ${ }^{1}$ Ukrainian Research Institute for Medicine of Transport, Ukraine; ${ }^{2}$ Training Center of Seafarers \\ AVANT
}

Objectives: The main purpose of the study was to define physiological and psychological covariates of adaptation capacity and degree of Professionally Essential Qualities (PEQ) developed in the contingent of Marine Academy cadets-graduates. The psychophysiological characteristics of cadets-graduates during examinations and influence of considerable intellectual loadings and emotional stress on dynamics of efficiency in the future occupational activity of seafarers were investigated.

Methods: Research was conducted during the final examination of marine cadets $(\mathrm{N}=58)$ on the Radar-Tracking Simulator (RTS) modeling real conditions of a vessel navigation examination by over than 30 parameters. Methods of memory estimation (Benton Visual retention Test), logic intellection (Raven Test), spatial visual perception ('Following the target' test), an emotional condition and personality features (projective tests) were applied. Investigation was conducted directly before and after passing of examination on RTS. Along with known valid and the standardized psychological methods we used experimental methodology and the questionnaires designed at Ukrainian Research Institute for Medicine of Transport; we also used methods of an expert psychological estimation of the emotional state \& situational behaviour of cadets.

Results: The analysis of risk tolerance as personal characteristics has great value for psychological forecasting of decision-making process in an uncertainty situation. According to the risk tolerance survey cadets have been divided on two groups: persons who prefer the work connected with risk (1) and persons who do not (2). Significant distinctions between these groups of examinees testify that group (2) is characterized by longer time of performance of logic intellectual tasks by Raven Test in comparison with group (1) $(t=3,2, p 0,01)$. For group (1) there were more signs of graphic organic symptoms complex $(t=3,2, p 0,01)$ and degree of double lining $(t=3,0, p 0,01)$. In the present research there was specified a group of 'demonstrative' persons $(n=11)$ who had significant distinctions from the other 'non-demonstrative', such as higher degree of emotional strain $(t=3,2, p 0,01)$, relatively worse psychophysiological status $(t=2,1, p 0,05)$, indicated more errors of memory retention $(t=2,0, p 0,05)$, and more degree of double lining $(t=2,4, p 0,05)$, and these 'demonstrative' cadets generally were not much successful passing examinations with average mark 3,72 $\pm 0,23$ (5 maximum). At the same time 'demonstrative' cadets on the moment of examination had significantly longer navigation experience $(t=2,1, p 0,05)$ in comparison with 'non-demonstrative'. On the basis of a correlation matrix the factorial analysis with a view of research of internal links between various elements of system has been conducted. On the basis of the data of the factorial analysis it was revealed four groups of personal characteristics and four groups of PEQ united in two groups of factors. We called the 1 st personal factor as 'emotional expressiveness' as it included indicators of anxiety, a psycho-emotional strain and emotional breadth. The 2nd factor was called as "psycho-vegetative" as united indicators of a functional condition of an organism, and could reflect type of psychophysiological adaptation to stressful environment. The 3rd factor "psychosomatic" was defined avoiding type of behaviour in stressful situations and somatic type of response to alarms and emotional pressure. The 4th 
factor of 'vegetative anxiety' included systolic pressure index and situational anxiety. Factors of PEQ have appeared grouped round following indicators: 1-success of performance of the Raven Test, a 2-error in 'Following the target', a 3-psycho-emotional strain, 4-signs graphic organic symptoms complex. According to the leading indicators, the factors of PEQ defined logic intellection, spatial perception, occupational health and adaptation capacity.

Conclusions: The data received as a result of the researches allow estimating the basic correlations between personal features of marine cadets and their successful performance of the simulator examination tasks. Persons with a high level of development of the majority of psychophysiological features have the best ability to qualitative and quantitative information processing and higher mental activity. The method of 'Following the target', the Visual Retention Benton Test and the Raven Test have appeared relevant for the purpose solution, and demand the further standardization for the contingent of marine cadets.

\title{
Does training improve participant well-being? The mediated moderation of work environment factors that influence the effects of training
}

\author{
${ }^{1}$ Nielsen, K. \& ${ }^{2}$ Daniels, K. \\ ${ }^{1}$ NRCWE, Denmark; ${ }^{2}$ Loughborough University, United Kingdom
}

Objectives: Many organisations devote considerable time and resources to establishing and developing teamwork. A recent review of task restructuring interventions, which included the implementation of teamwork, reported inconsistent results; some studies report positive effects whereas other report no or even negative effects. The authors suggested problems with implementation may account for these differences. One of the few studies to directly examine problems with team implementation found that important barriers for establishing effective teams were associated with managerial behaviours. The objectives of this study are two-fold: 1 ) to examine whether training middle managers (who were responsible for implementing teams) to implement and manage teams enables middle managers to deal better with the demands of the job and ensure their well-being, 2) to examine which factors in the working environment which may promote or hinder the transfer of training.

Methods: Fifty-eight middle managers and their employees participated in the study. A randomised controlled design was used where half of the middle managers received a tailored training course on how to implement and manage teams. Using electronic diaries middle managers completed questionnaires on their subjective well-being and their opportunities to use their skills pre-and post-intervention (each time over a two-week period, immediately prior to the intervention and 18 months post the intervention). Eighteen months post-intervention, employees reporting to each manager completed a questionnaire rating their previous experience with teamwork and their readiness for team implementation.

Results: Multi-level analyses explored the moderated mediating mechanisms of process factors. They revealed that middle managers who had received training in how to implement and manage teams experienced increases in opportunities to use their skills, challenge at work and subjective well-being than the control group if their employees reported not having worked in teams previously and welcomed the idea of team implementation (readiness for change). There was a subsequent 
decrease in the experience of skills/challenge for those middle managers who received training but managed groups with low readiness for change but with experience of working in teams. For these managers, decreases in skills/challenge were associated with lower levels of well-being.

Discussion: The results point to the importance of providing middle managers with the skills to plan and implement teams in order to ensure their subjective well-being through providing them with skills to cope with the changed nature of their job. However, it also points to the importance of considering the context; where employees were not receptive to teamwork middle managers experienced poorer well-being.

\title{
The work-stress relationship: Examining the independent contribution of organisational justice
}

\author{
'Noblet, A., ' Rodwell, J., 'Demir, D \& ${ }^{2}$ Steane, P. \\ ${ }^{1}$ Deakin University, Australia; ${ }^{2}$ Macquarie University, Australia
}

Objective: The overall objective of the current study was to examine the extent to which perceptions of organisational justice could account for variance in stress-related outcomes after controlling for the effects associated with more ubiquitous job-design stressors, namely, job demands, job control and social support. Conceptually, there is considerable overlap between the job design stressors and dimensions of justice and identifying the independent effects of fairness perceptions is critical for determining the extent to which job stress research should be guided by organisational justice theory.

Method: The study sample consisted of 146 administrative staff working in a medium to large healthcare organisation in Australia. Participants completed a survey measuring the justice and job design variables, as well as four stress-related outcomes (psychological wellbeing, psychological distress, job satisfaction and organisational commitment). A notable feature of the current study, in comparison to previous justice-stress research, was that the resource-oriented job design variables (i.e., social support and job control), were operationalized according to disaggregated definitions that allowed for increased levels of stressor-resource matching to occur.

Results: The results of the regressions indicated that the additive effects of demand, control and support accounted for the vast majority of the explained variance in all four health outcomes. The effect sizes of job control and social support were particularly strong in relation to job satisfaction and organisational commitment. Although the amount of adjusted R2 attributed to the additive effects of organisational justice was relatively small, the proportion of variance associated with the justice dimensions was significant for two of the outcome measures (job satisfaction and psychological wellbeing).

Implications: The results of the current study suggest that previous justice-stress research may have overestimated the role of justice in the stress process and that when disaggregated measures of the design variables are utilized, the effects associated with fairness perceptions are considerably smaller. 
Conclusion: Further research incorporating the disaggregated resource measures is required to develop a firmer understanding of the comparative influence of the job design and justice variables, particularly given the constraints of the current study (cross-sectional, single-method design). While there were strong indications that demand, control and support play key roles in the stress experienced by employees, there were also signs that dimensions of justice were closely associated with some of the employee-level outcomes. There is therefore potential for stressful working conditions to be overlooked if the measures of fairness are not taken into account.

\title{
Perspectives of workplace health promotion
}

\author{
Noehammer, E., Schusterschitz, C., \& Stummer, H. \\ University for Health Sciences, Austria
}

Objectives: Health promotion at the workplace is a wide-spread concept targeted at improving the health of the employees, encouraging mutual health related learning and the experience of self-efficacy, creating a health sustaining work environment, and a healthy organisation in the end (see for example ENWHP, 2007). However, the perspective of the workforce is largely ignored in research (Bödeker/Hüsing, 2008), which is why most concepts and approaches are based on experts' opinions. Frequently, the employee's wishes and desires are only taken into account in actual workplace health promotion (WHP) programs, but not in scholarly studies. This can lead to a misunderstanding regarding the determinants of participation in WHP, the possible barriers, and the potential effects and benefits of WHP. This research gap impedes progresses in understanding what makes WHP and other health promotion approaches attractive and effective for the target-group. Moreover, improvements in the programs based on such findings are delayed. In order to bridge the existing knowledge gap, our contribution contrasts the perspectives of selected experts in the field of WHP are with our findings regarding the employee's perspective.

Methods: The research, divided into a qualitative pre-study and a larger scale quantitative investigation, was conducted in Austria as an example of the German speaking countries in Europe, where a unique approach to WHP is taken.

Results and Implications: The results indicate that for example the socio-emotional and cognitive components related to health promotion in work settings are highly relevant for employees. Experts, at the same time, hint to a potential increase in the socio-emotional importance of the workplace due to a changing value system within society, which could promote active participation in WHP. Nevertheless, in addition to these re-enforcing tendencies, also potential pitfalls can be observed, as experts expect a reduction in the freedom of choice of whether to participate or not, which is a major criterion for interested staff.

Conclusion: Our contribution highlights the divergences as well as overlaps of expert's and employees' opinions concerning WHP programs and shows possible ways of integration. 


\title{
Creating and maintaining individual well-being: The potential of worksite health promotion
}

\author{
Noehammer, E., Schusterschitz, C., \& Stummer, H. \\ University for Health Sciences, Medical Informatics and Technology, Austria
}

Objectives: Drawing on motivation theory (Heckhausen \& Heckhausen, 2006), it can be assumed that well-being depends on personal disposition and situational context, which creates a dynamic interdependency. However, a multifaceted concept is not only complex and thus more difficult to understand and explain, but also offers various possibilities of intervention. Our contribution addresses this idea by analyzing the potential of worksite health promotion (WHP) to improve individual well-being from the employees' perspective, which is a new strand of research (Bödeker \& Hüsing, 2008).

So far, it has been assumed by experts that beneficial effects of WHP for individuals can be expected on the physical, emotional, and social level (Westermayer \& Wellendorf, 2001). An own qualitative study showed that employees also describe other aspects and evaluate WHP effects differently than experts.

Methods: In a quantitative study with 236 participants in four organisations the effects of WHP on well-being from the employees' perspective were further investigated.

Results: Our results not only show that WHP has a positive impact on employee well-being, but based on a factor analysis also that the emotional components of perceived benefits of participation are felt the most. Almost $50 \%$ of the respondents for example experience joy due to program participation and feel WHP helps them care better for their health. Feeling better physically also highly correlates with cognitive aspects like gaining health related knowledge. Social outcomes, like team climate improvement, are less perceivable.

Implications and Conclusions: Our results lead to the assumptions that (1) the current theoretical grouping of potential WHP effects should be revised, (2) research on WHP could gain from a consideration of the importance of emotions in the workplace and (3) strengthening the emotional components of WHP information and offer design could lead to higher participation rates.

\section{Managing Psychosocial Risks for Homeworkers}

\author{
O'Hara, R. \\ University of Sheffield, United Kingdom
}

Objectives: This paper details findings from research examining the management of psychosocial risks by organisations employing homeworkers. While the increased use of information technology (teleworking) has added to the numbers of people working at home, more traditional forms of homeworking such as sewing, packing, assembly, and soldering remain widespread. In the current difficult economic climate, organisations seeking ways to reduce costs are also increasingly likely to consider homeworking. The isolated nature of homeworking poses 
particular psychosocial risks and requires more specific risk management strategies than simply applying measures used for on-site workers. The aim of this research was to identify examples of such measures.

Methods: Semi-structured interviews were conducted with homeworkers and representatives from companies employing homeworkers, including owners, directors and health and safety managers. Twelve employing organisations were visited and 31 homeworkers were interviewed.

Results: Both homeworkers and their employers identified benefits and difficulties associated with homeworking. A number of psychosocial risks were identified in connection with the relative isolation of homeworkers compared to their on-site counterpart. These risks included lack of social interaction, lack of social support, lack of supervision and the potential for poor work scheduling. A number of measures designed to minimise these risk were identified. These included systems to facilitate formal and informal communication between employers and homeworkers. Also, measures that enabled homeworkers to communicate amongst themselves and with on-site employees. More detailed examples of these measures will be presented.

Implications and Conclusion: With effective risk management strategies homeworking can be a safe and not just a cost effective alternative to on-site working. Occupational Health Psychology practitioners have a role to play in raising awareness of the specific psychosocial risks faced by this less visible workforce and advising on management strategies. The findings from this research are intended to provide examples of such strategies.

\title{
Relaxed Entrepreneurs: Approaches to Stress Management and Burnout Prevention
}

\author{
Olos. L.
}

\author{
Free University Berlin, Germany
}

Business owners and self-employed individuals are, especially in the early years of their careers, highly committed and "burn" for their work. Against a background of work and time pressures, career uncertainty, and the dissolution of the boundaries between work and private life, however, this phase of life is often extremely demanding and may pose the risk of selfexploitation and "burnout". The planned presentation aims to discuss preventive measures such as stress management training.

The first part of the presentation will summarize findings of empirical studies showing that entrepreneurs and new business owners are exposed to particular risk factors, including long working hours, negotiations, conflicts in personal life, juggling multiple tasks, post-decision uncertainty, difficulty reconciling work and private life, and a precarious financial situation. The concentration of high-pressure situations increases continuously after founding a business and the autonomy of the owner can shift to a strategy of self-exploitation. Compared with employees, small business owners and self-employed individuals take greater health risks, and a considerable proportion of new business owners tend to demonstrate risky patterns of health behaviour. Given these risk factors and the associated danger of burnout, preventive measures are particularly important in this context. 
Drawing on the author's own empirical findings, the presentation will outline examples of both the risk factors identified and of the strategy of self-exploitation adopted by small business owners and by self-employed psychologists and physicians. The main part of the presentation focuses on ways of preventing burnout in entrepreneurs and the self-employed. Based on established health promotion measures, some approaches tailored especially to this target group will be proposed, using the example of a newly developed stress management training program for new business owners. Finally, possibilities of incorporating preventive measures into support programs for new business owners will be discussed.

\title{
Satisfaction with work-family balance, organisational culture, and stress reactions: Results from an academic institution
}

\author{
Omori, M. \\ Ochanomizu University, Department of Psychology, Japan
}

Objectives: The challenge of balancing work and life is relatively new to the Japanese society. The government finally provided a guideline concerning work-life balance in 2007, which started to direct attentions of individuals and organisations to this issue. In recent years, scientific efforts have demonstrated links between work-family balance or conflicts and psychological health. Although in the work-family literature, conflicts between work and "life" are often considered to a source of strain and stress, a positive side of work-life balance or satisfaction with work-family balance(SWFB) is not much examined. Therefore, focusing on SWFB, the present study was designed to examine whether SWFB mediate relationships between organisational characteristics and stress reactions. In doing so, the present study was conducted with employees at an academic institution because findings about occupational health of this population have not been sufficiently accumulated.

Methods: An anonymous survey was conducted at a national research institute specializing in environmental science and plant biology. Participants were 543 employees (155 female and 379 male; 64 executive managers, 191 administrators, and 199 research scientists, 67 technical staffs and others). The average age of the study participants was $43.4(\mathrm{SD}=10.1$ ). The average year at the institution was 16.1 year $(S D=10.1)$. Participants were asked to complete a packet of questionnaires measuring SWFB, job involvement, organisational commitment, work-family culture in the organisation, occupational stressors, and stress reactions.

Results: Organisational factors indicated interesting associations with different types of stressors and stress reactions. SWFB was negatively correlated with most of the stressors and stress reactions measured ( $r=-.11$ through -.47 for stressors; $r=-.24$ through -.42 for stress reactions). Similarly, job involvement and organisational commitment showed negative correlations stressors and stress reactions. It was implied that those who have positive view of the organisation tend to feel less stressed. A series of hierarchical regression analyses were conducted predictive in order to examine relationships among organisational culture, stress reactions, and SWFB. SWFB was found to mediate between organisational culture and stress reactions of employees. Implications of the findings will be discussed in terms of the development of organisational structures and work-life balance practices. 


\title{
The Working For Wellness Program: Evaluation of An Evidence-Based Employee Well-Being Program Using Rct
}

\author{
Page, K. \& Vella-Brodrick, D. \\ Monash University, Australia
}

Context: The study of employee well-being as a positive, psychological phenomenon is still in its infancy. Traditionally, occupational health psychology (OHP) has focused on psychological risk factors such as burnout and stress or on physical health and safety. This presentation will address this omission by describing a positive approach to defining and promoting employee well-being. This approach aligns with the positive psychology movement which defines employee well-being as the presence of positive feelings and functioning.

Objectives: To design and test an evidence-based employee well-being program using a randomized controlled trial (RCT). A key concern was whether employees could reliably and sustainably increase their well-being by applying positive psychology-based strategies.

Methods: Participants were 50 employees of a large, government health organisation $(80 \%$ female; mean age 41 years; mean tenure 10 years). Employees were randomly allocated into an intervention or control group. The intervention group participated in a six-week employee well-being program called the Working for Wellness Program. The control group did not participate in the Program. All participants completed questionnaires regarding their subjective, psychological and workplace well-being. Questionnaires were completed pre-intervention and post-intervention (one week, three month and six month follow-ups).

Results: Data were analysed using a 4 (time) x 2 (group) mixed ANOVA design. Results revealed significant time by group interaction effects for well-being. Specifically, participants in the intervention group showed a significant decrease in negative affect and significant increases in psychological well-being and work-related positive affect over time, in comparison to the control group.

Implications: Findings suggest that employees can learn effective strategies for sustainably increasing their well-being. This finding is encouraging for organisations and health professionals that strive to promote employee well-being as a positive psychological phenomena as well as the mitigation of psychological or physical risk.

Conclusion: OHP professionals recognise the importance of fostering employee mental health. However, research to date has tended to define employee well-being from an illness perspective rather than a health promotion perspective. Designing and testing methods that can reliably and sustainably increase employee well-being has been highlighted as a key area for future OHP research. This study describes the results of one such study, utilising an experimental design. It is hoped that future research replicates, extends and builds upon these results, with the aim of creating safe, healthy and well workplaces for current and future generations. 


\title{
Development of a survey for evaluating expert work in safety critical organisations
}

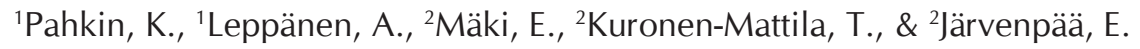 \\ ${ }^{1}$ Finnish Institute of Occupational Health, Finland; ${ }^{2} \mathrm{Helsinki}$ University of Technology, \\ Finland
}

Objectives: Maintaining and developing organisational expertise is an essential element of high-quality performance of safety critical organisations. Safety critical organisations (such as nuclear power plants) must pay extra attention to their employees' knowledge, expertise, and competencies. The objectives of this four-year research project are 1) to examine the nature of expert work in safety critical organisations, and 2) to discover how HR-functions can support the development and preservation of expertise in safety critical organisations.

Methods: To formulate and validate a questionnaire for analyzing expert work practices in safety critical organisation three datasets from different nuclear organisations will be used: A survey (in 2008) and its follow-up (in February 2010) and thematic interviews (in 2009).

Results: In 2008, a survey was carried out in order to analyze and evaluate the content of learning organisations e.g. organisation of work, actions of the supervisors and their connection to wellbeing, motivation and competence development of the experts. The survey called "Towards better working community" developed by the Finnish Institute of Occupational Health (FIOH) was used as a basic questionnaire. Because of the context in which the survey was conducted, the aspects of the safety critical environment had to be taken into account. This was done by developing new questions to be added to the survey. The construct and predictive validity of the new scales were tested. Internal consistency of the scales varied between 0.54 and 0.91 measured by Cronbach's alpha.

In 2009, the questions concerning safety critical environment/safety culture were evaluated. 12 persons from the target organisations were interviewed on how the safety critical aspect of their work should be taken into account in management and team working. These interviews suggested some modifications for the questions.

The follow-up of the survey will be carried out in February 2010. The construct and predictive validity of the modified scales will be then tested again.

Implications: The goal is to develop a tool (a set of survey questions) with which the safety critical organisations can evaluate their actions/operations safety promoting behaviour in different organisational levels.

Conclusion: The set of survey questions provides a basis for implementation of organisational development and interventions, and for documentation of changes in working conditions. This will strengthen the participative approach of work life improvement. 


\title{
Does the success of change management effect employees' well-being?
}

\author{
Pahkin, K., Leppänen, A., Ala-Laurinaho, A., \& Kajosaari, K. \\ Finnish Institute of Occupational Health, Finland
}

Objectives: At the beginning of 2008, the Finnish Institute of Occupational Health (FIOH) launched a two-year study to examine the current state of occupational well-being among paper industry workers. The project request came from the Finnish Forest Industries and the Finnish Paper Workers' Union, and the research is funded by the Finnish Working Environment Fund. Paying attention to occupational well-being was seen as important, at a time when the forest industry is going through an exceptionally extensive transition.

Methods: Eight production facilities took part in the study. In the spring of 2008, a questionnaire was sent to all blue-collar employees (response rate was $52 \%, \mathrm{n}=1$ 955) and in autumn 2009, a follow-up was carried out (response rate $48 \%, n=1518$ ). During the study period, all the participating units had faced organisational restructuring: In one unit downsizing was already going on during the first survey, three units were downsizing before the second survey, and one unit was sold to a foreign owner. The restructuring meant outsourcing, lay offs, notices. In addition, one of the units was closed down. To find out how successfully the management of change process was carried out, a set of questions related to the actions of the management and supervisors was formulated based on the literature and to the interviewees carried out in the project.

Results: The construct and predictive validity of the new scales were tested. Internal consistency of the scales was good (the Cronbach's alpha of management's actions was 0.92 and of supervisor's actions 0.94). Based on factor analysis, both sets of questions differed from other questions related to the management's and supervisors' actions at the workplace. Three groups based on the satisfaction of actions carried out were formed and the extreme groups (dissatisfied and satisfied) were taken in to the further analysis. All measures of employees' well-being (level of stress, work ability, job satisfaction, and trust to the future of work) were significantly better among group of employees who evaluated that the management or their supervisor had satisfactorily carried out the change process (t-test).

Implications: The set of questions formulated to measure success of different actors during the change process can be used as a tool to evaluate change management process, which is important when future actions are planned and carried out.

Conclusions: It is important to pay attention to the success of the change management because it is clearly related to well-being of employees. 


\title{
An Analysis Of Health Care Employees Experiences Of Mobbing Behaviours At Workplace; A Case Study Of Southern Marmara Region In Turkey
}

\author{
Palaz, S. \& Ozkan, S. \\ Balkesir University, Turkey
}

Objectives: To investigate the mobbing behaviours and its frequency among a group of health care employees, the prevalence of mobbing among participants, the association between workplace mobbing and demographic variables such as age, gender, education, seniority and consequences of mobbing behaviours on occupational health.

Methods: We conducted a questionnaire survey using a validated self-reported questionnaire among 582 full-time employees in the health sector both in private and public in Balikesir, Turkey. A Turkish adaptation of the revised Negative Acts Questionnaire NAQ was used to assess mobbing in workplace. The questionnaire was in three sections. The first part of the questionnaire aimed to identify the degree of mobbing using the NAQ scale which provides a 24-item measure of negative affectivity. The aim of the second part was to find out the reaction to mobbing incidents, effects of mobbing on occupational health and actions taken to escape from the mobbing. The third part consists of demographic information (age, gender, education and marital status), work-related data (seniority, job grade), and information on the relationships with superiors and colleagues. Results Findings indicate that a large percentage $(89.7 \%)$ of the hospital workers reported that they had experienced mobbing behaviours at workplace in the last 12 months. In addition, it is found that the most common mobbing behaviours were being humiliated and ignored followed by persistent criticism of your work and effort and being exposed to an unmanageable workload. Mobbing by colleagues and superior were more common. The most common reactions shown by participants to escape mobbing was "thinking of changing work area within the institution", "trying to rectify the injustice" and to tell their friends. Additionally, 66.9 per cent of participants who exposed mobbing stated that they "worked very carefully to avoid criticism".

Implications: The further and more research on mobbing at different sectors and in different cities of Turkey need to be conducted in order to generalize and enlarged the results and effect of mobbing to Turkish work environment. Then setting up a system of support for employees to prevent and assist against mobbing behaviours.

Conclusions: Our results show that mobbing is a serious problem among health care employees and may cause mental health problems, lack of self-esteem and confidence. Therefore an appropriate policies and procedures need to be taken into account to provide healthy and quality work life.

\section{Impact of Gender Identity and Marginality on Occupational Stress}

\author{
Palermo, J. \\ Deakin University, Australia
}

Objectives: Gender identity, when applied to organisational culture, is descriptive of the predominant beliefs about sex-roles, inherent within cultural values, mores and processes. 
Tenets of marginality and gender schema theory suggest that individuals who experience incongruence between their own gender identity and that of the organisation are more likely to experience adverse psychological effects. A common assumption of marginality theorists is that living in two cultures is psychologically undesirable because managing the complexity of dual reference points generates ambiguity, identity confusion and normlessness. This study aimed to investigate the impacts of marginality on occupational stress for women and men across diverse industry segments.

Methods: 251 participants from various organisations participated in this cross sectional study. Structural equation modelling analysis was used to investigate antecedents of occupational stress. Psychosocial factors and gender identity (masculinity and femininity) were used to predict occupational stress outcomes.

Results: The factor that most discriminated organisations on levels of marginality and vocational strain was gender identity congruence (i.e. marginality) in relation to values and practices in the organisation that embody nurturance (or more stereotypically feminine characteristics). Interesting the gender factor related to masculinity was not a significant predictor of occupational stress, yet participants described their organisations as more autonomous (masculine) than nurturing (feminine). In addition female employees, in particular, reported greater occupational stressors than males. For both males and females, a higher level of occupational strain was significantly related to increased marginality in relation to nurturance. These findings suggest that a plausible explanation for the higher levels of occupational stress in females may be due to greater incongruence felt by feminine individuals, and the requirement to behave in gender aschematic ways in masculine organisational environments

Implications: These findings demonstrate that occupational stress may be influenced more by organisational factors than by individual factors. This study has practical implications for the development of new organisational environments that are more balanced in relation to gendered culture as a way of addressing occupational stress factors for males and females at work.

Conclusions: Our study demonstrates that current stereotypically masculine or autonomous organisational environments may suppress the expression of more stereotypically feminine or nurturing values and behaviours in modern workplaces. It is this absence that may set conditions for poorer work environments in relation to health and productivity outcomes.

\title{
The Scientific Evidence on Occupational Health: A Meta-analysis on Burnout
}

\author{
${ }^{1}$ Palitti, T., ${ }^{1}$ Palermo, P., ${ }^{1}$ Tomei, G., Cinti, M.E., ${ }^{1}$ Rosati, M.V., ${ }^{1}$ Scimitto, L., ${ }^{1}$ Fiaschetti, M., \\ ${ }^{1}$ Schifano, M.P., ${ }^{1}$ Panfili, A., ${ }^{2}$ Cetica, C., ${ }^{3}$ Tomao, E., ${ }^{1}$ Fioravanti, M., ${ }^{1}$ Tomei, F., \& ${ }^{1}$ Sancini, A. \\ ${ }^{1}$ University of Rome 'Sapienza', Rome, Italy; ${ }^{2}$ Euristat, Rome, Italy; ${ }^{3}$ General Office for Military \\ Health-Defence General Staff, Italy
}

Objectives: According to scientific evidence, burnout can be described as a multi-dimensional syndrome with three fundamental dimensions: emotional exhaustion, depersonalization and (reduced) personal accomplishment. Since the attention to this negative reaction to occupational stress increased according to a fairly large amount of scientific production in recent years, we evaluated the scientific evidence on this matter with the application of the meta-analytic method. 
Methods: All comparative studies performed on burnout were identified and examined in order to compute the Effect Size (ES) of results. Forty studies were found compliant with the inclusion criteria to this analysis for a total of 2.031 burnout cases vs. 1.950 controls. Results: This meta-analysis reports the ES's of different issues such as the use of standardized questionnaires as methods for identifying burnout cases, the use of biological markers and cognitive predictors for the identification of burnout cases, and the effectiveness of prevention interventions on job satisfaction, intention to leave work and the various scores on burnout questionnaires.

Implications: A strong disparity emerges from our results due to the ways of selecting subjects with burnout. This problem evidences the lack of a standardized definition of burnout as a univocal clinical entity and, consequently, the lack of a consensus procedure for the identification of patients to be included in this diagnostic category.

Conclusions: The standardized questionnaires (in most cases the Maslach Burnout Inventory) are sensible in identifying burnout cases on a psychiatric basis (discriminating power and sensitivity of this psychological measures), some biological markers (Adreno Cortico Tropic Hormone, Growth Hormone, Aldosterone, urinary and salivary Cortisol) and the Continuous Performance Test are altered in burnout cases and the prevention interventions are useful in normalizing the scores of the burnout questionnaires. The wide heterogeneity among studies notably reduces the possibility of generalizing these results.

\title{
The project PAKT - Prevention of Back Pain in German Waste Management Companies
}

\author{
${ }^{1}$ Pangert, C., ${ }^{2}$ Herold, K., \& ${ }^{3}$ Lemke, P. \\ ${ }^{1}$ German Statutory Accident Insurance, Germany; ${ }^{2}$ uve GmbH fuer Managementberatung, \\ Germany; ${ }^{3}$ Institute for Economy and Prevention, Germany
}

Objectives: The paper describes the Project PAKT (funded by the Federal Ministry of Labor and Social Affairs; assisted by the Federal Institute for Occupational Safety and Health), which is intended to promote the effectiveness of an integrative prevention pro-gramme. This programme covers a variety of institutional and personal variables (e.g. work organisation, safety at work management, leadership, personal competencies) proved to be associated with health outcomes and is based on the $5 \times 5$ impact model (Wieland \& Hammes, 2008). The purpose of the project is to approach the problem of musculoskeletal disorders (MSDs) in high risk work arrangements. Within the project, we focus on 12 local waste management and sanitation companies in Germany, as em-ployees in this sector face severe physical as well as psychosocial demands resulting from diverse reasons. Therefore, the project aims at developing effective prevention measures in a participatory manner and implementing these on the different hierarchy levels.

Methods: To accurately determine the fields of action and deduce adequate measures, a mixture of methodological means is employed. This includes qualitative expert inter-views with company officials next to a quantitative questionnaire survey of the employ-ees and is completed by reflexive workshop sessions, inventory of safety at work man-agement, in particular risk assessments, and information recall activities. 
In addition, within the project a web-based communication platform had been installed and company representatives meet biannually to foster inter-company information and experience exchange. Furthermore, the project will be evaluated on different levels.

Results: Firstly, we were able to investigate employee-perceived leadership quality. Secondly, it was possible to assign the employees into three groups concerning their degree of back pain chronification, which helped us to offer specific recommendations. With in-depth content analysis of the data material, we gathered valuable knowledge about organisational hindrances to be overcome. There was evidence, that communica-tion (mode and manner of the information transfer), trust and appreciation (organisa-tional culture), workload as well as work-related latitudes are crucial. The safety at work management is well developed, but psychological risk factors are only slightly consid-ered.

Implications: It seems promising to let all interest groups participate on an equal level in the process of improving companies' workplace health management systems.

Conclusion: The prevention of MSDs requires a multi-channel approach including the development of personal competencies at all hierarchy levels, improvements concern-ing organisational variables (e.g. information management) and the establishment of assistance systems outside the company embedded in a corresponding organisational culture.

\title{
Qualitative investigation of the relationships between staff health and change management processes in the UK National Health Service
}

\author{
Parkin, T. \& Loretto, W. \\ University of Edinburgh, United Kingdom
}

Background: The concepts of organisational change and the effective management of change are pervasive within contemporary management literature. However, there has been relatively little focus on how change affects individual employees, especially their health and wellbeing. Studies to date have been limited to considering a uni-directional relationship, i.e. what aspects of organisational changes negatively influence employee health. There has been little investigation into how changes in staff health during times of change may mutually influence the ongoing development and outcomes of change management processes. There is a growing international literature on the management of organisational change in relation to health services (e.g. Bourbonnais et al., 2005; Hansson et al., 2008; Loretto et al 2009). Despite experiencing substantial and on-going change and consistently reporting higher levels of mental ill-health and distress, remarkably little attention has been paid to health service employees in managing change.

Objectives: Situated within a multi-methods research project in the UK National Health Service (NHS), this paper reports on a qualitative longitudinal study. It aims to explore the two-way nature of the relationships between organisational change and staff health.

Methods: Three in-depth interviews were held at yearly intervals with four teams from Reproductive Medicine and Cardiology in a UK NHS Hospital Trust. Interviewees also completed a GHQ12 (General Health Questionnaire 12) questionnaire at each interview to 
provide additional information on their mental health. This paper presents a case study of one of those teams, community based midwives $(n=9)$.

Results: A range of themes emerged, including: impact of workload, role of work location, role of support and action taken by staff in response to changes. From these, we developed a conceptual model to illustrate the two-way nature of the interactions between individuals and change. We used this, along with the GHQ results, to explore how the effects of change on individuals' health influenced their own change management strategies: to engage with, resist or avoid change. The longitudinal nature of our data allowed us to also consider the subsequent effects of these various strategies on future health of individuals and on the outcomes of the organisational change initiatives.

Implications: Findings exemplify why an individual level approach to organisational change, incorporating acknowledgment of staff health, should be considered by managers and professionals if they want to have successful organisational change outcomes.

Conclusions: This study identifies and demonstrates the two-way relationships between staff mental health and organisational change, a dynamic that has previously been under-explored.

\title{
A qualitative investigation of employees' perceptions of the impact of work on health behaviours
}

\author{
${ }^{1}$ Payne, N., ${ }^{2}$ Jones, F., \& ${ }^{3}$ Harris, P. \\ ${ }^{1}$ Middlesex University, United Kingdom; ${ }^{2}$ University of Leeds, United Kingdom; ${ }^{3}$ University of \\ Sheffield, United Kingdom
}

Research shows that work stress generally has a negative impact on health behaviours. However, most of the research in this area is quantitative and focuses on a narrow range of work factors. The present research aimed to provide a more complete picture of the disruptive and/or facilitative impact of work on health behaviours by asking employees about their experiences of four behaviours; smoking, alcohol consumption, exercise and eating.

We conducted semi-structured interviews with 24 employees of an information technology company. Four main themes were identified from a content analysis.

Individuals perceived that being busy at work led to a shortage of time for healthy behaviour, but had a positive impact on smoking. Business events such as lunches and travel were seen as disrupting employees' routines and changed the availability of factors that facilitate healthy behaviour. The work environment, for example, corporate gym membership and no smoking policy, was perceived as promoting healthy behaviour. However, a lack of availability of healthy food or on-site exercise facilities was unhelpful. Work stress was associated with health behaviours being used as a coping response, which in the case of exercise, may be considered an adaptive response. These findings have implications for worksite health promotion. 


\title{
A Hierarchical Model of the Internal Relationship Marketing Approach to Nurse Satisfaction and Loyalty
}

\author{
1Peltier, J, ${ }^{2}$ Schibrowsky, J.A., \& ${ }^{2}$ Nill, A. \\ ${ }^{1}$ University of Wisconsin-Whitewater, United States of America; ${ }^{2}$ University of Nevada, Las \\ Vegas, United States of America
}

Objectives: Due to demographic shifts and an aging population the health care industry has been growing rapidly in most developed nations. With more than 26 million jobs in healthcare in the European Union and the U.S., healthcare is one of the largest, fastest growing, and most important service industries. However, the viability of this industry has been threatened by difficulties to hire and retain qualified heath care workers due to a stable or even decreasing supply of nurses and a steadily growing demand. The purpose of this study is to empirically test a model of the proposed antecedents of nurse job satisfaction and loyalty based on the relationship marketing and internal marketing literature. The study investigates the degree to which structural, social, and financial bonding activities, and employees' perceived quality of care by the organisation influence nurses' job-satisfaction and the likelihood of remaining with the organisation. Though there is growing interest in exploring nurse satisfaction and retention, little research has focused on model development and testing of the relationship marketing and internal marketing constructs within the healthcare literature.

Methods: Following an extensive review of the literature, an internal relationship marketing model of nurse satisfaction and loyalty has been developed. Based on a sample of 309 nurses from two German and three US. health care institutions, the model has been tested using structural equation modeling software.

Results: The study resulted in two key findings: the hierarchical nature of structural, social, and financial bonding activities and the impact of nurses' perception of the care provided by their employer had a major impact on their job satisfaction and loyalty.

Implications: Service industries depending on front line employees to deliver high quality services are provided with innovative suggestions to improve job satisfaction and loyalty of its employees. The study provides organisations with empirical evidence regarding the synergistic effects of bonding activities other than increased pay, and the importance of communicating the quality of the service the firm provides to its own employees.

Conclusions: To the best of our knowledge, this is the first time a hierarchical model of the relationship bonds has been empirically tested. In addition, this study explores the impact of employees' perceptions of the service provided by their employer had a major impact on their job satisfaction and loyalty. Though there is a growing literature base concerning internal marketing, there are very few studies that demonstrate its importance to retention, loyalty, and satisfaction. 


\title{
The Direct and Indirect Antecedents of the Acceptance of Donation After Cardiac Death by Health Care Professionals: Psychological Concerns, Knowledge, and Engagement
}

\author{
${ }^{1}$ Peltier, J. \& ${ }^{2}$ Alessandro, A. \\ ${ }^{1}$ University of Wisconsin-Whitewater, United States of America; ${ }^{2}$ UWHC Organ Procurement \\ Organisation, United States of America
}

Objectives: Worldwide there is an ever-widening chasm between the supply and demand for organ donations. To reduce this shortage, efforts have begun to increase the supply of organs via Donations after Cardiac Death (DCD). As end-of-life decisions approach, caregivers have the unique ability to impede or assist organ donation through their willingness to identify potential donors and to provide referrals to the appropriate procurement agency, their willingness to approach bereaved families with the option to donate and the strength of their support for organ donation during the request process. Despite their importance in the DCD process, relatively little is known about the factors motivating or inhibiting their acceptance of DCD. A 3-yr study funded by the U.S. Department of Health and Human Services was conducted to identify potential barriers to and opportunities for increasing the number hospitals with donation after cardiac death (DCD) protocols, the support of DCD by individuals involved in the donation request process, and the number DCD donors recovered.

Methods: A survey was administered to each of the 1,143 certified requestors in the hospital's territory. A total of 806 surveys were completed and returned (70.5\% response). A structural equation model was used to analyze the direct and indirect relationships of the support of DCD by health care professionals using five dimensions: awareness, attitudes toward organ procurement organisation, ethical dilemmas, quality of life, and psychological concerns.

Results: The model was highly significant $(\mathrm{AGFI}=.984)$ and showed that the psychological support for DCD by health care professionals is a multi-faceted process. The strongest effects involved the extent to which DCD was seen as killing a patient, knowledge of DCD separate from brain death, and personal psychological concerns.

Implications: Based in part of the findings, a communications and relationship process targeting health care professionals was undertaken, resulting in increased knowledge and support for DCD an increase in DCD recoveries by nearly $200 \%$.

Conclusions: Our findings provide a better understanding of healthcare professionals' knowledge, attitudes, and behaviours regarding DCD. Understanding these issues is critical to the implementation of strategic plans for DCD programs. One of the biggest barriers to overcome is a lack of knowledge of DCD, which leads to misperceptions, which in turn contribute to negative attitudes and/or discomfort by healthcare professionals. Communication efforts that are able to educate healthcare professionals and eliminate misperceptions will increase support for DCD. Key to future success requires confident and well-trained DCD requesters. 


\title{
Social Stress Risk Model
}

\author{
Penati, V., Ferrari, F., Sinibaldi, F., \& Girard, A. \\ SIPISS, Italy
}

Objectives: In 2004, we started a research to identify work-related stress risk factors, in accordance with the European laws about the work-related stress. The purpose of this research was to identify factors that could be objective stress's sources and to measure them, to allow prevention managers to know which are the specific criticalities of each working environments.

Methods and Results: During our biennal project, we collaborated with several companies, studied the different contexts and roles, and interviewed more than 5000 people engaged in different sectors and tasks to identify the possible stress risk factors. Our research developed in three phases, involved 5020 people and lasted two years. In the first phase of the research we extracted a sample of 5020 workers that came from different sectors and subdivided them on the basis of their tasks.

We administered a questionnaire to the whole sample that observed five factors: communication, relation, environment, responsibility/role, personal ecology. From the initial sample, we extracted a new group of 2200 workers equally distributed on the basis of their tasks and sectors. Weadministrated to the whole sample the FerrariSinibaldi test,that gives two indexes: the level of anxiety/stress and the coping, that consists in strategies that people use in difficult or risky situations.

On the basis of the answers, we could measure the stress index for each person; if this value exceeded 7, the stress index was over the risk's threshold. 700 subjects had a Stress Index near to the risk's threshold. We identified 9 working environments -office, production, yard, shop, travel, warehouse, workshop, school, health service- that contained all the possible working realities, on the basis of the cultural and relational aspects, the kinds of task and the activities.

We placed the 2200 subjects in the different environments, and then we administrated them an interview about 10 risk factors extracted from the literature. Afterwards, we crossed the interview's data with the FerrariSinibaldi Test's results. After that in every environment it was possible to identify the stress's sources for each macro-factors (first phase) and with a frequency calculus we attributed a value from 1 to 10 to each micro-factors extracted.

Conclusions: From this psycho-social research, we created a model based on an objective observation of the environments that allows to identify the potentially critical factors, and to give them importance on the basis of the working context.

\section{Stress and Burnout Managing Model in Health Care Professionals}

\author{
Pereira, J., Rodrigues, J., \& Cunha, M.J. \\ Instituto Superior da Maia, Portugal
}

Introduction: Stress and Burnout are more and more the rule rather than the exception, presenting as concerning indicators, because they are reflected at emotional disorders level that severely damage the health of the individual that experiences it. Although currently there are already studies that show this problem in other professions, health care professionals, 
present themselves today as a group of extreme risk. The consequences arising from these experiences on the health care professionals are reflected in the quality of services provided to patients as well as their quality of life and their welfare. Analysing some variables such as work commitment and engagement, as a protective factor of vulnerability to stress and burnout.

\title{
Objectives:
}

1. Identify the presence of vulnerability to stress and burnout in both groups of health professionals;

2. Identify if the perception of greater workflow is an enhancer factor of vulnerability to stress and burnout among providers of both groups;

3. Verify wich is the role of vulnerability to stress in the development of burnout frameworks;

4. Understand the role of perception in the areas of work life and work commitment (Organisational Commitment) on the development of burnout frameworks ;

5. Identify the role of engagement as a mediator of the influence of the workflow on vulnerability stress and burnout in the study participants;

Results: The results show that the group of oncology health professionals (g1) has (13.9\%) of stress vulnerability and (7.6\%) of burnout. The group of health professionals from other services (g2), presents $(9.3 \%)$ of stress vulnerability and $(6.3 \%)$ of burnout. The results, highlight an important data in our study, the appearance of the variable involvement (engagement) develops, in health care oncology, significant values of vulnerability to stress and burnout frameworks, compared with health professionals from other services.

Implications: This study, at some point, presented itself as a source to new further investigations. With the advent of the variable engagement and values contrary to organisational Commitment, as grown the idea that it would be important analyze in the future and more detailed these variables, extending to other risk groups, as the health professionals of INEM, UCI and Urgency.

Conclusions: We conclude that health professionals in oncology are in situations of greater vulnerability to stress and to develop burnout frameworks. It is therefore important to note that due to low or no margin of error, these health professionals adopt defense mechanisms that leads them to submit distancing behaviours.

\section{Job dimensions, personal goal facilitation and burnout: a longitudinal analysis}

\author{
${ }^{1}$ Pisanti, R., ${ }^{2}$ van der Doef, M.P., ${ }^{2}$ Maes, S., 1 Violani, C., \& ${ }^{3}$ Lazzari, D. \\ ${ }^{1}$ University of Rome 'Sapienza', Italy; ${ }^{2}$ Leiden University, The Netherlands; ${ }^{3}$ Hospital "S. \\ Maria", Terni, Italy
}

Background: In literature, job dimensions described in the Job Demand-Control-Social Support Model (JDCS Model) are frequently presented as important predictors of burnout (Schaufeli, 2007; Van Der Doef \& Maes, 1999). Although findings have clearly demonstrated the predictive power of JDCS Model, several limitations and inconsistent results warrant the exploration of additional, complementary perspectives. From a self regulation perspective, (lack of) personal goal facilitation through work (e.g. Emmons, 1996) might play a role in the development of burnout. 
Objectives: This study aimed to test relationships between job demands, job resources (control and social support) and goal facilitation through work on the one hand, and the three burnout dimensions on the other, among Italian nurses. Moreover, given that previous longitudinal studies (e.g. Gelsema et al., 2006) have shown that nurses experience considerable changes in job conditions over time, we also included changes in work conditions in our model.

Methods: A longitudinal survey with a 1-year time interval yielded a panel group of 217 nurses (Female $=84.3 \%$; mean age of 42.0 years $-\mathrm{SD}=7.2-$ ) from the Hospital of Terni in Italy. Hierarchical regression analyses were applied, controlling for demographic variables and the dependent variable at Time 1 .

Results: In the final model changes in job conditions predicted respectively $14 \%, 12 \%$ and $6 \%$ of the variance in emotional exhaustion (Fchange $=14.2 ; \mathrm{p}=.000$ ), depersonalization (Fchange $=$ 10,$1 ; p=.000$ ), and personal accomplishment (Fchange $=5.2 ; p=.002$ ). Inclusion of personal goal facilitation improved the prediction of the burnout dimensions considerably, except in the case of depersonalization. More specifically, personal goal facilitation through work explained an additional $6 \%$ of the variance in emotional exhaustion (Fchange $=22.2 ; \mathrm{p}=.000$; Beta $=$ -.28 ), and $8 \%$ in personal accomplishment (Fchange $=22.8 ; p=.000$; Beta $=.33$ )

Conclusions and Implications: The inclusion of a self regulatory perspective may improve the predictive power of the JDCS model. Some suggestions regarding future research are discussed.

\title{
The dark side of high-skilled work. Assessing work-related stress risk in the Italian National Research Council
}

\author{
${ }^{1}$ Ponzini, G. \& ${ }^{2}$ Moccaldi, R. \\ ${ }^{1}$ National Research Council, Institute of Research on Population and Social Policy, Italy; \\ ${ }^{2}$ Prevention and Protection Service, National Research Council, Roma, Italy
}

Objectives: The paper presents the first results of an ongoing project mainly aimed at providing a risk assessment tool for work-related stress, applicable to research and administrative units of the Italian National Research Council (CNR), to comply with legislative decrees n. 81 of 2008 and n. 106 of 2009 and to identify strategies for tackling stress. The project has been designed and carried on by the Institute of Research on Population and Social Policy and by the Prevention and Protection Service of the CNR.

Methods: The methodology utilized is based on both a quantitative and qualitative approach, including objective data collection (i.e. on sickness absence or staff turnover), an online selfadministered questionnaire, and a set of focus group discussions and scenario planning sessions. In a first phase, the sample includes 12 research institutes and 3 central administration offices of the CNR. The questionnaire is based on the HSE standard, revised by inserting some items derived from the two-factor theory.

Results: First results of the project offer chance to better understand the relevance of differences arising from the allocation of workers over jobs with different requirements and characteristics (i.e. research staff vs. administrative staff) or from working in different scientific fields (i.e. biomedical, 
technological, theoretical). The project also offer a contribute to practically discriminate between endogenous and exogenous causes of stress. As a final result, the project will provide guidelines and recommendations to reduce the levels of work-related stress reported by the CNR staff.

Implications: On the basis of results emerging from the project, a simplified risk assessment tool for work-related stress and a standardized strategy for tackling the key causes of stress will be defined and made available to all the research and administrative units of $\mathrm{CNR}$.

Conclusions: The project focuses not only on the subjective perception of working conditions, but also on the subjective experience of organisational functioning and of formal and informal relationships in the workplace. In this way the relevance of endogenous stressors, typically belonging to the workplace, is underlined. Besides, the complex organisational structure that characterize the CNR, the variety of scientific fields covered, and the way in which workers are allocated over different jobs, could offer a chance to generalize (at least) some findings

\title{
Socioeconomic position and common mental disorders in a working age population
}

\author{
${ }^{1}$ Pulkki-Raback, L., ${ }^{1}$ Virtanen, M., ${ }^{1}$ Ahola, K., ${ }^{2}$ Elovainio, M, \& ${ }^{3}$ Kivimaki, M. \\ ${ }^{1}$ Finnish Institute of Occupational Health, Finland; ${ }^{2}$ National Institute for Health and Welfare, \\ Finland; ${ }^{3}$ University College of London, United Kingdom
}

Objective: Socioeconomic inequalities in mental health have been established but it is not known which components of socioeconomic position - income, education, or occupation have the strognest contribution to the inequalities. It has also been suggested that clustering of mental disorders (comorbidity) may be higher in lower socioeconomic classes but evidence is limited. We examined the socioeconomic patterning of common mental health disorders and their comorbidity in working-age Finns.

Method: The participants were a nationally representative sample of 4561 men and women aged 30 to 65 years. They were interviewed for 12-month DSM-IV diagnoses of depressive, anxiety, and alcohol use disorder using the Composite International Diagnostic Interview. Low socioeconomic position (SEP) was indicated by having manual occupational class, being relatively poor (income $50 \%$ of the national median), and low educational level. We examined the probability of having a disorder in low versus high SEP by logistic regression analyses.

Results: Separate associations of occupational class, relative poverty, and educational level differed for each type of mental disorder. When all of the socioeconomic indicators were entered into the same model at the same time as predictors, relative poverty remained the strongest predictor of mental disorder: poor individuals had higher odds for depressive disorder $(O R=1.73, p<0.0001)$, anxiety disorder $(O R=1.59, p<0.01)$, and psychiatric comorbidity (ORs from 2.12 to 2.39 , ps from $<0.0001$ to $<0.05$ ). In the same model, manual social class was associated with higher risk for alcohol use disorder $(O R=1.43, p<0.05)$ while educational level had no associations with any of the examined disorders.

Conclusion: Relative poverty seems to be the most important socioeconomic component of poor mental health in a high income country such as Finland. Occupational class and educational 
level has no remarkable explaining power on mental health over and above poverty. Our findings suggest that material deprivation may underlie poor mental health and the explaining mechanisms should be studies in the future.

\title{
Occupational and health conditions among victims of workplace bullying: a prospective study
}

\author{
${ }^{1}$ Punzi, S., ${ }^{2}$ Pavesi, A., ${ }^{1}$ Castellini, G., ${ }^{2}$ Conway, P.M., ${ }^{1}$ Militello, E., ${ }^{1}$ Cassitto, M.G., \& \\ ${ }^{1,2}$ Costa, G. \\ ${ }^{1}$ IRCCS Maggiore Hospital, Milan, Italy; ${ }^{2}$ University of Milan, Italy
}

Objectives: Study aim is to investigate short and long-term occupational and health conditions of a sample of subjects reporting exposure to workplace bullying and seeking health care at the Service for Stress and Harassment of the 'Clinica del Lavoro Luigi Devoto' in Milan.

Methods: A sample of 226 patients examined in 2003 was selected out of 2455 cases examined for suspect stress-related disorders from 1997 to 2003. The sample was composed mostly of women (53,1\%) and subjects aged 35-54 (76.5\%). Occupations were various, but especially white-collars $(53.5 \%)$. Through analysis of clinical records, we obtained information concerning characteristics of workplace bullying and health conditions. After 6 years, 100 subjects, out of 226, were invited to participate in a semi-structured telephone interview (response rate $91 \%$ ). The sample was representative of patients examined in 2003 with regards to the main socio-demographic and occupational characteristics. The interview investigated on present occupational conditions and career history since 2003, and also on current health status and quality of life, mainly in comparison with past conditions.

Results: As far as the baseline sample is concerned, workplace bullying occurred mostly during company reorganisations and changes in management staff. Most frequently, negative acts included social isolation and demotion. Major symptoms were exhaustion, sleep, mood and sexual disorders. At follow-up, 47.3\% declared they remained in the same workplace, $23.1 \%$ found another job, $22 \%$ resigned voluntarily, $17.6 \%$ retired, $11 \%$ were dismissed, while $9.9 \%$ were unemployed. Improved working conditions were reported by two third of the subjects that remained in the same workplace $(30.8 \%$ of the total sample) and by the majority of subjects that found another job. Among those who remained, 54.8\% have been transferred to another office/department and $19 \%$ changed supervisor. About two third reported an improvement in their health status. This occurred mostly among those with a new job, those who retired, and also among those, despite remaining at the same workplace, reported improved working conditions.

Implications: The present study confirms that bullying at work may result in short-term negative occupational and health effects. In the long-term three in four subjects reported that bullying ceased only after being moved away from exposure to the adverse situation. Improvement in health conditions and quality of life may suggest that bullying-related health impairment is highly contingent on objective occupational conditions.

Conclusions: Preventive interventions are highly recommended to avoid leaving the workplace to be the only way to recover from bullying-related health consequences. 


\title{
Age differences in stressors and strain
}

\author{
Rauschenbach, C. \& Hertel, G. \\ University of Münster, Organisational Psychology, Germany
}

Objectives: The age structure of Europe`s working population is changing. The employment rates of older workers in Europe are projected to increase massively from $40 \%$ in 2004 to $47 \%$ in 2010 and $59 \%$ in 2025 . To maintain and improve the work ability of their employees, organisations should adopt age-differentiated health management strategies. Therefore, knowledge about the influence of age on stressors and strain is necessary. Based on theories of emotional development which postulate an improved emotion regulation with age, we suggest that older workers experience less emotional and social stressors. Based on theoretical considerations, several relations between age and strain are possible: A positive linear relation, a negative linear relation, a curvilinear relation, or no systematic relation. In order to clarify and further explore the role of age, the present study examines age effects on stressors and strain.

Methods: Four hundred and fifty five employees from the public administration sector completed an online questionnaire. Participants were between 19 and 66 years old $(\mathrm{M}=$ 42.3; SD = 11.7; age groups: 19-35, 36-50, 51-66). The questionnaire included seven types of chronic job stressors: time pressure, work interruptions, uncertainty, concentration demands, problems of work organisation, social stressors, and emotional dissonance. Additionally, job control and coping strategies were measured. Strain was assessed with an irritation scale.

Results: There were no age differences reported in time pressure, uncertainty, concentration demands, problems of work organisation, social stressors and coping strategies. However, younger and middle-aged employees experienced more work interruptions compared to their older colleagues. Older workers experienced less emotional dissonance compared to younger workers. Furthermore, younger employees reported less control at work compared to middleaged and older employees. In addition, older workers experienced less irritation. Multiple mediation analysis revealed that the age effect on irritation was mediated by perceived control at work.

Implications \& Conclusion: This study revealed systematic age differences in stressors and strain. Interestingly, older workers experienced less emotional dissonance. In addition, older workers reported less irritation. This effect was mediated by higher work-related control older workers reported. Interestingly, this corresponds to earlier results showing that the importance of control and autonomy at work significantly increases with age. Therefore, providing autonomy and work-related control at work might be seen as particularly effective measure to reduce stress and increase well-being of older employees

\section{Attitudes to On-call Working and Impact on Family Life}

\author{
Reid, T., Earle, F., \& Clough, P. \\ University of Hull, United Kingdom
}

Objectives: A questionnaire study was designed to investigate attitudes to on-call working 
and the impact of this form of work scheduling on individuals from four diverse occupational groups.

Method: The study used a questionnaire methodology to ascertain each participant's subjective rating for the questions relating to their on-call work. Each participant was given a questionnaire booklet containing a bespoke on-call questionnaire (Earle \& Reid 2006). The dependent variables for the study were attitudes to on-call and impact on the family. The independent variables were job category (physiotherapists, anaesthetists, fire officers and police officers), and number of years worked on-call. The participants consisted of 22 Anaesthetists (8 females and 14 males), 37 Physiotherapists (34 females and 3 males), 37 Fire Officers (all male) and 31 Police Officers (17 females and 14 males). The participants ages ranged between 21-65, and years working oncall ranged between 1-10 years or over.

Results: The data were analysed using 4 (job category) x 5 (years worked on-call) between groups ANOVAs. This analysis revealed that physiotherapists were significantly more negative in their attitudes towards on-call working than the other 3 occupational groups. Attitudes to oncall working were not found to be affected by number of years worked on-call and there was no interaction. With regards to the impact of working on-call on family life, the physiotherapists and anaesthetists reported a greater negative impact on family life than police and fire officers, and there was a significant interaction between job category and number of years worked oncall for the police officers, with the impact on the family being greater with increasing number of years on-call service.

Implications: On-call working is now commonplace across a broad range of occupations. Operational working practices clearly differ across occupational groups and these differences will undoubtedly influence the impact of this type of work scheduling on the individual and their families. Greater understanding of these differences may lead to improved operational practices within such working communities.

Conclusions: While the research community has very limited knowledge and understanding of the issues related to on-call working, this study highlights that on-call working impacts differently on individuals from different occupations and that there can be a cost to this type of work scheduling not only for the individuals themselves but also for their families.

\title{
Evaluation of Job Stress Models for Predicting Health at Work
}

\author{
Reineholm, C., Ekberg, K., \& Gustavsson, M. \\ Linköping University, Sweden
}

Objectives: Development of a good work environment encompass both preventive and health promotive interventions. Methods for measurement of work conditions are developed from a risk factor perspective, rather than a work place health promotion (WHP) perspective. More knowledge is needed regarding what work conditions promote health in order to develop a good work environment. Are measures developed for assessment or risks useful in health promotion? The purpose of the present study was to elucidate how the Demand Control Support (DCS) model, the Effort-Reward Imbalance (ERI) model and the Job Characteristic Inventory (JCI) are interrelated and their ability to predict health and/or ill-health longitudinally. 
Methods: The study used questionnaire data from 662 civil servants, first at baseline and then a follow-up two years later. Health was measured with EQ5D, vitality in SF-36, and ill-health by global burnout in the Copenhagen Burnout Inventory. The data were analysed by simple and multiple linear regressions.

Results: Three clusters of work conditions emerged with high inter correlations between variables from the three job stress models: 'organisational work conditions' (demands, effort), 'social relations and interactions' (reward, social support, feedback) and 'health mediating work conditions' (variety, autonomy, control). Based on the results of a simple linear regression analysis, the three work condition variables from the three models with the highest score for each health measure instrument were used as predictors in a multiple stepwise regression analysis. The present study resulted in a new model based on effort, reward, and variety and with higher predictive power to predict health than the original models.

Implications: Social relations and health mediating work conditions needs to be considered as these conditions may enrich the job and function as social resources which indirectly promote employees' health. Health may be created in the social relations between employees at the workplace and employees with good health can also be assumed to contribute with good health through their interactions with others.

Conclusion: Traditional job stress-models capture important aspects of health promoting work conditions, but are better predictors for ill-health. To promote health at work, social relations and health mediating work conditions are of importance because these conditions may buffer health. Health can be assumed to be a resource which is created in everyday activities and interactions in workplaces. From a WHP perspective, there is a need of developing health measure instruments based on holistic health theories.

\title{
Collective coping within teams of low-qualified workers
}

\author{
Requa-Brueckner, G. \& Busch, C. \\ University of Hamburg, Germany
}

Until now, occupational stress research has been concerned almost exclusively with the individual stress management behaviour of qualified employees. Low-qualified workforces have been mainly neglected in occupational stress research. In this contribution a study will be presented on collective coping within teams of low-qualified workers. Fifty percent of lowqualified workers across Europe work in teams. This study investigates whether low-qualified team staff display collective coping behaviour at all; secondly, it looks into which kind of stress situations can be coped with collectively, and thirdly, it examines which forms of collective coping are to be found within this target group.

Teams of low-qualified workers from different industries, like manufacturing, kitchen work and waste disposal, and with different quality of teamwork were investigated by means of participatory observation. Stress situations and collective coping efforts were the focus of observation. The observations were evaluated by means of qualitative content analysis. 
The results clearly show that even in the case of low quality of teamwork low-qualified workers cope collectively with stress. A common mental model of stress situations and collective stress management is clearly demonstrated. Collective coping was shown particularly in task-related stress situations, for example when task fulfilment is interrupted by a defective machine. In this case, problem-focused collective coping efforts could be observed. On the other hand, lowqualified workers made hardly any effort to cope with stress situations when they were brought about by the social work environment or by the way work is organized; the situations were complained about without any attempt being made to cope with them. For stress management interventions for this target group the conclusions may be drawn, that measures should stress not only individual coping strategies but also collective coping efforts. Further, low-qualified workers should be supported in coping with stress related to the social work environment and to the way work is organized. An exemplary intervention is ReSuM, a team-based intervention for low-qualified workers, which stresses individual and collective coping and which is successfully evaluated in different industries.

\title{
Resources depletion model of Emotional Labour: Perceived Emotional Effort as key factor in Spanish-English samples
}

\author{
${ }^{1}$ Rodríguez-Carvajal, R., ${ }^{2}$ Quiñones-García, C., ${ }^{2}$ Clarke, N., ${ }^{1}$ Moreno-Jiménez, B., \& ${ }^{1}$ De \\ Rivas-Hermosilla, S.
}

${ }^{1}$ Autonoma University of Madrid, Spain; ${ }^{2}$ University of Southampton, United Kingdom

Emotional Labour (EL) and high turnover rates are central features of customer service roles within the Entertainment Industry. EL relates to the effort customer service employees have to exert in order to meet the emotional requirements of their job regardless of their own feelings. Evidence suggests that high leaving rates are the result of the stress associated to the EL employees have to perform. On the other hand, studies support that cultures differ in the extent to which they allow the free regulation of emotion. Impulsive cultures (e.g. Spain) encourage individuals to express their own emotions freely whereas in institutional cultures (e.g. UK) individuals tend not to express their own emotions and to regulate them in order to meet emotional rules.

Objectives: Based on a resources-depletion model, we examine the effect of perceived emotional effort to analyse the impact of EL at work within impulsive cultures (Spain) and institutional cultures (UK). Since EL often requires hiding own emotions to express those required by the organisation, and since Spain and the UK are examples of impulsive and institutional cultures respectively, it is expected that emotion labour will be a less harmful process for institutional cultures than for impulsive ones.

Methods: Through a self-questionnaire methodology, the sample consisted of 204 English employees and 211 Spanish employees working on the Entertainment industry. First, the theoretical model was assessed with equation regression modelling analyses. Subsequently, the hypothesized mediation effect of perceived emotional effort on the relationship between EL and turnover intention was analyzed controlling by age, sex, commitment to display rules, and emotion regulation as a trait. 
Results: The general model showed good fit indices that ranging from .938(NNFI) to .950(CFI) (SRMR .046). As regards the mediation analyses, EL, turnover intention, and perceived emotional effort were significantly correlated $(p<0.01)$. Path modelling showed that perceived emotional effort fully mediate the effects of EL on turnover intention.

Conclusions and Implications: This model improves on existing limitations in the literature by offering a comprehensive explanation of the EL impact through perceived emotional effort to fulfil the emotional requirement at work. The present study also revealed differences in the strain experienced by the Spanish sample (impulsive culture) compared to the UK sample (institutional culture). However, no differences were found related to fulfilling specific emotional requirements considering the emotional management as a process. Recommendations on cultural awareness on stress and emotional management and also on selection and training are given.

\title{
The development and validation of the Perceived Emotional Effort Scale
}

\author{
${ }^{1}$ Rodríguez-Carvajal, R., ${ }^{2}$ Quiñones-García, C., ${ }^{2}$ Clarke, N., ${ }^{1}$ Moreno-Jiménez, B., ${ }^{1}$ De Rivas- \\ Hermosilla, S. \& ${ }^{1}$ Alvarez-Bejarano, A. \\ ${ }^{1}$ Autonoma University of Madrid, Spain; ${ }^{2}$ University of Southampton, United Kingdom
}

Hochschild's study revealed that employees in customer service roles often engage in 'Emotional Labour' (EL) which eventually leads to burnout. EL refers to the effort involved in managing own emotions to display those required by the role. Despite the emphasis placed upon 'effort' instruments development was focused on the EL strategies 'deep acting' (i.e. changing your own feelings to achieve the required display) and 'surface acting' (i.e. changing only the outward display). Assessing the impact of EL with this approach is limited, as mixed evidence was found for deep and surface acting. The common assumption is that although both strategies involve effort surface acting is the actual predictor of burnout. Findings from qualitative studies where participants reported no effort and no stress associated to EL challenge this assumption, and support the assessment of 'effort' independently from the EL strategies. Martinez et al. developed an indirect measure of effort by interference with other tasks based on the Ego Depletion Theory. The scale had low reliability (.45) and did not take into account the role of 'direct effort'. Based on Lazarus stress theory we argue that in addition to indirect effort, individual's experience of stress is largely determined by how threatening (effortful) they perceive the emotional requirements of the role.

Objectives: To develop and validate the perceived emotional effort scale. The perceived emotional effort will be a 2-dimensional construct that distinguishes between direct and indirect effort. This model will fit the data well and better than a one-factor model both in the exploratory and confirmatory sample.

Methods: Through a self-administered questionnaire and cross-validation methodology, the sample consisted of 380 English and 308 Spanish customer service employees. Each sample was split randomly, the first half used for exploratory analysis and the second for confirmatory analysis. Exploratory analysis were informed by Kaiser's criterion, Cattel's Screeplot and Parallel Test. Confirmatory analysis were performed with SEM. 
Results: The 2 dimension structure found with exploratory analysis was replicated with the confirmatory analysis. Here, the 2 dimension model showed good fit indices ranging from $.947(\mathrm{NFI})$ to .984(CFI) (RMSEA .05). Reliabilities for each dimension ranged between .70 to .78

Conclusions and Implications: The need for a measure of perceived emotional effort as well as its development and validation has been presented. We believe that by measuring the effort we can overcome current limitations and demonstrate that the perceived effort is the actual predictor of burnout, regardless of the strate.

\title{
Monitoring of working and health conditions in victims of bullying at work
}

\author{
Romeo, L., Pelizza, L., Antonini, E., Ballottin, A., Cabianca, M., Tisato, S., \& Perbellini L. \\ University of Verona, Italy
}

Objectives: We periodically evaluate the working conditions and the health state of the victims of bullying, beginning from at least 12 months after the first observation. The personality traits of bullied workers has been found to be an important factor in the dynamics of workplace bullying and in the consequences on the health workers. The aim of the study is to find support for the hypothesis that the personality profiles of victims of bullying at work are susceptible to modification as a result of improvement of working conditions.

Methods: Between 2004 and 2008100 victims of bullying at work, were evaluated at the first time by means of medical and psychological assessment using also MMPI-2 questionnaire. A second evaluation of working and health conditions was performed 12-48 months after the first observation; MMPI2 questionnaire was administered again in 50 workers.

Results: Our data provide evidence that a significant number of workers actually develop mental disorders that are to be regarded as work-related. The study yielded evidence of a personality profile with higher scores in victims of personal bullying with high frequency of negative actions in those not receiving family support and suffering from psychological and psychosomatic pathologies that forced them to resort to daily pharmacological therapy.

Conclusions: Psychological support and improvements in the working environment bring notable benefits to these workers.

\section{Stressed and Fatigued on the Ground and in the Sky: Changes in civil aviation workers' conditions of work in the post 9/11 era}

\author{
${ }^{1}$ Rosskam, E., ${ }^{2}$ Greiner, B. \\ ${ }^{1}$ Graduate Institute of International and Development Studies, Switzerland; ${ }^{2}$ University of \\ Cork, Ireland
}

A global investigation of civil aviation workers in 116 countries in Africa, Asia/Pacific, Middle East, North America, Latin/South America, and Europe was completed in 2009. The study 
examined the direction of changes and trends that took place globally, between 2000 and 2007, resulting in the present pandemic of stress and fatigue among civil aviation workers. The contribution of changes in civil aviation workers' social and economic security has been given paramount importance in this study and is reflected in the identified stress and fatigue results. The year 2000 was used as a baseline to have an idea about conditions before 11 September 2001.

The study was conducted using Participatory Action Research methodology together with the International Transport Workers Federation, Civil Aviation Section. This methodology enabled the team (12 people, working in 6 different countries, including 4 members from the ITF's Civil Aviation Section) to develop collective knowledge of the causes and impact of emotional stress and fatigue on aviation workers.

Results will be presented of changes that have occurred since 2000 in the macro social and economic work environment, including general policy changes, changes in income security, representation security, labour market security, employment security, and work security.

The information obtained through this study provides the global affiliates of the International Transport Workers Federation with an empirical basis for developing policy recommendations and actions needed to address the general trends and problems identified.

\title{
Early-career burnout among nursing professionals: A three-year longitudinal observational study
}

\author{
Rudman, A. \& Gustavsson, P. \\ Karolinska Institutet, Sweden
}

Objectives: Cherniss's pioneering research on burnout focused specifically on competence crisis among new graduates, and identified negative attitude changes as the core phenomenon in the progression from competence crisis into early career burnout. The objectives of this study were to test the sequential-developmental model of burnout using a psychometric approach, and to identify common growth curves across the first years after graduation.

Methods: A sample of 933 early-career nursing professionals, recruited from a Swedish population-based cohort, were assessed up to three years after graduation, using items from a burnout inventory. Psychometric analyses were conducted using the one-parameter IRT measurement model. Classification of individual growth curves was conducted by means of cluster analysis.

Results: The psychometric tests showed that data adhere to a sequential-developmental model when examined using the one-parameter item response approach. When tested against external variables, the prevalence of low mood, low levels of job performance and health problems increased monotonically along this sequential-developmental model of early career burnout. Eight common growth curves were identified, explaining $77 \%$ of all individual variation. These different growth curves could be predicted by demographic variables, previous health problems, educational outcomes and current work load and life situation. 
Conclusion: Among early-career nursing professional's burnout may be operationalized as a one-dimensional sequential-developmental model. Longitudinal data revealed divergent developmental patterns associated with previous training, current socialization and work environment. The relative importance of different predictors of burnout as well as its consequences will be discussed.

Implications: The aim of this study was to elucidate some of the complexity associated with individual burnout growth curves during the three first years after graduation and to examine what individual and educational factors that predict different curves. The findings may be useful for practitioners, employees and educators in their effort to prevent burnout, improve nurse's health status and work environments. In a general sense, by showing factors that are associated with development of burnout in the transition between undergraduate studies and practice this can help facilitate a stable and healthy nursing workforce, and in turn high quality care and good patient outcomes.

\title{
The interaction between perceived job stress and rumination predicts long-term evening saliva cortisol secretion
}

\author{
${ }^{1}$ Rydstedt, L.W. \& ${ }^{2}$ Cropley, M. \\ ${ }^{1}$ Lillehammer University College, Norway; ${ }^{2}$ University of Surrey, United Kingdom
}

Objectives: to analyse the long-term main effects of perceived job stress and trait rumination as well as their interaction on long-term psycho-physiological reactivity, as indicated by morning and evening saliva cortisol.

Methods: The sample consisted of 76 white collar workers (52 men and 24 women) who four years earlier had participated in a survey where data on working conditions, strain reactions and personality traits was collected. Mean age was 45.8 years during the field study; the majority worked in professional occupations. Base-line trait rumination was assessed with the InhibitionRumination Scale (I-RS) of 18 items with true/false alternatives coded 0/1 additively with a mean of 5.3. Trait rumination was dichotomised by median split. A single item, which has been shown to be strongly associated with several critical job dimensions as well as to various health indicators, was used to measure perceived job stress. In the field study, carried out almost 4 years after the survey, saliva cortisol secretion was used as an indicator of psycho-physiological reactivity and measured over seven consecutive days. Two measures were taken each day: at awakening and at 10 PM.

Results: None of the independent variables showed any significant impact on morning saliva cortisol. In regard to evening saliva cortisol secretion, there was no significant main effect of job stress, while the main effect of trait rumination showed a none significant trend on evening saliva cortisol $(F=3.26 ; 1 ; 144 ; p<.10)$. On the other hand there was a significant interaction effect between perceived job stress and trait rumination $(F=5.23 ;$ df $1 ; 144 ; p<.05)$. Regardless of trait rumination, participants with low job strain had similar levels of evening saliva cortisol secretion levels, while participants with high perceived job stress and high trait rumination showed a markedly increase in evening saliva cortisol secretion. 
Implications: To our knowledge the extended time span between exposure and cortisol measure is unique for this study. Our findings suggest that perseverative cognition may be activated by stress exposure. An obvious restriction of this study was the limited sample size which did not permit gender separate analyses

Conclusions: The finding adds support to the growing data demonstrating the differential impacts of short- and long-term stress exposure. While emergent exposure increases morning saliva cortisol chronic exposure has in several studies been related to a flattening circadian curve with increased evening cortisol secretion

\title{
Psychological work well-being and occupational accidents in health professionals
}

\author{
'Santarém Semedo, C. \& ${ }^{2}$ Dominguez, J.L. \\ ${ }^{1}$ University of Évora, Portugal; ${ }^{2}$ University of Santiago de Compostela, Spain
}

Introduction: The growing concern shown by both organisations and academics with the health and safety of workers in the workplace has led to a large number of studies being carried out in the field. Objectives: The aim of this piece of research is to study the relationship between certain variables related to well-being and accidents at work in the context of the hospital, involving the study of well-being measures, work engagement, burnout, perceived organisational support and the organisational climate as perceived by workers.

Methods: The sample was collected in six public hospitals of Portugal. Before the relationship between the variables was analysed statistically, a psychometric study of measurement instruments was carried out, showing a good level of statistical reliability.

Results: Comparisons of averages and studies of correlations were carried out, which demonstrated results which were similar to those obtained in published studies: subjects who suffered accidents which did not have serious or immediate consequences did not present differences when compared with colleagues who did not have accidents. Logistic regression analysis carried out demonstrated dedication (engagement) and perceived organisational support as predictors of the increase in the number of accidents, while personal accomplishment (burnout) predicted the decrease of accidents.

Conclusions: In this kind of organisations, the study of psychological consequences of occupational accidents should probably be done by studying accidents with serious and immediate physical effects (e.g., falls, physical aggressions, etc). 


\title{
Health and motivation in nurses: A multi-national European study
}

\author{
${ }^{1}$ Schaufeli, W., ${ }^{2}$ Torrente, P., ${ }^{3}$ van der Heijden, B., \& ${ }^{4}$ Hasselhorn, H.M. \\ ${ }^{1}$ Utrecht University, the Netherlands; ${ }^{2}$ Universitat Jaume I, Castellon, Spain; ${ }^{3}$ Maastricht \\ School of Management, the Netherlands; ${ }^{4}$ University of Wuppertal, Germany
}

Hospital nurses work in stressful and challenging jobs, and for that very reason they have been studied intensively. Although nurses are by far the largest single professional group in health care in each country, comparative studies on their health and motivation across various national settings are lacking. The current study includes 9,435 nurses from seven different European countries, including Belgium $(N=684)$, Denmark $(N=1,267)$, Finland $(N=1,711)$, France $(N=1,030)$, The Netherlands $(N=751)$, and Poland $(N=1,369)$. The theoretical framework is constituted by the Job Demands Resources (JD-R) Model that assumes that in all work settings two types of job characteristics can be distinguished: job demands and job resources. The former refer to particular aspects of the job that require sustained physical and psychological effort, and are therefore associated with physiological and psychological costs. According to the JD-R model, prolonged exposure to high job demands may therefore lead to health impairment, for instance, burnout (the health impairment process). Job resources refer to those aspects of the job that may reduce job demands that are functional for achieving work goals, or that stimulate personal growth, learning, and development. In other words, these demands have motivational potential. According to the JD-R model, prolonged exposure to job resources may, for instance, lead to organisational commitment (the motivational process).

The current study tested the JD-R model in all seven European countries simultaneously, using the Multi-Group Method in Structural Equation Modeling. The latent job demands' factor was constituted by qualitative demands (uncertainty about patient treatment), quantitative demands, and work-home interference. The latent job resources' factor included opportunities for learning and development and social support from colleagues and supervisors. The latent health impairment factor included burnout, disabling back pain, and general health, whereas the latent motivation factor was constituted by organisational commitment, turnover intention, and job satisfaction.

Results indicated that the model fits well to the data of all seven countries (Ïキ2 = 1727.40; NFI, TLI, and CFI > .90; RMSEA < .03). As predicted by the JD-R model a positive relationship exists between job demands and health impairment (ranging between countries from .57 to . 80 ) and also between job resources and motivation (ranging from .45 to .73). In addition, an unexpected negative path was observed from job demands to motivation (ranging from -.37 to -.60). Obviously, job demands impair both nurses' health and motivation. Additional analyses showed that the paths of the model were not invariant across the seven counties.

In conclusion, the JD-R model was confirmed in a multi-national European sample, albeit that an additional unexpected negative relationship between job demands and motivation was found and that the strengths of the relationships differed between countries. 


\title{
Evaluating occupational health management: Development of the 'Health Index' and its relation to economic outcomes
}

\author{
Schraub, E.M., Buech, V., \& Sonntag, K. \\ University of Heidelberg, Germany
}

Being pressured by highly competitive markets and facing the demographic change, companies have recognized the value of healthy employees for maintaining high performance. Consequently, particularly large companies have implemented health management systems. Because these often lack evaluation, questions of cost effectiveness arise. The aim of the research project BiG ('Benchmarking in a health network') was, therefore, to provide managers with a simple but comprehensive overview on the current state of various job demands and resources, including health management characteristics, leadership and work characteristics: the 'Health Index'. We proposed that this index relates to economic outcomes such as productivity and absence rate.

Based on stress theory and prior research, we developed a questionnaire that assesses various perceived job demands and resources at work. With data from a norming sample of about 1800 employees of five large German companies, we developed a formula to calculate the Health Index. For that, we applied different statistical procedures such as factor and regression analyses. We examined in how far the perceived demands and resources were related to employee wellbeing before integrating them into the index and controlled for the contribution of each factor for employees' well-being by means of weighting. Using key data from the companies' reporting systems, we further analyzed the relation between the Health Index and economic outcomes.

Results show that the demands and resources contained in the index are differentially related to employees' well-being. Analyses of subsamples indicate that teams with higher Health Indices were higher in productivity and had lower accident rates.

Based on these first promising results, the Health Index and the economic outcomes need to be assessed in further samples to yield a validated measure, which could also be refined for medium-sized or even small companies.

The Health Index is an easy-to-use and thus practical tool to evaluate the perceived state of various job demands and resources. It can be applied to different levels of a company, such as departments and teams and serves for identifying improvement areas and for detecting changes in the respective demands and resources over time.

The project was funded by the German Federal Ministry of Education and Research (BMBF) and supported by the German Aerospace Center's Project Management Agency (PT-DLR).

\section{Team emotional intelligence as a predictor of employee strain and initiative: $\mathrm{A}$ conflict perspective}

\author{
Schraub, E.M. \& Schanz, K. \\ University of Heidelberg, Germany
}

This study examined the role of team emotional intelligence for employee strain and personal 
initiative with special focus on conflict within teams, which is one of the most bothering job stressors. While task conflict can be constructive for non-routine tasks, it often does not stay taskrelated but results in discomfort and frustration. Not handled well, conflict can thus jeopardize well-being and performance. However, it can be assumed that it is not the conflict itself that evokes strain, but rather the lack of resources employees can draw upon to manage emotions evoked during conflict. Therefore, we examined team emotional intelligence and individual coping as moderators of the relation between team conflict and employee strain. Based on the job-demands-resources model, we expected that interpersonal conflict leads to team members' strain if their problem-focused coping is low. Furthermore, we hypothesized that interpersonal conflict is negatively related to team members' personal initiative. Finally, we assumed that team emotional intelligence exerts positive effects by both reducing interpersonal conflict and by enhancing problem-focused coping.

We conducted a questionnaire-based team study including 300 members of 59 teams from different German organisations. With a repeated measurement procedure, we assessed interpersonal conflict, team emotional intelligence, coping and demographic data at one point of time. Strain and trait affectivity as control were assessed one week later, together with a colleague evaluation of personal initiative. All scales yielded sufficient reliability, and indices allowed for the aggregation of interpersonal conflict on a team level. We applied multilevel data analyses (HLM) for testing the hypotheses.

Results support our hypotheses: Team emotional intelligence was positively related to team members' problem-focused coping, which in turn buffered a positive effect of interpersonal conflict on strain. Also, interpersonal conflict negatively predicted personal initiative. Furthermore, teams with higher emotional intelligence experienced less interpersonal conflict.

In sum, our results confirm the detrimental effects of interpersonal conflict within teams, and underline the importance of emotional capacities as a resource in interactive work contexts. Concerning the occupational stress literature, a major contribution of our study is the identification of a mechanism that explains positive effects of team emotional intelligence. Further research should examine more closely in which specific contexts team emotional intelligence is an essential resource. Because team emotional intelligence can make a difference when it comes to productive and competitive teams, we suggest that practitioners in organisations should build up and strengthen the skills that characterize emotional intelligence.

\title{
Stress and Burnout in Canadian Midwives
}

\author{
Schryer LeBel, F. \& Day, A. \\ Saint Mary's University, Canada
}

Objectives: Although midwifery has long been part of health care in many European countries, modern Canadian midwifery only began in the 1990s (Bourgeault et al., 2004). The relatively recent emergence of Canadian midwifery exposes the country's midwives to a cluster of stressors that may exacerbate their experience of stress and burnout, and lead to poor work-life balance. Therefore, the purpose of this study is to identify and quantify aspects of the midwifery context related to midwives' stress, burnout, and work-life balance. 
Methods: Semi-structured interviews were conducted with midwives and subject matter experts to inform the construction of an on-line survey, which the Canadian Association of Midwives distributed to all registered midwives in Canada. Using a national sample of 233 midwives, we examined the impact of the identified demands (e.g., actual workload \& perceived work overload; role conflict and ambiguity; House et al., 1983; Rizzo et al., 1970; poor working relationships) and the direct and moderating impact of several resources (i.e., perceived job control; Dwyer \& Ganster, 1991; organisational support; Eisenberger et al., 1986; support from colleagues). We assessed several outcomes including burnout (Maslach et al., 1996), strain (Bartone et al., 1989), and work-life balance (Cannon et al., 2008).

Results: High role conflict, low compensation, and poor relationship quality were significantly correlated with most of the outcomes. Conversely, high job control and support were significantly correlated with lower strain and burnout, and with better work-life balance. Moderated regressions indicated partial evidence of a moderating effect for perceived job control, social support from colleagues, and organisational support. For example, job control moderated the relationships between the number of difficult births attended and cynicism. Both support from colleagues and organisational support moderated the relationship between being overloaded at work and emotional exhaustion.

Implications: We found both direct and moderating effects of job control and support on midwives' psychosocial outcomes. These results have practical implications for the practice of midwifery toward providing a supportive environment and allowing control over aspects of their practice.

Conclusion: This study provided a theoretical framework to identify job demands faced by midwives as well as the resources that are available to them. Control and support may be beneficial resources that can help reduce burnout, strain, and improve balance, and also mitigate the negative effects of demands on these outcomes.

\title{
Committed employees are healthy employees? Employee commitment and health relevant work attitudes and behaviours
}

\author{
${ }^{1}$ Schusterschitz, C., ${ }^{2}$ Geser, W., ${ }^{1}$ Noehammer, E., \& ${ }^{1}$ Stummer, H. \\ ${ }^{1}$ UMIT Private Universität für Gesundheitswissenschaften, Austria; ${ }^{2}$ Leopold-Franzens \\ Universität Innsbruck, Austria
}

Objectives: Following Kobasa (1979), psychological hardiness comprises of (1) the belief to be able to control or influence events, (2) a commitment to activities, and (3) the perception of change as a challenge rather than a threat. Considering this conceptualization, Jackson \& Rothman (2006) view organisational commitment as one component of hardiness (2006). Starting from that assumption and building on past research providing evidence for a positive impact of employee commitment on well being (Siu, 2002) and the experience of stress and strain (Meyer et al., 2002), the study at hand examines correlations between affective, normative and continuance commitment on the one hand and health relevant work attitudes and behaviours on the other.

Methods: Normative, affective and continuance commitment (Allen \& Meyer, 1997) were measured using the Commitment Scales by Schmidt, Hollmann and Sodenkamp (1998). Data 
on health relevant working attitudes and behaviours were collected by selected subscales of the AVEM (German abbreviation for Patterns of Work-Related Coping Behaviour) developed by Schaarschmidt and Fischer (2003). Research hypotheses were tested by means of correlation analyses.

Results: Regarding the tendency to exert, significant positive correlations with affective and highly significant positive correlations with continuance commitment were found. Thus, employees with high levels of affective and continuance commitment should show an increased inclination to overwork and to not take care of themselves. In addition, the current results reveal (highly) significant negative correlations between emotional distancing, an ability that plays a central role regarding burnout (Maslach, 2001), and continuance and normative commitment, respectively. Accordingly, the higher employees' continuance or normative commitment, the more difficult it is for them to enjoy the end of their work days and get work-related troubles off their mind. Finally, high continuance commitment is significantly negatively correlated with offensive coping. Consequently, high continuance commitment hinders employees to offensively and optimistically deal with problems at work.

Conclusions: The findings of the study at hand contradict past theoretical reasoning as well as past research, since they do not support the notion of employee commitment as one component of psychological hardiness. In contrast, the current results rather suggest negative health impacts of employee commitment, since the three commitment dimensions, and especially continuance commitment, lead to working behaviours that can be expected to have adverse health effects.

\title{
Good citizens are healthy citizens? - OCB and health-related attitudes and behaviours
}

\author{
${ }^{1}$ Schusterschitz, C., ${ }^{1}$ Stummer, H., ${ }^{1}$ Noehammer, E., \& ${ }^{2}$ Geser, W. \\ ${ }^{1}$ UMIT Private Universität für Gesundheitswissenschaften, Austria; ${ }^{2}$ Leopold-Franzens \\ Universität Innsbruck, Austria
}

Objectives: Past empirical studies in the field of organisational citizenship behaviours (OCB) mainly addressed antecedents of OCB. The smaller number of studies analyzing consequences of OCB focused on its contribution to organisational performance and success (Podsakoff \& MacKenzie, 2000). Therefore, also analyses of potential health relevant consequences of OCB are rare, although already addressed by Organ and Ryan (1955), in the form of overload. An exception is the work of Bolino \& Turnley (2005). They found the OCB-dimension individual initiative to be related to higher levels of role overload and job stress, thereby suggesting that OCB negatively affects health. The present work further addresses the existing research gap and examines whether different forms of OCB are related differently to health-related attitudes and behaviours.

Methods: OCB was measured by an OCB questionnaire developed by Staufenbiel und Hartz (2000), which allows for the measurement of OCB as a multidimensional construct. Health attitudes and behaviours of the study participants $(N=132)$ were collected by means of selected subscales of the AVEM (German abbreviation for Patterns of Work-Related Coping Behaviour) compiled by Schaarschmidt and Fischer (2003). 
Results: Regression analyses revealed that OCB-civic virtue, OCB-sportsmanship, OCB-altruism, OCB-conscientiousness, and OCB-loyalty represent highly significant predictors of employees' tendency to exert, their ability to emotionally distance themselves from work related problems in leisure, and of their ability offensively cope with difficulties at work. In detail, the higher individuals score on OCB-conscientiousness, OCB-civic virtue and OCB-altruism, the higher is their tendency to exert. OCB-sportsmanship in contrast, is negatively associated with the tendency to exert. Furthermore, our findings demonstrate that employees with high ratings on OCB-civic virtue have difficulties in emotionally distancing themselves from work related problems, even in leisure time. Finally, regarding the ability to offensively cope with problems at work, our findings show that the higher the scores on OCB-civic virtue and OCB-conscientiousness, the better is an employee's offensive coping.

Conclusions: The study at hand contributes to existing research by expanding the knowledge of health impacts of OCB. To the extent that the current findings provide evidence for some forms of OCB being related to negative health behaviours and attitudes, they are in line with Bolino and Turnley (2005). However, our results additionally suggest that OCB can have positive effects on employees' health-related attitudes and behaviour.

\title{
Adult attachment styles and health-related work attitudes and behaviours
}

\author{
${ }^{1}$ Schusterschitz, C., ${ }^{1}$ Stummer, H., ${ }^{1}$ Noehammer, E., \& ${ }^{2}$ Geser, W. \\ ${ }^{1}$ UMIT Private Universität für Gesundheitswissenschaften, Austria; ${ }^{2}$ Leopold-Franzens \\ Universität Innsbruck, Austria
}

Objectives: Building on previous findings on the influence of adult attachment orientations on experiencing work related stress and strain (Schirmer \& Lopez, 2001), the present study further examines whether Bartholomew \& Horrowitz's (1991) four styles of adult attachment contribute to an understanding of individual differences in health related work attitudes and behaviours.

Methods: To answer the above mentioned research question, full-time employees with different occupational backgrounds attending a course for professional development at the Management Center Innsbruck (Austria) were surveyed. Adult attachment was measured using the Scales to Measure Two Dimensions of Attachment Insecurity in Close Relationships (Grau, 1999), which allow for the respondents' classification into either the secure, the preoccupied, the fearfulavoidant, or the dismissive-avoidant attachment style. Data on health relevant working attitudes and behaviours were collected by selected subscales of the AVEM (German abbreviation for Patterns of Work-Related Coping Behaviour), developed by Schaarschmidt and Fischer (2003). Research hypotheses were tested using analysis of variance (ANOVA) and subsequent t-tests.

Results: Current results reveal that individuals with a secure attachment style differ significantly from those with a preoccupied one and by tendency also from those with a fearful-avoidant style regarding the tendency to exert, as secure individuals dispose of significantly lower levels of this tendency. Furthermore, secure attachment is associated with a trend towards higher levels of satisfaction with work than preoccupied attachment and significantly higher levels of satisfaction with work than fearful-avoidant attachment. Moreover, secure employees consider their past working life as more successful and feel more confident to be able to cope with future demands 
than employees showing one of the two insecure styles. Finally, the present findings provide evidence for the fact that secure individuals differ significantly from those with a fearful-avoidant attachment style in terms of emotional distancing, insofar as they report no problems to detract themselves from occupational problems and to enjoy leisure time.

Conclusions: Showing that secure employees not only positively evaluate their current and future work life but also dispose of behaviours favorable in terms of a positive work-life balance, the present findings imply that the secure attachment style is to be considered as the healthiest one. Thus, against the background of a salutogenetic approach (Antonovsky, 1987), secure attachment can be considered to present an important work-related health resource.

\title{
Job position and mental health: Do Jahoda's latent functions matter?
}

\author{
'Selenko, E., 'Batinic, B., 'Stiglbauer, B., \& ${ }^{2}$ Paul, K. \\ ${ }^{1}$ University of Linz, Austria; ${ }^{2}$ University of Erlangen-Nuremberg, Austria
}

Employed persons generally show better psychological well-being than unemployed persons, nevertheless psychological well-being can vary considerably between different kinds of professions. In the presented study we claim that the same mechanisms that explain why employed persons report better well-being than unemployed persons might also explain why some professions report better well-being than others. According to Jahoda's latent deprivation theory (1982) unemployed persons have less access to so-called latent functions of work, which explains why they also report less well-being than employed persons (Jahoda, 1982; Paul \& Batinic, 2009). We argue that also within the realm of employment, different professions provide with varying degrees of the latent functions, which might explain the differences in well-being found between them.

Data from two studies, one conducted on a representative German sample $(n=565)$ and the other from a large-scale online study $(n=827)$, largely support this argument. In both studies, persons in white-collar jobs report better well-being and more access to latent benefits than persons in blue-collar jobs. Furthermore, tests of multiple mediations indicate that the relation between profession and psychological well-being was partly mediated by access to latent benefits. The findings are discussed in view of the latent deprivation model as well as with regard to common approaches to job satisfaction.

\section{Witnessing workplace violence, psychological stress and job abandoning: Four case studies from Mexican State universities}

\author{
Sieglin, V., Coronado, M.Z., \& Ramos, M.E. \\ Universidad Autónoma de Nuevo León, Mexico
}

There is a broad literature about the health consequences of bullying at work in thw case of victims. However few studies have focused on the mental health outcomes of workers who witnessed 
systematical mistreatment and psychological violence towards colleagues. Although the reactions of witnesses towards bullying and their relationship with bullied colleagues and bullies may range from indifference to compassion and even active or passive participation in violent interaction, violence at workplace always infers mental and social wellbeing of the whole staff as it influences psychological climate of organisations.

This paper explores the reactions of scientific workers in Mexican State universities who witnessed during more than six months psychological and administrative violence towards colleagues by co-workers and administrators. The paper explores the different stages of spectators' emotional involvement, their relationship to victims and victimizers, the perception of psychological climate, and the configuration of their decision to leave. At the first stage witness observed somewhat indifferently the beginning of hostility and the victim's reactions. In the next stage witnesses tend to evaluate the legitimacy of aggression from an ethical standpoint or from their affective relationship towards victims and victimizers. When witnesses start to question hostile interaction and therefore are getting involved socially and emotionally within the conflict, aggressors also may react towards them especially when witnesses try to make public psychological and administrative mistreatment of colleagues in order to get it stopped by the intervention of major organisational authorities. Witnesses' intervention is not always welcome neither by the bullied colleague nor by organisational authorities who tend to protect political and power structures against any questioning. It is at this point when witnesses suffer intense stress and experiment deterioration of mental and social wellbeing. If this situation persists, witnesses may decide to move from their work department to another within the organisation or to leave organisation. This is particular critical for academic and scientific organisations as they are in danger to lose highly skilled workers.

The paper is a based on four case studies gathered from narrative interviews with female scientists who observed during more than six months intense administrative and psychological mistreatment of colleagues. The paper is part of a broader research project on organisational climate, stress and health in women scientists working in eleven Mexican State universities.

\title{
Institutional perspectives on psychosocial risk management in Danish elderly care centres
}

\author{
Simonsen, J.S.
}

\section{The National Research Centre for the Working Environment, Denmark}

Objectives: In Denmark it is a legal requirement to conduct a psychosocial risk management (PRM) procedure every three years. A study was conducted in 8 Danish elderly care centres examining their PRM practice. Context analysis indicated that these practices do not meet the legal criteria for PRM. This paper examines, using new-institutional theory, why clearly lacking PRM practices are being continued.

Methods: A case study approach was employed. All PRM documentation from each elderly care centre and 6 interviews with key persons on working environment issues was condensed to a case document. The PRM practices were then compared to the Danish legislation.

Results: The case studies show that several workplaces only analyse the working environment room by room via checklists which primarily focus on the physical working environment. Issues 
regarding the interpersonal and structural aspects of the psychosocial work environment were neglected in more than half of the centres. 6 of the 8 elderly care centres had PRM practices which clearly did not meet central criteria.

From a legal perspective this type of PRM practice is a serious issue, but there are other aspects of current PRM practice that are beneficial for the elderly care workers. From an institutional perspective the elderly care centres use the workplace assessment for several functions;

1. As a means to bridge different institutions and fields of practice, and legitimize their activities and needs.

2. The current PRM enables the elderly care centre to negotiate and legitimize ordering new equipment from the municipality,

3. It functions as a platform to communicate with the labour inspectorate

4. It provides the elderly care workers with a platform to legitimize ergonomic changes in the residents' homes.

When seen in this broader organisational context the PRM has many features which are necessary for the elderly care centre to function effectively.

Implications/conclusion: PRM should be seen as a institutionalized organisational tool in the workplace. Even though the PRM should be conducted in stricter accordance to the laws and regulations; the present PRM is a useful tool for the workplaces. Accordingly research and policy on PRM would benefit if the focus was broadened to include these dimensions. To understand PRM, it is necessary not only to see is as a framework for psychosocial work environment measures, but also as a organisational frame for interaction between elderly care centre and other institutions and actors.

\title{
Sustainability among managers in a public health care organisation: a prospective study
}

\author{
${ }^{1,2}$ Skagert, K., ${ }^{2}$ Ahlborg, G., \& ${ }^{1}$ Dellve, L.
}

${ }^{1}$ Göteborg University, Sweden; ${ }^{2}$ Institute of Stress Medicine, Region Västra Götaland, Sweden

Objectives: Leadership influence on employees health and performance has been examined in several studies. However, only a few studies have investigated health and sustainability among managers. There may be differences between male and female leaders regarding total workload (paid and unpaid) as well as possibilities in maintaining work-life balance. In this perspective being a leader could be more challenging for females compared to males. This could have an effect on sustainability in terms of turn over and health. The aim of the study was to describe female and male managers sustainability over time within a public health care organisation.

Methods: The present study is a prospective, descriptive study of managers ( $n=216$ at baseline 2004) within a cohort of employees working in a health care organisation. Sustainability was measured as being still a manager, having a balanced work attendance $(=<8$ days of sickness absence and $<2$ times of sickness attendance during the last 12 months) and showing no signs of burnout (= below highest quartile $(<3,6)$ ) of total score on the Shirom-Melamed Burnout Questionnaire) at follow-up 2006 and 2008. Predictive work factors were measured as low/ 
moderate demands (score below highest quartile) and moderate/high control (score above lowest quartile). Work-life balance was measured as having energy left for domestic work after a work day. Associations were expressed as prevalence ratios (PR) with 95\% confidence intervals $(\mathrm{Cl})$.

Results: Sixty-one per cent were still managers after 4 years. Balanced work attendance decreased from $62 \%$ to $52 \%$ during the studied period while the prevalence of no signs of burnout increased from $74 \%$ to $78 \%$. There were no significant differences between females and males except at follow up 2006 when the prevalence of no signs of burnout was lower among men (67 $\%$ respectively $88 \%$ for women). Low/moderate demands did not predict any of the outcomes at follow up. Moderate/high control predicted being still a manager at follow up 2008 (PR 1.8; CI 1.1-2.8), but neither balanced work attendance nor no signs of burnout. Work-life balance did not predict still being a manager or no signs of burnout, but predicted balanced work attendance at 2006 (PR 1.6; Cl 1.0-2.6) and at 2008 (PR 1.6; Cl 1.0-2.5).

Implications: Predictors for sustainability in position seem to be different from predictors of balanced work attendance.

Conclusions: The turn-over of managers was high. Work control, but not work demands, predicted sustainability in the manager position.

\section{Work and non-work determinants of Job burnout: a cross validation in time}

Smulders, P.G.W. \& Houtman, I.L.D.

TNO Work \& Employment, the Netherlands

Objectives: To date nonwork determinants of job burnout (demographic, personal, and personality characteristics) received little attention (Maslach, Schaufeli \& Leiter, 2004). This study aims at testing the impact of demographic variables (sex, age, education, ethnic origin), the home situation (household composition, hours spend to household and care activities) and the job situation on job burnout.

Methods: In 2007 and in 2008 national representative samples of about 23.000 Dutch employees, were surveyed with the Netherlands Working Conditions Survey. Both samples were analyzed separately in order to cross-validate results. The samples include about 49 percent males and 51 percent females. Additionally, the samples include permanent as well as temporary workers (93 and 7 percent respectively). Burnout was assessed with the Dutch version of the Emotional Exhaustion module (Schaufeli \& Van Dierendonck, 2000) of the Maslach Burnout InventoryGeneral Survey. Cronbach's alpha of the Emotional Exhaustion module (five questions) was 0,86 . The data were analyzed with logistic regressions.

Results: In both years sex, age and educational level showed no relation with burnout. But migrants from non-western countries showed significantly higher levels of burnout $(p<.001)$. More importantly, living with a partner turned out to protect against burnout $(p<.001)$, but the number of hours spend to household and care activities was not a significant factor. Furthermore, most of the job situational factors were related to job burnout. The most important were work 
pressure, emotional labor, number of working hours, job insecurity, working in shifts, at night and/or in weekends. Significant protection against burnout is received from job autonomy, job variety, and leader support. All these results $(p<.01)$ were found in both years.

Implications: Our study does not support earlier conclusions from burnout studies (Maslach, Schaufeli \& Leiter, 2004) as far as age and sex is concerned. In both years our analysis showed no significant differences between males and females and between age groups. Having a partner was confirmed as protective against burnout. As far as the job situation is concerned, the results of our study fit into the Job Demands-Job Resources-model (e.g., Schaufeli \& Bakker, 2004; Bakker, Demerouti \& Euwema, 2005). On the other hand one may question the model itself since lack of resources (e.g., lack of job control or lack of job security) may be included in the JD-JR-model.

\title{
Now I need a burger!" - Job stressors, self-control, and fastfood consumption
}

\author{
Sonnentag, S. \& Naegel, I.J. \\ University of Konstanz, Germany
}

Objectives: Research has shown that stress experiences are associated with unhealthy eating, particularly among persons who try to restrain their eating behaviour under normal (non-stressful) circumstances. Previously, it has been argued that the core underlying mechanism between stress experiences and unhealthy eating in restraint eaters refers to a breakdown of self-control in restraint eaters under stressful conditions. In the present study we specifically examined job stressors and aimed at testing the self-control hypothesis more explicitly by examining if dayspecific low levels of self-control moderate the relation between job stressors and unhealthy eating.

Methods: We conducted a daily-survey study over five consecutive working days with a sample of 105 employees from different occupational backgrounds. Participants reported the dayspecific level of job stressors (quantitative workload, situational constraints, emotional conflicts at work) and self-control at the end of the working day and fast-food consumption during afterwork hours at bedtime.

Results: Hierarchical linear modeling showed that emotional conflicts and situational constraints encountered at work were positively related to fast-food consumption during after-work hours, also when controlling for age, gender, and body-mass index. Quantitative workload showed no direct relation with fast-food consumption. Importantly, day-specific self-control moderated the relation between job stressors and fast-food consumption. On days when self-control was low, emotional conflicts and situational constraints showed a particularly strong relation with fast-food consumption, while on days when the level of self-control was high, the relation between emotional conflicts as well as situational constraints and fast-food consumption was non-significant. Level of day-specific self-control at the end of the working day was strongly predicted by the level of emotional conflicts experienced during the working day. However, day-specific level of low self-control in itself was not related to eating behaviour. It seems that it is the particular combination of a high level of job stressors experienced during the day and lack of self-control that explains unhealthy eating behaviour during after-work hours. 
Implications and Conclusions: As unhealthy eating is a risk factor for many illnesses, attention should be paid to job stressors and self-control that -in combination- account for unhealthy eating. It is important to support employees in managing emotional conflicts and situational constraints at work. In addition, more attention should be paid to the protection of self-control, particularly under stressful work situations.

\title{
Workplace Conflicts and Employee Well-Being: The Moderating Role of Detachment from Work during Off-job Time
}

\author{
Sonnentag, S., Naegel, I.J., \& Unger, D. \\ University of Konstanz, Germany
}

Objectives: Workplace conflicts are a widespread phenomenon in organisational life and belong to the most severe job stressors. Empirical research has shown that employees who experience workplace conflicts suffer from strain symptoms. Despite this empirical evidence, crucial questions remain unanswered. First, most studies on well-being consequences of workplace conflicts used rather general measures of conflicts and did not differentiate between various conflict types such as task conflicts and relationship conflicts. Thus, until now we know only very little about the specific relations between task versus relationship conflicts on the one hand and employee well-being on the other hand. Second, our knowledge about moderators of the relation between workplace conflicts and impaired well-being is still limited. Although personality and conflict management strategies at the workplace have been addressed as potential moderators, it is unknown if employees are less negatively affected by workplace conflicts when they detach from work during non-work time. Therefore, we tested if psychological detachment from work during non-work time moderates the relation between workplace conflicts and poor well-being.

Methods: We conducted an empirical study with 291 employees from a construction company to address these gaps in the literature. Participants completed survey measures of task conflicts, relationship conflicts, psychological detachment from work during non-work time, and well-being. As control variables we additionally assessed job demands and job control. Confirmatory factor analysis demonstrated good construct validity of the core measures.

Results: Multiple regression analyses showed that task conflicts, relationship conflicts, and low psychological detachment were associated with poor well-being. Importantly, psychological detachment moderated the relation between relationship conflicts and poor well-being. Employees experiencing a high level of relationship conflicts suffered from poor wellbeing when they did not detach from work when being at home; for employees detaching from work when being at work relationship conflicts were not related to poor well-being.

Implications and Conclusions: In sum, our study demonstrates that workplace conflicts are related to poor well-being, also when controlling for other workplace factors such as time pressure and job control. Psychological detachment seems to play an important role for protecting employee well-being, showing both a direct relation to well-being and acting as a moderator. Therefore, employees should be encouraged to detach from work when being at home. 


\title{
How to stand emotionally demanding work settings: Workload, Emotion Regulation and Emotional Exhaustion among German physicians
}

\author{
${ }^{1}$ Staechele, T., ${ }^{1}$ Ensinger, K., \& ${ }^{2}$ Schuepbach, H. \\ ${ }^{1}$ University of Freiburg, Germany; ${ }^{2}$ University of Applied Sciences Northwestern Switzerland, \\ Switzerland
}

Objectives: There is evidence that emotion regulation strategies help to understand the relationship between emotionally demanding work settings and emotional exhaustion. One objective is to study this relation among physicians, who have to face high emotional requirements and a risk of emotional exhaustion. To keep well, physicians in hospitals successfully have to deal with high workload, time pressure and excessively long working hours. Thus we are interested in the relation of emotion regulation strategies and workload. A second objective is to examine the role of emotion regulation strategies in the process of emotional exhaustion. We expect surface acting strategies to better forecast emotional exhaustion than deep acting strategies. Furthermore we like to investigate the influence of mindfulness.

Methods: In autumn 2009 we collected data from 410 physicians (assistant and senior level) in a university hospital using a self-reporting questionnaire. The questionnaire contained validated scales (some of the items re-formulated for clinician work settings), measuring working conditions (stressors and resources, e.g. based on ISTA), emotion regulation processes (e.g. ELS, ERQ) and strain/ health parameters (e.g. Irritation, Emotional Exhaustion, Quality of sleep). Additional aspects like Mindfulness (MAAS), aspiration level or self-control were integrated.

Results: (preliminary findings $\mathrm{N}=210)$ Weekly working hours $(\mathrm{m}=55.4 \mathrm{~h} /$ week $\mathrm{s}=12.9)$ are not associated with emotional exhaustion or health. But perceived time pressure is highly correlated with emotional exhaustion $\left(r=.45^{* *}\right)$ also emotional dissonance $\left(r=.46^{* *}\right)$. About $65 \%$ of the physicians strongly agree or agree that emotions play an important role within the job. Analyses of the emotion regulation strategies show the expected results: surface acting goes significantly with emotional exhaustion $\left(r=.29^{* *}\right)$ while deep acting has no relation to it. The investigation of mindfulness (MAAS) shows noticeable negative correlations with perceived work load (e.g. with time pressure $r=.-42 * *$ ) as well as with strain parameters (e.g. with emotional exhaustion $r=.52 * *)$. Positive correlation consist to health $(r=.42 * *)$ and resources of the work setting (e.g. autonomy $r=.27^{* *}$ ).

Conclusions: German physicians are faced with emotionally demanding work settings. The kind of emotion regulation strategy has an impact on emotional exhaustion. Surface acting as a strategy to deal with emotional dissonance is one important factor in the process of explaining emotional exhaustion among physicians. We did not find working hours but workload and perceived time pressure related with emotional exhaustion. Mindfulness as a rarely used construct in occupational health psychology among physicians likely helps to understand more of ways to keep well. 


\title{
Workplace changes among Norwegian employees - does information and participation during the change process affect health and well-being?
}

\author{
Sterud, T. \\ National Institute of Occupational Health, Norway
}

Objectives: To examine the outcome of downsizing and internal reorganisation on job insecurity, job satisfaction and work related health problems among workers in Norway. For workers who had experienced downsizing in their present department, we examined the effect of receiving information and participation during the process on the consequences for the workers involved.

Methods: The analyses are based on data from the Level of Living Survey: Working Conditions 2006, which is a nationwide survey of Norwegian employees conducted by Statistics Norway ( $\mathrm{n}=9961$, answer rate $=67 \%$ ). The questionnaire included measures of reorganisation, downsizing, job insecurity, job dissatisfaction, mental and musculoskeletal complaints attributed to work. Several multiple regression models were estimated, and a number of control variables (e.g. occupation, sector, work hours, seniority etc.) were included in addition to the workplace change variables.

Results: In total 29 percent had experienced downsizing during a 3-years period, and 34 percent had been reorganized. Among employees reporting downsizing in their present department (18 percent), 70 percent reported insufficient information and/or no opportunity to participate in the process. In the adjusted model downsizing was associated with job uncertainty $(\mathrm{OR}=3.895 \%$ $\mathrm{Cl} 3.2-4.6)$, job dissatisfaction $(\mathrm{OR}=1.895 \% \mathrm{Cl} 1.3-2.1)$, job-related mental and musculoskeletal complaints $(\mathrm{OR}=1.595 \% \mathrm{Cl} 1.2-1.8$ and $\mathrm{OR}=1.395 \% \mathrm{Cl}$ 1.2-1.5). Similar finding were found for reorganisation. Separate analyses were conducted among people who had experienced downsizing in their present department. In the adjusted models statistically significant higher prevalence of job uncertainty, job dissatisfaction, and job-related health complaints were found among the group who had experienced inadequate information and participation during the process. The group who reported adequate information and participation during the process did not differ systematically from the reference group reporting no downsizing, except for a higher prevalence of job uncertainty $(\mathrm{OR}=2.895 \% \mathrm{Cl} 2.1-3.8)$.

Implications: Access to information and employee participation during change processes may have a significant impact on the health and well-being of the workers involved and should be considered in future studies and policy making on health and safety management.

Conclusions: This study confirms earlier findings and suggests that workplace changes in Norway generally are associated with a variety of negative consequences. However, among people who had experienced downsizing in their present department information and participation during the process were associated with lower levels of job insecurity, job dissatisfaction and job related health complaints. 


\title{
Humour Style in Relationship to Stress and Well-being: Four Different Personalities
}

\author{
Stokenberga, L. \\ University of Latvia, Latvia
}

\begin{abstract}
Humour has been recognized as effective coping strategy in variety of scientific and self-help literature. Objective of the present study was to explore the role of individual differences of humour style in coping and well-being, distinguishing potentially adaptive and maladaptive humour use. Undergraduates $(\mathrm{N}=183)$ and employed adults $(\mathrm{N}=316)$ participated in this study and completed measures of daily hassles, perceived stress, humour style, several well-being scales and measure of personality.
\end{abstract}

Results suggest four different portraits of humourous personality and their coping characteristics on the basis of the results of the present study. The first, 'soul of the company', is a highly extraverted person, quite open to experience and in a good mood. His of her ability to cope effectively depends on resources utilized from the group - social support, shared good mood and membership. The second is an emotionally stable, extraverted individual in a good mood, internally open to new, unusual experiences, and quite agreeable. This person has a 'humourous view of life' and has adequate individual cognitive resources readiness and ability to take new perspectives and to laugh at everyday life and its absurdities. The third is 'the bad boy' who is neither agreeable, nor conscientious. He or she uses rude, hostile, and cynical jokes in the group; however using humour in order to laugh at others does not improve his or her long-term coping efficacy. The fourth character, 'The sad clown', is a stressed, depressed and anxious person, with quite a low sense of conscientiousness overwhelmed with daily problems. He or she has difficulty coping with stress and allows and provokes others to ridicule him or her in order to gain at least some degree of attention from others.

In conclusion, it was found that different humour style dimensions have different patterns of relation to the coping with stress and well-being. Differences in the humour use should be acknowledged when promoting humour as an effective coping resource.

\section{Leadership and Health - Conceptual Considerations and Empirical Evidence}

\author{
Stummer, H., Noehammer, E., \& Schusterschitz, C. \\ UMIT Private Universität für Gesundheitswissenschaften, Austria
}

Objectives: Workplace health promotion programs (WHPPs) are widely discussed in Continental European companies. Especially in Scandinavian and German-speaking literature, individual and organisational actions are both seen to be important factors in promoting health and well-being. Individual behaviour with regard to health ranges from nutrition and exercise to individual stress trainings. Organisational factors, such as organisational justice seem to be supportive for salutogenic processes/ behaviour and are the second large category to influence health of the workforce (Badura/Hehlmann, 2003; Lowe, 2003).

Methods: Following the conceptual considerations, empirical evidence for those assumptions 
is shown using the results of a longitudinal leadership study and combines it with by two in-depth case studies investigating the impact of leadership, desolidarization and health.

Results: Analyzing the longitudinal data of leadership behaviour from the Vroom/Yettonstudies, a de-solidarization of leadership behaviour has been observed in Germany and Austria since the year 2000 after being extremely stable since the beginning of the studies in 1985. Managers and leaders have been violating acceptance rules more and more since the start of the new millennium, a trend can be assumed (Auer-Rizzi et al., 2005).

Comparing the data with health studies and workplace sickness models, especially the effortreward imbalance (Siegrist/Dragano, 2006) and the findings along the concept of social support (Stummer, 2007) gives a clear picture. As the direct leader is the "significant other" (Weick, 1995) and on the one hand influences the actions of the employees strongly, on the other hand acts as a role model, a serious impact on individual behaviour and a severe effect on employee health and well-being can be assumed.

Implications: A recursive paradox seems to happen: On the one hand, German and Austrian companies are investing more and more money in WHPPs, on the other hand, the change in leadership behaviour might make employees sicker. The paper conceptually combines the findings from different theoretical models and large-scale empirical studies in order to develop propositions and hypotheses as to which effects may come about if the trend observed in the Vroom/ Yetton studies is long lasting. The findings show a tendency towards desolidarization and a trend toward pathogenous working conditions can be assumed which can be partly verified by the two case studies.

\title{
The mediating role of work engagement between job characteristics, and positive and negative non-task behaviours
}

\author{
${ }^{1}$ Sulea, C., ${ }^{1}$ Maricutoiu, L.P., ${ }^{1}$ Virga, D., ${ }^{2}$ Schaufeli, W., ${ }^{1}$ Dumitru, C.Z. \\ ${ }^{1}$ West University of Timisoara, Romania; ${ }^{2}$ Utrecht University, Utrecht, the Netherlands
}

Objectives: Employee performance may be a function of two broad classes of behaviour: task performance and non-task performance - that includes positive behaviour, such as citizenship (OCB), and negative behaviour, such as counterproductive work behaviour (CWB). The aim of the current study is to show, within the frame of Job Demands-Resources (JD-R) model that organisational factors (i.e. interpersonal conflicts at work and perceived organisational support) and personal factors (i.e. conscientiousness) have a direct and indirect relation with non-task behaviours, through work engagement. Our study seeks to uncover the affective-motivational underpinnings of organisational citizenship and counterproductive work behaviour.

Methods: The sample $(n=258)$ consists of Romanian employees from several industries. Structural equation modeling has been used to test two path models, a (1) fully-mediated model, and (2) a partially mediated model that specifies both direct and mediated relations between predictors and criteria.

Results: The fit of the partial model was found to be very good with all fit indices satisfying their respective criteria. Overall, mediation effects were stronger for OCB than for CWB. Work 
engagement mediated the relation between conscientiousness and CWB, on the one hand, and OCB on the other hand. In addition, work engagement played a mediating role between perceived organisational support and OCB, and between interpersonal conflicts at work and CWB. Results show that job resources and personal resources are related to non-task behaviours, and that employees' affective-motivational state plays a mediating role in this association.

Implications: Concerning personal resources, we have found that conscientious employees are likely to feel energetic, dedicated and absorbed in their work and will therefore tend to display citizenship behaviours, whereas low levels of conscientiousness are related, via engagement, to frequent displays of counterproductive behaviours. Regarding job resources and job demands, employees who perceive organisational support are likely to experience work engagement and will therefore manifest more organisational citizenship behaviour. In contrast, when employees experience interpersonal conflicts at work feel less engaged and therefore more inclined to involve in counterproductive work behaviours.

Conclusions: The current research demonstrated the crucial role that work engagement plays in the occurrence of positive and negative non-task behaviours in organisations. Also our study draws attention on conscientiousness and workplace factors in explaining these non-task behaviours. The present results expand the JD-R model and provide practical suggestions for organisations how to increase positive non-task behaviours and decrease the negative ones; thereby at the same time enhancing work engagement.

\title{
Cultural variation and motivation of Indian and Korean employees: A cross- cultural study
}

\author{
Kamalanabhan, T.J. \& Deug Kim, H. \\ IIT Madras, India
}

This study evaluates the factors of motivation that determine overall motivation among Indian employees of Indian companies in India (INDIAN), Indian employees of Korean companies in India (INDO-KOREAN), and Korean employees of Korean companies in Korea (KOREAN), and then examine how cultural variations affect the factors of employees' motivation. Cross-cultural motivation has been highlighted recently, but there are not many studies available concerning two developing nations such as India and Korea. This study was conducted to understand the role of culture on motivation factors among the employees between India and Korea.

A questionnaire survey was used to collect the data. Culture was measured by seven dimensions which was developed by Hofstede et al., (2008). A five point Likert scale to find out the level of various responses such as importance, agreement and its frequencies are attached to each of the cultural dimensions.

The independent variables are demography (3 demographic variables - Gender, Age, Education) and motivation factors (using seven constructs - Interesting Job, Equity at Work, Salary, Physical Environment, Goals, Feedback, and Bonus). The employee motivation scale was originally developed by Fey (2005) and modified according to the purpose of this research. Motivation factors were measured by using a five point likert scale to find out the level of agreement attached to each of the factors of motivation ( 1 being- strongly disagree to 5 being strongly agree). Reliability was established through Cronbach's alpha. 
A measurement model was specified and tested through LISREL for each critical factor. The dependent variable, overall motivation (using five items) is measured by using a 5 point likert scale to gauge the level of agreement attached to each of the factors by motivation ( 1 beingstrongly disagree to 5 being strongly agree). Cultural similarities found that all three groups are collectivistic, high power distance, feminine, restraint, and long-term oriented cultures. These similarities influence the three groups to respond in a similar pattern to particular motivation factors such as interesting job, provision of goals, equity, salary, and bonus. Cultural differences found in two cultural dimensions such as monumentalism/flexumility and uncertainty avoidance that can explain why motivation factors such as provision of feedback and physical environment has different ranks among the groups.

The results of this research confirmed that culture influences motivation and provides important insights to draw effective cross-cultural motivation strategies. The research findings are not only important for Korean companies but also for multinational companies entering India since this research provided insights on a culture-based model of work motivation that serves as a useful conceptual framework for evaluating the motivational techniques across cultures.

\title{
A Study of Social Capital and Health and Safety Climate-Behaviour-Outcome Relationship in education sector
}

\author{
${ }^{1}$ Tang, J.J., ${ }^{1}$ Hunt, N., ${ }^{1}$ Leka, S., \& ${ }^{2}$ McClellan, S.J. \\ ${ }^{1}$ University of Nottingham, United Kingdom; ${ }^{2}$ University of Aberdeen, United Kingdom
}

Objectives: It is widely acknowledged that Hong Kong (HK) teachers are suffering from work related health problems (HK Professional Teachers' Union, 2007). Reviews of psychosocial work and health research suggest that there is a wealth of scientific data on work stress (Cox et al., 2000) to establish both national and international legislation that underlines the importance of risk management. With the results from previous interviews with 38 key stakeholders in HK education sector, including policy makers, policy implementers and school stakeholders. Potential leading factors to the occupational safety and health (OSH) outcomes in HK schools were identified. These factors included trust, communication, management values and knowledge of OSH issues. This study examined the social capital and OSH climate-behaviouroutcome relationship in HK educational sector. The role of OSH knowledge as the mediator of the OSH climate-behaviour-outcome relationship was also investigated.

Methods: Seven hundred and four teachers completed a range of questionnaires. Few instruments were used in this study, they included: OSH climate, General Health Questionnaire 12 (GHQ-12), OSH knowledge, OSH behaviour (compliance and participation). Items in the $\mathrm{OSH}$ climate, $\mathrm{OSH}$ behaviour, and OSH knowledge scales were modified from (Griffin \& Neal, 2000; Neal \& Griffin, 2006) safety climate, safety behaviour and safety knowledge scales. All questions, except GHQ-12 were translated into traditional Chinese by using back translation technique. Structural Equation Modelling (SEM) was used to analyze the relationships between predictive variables and outcome variables.

Results: SEM analysis revealed a good fit of the proposed model. Social capital, OSH climate, OSH knowledge and OSH behaviour predicted all outcome variables, namely as OSH complaints and 
general well being direct and indirectly. In addition, it was found that knowledge is the mediator of OSH climate-behaviour-outcome relationship while social capital is the antecedent.

Implications and conclusions: Most of the empirical work on safety climate has been preoccupied with the research in industrial sectors. This paper makes a contribution to the climate literature by empirically assessing the $\mathrm{OSH}$ climate-behaviour-outcome relationship in educational sector. Additionally, this study also enumerates the importance of school management role and knowledge of OSH issues to improve teachers' health and safety. These findings have important implications for providing the HK government with information on the effectiveness of implementation of occupational safety and health policies and services.

\title{
Measurement and evaluation of perceived stress among students: What supports in an university context?
}

\author{
${ }^{1}$ Tavolacci, M.P., ${ }^{1}$ Grigioni, S., ${ }^{2}$ Villet, H., ${ }^{1}$ Dechelotte, P, \& ${ }^{1}$ Ladner, J. \\ ${ }^{1}$ Rouen University Hospital, Rouen, France; ${ }^{2}$ Upper Normandy Health Observatory, France
}

Objectives: To study the prevalence of mental stress in students in higher education (university) and to identify associated risk factors and risk comportments associated to stress.

Methods: A cross-sectional survey was conducted in university students in Upper Normandy region (France), in 7 campus. This survey was conducted online. Students must complete an anonymous questionnaire as soon as their first connection to the Web site www.tasanteenunclic. org. This questionnaire collected age, gender, to have a grant, a job, alcohol consumption using ADOSPA test, tobacco smoking cannabis use, practice of sport, eating disorders (Scoff questionnaire), the tendency of cyber addiction (Orman test), consumption of anxiolytic drugs and sleep disorders (sleep or waking). The level of stress was measured by Cohen Perceived Stress Scale (with a total of 40).

Results: A total of 508 students were included in the study. The mean age was 20.8 year (standard deviation $[S D]=2.3$ ), sex ratio $(\mathrm{M}: \mathrm{F})$ of 0.44 . The mean score of stress according to Cohen score was $16.0(\mathrm{SD}=7.8)$. The median score was 16 and the 3rd quartile was 21. Women were more stressed than men (respective means of 17.4 and $13.0 ; p<10-4$ ). Students who had trouble falling asleep and those who woke early in the morning were more stressed than students who did not have these sleep disorders (respectively (19.4 and 14.2, $\mathrm{p}<10-4$ and 19.7 and 13.7; $<<10-4) .8 .1 \%$ of students were taking anxiolytic, these students were more stressed than non-users (22.4 and 15.5, p <10-4). More quarter of students $(28,9 \%)$ had tendency of cyber addiction. After logistic regression, perceived stress was significantly associated to female gender $A O R=4.5 ; 95 \%[2,3-8,9]$, no practice of sports $\mathrm{AOR}=2.2 ; 95 \%[1,2-3,9]$, positive ADOSPA $\mathrm{AOR}=2.3 ; 95 \%[1,3-4,1]$, tendency to cyber addiction $A O R=3.8 ; 95 \%[2,0-7,2]$ and positive Scoff $A O R=8.1 ; 95 \%[3,8-17,5]$. To have a grant, job, smoking tobacco or cannabis use were not associated with the perceived stress.

Implications: The higher level of stress morbidity warrants need for interventions like social and psychological support to improve the quality of life for theses students. Student advisors and counsellors may train students about stress management. 
Conclusion: The important findings of this study reveal serious stress disorders in a population of students in France with risk factors and risk comportments. Cyber addiction appears as a new comportment among students which have now almost all a personal computer and was a factor associated with perceived stress.

\title{
The Incidence of Secondary Traumatic Stress in workers dealing with traumatising materials, victims and perpetrators
}

\author{
Tehrani, N. \\ Noreen Tehrani Associates, United Kingdom
}

Background: The subjects in this study are worker involved in law enforcement and required to engage in viewing images of child abuse, reading testimonies relating to sexual crimes, viewing pictures of scenes of murders and post-mortems.

Objectives: To identify whether secondarily traumatised (ST) workers experience work related distress

Methods: 192 ST workers were involved in this study, 101 females and 81 males. The research process involved the psychological assessment of the ST workers. The assessment procedure included a structured interview which examined the workers current state of well-being, family history, relationships, personal style, education, employment and current stresses. The workers also completed a set of clinical and occupational questionnaires. Feedback was provided on the questionnaire results and where appropriate information, support or counselling.

Results: The results showed that the ST workers were not more anxious, depressed or burn-out than workers in non ST roles. However, the mean scores for PTSD were higher. A 20\% of ST workers experienced emotional exhaustion and low personal achievement but only 6 per cent had high levels of depersonalisation suggesting that ST workers are able to maintain their emotional connection with the primary trauma victims. In an exploratory factor analysis of the data dissociation was not associated with any of the other symptoms including depersonalization. There was a high correlation between neuroticism and the physical and emotional awareness responses $(p<001)$ suggesting that there may be a link between neuroticism, hyper-vigilance and reactivity to the psychophysical signs of distress. The factor analysis also confirmed Adams et al. (2006) findings that secondary traumatic stress and burn-out are separate factors.

Implications: An increasing number of crime analysts and law enforcement personnel are being exposed to traumatic materials; this study is a first step in finding out about the impact of ST on workers. The findings show that while some workers suffer ill effects others cope well.

Conclusions: The assessment of the ST workers provided an opportunity to consider the impact of working with traumatising material; however, there is a need to examine some of the emerging issues including: Is there a benefit to recognizing early physical or emotional responses to traumatic material? Does dissociation prevent investigators and analysts from noticing subtle trauma related evidence? Is there a way to identify the most appropriate coping skills? Is it possible to develop an organisational culture which promotes post-trauma growth? 


\title{
The family demands resource model
}

\author{
${ }^{1}$ ten Brummelhuis, L. \& ${ }^{2}$ ter Hoeven, C.L. \\ ${ }^{1}$ Erasmus University Rotterdam, the Netherlands; ${ }^{2}$ University of Amsterdam, the Netherlands
}

Introduction: The Job-Demands-Resource model (JDR model) has proven itself as a robust model explaining work outcomes (Demerouti, Bakker, Nachreiner \& Schaufeli, 2001). This model shows that employees with heavy job demands risk exhaustion and worsened in-role performance, whereas job resources contribute to their energy at work, and consequently, extrarole performance (Bakker, Demerouti \& Verbeke, 2004). However, the JDR model does not take into account that today's workforce often occupies a dual role, combining work and family. As for work, employees have demands and resources in their family life that affect their energy level (Ten Brummelhuis, Van der Lippe \& Kluwer, 2009). The current study investigates whether the assumptions of the JDR model can also be applied to the family domain. In line with the JDR model, we examine two pathways. First, we test whether family demands (i.e. perceived burden at home) affect the employee's in-role performance at work through emotional exhaustion. Second, we investigate whether family resources (i.e. quality time with family members) contribute to the employee's extra-role performance via engagement. We also evaluate the interaction effects of family demands and family resources on the work outcomes.

Methods \& Results: We analyzed the hypothesized model with Structural Equation Modeling (Arbuckle, 1997) using a sample of 1014 employees from a Dutch consultancy firm. The results confirm our expectations that family demands reduce in-role performance via increased exhaustion, whereas family resources enhance extra-role performance via increased engagement. In addition, we found that family demands decrease engagement, thereby diminishing extra-role performance, whereas family resources also decrease exhaustion and in turn in-role performance. A more detailed investigation of the model showed that employees who spent more time with their family experienced less exhaustion and performed better than employees who spent less time with family members. Finally, we found that the positive relationship between family demands and exhaustion was stronger for employees who spent more time with the family.

Conclusion: Our results show that the JDR model is applicable to the family domain. Whereas a heavy family burden hinders work outcomes, spending time with family members helps employees to perform better at work. However, unlike the JDR model, we did not find two separate processes. Both family demands and family resources affected exhaustion and engagement at work, resulting in reduced or enhanced in-role and extra-role performance. Finally, we note that spending time with family members functioned as a resource, unless people experience their family life as burdensome. 


\title{
Depression at work: The moderating role of depressive symptoms on the association between information flow and work outcomes
}

\author{
${ }^{1}$ ter Hoeven, C., ${ }^{2}$ ten Brummelhuis, L.L., \& ${ }^{2}$ Peper, B. \\ ${ }^{1}$ Amsterdam School of Communication Research ASCoR, the Netherlands; ${ }^{2}$ Erasmus \\ University Rotterdam, the Netherlands
}

Introduction: Depression has become one of the most critical public health challenges in the world (Ustun et al., 2004). It affects people's mood, expectations, and motivation and is one of the most common, but often unrecognized- mental disorders (Prins et al., 2008). Due to its prevalence depression is also a major workplace issue. However, depression is something that is not dealt with at work in an effective way. On the one hand employees feel that their working environment is not a safe place to talk about their depression and on the other hand employers might not know how to communicate with possibly depressed employees (Fitter \& Gulas, 2002). A possible explanation for the miscommunications between employers and depressed employees might be different attributional tendencies. It has been found that depressed people perceive information differently as opposed to nondepressed people (see Taylor \& Brown, 1988, for a summary). The current study starts by investigating whether depressed employees indeed perceive communication at work differently and how this affects their work outcomes. Consequently, the following research question will be addressed: Has organisational communication a different effect on work outcomes (i.e. helping behaviour and somatic tension) for employees who suffer from symptoms of depression as opposed to those who do not?

Methods \& Results: For the measurement, we used a sample of 1014 employees from a Dutch consultancy firm. An interaction effect was observed between perceived information flow (from the Organisational Culture Survey; Glasner \& Zamanou, 1987) and depression (CES-D; Kohout, Berkman, Evans, \& Cornoni-Huntley, 1995) on both employees' somatic tension and helping behaviour. Simple slope analyses further revealed that the effect of information flow on work outcomes is more pronounced for people who perceive themselves nondepressed or moderately depressed as opposed to depressed. In other words, a positive evaluation of the information flow in the organisation was associated with better work outcomes for nondepressed employees whereas a positive evaluation of the information flow for depressed had no influence on work outcomes.

Conclusion: The results confirm our expectations that information has a different effect on work outcomes for people who suffer from depression as opposed to those who do not. Organisational communication is often used to motivate employees and let organisational processes go efficiently. The results of this study suggest that different communication strategies are needed when communicating with depressed employees as opposed to non depressed employees. 


\title{
The Development and Validation of the Job Crafting Scale
}

\author{
Tims, M., Bakker, A.B., \& Derks, D. \\ Erasmus University Rotterdam, the Netherlands
}

\begin{abstract}
Objectives: The aim of the present study is to develop and test a scale to measure job crafting. Job crafting refers to the active changes that employees make in their own job design to optimize their person-job fit. On the basis of the Job Demands-Resources model, three dimensions were formulated to capture the changes that employees may make in their jobs. The first dimension, called 'increasing job resources', is suggested because research has shown that job resources are important antecedents of work engagement and that they may buffer the negative impact of high job demands. The second dimension, 'increasing job demands', may enable employees who have knowledge and skills to perform more difficult tasks to prevent boredom at work. The third dimension, 'decreasing job demands' is suggested because employees may lower the level of job demands when they perceive that these demands are too high for them. Thus, in some cases it will be worthwhile to lower the amount of job demands whereas in other cases they should be increased.
\end{abstract}

Methods: We performed two studies. In the first study $(\mathrm{N}=375)$ we used explorative factor analysis with varimax rotation in SPSS to investigate whether the three factors could be meaningfully distinguished from each other. In the second study $(N=294$ and $N=196)$ we aimed to cross-validate the findings of Study 1 in two new samples.

Results: The results of Study 1 provided evidence for the three dimensions of job crafting that we hypothesized. However, the dimension 'increasing job resources' turned out to consist of two separate factors. The first factor contained the items for increasing task variety, autonomy, and opportunity to develop oneself ('increasing structural job resources') and the second factor consisted of the items for increasing social support, feedback, and coaching ('increasing social job resources'). The four dimensions of job crafting could be reliably measured using 21 items. Study 2 showed that we could replicate this factor structure reliably in two additional datasets.

Implications: The availability of a job crafting scale may enhance research on this topic.

Conclusion: It may be concluded that this job crafting scale is a brief, internally consistent selfreport measure of job crafting behaviour that may be useful to researchers interested in proactive behaviour of employees.

\section{Do Transformational Leaders Enhance their Followers' Daily Work Engagement?}

\author{
Tims, M., Bakker, A.B., \& Xanthopoulou D. \\ Erasmus University Rotterdam, the Netherlands
}

Objectives: The aim of the present study is to investigate whether and how supervisors' leadership style influences followers' daily work engagement. Previous studies have shown that job resources and personal resources are important antecedents of work engagement. However, the role of the leader in fostering work engagement has received limited research attention. 
On the basis of leadership theories and the Job Demands-Resources model, we predicted that a transformational leadership style enhances employees' work engagement through the mediation of self-efficacy (Hypothesis 1) and optimism (Hypothesis 2), on a day-to-day basis. That is, employees working in a resourceful work environment (e.g., characterized by quality coaching, feedback, support) are likely to feel optimistic and to believe that they are able to achieve their work goals. Furthermore, it is proposed that transformational leaders enhance their subordinates' self-efficacy by expressing high expectations for them and confidence in their abilities to meet such expectations. Consequently, their work engagement may be enhanced.

Methods: We performed a diary study, in order to capture between- and within-person variations in the variables of interest. Forty-two employees first filled in a general questionnaire, and then a diary survey over five consecutive workdays. The trait (betweenperson) measures of the variables of interest were used as control variables in this study.

Results: The results of multilevel analyses offered partial support for our hypotheses. Daily transformational leadership related positively to employees' daily engagement, and daylevels of optimism fully mediated this relationship. However, daily self-efficacy did not act as a mediator in the relationship between transformational leadership and work engagement.

Implications: These findings expand theory and previous research by illuminating the role of transformational leaders in fostering employee work engagement through the enhancement of personal resources (e.g., optimism). The diary design of the present study offers the opportunity to capture work events and experiences as they unfold in the work environment. By examining day-level transformational leadership style as an antecedent of day-level work engagement, we gain more insight in the role of the leader in the daily process that explains employee engagement at work.

Conclusion: The present study demonstrates that daily fluctuations in transformational leadership may also influence employees' self-beliefs (i.e., personal resources) and work experiences (i.e., work engagement). Considering the strong positive link between work engagement and performance found in previous studies it is likely that their followers will perform better.

\title{
A study of personal and collective efficacy at work in an individualistic culture
}

\author{
Torkelson, E. \\ Lund University, Sweden
}

In the past, research about stress has mainly had an individualistic focus where the individual's appraisal has been seen as the core of the process. While several studies emphasize the role of personal resources such as self-efficacy (the personal cognitive estimate of ones capacity to perform), little attention has been paid to collective aspects such as collective efficacy. Selfconcepts are regulated, in part, by cultural orientations and in a collectivistic culture, people base their self-understanding on the reactions of others around them. In an individualistic culture, people are more prone to look to their own actions. Self-efficacy has in earlier studies shown to be inversely related to depression, pain and burnout. 
Objectives: The aim of the study was to contribute with further knowledge about individualistic collective aspects of efficacy at work and how these are related to mental health. The hypothesis was that in Sweden, regarded by Hofstede to be an individualistic country, personal efficacy beliefs would be negatively related to mental health problems whereas no link would be found between collective efficacy beliefs and mental health problems.

Methods: An internet-based questionnaire was sent to 240 employees working as administrators in a large Swedish construction company. A total of 133 employees (19 female and 112 male) completed surveys were received, resulting in a response rate of $55 \%$. The questionnaire included demographic items and scales measuring personal efficacy belief, collective efficacy belief, social support, control, quantitative demands, cognitive demands, job insecurity and mental health problems.

Results: The results showed contrary to the hypothesis that both personal efficacy beliefs and collective efficacy beliefs were negatively related to mental health problems. However, when all the study variables linked to mental health problems, were entered in the hierarchical regression analyses, only two predictors of mental health problems were found. The results showed that only number of children and quantitative demands predicted mental health problems.

Conclusions: Both personal efficacy beliefs and collective efficacy beliefs were negatively related to mental health problems. However, the main predictors of mental health problems were the number of children living at home and quantitative demands.

Implications: The need to consider collective and individual components in the stress process in future studies is addressed.

\title{
How positive psychosocial work factors may promote caring efficacy and mental health among psychiatric nurses
}

\author{
${ }^{1}$ Torp, S., ${ }^{1}$ Grimsmo, A., \& ${ }^{2}$ Bredrup, O.J. \\ ${ }^{1}$ Vestfold Mental Health Care Trust, Norway; ${ }^{2}$ Work Research Institute, Oslo, Norway
}

Background: Nurses have strenuous jobs and many suffer from adverse work-related disorders and diseases. Few studies have focused in particular on psychiatric nurses' work environment and health defined as something else than the absence of disease. The tensions of nursing may be of both psychosocial and physical character, but for nurses working in psychiatric wards the psychosocial measures may be the most important, since psychiatric nurses are often exposed to aggression, physical violence and threats from patients. Objective: The objective of this study was to investigate the relationships between work-related psychological demands, social support and decision latitude/control and psychiatric nurses' health, measured as caring efficacy and mental health. A particular objective was to investigate whether caring efficacy mediated the possible effects of the work factors on mental health.

Methods: A cross-sectional questionnaire study was performed among all psychiatric nurses in a Norwegian mental health care trust $(n=258)$. The Demands-Control-Support Questionnaire (DCSQ), a modified version of the Caring Efficacy Scale, and the mental component of the SF-12 Health Survey were used to measure the different independent and dependent variables. 
Results: A total of 196 nurses (76\%) responded. Nurses reporting high control and social support had significantly higher caring efficacy compared to nurses reporting low control and social support. Control was the most important psychosocial work factor positively associated with the psychiatric nurses' mental health. Caring efficacy correlated positively with mental health, but did not seem to mediate the effect of the psychosocial work factors on mental health. There was a negative correlation between mental health and the cross-product of caring efficacy and psychological demands, indicating that demands affect mental health negatively among nurses with high caring efficacy, but not among nurses with low caring efficacy.

Conclusion: Psychosocial work factors seem important for psychiatric nurses' positive health measured as caring efficacy and mental health. It is of particular importance to focus on improvements of the work environment in the health care sector because the forthcoming boom of elderly will result in increased strain on the sector and possibly on the personnel working there.

\title{
Task performance and organisational citizenship behaviour among Chinese nurses: The moderating effects of emotional exhaustion and organisational support.
}

\author{
'Tourigny, L., ${ }^{2}$ Baba, V.V., ${ }^{3}$ Han, J., \& ${ }^{4}$ Wang, X. \\ ${ }^{1}$ University of Wisconsin, Whitewater, United States of America; ${ }^{2}$ DeGroote School of \\ Business, Canada; ${ }^{3}$ China Europe International Business School, Shanghai; ${ }^{4}$.H. Asper School \\ of Business, University of Manitoba, Canada
}

We investigate the impact of task performance on organisational citizenship behaviour (OCB) among nurses in Mainland China. We explore how emotional exhaustion and organisational support moderate the relationship. We select OCB-Altruism as indicator of OCB oriented toward other individuals (OCB-I) and OCB-conscientiousness as indicator of OCB oriented toward the organisation (OCB-O) as criterion variables. We propose that in a collectivist society such as China, the relationship between task performance and OCB-O will be relatively stable, whereas the relationship between task performance and OCB-I may vary depending upon personal and contextual contingencies.

The sample consists of nurses and physicians from three large hospitals in Mainland China. Matched surveys were distributed to nurses and their supervising physicians. A total of 197 nurses with matched performance and OCB data from supervising physicians constitute the sample for this study. Validity and reliability tests are conducted with all measures. Confirmatory factor analysis endorses the validity of the OCB dimensions. All measures display satisfactory psychometric properties. Hierarchical moderated linear regression analysis is used to analyze the data. As expected, task performance is positively related to OCB-conscientiousness and to OCBaltruism. In line with the collectivist society argument, the 3-way interaction term is significant only for OCB-Altruism revealing that the relationship between task performance and OCB-I is both complex and contingent. Indeed, the pattern of this relationship changes depending upon how emotionally exhausted the nurses are and how much organisational support they receive. Among nurses reporting low organisational support, there is a positive relationship between task performance and OCB-altruism only when emotional exhaustion is low. When the nurses are emotionally exhausted, task performance does not spill over to altruistic OCBs. On the 
other hand, when the nurses receive higher levels of organisational support, the relationship between task performance and OCB-altruism is positive under conditions of high emotional exhaustion. This seems to suggest that organisational support facilitates the spillover effect of task performance on OCB-I by neutralizing emotional exhaustion. Given that the measures of task performance and OCB were provided by the supervisor and the measures of emotional exhaustion and organisational support were provided by the incumbent, we aver that the findings are free of single source method bias and are consequently more convincing. We also believe that they are useful for the practice of management in China's hospitals.

\title{
Healthy Change Process Index (HCPI) as a predictor of workplace bullying during organisational change
}

\author{
${ }^{1}$ Tvedt, S.D., ${ }^{2}$ Nielsen, M.B., \& ${ }^{3}$ Lau, B. \\ ${ }^{1}$ NTNU, Norway; ${ }^{2}$ University of Bergen, Norway; ${ }^{3}$ The National Institute of Occupational \\ Health, Norway
}

Objectives: The literature on bullying lists several risk factors in the working environment for workplace bullying: Authoritarian and aggressive leadership style, lacking role clarification, absent or evasive management, poor conflict management, and low control (Einarsen, et al, 2007). There is a striking resemblance between these and the factors related to psychosocial work environment and stress included in studies of the Healthy Change Process Index: Awareness of diversity in reactions to change, manager availability, role clarification, constructive conflicts and control (Tvedt, Saksvik, \& Nytro, 2009). Furthermore, in as much as workplace bullying can be seen as a symptom of an impaired psychosocial work environment, workplace bullying during organisational change needs special attention. However, there exist at present no studies on the relationship between $\mathrm{HCPI}$ and workplace bullying. Thus, the present study set out to test if HCPI can predict workplace bullying.

Methods: H1: Change process healthiness, as measured by the Healthy Change Process Index, directly reduces Bullying. $\mathrm{H}$ 2: The change process healthiness also has indirectly reducing effects on Bullying mediated by Control and Support, but not mediated by Demands. H3: Demands does not directly affect Bullying. The hypotheses were tested on sample consisting of four enterprises with a total $N=426$. Structural Equation Modelling (SEM) analysis was used by means of AMOS 7.0 software to test the hypotheses. The sample was assessed and found to be moderately non-normal, hence the Bootstrapping procedure was employed in the analysis.

Results: A model incorporating $\mathrm{H} 1, \mathrm{H} 2$, and $\mathrm{H} 3$ was achieved with reasonable fit (CMIN/DF $=2.805, \mathrm{GFI}=.968, \mathrm{AGFI}=.931, \mathrm{CFI}=.967, \mathrm{RMSEA}=.071)$. The model explained $11 \%, 44 \%$, and $19 \%$, of the variance in Control, Support, and Bullying respectively. All three hypotheses were fully supported.

Conclusions: The SEM analyses used in the present study should not be taken as confirmation of the causal nature of the models, and the moderate sizes and clustered nature of the samples limits the ability to make generalisations. However, the results indicate that healthy change process can reduce the experience of Stress and facilitate the long term coping of Stress from change related demands through enhancing Support and Control. These findings show that the 
HCPI together with the control and support dimensions of Karasek and Theorell's (1990) can be a good approximation to the psychosocial risk factors listed by the bullying literature, promising a fruitful new vista of research by examining workplace bullying during organisational change.

\title{
The importance of change content: A study of different change contents in the Norwegian food processing industry
}

\author{
Tvedt, S.D. \\ NTNU, Norway
}

Objectives: There is an increasing awareness concerning the need to differentiate between process and content in the study of organisational change (e.g., Burke, 2002; Murphy, 1989). However, systematic inquiries into the nature of change content are not easily found. Earlier research indicate that the relevance of organisational changes to co-workers' own everyday work experience is a powerful predictor of change-related stress (Nielsen, Randall, \& Albertsen, 2007; Saksvik \& Tvedt, 2009). And in the downsizing literature, experiences of threat experiences have been (see e.g. De Witte, 1999). Thus, the present study aims to test the viability of treating the co-workers' experience of concrete change contents in three general dimensions as predictors of change-related stress: 1) the number of changes 2) the personal relevance of the changes 3 ) the degree of personal threat posed by the changes.

Methods: Three hypotheses were tested in the present study by regression analyses. H1: The number of categories of change content perceived predicts more stress than the presence of any given category of change content. $\mathrm{H} 2$ : Change content categories high on personal relevance will predict the most stress. H3: Of categories that are high in personal relevance, those most threatening will predict the most stress. The sample was a representative sample of the employees in the Norwegian food processing industry $(\mathrm{N}=933)$. Indexes for the three dimensions were computed by categorizing eleven yes/no questions regarding concrete categories and dividing the response by the number of items for each additive index.

Results: H1 was partially supported; with the exception of Intensification of work - the single most reported change content, no single category of change content predicted more stress than the combined index of Extent of change. $\mathrm{H} 2$ and $\mathrm{H} 3$ were fully supported.

Conclusions: The cross-sectional character of the data in the present study, and especially the use of nominal questions, limits statistics and conclusions. However, the results indicate that it is possible to treat change content in general dimensions that can be combined to describe any change content adequately. Paradoxically, moving from specific questions about particular categories of change to general dimensions concerning the psychosocial nature of the change content, may allow for more accurate risk evaluations of change-related stress. The results of the present study points to three possible dimensions in such change-content evaluations: 1) the extent, or number, of changes 2) the personal relevance of the changes 3) the degree of personal threat posed by the changes. 


\title{
Darling, it's gonna be late tonight: The relationship of work time on relationship quality
}

\author{
Unger, D., Niessen, C., Sonnentag, S., \& Neff, A. \\ University of Konstanz, Germany
}

\begin{abstract}
As more women take part in the job market there are more couples in which both partners pursue independent careers. For both partners in these dual-career couples time investment is a key strategy in order to progress with their careers. From a work-family-conflict perspective less time left for the partner should be associated with an impaired fulfilment of one's role demands within the relationship. So it is extremely important for dual-career couples to cope with time scarcity. In our study we focus on two goals. First, we investigated the relationship of work time and relationship quality on a daily basis. Second, we studied the attenuating effects of detachment from work on the association of work time and relationship quality. Detachment is the mental disengagement from work during non-work time. Knowing moderators is critical if we want to help dual-career couples establishing work-life balance although they face time scarcity.
\end{abstract}

We conducted a diary study with 73 dual career couples. On five consecutive workdays participants completed online survey measures of work time, quality of marriage, relationship conflict, and detachment from work. Applying the actor-partner interdependence model and controlling for the general level of quality of marriage, gender, and relationship length we conducted multi-level data analyses.

First, we examined the main effects of the actor's and partner's overall work-time on the time the couple spends together. The longer both partners work the less time they spend together. Second, we used the time spent together as a predictor of daily quality of marriage and relationship conflict. The time the couple spends together significantly predicts higher partner reports on quality of marriage. This association was not found in the case of relationship conflict. Third, we examined detachment from work as a moderator of the relationship of time spent together and quality of marriage respective relationship conflict. Detachment from work significantly moderates the association of time spent together and relationship conflict. If actors are high in detachment from work, partners report more relationship conflict as the couple spends more time together. For actors low in detachment from work this pattern is reversed. Detachment from work does not moderate the relationship of time spent together and quality of marriage.

In sum, detachment from work proves itself being beneficial for employees' work-life balance. Especially for couples with time scarcity actor's detachment from work has salutary effects on the relationship quality as partners report less conflicts.

\section{A daily study of effort and recovery: examining the role of time, effort and pleasure}

\author{
${ }^{1}$ van Hooff, M., ${ }^{2}$ Geurts, S., ${ }^{2}$ Beckers, D., \& ${ }^{2}$ Kompier, M. \\ ${ }^{1}$ University of Amsterdam, the Netherlands; ${ }^{2}$ Radboud University Nijmegen, the Netherlands
}

Objectives: According to effort-recovery theory (Meijman \& Mulder, 1998) an enduring lack 
of recovery opportunities after effort investment at work will eventually result in adverse health outcomes. However, the daily process of effort and recovery is still not very well understood. The present study aims to advance insight in this process by examining if and how daily positive experiences, daily effort expenditure and daily time investments in the work and non-work domain relate to recovery indicators both at the end of the work day and before going to bed.

Methods: A daily diary design covering five consecutive workdays was employed to answer the research questions. Participants were 120 university faculty members, who completed three questionnaires daily, addressing the time spent on work activities and on various home activities (i.e. overtime work, domestic activities, active leisure activities and low-effort activities), as well as the effort expended and the pleasure experienced while engaging in each of these activities. Fatigue and vigour were included as indicators of (a lack of) recovery. Data were analysed by means of multilevel analysis.

Results: The time spent daily on work activities was positively related to fatigue, but was unrelated to vigour. Work-related effort showed no relationship with both indicators of recovery. Pleasure experienced at work turned out to be positively related to vigour and negatively to fatigue. In the home domain, the time spent on each of the activities was not associated with both recovery indicators. Effort expended in the non-work domain was not related to the indicators of recovery either, but pleasure showed a positive relationship with vigour and a negative relationship with fatigue before going to bed.

Conclusion: Whereas the time spent on work activities turned out to increase employees' need for recovery, the time spent on home activities shows no relationship with recovery indicators. This demonstrates that focusing exclusively on activity patterns might not be sufficient to explain the process of effort and recovery. Instead, the psychological characteristics of these activities might be more important. Of these characteristics, effort expenditure does not by definition impede employee well-being. Both in the work and home domain however, the experiencing pleasure turned out to significantly contribute to recovery.

Implications: To foster their well-being, employees should not by definition refrain from effortful activities. They can nonetheless be advised to engage in pleasurable activities both in the work and in the home domain.

\title{
HRM, psychological employee well-being \& organisational performance: a 2-1-2 multilevel mediational analysis
}

\author{
${ }^{1}$ van Veldhoven, ${ }^{1}$ M., ${ }^{1}$ Croon, M., ${ }^{2}$ Peccei, R., \& ${ }^{3}$ Wood, S. \\ ${ }^{1}$ Tilburg University, the Netherlands; ${ }^{2}$ King's College London, United Kingdom; ${ }^{3}$ Institute of \\ Work Psychology Sheffield, United Kingdom
}

Objectives: Theories about organisational processes often try to connect multiple levels, and in research based on such theories data structures can arise which classify as multilevel mediation models. Multilevel mediation is of particular importance to research on Human Resource Management and organisational performance. Typically, the focus in this research area is on examining the effects that organisational policies, practices or interventions have on various aspects of organisational performance through a range of employee-level variables. There is 
considerable debate about which variables should be included in organisational (level 2) and individual (level 1) levels of analyses, but in general a 2-1-2 (organisation-individual-organisation) process holds in many instances (Nishii \& Wright, 2008). The development of methods and statistics for dealing with 2-1-2 mediational data has lagged behind. The first objective of this paper is to present an analytic solution for dealing with such data and illustrate it with a concrete example.

Furthermore, in the literature two competing views can be found with respect to the role of psychological employee well-being in relation to HRM and performance. In the mutual gains view, HRM is positive for employee well-being as well as organisational performance. Indeed, increased employee well-being is often thought to mediate the performance benefits of HRM for the organisation. In the conflicting outcomes-view, HRM achieves benefits for organisational performance at the expense of reduced employee well-being. The second objective of this paper is to research empirically the evidence for the mutual gains and confllicting outcomes views.

Methods: Data from the 2004 edition of the UK Workplace Employment Relations Survey are used. Mplus is used to analyze a 2-1-2 multi-level mediation model, based on an extended version of the procedure proposed by Croon \& van Veldhoven (2007). HRM and performance data were derived from management interviews in each of $>1,200$ workplaces. Employee wellbeing was assessed by means of a survey among $>15,000$ employees in these workplaces.

Results: We find that workplace level work enrichment initiatives are related to increased performance (all 4 types) through increased job satisfaction. Work enrichment is not related to anxiety among workers. A direct effect on performance of work enrichment is only found for 1 type of organisational performance. Workplace level high-involvement management is related to anxiety, but not to job satisfaction. Performance effects of high-involvement management are mostly direct, and are established for 3 types of organisational performance indicators.

Implications: It is now technically feasible to run multilevel mediation models which enable researchers to connect individual level variables about worker well-being (including health) to organisational level antecedents as well as outcomes.

Furthermore, results show that the WERS data from the UK support both mutual gains and conflicting outcomes views, depending on the linkage investigated. The results on work enrichment, job satisfaction \& performance fit a mutual gains view. Results on high-involvement management, job anxiety \& performance are more in line with a conflicting outcomes view. The results support a differentiated view of the linkages between HRM, well-being and performance.

\title{
Effectiveness of an empowerment based job retention program for employees with a chronic disease; a randomised controlled trial.
}

\author{
Varekamp, I., \& van Dijk, F.
}

Coronel Institute of Occupational Health, the Netherlands.

Objectives: A growing number of persons aged 16-65 have a chronic somatic disease. If employed, many of them are hampered in job performance. Emotional distress, fatigue, insufficient social support and lack of work accommodations may lead to unnecessary job loss. We investigated 
the effectiveness of a group training programme aimed at a) exploring and clarifying practical, social and psychological work-related problems, b) communication at work, and c) thinking out and realising solutions.

Methods: Participants were randomly assigned to the intervention group $(n=64)$ or the control group ( $n=58$ ). Self report questionnaires were filled in at baseline, after 4, 8, 12 and 24 months. Outcome measures are work related self-efficacy, fatigue, job dissatisfaction and job retention.

Results: Three participants dropped out of the training programme halfway. Loss to research follow-up was 0/122, 3/122 and 6/122 after 4, 8 and 12 months. After one year, self efficacy had increased significantly more for the experimental group, fatigue decreased more for the experimental than for the control group but not statistically significant, and job dissatisfaction decreased in the experimental group and increased in the control group, but the difference was not statistically significant. Only a few persons in both groups lost their job. The participants of the training group evaluated the programme with a mean score of 8.1. Especially the focus on feelings and thoughts about having a chronic disease was highly valued, as were the exchange of experiences and role-playing directed at more assertive communication. Sixty-one percent of the control group stated that they or others had undertaken measures to solve work-related problems because they were randomised to the control group.

Implications: This study points to the importance of addressing psychosocial aspects of working with a chronic disease beside practical problems.

Conclusions: An empowerment based job retention program is feasible and is perceived to be effective by the participants. Statistically significant differences are found on some, but not all outcome measures. For vocational rehabilitation interventions, where blinding participants is not possible and participants may find other solutions to their problems at work, the rct-design has serious shortcomings.

\title{
Work-Related Stress Assessment: An Organisational Approach
}

\author{
Vecchio, G.M., Fiaschi, S., Pacelli, F., \& Pacelli, E. \\ University of Rome 'Sapienza', Italy
}

Objectives: According to the European Framework agreement on Work-Related Stress of 10/8/2004, Work-Related Stress regards aspects relating to the planning, organisation and management of work, besides their environmental and social contexts that have the potential to give rise to physical, social, and psychological damages. The European Agency for Safety and Health at Work (2000) identified psychosocial risk factors related both to the intrinsic characteristics of work and those concerned to organisational contexts.

This paper describes a protocol to assess risks from Work-Related Stress (according to current Italian Laws) that, according to the model proposed by the W.H.O., is characterized by: a) focus on primary prevention (including both risk and protective factors); b) partecipative approach; c) organisational perspective (by examining both organisational structures and processes with qualitative and quantitative methods) 
Methods: The protocol follows 6 steps:

1. Start-up. Preliminary meetings about intervention methodologies and procedures with all stakeholders involved by Italian laws;

2. Quantitative data collection. Desk analysis on organisational structure and safety, collective administration of the Multidimensional Organisational Health Questionaire (MOHQ);

3. Preliminary results. Findings are discussed with the stakeholders.

4. Qualitative data collection. Focus Group sessions with homogeneous work teams to deepen the results.

5. Final Report on Work-Related Stress, including results, an action plan to correct negative aspects and enhance positive aspects, and promotional activities of the results to all workers.

6. Follow-up. After six months, a meeting is proposed to monitor advancement of actions proposed.

Results: Over the last year, the protocol was applied on 12 Italian companies located in Lazio, of different ATECO sectors, for a total sample of 1456 workers (708 men, 715 women).

Findings are discussed about the differences on perception of risk factors, protective factors and job satisfaction by gender, age and organisational size.

Implications: The described protocol allows the identification of specific measures of prevention and protection for homogeneous work groups, and provides elements for organisational development of the whole company.

Conclusion: The protocol described provides the other company divisions (eg Human Resources) precise management strategies about Organisational Health dimensions, both in general and on homogeneous groups of workers. Moreover, the involvement and participation of all workers sets up the start of a process of development of organisational citizenship. Among the limitations of the protocol, we point out the difficulties of dealing with a bureaucratic approach to work safety by some company, and high costs related to the complexity of the actions proposed.

\title{
Workplace and couple relationship: how are they related?
}

\author{
Vintila, M., Goian, C., \& Cintar, D. \\ West University of Timisoara, Romania
}

Background: There is no doubt that men and women have different needs and expectancies in a relationship. This makes us search for the right partner. One of the most common places to find a partner is at work. In the present paper we assess some relational differences between couples who work at the same place and those who have different jobs.

Methods: a number of 40 subjects who have the same work place as there partner were compared with 40 who work in different organisations. We assessed there level of jealousy, perception of sex-life and perception of happiness by using: the sexual relationship scale, the Lock-Wallace Marital Adjustment test, the hypothetical jealousy - producing events scale and the dominance - accommodation scale. 
Results: We found out that the jealousy level is higher for those who work in different places compared to those who work together, $t=-13.164$ at $p<.01$. The average value for those who work at separate work places was 67.07 compare to 47.79 of the other group. This is confirmed when we compare results taking into consideration gender. Neither on the whole sample, nor when analyzed by gender could we find differences related to the perception of sex life. Perception of happiness was found to present no differences between the two groups.

Conclusions: The only significant difference between the assessed parameters concerns the level of jealousy. Those couples who work in the same place have a lower jealousy level due to the fact that they know exactly the persons the partner is interacting with, so their level of trust is higher and the level of jealousy is normal. Also, the compatibility between the partners is higher as partners where chosen on criteria of similarity. The lack of differences on sexual relationship and happiness can be explained through the fact that sex is a basic human need, so that physical attraction is the first criteria of selection, common at all people regardless of their work place, as a filter comes afterwards the psychological criteria. This second one will than contribute to the decision of building and maintaining a serious relationship or move on to another experience.

\title{
Work-Family Conflict and Supervisor Support: The Role of Perceived Similarity
}

\author{
Waite, E., Rubino, C., \& Avery, D.R. \\ University of Houston, United States of America
}

Objectives: Research on work-family conflict (WFC) has shown that tension between work and family roles leads to an array of negative outcomes such as increased turnover intentions, lower perceived career success, and substance abuse (see Eby et al., 2005 for a review). Although studies have found that support decreases WFC (Adams, King, \& King, 1996; Foley, Linnehan, Greenhaus \& Weer, 2006), studies have not looked at the joint effects of supervisor racioethnic similarity and supervisor support. Thus, the present study seeks to extend the perceived organisational support (POS) literature and the WF interface for racioethnic minorities by examining how racioethnic similarity and support in the workplace independently and interactively relate to WFC among Black and White employees. We hypothesize that perceived supervisor support will moderate the supervisor racioethnic similarity - WFC relationship for Black employees, such that the negative relationship between supervisor racioethnic similarity and WFC will be strengthened if there is high perceived supervisor support.

Methods: The study retained data collected by the Families and Work Institute for the 2002 National Study of the Changing Workforce $(\mathrm{N}=3,552)$ (Bond, Thompson, Galinsky, \& Prottas, 2002).

Results: The hypothesis was tested using hierarchical moderated multiple regression. We entered the controls (i.e., work hours, tenure, education, age, and sex) in step 1. We then entered supervisor racioethnic similarity and supervisor support in step 2, and the supervisor racioethnic similarity $x$ supervisor support interaction in step 3 . We conducted this analysis separately for both Black and White employees. The supervisor racioethnic similarity $x$ supervisor support interaction was significant for Black employees $(B=.56, p<.01)$, but not for White employees $(B=.05, \mathrm{p}=$ n.s. $)$. 
Conclusion: For both Black and White employees, high levels of supervisor support resulted in less WFC. Racioethnic similarity differentially impacts WFC depending on one's racioethnicity and the domain of support. Whereas for Black employees, the negative relationship between support and WFC was strengthened when the supervisor was racioethnically similar, this was not the case for White employees. Although a model for WFC exists, it does not highlight how work demographics can affect conflict. The similarity-attraction paradigm asserts that racioethnic similarity can lead to increased positivity in relationships (Byrne, 1971), which may be helpful in reducing WFC for Black employees. Organisations should focus on supportive supervision for all employees, but also investigate the benefits of racioethnically congruent employee-supervisor relationships for minority employees.

\title{
The effect of risk perception and optimistic bias on safety climate attitudes and behaviour
}

\author{
Walker, A. \& Pearce, M. \\ Deakin University, Australia
}

Objectives: Employees' perception of injury risk has been identified as an antecedent to safety climate attitudes and behaviour in the workplace. Perceptions of risk in the workplace vary between individuals and have been shown to be influenced by a range of factors. In relation to personal risk, individuals have been shown to underestimate their level of risk associated with a range of life events compared to others. This is termed the optimistic bias. There is evidence that the optimistic bias can increase risk-taking behaviour. For example, road safety research has demonstrated a negative relationship between optimistic bias and wearing a seat belt, and a positive relationship between optimistic bias and running a red light. However, there is little known research examining how the optimistic bias may influence occupational safety attitudes and behaviour, or perceptions of risk in the workplace. The present study aimed to examine the influence of risk perception and optimistic bias on safety climate attitudes and behaviour.

Methods: Participants were 153 power generation workers recruited from three large electrical power stations located in Victoria, Australia. A self-report questionnaire measuring safety climate attitudes, safety behaviour, risk perception and the optimistic bias was completed. Participant demographic information was also collected.

Results: Regression analyses found that risk perception, but not optimistic bias significantly influenced safety climate attitudes. Risk perception was also found to fully mediate the relationship between optimistic bias and safety climate attitudes in the predicted direction. Contrary to expectations, neither risk perception nor optimistic bias was found to influence safety behaviour.

Conclusion and Implications: Contrary to findings in road safety research, optimistic bias was not found to be directly related to occupational safety outcomes. This is surprising as it might be assumed that employees who believe they are invincible are also more likely to engage in unsafe work practices and to hold poor safety attitudes. Rather, the current study found that perceptions of overall workplace risk were associated with poor safety attitudes. The implications of this research for occupational safety research are explored. 


\title{
The Effects of Job Demand and Job Control on Perceived Stress and Motivators among Information Technology (IT) Consultants
}

\author{
Wallgren, L.G. \\ University of Gothenburg, Sweden
}

Objectives: It is generally believed that knowledge firms that are dependent on the latest technology and a highly competent workforce (e.g., IT consultancy firms) represent the future in business and working life. The aim of this study was to explore the relationship between the combined effects of control and demand part's on perceived stress and part's on motivators (e.g., responsibility, recognition, achievement, and possibility for growth).

Methods: A web-based questionnaire survey was conducted among IT consultants employed at ten IT consultancy firms in Sweden $(\mathrm{N}=380)$. Two models consisted of demands and control as independent factors with perceived stress and motivators as dependent factors respectively. Subjects were categorised by dichotomising all factors to "low" (first half) and "high" (second half) groups. In the models I have used the theoretically lowest strain group (i.e. low psychological load and high decision latitude) as a reference category. Prevalence rate ratio ("relative risk") (RR) with $95 \%$ confidence intervals according to Mantel-Haenszel weighted RR were calculated.

Results: All three combinations produced significantly increased RR for perceived stress; high level of demands and low level of control $(R R=4.05, C l=2.82-5.83)$, high level of demands and high level of control $(\mathrm{RR}=3.08, \mathrm{Cl}=2.08-4.57)$ and low level of demand and low level of control $(\mathrm{RR}=2.13, \mathrm{Cl}=1.39-3.26)$. Furthermore, two of three combinations produced significantly difference to motivators, according the reference group with high motivators $=69.6 \%$; high level of demands and low level of control (High motivators $=41.1 \%, \chi^{2}=17.89, p<.001$ ) and low level of demands and low level of control (High motivators $=37.9 \%, \chi^{2}=20.85, p<.001$ ). Nonsignificant difference; high level of demands and high level of control (High motivators $=57.5 \%$, $\left.\chi^{2}=2.94, p=.086\right)$

Implications: Since managers have a substantial influence on the work organisation, it is important to involve and educate managers on the factors related to stress among their subordinates. The practical implications are that managers must find a management or leadership style which in interaction with the organisation creates a healthy work organisation which increases a sustainable motivating environment.

Conclusions: The study concludes that the work characteristics of job demands and job control are important factors in explaining the genesis of perceived stress and motivators among IT consultants. Thus, compared to the theoretically lowest strain group the group with the combination of high level of demand and a low level of control had a four times higher "risk" of experiencing a high level of perceived stress and the two groups with low level of control 


\title{
Bunrnout and secondary trauma: a review of the emotional costs of nursing
}

\author{
Watts, J. \& Robertson, N. \\ University of Leicester, United Kingdom
}

Objectives: There are occupational health implications for nurses treating those in pain. Researchers have observed that nurses may exhibit symptoms of secondary trauma following interaction with a patient in emotional or psychological distress. This systematic literature review sought to determine current understanding and provide a summary of risk factors and sources of resilience in order to inform future research and policy.

Methods: 6 electronic databases were searched using 19 keys terms to reveal a total of 2156 abstracts. After removing duplicates, non-empirical investigations and reports of primary trauma, 23 relevant papers emerged.

Results: A data extraction form was used to determine how nurses' distress had been measured and perceived. In addition, the sample characteristics, observed consequences to nurses, predictive variables and coping strategies were also noted. The review revealed that many concepts have been used to describe nurses' distress, however, they described very similar experiences. The most common terms were Compassion Fatigue, Burnout and Posttraumatic Stress Disorder. A vast range of specialities and locations have been studied from both acute and chronic wards. However, the review revealed a lack of research into non-hospital care environments. There is also a need for further quantitative research. Factors associated with burnout and secondary traumatic stress included the type of ward, hours of patient contact, interpersonal conflict and empathy experienced for the patients. Many authors claimed that empathy was vital to the development of secondary traumatic stress reactions. Patient characteristics appear to moderate nurses' vulnerability with heightened responses to suffering children, patients with similarity to the nurse and patients who resemble loved ones. Coping strategies suggested by nurses and researchers included maintaining professional boundaries during patient care. However, coping strategies and sources of resilience have not been thoroughly investigated.

Implications: There is scope for further research specifically involving nurses not working within a hospital setting. In addition it is important to determine how nurses can maintain a good quality of care but have sufficient boundaries between the patient to reduce potential vulnerability to secondary traumatic stress or burnout.

Conclusions: The literature review suggested that patient characteristics can be highly influential in the development of burnout and secondary traumatic stress in the carer. Accordingly, empathy for the patient is thought to be vital to the pathway that can lead nurses to become vicariously traumatized. Therefore this relationship warrants further investigation in order to inform occupational policy and staff training. 


\title{
Socio-demographic implications of extended working life
}

\author{
Weyman, A. \\ University of Bath, United Kingdom
}

This review paper relates to futures research undertaken for the UK Health and Safety Executive aimed at mapping and articulating the implications for the quality of working life arising from projected increases in the age of retirement. It seeks to assimilate and draw inference from empirical research; published discourses within the policy policy domain and commentaries offered by employers and trades unions.

Objectives: To review published findings on the quality of working life for employees and the wider socio-economic implications of extending the age of retirement beyond 60/65yrs. To identify and articulable public policy relevant evidence gaps and research needs. To highlight and articulate the implications for policy makers in terms of promoting and maintaining the health and well-being of older workers.

Methods: Review \& thematic analysis of relevant academic literatures; published government/ agency policy documents; employer associations, trades unions and other NGO commentaries from the grey literature and web based publications.

Results: There is notable alignment between key stakeholders over the benefits of extending working life; specifically government perspectives, dominated by economic implications of funding worthlessness; employers desire for labour force flexibility and advocacy groups pursuing choice and social inclusion agendas.

Managerial orientations to older workers are widely viewed as the most significant barriers to the employment, negative associations surrounding trianability; return on investment; creativity, cautiousness, productivity physical capabilities and accident-proneness. The government policy perspective is oriented around employer self-regulation and corporate social responsibility, with much faith vested in education initiatives aimed at propagating evidence of a business case employing of older workers.

The public policy and focus is on work and worklessness, rather than quality of work, with modest attention to psycho-social aspects.

Implications \& Conclusions: Overwhelmingly, contemporary evidence is of older workers being disproportionately excluded from work and over-represented in low skilled, less secure, low paid work. In the absence of legislative intervention, a change in this profile rests upon the impact of government initiatives aimed changing employer orientations. There are grounds for inferring that extended working life, rather than reducing social differences may have the effect of increasing inequality in old age. Forseeably, there will be marked differences in patterns and employee options for the extension of working life by profession and sector.

Good practice is evident amongst some employers, despite the absence of strong evidence of a demonstrable business case. Research is needed to understand what motivates this good practice. 


\title{
Effects of a stress management intervention on absenteeism and return to work - results from a randomized wait-list controlled trial
}

\author{
${ }^{1}$ Willert, M.V., ${ }^{2}$ Thulstrup, A.M., \& ${ }^{3}$ Bonde, J.P. \\ ${ }^{1}$ Aarhus University Hospital, Denmark; ${ }^{2}$ Aarhus University Hospital, Denmark; ${ }^{3}$ Bispebjerg \\ Hospital, Denmark
}

Objectives: High levels of work-related stress are associated with increased absenteeism from work and reduced work ability. The effects of a stress management intervention on absenteeism is investigated.

Methods: 102 participants were randomized to either the Intervention (I) or the Wait-list control (WLC) group. The I-group received the intervention in weeks 1-16 from baseline, and the WLC-group received the intervention in weeks 17-32. Self-reported data on absenteeism, defined as days full or part time absent from work within the previous three months, were obtained at 16, 32 and 48 weeks follow-up from baseline. Register-based data on long term absence from work were drawn from the DREAM database from baseline and 48 weeks onwards. The DREAM database contains weekly information on on long term sickness absence compensation and other public transfer payments. Threshold to enter DREAM is sick leave for two consecutive weeks. Data are reported with 95\% confidence intervals in parantheses.

Results: On self-reported absenteeism the I-group median was 11(3-25) days absent in the three months preceding the post-intervention measurement. The median of the WLCgroup in the same interval was 45(19-60) days. This difference was statistically significant $(p=0.02)$. On register-based long term absence from work (cumulative weeks in the DREAM database, weeks 1-16, the I-group had a median of 6(0-11) weeks, while the WLC-group median was 12(8-16) weeks. This difference fell short of reaching statistical significance $(p=0.06)$. Regarding the rate of lasting return to work in weeks $1-48$ a hazard ratio of 1.58(0.89-2.81) favouring the I-group was found, with a corresponding $p$-value of $p=0.12$.

Implications: The observed reduction in absenteeism from work from the intervention, has potential clinical and practical implications, since costs associated with absenteeism from work is a major concern for employers, and society as a whole. Reduced absenteeism from work may result in a more stable productivity output, less strain on co-workers, less reliance on temps, less expenditure on sickness compensation benefits, less use of health care benefits, and reflect improved psychological health of workers.

Conclusions: The intervention is effective in lowering self-reported absenteeism from work. No conclusive evidence on long-term absence from work was found from the register-based data. No conclusive evidence was found regarding rate of return to work. 


\title{
The Three-Level Model of Psychological Stressors - Limitations in Workplace Prevention of Psychological Stress
}

\author{
Windemuth, D. \\ BGAG-DGUV, Germany
}

Psychological stressors are a normal part of human life - not only in the workplace. Included amongst important positive consequences of psychological stress are stimulation, variety, learning progress and training effects. Possible negative consequences include short and long term strain in physical, psychological and behavioural terms. Whether negative consequences result is dependant on one hand by the extent, that is, the intensity and duration of the psychological stressor and on the other hand by the capability of the affected person to deal with psychological stress.

Much has already been discussed in the literature concerning possibilities for prevention. However, limitations in prevention have received less attention. This results from the fact that:

1. companies operate in a social context;

2. people are also exposed to psychological stress in their private lives and;

3. illnesses are caused by multiple factors.

The first two points are explained through the Three-Level Model. The third point through current etiopathogenetic models.

\section{Workplace Bullying and Staff Well-Being: A Mixed Methodological Approach}

\author{
Woodrow, C. \& Guest, D. \\ King's College London, United Kingdom
}

Objectives: A growing body of research evidence has identified workplace bullying as a serious threat to employee well-being. Much of the existing research in this area is based on crosssectional survey methods and focuses on the victims of bullying. As a result, our understanding of the process through bullying affects well-being remains incomplete, and it is therefore difficult for organisations to manage the problem effectively. To address this issue, experts have called for more innovative approaches to the study of workplace bullying. This study aimed to answer these calls by examining the process through which workplace bullying is related to staff wellbeing using a unique mixed methodological approach.

Methods: The research was undertaken in two healthcare organisations in the UK with particularly high reported levels of staff bullying, and consisted of two primary elements. First, statistical analysis of staff attitudes survey data from the host organisations was conducted to examine associations between bullying behaviour and measures of work-related well-being (such as stress). Second, a series of semi-structured interviews, based on the critical incident technique, were conducted with organisational staff. Interview participants included victims of bullying, witnesses of bullying and individuals who had been accused of bullying. 
Results: The statistical analysis revealed that bullying behaviour was related to poorer levels of well-being in victims. The interview series provided a number of additional insights into this relationship. A variety of damaging effects of bullying emerged, both emotional (including feelings of humiliation, disempowerment and isolation) and behavioural (including poorer relationships with colleagues and poorer quality interactions with service users). Importantly, the consequences of bullying were not limited to victims, but were also evident in those who were accused of bullying and others in the work group.

Implications: These results suggest that in addressing bullying, there is a need for organisations to consider well-being beyond that of the staff who are victims and to take account of the complex emotional and behavioural reactions to the behaviour.

Conclusion: This study further highlight the highly damaging nature of workplace bullying and provides new insights into the way in which staff well-being is harmed. Additionally, the results imply that survey research is a necessary but insufficient method for studying workplace bullying, and suggest that the use of more innovative methodologies will produce further insights into this complex issue.

\title{
Relaxation or Psychological Detachment? A Diary Study on Recovery among Workaholics
}

\author{
${ }^{1}$ Xanthopoulou, D. \& ${ }^{2}$ Bakker, A.B. \\ ${ }^{1}$ University of Crete, Greece; ${ }^{2}$ Erasmus University, the Netherlands
}

The purpose of the present diary study was to examine the daily recovery of workaholics. In general, resource expenditure during work enhances employees' need for recovery after work. Recovery is the process during which individuals restore the resources that have been used up during work hours. Due to energy loss, the higher the need for recovery, the more difficult it is for individuals to engage in activities that help them unwind. Effective recovery can be particularly difficult for workaholics, who work beyond what is reasonably expected from them. Workaholics spend long hours working not because they enjoy it, but because of an obsessive inner drive. Therefore, employees high (vs. low) in workaholism are more likely to ignore their need for recovery and they continue working or ruminating about work during off-job hours thus, avoiding engagement in recovery activities. Based on the effort-recovery model, we hypothesized that the negative relationship between daily need for recovery after work and recovery experiences (relaxation and psychological detachment) during off-job hours is particularly strong for employees high (vs. low) in workaholism. Furthermore, it was hypothesized that relaxation and psychological detachment after work have a positive effect on actual recovery as reported the next day.

To test these hypotheses, a diary study was conducted among 42 Dutch primary school teachers. Participants filled out first a survey, and then a diary for five consecutive work days, twice per day: after leaving work and before going to bed. Multilevel analyses supported our hypotheses for relaxation but not for psychological detachment. Specifically, daily need for recovery (as reported right after work) interacted with workaholism in predicting relaxation after work (as reported in the evening). 
In line with predictions, plotting procedures showed that the negative relationship between need for recovery and relaxation was particularly strong for workaholics. In turn, relaxation was positively related to next day's recovery. Additional analyses suggested that workaholism had an indirect negative effect on next day's recovery through previous night's lack of relaxation.

These results are important from a theoretical point of view because they explain why workaholics find it hard to recover on a day-to-day basis. It is suggested that trainings that help workaholics recognizing their need to recover after work may be particularly beneficial for their well-being.

\title{
An Innovative Approach to Address Workplace Stress in Healthcare
}

\author{
Zogran, C. \\ Virginia Commonwealth University, United States of America
}

This paper is intended to present an innovative educational approach developed for undergraduate nursing students at Virginia Commonwealth University in Richmond, Virginia to address workplace violence in the health care industry. The objective of this program is to provide a comprehensive understanding of the various stressors health care professionals may encounter prior to their entering clinical practice. Strategies for self-care and promotion of effective stress management practices are covered from both a didactic and experiential approach. This program could easily be adapted for staff development and health promotion programs in a variety of occupational settings.

Recent studies indicate that workplace violence in the healthcare industry have increased in frequency and severity. In a 2009 survey conducted by the American College of Physician Executives, over $97 \%$ of the nurses and doctors reported experiencing behaviour problems from co-workers in the health care environment. The range of negative behaviours experienced varies on a spectrum of a "hostile work environment" up to actual workplace violence. The majority of the behaviours were in the realm of verbal abuse including degrading comments, inappropriate joking, yelling, and cursing at another co-worker. Other behaviours included refusing to speak or work with one another, spreading malicious rumors, or trying to get someone fired. Although physical assaults were less frequent (reported by less than $3 \%$ of the respondents), they did occur. The frequency of the behaviours was reported to occur on a weekly basis by one third of the respondents and ten percent stated that they occurred daily. The study cited only dealt with behavioural problems between co-workers. The additional stressors stemming from patients who are confused, intoxicated, or psychotic also pose a threat to the health and safety of staff.

As a faculty member working with students who are preparing to enter this environment, I have developed a four-hour class that integrates information on assertiveness training, leadership skills, group process, interpersonal communication, relaxation techniques and lifestyle management. The content of this course is largely based on research from the fields of mind-body medicine, the neurobiology of stress, and positive psychology. Students learn about the stress response, how to practice relaxation techniques, how to identify their style of communicating under stress, the characteristics of groups, effective leadership and good communication skills. The overriding goals are to increase self-awareness, especially in relation to how each person perceives and copes with stressful situations, and the promotion of healthy self-care practices. 


\title{
Work-related stress assessment: organisational demands and methodological considerations
}

\author{
Zuffo, R.G. \& Cortini, M. \\ University of Chieti and Pescara "G. D'Annunzio, Italy
}

Objectives: The legislative decree 81/08 established a deadline (many times postponed, today the deadline is august the 1st, 2010) in order to analyze work-related stress: it is the first time that Italian legislative system imply 'something psychological' in the organisational context, which is not connected to the occupational medicine or to some work specificities (such as for aviators).

There are a lot of doubts about this legislative decree, because of its many integrations (legislative decree 106/2009) and postponements, the deficiency of protocols and of penalties. These gaps gave rise to worries and explanation demands for organisations, which addressed themselves to trade associations and to employers' associations, but they are providing doubtful information. So, through our research we would to do more clearness.

Methods: This work derived from an academic/professional activity, whose aim is to produce specific tools. In particular, a questionnaire was built: it can be administered on-line, it has been validated (on 2314 subjects, in hundreds of organisations) and it is nowadays proposed to organisations with the aim to investigate objective but also subjective information.

Results and implications: This work could be a first consideration in order to progressively set up a research-intervention more structured that could make managers able to control anxieties, difficulties and, sometimes, their lack of clearness in facing these problems. Nevertheless, it is nowadays clear that communication strategies, intervention proceedings and methods (i.e. focus group, questionnaires, should be formulated on the basis of organisational cultures and managerial models. Shein's contributions about organisational culture (1999) and process advices (1999) are the main theoretical references for this topic. 



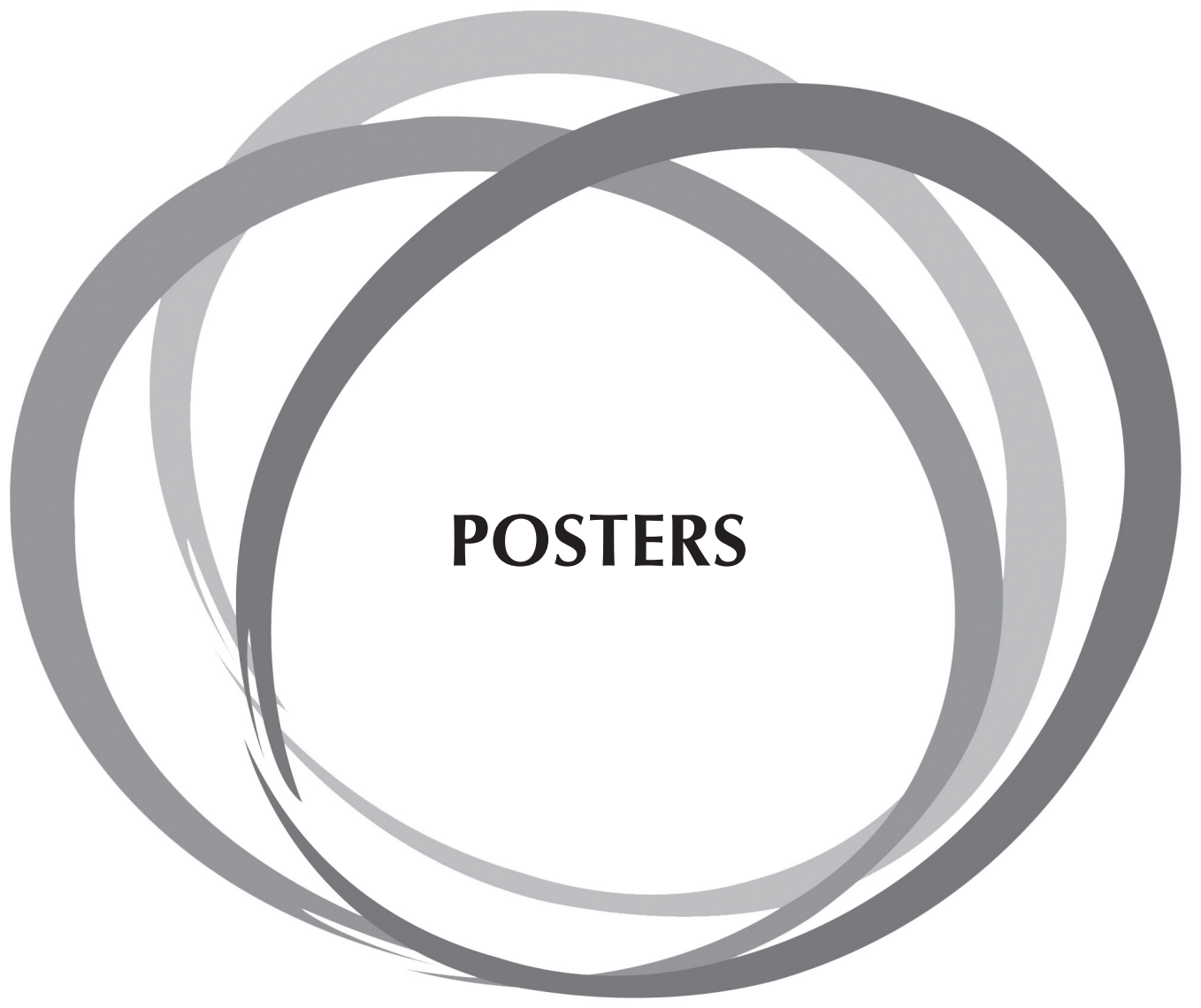




\title{
A Study Investigating if Emotional Labour and Other Work Strains Impact on Wellbeing in Cabin Crew
}

\author{
Al Serkal, A. \\ du (Emirates Integrated Telecommunications Company (PJSC)), United Arab Emirates
}

Objectives: Since the study of Hochschild (1983), scant research has been conducted on airline cabin crew with regards to Emotional Labour and its effect on wellbeing. The aim of the study is to investigate if Emotional Labour and other work strains impact on the wellbeing of cabin crew.

Methods: A questionnaire which included measures of Emotional Labour (FEWS) (Display of Positive Emotions, Display of Negative Emotions, Norms Regarding Emotions, Emotional Control, Emotional Dissonance), Organisational Variables (Job Satisfaction, Decision Making, Role Clarity, Role Conflict, Autonomy and Control and Peer Support), the experience of Physical Symptoms (PSI) and General Health Questionnaire (GHQ), were administered to cabin crew $(\mathrm{N}=68)$ who were working in an airline based in the Middle East. The average age of crew was 27.53 years (SD 3.42), 79\% were women. Thirty one nationalities participated in this study.

Results: Participants scored high on the PSI (Mean $=6.05$, SD 4.20) indicating that they experienced psychosomatic symptoms. The GHQ results indicate that $25 \%$ of all the participants presented some form of psychological morbidity. Regression analysis indicated that Norms Regarding Emotions, Emotional Control, Role Conflict and Decision Making predict Job Satisfaction. The present study clearly indicates Displaying Negative Emotions was a predictor for lower psychological wellbeing. It was also observed that experiencing Emotional Dissonance, and the display of Negative Emotions, predicts a greater experience of Physical Symptoms. In addition, it was observed that Role Conflict and Displaying Negative Emotions predict Emotional Dissonance. When Emotional Dissonance was used as a mediator for Job Satisfaction and Role Conflict, results were significant, as they clearly indicate that Emotional Dissonance is a mediator between Job Satisfaction and Role conflict. In addition, the Display of Negative Emotions and Role Clarity predict lower psychological well being and that the experience of Emotional Dissonance and the Display of Negative Emotions predict a greater experience of Physical Symptoms.

Conclusion and Implications: In conclusion, the study indicated that the experience of Emotional Labour and orgnisational factors does have an impact on physical and psychological well being. From the overall results observed, cabin crew would need to be trained in being able to handle and cope better with the experience of Emotional Dissonance. If crew were able to understand the concept of Emotional Dissonance and the possible consequences, then they may be able to tackle the discrepancy before they got emotionally exhausted, which could manifest itself as being de-motivated on the job. 


\title{
Family Systems in Parents of Children with Generalized Anxiety Disorder (GAS)
}

\author{
Aleyasin (Al yasin), S.A. \& Salehi, B. \\ Islamic azad Uiversity of Ashtiyan, Iran
}

The purpose of the study was to investigate the role of family systems among parents of children with Generalized Anxiety Disorder (GAD). A cross-sectional comparison design was used to administer two questionnaires: (i) Socio Demographic Questionnaire (SDQ); and, (ii) Family Adaptability and Cohesion Evaluation Scale (FACES III) with two main subscales as cohesion and adaptability.

The total sample consisted of 401 parents - 209 parents with normal children and 192 parents with GAD children. The research hypotheses discussed the significant differences between the two groups regarding their family systems and associated variables. Later, the data collected were subjected to statistical techniques such as General Linear Model-Multivariate Analysis of Variance.

There was a significant difference between parents of GAD and normal children on cohesion, adaptability and total FACES scores. Parents of normal children experienced a flexibly connected family system, while parents of GAD children perceived themselves as rigidly enmeshed family system. These differences between the groups are influenced by socio demographic characteristics of the respondents .These findings support the hypothesis that GAD could be viewed as a familial disorder.

Overall, the study showed that GAD associated with high degrees of parental psychopathology. These findings support results of other psychopathological researches that revealed GAD is a familial disorder.

\section{Prevalence of the burnout syndrome in top managers sent by multinational industrial companies on long-term working stays to foreign culture}

\author{
${ }^{1}$ Alfoldy, S. \& ${ }^{2}$ Gil-Monte, P.R. \\ ${ }^{1}$ Charles University in Prague, Czech Republic; ${ }^{2}$ University of Valencia, Spain
}

Introduction: This project's aim has been to evaluate the prevalence of the burnout syndrome in Czech top managers sent by their multinational industrial companies on long-term working stays abroad to China, India, Russia, etc. Working in a foreign culture is a demanding and stressful life situation, especially when the culture in question is significantly different to the native culture. The managers are exposed to extreme and long-term stress, since the job stress is here combined with the acculturation stress which occurs in the phase of culture shock during the course of the adaptation process.

Methods: Sample: 180 Czech top managers from multinational companies, mainly from the automotive industry, who are at the moment on a long-term working stay in a significantly 
different foreign culture in India, China, Russia, and other countries. Instruments: To evaluate the burnout syndrome, the 'Spanish Burnout Inventory' (SBI) method will be applied (Gil-Monte, 2005; Gil-Monte, Unda \& Sandoval, 2009). This instrument contains 20 items distributed into four dimensions called: 1 . Enthusiasm toward the job (5 items); 2. Psychological exhaustion (4 items); 3. Indolence (6 items); 4. Guilt (5 items). Items are answered on a five-point frequency scale, ranging from 0 (Never) to 4 (Very frequently: every day). Low scores on Enthusiasm toward the job, together with high scores on Psychological Exhaustion and Indolence, as well as on Guilt, indicate high levels of burnout.

Results: The results of the research acquired through the SBI will clarify:

- Whether, and to what extent, the job and acculturation stress in a foreign culture is connected with the occurrence of burnout symptoms.

- Whether the symptoms of the burnout syndrome, which originated as a result of job and acculturation stress during a long-term working stay in a foreign culture, are specific in any respect.

- Possibilities and instruments of prevention.

Conclusion: The current research has shown that the burnout syndrome can occur in all working areas, if a person is disproportionately stressed and exposed to some other detrimental impacts. At the present time, burnout also occurs in very capable managers who have worked with full engagement in unfavourable conditions over the long term. The results of the research acquired by means of the 'Spanish Burnout Inventory' (SBI) will show the scale of the occurrence of burnout in top managers amid the mentioned conditions, thus contributing to early inclusion of effective prevention.

\title{
Stress, Burnout and Emotional Disorders in the Emergency Medical Volunteers
}

\author{
Alheiro, A.S. \& Cunha, M. J. \\ Instituto Superior da Maia, Portugal
}

If exposure to traumatic events increases the likelihood to develop emotional instability, the emergency medical technicians and including volunteers are more likely to develop some emotional distress for the rest of the population. With the increase of volunteers in health, it's necessary check the characteristics highlighted in this population. This article addresses the topic of stress, burnout and disorders emotional in Volunteer Emergency Medicine. Attempts characterize a group of volunteer emergency medical about variables psychological as vulnerability to stress, symptoms psychopathology, burnout and quality of life, study the relationship between vulnerability to stress, symptoms psychopathology, burnout and quality of life and the relationship between sex and the psychological variables under study.

The sample is composed of 42 Volunteer Emergency Medical of Cross red Portuguese, with aged between 18 and 54 years (28.88), is that $54.8 \%$ are male. The instruments used are the Brief Symptom Inventory (BSI), the scale of 23 Questions for Vulnerability the Stress (23QVS), the Maslach Burnout Inventory (MBI) and World Health Organisation Quality of Life (WHOQOL-BREF). 
On average, the volunteers of Emergency Medicine didn't present emotionally disturbed. The psychological symptomatology more present are: obsession-compulsion, paranoid ideation, feelings Interpersonal hostility and depression. About $50 \%$ of the volunteers are vulnerable to stress. These present higher levels of depersonalization, somatization, anxiety and psychoticism. The non-vulnerable evaluate more positive its quality of life, assuming a positive effect of vulnerability to stress in development of emotional disturbances. With regard to sex, although the differences aren't significant, women showed better quality of life, while men show higher levels of burnout.

In general the results indicate no disturbance emotional in emergency medical volunteers. This may explained by the well-being behind the volunteer. This work isn't seen as a stressful job, but probably as an escape from problems individual.

The reduced time, and consequently much less exposure to stress, may assist in this matter, is underscored only the positive feelings inherent volunteering, as personal growth, the social recognition, the expression of values, a defense of self, the increase knowledge, preparation for a career, as well as aggrandizement. Thus, it could call into the question the motivations inherent in volunteerism and the importance attached in relation to the stress experienced during emergencies.

\title{
Coping and Quality of Life in Informal Caregivers of Individuals
}

\author{
Alves, J. \& Machado, F. \\ Instituto Superior da Maia, Portugal
}

The present investigation tries aimed to learn more about the Coping Strategies (CS) and Quality of Life (QOL) of Informal Caregivers (IC) of subjects with Spinal Cord Injury (SCI). The relatively short supply of studies in the care area, particularly in Portugal, makes it critical to conduct research that can contribute to this important area of health care.

Our group of subjects consisted of 44 Informal Caregivers of subjects with Spinal Cord Injury that were accompanying SCI victims attending health care services in a General Hospital in Porto, Portugal. Our main objective was to investigate which Coping Strategies are used by IC to deal with the difficulties posed by their caring tasks as well as their perception of QOL, considering the potential negative consequences of the demanding and permanent nature of their role as IC. Accordingly with the latest research in this scientific domain and our theoretical framework, the analysis of the role of Anxiety and Depression symptoms and the IC overload were also included in the objectives of our study. To gather the necessary data in order to achieve our objectives the following instruments were used: Brief COPE (Coping Orientations to Problems Experienced Scale), the HADS (Hospital Anxiety and Depression Scale), WHOQOL-100 (World Health Organisation Quality of Life) and the QASCI (Informal Caregiver Overload Questionnaire).

Results show that a majority of IC tends to use more effective CS, namely, Active Coping, Acceptance and Planning, making much less use of more ineffective CS such as Substance Use. The CS of Positive Reinterpretation and Humour (specially in IC with higher levels of Anxiety 
and Depression symptoms) was found to be associated with lower overload levels and Anxiety and Depression symptoms in IC, reinforcing it's potential importance in intervention programs with these subjects. The use of Denial, Religion and Behavioural Disinvestment as CS was found to be associated to lower levels of QOL.

\title{
Psychiatric disabilities and employment discrimination: An empirical analysis of EEOC ADA Title I charges
}

\author{
${ }^{1}$ An, S. \& ${ }^{2}$ Roessler, R. \\ ${ }^{1}$ Korea University Anam Hospital, South Korea; ${ }^{2}$ University of Arkansas, United States of America
}

Although employment plays a vital role in the rehabilitation of people with psychiatric disabilities, many individuals with psychiatric disabilities encounter barriers to employment in the form of employer and coworker discrimination. The extent of this alleged discrimination is revealed in charges filed with the US Equal Employment Opportunity Commission (EEOC) under Title I of the Americans with Disability Act (ADA). People with psychiatric disabilities are the second largest group filing employment discrimination charges under Title I. The purpose of this study was to explore the profiles of adults with psychiatric disabilities with respect to the nature, scope, and impact of employment discrimination in the U.S. as evidenced in ADA Title I charges filed with the EEOC.

A total of 369,183 allegations were extracted from the EEOC IMS database, and these cases were arranged into two groups, a psychiatric disability group (PSYGROUP) and a general disability group (GENDIS). PSYGROUP included schizophrenia, affective disorders, and anxiety disorders. GENDIS included physical, sensory, and neurological impairments.

White females in the age range from 40 to 61 years appeared more frequently as Charging Parties in PSYGROUP; Service industries, larger employers with 501 or more employees, and the Southern region of the U.S. were descriptive of Responding Parties responding to the alleged discrimination. Discharge followed by failure to provide reasonable accommodations were the two most frequent allegations of discrimination; only $18.7 \%$ of psychiatric disability charges were closed with merit. Compared to GENDIS, allegations based on psychiatric disabilities occurred more often among CPs who were younger, female, and White. PSYGROUP was significantly more likely than GENDIS to occur in the Transportation and Utilities, Finance/Insurance/ Real Estate, Services, and Public Administration. PSYGROUP was more likely than GENDIS in larger employers and Northeast and West regions. PSYGROUP was 2.88 times as likely as GENDIS to file charges of Posting Notice. Allegations of discrimination made by individuals with psychiatric disabilities were significantly less likely to be deemed meritorious by the EEOC than those filed by persons in GENDIS. Gender, types of industry, size, and region were significant predictors of merit closure of PSYGROUP. Waiver of ADEA Suit Right was a very strong predictor of merit closure, followed by Prohibited Medical Inquiry and Benefits/Insurance.

As the first study to examine allegations of discrimination filed by people with psychiatric disabilities, this research provides useful information for rehabilitation practitioner, researchers, and policy makers working with people with psychiatric disabilities. 


\title{
Study of psychological health status in the dental students of the Qazvin University Of Medical Sciences (second semester of the year 2007-2008)
}

\author{
Ansari Ramandi, S., Ramandi, M.M.A., Yazdizadeh, M., \& Sarichlo, M.E. \\ Qazvin University of medical sciences, Iran
}

Background: By taking in mind the prevalence of psychological disorders in the society( according to the statistical studies) and the major role of medical students in estabilishing and maintaining the future health of the society, we decided to evaluate the psychological well being of the dental students of Qazvin university of medical sciences.

Methods: This study was done on the students studying at the university in the second semester of the year 2007-2008 .In this research two questionnaries were used one for the personal information of the students and the other one for determining their psychological health(symptoms check list-90-revised).the number of students included in the research were 165 from which $118(71 / 5 \%)$ were females and $47(28 / 5 \%)$ were males.

Results: Regarding somatization $28 \%$ of the students suffered from its symptoms.Regarding obsession and compulsion $29 / 7 \%$ of the subjects had symptoms of it. Regarding depression $26 \%$ of the subjects experienced depression.Regarding interpersonal sensitivity $8 / 5 \%$ of the subjects suffered from it.Regarding anxiety $18 / 18 \%$ of the subjects had anxiety. $12 / 7 \%$ of the subjects had hostility.Corresponding to phobic anxiety $3 / 6 \%$ of the subjects experienced it. $9 / 7 \%$ of the subjects had paranoid ideation. Corresponding to psychoticism $35 / 1 \%$ of the subjects suffered from it

Conclusion: According to the result of this research it can be concluded that a significant number of the dental students of Qazvin university suffer from psychological disorders.Also it can be concluded that some of the psychological disorders have relations with factors such az marital status, place of living,stage of study,occupation status and being aborigine or not.

\section{Psychosocial Risk Assessment: a pilot study in an Administration and Coordination Operative Unit}

\author{
${ }^{1}$ Atzori, E. \& ${ }^{2}$ Tagliaferri, C. \\ ${ }^{1}$ Azienda Complesso Ospedaliero San Filippo Neri, Roma, Italy; Collaboratrice del Servizio \\ Prevenzione Protezione Rischi, Italy
}

Objectives: Identify and reduce psychosocial risks for health workers, starting from the hypothesis that it may be caused by the gap between organizzative culture and professional objectives. Trace the Operative Unit's development lines in order to increase the costumer satisfaction perspective. Promote a safety approach inside the management project, as an integration with the productive process.

Methods: Following an action-research methodology, into a psychosocial perspective, were used focus group and interviews in order to explorate the nurse coordinators' issues about 
psychosocial risks. Then was realized a factorial analysis of texts, through the Text Emotional Analysis (TEA), a methodology supported by informatic and statistic, that allows to outline collusive processes as mode of treating emotional collusive dimensions into different contexts by a social group. Finally, client commissionings and costumers received analisys' feedback.

Results: Text analysis revealed three clusters of words that represent the different cultural repertoires, i.e. the different emotional representations that people have of reality on which were invited to talk. Each representation sets different organisational behaviours:

- Organisation "Mother": emotionally, operators know in advance their patients: I know what you need, like a mother with her child. Everyone does everything, disappear professional roles, functions and skills.

- Organisation "Closed": there's an asymmetric, conflicting relationship with clients. The changes tend to be restrained in order to restore the fixity of the dominant hierarchical system.

- Organisation "Empty": the company is experienced as a sum of structures and roles: operators and patients are absent, so coordinators' activity is a title, a label, a continuous "doing" emptied of meaning: it' s like a glove without hand.

Implications: The change is inconceivable outside of expectations, attitudes, behaviours, goals of those involved. With the assessment of psychosocial risk is analyzed cultural representations of the context, that organize customer relationships "acted out". We dealt with the issues that have arisen as a psychosocial intervention training in which they were taken into account the different problem areas that hinder the coordinators in their work.

Conclusion: Working to reduce the risk psychosocial means, in our opinion, to increase the organisational competence of operators in relation to:

- who: promoting customer orientation;

- where: to introduce organisational tools that allow to contextualize actions and professional roles;

- with whom: to create connections and relationships, in order to promote the integration of services.

\title{
The Relationship between Perceived Compensation, Organisational Commitment and Job Satisfaction: The Case of Expatriates in South Africa
}

\author{
Barkhuizen, N. \& Sage, N. \\ University of Pretoria, South Africa
}

Objectives: Research continues to focus attention on expatriate needs, concerns and extensive efforts in understanding the ideal environment for the expatriate. One of the key outcomes of previous research is that the process of expatriate adjustment and an appropriate compensation package (Skowronski, 2007) are important factors for expatriate success. Literature also indicates that the expatriate achievement is heavily reliant on the Home Country Workforce 
(HCW) (Jassawalla, Garvey \& Truglia, 2004). Research at present however has seemed to over look the importance of the HCW perception of the expatriate's compensation and the influences these perceptions may have. The aim of the research was first to determine the perceived compensation gap between the South African HCW and the referent expatriate within organisations and second, to determine the effect of the compensation gap on the organisational commitment and job satisfaction of HCW.

Methods: A cross-sectional survey design was used with a purposive sample taken from professionals and managers $(\mathrm{N}=500)$ interacting with expatriates in South African organisations. The Compensation Disparity Questionnaire, Organisational Commitment Questionnaire and Minnesota Job Satisfaction Questionnaire - short form were administered.

Results: Preliminary results show that the South African HCW perceives lower compensation compared to expatriates. The compensation gap between HCW and expatriates is negatively related to affective and continuance commitment. A positive relationship exists between HCW organisational commitment and job satisfaction. More results will be presented.

Implications: HCW are a valuable source of social support, assistance and friendship to freshly arrived expatriates (Black, Mendenhall, \& Oddou, 1991). HCW is a linchpin in expatriate adjustment; therefore all negative behavioural manifestations that may prohibit a good interaction between HCW and expatriate should be identified and possibly reduced. If the HCW observe expatriates receiving a substantially larger compensation package for almost equal responsibilities he/she may have a belief of inequality in the organisation and these accumulative negative behaviours in turn could have devastating effects on two vital counterparts; the expatriate and more importantly the home organisation (Leung, Smith \& Wang, 2001)

Conclusion: The expatriate's job success is inclusive and limited to the adjustment with the Host Country Workforce. Therefore, it is not only critical to determine the appropriate salary for the expatriate but also to ensure local workforces are part of this strategic decision. The compensation offered to expatriates may have effects on the local employee and turn may affect the overall business success.

\title{
The Relationship between Personality Traits, Job Satisfaction and Job Performance of South African Retail Store Managers
}

\author{
Barkhuizen, N., Ermakova, J., \& van der Walt, R. \\ University of Pretoria, South Africa
}

Objectives: Consumer goods retailing is a fast-moving and results-orientated industry and, as such, places high demands on people across all levels in the business. Most importantly, however, it requires exceptional performance from people occupying managerial positions in the retail stores. This research aimed at investigating the relationship of personality traits (Extraversion, Emotional Stability, Conscientiousness, and Core Self-evaluations) to Job Satisfaction and Job Performance for the occupational class of retail store managers in South Africa. The rationale for the study arouse from the believe that by using these personality traits as predictors of job satisfaction and 
job performance, the study may contribute to the identification of predispositional "ey success factors" that will ensure the desired job outcomes of retail store managers.

Methods: A cross-sectional survey design was used with a purposive selected sample taken from managers $(\mathrm{N}=89)$ of South African Retail Stores. The $15 \mathrm{FQ}+$, Core Self-Evaluations Scale and Job Diagnostic Survey were administered. Job performance was measured by means of supervisory ratings.

Results: Preliminary results show that extraversion and conscientiousness is positively related to the job performance of retail store managers. Emotional stability has a positive effect on job satisfaction, while neuroticism has a negative effect on job satisfaction. Extraversion and core self-evaluations are positively related to job satisfaction. Job satisfaction is positively related to job performance. Job satisfaction mediates the relationship between emotional stability, extraversion, core self-evaluations and job performance.

Implications: Retail is characterised as a highly pressurised, result-orientated and demanding environment. The identification of key success factors such as personality types is thus important for the job satisfaction and hence successful performance of retail store managers. The results in turn, can assist in the selection of the 'right' candidate who is perfectly fit for the management job in the challenging retail environment.

Conclusion: The research concludes with a predictive model relating personality traits, job satisfaction and job performance for the success of retail store managers. The results thus provide some insights for other retail organisations into the identification of those personality traits that predict job performance and affect job satisfaction of the store managers. The research also contributes to the optimisation of personnel recruitment and selection processes as well as talent management strategies in retail industry.

\title{
Leadership Competencies in Emerging Markets: A South African Study
}

\author{
Barkhuizen, N. \& McMahon, G. \\ University of Pretoria, South Africa
}

Objectives: Although fifteen years have passed since South Africa's first democratic election, there are still many complex social, economic and political issues facing the country and its businesses. South African leaders are trying to adapt to a changing organisational culture that is largely influenced by globalisation, technology, diversity, skills, education and legislation centred on affirmative action and black economic empowerment (Luthans, Van Wyk \& Walumbwa, 2004). Research indicates that if businesses want to survive and prosper in the coming era, they have to develop world-class thinking and innovative ways of doing business. Thus, major paradigm shifts in leadership are required if organisations in South Africa want to become more competitive and sustainable in a global economy (Denton and Vloeberghs, 2003). This study aims to establish the corporate leadership competencies required to sustain and grow businesses within the context of South Africa, both as a developing country and one that is currently experiencing vast social and economic issues. As a result, a new set of competencies are to be identified. 
Methods: A qualitative research approach is followed with six in-depth interviews being conducted with corporate leaders in the South African private business sector.

Results: Preliminary findings show that leaders need to re-evaluate their leadership approach and reposition their businesses. This repositioning of businesses and business leaders include leaders being more responsible and resourceful, playing the role of change agent and teacher and most importantly demonstrating cultural awareness.

Implications: In line with the preliminary findings, it is evident that if leaders in South Africa want to succeed in growing and sustaining their businesses in a tough but highly potential market, they would need to re-evaluate their leadership approach and reposition their businesses. This requires that South African business leaders be brave enough to make decisions and implement leadership practices within the broader context of South Africa and effectively manage the organisation's wider impacts and contributions to society.

Conclusion: In conclusion, developing an understanding of the challenges and future demands of leadership will provide insight into the requirements of the South African leader. Mastering the ability to lead and grow successful businesses in an emerging market will not only set the benchmark for leaders in other developing countries but enable these leaders to successfully manage and grow businesses in any market.

\title{
The relationship between masculine financial identity, workgroup aggression normalization, and incivility perpetration: a discriminant function analysis
}

\author{
Bauerle, T. \& Magley, V.J. \\ University of Connecticut, United States of America
}

The negative consequences of conforming to the traditional masculine gender role have mostly been evaluated within the context of counseling psychology $\left(\mathrm{O}^{\prime} \mathrm{Neil}, 2008\right)$. More research is needed to bridge the gap between organisational psychology and the psychology of men and masculinity, namely with regard to how established connections between masculine identity and aggression (Anderson \& Anderson, 2008; Cohn \& Zeichner, 2006; Jakupcak, 2003) play out in the workplace. Our objective was to assess the impact of masculine financial identity 'a facet of masculine identity' on the perpetration of workplace incivility. In particular, we wanted to contrast this effect of masculine identity with that from general organisational norms for aggression.

Methods \& Results: The outcomes associated with the link between masculinity and finances provided a substantial basis to both examine masculinity uniquely in the workplace and build on Spector's organisational frustration-aggression model (1997). An online survey was administered to a convenience sample of 68 men. Two distinct factors (each with three items) emerged from a principal components analysis of the masculine identity items: masculine financial identity (MFI) and financial comparison (FC). These two factors accounted for approximately $70 \%$ of the variance in the items and had alpha reliabilities of .83 and .62, respectively. 
Based on responses to 20 items assessing incivility perpetration, we trichotomized respondents into low, average, and high perpetrators. Two significant functions emerged from a discriminant function analysis (DFA) that accounted for $44 \%$ of the variability in differentiating among the three perpetrator groups based on linear combinations of the following variables: masculine financial identity (MFI and FC), workgroup aggression normalization, earner status, financial strain, and perceived job stress. Group centroids and correlations of measures with the discriminant functions suggest that workgroup aggression normalization best accounts for low and high levels of incivility perpetration, whereas masculine financial identity (both MFI and FC) and stress account for moderate levels of incivility perpetration.

Implications \& Conclusions: Our findings suggest that individual pressures (e.g., to earn and keep a salary) drive perpetrated workplace aggression when an aggressive climate is average or ambiguous. As such, the results confirm theories underlying traditional masculine gender role norm adherence in an organisational setting, that the frustration caused by gender role adherence results in negative consequences for both men and their coworkers.

\title{
Continuance commitment mediating the effect of masculine financial identity on burnout and stress: a case for masculine occupational health.
}

\author{
Bauerle, T. \& Magley, V.J. \\ University of Connecticut, United States of America
}

Objectives: Filling in the pieces between occupational health psychology and the psychology of men is both daunting and necessary, especially considering the hazards of masculine work (e.g., Farrell, 2001). The objective of the current study was to measure a unique masculine identity that would tap into a masculine drive to perform in the workplace, and to examine how these identities were related to health.

Methods \& Results: The link between masculinity and finances provided a basis to incorporate previous conceptualizations of success, power, and competition in masculine ideology (O'Neil, 1981; Eisler \& Skidmore, 1987). Unique items were created and administered to a convenience sample of 68 men. Two distinct factors (each with three items) emerged from a principal components analysis: masculine financial identity (MFI) and financial comparison (FC). These two factors accounted for approximately $70 \%$ of the variance in the items and had alpha reliabilities of .83 and .62 , respectively.

Because continuance commitment (CC) is a type of organisational commitment based on potential costs (both economic and social) of losing organisational membership, we hypothesized that CC would be related to both financial identities and health outcomes as men who have higher financial identities may evaluate the social costs of job loss more severely, thereby increasing pressure and stress. We used Preacher and Hayes's (2008) SPSS macro for testing the indirect effect of financial identities on burnout and stress through CC. Several meaningful trends emerged: FC to exhaustion was partially mediated by CC, and CC fully mediated MFI to exhaustion. Although $\mathrm{CC}$ did not mediate the effect of MFI on disengagement, it did fully mediate the effect of FC on disengagement. Finally, CC did not mediate the effect of FC on stress, although it did partially mediate the effect of MFI on stress. 
Implications \& Conclusions: This research constitutes the first work examining the role that men's masculine financial identity has on their occupational health. Although our sample size was small and perhaps the main reason why significant indirect effects $(p<.05)$ were not found, we argue that the test of joint significance is appropriate (Mallinckrodt et al., 2006) and that the patterned results certainly should be used as a springboard for further research and replication.

\title{
Job burnout and work fatigue in the context of organisational stress among nurses
}

\author{
${ }^{1}$ Beata, B. \& ${ }^{2}$ Wilczek-Ruzyczka, E. \\ ${ }^{1}$ Gdansk University of Technology, Poland; ' 2 Jagiellonian University of Cracow, Poland
}

Objectives: The fatigue at work and job burnout are two interrelated, but distinct phenomena. In this study the problem of work fatigue and job burnout in the context of shift-work of nurses Has been discussed. We suspected that the efforts made in shift-work would cause the fatigue where as job burnout would be a result of the Effort-Reward Imbalance.

Methods: The research was conducted in two stages in the time span of one month. The group consisted of 56 nurses (rate of return In time 2 took out 59\%) with an average working experience 16 years.

The following methods were used:

(a) rganisational stress: Effort-Reward Imbalance (time 1): the discrepancy term and the ration term,

(b) fatigue at work (time 1, time 2): Athens Insomnia Scale and Job-Fatigue Index,

(c) burnout (time 2): Maslach Burnout Inventory,

(d) shift-work and satisfaction with this system.

Results: A moderate relation between fatigue and emotional exhaustion was observed as well as between depersonalization and insomnia. Professional competence did not interrelate with fatigue measures. Results for shift-working nurses did not differ from those working during the day. The results of hierarchical regression analyses demonstrated that efforts at work contributed to fatigue. Effort-Reward Imbalance (the ratio term) and lack of reward (the discrepancy term) caused the emotional exhaustion. When checking satisfaction of the shift-work system, the insomnia did not mediate between organisational stress and emotional exhaustion, but together with the stress they created the main effect.

Implications: The research show the need for proper diagnosis to differentiate fatigue and job burnout and selection of adequate therapeutic methods. The above findings have implications for organisational and therapeutic intervention programs for nurses. 


\title{
Worksite health promotion program for physicians: the assessment and management of short-term stress
}

\author{
${ }^{1}$ Berezovskaya, R. \& ${ }^{2}$ Shabelnik, O. \\ ${ }^{1}$ Saint-Petersburg State University, Russia; ${ }^{2}$ Medical multiple-profile clinic MEDI, Russia
}

This paper presents the results of a study investigating psycho-social aspects of Occupational Health Management (OHM) practice as a part of strategic human resource management (HRM) in Russia. A large number of studies supports the notion that there is a positive relationship between "human capital enhancing"; practices and firm performance or employee productivity. However, while the impact of some HR practices such as employee training or performancebased compensation programs have been extensively studied (Fey et al., 2007), little is known about the effect of occupational health-management process especially in medium-sized enterprises.

According Bauer \& Jenny (2007) OHM is the continuous participatory analysis and optimization of organisational structures and processes that have a direct or indirect impact on the health of the employees and thus influence the organisation's business outcomes. Hence health promotion programs at the workplace with the focus on stress-management training are receiving increased attention in Russia now.

Objectives: This study examines the level of short-term stress among employees of private medical clinic on the one hand and on the other hand the stress-management practice in it. Methods: The participants involved in the current study were physicians of private medical multiple-profile clinic in Saint-Petersburg. The variables were assessed using three instruments: (1) Plath and Richter- BMS II (Russian version adopted by Leonova \& Velichkovskaya) which evaluate short-term stress; (2) Hardiness Survey (Maddi/Leontiev \& Rasskazova); (3) assessment tools. Moreover, control variables such as gender, length of experience and specialization (dentistry vs. aesthetic medicine) were derived from employers'; registers.

Results: The study produced several significant findings. First, the assessment show that employees report, generally, an average degree of short-term stress: a low level of monotony and satiation; and a moderate level of fatigue and stress. Second, no significant gender differences emerged in response patterns to stress in this survey. However, we received some differences connecting with the physicians' specialization. Third, after conducting a Pearson correlation, significant associations were observed between the overall hardiness score and fatigue $(r=-.62)$ and satiation $(r=-.65)$. Correlations of stress and monotony with hardiness did not reach the level of significance $(p>.01)$.

Conclusions: The results of our study provide a useful first insight into health promotion program at the workplace and support the notion that it has important practical implications for the employee well-being. The significance of these results and areas of future research will be discussed. 


\title{
Psychological Capital and Safety Climate: A study of Norwegian Air Traffic Controllers
}

\author{
Bergheim, K., Hystad, S.W., \& Eid, J. \\ University of Bergen, Norway
}

Objectives: Considerable research has demonstrated that the emerging positive core construct of psychological capital (PsyCap) is related to desired employee attitudinal, behavioural, and performance outcomes. This study is the first to examine if PsyCap is related to perceptions of safety climate.

Methods: The overall PsyCap and each of its components (efficacy, optimism, hope and resiliency) of a sample of Norwegian air traffic controllers $(N=77)$ were correlated with their perception of safety climate (both vertical and horizontal).

Results: After controlling for age and organisational affiliation, PsyCap explained 30\% and 28\% of the variance in vertical and horizontal safety climate, respectively. In addition, the factors of optimism, hope and resiliency each explained between $4-5 \%$ of one or both of the safety climate dimensions.

Conclusions: The present study extends previous PsyCap research into safety science, and provides first-time empirical evidence linking PsyCap to safety climate in high reliability organisations.

\section{Need for achievement as a vulnerability factor in a politicized work setting}

\author{
Berset, M., Henseleit, J., Omlin, F., Willi, S., Semmer, N.K., \& Elfering, A. \\ University of Bern, Switzerland
}

Objectives: Organisational politics has been shown being detrimental for well-being (e.g. Huang et al., 2003). We propose that employees having a high need for achievement are especially prone to suffer from organisational politics. A highly political setting is characterized by having to agree with powerful others and the use of social influence to protect self-interests (Kacmar \& Carlson, 1997). In addition, rewards are assigned based on interpersonal aspects, thus as sympathy, more than on job performance. Subjects being high in need for achievement like to work on difficult and demanding task and want to spend their time working on those tasks. Spending time on political behaviour is shortening the time left for the actual core tasks and is, therefore, a threat to the goal of performing well. Furthermore, not being rewarded for actual performance, and seeing others being rewarded in spite of not excelling, is likely to be perceived as unjust, and as an offense to the self. Threats to important goals are assumed to be related to rumination (Martin \& Tesser, 1996) and irritability (Mohr et al., 2006). Therefore, we assume that organisational politics should be associated with irritability and rumination among individuals high in need for achievement more strongly than among people low in need for achievement. 
Methods: Participants filled in a questionnaire three times, with one month in between. Having repeated measures, and, therefore, a nested data structure, we ran multilevel analyses with subjects on level 2 (HLM 6.0, Raudenbush et al., 2004). We modeled the main effects of organisational politics and need for achievement and their interaction on level $1(\mathrm{~N}=586$; Level 2: $N=261$ ). Dependent variables were the two subscales emotional irritation and cognitive irritation of Mohr's irritation scale (Mohr et al.2006), indicating irritability and rumination, respectively.

Results: As expected, organisational politics was related to both outcome variables, whereas need for achievement was not. The main effect of organisational politics was, however, qualified by a significant interaction, indicating that organisational politics had a stronger impact on both dependent variables for participants high in need for achievement. Thus, our assumptions were supported.

Implications and conclusions: Organisational politics is detrimental to the organisation as well as to employees. Our results indicate that it is those participants who tend to be the best performers, that is, people high in need for achievement, who may suffer the most from organisational politics.

\title{
Shift work and health: How personality and circadian typologies affects the relationship between shift work and mental health
}

\author{
Berthelsen, M., Ljoså, C.H., \& Lau, B. \\ National Institute of Occupational Health, Norway
}

Objectives: Aim of the study is to test the relations between shift work, circadian typologies, personality and their effects on workers mental health. Mismatch between circadian typology and daily shift schedule may result in psychological and psychosomatic disturbances (Costa, Lievore, Casalleti, Gaffuri and Folkard, 1989). Further, research on personality and circadian typology indicates that evening-type subjects are more extraverted, impulsive, and noveltyseeking, while morning ones tend to be more introverted and emotionally stable.

Methods: The research design was cross sectional and used a web-based questionnaire. One thousand and ninety seven completed questionnaires from 3038 invited employees of a Norwegian oil and gas company (2006) were received, yielding a response rate of $55.9 \%$. Mental health was measured on a five point scale using HCL-5. Circadian typology was measured by one item from the Composite Morningness Questionnaire: One hears about "morning" and "evening" types of people. Which one of these types do you consider yourself to be? 'Definitely a morning type', 'more a morning than an evening type', 'more an evening than a morning type' and 'definitely an evening type'. Personality was measured using Eysenck's (EPI-12).

Results: Two-way ANOVA showed a effect of shift schedule on mental health, where workers with both day and night schedules showed poorer mental health compared to workers with day schedules only $(F(1,784)=9.43, p<.01)$. No significant interaction of shift schedule and circadian typology on mental health was found, nor a significant effect of circadian typology. To test the effects of neuroticism and extraversion on the relation between shift schedule 
and circadian typology on mental health, two additional ANOVA's were conducted, adding first extroversion, then neuroticism to the model. The results showed an significant effect of extraversion $(F(1,780)=40.96, p<.001)$, and a significant effect of shift schedule on mental health $(F(1,780)=8.36, p<.01)$. Adding neuroticism to the model, the relation between shift schedule and mental health was no longer significant $(F(1,780)=0.61, p>.05)$, leaving only a significant relation between neuroticism and mental health $(F(1,780)=461.44, p<.001)$.

Conclusion: Circadian typology does not seem to affect offshore workers mental health. However a more stressful shift schedule does. Offshore workers working day/night schedule have poorer mental health compared to day shift offshore workers, independent of circadian typology. However, adding neuroticism to the model removes the effect of shift schedule on mental health.

\title{
The Effectiveness of Psychological characteristic and Skill Training on Teacher Supervision for Enhancing Responsibility among Early Adolescent Students
}

\author{
Boonprakob, P., Boonprakob, M., Intarakamhang, U., \& Thongpukdee, T. \\ Srinakharinwirot University, Thailand
}

The objectives of this study were 1) to examine the effectiveness of teachers' psychological characteristic and enhancing skill training for fostering responsible behaviour among students 2) to study the predictive power of teachers' enhancing students' responsible behaviour and students' responsible behaviour through the training, together with the teachers' psychological traits and the situational factors.

The 2 sample groups comprised 48 teachers, who taught 993 secondary students in grade 5 and 6 at 3 schools and these students from 24 classrooms in Bangkok, Thailand. Three classrooms were set as the experimental group and one classroom was the control group. Three experimental groups were designated as room $A$, roomB and room $A B$. The control group was roomC. Teachers in roomA were trained on psychological characteristic (internal locus of control). In roomB, the teachers were trained on enhancing skills (love and reasoning oriented and enhancing approaches). Teachers were given training on both types in room $\mathrm{AB}$. In the control group or room $C$, teachers weren't trained. The research instruments were 1) training modules A and B for teachers 2) seven measurements for teachers, and 3) four measurements for students. There were 3 measurement periods consisting of pre-test, immediate post-test and post- test after 3 months. Data were analyzed by using Pearson Correlation, MANOVA, TWOWAY ANOVA and Multiple Regression Analysis.

The research findings were as follows:

1. Training results on students' responsible behaviour were significantly higher than those of the control group.

2. Training results on teachers' internal locus of control, the readiness of teachers' love and reason orientation and fostering students' responsible behaviours between the trained group and the untrained one were not different.

3. In group $A B$, teachers' internal locus of control and the readiness of teachers' love and 
reason orientation could predict teachers' enhancing students' responsible behaviours by 49-69 percent.

4. In group $A B$, group $A$ and groupB, mental health, relationship between student and teacher and role model by teacher could predict students' responsible behaviours by 10.7 percent, 12.5 percent and 26.0 percent respectively.

5. In the experimental group, teachers' scores on locus of control and love and reason orientation measured directly after the treatment and 3 months later were higher than the pre-test scores.

These research results showed that training for teachers could develop both psychological characteristic and knowledge in skill fostering the responsibility among students. Besides, the relationship between the teacher and student and teachers' role model are also should be interested by the teacher college.

\title{
Psychopathological Screening and Assessment of Psychological Discomfort in a Sample of Italian School Teachers
}

\author{
'Borrelli, I., 'Bergamaschi, A., ${ }^{2}$ di Giannantonio, M., \& ${ }^{1}$ Pozzi, G. \\ ${ }^{1}$ Catholic University of Sacred Heart, Italy; ${ }^{2}$ University of Chieti, Italy
}

Objectives: In all the countries of the world great attention is paid to handling and evaluating the problems regarding school workers. Italian scholastic system is high level but teachers complain about an insufficient recognition of their professionalism and reach the working stability after many years of job insecurity. This preliminary analysis is a part of a research program on the health and job conditions of the Italian teachers. In the present study we preliminary assess anxiety and depression as well as sleep troubles sleep and alcohol-related problems.

Methods: Self-administered questionnaires have been employed in anonymous among the personnel of four school (all of them including day nursery, primary and secondary school). The questionnaires include the following tools in Italian version:

- Epworth Sleeping Scale (ESS): 10-items (score 0 -18) identifying subjects with excessive diurnal drowsiness (for scores $>10$ );

- Center for Epidemiologic Studies Depression Scale (CES-D): 20-items for the epidemiological screening of depression (score 0-60, cut-off 16);

- Zung Self-rating Anxiety Scale (SAS): 20-items (score 20-80) identifying pathological anxiety (for scores > 45);

- CAGE Assessment (Alcohol Abuse): 4-item (score 0 -4) catching behaviours at risk for alcoholism (for scores $>1$ ).

Results: Ninety-four workers have returned the completed questionnaire (i.e. $52 \%$ answer rate). The mean age of the sample is 50.7 , with a seniority of 24.8 on average. The average time of weekly employment is 23.5 hours; $80 \%$ of workers report housework for 5.3 hours/week, while $38 \%$ work overtime 4 hours on average.

Questionnaire results were: 
- ESS: $10 \%$ with a score $>10$ (6\% refused this section);

- CES-D: the mean score was 16.8 ; of $45 \%$ over 16 , the average score was 22.7 (5\% refused this section);

- SAS: the mean score was 37.6 ; of $13 \%$ over 45 , the average score was 50.5 (5\% refused this section);

- CAGE: $10 \%$ reported a value $>1$ (25\% refused this section).

Implications: About half of the questionnaires were returned. Among the responders, an elevated proportion suffered from depressive symptoms; the missed compilation was more frequent for the screening questions on alcohol-related problems.

Conclusions: Preliminary data appear interesting; as soon as that sample is expanded to a greater number of schools, the results will be analyzed with reference to demographic and environmental factors as potential correlates of the psychological discomfort.

\title{
Psychometric Properties of the "Spanish Burnout Inventory" in German Professionals: Preliminary Results
}

\author{
${ }^{1}$ Bosle, A., ${ }^{2}$ Gil-Monte, P.R., \& ${ }^{2}$ Figueiredo-Ferraz, H. \\ ${ }^{1}$ University of Mannheim, Germany; ${ }^{2}$ University of Valencia, Spain
}

The purpose of this study was to assess the factorial validity and the internal consistency of a German translation of the "Spanish Burnout Inventory". A model of four factors was hypothesized, similar to the original structure.

The sample consisted of 115 human services professionals in Germany. Confirmative factor analysis was conducted with the LISREL 8.30 program.

The hypothesized four factor model obtained an adequate data fit for the sample: ChiA $\hat{A}^{2}=$ $272.47(p=.000), \mathrm{CFI}=.91, \mathrm{NNFI}=.90, \mathrm{RMSEA}=.069$. Cronbach's alpha values for all subscales were higher than .70 .

The results indicate that the SBI offers factorial validity and its scales present internal consistency to evaluate burnout in German human services professionals. **Hugo Figueiredo-Ferraz was supported by grant SFRH / BD / 45899 / 2008, of the Fundação para a Ciência e a Tecnologia, Ministerio da Ciência, Tecnologia e Ensino Superior, Govemment of the Portuguese Republic.

\section{Moral Agency and Workplace Aggression}

\author{
Bozeman, J. \& Hershcovis, S. \\ University of Manitoba, Asper School of Business, Canada
}

Workplace aggression, defined as efforts by individuals to harm the organisation, or its members (Neuman \& Baron, 2005) is a serious issue facing organisations. However, although most forms 
of aggression have a moral dimension, the moral agency of the perpetrator (i.e., the process of moral decision-making and conduct; Bandura, 1986), has received little attention. The very definition of workplace aggression suggests that an understanding of the processes of moral conduct might help explain when and why individuals engage in deviant acts. Therefore, the purpose of this paper is to illustrate the relationship between moral agency and employee deviance; intentional threatening behaviour that goes against organisational norms directed towards employees and/or the organisation (Robinson \& Bennett, 1995).

The process of moral conduct that leads individuals to behave either morally or not encompasses aspects of individual traits, cognitive processes, and contextual factors. That is, moral behaviour will be a function of a person's predisposition to behave morally, their cognitions about the actions they are about to take, and the extent to which the situation demands morality. We therefore consider moral disengagement (Bandura, 1999), moral reasoning (Kohlberg, 1969), and moral intensity (Jones, 1991) to help us understand the conditions under which individuals may be more or less likely to engage in deviance.

Moral disengagement describes the process by which individuals allow themselves to act in a manner that is not necessarily congruent with their moral standards, for example, using euphemistic labelling to enable stealing by instead labelling it as borrowing (Bandura, 1999). In this article, we theorize that moral disengagement is an important antecedent to employee workplace deviance. Examining the various disengagement methods proposed by Bandura (1999), we posit that individuals who implement these disengagement techniques are more likely to act aggressively towards their organisations and coworkers.

We further argue that moral reasoning, the individual process by which individuals decide how to act when faced with a moral dilemma (using Kohlberg's model of cognitive moral development, 1969), and moral intensity, the extent to which the situation demands moral action (using Jones' model of moral intensity, 1991) will moderate the relationship between morally disengagement and workplace deviance. We develop a theoretical model and present a series of hypotheses that demonstrate the direct relationship between moral disengagement and deviance, and the moderating effects of moral reasoning and intensity. Practical implications and future directions are discussed.

\title{
Work-life balance - despite or because of volunteering. A comparative, explorative data analysis in Switzerland
}

\author{
Brauchli, R., Bauer, G., Wehner, T., \& Hämmig, O \\ University of Zurich and ETH Zurich, Switzerland
}

Objectives: The growing research literature on negative spillover from work to family (and vice versa) is limited to role conflicts between paid work and home. Unpaid voluntary work has not been considered in this research field so far. Volunteering is defined as organised social work (i.e. a personal and charitable activity) that requires giving of one's time and could, in principle, also be paid for and carried out by someone else. This study aims to investigate if unpaid voluntary work in addition to paid work and gainful employment is beneficial or detrimental for one's work-life balance. In fact, volunteering is expected to be more than just an 
additional time-consuming social role leading to temporal overload and increasing role conflict. On the contrary, it is expected to be a psychosocial resource improving one's work-life balance and well-being rather than impairing it.

Methods: To test whether and how work-life balance or imbalance (measured by indicators of work-life conflict) and volunteering are associated, different data sources were used. On the one hand data were taken from the Swiss Household Panel (a representative survey with a sample of 5771 employees) and on the other were collected in the context of a survey among the workforces of four large and well-known Swiss companies of different industrial sectors (health-care system, financial sector, transportation sector) covering a sample of more than 6000 interviewed employees in total. Some analyses of variance have been done already and some additional and advanced statistical analyses still have to be performed.

Results: Preliminary results indicate that, overall, there is a non-linear relationship between work-life conflict and volunteering. However, if the two causal directions of work-life conflict (work-to-life and life-to-work conflict) are analysed separately, weak but significant associations can be found between work-to-life conflict as well as life-to-work conflict and volunteering, but with reverse algebraic signs surprisingly: By tendency, the more often voluntary work is done the weaker is work-to-life conflict but the stronger is life-to-work conflict.

Conclusions: Our results apparently confirm that volunteering figures as a resource concerning work-to-life conflict, but obviously not concerning the other causal direction, i.e. life-to-work conflict. In light of this inconsistent but very interesting finding additional investigation is needed before drawing any conclusions or practical implications.

\title{
Factors important for a good self-rated health last year in higher education: a nationwide longitudinal study among Swedish nursing students
}

\author{
${ }^{1}$ Bruce, M., ${ }^{1}$ Gustavsson, P.J. \& ${ }^{2}$ Omne-Pontén, M. \\ ${ }^{1}$ Karolinska Institutet, Sweden; ${ }^{2}$ Centre for Clinical Research Dalarna, Sweden
}

Background: Students experiences' during higher education may have an impact on future selfrated health $(\mathrm{SRH})$ and health behaviour after graduation. Health care professionals, like nurses, are often seen as role models for healthy living, which could encourage patients to do the same. The aims with this study were to investigate Swedish nursing students' SRH and health behaviour with a longitudinal design and to study variables associated with a good SRH health during last year in education.

Methods: This nationwide descriptive study surveyed 1,291 nursing students from 24 universities and university colleges in Sweden. Odds ratios (ORs) and $95 \%$ confidence intervals (Cls) were computed using multivariate logistic regression.

Results: Impairment in SRH was found, but most of the nursing students reported to have an overall good or somewhat good SRH throughout their education $(91 \%$ year one and $88 \%$ year three). Health behaviours that improved over time were alcohol consumption, smoking, backache and exercise. Variables deteriorating over time were: sleep quality, experienced stress 
regarding the future, problems with headache and stomach ache. To have a good SRH last year in education was found to have the strongest associations with: 'good sleep quality year one' (OR 2.25, Cl 1.58-3.2), 'to have high emotional engagement for the studies year three' (OR 1.87, $\mathrm{Cl} 1.43-2.44)$ and 'high nursing self-efficacy year three' (OR 1.75, Cl 1.25-2.44).

Conclusions: The majority of Swedish nursing students seem to have a good SRH throughout their education, but some of their health behaviour could be improved. More of attention could be given to higher educational variables important for a good SRH last year in education.

Implications: Those working with nursing education can pay more attention to students' own health behaviour, their study engagement and their development of professional competence. The newly registered nurses' preparedness for the coming demands in their professional role would benefit if these positive strengths were incorporated already during higher education.

\title{
Investigating the causes of burnout in police officers
}

\author{
Burba, $M$. \\ University of Lausanne, Switzerland
}

Objectives: Several studies have shown that burnout is caused by multiple factors. The purpose of this study was to explore the predominant causes of stress leading to potential burnout in two Swiss cantonal police institutions. Participants included ten cantonal police officers who volunteered to talk about their careers and the professional difficulties they have experienced.

Methods: The investigation combined qualitative and quantitative methods. Qualitatively, a one-hour semi-structured interview was conducted with ten Swiss cantonal police officers. Participants were asked open-ended questions regarding their career and their professional difficulties. The researcher asked other open-ended questions to further probe participants' experiences with regard to major themes in the literature that were not spontaneously expressed. Quantitatively, the Maslach Burnout Inventory-General Survey was used to measure participants' risk for burnout. This instrument gives a global burnout score and three subscores: emotional exhaustion, cynicism, and professional accomplishment. Results from this instrument classify individuals' responses into three levels of risk for burnout: low, moderate, and elevated. Qualitative and quantitative results will be compared across participants in order to better understand and describe the factors that police officers at different levels of risk for burnout experience. Additionally, particularly representative quotations will be chosen to more concretely illustrate these differences.

Results: Quantitative results showed that the participants in this study are at different levels of risk for burnout. Qualitative analyses are still being conducted. However, preliminary results attest to the following difficulties: insufficient staffing; lack of recognition for the job they do and the feeling that they are not being heard when they express their concerns to their superior officers; and the fierce and abhorrent manner in which other officers compete for promotion.

Implications: The findings should help us to determine the predominant factors leading cantonal police officers to burnout. Using both quantitative and qualitative findings, the author 
will identify police officers at moderate and elevated risk for burn out and develop prevention programs that reduce the personal and organisational stressors of these officers.

Conclusions: Using mixed methods, the stressors police officers face during their career can be identified and classified according to different levels of risk for burnout. By applying the findings of this research, prevention programs will be developed in order to suggest changes that reduce the risk of burnout for current and future police officers and their subsequent resignation from the field.

\title{
Risk stress work-correlated: the results about 6000 Italian workers
}

\author{
Bertana, E., Bisio, C., Menighini, A., Muratore, M., Nocera, A., \& Riva, P. \\ Centro per lo sviluppo e il benessere organizzativo, Italy
}

The poster represents the results of some preliminary analysis phases for the assessment of the risk stress work-correlated, carried out collecting objective data (impact indicators, index of ergonomical load and organisational process quality) on 29 sites of 9 companies (altogether approx. 6000 workers) of different dimensions and activity sectors present on the Italian territory. The impact indicator and the stress risk factor are described (ergonomical and organisational); besides the most interesting relations detected among the results are shown.

\section{The experience of Work Engagement: Examining Cross-National Differences in Hardy Personality, Optimism and Subjective Well-Being among Nurses from China and Spain}

\author{
Carmona, I., Garrosa, E., Ladstätter, F., Moreno- Jiménez, B., \& Gan, E. \\ Universidad Autónoma de Madrid, Spain
}

Objectives: To analyse the experience of work engagement, examining cross-cultural differences in hardy personality, optimism and subjective well-being (SWB) among nurses from China and Spain.

Methods: Cross-cultural design with a sample of 154 nurses from Beijing (China) and 164 nurses from Madrid (Spain) that completed the engagement, hardy personality, optimism, and SWB scales. The data were analysed using Pearson correlations, Student's t-tests and hierarchical multiple regression. Results: The personality characteristics in these countries are different, as are the variables that explain engagement. In both samples, the variable most closely associated with engagement is commitment. Chinese nurses are characterized by higher scores in hardy personality, optimism, and positive affect, but by lower scores in engagement. Nurses who interacted more with patients obtained the lowest scores in engagement. This result was not obtained in the sample of Spanish nurses. Optimism was another predictor variable, but subjective well-being was only a predictor for Spanish nurses. 
Implications: Cross-cultural nursing research is relevant for nursing knowledge. This study is a first approximation to understand the function of hardy personality, optimism and subjective well-being as personal resources that affect the way in which nurses interact with their working environment and improve engagement. Conclusions: From a cross-cultural perspective, the main effects of commitment and optimism on engagement are revealed. Commitment and optimistic nursing in both countries have more engagement. These results may contribute to the achievement of healthier organisations.

\section{Stress, Alcohol and Drug Addiction in workers employed in the building of the great infrastructures}

${ }^{1}$ Carpentiero, L., ${ }^{2}$ Costa, G., ${ }^{3}$ De Luca, G., ${ }^{1}$ Piovanelli, S., ${ }^{1}$ Vene, D., ${ }^{3}$ Righini, S., ${ }^{3}$ Marini, M., ${ }^{3}$ Baldanzi, S., ${ }^{3}$ Selvi, A., ${ }^{3}$ Scalas, S., ${ }^{1}$ Cassitto, M.G., ${ }^{2}$ Sartori, S., ${ }^{3}$ Lacangellera, D., \& ${ }^{3}$ Bosco, A.

${ }^{1}$ Azienda Sanitaria Firenze, Italy; ${ }^{2}$ University of Milan, Italy; ${ }^{3}$ Cooperative Study and Social Research Marcella via della Pace, Italy

Objectives: The research on stress, alcohol and substance dependence in workers employed in the building of the great infrastructures is part of the project Euridice-Ten; the project's mission is to provide answers to the workers on issues of stress, distress, mobbing, alcohol and drugs

Methods: The $75 \%$ of the workers employed in the macro-builder's yard of the Florence area took part in the research through an anonymous and structured questionnaire. The questionnaire consists of 85 items taken from the existing questionnaires from: The Work Ability Index, the Karasek JCQ, the CDL2; the Standard Shiftwork Index; Euridice on alcohol, drugs, smoking and gambling. The Clinica del Lavoro of the University of Milan elaborated the data. Among the objective means were used focus groups based on check list to managers and workers involved in prevention system business, workers safety and union representatives.

Results: The initial results produced on 773 valid questionnaires it was found:. $55 \%$ is used as a more onerous tasks (tunnel diggers, carpenter, machine operator, driver, laborer). 45\% less onerous tasks (warehouses, maintenance-Equipment, provost, employee, geology, topography). The workers live in base camps dormitory, $87 \%$ of workers in overtime with different motivations. They point out a high risk of stress, a low level of control, a marked risk of mobbing, a high chronic fatigue in the most exhausting work conditions.

$15 \%$ of workers suffer from illnesses related to stress and distress: high blood pressure, anxiety, depression, insomnia, gastritis and colitis. Particularly data underline that the $34,7 \%$ of workers abuse of alcohol and that the $9 \%$ of the workers know colleagues who use cocaine.

The results of focus groups with businesses showed the delay is becoming an integrated system of safety at work assessing the risk organisational.

Companies take shifts heavy (continuous cycle) with sequence 6 nights a rest, 6 afternoons a rest, 6 mornings 4 rest 
Implications: The paper discusses the probable link between the work organisation, stress conditions, alcohol and drug abuse in workers employed in the building of the great infrastructures. An action plan to reduce the pathological work organisation is also proposed.

Conclusions: It provides for the implementation of a program of corrective and preventive measures that companies should adopt with the participation of workers to improve work organisation and combat stress, mobbing, and the use of alcohol and drugs.

\title{
Good Governance of Thai Small and Medium Sized Enterprises
}

\author{
Chongvisal, R. \\ Kasetsart University, Thailand
}

The purpose of this study was to study good governance in Thai SMEs and to examine the structural relation among psychological factors; leadership, instinct motivation and attitude toward good governance, affecting good governance and success in business. The sample were 435 SMEs entrepreneurs in three types of enterprises comprising of manufacturing, wholesale and retail selling, and providing service enterprises. They were selected by simple random sampling. Instruments for data collection were one questionnaire for biosocial data and 5 measurement tools for leadership, instinct motivation and attitude toward good governance, good governance and success in business. Data were analyzed by Mean, Standard Deviation, Variance, Correlation and Structural Equation Model.

The findings from the study were found that good governance in Thai SMEs had high level including their three factors; the performance on employees, consumers and social and environment, had high level as well. In addition, instinct motivation, attitude toward good governance and success in business also had high level The structural relation model fitted the empirical data $(2=510.93, \mathrm{df}=175, \mathrm{p}=0.00, \mathrm{CFI}=0.93$, GFI $=$ 0.90 , RMSEA $=0.07$ ). In terms of effect size, leadership of entrepreneurs had positive direct effect on attitude toward good governance and good governance at .05 statistic significant level. Their standardized coefficient were .35 and .48 respectively including had positive indirect effect on success in business through attitude toward good governance and good governance. Its standardized coefficient was .16. Besides, Instinct motivation had positive effect on attitude toward good governance, good governance and success in business. Their standardized coefficient were .29,.15 and .25 respectively. Attitude toward good governance had positive effect on good governance. Its standardized coefficient was .35. Moreover, good governance had a positive effect on success in business, its standardized coefficient was .65.

According to research results, organisations should promote and develop leadership, instinct motivation and attitude toward good governance to SMEs entrepreneurs by activities such as workshop or case study. Furthermore, they should be supported, promoted and realized in ethics in business through training or activities, for example; to advise by experts or mentors in good governance development, to create the entrepreneur network for knowledge sharing and to publish information data declared that if organisations had high good governance, it could lead to be success in business and economic development in country and global level. 


\title{
Burn out syndrome and contributory work environment factors among psychiatry nurses
}

\author{
Circenis, K., Deklava, L., \& Millere, I \\ Riga Stradiņš University, Latvia
}

The subject of burn out syndrome is considered to be one of the most critical problems in the 21 st century. The objective of the research was to identify the existence of the burn-out syndrome and its contributory factors in the working environment of psychiatry nurses. The research instruments was questionnaire by $\mathrm{C}$. Maslach to define the burn out syndrome of psychiatry nurses and form to identify the factors in the working environment that cause the burn out syndrome of psychiatry nurses. With test were made assession for three burn out components: emotional exhaustion, depersonalization and rank of personal success for psychiatric nurses.

The participants of the study were 200 psychiatric nurses from several Psychiatric hospitals in Latvia. Results of the research indicates burnout presence among psychiatric nurses. A number of factors contributing to burn-out syndrome have been identified, e.g. inadequate salary, psychological pressure working with psychiatric patients and the professional achievement of nurses, which are often underestimated.

\section{Job Satisfaction and Stress in Doctors of Public Tertiary Medical Care in Mexico}

\author{
${ }^{1}$ Colunga Rodriguez, C., ${ }^{2}$ Rendon-Manjarrez, J.I., ${ }^{2}$ Dominguez-Diaz, R., \& ${ }^{3}$ Angel-Gonzalez, M. \\ ${ }^{1}$ Instituto Mexicano Del Seguro Social, Mexico; ${ }^{2}$ Nacional de Occidente del Instituto \\ Mexicano del Seguro Social, Mexico; ${ }^{3}$ Gobierno de Jalisco - Secretaría de Educación, Mexico
}

Objectives: To determine association between job satisfaction and stress among physicians in a tertiary care public hospital in Guadalajara, Jalisco, Mexico.

Methods: Using cross-sectional analysis as a probability sample of 99 general practitioners, were applied during the working day, 2 validated scales to assess stress and job satisfaction (Seppo Aro and Melia 26's, respectively), both alpha chronbach than 0.88. Data were collected sociodemographic and labor, we use descriptive statistics and Chi square and association as odds ratio, through the SPSS version 17 , was taken as significant a P less than 0.05 , participants were asked to consent verbally.

Results: $60 \%$ are males; schooling of $45 \%$ with postgraduate education. Average age 37 years. $56 \%$ people have a partner. As for working shifts $41 \%$ for the morning, evening and $42 \%$ at $17 \%$ per night. In psychosocial variables to $26 \%$ and $41 \%$ presented stress job dissatisfaction, the institutional average age is 10 years with a standard deviation of 7.6; in the last year 12 people had some disability from disease and 2 have multi-employment.

We find statistically significant differences ( $p<0.05$ ) between stress with gender, age and institutional antiquity. With men more affected by stress in a ratio 4 to 1 ( $20 \%$ of sufferers are men and $5 \%$ female) this with odds ratio of $3.87(95 \% \mathrm{Cl} 1.20-13.2)$, $\mathrm{P}$ value $=0.01$; in terms 
of institutional antiquity, found that physicians with less than 10 years have a higher stress ratio $(\mathrm{OR}) 2,58(95 \% \mathrm{Cl} 0.91-7.47)$ with $\mathrm{P}$ value $=0.04$. The relation between stress and have not partner with a probability of three to one (OR 3.22) $(95 \% \mathrm{Cl} 1.15-9.16)$ with $\mathrm{P}$ value $=0.01$. Moreover, stress and dissatisfaction, showed no association.

Implications: should to attend groups vulnerable to stress to reduce the harm to overall health, according to this study are men without partners and less than 10 years old.

Conclusions: The socio-demographic variables, work and stress, showed no association with job satisfaction in the study doctors. The only stress was associated with sex, age and marital status, with males more affected than women, without partners and who are under 10 years of exposure to stressors of work.

\title{
Predictors and Mediators of Psychosocial Wellbeing and Discomfort across Different Occupational Sectors. First Research Results
}

\author{
Converso, D., Gattino, S., Loera, B., Viotti, S., \& Hindrichs, I. \\ University of Turin, Italy
}

Introduction: A systematic review of literature, regarding the evaluation of psychosocial risk factors and job-related stress (cfr. Tabanelli, et al., 2008), highlights the large amount of tools developed and the specificity of many of those in connection with the selected analysis level (individual, relational and organisational job characteristics). At the same time, the amount of stress sources (Cox et al., 2000) suggests the choice of tools suitable for the complexity of the phenomena here examined. In Italy, the recent DL 81/08 (art. 28 comma 1), which imposes a mass evaluation of work-related stress, urges the adoption of common instruments and defined protocols. The strengthening of these practices is not facilitated by the difficulty to conjugate the purpose of using a multifactor and multilevel perspective and by the constraints (e.g. time, place etc) imposed by data collection.

Objectives: On these grounds, in 2009 our research group systematically compared the most recent researches, in order to identify the main available questionnaires for the assessment of work-related psychosocial factors and stress.

Methods: Finally, a questionnaire was prepared and administered to 800 workers, in order to explore the complex plot of variables that describe workers' perceptions and experiences on an organisational, relational and individual level. Moreover it was aimed at determining their influence on workers wellbeing or psychosocial discomfort. Particularly, at the organisational level, the following factors were examined: the perception of organisational equity (OCS, Maslach \& Leiter, 2000), organisational justice (JCQ, Karasek, 2007), workers' agreement with organisational values (OCS, Maslach \& Leiter, 2000), organisational support (JCQ, Karasek, 2007), organisational conflict management (MOHQ, Avallone \& Paplomatas, 2005), the clarity of organisational objectives (MOHQ, Avallone \& Paplomatas, 2005), organisational communication and decisional processes (COPSOQ, Kristensen, 2005), reward (ERI, Siegrist 1996), work-life balance (SWING, Geurts, 2005), investment perceived in terms of safety policies and work hours (OPRA, Magnani e Mancini,2008); at the relational level: relationship with superiors 
(JCQ, Karasek, 2007), with colleagues (JCQ, Karasek, 2007), and with own workgroup (JCQ, Karasek, 2007); at the job characteristics level: decisional authority (JCQ, Karasek, 2007), skill discretion (JCQ, Karasek, 2007), job demands (emotional, cognitive, quantitative psychological and physical: JCQ, Karasek, 2007), job meaning (COPSOQ, Kristensen, 2005), role clarity, and conflict (COPSOQ, Kristensen, 2005); at the individual level: coping strategies (COPSOQ, Kristensen, 2005) and self-sufficiency (Caprara, 1996). Finally, in order to evaluate the outcomes on workers' psychosocial health, the following factors were taken into account: burnout (MBI, Maslach, Jackson \& Leiter, 1996), satisfaction (COPSOQ, Kristensen, 2005), organisational commitment (Allen \& Meyer 1996), affective wellbeing (Daniels, 2000), organisational cynicism (Brandes, Dharwadkar \& Dean, 1999), turnover intention, and, finally, a check-list aimed at detecting psychosomatic symptoms which are commonly referred in literature to the individual stress experience.

Results: The first results, regarding both the reliability and psychometric validity of the questionnaire, and the main comparisons between workers belonging to different occupational sectors( public health, advanced tertiary sector in the first place), will be presented in the poster. Moreover, individual, group and organisational characteristics and psychological work (positive and negative) reactions will be presented.

\title{
Vulnerability to stress, burnout, emotional disorders and quality life in occupational health care providers in women with breast cancer
}

\author{
${ }^{1}$ Cunha, M.J., ${ }^{1}$ Costa, M., ${ }^{1}$ Pereira, J.P., \& ${ }^{2}$ Gascon, S. \\ ${ }^{1}$ Instituto Superior da Maia, Portugal; ${ }^{2}$ Universidade de Zaragoza, Spain
}

Background: Cancer is considered a public health problem that affects people who suffer from it, but also their care-givers. Oncology professionals are in a high-risk group for the development of burnout and emotional disorders.

Objectives: This study aims to investigate the vulnerability to stress, burnout, the emotional disorders and quality life of oncology health professionals in the area of oncology, and dealing with the issue of breast cancer, for studying the impact when there is identification gender. Method: A convenience sample, consists of 103 health professionals, aged between 23 and 57 years (mean = 34.83). Instruments: 23QVS, MBI, BSI, WHOQOL-Bref, GHQ-12.

Results: $14 \%$ of these health professionals are vulnerable to stress and $12 \%$ have been emotionally disturbed. The professional men have higher rates of somatization and Depersonalization and the female, had higher rates of phobic anxiety than men.

Conclusions: The professionals vulnerable to stress had higher rates of psychopathological symptoms than those who were not vulnerable, and a poorer perception of quality of life poor, and those not working in shifts have a more satisfactory understanding of their health. Those who have worked from 3 to 6 years are those with greater personal fulfillment, while those working for less than 1 year had lower levels of hostility and paranoid ideation. 
Implications: In Portugal, where oncology diseases, and more specifically, breast cancer are concerned psychological interventions are most often planned according to the patient himself, and do not include health professionals that are in daily contact with this problem. Thus studying the stress, burnout and the emotional disorders of these professionals is an essential step towards the future design of psychological interventions in accordance with their specific needs.

\title{
Organisational Justice as Predictor of Incivility and Verbal Abuse at the Workplace: The Role of Recovery Experiences
}

\author{
Diaz-Gracia, L., Garrosa, E., Diaz, L., Moreno- Jiménez, B., \& Rodriguez- Muñoz, A. \\ Universidad Autónoma de Madrid, Spain
}

Organisational justice is an issue that is becoming increasingly important in managing aggression at the workplace. Several studies have shown that the perception of by workers predict the emergence of aggressive behaviours, psychological symptoms, turnover intentions and counterproductive work behaviours (Aquino et al., 2001; Francis, Kelloway, Barling \& Keeley, 2003; Skarlicki, Folger \& Tesluk, 1999).

Objectives: The aims of the present study are to examine the predictive role of organisational justice on incivility and verbal abuse and to assess the moderating role of recovery processes on the relation between organisational justice and work aggression.

Methods: Cross-sectional effects were evaluated in a sample of 300 workers from service sector of Madrid, Spain. From the whole sample, 43.5\% are men and 56.5\% are women. Participants' mean age was 36 years, minimum age 18 years and maximum 67 years. Regarding to personal relationships, most of the participants had a partner (54\%), followed by single participants $(36.3 \%)$, and participants with no children $(61.2 \%)$.

A stepwise hierarchical multiple regression analysis was carried out to assess the role of organisational justice (distributive, procedural, interpersonal and informational) and the recovery experiences (psychological detachment, relaxation, mastery experiences and control) on variables of work aggression: incivility and verbal abuse. The interaction terms of recovery experiences with justice variables were included to test for the hypothesized moderating effect of recovery experiences on the relation between organisational justice and work aggression.

Results: Data from 300 workers showed that both organisational justice and recovery processes were related to work aggression. Specifically, organisational justice has a main negative effect on incivility and verbal abuse. Recovery experiences showed a moderator effect on the relation between organisational justice and work aggression.

Implications: From a practical point of view in the workplace, it is important to promote organisational justice as like a protector factor of work aggression. Moreover, it is necessary to examine in depth the role of recovery experiences processes, studying their positive consequences in the approach of diverse work-related problems such as work aggression. 
Conclusions: The study provides additional support about organisational justice as an important predictor of incivility and verbal abuse. In the other hand, recovery processes play a role in the relationship between organisational justice and work aggression.

\title{
Cultural enablers and barriers for promoting organisational health and safety in aviation companies
}

\author{
Diaz-Cabrera, D., Hernandez-Fernaud, E., Ramos-Sapena, Y., \& Casenave, S. \\ University of La Laguna, Spain
}

This research has been undertaken within the 6th framework of the HILAS Project (Human Integration into the Lifecycle of Aviation Systems - AIP4-CT-2005-516181) supported by the European Union.

This poster shows the role of organisational culture and the implementation of knowledge management systems in the field of health and safety promotion. Specifically, the purpose of this research is: 1) to report the role of organisational culture as an enabler or potential barrier in the implementation of knowledge management systems and, 2) put forward a proposal about cultural barriers and enablers that influence the development of a specific knowledge management system within the framework of a research project.

In the first phase of this research, a number of semi-structured exploratory interviews were designed and eighty-one workers from airlines, maintenance, and airport services from six companies in China, Ireland, Norway, and Spain participated in this study. In the second phase, a questionnaire was developed with the aim of undertaking quantitative evaluations that would lead to more complex statistical analyses, thus allowing us to compare organisations. Eighty-six workers from flight operations and maintenance services from two companies in Italy and Slovenia responded the questionnaire in order to test its structure.

Through the initial interviews and the developed questionnaire there were identified a serie of barriers and enablers as well as a serie of modulatory cultural characteristics. The cultural characteristics are: communication and information processes; learning capacity value; adaptability to change and innovation; information/communication/coordination systems; information and communication about change; organisational practices directed at changes and innovation; resistance to change; successful processes of change and sustainability of change initiatives. Besides, the modulatory cultural characteristics are: individual and group perceptios related to values related to work, adaptability to change and innovation, resistance to change and organisational commitment; trust in managers and organisation; and organisational and proactive climate.

The use of knowledge management systems in the field of health and safety promotion is highly beneficial for the organisation and its members. It fosters information integration from several evaluations and intervention programs, leading to more efficient long-term management that pervades all organisational processes and practices. This research shows the importance of an appropriate organisational culture to be successful in the implementation of this kind of systems. 


\title{
Vacation Effect: Reduction of Load and Addition of Pleasure?
}

\author{
de Bloom, J., Geurts, S.A.E., Sonnentag, S., \& Kompier, M.A.J. \\ Radboud University Nijmegen, the Netherlands
}

In general, health and well-being $(\mathrm{H} \& \mathrm{~W})$ improve during a vacation period. However, it is unclear, if this general development applies to every vacationer. Moreover, little is know about the underlying processes causing the improvement in $\mathrm{H} \& \mathrm{~W}$ during a vacation period. Therefore, this study focused on 2 questions: 1) Is a positive vacation effect a universal phenomenon?, and 2) Why do vacationers experience a vacation effect? We proposed a passive and an active mechanism jointly underpinning the improvement in $\mathrm{H} \& \mathrm{~W}$ during vacation: reduction of load and addition of pleasure.

In a longitudinal field study covering 7 weeks, 96 workers reported their H\&W 2 weeks before a winter sports vacation, during, and 1 week, 2 weeks and 4 weeks after vacation on 6 single indicators (health status, mood, fatigue, tension, energy level, satisfaction), which we combined into $1 \mathrm{H} \& \mathrm{~W}$ indicator.

The majority of the sample (60\%) experienced substantial improvement of $\mathrm{H} \& \mathrm{~W}$ during vacation and, still, experienced a slightly improved H\&W after 2 weeks of work resumption. Yet, a small group experienced no $(23 \%)$ or a negative vacation effect $(17 \%)$. H\&W during vacation was significantly related to the pleasure derived from vacation activities, the number of hours spent on physical and passive activities, and the absence of negative incidents during vacation. Prevacation workload was not directly related to $\mathrm{H} \& \mathrm{~W}$ during vacation. A model consisting of pleasure derived from vacation activities, passive activities and negative incidents explained $42 \%$ of the variance in the vacation effect.

In conclusion, although vacation had a positive effect for the majority, that lasted even 2 weeks after work resumption, it is not invariably positive for everybody. Because changes in $\mathrm{H} \& \mathrm{~W}$ during vacation were unrelated to workload, liberation from demands (passive mechanism) does not seem to have played a major role. Instead, there was stronger evidence for the active mechanism of choosing for and engaging in pleasurable leisure activities. Future research should focus on the role of autonomy as a core element of the vacation effect.

\section{Work Contexts and their effects at an individual level}

\author{
Deitinger. P., Nardella, C., Bentivenga, R., Ghelli, M., \& Bonafede, M. \\ National Institute of Occupational Safety and Prevention (ISPESL), Italy
}

The quality of work and the social relations developed in workplaces strongly influence the health and wellbeing of a worker. Many studies show that physical or social isolation, personnel conflicts and lack of support act as sources of stress able to produce psycho-physical diseases, exactly like other precursors of work related stress, as workload or shifts, etc. (Levi, 1971). Leyman (1996) highlighted the circular relation between work related stress and mobbing, ascribing to the last a double role: effect and source of individual and social stress. 
Mobbing is defined as - a type of persecution or psychological violence almost always practiced with the intent of damaging someone in systematically and repeatedly different ways; the action is performed over a certain period and it depends on conditions and realization and on the subjects' personality and its aim is to remove the person from that workplace.

Objectives: The aim of the present study is to identify the organisational and individual variables that constitute the mobbing process, using the MMPI2 and the Italian Val.Mob Scale. The MMPI2 was selected because of its proven validity and reliability and the general scientific consensus it enjoys, the Val.Mob.scale for its validity and reliability to assess individual and organisational variables.

Methods: Correlations analysis among some factors of MMPI2 and some scales of Val.Mob has been carried out for this study.

Results: Participants were selected in 2008 and 2009 when they requested a medical consultation, complaining of mobbing (bullying) at work for at least six months. The sample comprised 71 people, balanced for age, gender, education. Correlations analysis gave the following picture:

- positive correlations ( $p=, 05$ or $p=, 01$ ) between the factor Relational in the Val.Mob. scale and some of the MMPI-2 scales and a negative correlation between the factor Relational and the Es-Ego Strength Scale.

- positive correlations ( $p=, 05$ or $=, 01$ ) between the factor Intrusiveness/Interference on the Val.Mob. scale and some of the MMPI-2 scales and a negative correlation between the factor Intrusiveness/Interference and the Re-Social Responsibility scale.

- positive correlations between the Val.Mob. Symptomatological Scale $(p=, 01)$ and many of the MMPI-2 scales and a negative correlation between the Symptomatological Scale and the Es-Ego Strength Scale.

Conclusion: The two evaluation tools significantly correlate in some of their scales and consent a better understanding of the case histories and a differential diagnosis among real mobbing cases and other situations of suffering deriving from other types of work related stress.

\title{
Reference regulation and good practices in the assessment of psychosocial risk factors in Italy
}

\author{
Deitinger. P., Nardella, C., Bentivenga, R., Ghelli, M., Persechino, B., \& lavicoli, S \\ National Institute of Occupational Safety and Prevention (ISPESL), Italy
}

The topic of work-related stress and, in general, the management of psychosocial risks in the workplace have become pivotal and involve different disciplinary domains and intervention areas such as medicine, engineering, psychology and sociology. Although the directive 89/391/ CEE does not clearly refer to the stress as such, it obliges the employers "to safeguard the safety and health of workers within their field of activity". The Italian implementation of this directive. Decree 626/94 - introduced important changes on occupational health and safety through "improved" safeguarding regulations, as provided by the national legislation in the 50-60's. The above mentioned decree, successively modified and integrated, identified those who are 
still referred to as "OSH professionals" and also highlighted the key role of workers in managing health and safety at work. The decree also acknowledged that the safeguard of health and safety in the workplace requires integration and involvement of the workers themselves. Today, the laws on occupational health and safety have been redefined by the Lgs. Decree 81/08 clearly underlining, in Art. 28, that the risk management must involve all risks to health and safety of workers, including work-related risks, as provided by the European Agreement of 8 October 2004.

Objectives: The objective of this paper is to examine the assessment tools for psychosocial risk factors in Italy.

Methods: An accurate review of assessment models and tools acknowledged by the scientific literacy has been outlined, based upon a mainly documentary method. The psychometric qualities of the examined tools such as reliability, statistical significance and representative sampling have been accurately assessed.

Results: 10 different tools have been identified; some of them have been borrowed from foreign questionnaires and adapted (40\%), others have been directly defined in Italy (60\%). Every assessment tool is based on a specific theoretical approach or model and shows specific characteristics; such tools have been tested on different samples and usually target some kind of organisational variables rather than others.

Implications: The Italian case is particularly interesting as SMEs account for approximately $90 \%$ of the economic activity and in many cases they have less than 10 workers. Therefore, the implementation of tools conceived for a large number of people might lead to critical problems.

Conclusions: The work-related stress assessment is not an easy issue to solve both due to the multifaceted nature of stress and the context specificity which needs to be considered when choosing an efficient assessment tool.

\title{
Workload and job burnout among prison educators
}

\author{
Deitinger. P., Nardella, C., Bentivenga, R., \& Ghelli, M. \\ National Institute of Occupational Safety and Prevention (ISPESL), Italy
}

Introduction: Studies show that prison personnel experiment high levels of stress and burnout due to the intense workload. However, significant differences in stress reactions are found among different sectors such as Security, Administration and Treatment. The job burnout is a syndrome characterized by emotional exhaustion, disaffection and a reduced professional efficacy. It may affect subjects who get highly emotionally involved with their work.

Objectives: Our study focuses on the assessment of the Demand-Control-Support model (DCS; Karasek \& Theorell, 1990) scales and some aspects of job burnout such as emotional exhaustion, disaffection and professional efficacy among educators employed in the Treatment sector of an Italian district penitentiary. 
Methods: The Karasek JCQ and the Maslech MBI-GS were administered to a group of 16 prison educators from a district penitentiary.

Results: Survey subjects were $62,5 \%$ female and $27,5 \%$ male. Women, indeed, are usually more involved in this work. The mean age was approximately 51 years $(\mathrm{m}=50,8 ; \mathrm{sd}=8,1)$. They were all highly educated: $56,2 \%$ graduated or post graduated; $6,3 \%$ got an academic degree and the remaining $37 \%$ got a high school diploma. The average professional seniority was 16 years $(m=16,56 ; s d=11,73)$. Significant correlation emerged between D-C-S and job burnout scales.

Conclusion: During the protocol, the district penitentiary hosted 1492 prisoners; 877 of them were condemned prisoners and the ratio between educators and condemned prisoners was 1 to 55. That being so, the study of the Karasek and Theorell model scales integrated with job burnout variables permitted to evaluate the correlation between the perception of work related stress and distress scales among prison educators.

\title{
Personality factors and professionally relevant behaviour in health care
}

\author{
Deklavs, L., Millere, I., \& Circenis, K. \\ Riga Stradins University, Lativa
}

Medicine is one of those branches where the issue of human resources is essential and important. The behaviour at work in general, and personality factors in particular, play a significant role in health care. Work behaviour characterizes such factors as job satisfaction, attainment of motivation, empathy, adequate behaviour in a given situation, ability to cooperate, work ethics respect, creative approuch to work and others. Personality dimensions - coping strategies, emotional intelligence, locus of control related with behaviour at work.

Research objectives and research aim: The aim of the paper is 'Personality factors and professionally relevant behaviour in health care' to study individuality characterizing factors for emotional intellectuals, locus of control, coping strategies and health care workers behaviour.

Research material and methods: Respondents were nurses from different areas of hospitals and out patient health care services. The study in using such tools as Lazarus and Folkmans Ways of Coping Checklist, Questionnaire about Professionally Relevant behaviour, obtaining demographic data, Subjective control level determination test and Questionnaire of emotional intellectuals.

Results: Data about respondents on the locus of control show that for $57 \%$ typical is the internal locus of control, but $26 \%$ external. Emotional intelligence indicator average interval is from 2.8 - 4.1 points (max5). Largest group of respondents have a total emotional intelligence average 3.2 points. All the emotional intelligence competencies average rates range from 3.15 for intrapersonal competence till 3.27 for between interpersonal competence. The most common used stress overcome types are positive self-esteem (51,6\%), elective solving of situation (51\%), control of self $(47,6 \%)$, accepting responsibility $(43,8 \%)$ and search for social support $(43,7 \%)$. The highest average rates 3,71-3,61 (max 4) has the following professional relevance to behaviour parameters: adequate behaviour in a given situation, responsibility and emotional stability, ability 
to acknowledge their guiltiness, concede, if it necessary, concentration, optimism and ability to cooperate, truth, openness, reliability and ability to improve and develop their practical skills.

Conclusion: Health care workers use the emotion focused coping overcome types, the most common is the use of positive self-esteem, a planned settlement of the situation and self-regulation. They are characterized with external locus of control. Leading indicators of Emotional intelligence competence is interpersonal competence, stress management and adaptive competencies.

\title{
Till' 5pm Do Us Part: Intimate Relations in the Workplace
}

\author{
Donohoe, $M$ \\ Saint Mary's University, Canada
}

The lines between career and personal life are increasingly blurred in today's workplace due to increased hours spent on the job as a result of job travel, overtime, flextime and shift work (Carson \& Barling, 2008). Having someone at work with whom one can share the daily pressures, frustrations, and joys of the job has led to more people pursuing close, intimate relationships in the workplace. Two types of intimate relationships in the workplace are currently well documented: the close friendship and romantic relationship. A third type of relationship, the work spouse (a platonic workplace relationship that resembles a marriage), has recently been gaining attention in the popular media and, until now, has not been examined empirically. In the current study, we seek to characterize the work spouse relationship through the use of Sternberg's triangular theory of love and determine if this new phenomenon is any different than a close friendship or romantic relationship at work.

Role conflict and role facilitation occur as a result of juggling several roles at once. Individuals who are part of intimate relationships in the workplace in particular may be required to balance their role in the relationship while meeting the demands of their organisational role. Factors such as the relationship motive, power distance and social support may impact the extent to which role conflict or facilitation occurs. Both benefits and costs are associated with the occupation of multiple roles in the workplace; therefore the present study will also examine the consequences on health and well-being of those individuals involved in intimate workplace relationships.

A survey study will be used to gather data from 400 participants who are currently working. Data will be analyzed through the use of 3 one-way ANOVA's as well as other post-hoc analyses to address the exploratory nature of this study.

\section{Work related stress evaluation: two cases studied}

\author{
Dusi, P. \& Servadio, M.
}

A work related stress evaluation can and must be a great opportunity for the business development of the company finalized to increase the organisational well-being and therefore 
to improve own performances (working in a comfortable atmosphere limits the risk to produce illness, reduces the phenomenon of the absences for disease and increases the workers' performance). To take advantage of this opportunity is necessary managing the evaluation process and its outcomes/effects (preventive or improvement actions) in a competent way making use of appropriate methodologies and tolls by professionals specifically trained. Our intervention will start with a short explanation of the methodologies, tools and skills necessary to realize the work related stress evaluation, through standardized models and new approaches (both one-step or multiple steps) finalized to draft the documents required Documento di valutazione dei rischi DVR Stress Lavoro correlate.

Following, will be presented two cases of evaluation conducted in Italy during the 2008-2009, practical experiences related to small, medium and large companies (possibly the choice of case studies will be agreed with you). All the experiences presented included also the subsequent preventive or corrective action planning oriented to job design, work organisation and management.

This paper will be designed and will be realized according to the European Agreement, to the European and Italian UNI EN ISO norms (since 2008 we work with Dr. Paola Cenni, Italian supervisor of UNI Group of study on stress work related stress) and also to other Italian norms, laws, best practices or guide lines (for example: ISPESL, SPISAL, AUSLL; Confindustria, ecc.).

\title{
Stress and coping of unemployed before and after interaction with job coaches
}

Eisele, P.

\author{
Blekinge Institute of Technology. Sweden
}

The main aim of the present study was to investigate if unemployed experiences of stress are affected by their meetings wih job coaches.

The Percieved Stress Scale (PSS) and a coping style questionnaire were used before and after job coaching. Personal experrence of stress was not decreased by job coaching. Rather, the use of more and new coping strategies decreased self-reported stress.

The result has implication for the choice between different coaching styels. "Coping-related" coaching is perhaps better than "job-related" coaching.

\section{Psychological and social factors at work in relation to musculoskeletal disorders among employees in the Norwegian meat process industry}

Elka, S., Christensen, J.O., Emberland, J.S., Vindsetmo, K., Pettersen, T., Aune, M., \& Lau, B. National Institute of Occupational Health, Norway

Objectives: Musculoskeletal disorders and their associations with physical workplace factors have been examined in various studies. However, there is limited understanding of the exact 
roles that psychosocial factors play in development of these disorders. Consequently, in this study we wished to investigate musculoskeletal problems among workers in the Norwegian meat processing industry, an industry known to have a high prevalence of musculoskeletal problems. We were expecting a high prevalence of musculoskeletal problems in this industry, and an even higher prevalence among production worker compared to administrative personnel. We also wished to investigate the significance of psychological and organisational factors for occurrence of musculoskeletal problems.

Methods: A total of 1015 employees from eight meat processing enterprises from different regions in Norway participated in the study. Data were collected by a web-based questionnaire. The measurement instrument was based on the General Nordic Questionnaire for Psychological and Social Factors at Work (QPSNordic), including 10 scales measuring psychosocial and organisational factors, and the 'musculoskeletal pain' scales from the Subjective Health Complaint Inventory. The response rate was $62.6 \%$.

Results: After adjusting for factors independently associated with musculoskeletal problems (age and gender), we found more musculoskeletal complaints among process workers, compared to administrative personnel. Further, the results showed an association between psychosocial and organisational factors and musculoskeletal problems among process workers. In multiple regression analyses, adjusted for age and gender, the results showed positive associations between musculoskeletal complaints and quantitative demands and decision demand, respectively, and negative associations between musculoskeletal complaints and control of decisions, control of work pacing, support from coworkers and superior, and fair leadership and empowering leadership, respectively.

Implications: The results indicating that psychosocial factors are of importance to musculoskeletal complaints among the process workers in this industry, implicate that the psychosocial work environment should be more in focus in order to reduce musculoskeletal complaints in this industry.

Conclusions: The findings emphasize the importance of psychosocial factors for musculoskeletal problems among meat processing workers. However, longitudinal studies are required in order to gain better knowledge of the relationship between work environment and musculoskeletal problems.

\title{
Working in a call centre: the role of personal and organisational resources on positive and negative emotions at work
}

\author{
Emanuel, F., Ricotta, S., Ghislieri, C., Gatti, P., \& Colombo, L. \\ Università degli Studi di Torino, Italy
}

Objectives: Several studies identify call centres as a work environment which can determine negative consequences for employees' well-being. Referring to job demands-resources model (JD-R, Demerouti, Bakker, Nachreiner, \& Schaufeli, 2001), this contribution focuses on resources which can have an influence on positive and negative emotions in call centre employees. Bakker, Demerouti, and Schaufeli (2003) already adopted JD-R model to investigate employees' health 
problems and motivation in call centres. The present study, in particular, inquires the effects of personal resources (internal locus of control and optimism) and organisational resources (colleagues support and supervisors support) on positive emotions at work (psychological wellbeing) and negative emotions at work (psychological discomfort) in call centre employees.

Methods: This research was conducted through a questionnaire filled out individually by 525 respondents from different Italian call centres of the same telecommunication firm. The questionnaire consisted in six measures: positive emotions at work (6 items, $\alpha .89$ ); negative emotions at work (6 items, $\alpha$.87); optimism (5 items, $\alpha .73$ ); internal locus of control (5 items, $\alpha$ .90); supervisors support (4 items, $\alpha .94$ ); colleagues support (4 items, $\alpha .92$ ). Data analysis was performed with SPSS 15: to test the reliability of the scales the Cronbach's alpha was calculated, and multiple regressions were used to understand causal relations among variables.

Results: Positive emotions at work are influenced (32\% explained variance) by supervisors support, optimism and internal locus of control. Negative emotions at work are influenced (17\% explained variance) by optimism, supervisors support and internal locus of control. The results highlight that personal and organisational resources have a differential impact on positive and negative emotions at work. Among personal resources, optimism has a stronger influence on the dependent variables than locus of control. Among organisational resources, supervisors support has a stronger influence on positive than on negative emotions, whereas impact of colleagues support is not significant.

Implications and Conclusions: This research project deepens the comprehension of different resources influencing psychological well-being and discomfort at work. The personal resource of optimism has a higher impact than locus of control on both dependent variables, according to their common affective dimension. The results have useful implications for organisational politics and investments. Implications for human resource management refers first to recruitment - that could consider recruits' personal characteristics and secondly to supervisors training.

\title{
Results of a psycho - educative intervention about the stress symptoms, stressors at work and metabolic indicators in nurses (male and female) of attention of third level
}

\author{
Enriquez Hernandez, C. B., Colunga Rodriguez, C., Preciado Serrano Maria del, L., Angel \\ Gonzalez, M., \& Dominguez Diaz, R. \\ Universidad Veracruzana and Universidad de Guadalajara, Mexico
}

Objectives: To establish the results of a psycho - educative intervention focusing on the cognitive and behaviour on stress symptoms and the stressors at work in the nursing staff of attention of third level. To know the results of a psycho - educative intervention focusing on the cognitive and behaviour stress of some metabolic indicators such as glucose and cortisol in the nursing staff of attention of third level.

Methods: To intervene with measurements before and after, taking the group as its own control. It was designed and applied a cognitive and behaviour psycho-educative program in 5 sessions of 3 hours. It included 8 nurses who attended the sessions, at 100\%, these sessions were held by a psychologist. The nurses presented stress symptoms and full filled the psychometric 
instruments and lab samples. They were applied the Aro Seppo's instrument of stress symptoms with 18 reactive which explore symptoms associated with the stress of psycho somatic kind, emotional and knowledge with Cronbach alpha of 0.83, the Nursing Stress Scale of 34 items which describes potential stressing working situations in nursing, they were added social demographic and labor data and the glucose in fast was measured, it was also measured cortisol before and after the intervention. The data analysis was made with the S. P. S. S. program version 15.0. To compare results it was used the student $\mathrm{T}$. It was taken in consideration the confidentiality and agreement.

Results: the average age was 37 years old, marital status 4 of them were married or with partner, 3 singles and a single mother. All of them specialist nurses working full time with 5 years seniority in the hospital. This intervention influenced notoriously in the dropping of the stress symptoms, the pre intervention against the immediately post intervention results reported a significant statistic of $t=3.1 ; p=0.01$. According to the labor stressors variable, the intervention influence in the dropping of the stressors perceived the pre intervention against the immediately post intervention results reported a significant statistic of $t=2.55, p=0.03$ in the same way in the metabolic indicators which were also studied, it was observed a significant statistic in the glucose before breakfast: $\mathrm{t}=3.47 ; \mathrm{p}=0.01$; cortisol; $\mathrm{t}=2.46 ; \mathrm{p}=0.04$.

Conclusions: the intervention showed results with statistic significant dropping the stress symptoms, the labor stressors perceived; as well as; the metabolic indicators studied in the group considered.

\title{
Structure matrix of work related stress based on "Framework agreement on work- related stress" of October 8th 2004
}

\author{
Erba, A.M. \& Carlo Picchi, M.P. \\ ECoNA (Interuniversitary Centre for research on cognitive processing in Natural and Artificial \\ Systems), Italy
}

Objectives: The "Framework agreement on work-related stress" is probably the stronger reference for work related stress evaluation, and it is clearly mentioned in the art. 28 of Italian law on safety at work (d.lgs. 81/08).

The "Framework agreement on work-related stress" suggests the importance of four so-called "potential stress indicators": high absenteeism, staff turnover, frequent interpersonal conflicts and complaints by workers; and four "risk factors": work organisation and processes, working conditions and environment, communication and subjective factors. There are at the moment a lot of questionnaires issued to evaluate the work related stress but no one of these is in strict accordance with the "Framework agreement on work-related stress". We asked ourselves if it was possible to theorize an inventory with construction validity referred to this agreement and with a structure matrix (as results of a factorial analysis) confirming the main groups of indicators and factors described.

Methods: We created a check list of 36 items, strictly referred to the indicators and factors stated by the "Framework agreement on work-related stress", these lists of items have been filled up by 
153 workers in 19 different organisations. Everyone had high experience in job, high knowledge of working groups and received two hours training on work related stress main assumptions.

Results: We have submitted the answers to the check lists (expressed in Likert scale from 1 to 5) to a factorial analysis (Principal Component Analysis with Oblimin Rotation), obtaining a Structure Matrix with five main factors (wellness, conflicts, environment, organisation/skills and stillness) showing $48,78 \%$ of variance.

Implications: The Structure Matrix obtained partially confirms the main groups of indicators and factors described in the "Framework agreement on work-related stress"; but the five factors selected by the factorial analysis do not allow an assessment strictly relating to its contents.

Conclusions: As in Italy the law on safety at work quotes the "Framework agreement on workrelated stress" as the main reference for the evaluation of the work-related stress, this agreement has probably to be considered as an "external standard" for any questionnaire or check list to be used. Our research shows that is probably more simple to use a single scale for each indicator and factor or a simple check list than to arrange a questionnaire with a complex internal structure.

\title{
Participation in everyday activities - a comparison of people on sick leave and a reference group from the Swedish population
}

\author{
Eriksson, T., Jonsson, H., Tham, K. \& Eriksson, G. \\ Karolisnka Institutet, Sweden
}

Objectives: Musculoskeletal disorders and stress-related ill health are conditions that generate extended periods of sick leaves in the European society. Rehabilitation interventions focusing on work are most commonly used within this group. However, it is known that various aspects need to be considered in facilitating return to work and not just work issues. The aim was to describe participation in everyday occupations in people with musculoskeletal disorders or stress-related ill health compared to a reference group from the Swedish population by examining occupational gaps

Methods: A cross-sectional study of 72 persons in a rehabilitation group and 497 persons in a reference group. A survey with the Occupational Gaps Questionnaire (OGQ) was answered. OGQ is a self-report questionnaire that assesses the presence of occupational gaps in 28 everyday activities in instrumental ADL, social life, leisure and work. An occupational gap is defined as the discrepancy between what the person wants and needs to do and what he or she actually does.

Results: Occupational gaps were experienced three times more often in the rehabilitation group than in the reference group. Most occupational gaps were reported in leisure activities. The majority of occupational gaps reported by both groups occurred because they did not do the activities that they wanted to do. Occupational gaps also occurred because the participants did activities that they did not want to do. This was most apparent in instrumental ADL. However, the participants that had been on sick leave for more than a year reported that they did not do the activities that they wanted to do to a higher extent 
in instrumental ADL than those that had been on sick leave for a shorter period of time. Implications: There is a need of focusing on all everyday activities in rehabilitation and not just work related activities to facilitate the process of return to work. Furthermore, it is important to emphasize the individually valued activities in everyday life when designing rehabilitation interventions as they seem to shift meaning according to length of sick leave.

Conclusions: The results showed that everyday activities are highly affected, especially leisure activities. The occupational repertoire seems to decrease as the period of sick leave extends and the occupational arenas, such as work or social life, are absent. In this situation the activities that still are present in everyday life becomes more important, such as instrumental ADL, and that has to be acknowledged in rehabilitation.

\title{
Conflict Management Styles and Performance Relations Among Voluntary Health Workers
}

\author{
Erturk, E.M \\ Uludag University, Turkey
}

Objectives: Conflict is considered as a part of social life. Especially in work environments requiring group work, conflict is inevitable. Non-existence of conflict in a group work may even create a negative effect in the group. A healthy dose of conflict has positive effects, such as activation and motivation of group members, however, while its exceeding an acceptable level may lead to undesirable results. Therefore, management of conflict is essential for the group work to be fruitful. The aim of this study is to investigate how the styles of conflict management effect job satisfaction for voluntary groups, which is not much studied. Conflict management styles considered are, forcing, avoiding, yielding, problem solving, and compromising.

Methods: The research is carried out by survey method on 263 voluntary health workers, chosen among those participating camps of National Medical Rescue Team (NMRT) established by volunteers in the body of the Ministry of Health, to provide assistancy in medical rescue activities in case of disaster. Dutch Test for Conflict Handling (DUTCH), based on five factor conflict management styles model, developed and revised by Van De Vliert (1997), is used in the study. Spector's Job Satisfaction Scale is used to measure job satisfaction (Keser, 2006; Spector, 2009). Since the sampling group is composed of voluntary workers, three sub-scales including pay, promotion and fringe benefits are excluded while preparing the questionnaire form.

Results: Of the 263 volunteers taking part in the survey, 97 are women, 163 are men (3 people has not mentioned their sex). They belong to various medical occupational groups such as health officers, emergency medical technicians, nurses, doctors, midwives, laboratory technicians, anesthetic technicians. 55 percent of the participants expressed experiencing conflict in the team. Significant correlations are observed between conflict management styles and satisfaction they get from their voluntary work.

Implications: The most used conflict management style among the voluntary workers is seen to be compromising, among both women and men. Job satisfaction is found to be correlated positively with compromising, but negatively with forcing and avoiding styles of conflict managements. 
Conclusion: The styles of conflict management effect the satisfaction people get from their voluntary works. Those who adopt a compromising attitude are more satisfied with their works, while those who try to resolve conflicts by forcing or avoiding styles get less satisfaction from their works.

\title{
Some remarks about the function of ergonomics when facing psychosocial risks: an interactive approach aggregating clinical, organisational and activity-centered conceptions of field interventions
}

\author{
Favaro, M. \\ INRS (Institut National de Recherche et de Sécurité), France
}

We propose a discussion about the role of ergonomics when facing psychosocial risks. After pointing out that influent French work psychopathology schools in such an occupational prevention field can be considered as sub-parts of a more traditional ergonomics trend, some characteristics of field interventions when implemented into labour contexts faced with psychosocial risks will be put under discussion.

To do this, we will present three levels of such characteristics dealing in an interactive way together with clinical, organisational and activity-centered analysis:

1. Contextual vs individuals factors,

2. Management vs activity,

3. 'Profesional involvment' vs 'organisational adherence'.

A short illustration of an intervention made in context of external violence against French railway personnel will conclude our presentation.

\section{Assessment of Occupational Stress Among Shopping Centre Employees}

\author{
${ }^{1}$ Fenga, C., ${ }^{1}$ Di Nola, C., ${ }^{1}$ Cacciola, A., \& ${ }^{3}$ Nardella, C. \\ ${ }^{1}$ Universita' Degli Studi Di Messina, Italy; ${ }^{2}$ SPPESL, Italy
}

Objectives: Objective of the present study was to evaluate the occupational stress level of a shopping centre employees.

Methods: A sample of 50 workers, $54 \%$ male $(n=27)$ and $46 \%$ female $(n=23)$ underwent to the Occupational Stress Index (OSI) questionnaire, used to examine occupational stress level of employees through the evaluation of work organisation, individual characteristics, coping strategies, and psychosomatic effects of the stress.

Results: The results of OSI were compared to the stress levels of a reference population. Results of data analysis provided evidence that the work organisation of the examined shopping centre was good and there was no correlation between work characteristics, personality patterns, coping strategies and the psychosomatic effects of the stress. In addition a negative relationship 
between job satisfaction and length of service was found. Improvements in working conditions as well as early diagnosis are essential for preventing further increases in these disorders among workers.

Conclusions: The next step would be multidisciplinary intervention studies aimed at the work environment, with the goal of identifying scientifically-based strategies that are most effective in preventing occupational stress.

\title{
Well-being Survey in an Italian Research Institute
}

\author{
Ferravante, D.G. \& Gloriani, I. \\ ENEA, Italy
}

This proposal concerns the results of the well-being survey carried out in an Italian public agency, in different sites of the company (North, South and Middle of Italy).

The objectives were to identify if there are differences, about strengths and weakness of the work environment, between the Research Institutes due to the number of people or to the geographic area.

The questionnaire we've used allows to check 14 factors about safety and health at work e.g. stress, interpersonal relationship and conflict, leadership, enhancement, etc. The study was conducted in several phases, in different Research Institute, with different number of people, in three geographic areas (North, Middle and South of Italy).

In the first step have been involved 122 people, in the second step 272 people and, in the last step, 108 people.

The survey was carried out in several phases: 1) communication at the people involved about the objectives of the survey, the methods, the contents, etc.; 2 ) the administration of the anonymous survey in group of ,at least, 60 people, 3) data entry; 4) data analysis 5) feedback to the participants about the results of the survey and the following action to manage weak points.

The comparative analysis have been implemented. The study allows us to examine the data and results in smaller and different units and the intercorrelations among the indicators of the survey.

\section{Quality of Work Life Assessment of a Brazilian Public Company}

\author{
${ }^{1}$ Ferreira, M.C., ${ }^{1}$ Ferreira, R.R. \& ${ }^{2}$ Seidl, J. \\ ${ }^{1}$ University of Brasilia, Brazil; ${ }^{2}$ University of Coimbra, Brazil
}

The research was carried out in a Brazilian public company in which managers were interested on designing and implementing a Quality of Work Life Program (QWLP). The study aimed 
to analyze the Quality of Work Life of the mentioned enterprise based on the employees' opinion.

Four hundred and four respondents $(\mathrm{N}=723,55.9 \%)$ participated voluntarily of the study. They can be characterized by the following profile: $53.4 \%$ were male; $52.4 \%$ married; $33.8 \%$ graduated; and $41 \%$ aged between 41 and 50 years-old. Data was collected applying the Quality of Work Life Assessment Inventory (IA_QVT, $\alpha=0.94$ ), validated by Ferreira (2009). This Inventory was available to the participants in the internal computer based communication system of the company for three weeks. Employees should judge the items accordingly to a Likert scale of 11 points, from " $0=$ totally disagree to $10=$ totally agree". Confidentiality and anonymity were guaranteed to all participants when results were presented. Descriptive statistical analyses (mean, standard deviation, minimum and maximum values) were performed using the Statistical Package for Social Science, version 15.0.

Results showed a critical perception of the employees regarding the Quality of Life ( $M=5.6$, $\mathrm{SD}=0.67$, indicating an alert state that requires management procedures in a short and medium term to remove the negative aspects that are affecting the QWL of the company. The factor rated as more negative - demonstrating serious risk of illness and requiring immediate management action - was "Work Organisation" (9 items, $\alpha=0.73)$, mean of $4.8(\mathrm{SD}=0.89)$. The respondents $(\mathrm{n}=358)$ complained of "work performed under pressure", "heavy workloads", "excessive work rate", "strong charging for results" and "repetitive tasks". The factor rated as the most positive - indicating situation which promotes health and well-being at the workplace and requiring managerial action of maintenance and reinforcement - was the "Work-Social Life Link" (10 items, $\alpha=0.80)$, average of $7.2(\mathrm{SD}=0.73)$. Participants $(\mathrm{n}=356)$ stated that "the work is useful for the society" and "the work itself represents a source of pleasure greater than the relationship with their co-workers".

These results provided the main empirical basis to design the organisation's Quality of Work Life Program (QWLP) and denoted indicators that can promote well-being at work, efficiency and effectiveness of the company's processes.

\title{
What is Quality of Work Life? A Research Based on the Workers' Point of View of a Brazilian Public Company
}

\author{
${ }^{1}$ Ferreira, M.C., ${ }^{1}$ Ferreira, R.R. \& ${ }^{2}$ Seidl, J. \\ ${ }^{1}$ University of Brasilia, Brazil; ${ }^{2}$ University of Barcelona, Spain
}

Regarding the present mutable work context and specially its negative consequences to the organisations, the issue Quality of Work Life (QWL) has been of increasing interest to leaders, managers and researchers. The research aimed to identify the main elements of the Quality of Work Life concept based on the employees' perspective of a Brazilian public company. Three hundred and sixteen workers $(\mathrm{N}=723,43,7 \%)$ participated of the study and presented the following profile: 53,4\% were male; 52,4\% married; 33,8\% graduated; and $45 \%$ aged between 41 and 50 years-old. The question "In my opinion, quality of work life is" was available during three weeks at the company's internal computer-based communication system. Data analysis was conducted using the software Alceste (Analyse Lexicale par Contexte d'un Ensemble de 
Segments de Texte). Results indicated three core categories in participants' speech, expressing the main elements of the Quality of Work Life's concept. Quality of Work Life stands for "Wellbeing at Work" (69\%, 254 uce's) as the first core category, understood as crucial to employees' health maintenance, the promotion of pleasure at work and as an essential requirement for good performance and for the quality of the services provided. The second category referred to "Recognition, Opportunities and Fair Salary" (24,86\%, 92 uce's), denoting the importance of equality of opportunities, potential development and of compatibility between salary and career. The third core category expressed the idea of "Happiness at Work" (6,49\% 24 uce's) understood as feeling well-being and satisfaction at work, perception of accomplishment and desire to work. Results provided empirical basis to monitor the QWL concept accordingly to the workers' point of view and to conceive the company's Quality of Work Life Program.

\title{
Workplace bullying and personal and organisational well-being
}

\author{
${ }^{1}$ Fida, R., ${ }^{2}$ Natali, E., 'Mamazza, L., \& ${ }^{1}$ Barbaranelli, C. \\ ${ }^{1}$ University of Rome 'Sapienza', Italy; ${ }^{2}$ National Institute for Occupational Safety and \\ Prevention (ISPESL), Italy
}

Objectives: Bullying at work, the systematic exposure to negative acts (e.g., verbal abuse, offensive remarks, ridicule, slander, or social exclusion) from co-workers, supervisor, or subordinates (Einarsen, 2000), emerged as an important risk factor or stressor on many employees (Dollard et al., 2007; Einarsen \& Raknes, 1997; Spector \& Jex, 1998). The Fourth European Survey of Working Conditions reports that $5 \%$ of the European workforce has been exposed to bullying or harassment at work in the previous 12 months, whereas in Italy the percentage decreased at 2\% (European Foundation, 2007; ESWC, 2007). Some recent studies highlighted a significant association between bullying and physical illness and injury (Bowling \& Beehr, 2006; De Pedro, SÃ nchez, Navarro \& Izquierdo, 2008; Mikkelsen \& Einarsen, 2001; Mikkelsen \& Einarsen, 2002), as well as decreased job satisfaction (Argevold \& Mikkelsen, 2004; Bowling \& Beehr, 2006; Hershcovis et al., 2007; Hoel \& Cooper, 2000; De Pedro, SÃ nchez, Navarro \& Izquierdo, 2008;) and decreased organisational commitment (Argevold \& Mikkelsen, 2004; Bowling \& Beehr, 2006; Hoel \& Cooper, 2000). According to these results, the aim of the present study is to investigate the effects of workplace bullying on job commitment, job satisfaction, burnout and worker's health.

Methods: A sample of about 500 Italian workers (70\% male) ranging in age from 21 to 68 (Mean age 40.6, SD 10.05) was administered a set of self report Likert scales to assess the key constructs of the study. In particular job satisfaction was measured by 24 items (Avallone \& Paplomatas, 2005), job burnout by 16 items (Demerouti, Bakker, Nachreiner, \& Schaufeli, 2001), workplace bullying by 32 items (Negative Acts Questionnaire developed by Einarsen, Raknes, \& Mattheisen, 1994), workplace commitment by 6 items (Allen \& Meyer, 1990) and finally psychosomatic complaints by 8 items (Avallone \& Paplomatas, 2005).

Results: Exploratory factor analysis revealed three distinct but correlated dimensions of workplace bullying: personal derogation, abuse/harassment and work-related harassment. A structural equation model approach was used to investigate how workplace bullying influence job satisfaction, organisational commitment, job burnout and psychosomatic complaints. Results 
confirm that workplace bullying predicts job satisfaction. Moreover it influences psychosomatic complaints both directly and indirectly through job satisfaction, commitment and burnout.

Implications and conclusions: This study demonstrated that victims of bullying are less satisfied and have higher level of psychosomatic problems. These results reveal the importance of arranging programs for the prevention and management of workplace bullying.

\title{
Relationship between cognitive behavioural treatment and cortisol. A longitudinal study
}

\author{
${ }^{1}$ Figueiredo-Ferraz, H., ${ }^{1}$ Gil-Monte, R., \& ${ }^{2}$ Ribeiro do Couto, B. \\ ${ }^{1}$ University of Valencia, Spain; ${ }^{2}$ University of Murcia, Spain
}

Objectives: Cortisol is a glucocorticoid produced by the adrenal cortex, which is part of the adrenal gland. According to the literature, cortisol is commonly known "stress hormone" as it is involved in response to stress and anxiety, controlled by the Corticotropin-releasing hormone $(\mathrm{CRH})$. Some studies have linked cognitive behavioural treatment and cortisol and after the program, cortisol levels increase significantly (Mommersteeg et al., 2006). The aim of this study is to examine the effectiveness of a cognitive - behavioural program and their impact on cortisol levels in saliva during a period of 9 months in a sample of teachers.

Methods: A total of 31 (10 male) participants were investigated. The sample was divided into two groups, one experimental group (EG)/ (17 participants) and a control group (CG)/ (14 participants).

Instruments: Salivary cortisol was collected by a salivette - is a tube containing a swab used to absorb the saliva.

Procedure: This is a longitudinal study. Time 2 was collected 6 months after time 1, and time 3 will be collected 3 months after time 2. Saliva sample were collected in two different moments. Time 1: All samples were collected at the same time at the 3 times (11.30); Between the time 1 and time 2, the experimental group received a cognitive - behavioural treatment. The program consisted of five sessions of four hours everyone. The agenda was as follows:

a) Training on the conceptualization of stress,

b) Training on cognitive skills: It explains the technique of cognitive restructuring as the primary means to control our thoughts.

c) Training on the physiological techniques: learning about breathing and relaxation.

d) Training in control of emotions and aggressiveness. Between the time 2 and time 3, the experimental group received the last session of - behavioural treatment:

e) Training on assertiveness: Training in social skills and techniques to be more assertive.

Results: In Time 1, levels of cortisol of EG were similar to CG. After the first part of cognitive behavioural treatment (Time 2), in EG, there was a significant rise in cortisol levels from Time 1 to Time 2. From T1 to Time 2, there was no significant difference in cortisol levels in CG. However, there was a significant differences between EG and CG. In the EG, there was a 
significant decrease in cortisol levels from Time 2 to Time 3. On the other hand, in CG from Time 2 to Time 3 there was no significant difference in cortisol levels. Therefore, differences between EG and CG disappeared. Between Time 1 and Time 3, there was a significant difference in the cortisol levels in EG.

Conclusion: We conclude the treatment was effective to increase the levels of cortisol in EG.

\title{
Job insecurity, job predictability and mental health: a prospective study of Norwegian employees
}

\author{
Finne, L.B. \& Lau, B. \\ National Institute of Occupational Health, Norway
}

Objectives: Research has shown that job insecurity (see e.g. De Witte, 1999; Ferrie, Shipley, Stansfeld \& Marmot, 2002; Ferrie, Shipley, Marmot, Stansfeld \& Smith, 1998) and job predictability (Lau \& Knardahl, 2008) is related to poor mental health. The aim of this study was to investigate the relation between job insecurity, job predictability and mental health with a prospective design.

Methods: 6361 Norwegian workers were invited twice with a time-span of two years. 2835 $(44,6 \%)$ answered at both baseline and follow-up. 64\% were women and mean age was 45.04 (SD 9.61). Job insecurity was measured by one single item from the QPS-Nordic Questionnaire: 'Are you confident that, in 2 years from now, you will have a job that you consider as attractive as your present job?'. Job predictability was measured with two scales from the QPS-Nordic Questionnaire: Predictability during the next month scale and Predictability of next two years scale. Mental health (psychological distress) was measured by the Hopkins Symptom Check List (HSCL-10).

Results: Pearson's correlations showed that score on HSCL-10 was significantly associated with confidence in having a good job in 2 years at both T1 $(-.23, \mathrm{p}<.01)$ and T2 $(-.23, \mathrm{p}<.01)$, predictability during the next month at both T1 $(-.10, \mathrm{p}<.01)$ and T2 $(-.12, \mathrm{p}<.01)$, and predictability of next two years at T1 $(-.14, \mathrm{p}<.01)$ and at T2 $(-.15, \mathrm{p}<.01)$. A paired sample t-test showed that there was a significant increase in confidence in having a good job in 2 years from baseline $(M=3.6, S D=1.13)$ to follow-up $(M=3.7, S D=1.06) ; t(2083)=-4.52, p<.001$. Furthermore, paired sample t-tests also showed that there were no significant change in either predictability during next month from $\mathrm{T} 1(\mathrm{M}=4.3, \mathrm{SD}=0.70)$ to $\mathrm{T} 2(\mathrm{M}=4.3, \mathrm{SD}=0.70)$; $t(2633)=.18, p>.05$, or predictability of next two years from baseline $(M=3.0, S D=1.12)$ to follow-up $(M=3.1, S D=1.08) ; t(2090)=-1.58, p>.05$. Linear regression analyses showed that a increase in confidence in having a good job in 2 years significantly predicted a decrease in HSCL-10 score $(-.12, p<.001)$, a increase in predictability during the next month significantly predicted a decrease in HSCL-10 score $(-.11, \mathrm{p}<.001)$, and lastly that a increase in predictability of next two years also predicted a decrease in HSCL-10 score $(-.06, \mathrm{p}<.01)$.

Conclusion: Job insecurity and job predictability was associated with mental health in workers. A decrease in insecurity and an increase in predictability at work over time can lead to better mental health for the workers. 


\title{
Strike experiences and the post-strike workplace: The strikers' perspective
}

\author{
Francis, L., Kelloway, K., Donohoe, M. Catano, V.M., \& Gilin, D. \\ Saint Mary's University, Canada
}

Objectives: Although workers are expected to return to 'business as usual' when a labour dispute ends, experiences during the strike may contribute to a negative post-strike work environment. For instance, there may be increased conflict or decreased team work among returning strikers and managers who worked throughout the dispute (e.g., Barnett, 1994; Dannemiller et al., 1982).

Methods: We examined strike experiences and qualities of post-strike workplaces via long interviews with 2 males and 6 females from the telecommunications industry, universities, and government agencies who had been on strike at some point in the previous 5 years. Strike duration ranged from 3 weeks to more than 4 months.

Results: Strike Experiences: Stress, including frustration from feeling unproductive, financial pressure, and rumination about the strike, were commonly discussed. That said, respondents unanimously reported positive aspects to striking, including pride, camaraderie, and relationship building. Regarding people crossing the picket line, respondents understood people crossing because they were bound by corporate policy. But, strikers were particularly buoyed when individuals did not cross as a show of solidarity.

Reports of strike experiences differed between individuals who played active union roles, either currently or in the past, and those who had not. Those involved in unions focused their recounts on the actions of the 'other side'. In contrast those who had not been involved focused on their own feelings about the strike, such as embarrassment.

Post-Strike Experiences: Most respondents were happy to return to work post-strike, but they also felt nervous or overwhelmed. Some observed that strained relationships between union and management characterized a 'chilly' post-strike work climate. Respondents experienced negative changes in their organisational attitudes such as decreased loyalty and less job satisfaction, which were reflected in behaviour such as ignoring certain managers or working to rule. Some reported increased union militancy. Respondents also reported organisational changes, such as stricter enforcement of organisational policies, post-strike. Fully 7 out of 8 respondents said that their workplaces have not returned to its pre-strike state, even though for some the strikes had occurred up to 5 years previous.

Implications and Conclusions: Labour disputes appear to affect strikers' organisational attitudes following the strike. It seems that strikes leave a stamp on the interpersonal relationships in the post-strike work environment. Future research should consider this issue further with studies that include managers' perspectives and with longitudinal investigations that follow individuals' experiences throughout a strike and return to work. 


\title{
Solutions to university faculty work overload: A study of job sharing
}

\author{
${ }^{1}$ Freeman, B. \& ${ }^{2}$ Coll, K. \\ ${ }^{1}$ Northwest Nazarene University, United States of America; ${ }^{2}$ Boise State University, United \\ States of America
}

Objectives: The purpose of this study is to provide a review of work overload and job sharing, especially focused on university faculty; and then investigate the opinions of sampled university department heads (chairs) and college deans regarding the advantages and disadvantages of jobsharing. The American work place has shown a marked preference toward pushing full-time employees to work more and more hours. The full-time American workers are now working the equivalent of one month more per year than Americans 20 years ago, and a full nine weeks (about 320 hours) more per year than their western European counterparts (DeGraaf, 2003; Schneider, 2000; Schor, 1991). Higher education, like business and industry, has been impacted by higher work demands. Tenured faculty positions in higher education may be stereotyped as having a low workload; however, Tommerdahl (1998) found that the average faculty member works 60 clock hours per week, despite tenure. The tension between teaching and research, continuous conflict between the demands of personal/family life and the requirements for professional success produce significant stress (Leinbaugh, Hazler, Bradley, \& Hill, 2003). More flexible work paradigms could help faculty to succeed both at work and at home. One type of flexible work contract that has received little attention in universities is job sharing. A job share is an upgraded part-time contract in which two faculty members are jointly responsible for the workload of one position, and both individuals have rank, advancement opportunities, and benefits consistent with those of a full time faculty member.

Method: This study focused on a national U.S. survey of counselor education department chairs and college of education deans. The participant department chair and deans were randomly selected from U.S. programs and universities. The survey was developed by the authors from the content of an extensive review of job sharing related literature.

Results: Only $11 \%$ of the chairs and $15 \%$ of deans had worked in a department with a job sharing arrangement, and most chairs $(72 \%)$ and deans ( $81 \%$ ) believed that job-sharing would not be desirable for committed professionals. Chairs and deans may have similar perceptions to U.S. employers in general, according to a recent nation-wide study (Spherion, 2005).

Conclusion: Perceptions of those administrators do not support job sharing, and may deter faculty members from seeking job shares or other flexible work paradigms.

\section{Positive and Negative affect: Diverse pathways on emotion management}

\author{
Garrosa. E., Blanco, L. M., Rodriguez-Carvajal, R., Sanz-Vergel, A.I., \& Rodriguez-Muñoz, A. \\ Autonoma University of Madrid, Spain
}

Introduction: In spite of available evidence regarding of diverse pathways to positive and negative affect in adulthood, a scant number of studies systematically assess these differences on emotion 
management process. In this study we examined the relationship between emotional regulation, emotional intensity, emotional stability and sense of coherence in order to predict positive and negative affect.

Methods: Through a self-questionnaire methodology, two hundred and eleven undergraduate students completed the survey, with 20.31 years old of average.

Results: A series of hierarchical regression analyses were performed to explore the influence of these variables in order to predict positive and negative affect. Variables were entered into the regression analysis in four steps. Demographic variables (gender, and age) in Step 1. Reappraisal and Suppression strategies as key mechanisms on emotional regulation were entered in Step 2. Emotional intensity and emotional stability (emotion and impulse control dimensions) were entered in Step 3. Finally, manageability, comprehensibility and meaningfulness, the three dimensions of sense of coherence were entered in Step 4. All independent variables were centered prior to their entry in regression equations, and tests for possible presence of multicollinearity demonstrated no violations of assumptions underlying the regressions. Results of hierarchical regression analyses showed that just emotional intensity $(\beta=.27, \mathrm{p}<.01)$ and meaningfulness in life $(\beta=.45, \mathrm{p}<$ .001) were significant predictors of positive affect. In the first step both reappraisal $(B=.23, p<$ $.01)$ and suppression $(B=-.24, p<.01)$ were significantly related to positive affect. However, its significance diminishes to a non-significant level after the former personality variables were entered into the regression analysis. Testing the possible mediation effects, the results showed that meaningfulness in life fully mediated the relationship between reappraisal and positive affect, and emotional intensity fully mediated the relationship between both reappraisal and suppression strategies and the criterion variable. However, in order to predict levels of negative affect, emotional stability (emotional control dimension: $\beta=-.33, p<.01)$ and comprehensibility $(\beta=-.33, p<$ .01) emerge as significant variables.

Conclusions and Implications: Results are discussed in terms of their implication for emotion management programs in order to handle positive or negative emotions. Discussion also emphasized the need to focus on these personal variables in order to predict levels of positive and negative affect as more than poles apart.

\title{
Post Traumatic Stress Symptoms and Burnout in Doctors and Nurses Suffering Aggressions
}

\author{
'Gascon, S., ${ }^{2}$ Pereira, J.P., ${ }^{2}$ Cunha, M.J., ${ }^{3}$ Santed, M.A., \& ${ }^{1}$ Martinez-Jarreta, B. \\ 'Zaragoza University, Spain; ${ }^{2}$ Insituto Superior de Maia, Portugal; ${ }^{3}$ Universidad Nacional de \\ Educación a Distancia., Spain
}

Aggression against healthcare workers is a problem of important consequences which is becoming a focus of research. However, its possible effects on psychological health have not been studied sufficiently in spite of the fact that they may be of importance even in the absence of physical aggression.

Methods: Sample: cross-sectional multicentre study of 1,845 healthcare workers belonging to rural and urban healthcare centres and hospitals. 
Instruments:

- Record of demographic, family and work data.

- Questionnaire on aggressions (physical, threat, verbal abuse, etc.)

- Record of psychological health, possible psychological symptoms caused by violence

- Maslach Burnout Inventory (MBI)

Results: Eleven percent of healthcare workers reported having suffered at least one episode of physical aggression. Non-physical aggression (insults, threats, coercion) affected $64 \%$ of the sample. These rates were much higher in large hospitals and in Services such as Casualty and Psychiatry.

No statistically significant association was found between physical aggression and psychological symptoms. On the other hand, the association between non-physical violence and anxiety and symptoms of Post-traumatic Stress Syndrome was seen to be statistically significant. Both forms of violence showed an identical negative impact in terms of burnout and work dissatisfaction. Only two out ten healthcare workers felt they were supported by the management or administration in cases of aggression and accusations. This variable was seen to be a modulating factor of the psychological effect of aggression.

The data provide scientific evidence as to the severity of the acts of violence studied, due to both their frequency and their consequences for health. At the same time they demonstrate the necessity for the Administration to pay special attention to these acts and for the elaboration of medical and legal action protocols in cases of aggression which would include psychological support of the victim and the necessary medical and legal counselling.

\title{
Stress factors in the medical profession. Measure of the imbalance in assistance relationships. TEEM questionnaire
}

\author{
${ }^{1}$ Gascon, S., ${ }^{2}$ Pereira, J.P., ${ }^{2}$ Cunha, M.J., ${ }^{3}$ Santed, M.A., \& ${ }^{3}$ Martinez-Jarreta, B. \\ 'Zaragoza University, Spain; ${ }^{2}$ Insituto Superior de Maia, Portugal; ${ }^{3}$ Universidad Nacional de \\ Educación a Distancia.,Spain
}

Healthcare practice supposes a constellation of stressful elements: living together with pain and death, feelings of failure, the sensation of impotence faced by a terminal illness, the pressure provoked by having to attend many patients in too little time, often in small and inadequate spaces without the necessary intimacy, having to attend patients' increasingly troubled demands and to face the increase in official complaints and, especially, increasingly frequent episodes of physical and verbal aggression. This contributes to the constant deterioration, even to the loss of this necessary confidence between the patient and the healthcare professional and has turned this profession into one of the labour areas with greatest psychological morbidity.

This can lead to a loss of quality of healthcare and the relationship with the patient can become the main source of stress for the professional, when, in a normal situation, the relationship with the patient can be one of the most rewarding aspects of the medical professions. Very 
negative consequences can result from the damage to such an essential aspect in the healthcare professional-patient relationship, as is trust, which will damage not only the patient, but also the healthcare professional.

The TEEM questionnaire was designed to measure the degree of imbalance in assistance relationships.

Methods: The development of the TEEM questionnaire was carried out using quantitative methodology. A pilot test was made of the questionnaire with 211 healthcare professionals.

Participants: 1,845 healthcare professionals. Materials:

- $\quad$ TEEM Questionnaire (Gascon \& Martinez Jarreta, 2006)

- $\quad$ MBI. Maslach Burnout Inventory (Maslach, Jackson, Leiter, 1996)

- $\quad$ - List of psychological symptoms (Martinez-Jarreta \& Gascon, 2006)

Results: Alpha coefficient $(\alpha=0.91)$. Statistically significant correlations between the TEEM and the burnout dimensions. By means of COR, TEEM high rates of diagnostic specificity/ sensitivity were shown. By means of a logistical regression analysis, emotional exhaustion (CE), depersonalization (DP) and personal accomplishment (RP) were predicted. A factorial analysis showed that the questionnaire had two dimensions: F1 -fear of being harmed- (30.81\% variance), and F2 -fear of being accused of malpractice (29.36\% variance).

\title{
A quali-quantitative approach for the survey of job stress and the Organisational Development in the organisations
}

\author{
${ }^{1}$ Gattai, A., ${ }^{2}$ Scatolini, E., ${ }^{2}$ Magnani, M., ${ }^{2}$ Mancini, G.A., ${ }^{2}$ Majer, V., \& ${ }^{2}$ Marocci, G. \\ 'Universita' di Verona, Italy; ${ }^{2}$ Università degli Studi di Firenze, Italy
}

The recent law for safety at work (D.Lgs. 81/08) imposes an interesting reflection as regards some psycho-sociological approaches to the health/safety of workers and it allows us to re-think the praxes of participation in the organisations. In fact, the ratio of the legislator of this new law is dealing the safety with a logic of primary prevention, promoting 'safety culture' .

Objectives: In this study we introduce an intervention in a municipal company of the public service. By thinking to the organisation as an interdependent system (organisational and relational variables) we can consider the survey of the Stress Job Correlated factors (SLC) like an Organisational Development (OR) opportunity and, therefore, like a kind of intervention in the organisations. To such aim it has been elaborated a protocol of intervention for the survey of the SLC that allows a psycho-social risk analysis and, above all, a 'participation increased' on the work safety topic.

Methods: Group interviews.

Ad hoc questionnaire for the 'Organisational Wellness' analysis.

Maslach Burnout Inventory-General Survey (Schaufeli, Leiter, Maslach, Jackson, 1996). 
Conclusions: The protection of the safety and health of workers is organisations' duty that surpasses the idea of law obligation and a participatory intervention can facilitate the passage from a culture of the normative implementation to a culture of promotion and spread of safety at work.

\title{
Job attendance in spite of musculoskeletal pain - a qualitative study on the role of work-related sense of coherence
}

\author{
Geving, G., Torp, S., Hagen, S., \& Vinje, H. \\ 1 Hjelp 24 Occupational Health Service, Norway
}

Background: The core of the sense of coherence (SOC) concept is to perceive the world as predictable, manageable and meaningful. According to Antonovsky (1987), workers with a strong SOC have the capacity to thrive and experience a sense of wellbeing despite stressors in life. Musculoskeletal pain is common and is the most important cause of sick leave in Norway. Nevertheless, there are some workers experiencing longstanding musculoskeletal pain who seem to thrive at work and attend work despite their physical impairments.

Objective: The main objective of this study was to investigate what work resources are associated with work-related sense of coherence and job attendance among workers with musculoskeletal pain.

Methods: Qualitative in-depth interviews were performed with five workers experiencing longstanding musculoskeletal pain without being absent from work. All were skilled information and communication technology (ICT) workers employed in the same ICT-enterprise. The study had a descriptive and explorative design, positioned as phenomenological-hermeneutic research. The data were analyzed by use of content analysis inspired by Giorgi (1999).

Results: The analysis revealed that job community, empowerment, and development seem to function as important resources for experiencing SOC at work in general. An enduring pattern of meaningfulness seems particularly important for the workers' job engagement and ultimately for their job attendance. In addition to the factors associated with SOC, all the informants described some kind of work-related self-efficacy as most important for thriving at work, and thereby attending work despite musculoskeletal pain.

Conclusion: A salutogenic process, in which work engagement plays a central role, can be stimulated by meaningful work tasks and thus result in job attendance among workers with musculoskeletal pain. This is in line with the Job Demands-Resources Model (Bakker \& Demerouti, 2006), which holds that work resources have a motivational potential and lead to high work engagement despite high psychological demands. In addition, self-efficacy can play a critical role. To increase work attendance among workers with musculoskeletal pain it seems worthwhile to apply a settings approach for health promotion in which both individual, social, and organisational factors are focused. 


\title{
Comparative analysis of stress factors and their influences on employees in a Romanian telecommunication company
}

\author{
Goian, C. \& Vintila, M. \\ West University of Timisoara, Romania
}

Background: The level of stress has raised significantly in the last years in Romanian telephone communication companies. While until a few years back there was just one state company, now this is privatized and in acute competition with several private ones.

Methods: Employees of two departments of the company the Call Centre (CC) and the Billing Back Office (BBO) were studied comparatively. Each department has 25 persons. BBO consists of 25 employees, aged between 35 and 55 . The CC has a significantly younger average age, 14 out of the 25 subjects are under 30 years of age. The test battery consisted of: Epstein Stress Management Inventory (ESMI-i), Type A personality Test by Friedman and Roseman, Stressful Work Environment Survey and the Symptom Evaluation Scale (SCL-90-R).

Results: The essential difference between the two departments is the direct contact with the beneficiaries of their services at those from CC, while those from BBO do not confront themselves with clients in a direct manner. This can explain the fact that while $32 \%$ of those from BBO perceive their work environment as stressful, $64 \%$, exactly the double number of employees from CC consider their work environment as a source of stress. All subjects scored poor on stress management techniques, which show a constant need for improving these aspects. The subjects from the BBO department have higher perseverance, lower irritability and competitivity levels than those from CC. Somatization proves to be higher in the BBO department, where we found a lower stress level, this might be due to the higher average age of the group.

Conclusions: The fact that the company is in a process of restructuring and there is a fear of becoming unemployed represents a significant source of stress. The employees consider that the measures to stimulate them professionally are missing; they are also affected by the fact that they just get feedback when doing something wrong never get positive feedback. Internal evaluation of the personal from the CC is more often done than that in the BBO department, and this is also perceived as a source of stress for the subjects.

\section{Explaining an Unexpected Interactive Relationship Between Gender Harassment and Union Tolerance for Harassment in Relation to Mental Health: The Role of Conflict}

\author{
Golay, L., Mellor, S., \& Magely, V.J. \\ University of Connecticut, United States of America
}

In 2001, Bulger examined the relationship between gender harassment $(\mathrm{GH})$ and negative mental health in relation to perceived union tolerance for sexual harassment (UTSH). She predicted that when women union members perceived low UTSH, GH would be less positively related to negative mental health outcomes. However, she found the opposite: when low UTSH was 
perceived, $\mathrm{GH}$ was more positively related to negative health outcomes. The present study sought to explain this unexpected finding using cognitive dissonance theory, proposing a threeway interactive relationship between $\mathrm{GH}, \mathrm{UTSH}$, and perceived union support (PUS; defined as perceived care and support from one's union). Assuming that dissonance arousal between conflicting perceptions would contribute to harassment trauma and delayed recovery, we hypothesized when UTSH and PUS conflict, GH and negative mental health would be more positively related. In effect, our hypothesized model suggested that the GH-negative mental health relationship was moderated by UTSH in relation to PUS.

The model was tested with a sample of 45 U.S. women union members based on their responses to validated scales for the variables.

The data were analyzed using a hierarchical multiple regression, where the main effects $(\mathrm{GH}$, UTSH, and PUS) were entered first, followed by the two-way interactions (GH X UTSH, GH X PUS, and UTSH X PUS), and then the three-way interaction (GH X UTSH X PUS). The R-square change for the three-way interaction was significant $(p<.05)$ and explained $7 \%$ of the negative mental health variance above and beyond the main effects and two-way interactions.

Inspection of the simple slopes based on subgroup regressions supported the hypothesized model. Conflict slopes between $\mathrm{GH}$ and negative mental health were positive and strong. The unstandardized coefficient for high UTSH and high PUS was significant $(B=1.530, p<.05)$ and the unstandardized coefficient for low UTSH and low PUS was sizable, but nonsignificant $(\mathrm{B}=$ 1.213 , ns). In contrast, the non-conflict slopes were less positive and weak; the unstandardized coefficient for low UTSH and high PUS was nonsignificant ( $=.414, \mathrm{~ns})$ and the unstandardized coefficient for high UTSH and low PUS approached zero ( $\mathrm{B}=-.053, \mathrm{~ns})$.

The results are discussed in terms of the relationship between dissonance arousal and delayed mental health recovery in relation to gender harassment, with respect to pernicious situations defined by perceptions of union tolerance for harassment in conflict with perceptions of union care and support. Implications for unions are discussed.

\title{
The Effects of Task Interdependence, Workgroup Efficiency, and Perceived Supervisor Support on Safe Social Status: A Moderated Mediation
}

\author{
Golay, L., Reeves, D.W., Magley, V.J., \& Gilson, L.L. \\ University of Connecticut, United States of America
}

As organisations move away from individual-level tasks and become increasingly reliant on workgroups, understanding how this shift affects workplace outcomes becomes paramount. Safe Social Status (SSS; a dimension of Safety Climate emphasizing workgroup safety norms) and Workgroup Efficiency (WGE) are organisational aspects that could be particularly affected by this trend. As jobs become more interdependent, coworkers must rely on each other to adhere to organisational safety policies and ensure a safe working environment. However, the team must remain efficient, and coordinate to complete tasks successfully. Some suggest that pressure to perform at high levels in a co-dependent workgroup reduces safety emphasis. But others propose that because roles that are highly interdependent promote group cohesion and responsibility, they can be used to foster individual accountability, which extends to safety 
behaviours. Therefore, we propose that the increased Task Interdependence (TI) will promote WGE, which will cultivate positive SSS.

The role of the supervisor is also important. A supportive supervisor can encourage team cohesion and cooperation. We suggest that the relationship between TI and WGE will be dependent upon Perceived Supervisor Support (PSS). As such, team members will be more inclined to promote responsible behaviours (safety compliance) to other co-workers.

In sum, we hypothesize that increased TI will lead to greater WGE, which will lead to higher SSS. This indirect relationship between TI and SSS through WGE will be dependent upon PSS, such that the relationship will only exist when PSS is high.

Data were collected from a state agency in the U.S. $(N=724)$. All measures came from published, validated scales, except WGE, which was created for this study. The conditional indirect effect was tested using Preacher's (2007) moderated-mediation macro for SPSS.

Results showed an indirect effect of TI on SSS through WGE, but only when PSS is high. As PSS varies from one standard deviation above the mean to one below, the indirect effect drops to non-significance $(p<.001, p=.34$, respectively).

The cost of injuries due to non-adherence to safety regulations is significant, so it is important to examine how organisations can increase employees' adherence to safety policies. We showed that TI has an indirect effect on SSS through WGE. Notably, this effect exists only when employees have high PSS. Therefore, organisations should stress to supervisors that being supportive of subordinates, even when not directly stressing safety, can lead to increase safety adherence. Further analyses will explore the multi-level nature of the constructs.

\title{
Stress sources in police officers
}

\author{
Goncalves, S., Neves, J., \& Morin, E. \\ CIS/ ISCTE-IUL, Portugal
}

Professional stress has been motive of concern and it has been studied among several professional groups, such as teachers, medical doctors and nurses (Marques Pinto, 2000). In police forces, there are a reduced number of studies. However, they suggest the existence of high levels of stress within these professionals due to the specific nature of their functions (Violanti, 1983; Brown \& Campbell, 1994). The knowledge of the specific sources of stress of this professional group could support interventions towards better work conditions. Having this goal in mind, we have developed the Scale of Stress Sources in Police Forces (EFSCP). In this article, we present the scale and their psychometric properties.

Results obtained from a sample of 355 Portuguese policemen indicate adequate internal consistency, discrimination and validity of the EFSCP. Factor analysis of EFSCP reveals the existence of seven sources of stress: Internal management, operational tasks, work-family balance, demands of the work environment, social image, role ambiguity, and interpersonal relationship. 


\title{
Longitudinal study with Job Demands-Resources Model in police context: Ongoing study
}

\author{
Goncalves, S., Neves, J., \& Morin, E. \\ CIS/ ISCTE-IUL, Portugal
}

In last decades we have assisted changes in the work environment. These changes have had consequences on people's lives and organisations, for instance related with work stress. It is now accepted that work and the conditions under which it is carried out have an impact on physical and mental health (Kasl, 1992). From both research and practical perspectives, it is important to consider how organisations can alleviate any negative outcomes that may be associated with demanding work. The present study has as theoretical background the recent model of Job Demands-Resources Model, founded on Karasek's (1979) demands-control model. The studies with the job demands-resource model suggests that the consequences of demanding work conditions can be offset by available resources, including job control, job efficacy or personality characteristics. This study goal is to test the Job Demands-Resources Model in a longitudinal study. The sample comprises of 300 police officers. Results appear to support this model in this context.

\section{Analysis of a Subjective Mental Workload Scale and the influence of mental work load in occupational health}

\author{
Hernandez-Fernaud, E., Diaz-Cabrera, D., \& Rolo-Gonzalez, G. \\ University of La Laguna, Spain
}

The relevance of the concept of mental workload is determined for the cognitive demands required by a broad number of jobs and their associated influence on physical and psychological well-being of workers. Workload is influenced by the interaction between the level of task requirements and the physical and mental effort developed by workers in order to perform the task. This concept is related to objective task requirements, subjective worker perceptions and limitations in the information processing capacity.

The subjective multidimensional methods are the evaluation procedures more generally used because of low intrusiveness and implementation requirements, as well as the good level of participants' acceptance and its low cost. The aim of this research is to develop a subjective evaluation scale of mental workload (ESCAM), and evaluate its empirical structure.

The sample consisted of 875 workers that were working in different jobs in a variety of enterprises. The average age of participants was 34 years $(s d=10.46$; range: $18-61) .39 .7 \%$ were men and $60.3 \%$ were women.

A multidimensional scale to evaluate the subjective mental workload was developed. The scale is composed of 31 items that would be ranked from one to five points on a Likert scale. 
In the factor analysis we obtained a coefficient of sampling adequacy (KMO) of 0.860 and $\ddot{i}$ $£ 2(465)=7712.658$, pâ\%o 0.001 with Bartlett's test of sphericity. The solution obtained grouped the items into nine factors that explained $59.07 \%$ of variance. However, the factors 7,8 and 9, explaining $3.58 \%, 3.36 \%$ and $3.23 \%$ of variance, respectively, were not considered as they show low internal consistency (Russel, 2002). Factors obtained were Cognitive requirements and information complexity, Temporal organisation of work, Health consequences for workers, Task characteristics, Work rate and External feedback.

The empirical structure obtained is similar to the theoretical dimensions used for the scale development. However, some of the theoretical dimensions are grouped together in one factor only whereas others dimensions are divided into two different factors. Cognitive requirements and information complexity, Temporal organisation of work, Task characteristics and External feedback factors contribute to significantly explain Health consequences for workers.

\title{
Democratic Participation in NPO as a Factor of Organisational Wellbeing. First Research Results between Italy and Mexico
}

\author{
Hindrichs, I. \& Converso, D. \\ Università degli Studi di Torino, Italy
}

Objectives: Several researches and theories suggest that democratic leadership and participation in organisational decision-making and governance increase a sense of affiliation and satisfaction, and represent a protecting factor against organisational disease (Harris, Kacmar, 2005; Zotti et al., 2008). Democratic governance is a key-issue in Non-profit-Organisations (NPO): it represents a core value and defines the identity and quality of work-life and the outcome of NPO (Lombardi et al. 1999; Converso, Piccardo, 2003). However we can also observe difficulties in translating democratic values in organisational praxis, especially after the pioneer years of NPO (Pagliarani, 1973; Acler, 1995; Hindrichs, 2008). Regarding our hypothesis, democratic participation can be improved by developing a double empowerment strategy (Converso, Piccardo, 2003; Piccardo, Martini, 2004; Converso, Hindrichs, 2009): in order to sustain the empowerment of 'others', people working in this kind of organisations need to sustain their own empowerment both at a psychological and at an organisational level.

Methods: A questionnaire was purposely developed on the bases of a former qualitative study between NPO in Mexico and Italy (Hindrichs, 2008) and the work of Forcadell (2005) and Avolio and Bass (1992, 2004), in order to measure the perceived democratic participation and leadership in NPO. In a pilot-study we submitted the questionnaire to the workers of two Mexican $(N=100)$ and three Italian $(N=150)$ NPO. In one of the Italian NPO $(N=100)$, we also administered some subscales of our instrument to evaluate psychosocial risks (see abstract Converso, Gattino, Loera, Viotti, Hindrichs) in order to verify the link between democratic governance and organisational wellbeing.

Results: The first results seem to confirm the hypothesis of a growing lack of participation in the organisations here examined: significant differences were found between younger and newer members and founders of organisations in affiliation and psychological empowerment, and a minor perception of democratic values and processes in older organisations that experienced a 
kind of institutionalization. Furthermore, whoever perceives less possibility and willingness to participate seems to be exposed to higher psychosocial risks, especially burnout syndrome.

Implications: We shared the results with the research-participants in data-restitution-sessions focused on possible implications of minor democratic participation, finding generally the desire to invest in intervention that can improve the organisational affiliation and the worker's empowerment in order to contrast psychosocial risks in a work that requires relationship skills and commitment to a social mission.

Conclusions: We are aware of the limits of our first results caused by the low number of respondents. Indeed, to validate our questionnaire data-collection improvements are required. However, a comparison with research-participants confirmed our hypothesis of the need of double empowerment. Our aim is to develop tools for action-research that will facilitate interventions to overcome the participation-dilemma, not only as an issue of political value, but also as a tool for developing organisational wellbeing.

\title{
Does ethical organisational culture associate with managers' occupational well- being?
}

\author{
${ }^{1}$ Huhtala, M., ${ }^{2}$ Kaptein, M., ${ }^{1}$ Mauno, S., ${ }^{1}$ Hyvonen, K., \& ${ }^{1}$ Feldt, T. \\ ${ }^{1}$ University of Jyväskylän, Finland; ${ }^{2}$ RSM Erasmus University, Finland
}

Objectives: The aim of the present study was to test the structural validity of the 58-item Corporate Ethical Virtues-scale (CEV; Kaptein, 2008) in a large-scale sample of Finnish managers. The CEV is designed to include eight dimensions: clarity (10 items), congruency of supervisors (6 items), congruency of management (4 items), feasibility (6 items), supportability (6 items), transparency (7 items), discussability (10 items) and sanctionability (9 items). The second aim was to examine the associations between ethical organisational culture and managers' occupational well-being indicated by burnout and work engagement.

Methods: The participants consisted of technical and commercial managers who responded to a postal questionnaire study in Autumn 2009. The sample $(n=3000)$ was randomly selected from the membership registers of two Finnish national labour unions (The Finnish Association of Business School Graduates and The Finnish Association of Graduate Engineers). The respondents represented top and middle management. In addition to the CEV scale, the questionnaire included the nine item Utrecht Engagement Scale (UWES-9; Schaufeli, Bakker, \& Salanova, 2006) and the items of emotional exhaustion and cynicism from Bergen Burnout Indicator 15 (BBI-15; Tekijä, Näätänen, Aro, Matthiesen, \& Salmela-Aro, 2003). Once data collection is completed (currently in process), we will perform a confirmatory factor analysis with the Mplus 5.1 program (Muthén \& Muthén, 2007) to test the hypothesized eight-factor structure of the CEV scale. Further analyses will be conducted with Structural Equation Modelling to investigate the associations between the dimensions of ethical culture and occupational well-being.

Conclusions: The validation of the CEV-questionnaire (Kaptein, 2008) in the Finnish context enables defining the ethical culture at an organisational level. This is an important contribution to the research of organisational culture and its influences. 


\title{
Burnout and work engagement during the transition from education into work life
}

\author{
Hultell, D. \& Gustavsson, J.P. \\ Karolinska Institutet, Sweden
}

The main objective was to study which factors that affect burnout and work engagement during the transition from education to work life for beginning teachers. However, in order to do so an instrument which measures the state mood of burnout and work engagement first needed to be developed and evaluated. The first objective was therefore to evaluate the psychometric properties of the Scale of Work Engagement and Burnout (SWEBO). The second objective was to study how individual characteristics, the educational context, and the work context predicted levels of burnout and work engagement during the transition period from higher education into work life.

When evaluating the SWEBO a national sample consisting of over 2000 newly graduated Swedish nurses and teachers was used. Measurement models of both burnout and work engagement were evaluated using confirmatory factor analysis (CFA). Both burnout and work engagement were also tested for measurement invariance across occupation and age. The fit of the measurement model of burnout was satisfactory and it was invariant across both occupation and age. The measurement model of work engagement as initially defined did not fit the data satisfactorily, and the model was therefore revised and reanalyzed. The revised model had a satisfactory fit and was invariant across occupation. Analysis of its invariance across age, however, gave ambiguous results that were difficult to interpret. The results show that the SWEBO presents a psychometrically sound alternative for measuring the state mood of burnout and work engagement.

When studying the transition period a national sample comprising over 1200 beginning teachers was used. The data was analyzed using hierarchical regression analyses, one with burnout as the outcome variable and a second with work engagement as the outcome variables. The results show that job demands and job resources accounted for the largest amount of explained variance in both burnout and work engagement; and unmet expectations and mastery of skills were the predictors with the greatest relative influence on both burnout and work engagement. Implications of the results are that student teachers should be given a more comprehensive picture of the actual conditions of work life in order to reduce the negative impact of a reality shock. Furthermore, preparing the student teachers in work related tasks will likely result in higher self efficacy and feelings of competence. The results shine new light on the difficulties of transition from higher education into work life.

\section{Leadership, Work Environment, Health and Effectiveness - Longitudinal Study}

\author{
Hytter, A.
}

\author{
Linnaeus University, Sweden
}

Objectives: If you search for literature on work environment and health you will end up with a huge number of studies. When adding leadership to the search, the number of studies is however quickly reduced. The lack of knowledge in this intersection of areas becomes even 
more evident if you also add the concept effectiveness to the search. This study was conceived in order to create knowledge that will help explaining this area.

The objective of the study is to explore and explain the possible links between Leadership, Work Environment, Health and Effectiveness.

Methods: Two instruments, one on work environment and health and one on managerial behaviour have been deployed in four Swedish public organisations. 2600 employees and 153 managers returned the surveys. At the time of writing this abstract (December 2009), the second data collection has just been finished in two of the organisations. The two other organisations will follow in late Spring and Autumn 2010

Furthermore the same instruments will be deployed in a private national company in the construction industry in Spring 2010. Negotiations are also under way with a national cooperative organisation concerning their participation in the study.

The work environment and health instrument has been based on the following studies/ instruments: COPSOQ, SLOSH, and New Working Life.

In order to avoid the risk of limiting the study to too few aspects of managerial behaviour, the second instrument covers nineteen different areas of managers' behaviour. This includes classical ones' such as feedback, relation orientation, power orientation, etc. It also includes unconventional ones' such as integrity and narcissism.

Results: The study is still work in progress, although reports based on the study are starting to come out. Focus so far has been on how managers' behaviour influences employee health. Furthermore a report is now being prepared on managers' health. Work to come: The next phase includes doing the second data collection within the two remaining public organisations, together with the first data collection from the private company. Furthermore it includes to collect data on effectiveness and to perform longitudinal analysis on the first part of the data.

\title{
Nanotechnologies at the Workplaces: Social Impact and Risk Perception
}

\author{
lavicoli, S., Boccuni, F., \& Ronchetti, M. \\ National Institute for Occupational Safety and Prevention (ISPESL), Italy
}

Objectives: Nanotechnologies offer manifold opportunities in the development of materials, structures and devices with new properties and features; great expectations for this development are still present in various market and work sectors. At the same time, the international scientific community is focusing its attention on the potential risks for the environment and the human health posed by nanotechnologies, with particular focus on occupational exposure. Main objective of the present study is to analyze the impact of nanotechnologies on the society and the world of work, taking into account the risk perception of this emerging issue.

Methods: The main fields of nanotechnologies application have been examined in order to determine their current degree of diffusion in the world of work and to suppose the possible future scenarios. Furthermore, some studies on risk perception related to nanotechnology 
have been analysed with the aim of defining the ways in which population interacts with this technology also considering the experiences gained from the occupational medicine in the management of emerging risks.

Results: The analysis conducted on population risk perception also related to the impact of nanotechnologies reveals both a general enthusiasm about the potential of nanotechnologies medical and commercial applications and a low level of knowledge with subsequent low levels of the related risk perception. Furthermore, some well-known cases/examples of risk management provide useful information on how society reacted in the past to technological innovations (asbestos, biotechnologies and mobile phones). These information can be assimilated to the current development conditions of nanotechnologies in the changing word of work.

Implications: Public, research and scientific organisations are working hard to ensure a responsible nanotechnology development; there is a widespread agreement about the need for new regulatory instruments controlling the production and diffusion of nanotechnological products and shared strategies for risk assessment and management taking into account also the population risk perception degree in order to prevent and reduce its negative impact on users' and workers' health and to improve the quality of communication among the involved stakeholders.

Conclusions: In this view some criticisms emerged, connected both with intrinsic features of nanotechnology risk which can influence the perception of population as well as with the social impact of nanotechnologies. It is therefore necessary to consider and develop these aspects in order to integrate and complete the process of nanotechnologies risk management.

\title{
Assessing impact of work organisation on nursing: migration, outsourcing and early retirement
}

\author{
lavicoli, S., Boccuni, F., Natali, E., Petyx, C., Ronchetti, M., \& Valenti, A. \\ National Institute for Occupational Safety and Prevention (ISPESL), Italy
}

Objectives: The aim of the present study is to analyze the changes in the nursing sector and its impact on occupational safety and health $(\mathrm{OSH})$ with a view to identifying 'gaps and needs' for nurses in the OSH area.

Methods: We analyzed the scientific literature and some studies on the subject (eg. The European NEXT - Study) to investigate the relation between early retirement, outsourcing and migration. Also, a specific questionnaire has been created and will be administered to Italian nurses in order to study the nursing profession and to identify 'gaps and needs' in OSH for nursing sector.

Results: In the last few decades, the global labour market has been characterized by huge changes in the organisation of work: Workforce is rapidly changing, particularly in nursing sector: nursing shortage is reported to be an increasing problem worldwide due mainly to early retirement from work and insufficient turnover of nurses coming from educational programmes. In particular, the number of nurses in Italy (5,4/1000 inhabitants) is much lower than the average as predicted by the Organisation for Economic Co-operation and Development (OECD) for industrial countries (6,9/1000 inhabitants). Work flexibility by acquiring professionals through 
outsourcing (social cooperatives and work supply) and facilitation of migration flow of foreign nurses have been introduced to tackle the problem. The presence of foreign nurses in Italian hospitals has tripled in the period 2002-2005.

Implications: The effectiveness of health systems and the quality of health services depend on the efficiency of the professionals which is derived from their know-how, skills and motivation. The growing shortage of nurses increases nurse workload, stress at work and clinical risk, reducing working satisfaction and the quality of patient care. According to the National Insurance Institute for Employment Injuries (INAIL), the health sector has been affected, in the period 2001-2005, by a $21 \%$ increase in accidents, particularly in nursing sector.

Conclusions: It is necessary to identify key priorities for developing strategies and tools for better work organisation in nursing sector with a special focus on migration. In fact, the study shows a higher vulnerability of migrated nurses and nurses hired through cooperatives. Therefore urgent measures are needed to promote their integration and assess educational and training needs in the field of health and safety at work.

\title{
Work/family conflict and work stress outcomes in working shifts nurses
}

\author{
Iskra-Golec, I. \\ Jagiellonian University, Poland
}

Objectives: There is a shortage of research on work/family conflict in shiftworking populations. However, work and non-work periods in shiftworking are out of step with the normal social environment what often makes people working shifts unable to fulfill their family commitments. The aim of this study was to compare perception of work background variables and work stress outcomes in nurses different with regard to the amount of work-family conflict.

Methods: Two hundred and ninety three shiftowrking nurses aged between 26 and 58 years ( mean $=39.36, \mathrm{SD}=9.07$ ) employed in a few hospitals of a big town participated in the study. They completed Survey of Work and Time and (SWAT) to measure on-the-job and off-the-job stressors, stress, stress outcomes, and work/family conflict as well as Maslach Burnout Inventory to measure professional burnout. The nurses were divided into three groups according to their answers to the questions concerning work/family conflict of SWAT (low - (LWFC) intermediate(IWFC) and high (HWFC) work/family conflict). The groups were compared with regard to their scores in $\mathrm{MBI}$ scales and work stress outcomes using one way analyses of variance ANOVA.

Results: The number of days of bad mental health, of feeling depressed, worried and tired was significantly higher in the group of HWFC when compared to the other groups. The HWFC nurses assessed significantly higher their work demands and significantly lower social support at work than the nurses of the other groups. They also felt more tired after a work day and had lower job satisfaction and higher emotional exhaustion than nurses of LWFC and IWFC. The nurses of HWFC group were statistically significantly less satisfied than the other groups with the time left for off-the-job activities. The nurses of HWFC group when compared to nurses of IWFC and LWFC groups worked more hours per week and significantly more hours of additional job per week. The nurses did not differ significantly with regard to the age and shiftwork experience, and family situation. 
Implications: The results are in agreement with the studies among day workers showing negative effect of work-family conflict on health (especially mental and emotional) and well-being. The results are also in line with the other studies findings showing a relationship between burnout and work/family conflict.

Conlusions: Higher work family conflict is accompanied by higher indices of work stress outcomes in shiftworking nurses. Both, work family conflict and work stress outcomes are higher in the groups of nurses working more hours per week.

\title{
Knowledge Management Systems for promoting Healthy Organisations
}

\author{
Isla-Diaz, R., Hernandez-Fernaud, E., Diaz-Cabrera, D., Ramos-Sapena, Y., \& Casenave, S. \\ University of La Laguna, Spain
}

This poster explores the use of Knowledge Management System (KMS) for promoting healthy organisations. Most people consider good health to be a key factor when defining quality of life. Work is also a crucial factor for psychological wellbeing, since it contributes to a sense of identity and social benefit, and occupies a large part of a person's time. Therefore, working conditions and their impact on health play an important role in quality of life. Healthy organisations are founded on explicit organisational and job design practices, and on specific relational dynamics between all members. In particular, they are based on aspects of the working environment that encourage the attainment of organisational objectives, which decrease the job demands resulting from physiological and/or psychosocial costs, and which stimulate personal growth, learning, and professional development. Healthy organisations must accept that organisational health falls within their area of action and responsibility. Accordingly, optimum organisational health management calls for a holistic, comprehensive approach to processing information about health in organisations, so that proactive intervention and organisational learning is fostered. Occupational health management is the optimization of organisational processes and structures that have a direct and indirect impact on workers' health and eventually influence organisational results. Dialogue and communication between all organisational units is essential for organisational health management and fosters the capacity to be a healthy organisation that learns. In this respect, knowledge management systems play a central role. These systems facilitate the creation, identification, acquisition, development, preservation, dissemination and, finally, the utilization of the various facets of healthcare knowledge.

This poster centers on social learning or people-driven initiatives. In our opinion, successful knowledge management depends on a suitable socially-based or people-driven KMS. However, the social aspects of evaluation processes and organisational change, which may be required for KMS implementation, are often overlooked, and the attention mainly falls on technological aspects. At this respect, this work describes the main characteristics in the implementation of a successful KMS, centered on the social requirements. 


\title{
Embitterment at Work and Withdrawal
}

\author{
Jacobshagen, N., Rutz, J., Elfering, A., \& Semmer, N.K. \\ University of Bern, Switzerland
}

Objectives: Justice Theory postulates that perceived unfairness at work evokes negative feelings (Kohler \& Matthieu, 1993), and that these feelings lead to withdrawal (absenteeism, intention to quit and reduced commitment (Geurts, Schaufeli, \& Rutte, 1999). Embitterment in general can be considered as negative affect and is associated with a burning sense of unfairness or injustice, a protesting feeling of having been wronged without cause, or at least without sufficient cause (Alexander, 1960, p. 34). The question remains if unfairness also provokes embittered cognitions and behaviours of employees which may also trigger the withdrawal actions. Our goal was to construe a scale measuring the cognitive and behaviour components of work-related embitterment and test its influence on the relationships between stressors and withdrawal.

Methods: Questionnaire study; 147 Swiss service employees filled in a questionnaire. The scale work-related embitterment contained 10 items measuring cognitive embitterment (One mistake - and years of work do not count anymore) as well as behavioural embitterment (I just do what I must do). Two stressors were assessed: a) Illegitimate tasks (Semmer, Jacobshagen, Meier, \& Elfering, 2007; asks to what extent participants have to carry out tasks they consider unreasonable or unnecessary), b) effort-reward-imbalance (Van Yperen, 1996). Three withdrawal constructs were assessed: a) Absenteeism (one item), b) affective commitment (seven items, Allan \& Meier, 1990), and c) intention to quit (one item - Baillod, 1992). Correlation and regression analysis as well as path analysis using AMOS were employed to test the relationships.

Results: After deleting one item the scale measuring work-related embitterment showed a good internal consistency $(\hat{I} \pm=.91)$. Also, work-related embitterment was positively correlated to illegitimate tasks and effort-reward-imbalance as well as to the three outcomes. Mediation analyses revealed that work-related embitterment fully mediated the relationships between illegitimate tasks and all three outcomes and the relationship between effort-reward imbalance and commitment. A final path analysis confirmed the mediating function of work-related embitterment between illegitimate tasks and the three outcomes $(\mathrm{GFI}=.98, \mathrm{AGFI}=.92, \mathrm{CFI}=.92, \mathrm{TLI}=.89)$.

Conclusions: The results confirm our proposition that cognitive and behavioural components of embitterment play a significant role. Illegitimate task refer to tasks that violate norms about what can reasonably be expected from a person in a given job and, therefore, can be considered as a more specific form of injustice than the more general effort-reward imbalance (Semmer et al., 2007). Both were associated with embitterment and, through embitterment, with absenteeism, affective commitment, and intention to quit. Work-related embitterment, therefore, is a promising concept. The associations found, though, need further confirmation in longitudinal studies. 


\title{
Promoting Safety Performance
}

\author{
Jones, C., Cox, T., \& Griffiths, A. \\ University of Nottingham, United Kingdom
}

Research has established the nature and structure of organisational safety cultures and their role in determining safety performance. A positive and strong safety culture is necessary for good safety performance.

The purpose of the study is to develop holistic approach to the promotion of safety within a high hazard industry. The organisation is a construction and engineering organisation with employees working at height. The intervention strategy will combine; a bottom up approach focussed on behavioural safety interventions with foremen, a middle tier approach focussed on managers and their awareness and knowledge of safety, a top down approach focussed on culture change through senior and middle managers.

This study utilises an evidence-based and participatory problem solving approach. This paper reports on the first stage of the holistic process, understanding the factors that influence performance and error causes and mechanisms both at an organisational and individual level. This has been achieved through analysing accident, incident and near miss data and conducting interviews with those employees on site who work at height. The implications of the results are that by utilising an evidence based participatory approach and through gaining a more in-depth understanding of the factors that influence safety performance any solutions developed to promote safety performance can be more accurately tailored to the needs of this high hazard organisation.

\section{Longitudinal effects in the treatment of occupational stress: Controlled evaluation of an inpatient group-therapy program (7-years follow-up)}

\author{
${ }^{1}$ Koch, S., ${ }^{2}$ Schaaf, V., \& ${ }^{1}$ Hillert, A. \\ ${ }^{1}$ Roseneck Center of Behavioural Medicine, Prien, Germany; ${ }^{2}$ Technical University of \\ Braunschweig, Germany
}

Objectives: Traditionally the importance of occupational stress in the development and maintenance of psychological disorders was of minor importance. Only recently there is an increasing interest in job-related interventions for clinical target-groups. Nevertheless little is known about long-term effects of occupational stress-interventions, especially for high-risk populations (tertiary prevention). In addition to surveys up to twelve months post-treatment (Koch et al., 2007), the aim of this longitudinal study was to investigate the development of coping with occupational stress (7-years follow-up, $n=340$ ) in a clinical high-risk group. As a second objective, differential effects of an eight-session inpatient group-intervention in addition to treatment-as-usual (TAU) $(n=131)$ were compared to patients in TAU only $(n=209)$.

Methods: At admission participants were an average of 37 years old (58\% females), they were employed predominantly full-time (64\%) or part-time (13\%), 19\% were unemployed. Patients had an average of 71 days of inability-to-work per year. The objective work situation, subjective 
estimates of work stressors and of individual coping skills were assessed. The manualized intervention included: motivation and goal-setting for changes in stress-relevant coping behaviour, job-specific social skills and coping with social conflicts at work, coping with acute and chronic stress at work and job perspectives, e.g. issues of successful job applications.

Results: At 7 years follow-up (return rate: 61\%) inability-to-work dropped significantly (39 days per year). 20\% were retired early. Overall longitudinal outcomes in psychosomatic health (SCL90-R), occupational coping and well-being with their work-situations attained positive medium to high effect sizes. There were no differential effects between the additional job-specific groupprogram (intervention) and treatment-as-usual (controls).

Conclusions: Results confirm that employees with mental disorders have a clearly elevated risk to drop out of active working live through early retirement. Since continuously working patients of both treatment conditions equally reported moderate to high long-term effects in health and coping with occupational stress, only little specific effects of an additional job-related groupprogram could be detected. The present study will help to specify occupational perspectives of patients with mental disorders and to design job-specific prevention programs. Further investigations will focus on variables that may allow to predict a successful reintegration into work or early retirement respectively.

\title{
Literature Review: Psychosocial Aspects of Injury-Related Occupational Leave and Implications for the Military Context
}

\author{
Lee, J.E.C.
}

\section{Defence Research and Development Canada \& Canadian Forces Health Services Group}

Background: Non-battle injuries represent a significant cause of morbidity among military personnel, and often result in activity restrictions and lost work days. There is clear interest in identifying factors that facilitate or impede return to work among injured personnel excused from duty. In the civilian sector, there is a growing body of research suggesting that return to work is related to psychosocial factors. The aim of this literature review was to determine which psychosocial factors have been examined in relation to injury-related occupational leave, evaluate the weight of the evidence, identify theoretical frameworks that have been applied in this area, and discuss potential implications for the military context.

Method: A literature review was carried out of peer-reviewed French- or English-language articles pertaining to physical injury-related occupational leave. Only empirical studies or reviews of empirical studies that examined psychosocial correlates or predictors were reviewed. This strategy yielded a sample of 71 studies, among which 11 were review papers.

Results: Psychosocial variables examined in empirical studies fell in the broader domains of i) health and well-being (mental health, perceived health/wellbeing, stress), ii) injury and/or painrelated beliefs (pain catastrophizing, recovery expectations, fear avoidance, perceived pain and disability, causal attributions), iii) intrapersonal factors (personality, self-efficacy, coping), iv) interpersonal factors (social support), v) occupational stress (fear of job loss, job stress, barriers in the workplace), vi) treatment (perception of injury-related care), and vii) compensation issues (compensation receipt, involvement in litigation). Evidence was most reliable for recovery 
expectations, fear avoidance, perceived pain and disability as correlates/predictors of injuryrelated occupational leave. Mental health and occupational stress were also significant correlates/ predictors, albeit less reliable. Theoretical frameworks most commonly applied included the biopsychosocial model, as well as various models of disability or occupational stress. None of the studies in the study sample focused on military personnel.

Conclusion: Injury-related occupational leave and return to work appear to be strongly linked to psychosocial factors, such as those reflecting injury and/or pain-related beliefs. Occupational stress and mental health may also be involved, although their relationship may be mediated or moderated by these more proximal factors. Multifactorial frameworks of occupational disability that account for biomedical, occupational, and psychological factors and their interactions show promise for understanding injury-related occupational leave and return to work in the civilian sector. However, more research on military personnel is needed to determine if similar factors or frameworks are applicable in the military context.

\title{
Risks and hazards affecting Health \& Safety Advisors
}

\author{
Leitão Alexandre, S. \& Greiner, B.A. \\ Department of Epidemiology \& Public health, Ireland
}

Objectives: The research on psychosocial risk factors affecting health and safety at work has shown great progress in the last decades. There is almost no research on the health and safety work aspects of health and safety professionals. Whereas previous research with a number of occupational groups showed associations between safety climate and health and safety indicators, it is hypothesised that the safety climate in an organisation is an important determinant of the health and well being of Health and Safety (H\&S) Advisors. The study focussed on describing the main risks and hazards (physical or psychosocial) affecting H\&S Advisers. It explored the key obstacles and factors impacting on these professionals and considered the influence of Safety Climate (SC) on their work, specifically on work demands, control, social support, and on their health and wellbeing $(\mathrm{H} \& W)$.

Methods: A new conceptual model was proposed to investigate the association between SC on either demands, control, or social support and on $\mathrm{H} \& \mathrm{~W}$ of the H\&S Advisers.

Qualitative interviews were carried out to inform the design of a quantitative questionnaire. Scales from the Copenhagen Psychosocial Questionnaire (COPSOQ), the safety climate toolkit (Cox \& Chayne, 2000) and Health Perceptions Questionnaires were also added to the survey instrument. Data was collected through a survey with $45 \mathrm{H} \& \mathrm{~S}$ Advisers from local authorities in 18 counties in Ireland.

Results: Key Physical Hazards were VDU use, extensive driving and work posture. The main psychosocial hazards were excessive task variety and quantitative and cognitive demands. Bivariate correlations supported the Conceptual Model and associations were found between: Safety Climate and H\&W ( $r=0,391$; sig $=0,010)$; Safety Climate and Demands $(r=-0,304$; $\operatorname{sig}=0,056)$; Safety Climate and Control $(r=0,600$; sig $=0,000)$; Safety Climate and Social Support ( $r=0,477$; sig $=0,001)$; Demands and H\&W ( $r=-0,241$; sig $=0,124)$; Control and 
$H \& W(r=0,562 ; \operatorname{sig}=0,000)$ and; Social Support and $H \& W(r=0,314 ; \operatorname{sig}=0,038)$.

Implications: The results suggest that a poor safety climate may be considered as a psychosocial health hazard for h\&s professionals. One explanation could be that a poor safety climate makes their professional task more difficult as it affects demands, control and social support.

Conclusions: Ultimately, a strong association between Safety Climate and Health \& Wellbeing was identified. This suggests that in organisations with a good Safety Climate, the Health \& Wellbeing of the H\&S Advisers will show positive levels as well.

\title{
Shiftwork and mental health - a cross sectional study of shiftworkers in the Norwegian petroleum sector
}

\author{
Ljoså, C.H \\ National Institute of Occupational Health, Norway
}

Background: There are several factors that affect the psychological wellbeing of a worker. First there are individual characteristics, like gender, age and marital status. Further there are individual differences, like personality, locus of control and how the workers cope. Finally there are several organisational factors, like job demand, control, support and reward that may affect the workers mental health. Work schedules and shiftwork may have negative impact on employees mental health. Several studies conclude that there are unfavorable psychological effect associated with extended workhours, shiftwork and nightwork.

Objectives: The research referred here is mainly done in health care and service industry, and cannot be generalized to the petroleum industry. Consequently, we wish to examine how different shift arrangements in a large Norwegian petroleum company influence mental health. Secondly, we wish to examine whether individual differences, locus of control and work factors influence this relationship.

Methods: All 3038 employees of a large Norwegian oil and gas company, who worked onshore and offshore during a two-week period in August 2006, were invited to participate in the study. The research design used a web-based questionnaire. Complete questionnaires were received from 1697 employees, yielding a response rate of 55.9\%.

Results: Unianova analysis show that there are some differences in the association between mental health and shift schedules. Employees working offshore (day and night) report the highest scores on mental distress. These scores differ significantly from the scores reported by employees working daytime offshore.

Further there are individual differences. The women report fewer decisional demands and less control than the men. On the other hand, they report receiving more social support than the men. The youngest employees report more social support than the employees over forty, and less quantitative demands than all the other age groups. The married/cohabitants report more quantitative demands, more decisional demands, and less social support than the single employees. 
Results from a regression analysis shows that three factors seem to be importance regarding mental health. Quantitative demands and social support are significantly associated with mental health when controlled for gender, age, marital status, shift arrangements, organisational factors and neuroticism. Further, neuroticism is highly correlated with mental health, also when controlled for other factors.

Preliminary conclusions: When studying the effect of shiftwork on mental health, it is important to take individual factors and work factors into account.

\title{
Moderated effects of Job Insecurity on Work Engagement and Distress
}

\author{
${ }^{1}$ Lo Presti, A., ${ }^{2}$ Nonnis, $M$. \\ ${ }^{1}$ Seconda Università degli studi di Napoli, Italy; ${ }^{2}$ Università degli studi di Cagliari, Italy
}

Objectives: On the basis of the literature, the examination of potential moderators of the effects of job insecurity has been carried out partially and unrehearsedly. This study was concerned about examining a wide array of potential moderated effects in the relation between job insecurity and outcomes such work engagement and distress, including the role of either biographical variables (gender, marital and occupational status, kind of contract, unionization) and continuous ones (perceived employability, career confidence and readiness).

Methods: Recurring to a self report questionnaire including different variables on Likert scale response sets, data were collected on a sample of 536 Italian employees working inside different organisations. Analyses were carried out recurring to hierarchical multiple regressions to test for two-way interactions between insecurity and potential moderators.

Results: Consistent to previous findings, a single two-way interaction of Job Insecurity and Marital Status on Engagement was found, while neither Occupational Status nor Union Membership buffered the effects of Insecurity. Individual career planning variables such Confidence and Readiness confirm to be important intervening variables. As regards to Distress, higher levels of them compensate increases in Job Insecurity; while as regards to Engagement, only the role of Confidence can be stressed, which lets workers to decrease their Engagement (while Insecurity increases) probably to let them psychologically separate from their actual organisation and take an active role in their career planning in view of a possible new job-search.

Implications and Conclusions: From a theoretical point of view, the present study offers a comprehensive analysis of two- way interactions between job insecurity and other variables on traditional outcomes like engagement and distress, including both traditional (biographical) and original (Self-efficacy, readiness, employability) variables. As concerns with practitioners, either managers involved in mergers/lay-off and counsellor implied in career interventions, they are invited to consider, on one side the role of biographical variables such Marital Status, Union Membership, Kind of Contract and Occupational Status, and on the other side to concentrate on the development, through dedicated interventions, of career planning skills like Self-efficacy and Readiness, to buffer the negative effects of Job Insecurity. 


\title{
Multicentric Italian study on gender work related stress among health workers in rehabilitation hospital departments: assessment and management
}

\author{
d'Ettorre, G., Pati, C., Mazzotta, M., \& Russo, L. \\ University of Bari and Local Health Authority BR, Brindisi, Italy
}

Background: Job related stress is considered the second most important problem by European workers and it is the cause of 50-60\% of lost workdays. Scientific literature reports that healthcare workers employed in rehabilitation departments are exposed to high work-related psychosocial risk.

Objectives: the purposes of this study were: to evaluate the gender susceptibility to work-related stress and the coping attitude between a sample of healthcare workers employed in rehabilitation hospitals of the Salento, in Italy; to study a primary prevention program in the management of work-related stress in the rehabilitation hospital departments.

Methods: the Rapid Stress Assessment Scale of Tarsitani and Biondi, a self-rated tool, was administered to 150 physiotherapists (70 M, $80 \mathrm{~F}$ ) employed in the rehabilitation hospitals, as exposed, and to135 white-collar workers (60 M, $75 \mathrm{~F})$, as controls. Exposed and controls subjects were compared by age, length of service, smoking habit and alcohol comsumption. For the evaluation of occupational risk stress factors the authors have used the Kasl questionnaire.

Results: The total score of scale RSA and the scores for clusters of anxiety, depression and social support were significantly higher in the female exposed workers than male exposed $(p<0,05)$ and female non exposed workers $(p<0,05)$. No statistically significant difference in total score of RSA and clusters for depression, anxiety, social support and somatization was found between the male exposed vs the male non exposed workers. Older and more experienced female physiotherapists, showed higher total score of scale RSA than younger and less experienced female physiotherapists. No statistically significant difference in total score of RSA was found between male physiotherapists compared by age and length of service. Implications and conclusions: The study showed that stress management programs based on gender specific approaches, for female physiotherapists employed in rehabilitation hospitals, have to be priority developed; the authors suggest job organisation interventions as instrument of primary prevention in order to guarantee the worker's wellness, with regard to improve the team development, the reflective dialogue and feedback, decision making policies and processes; the rehabilitation hospital departments should offer safety training programs to assist the physiotherapists to adopt constructive stress coping strategies.

\section{Psychosocial risk in finance and banking sector:active participation as a prevention instrument. Application of a multivariate analysis technique (Multiple Correspondence Analysis - MCA)}

\author{
Macciocu, L., Rossi, I, Nardella, C., Ferrante, P., Buresti, G., \& lavicoli, S. \\ National Institute for Occupational Safety and Prevention (ISPESL), Italy
}

Objectives: In the European context the development of professions and increasing of accidents 
at work and professional diseases, request the improvement of health and safety conditions. The new health concept consists of a state of total well-being.

D.Lgs.81/08 on Health and Safety needs (Section. 15, 1st paragraph) the active participation and consultation of workers. In order to prevent, eliminate or reduce stress at work, the European Agreement (8/10/2004) introduces information, employees' and/or their representatives' consultation as an example of preventive measures.

The aim of this research is to examine the level of participation in health and safety in the Italian banking sector, focusing also on issues related to well-being, with particular reference to work situations experienced as critical, such as robbery and theft, organisation processes.

Methods: Survey construction refers to Cox/Griffths/Rial-Gonzalez theoretical model on psychosocial risks and on concept of active participation at work as a means of prevention. A questionnaire was administered to 2.100 subjects from the credit sector consisting of 64 items.

The Multiple Correspondence Analysis - MCA has been used to simplify the reading of data. Socio-Demographic variables, projected on the factorial plan, identified 5 banking operator's profiles: Employees, Managers, Financial, Youth, Back-office.

Results: The highest level of ILLNESS is found in Employees (mainly front office task and money handling) with high seniority, permanent contract, medium/high RESPONSIBILITY. The Critical Situation's area is located on medium/high RESPONSIBILITY and ILLNESS level. 1165 subjects had experienced critical situations at work, 58,8\% of which stated they had been robbery victims. The Italian rate of front office robbery is 20 times higher than Switzerland, 10 times than Greece, 6 times than Germany and UK and 4 times than France. The wellness level at work is also connected to the quality of working relationships as a starting point in building collaborative bonds with others; $89 \%$ of subjects affirmed having collaborative relationships with colleagues but relations with superiors are more formal and reach peaks of conflict. The high percentage $(24 \%)$ of operators that felt intentionally treated poorly by their superiors for at least a period of six months confirms this statement.

Implications: The comparison and exchange between the parties is the stimulus to improve the organisational environment that affects welfare conditions of individual/group/ organisation.

Conclusions: The active participation becomes a tool for the prevention of risk if implemented by integrating the interests of different players in the system for managing health and safety at work.

\title{
Quality of Life in subjects with Spinal Cord Injury and their Informal Caregivers
}

\author{
Machado, F., Teixeira, A., \& Alves, J. \\ Instituto Superior da Maia, Portugal
}

Faced with an unexpected, shocking and traumatic change in their lives, Informal Caregivers (IC) of Spinal Cord Injury (SCl) victims go through a turmoil of emotions, difficulties and wearing 
conditions while caring for their subjects, frequently sacrificing their own health, Quality of Life (QOL) and well-being.

Finding that there's a deficit of studies in this particular area of Health and Occupational Health Psychology, we find that's essential to increase our knowledge about what entails to be a IC in order to attend to the special needs of this role and consequently help to improve not only the life conditions of the IC, but also the quality of the care giving per se. Our investigation was conducted with $44 \mathrm{SCl}$ victims and their respective IC, while attending health care services in a General Hospital in Porto, Portugal. Anxiety and Depressive symptoms and their QOL were evaluated using HADS (Hospital Anxiety and Depression Scale) and the WHOQOL-100 (World Health Organisation Quality of Life). Results show that the IC present higher levels of anxiety and depression than the $\mathrm{SCl}$ victims whom they care for. This result is especially significative for anxiety. Relating to the QOL, it was found that although IC present higher levels of QOL, the difference is not significative comparatively to the QOL levels presented by SCI victims. Our findings reinforce the belief that the IC (mental health and psychological adjustment) should be included when planning the therapeutic (medical and psychological) intervention with $\mathrm{SCl}$ victims, attending to the fact that these caregivers play a fundamental role not only in the physical recovery of the patient, but also in his psychosocial adjustment. Ignoring the psychological health and adjustment of the IC might constitute a real impairment to the effectiveness and success of the recovery process of the SCI victims.

\title{
Stress prevention for better health, better health to reduce the social costs
}

\author{
${ }^{1}$ Magnani, M., ${ }^{1}$ Mancini, G.A., ${ }^{1}$ Scatolini, E., ${ }^{2}$ Gattai, A., ${ }^{1}$ Marocci, G., \& ${ }^{1}$ Majer, V. \\ ${ }^{1}$ University of Florence, Italy; ${ }^{2}$ University of Verona, Italy
}

Objectives: Occupational desease its every change of the state of mental and physical health which may involve the injury or illness. According to data provided by the European Agency for Safety and Health at Work and reported by INAIL (2009), the victims of work-related stress in Europe would be about 40 million, affected by diseases such as cardiovascular and gastrointestinal disorders, fatigue and depression. The social costs are very high in terms of working days lost each year (50-60\%), equivalent to around 20 billion euros, or between 3 and $4 \%$ of gross national product in Europe.

In the wake of research promoted by the World Health Organisation in order to control the health effects resulting from stressful situations, this work will occur, using Organisational \& Psychosocial Risk Assessment questionnaire (OPRA, Magnani, Mancini and Majer, 2009) the impact of the 9 risk sources on the mental and physical health

Methods: Using logistic regression, CHAID and SEM and the relationship between 2 health factors and 5 indicators of organisational malaise (job satisfaction, identification with the workgroup, identification with the company, organisational trust and intention to leave). The sample consisted of 1100 subjects, of companies both public and private.

Results: A linear relationship was confirmed between the five indicators and scales for the mental and physical health, especially with job satisfaction and identification with the workgroup. 
Implications and Conclusion: Psychosocial risk management paths should concern increasing job satisfaction and good relationships within workgroup for effective prevention and to preserve mental and physical health.

\title{
Workplace Violence in Health Care Workers
}

\author{
Magnavita, N. \\ Catholic University, Italy
}

Background: Workplace violence in the health care is increasing. The Italian Ministry of Health released a Recommendation in 2007, November, for the establishment of a comprehensive workplace violence program.

Objectives: Epidemiological analysis of workplace prevalence and incidence rates, and study of risk factors, are the first step of prevention and control programs.

Methods: Information was collected through interviews during medical visit of workers in 4 health-care units (569 workers) and two nursing schools (96 students). 50 office workers were studied as control.

Results: Physical violence had been experienced in the last year by $7.7 \%$ of health care workers and $3.1 \%$ of students, but none of controls. $26.1 \%$ of health personnel had experienced at least one incident of physical violence in their professional life. Threats, or other forms of psychological violence, were reported by over a third of workers in all professional groups in the health sector, but only by $8.2 \%$ of office workers.

Implications and Conclusions: Precautions to prevent workplace violence in health care are urgently needed.

\section{The Hazardous Physician}

\author{
Magnavita, $\mathrm{N}$. \\ Catholic University, Italy
}

Background: Physicians, if affected by transmissible or impairing diseases, may be hazardous for their patients. Policies dealing with this argument, are facing the ethical dilemma between patient's and sick physician's rights.

Objectives: To outline a way of managing risk related to physician's illnesses, ageing and other factors which may lead to impairment (e.g.: addictive behaviours).

Methods: Through the analysis of literature, and the examination of clinical cases, a consensus has been developed within the La.R.A. study group, an association of physicians from different specialties, jurists, bioethicists, employers and employees. 
Results and Implications: The management of the unhealthy doctor implies an original methodological approach, involving full cooperation of employer, employees, and health and safety consultants in a process of consensus building that leads to hospital wide policies. The controversial issues of safeguarding both patient's and diseased worker's health may lead to different and equally reasonable solutions, depending on how much weight the deliberative process gives to any of the substantive principles that are proper to the health care organisation's role. Policies concerning informed consent, non compliance, confidentiality, responsibility of workers, disclosure of risk to customers, non-discrimination and counselling of workers, should be clarified. Costs and targets of the policy need to be stated, plus the means of recovering costs to ensure viability.

Conclusions: To solve the apparent chiasm between patient's and sick worker's rights, a consensus building process leading to hospital wide policies is the better alternative to individual decision making. Policies have to balance the rights of the sick worker, the right of the other workers, patients and customers, and society's expectations.

\title{
Psychogenic Mass Syndrome in Anti-Cancer Drug Exposure
}

\author{
Magnavita, N., lavicoli, I., Leso, V., \& Bergamaschi, A. \\ Catholic University, Italy
}

Background: Psychogenic mass illness (PMI) is probably the most dramatic emergency in occupational health. The sudden spread of symptoms in a large percentage of workers often poses at stakes the occupational health service. Early recognition of psychogenic factors is the key factor in clinical management of this condition.

Objectives: Through the analysis of a case, criteria for clinical management of PMI are proposed.

Methods: A case of PMI, involving 5 health care workers (physicians and nurses) working in an Oncology Unit, is reported.

Results: After water infiltration, unidentified liquids spilled out from the laminar flow hood of an hospital anti-cancer drug unit. At the end of the day work, workers in the unit become to complain for urinary complaints; they called by telephone each other, and shared symptoms. Workers were feeling the need to urinate frequently, and passing only a tiny amount of urine even when the urge to urinate is strong; they had no pain or a burning feeling during urination, nor altered appearance of the urine. Workers also complained for tachycardia, increased ventilation rate, general malaise and fatigue. They all were admitted to a general hospital, and underwent a wide range of clinical and toxicological examinations, which showed no abnormality. Workers were subsequently send to a specialized occupational health center, which confirmed the absence of toxicological effects of anti-cancer drugs. Symptoms persisted for the entire period of medical examination.

Implications: Workers complained for a symptom pattern which was clearly unrelated to anticancer drug effects. The most important symptom, i.e. the increased frequency of urination, in 
the absence of infection, may well be related to autonomic nervous system activation; the same is true for other reported symptoms. It is worth to note that a common recommendation that medical personnel in anti-cancer drug units make to patients, is to increase drinking water (and urine quantity) after therapy. We hypothesized that workers, supposing an exposure to anticancer drugs, unconsciously adopted the behaviour they had suggested to patients. Telephone calls increased anxiety and consented the sharing of symptoms and behaviours.

Conclusions: PMI affects healthy workers, under abnormal stress conditions. The occurrence of a real exposure to toxic substances at very low doses acts as a trigger for psychogenic manifestations. Inappropriate management of the syndrome will increase both the spread of symptoms and the duration of illness. The occupational health physician.

\title{
Promote organisational wellness through the reduction of constraints and the development of organisational resilience
}

\author{
Maisetti, M., Bonzi, F., Fattori, M., \& Camicia, B. \\ ASP-Associazione Italiana Psicologi, Italy
}

Continuous and accelerated changes force companies to quickly align their strategy to all environmental needs. Introducing "Organisational Wellness" policies represents the so-called 'egg of Columbus', but they need a management focused on complexity of systemic organisations. First need is to remove or streamline "Organisational Constraints" in order to maximize the capabilities to withstand all discontinuity experienced by the company, namely to strengthen the "Organisational Resilience".

Objectives: Authors propose an application model starting from several researches and contemporary theories. The goal is to promote a diagnostic model designed to identify strengths and improvement areas of whole company.

\section{Definitions:}

OC "Organisational Constraints": those elements limiting organisation to achieve higher levels of performance. Constraints determine system's upper limit of performance (Goldratt's Theory of Constraints).

OR "Organisational Resilience": is the capacity of an organisation to adapt, to recover and to resist in providing a positive level of functioning, despite economic and systemic risks.

OSF "Objective Stress Factors": those measurable factors that can cause uncomfortable situations in the company. Organisational data are represented by clusters.

OHF "Organisational Health Factors": those measurable factors of OSF subjective perception, organized in aggregated data and representing the whole-system company.

OW "Organisational Wellness": organisational strategy aimed to optimize OC management and to increase OR.

Methods: Analyzing both OSF and OHF we are able to identify best improvement actions to introduce OW policies. 
OSF can become the most limiting constraints and potential cause of OHF deterioration.

OSF can cause individual stress, decreasing performance level of human resource: thus OSF become OC.

But, paradoxically, OSF may become a development opportunity.

OSF generates individual stress only if they result dysfunctional for employee in achieving his personal and/or professional goals. Only in this occurrence, OSF really become OC.

OR can be increased through identification and optimization of OC, eliminating OSF or leverage them as tools for OC reduction.

Conclusions: Application model is represented by the following empirical function:

$\mathrm{OW}=\mathrm{f}(\mathrm{OC}+\mathrm{OR})$ where $\mathrm{OC}=\mathrm{f}(\mathrm{OSF}+\mathrm{OHF})$ and $\mathrm{OR}=\mathrm{f}(\mathrm{OSF}+\mathrm{OHF})$

More sum of OC and OR is positive, more widespread prosperity (so called wellness) is possible.

With a quantitative diagnosis of OSF and OHF and a direct comparison of results, it's possible to know both presence of OC and level of OR. Then, we can identify the most effective improvement actions to limit OC and increase OR, leading company towards ORGANISATIONAL WELLNESS.

\title{
The assessment of workaholism as a work-life imbalance: "Time Usage Inventory" development and preliminary empirical testing
}

\author{
Malinowska, D., Tokarz A. \& Jochymek, S \\ Jagiellonian University, Poland
}

The goal of the presented study was the development and preliminary empirical testing of Time Usage Inventory designed to assess work-life imbalance. Work-life imbalance concept is an important aspect of workaholism that is defined as an excessive involvement with work evidenced by neglecting other areas of life. Work-life imbalance and workaholism are strictly related to quality of life, satisfaction and well-being. Both concepts are the topics of contemporary interest. Time Usage Inventory includes ten life-domains such as: Sleeping, Hobbies, Leisure, Household Matters, Family, Work, Education and Training, Spirituality, Friends and Acquaintances, Other Areas (not included above). The inventory consists of three scales: "Doing", "Thinking" and "Emotions". The scales were design to assess behavioural, cognitive and emotional aspect of work-life imbalance. "Doing" scale assessed time committed to action in each of ten life domain. "Thinking" scale assessed time committed to thinking (reflection) about each life domains. "Emotions" scale assessed positive or negative emotions related to each life domains.

Evidence from a sample of 371 employed respondents indicated, that inventory is internally consistent and valid measure of work-life imbalance. Work-life imbalance concept is distinguishable from other aspects of workaholism, for example from inner drive to work. In summary, Time Usage Inventory developed for this investigation, appears both reliable and valid and thus potentially able to shed light on the phenomenon of workaholism. The inventory could be also used in the study of the relation between work and the rest of life. 


\title{
An investigation of workaholism components in relation to work and life values and satisfaction
}

\author{
Kasprzak, M., Tokarz, A., \& Malinowska, D. \\ Jagiellonian University, Poland
}

The goal of this study was to identify the relationship between people's values and their attitude towards work and life. It examined the associations between workaholism components proposed by Spence and Robbins (1992) - work involvement, feeling driven to work, work enjoyment, and work and life values. Building on previous research on sources of motivation (e.g., the effects of intrinsic versus extrinsic goals) and different processes or motivations for realizing these goals (e.g., internal versus external motivations) which showed that individuals motivated by extrinsic goals and external sources of motivation report lower levels of satisfaction and psychological health (Deci and Ryan, 1985, 2000; Deci et al., 1999; Ryan and Deci, 2000), was developed a hypothesis that values related to internal work characteristics will be positively connected with work involvement and work enjoyment. Extrinsic values, such as salary, will be negatively correlated with those dimensions. Analogous assumption was made towards life values. Extrinsic values will be negatively related with work enjoyment, intrinsic - positively.

Data were collected from a sample of 72 workers of Polish production company. The set of questionnaires was used: Workaholism Battery, Work Values Orientation Questionnaire, Scheler Value Scale and Time Usage Inventory. The predictions were supported in a study. It confirmed the associations between intrinsic work values and work satisfaction and also general life satisfaction. Non relationship between life values and satisfaction were found. There was also no correlation between global satisfaction and workoholism components. Positive correlation has been found between high probability of workaholism and intrinsic work values. Work enjoyment was positively related with intrinsic work values. Work involvement appeared to be negatively correlated with extrinsic work values. Only one significant correlation between life values have been found. Work involvement was negatively correlated with hedonic values. These results could be discussed in the context of human resources management and could be successfully used in projecting career planes and improving organisational climate.

\section{Job insecurity, employability and wellbeing: Evidence from an Italian study}

\author{
Manuti, A., Rosa Pia, F., \& Mariangela, M. \\ University of Bari, Italy
}

Objectives: In recent years, research has shown a growing interest in the study of job insecurity, defined as 'the perceived powerlessness to maintain desired continuity in a threatened job situation' (Greenhalgh \& Rosenblatt, 1984: 438). Such construct has become even more relevant in the analysis of the person/organisation relationship, as long as labor markets are experiencing growing instability and workers are forced to cope with flexibility. In this frame, employability becomes a key work as well as a competence to successfully manage labor market transitions. Moreover, empirical evidence has showed employability to be favorably related to employees' wellbeing, since it may promote feelings of being in control with one's own career and thus of 
being less vulnerable to changes in times of economic recession. Moving from such framework, the aim of the study was to investigate the relationship between employability and job related and general wellbeing (measured as life satisfaction and work engagement), hypothesizing that job insecurity might be a mediating factor (De Cuyper et al., 2008).

Methods: Participants, 100 workers employed at an Italian retail organisation, filled in a questionnaire which included the job insecurity scale (De Witte, 2000), the employability scale (Van Dam, 2004) and the Utrecht work engagement scale (Schaufeli \& Bakker, 2003).

Results and Implications: Results showed the role played by employability in securing one's own job position and thus in increasing both general and work related wellbeing. Future implications of this study are linked to the promotion of employees' wellbeing also by the encouragement of their employability.

\title{
Impact of exposure to critical incidents in health and psychological well-being of Emergency Ambulance Personnel
}

\author{
${ }^{1}$ Marcelino, D., ${ }^{2}$ Figueiras, J., \& ${ }^{1}$ Claudino, A. \\ ${ }^{1}$ University of Evora, Portugal; ${ }^{2}$ Instituto Piaget de Almada, Portugal
}

Objectives: The aim of this study is to investigate the influence of post-traumatic stress disorder (PTSD), peritraumatic dissociation, psychological distress and subjective health complaints in the psychological well-being of the emergency ambulance personnel.

Methods: This is a cross-sectional study in which 250 ambulance personnel; both sexes completed a self-administered questionnaire, with measures of post-traumatic stress disorder, peritraumatic dissociation, psychological distress, subjective health complaints, psychological well-being and socio-demographic variables.

Results: The results indicated that ambulance personnel have on average some PTSD symptoms, while $10 \%$ have a clinical diagnosis of PTSD, peritraumatic symptoms are also significant, as well as psychological distress and health complaints. Correlational analyses indicated a negative significant association between symptoms and psychological well-being. The peritraumatic dissociation, the subjective health complaints, psychological distress and sex were found to be predictors of the post-traumatic stress disorder. There were significant differences concerning the socio-demographic variables in relation to the dependent variables.

Implications: The results of this study highlight relevant indicators of the need to develop and implement intervention programs to alleviate the psychological suffering, promoting the adjustment and the physical and psychological well-being in emergency ambulance personnel.

Conclusions: This study presents a contribution to understanding the psychological consequences of daily exposure to traumatic incidents, and its impact on health and well-being of emergency ambulance personnel. 


\title{
Assessing the Role of Climate for Mentoring and Distributive Justice in the Relationship Between Perceived Organisational Support and Job Satisfaction: A Moderated Mediation
}

\author{
Marmet, M., Magley, V., \& Gilson, L. \\ University of Connecticut, United States of America
}

Objectives: Organisational support has been widely touted as influencing employee well-being. Two forms of such support "perceived organisational support (POS) and mentoring support" have both been shown to influence employee well-being, as evidenced by their positive relationship with job satisfaction. We argue that both forms of support affect job satisfaction in light of additional perceptions that organisational resources are fairly distributed. In particular, such distributive justice perceptions should moderate the influence that support has on job satisfaction, such that perceptions of just treatment could buffer the potentially negative impact of perceived lower support. Additionally, we argue that, because mentor programs are often housed within a larger support system, the climate for mentoring will be positively influenced by the presence of a larger supportive organisation. In sum, we hypothesized that climate for mentoring will mediate the relationship between POS and job satisfaction and that distributive justice will moderate this mediated relationship.

Methods: Members of a large state agency were surveyed $(\mathrm{N}=752)$ using measures from previously validated scales. The mediation was tested using the Baron \& Kenny (1986) approach. The significance of this indirect effect was assessed using the Sobel Test of significance. The moderated mediation was tested using an SPSS macro developed by Preacher, Rucker, \& Hayes (2007).

Results: The mediation analyses revealed that climate for mentoring did in fact partially mediate the relationship between perceived organisational support and job satisfaction $(p<.001)$. The subsequent moderated mediation analysis however, showed that distributive justice did not moderate the overall proposed relationship.

Discussion: Although perceived organisational support has been linked to job satisfaction in the past, the current study offers an expansion on this literature by examining the mediating role of climate for mentoring in this relationship. Although distributive justice failed to moderate this overall relationship, this null finding may provide avenues for future research.

Implications: From these research findings we see that regardless of the level of distributive justice, POS has an indirect effect on job satisfaction through climate for mentoring. This may shed some light on the importance of establishing a positive mentoring climate in one's organisation, as this may outweigh other factors that could potentially influence job satisfaction.

\section{Burn-out and psychosomatic health complaints in a hospital in Piemonte region}

\author{
Martini, M. \& Converso, D. \\ University of Turin, Italy
}

Introduction: Care professionals are particularly exposed to risk of burn-out and psychosomatic 
health complaints as psychological reactions to stressful work conditions (Tummers, Landeweerd, \& van Merode, 2002).

The JDC-S model (Karasek, 1979; Johnson \& Hall, 1988) is the most common one to investigate the antecedents of these reactions. As Verhoven and colleagues (2003) evidence, it is, nevertheless, quite a simplistic model. They suggest to keep in consideration also other work and organisational conditions. Simon and colleagues (2004) then, underline the importance of aspects related to other domains of life, mainly the work-family relationship, considered as potential buffering or stressful aspect. Work-family relationship can be expressed by the two complementary constructs of conflict and enrichment (Rothbard, 2001; Lapierre \& Allen, 2006).

Objectives: Aim of this study is to describe the perception of burn-out and psychosomatic health complaints by the population of a hospital specialized in obstetrics and gynaecology in Piemonte region.

In particular, we want to individuate differences among groups of professionals (nurses, physicians) in the perception of psychological stress reactions and to understand how these reactions are influenced by the perception of some work and organisational characteristics and of work-family relationship.

Methods: 105 operators filled a self-report questionnaire composed by measures to evaluate: burn-out (EE, PA, DP; Maslach \& Jackson, 1981) and psychosomatic health complaints, work and organisational characteristics, work-family conflict and enrichment.

After Cronbach's alpha coefficient of the scales, Analises Of Variance (ANOVA) was calculated to individuate differences among groups. Correlations and hierarchical regressions were estimated to see influences on burnout and psychosomatic symptoms. In the first step we put, as determinants, work and organisational aspects and, in the second step, the work-family conflict and enrichment.

Results: Burn-out and psychosomatic health complaints are not perceived as particularly serious by workers in this hospital. Some differences are perceived in burn-out factors and organisational aspects among professionals. Both work and organisational characteristics have an influence on psychological stress reactions, but the introduction of work-family relationship in the regressions models has a significant impact.

Implications and conclusions: The research has some limitations, in particular the participants are not very numerous and from an only hospital. In the future the questionnaire will be distributed in other contexts.

Even if the work stress reactions are not so intense in this hospital, keeping in exam workfamily relationship jointly to work and organisational conditions can be useful in order to sustain positive psychological work reactions. 


\title{
The influence of 'significant others' on back pain disability and return-to-work: a qualitative pilot study of illness perceptions
}

\author{
McCluskey, S., Brooks, J., Burton, K., \& King, N. \\ University of Huddersfield, United Kingdom
}

\begin{abstract}
Background: There is little understanding of the individual and social influences involved in the everyday life experiences of those with disabling back pain which may act as obstacles to recovery, and ultimately, a return-to-work. Illness perceptions have been acknowledged as important influences in the course of, and recovery from, back pain. However, an in-depth exploration of the influence of illness perceptions, particularly those of 'significant others' (close family members), on back pain disability has yet to be carried out.
\end{abstract}

Objectives: We are proposing to design and validate a method which will allow a more in-depth examination of the illness perceptions of both individuals who are disabled due to back pain, and those of their 'significant others'. The aims of this pilot study are:

1. to design and validate a semi-structured interview for individuals disabled due to back pain and their 'significant others' to explore their illness perceptions in relation to the individual's back condition and work function;

2. to compare the beliefs of such individuals and their significant others with a view to designing a large-scale mixed-methods research project exploring the impact of significant others(beliefs on individuals) own beliefs about their disability and work function.

Methods: Interviews based on The Revised IIIness Perception Questionnaire - Chronic Pain Version (IPQ-R) (Moss-Morris et al, 2002), and The Work and Social Adjustment Scale (WSAS) (Mundt et al, 2002) will be carried out with a convenience sample of incapacity benefit claimants experiencing chronic back pain (and their significant others). Data will be analysed using qualitative techniques, and themes will be extracted and explored in relation to disability and work function, and compared between claimants and their 'significant others'.

Outcomes: The influence of 'significant others' on back pain disability is largely unexplored. This pilot study aims to provide a method which will enable further understanding of these psychosocial influences, with the aim of informing future back pain research and return-to-work programs.

\section{"Preliminary study on the welfare of a community Nosocomial of the province of Rome conversational approach and methodology"}

\author{
Messineo, A., Panunzi, M., Visconti, S., \& Ciconte, A.R. \\ Azienda USL RM H (Albano Laziale), Italy; SPISAL ASP, Italy
}

Introduction: A Project of welfare organisation implemented aimed at medical staff of a company in an ASL-year project (2008-2010) aims to identify potential discomfort workers have in their workplaces. In order to have a point of comparison with other surveys conducted in 
the public sphere has been diffused the questionnaire on organisational well-being developed by the Department of Public Administration in collaboration with the Chair of Occupational Psychology of the Faculty of Psychology 2 University "La Sapienza" of Rome.

The results obtained have confirmed the usefulness of continuous monitoring of critical points emerged through additional survey instruments and a 'corporate approach to business counseling.

Methods: Operations of the Department of Prevention and the Office of Quality as an ASL of the Province of Rome have launched a project that includes:

1. A training phase prepared by a 16-hour business seminar in 2 days having as its object the organisational culture and psychological well-being in the workplace. This seminar, aimed primarily at nursing staff coordination, later also involved occupational physicians, occupational physicians and executives of various business services for a total of 315 participants;

2. A subsequent phase with the diffusion of the questionnaire during the training course, with the methodology of the "Programma Cantieri".

3. Resuming after the critical points raised through the conversational approach and counseling in various business units.

4.

The data, compared with those obtained following a single diffusion of questionnaires in the ASL of Calabria, were entered into a database, analyzed and processed statistically.

Results: These achievements have provided useful elements to improve the quality of life and relationships in the workplace, having allowed the implementation of improvement measures, having spread the culture of participation within their organisation and having strengthened and developed the concept of prevention of psycho-physical and optimized the performance and the quality of services. Of particular interest were the indications provided Strategic Direction on the conflict exists, the value attributed by employees to their work, the need to use the experiences of individuals. It 'was suggested, based on the growing demands of the employees sensitized by the course, to formalize moments of supervision and analysis of organisational climate for example through one-stop listening business.

Conclusions: An analysis of data showed the need to continue fostering a culture of wellness and risk prevention in the workplace through: continuing education, counseling and the establishment of business circles of listening with the active involvement of the operators themselves.

\title{
A Global Look at Workplace Incivility: Does Personality Play a Role Throughout the World?
}

\author{
${ }^{1}$ Milam, A., ${ }^{2}$ Sulea, C., ${ }^{1}$ Hanif, R., ${ }^{1}$ Spitzmueller, C. \\ ${ }^{1}$ University of Houston, United States of America; ${ }^{2}$ West University of Timisoara, Romania
}

Objectives: One of the most prevalent counterproductive work behaviours studied is workplace incivility, which typically manifests itself in the form of disrespect, condescension, and degradation 
(Andersson \& Pearson, 1999; Burnfield et al., 2004; Robinson \& Bennett, 1995), and is prevalent and detrimental for organisations and individuals (Cortina, Magley, Williams, \& Langout, 2001), contributing to poor job attitudes, malaise and job-related strain (Notelaers, Einarsen, De Witte, \& Vermunt, 2006). In a study recently conducted in the U.S., Milam, Spitzmuller, and Penney (2009) found that low agreeableness and high neuroticism predicts whether one is likely to become a target of workplace incivility, and further, that these personality characteristics tend to provoke uncivil treatment by others. The present paper aims to replicate and expand the Milam et al. paper by looking at other measures of workplace incivility and examining other parts of the world, namely Pakistan and Romania to see if this phenomenon is universal. Review of literature has established that five factor model of personality is a universally valid taxonomy of traits (Hofstede \& MacCrae, 2004), and these are fundamental dimensions of personality that are independent of culture (McCrae et al., 2000).

Methods: There were three samples: 323 working undergraduates from a large U.S. university, 141 working undergradutes from a large Romanian university, and 314 working adults in a large city in Pakistan. We also obtained coworker data for each participant. Workplace Incivility was measured three ways (Burnfield, Clark, Devendorf, \& Jex, 2004; Cortina et al., 2001; Ferris, Brown, Berry, \& Lian, 2008), and Big Five personality measures were utilized (IPIP; Goldberg, 1999).

Results: Hierarchical multiple regression analyses were performed with gender as a covariate in the first step of every model test. Hypothesis 1 proposed a negative relation between agreeableness and workplace incivility, and was supported for all three countries. Hypothesis 2 proposed a positive relation between neuroticism and workplace incivility, and was also supported in all three countries.

Implications: The findings have implications both for researchers and practitioners alike. For researchers it suggests that the relation between personality and workplace incivility is universal. Organisations can use the present results to create work climates where high levels of agreeableness and low levels of neuroticism are encouraged and rewarded.

Conclusion: Personality plays a significant role in predicting workplace incivility regardless of culture and ethnicity.

\title{
Shaping of Well Being with Job Satisfaction
}

\author{
Molan, M. \& Molan, G. \\ University Medical Centre Ljubljana, Slovenia
}

Objectives: Perception of well being is shaped with the influence of working environment and with individual perception of workers' availability, according to the $\mathrm{AH}$ model. Adequate vocational choice is one of the most important decisions in the life of individual with long term consequences. Identification of connections between job satisfaction, vocational choice and perception of actual availability after more than 10 years of work is our main research goal. 


\title{
Methods:
}

- Questionnaire of actual availability (QAA) from the AH model for identification of perceived well being during normal every day work.

- Questionnaire of vocational decision satisfaction (QVDS) for determination of vocational decision satisfaction in the youth age.

- Questionnaire of anxiety from Spielberg for identification of manifested anxiety level. Sample is 58 office workers on different working places in financial and in public sector.

Results: Perception of well being in different age group is according to the $\mathrm{AH}$ model standards in the interval of fatigue in the age groups (30-39) and (40-49). After 50 there is more fatigue and after 60 there is the exhaustion. According to the results of QVDS, 65\% of workers would choose the other profession, no more the same one. For $47 \%$ of workers the present job is no related with the basic profession. Minority of workers (19\%) are satisfied with their profession with no wish to do something else. Majority of workers (57\%) from the sample have not finished basis education. They are statistically more overload than workers worker's with higher education. They report also less motivation for work. There is an important correlation between basic education and present job. There is also significant relation $(0,683)$ between unsatisfaction with the profession and their job. People who did not finish their education would prefer same other profession $(0,633)$.

Implications: Perception of well being is decreased with ages. People, who did not finish their started education, now perform jobs not related with those educations. Workers unsatisfied with their profession report higher level of anxiety. They are more exhausted and depressed. Those manifestations are shaped with ages.

Conclusions: Unsatisfaction with their professional decision reflected in success in education process. The important influence of unsatisfaction and unmotivation for their profession reflect in perception of unmotivation, overload and stress. Adequate professional decision is one of the most important one in the life of individual. It shapes perception of well being also in ageing period.

\section{Stress Management Interventions in Polish Companies}

\author{
Molek-Winiarska, D. \\ Wroclaw University of Economics, Poland
}

The research carried out by the author in 2002-2003 showed that 70\% of Polish workers suffer from high or medium level of occupational stress. Many of them can point to the source of stress correctly and they are usually aware of the psychological cost of this state. The main aims of this study are to find if top management of Polish companies know how to evaluate occupational stress (its sources, level, costs and coping) and know of any stress management interventions both at organisational and individual level.

Preliminary data shows that polish HR Managers usually have not got solid tools to measure the level, the cost of stress or the way of coping with stress. It also appears that the idea of stress management and different kinds of interventions are hardly used by Polish companies. 
However, there are some enterprises, which are interested in reduction in occupational stress. They usually implement different programs of interventions directed to their employees (individual level interventions).

There is tremendous need to inform polish HR Managers about different ways of measuring occupational stress, such as professional psychological tests and questionnaires. It is also important to collect data about results of stress and costs such as work absence, damage, lost output, cost of covering for sick leave etc. Finally, one should strive to foster cooperation between top managers and stress management intervention specialists to prepare, implement and control suitable programs of intervention in Polish companies.

\title{
Non-formal learning: proposal for an occupational health and safety-oriented approach
}

\author{
Nardella, C. Pizzuti, A., \& Deitinger, P. \\ National Institute of Occupational Safety and Prevention (ISPESL), Italy
}

In a system as complex as today's work organisations, workers develop an organisational culture where socializing and informal learning are more important and meaningful than formal training. This is particularly so in safety training, which is all too often reduced to simply communicating rules and regulations with too little consideration of the overall level of attention to safety in the company.

Starting from theoretical concepts of Non-formal learning (Eraut, 2000) and Workplace Learning (Eraut, 2004), we questioned whether a non-formal learning approach could serve to develop a safety culture in the workplace, and to what extent less formal training strategies could ensure socially acceptable safe behaviour that can be adopted throughout an organisation.

Objectives: To propose a method for establishing a "safety culture" within a company, by a process of non-formal learning.

Methods: We reviewed the main reports of non-formal learning in relation to safety training in Italy.

Results: This initial analysis of the Italian situation did not bring to light any significant reports of training using a non-formal learning approach.

Implications: The Italian situation is particularly interesting because some of the current labor contracts (occupational apprenticeships, training/work contracts, and "deferred hiring" contracts), combining a mix of training and work, should lend themselves well to unstructured training methods.

Conclusions: A model of non-formal learning to foster a safety culture in a company should be applicable, particularly for workers with the types of contracts mentioned, through mentoring and work alongside people with occupational health and safety skills. This way goals could be achieved not by training alone, but through actual work (Lave, Wenger, 1991). The idea, therefore, is to move towards intentional but informal education. This form of non-formal 
learning would be based on a non-asymmetric relation between the worker-pupil and the person transmitting knowledge, values and behaviour, facilitating a serene organisational climate, and influencing workers' values, hence also their behaviour.

\title{
Coping strategies and positive affect through gender dimension
}

\author{
Niculaes, A. \\ Renes Descartes University, France
}

\begin{abstract}
Most stress research has focused on testing the effects of coping strategies on negative outcomes such as distress and anxiety. The present study focused on the effects of coping styles on the positive affect. Its aims were to examine which coping strategies lead to positive affect and to examine through gender dimension which coping strategies lead to positive affect.
\end{abstract}

Participants were 40 female and 40 male international students, who were randomly selected from Cité internationale universitaire de Paris. Participants completed Coping Inventory for Stressful Situations and Cognitive Emotion Regulation Questionnaire taking into consideration unpleasant and negative experiences since they have arrived in France. Also they completed Positive and Negative Affect Schedule being asked to indicate to what extent they had felt this way during the past week.

The main results based on correlations, ANOVA and linear regression analysis showed that positive reappraisal, refocus on planning and task oriented coping strategies were positively related to positive affect. There were found gender differences in positive reappraisal and social support favouring female positive affect and task oriented coping strategy favouring male positive affect. Theoretical and applied implications of the results were discussed.

\section{The mediating effects of self-efficacy on the relationship between transformational leadership and psychological well-being: A longitudinal field study}

\author{
${ }^{1}$ Nielsen, K. \& ${ }^{2}$ Munir, F. \\ ${ }^{1}$ NRCWE, Denmark; ${ }^{2}$ Loughborough University, United Kingdom
}

Introduction: Transformational leaders employ a visionary and creative style of leadership that inspires employees to make independent decisions and develop in their work (Bass, 1990; 1999). There is some evidence that transformational leadership style is linked to employee health and well-being (Dunham-Taylor, 2000; Shieh, et al, 2001, Sosik, \& Godshalk, 2000 and Seltzer et al, 1989). However, there is less research that has focused on the psychological mechanisms that may explain this link. It is possible that transformational leaders influence their followers' self-efficacy and thereby influencing psychological well-being in followers. This study aims to extend previous work by examining the validity of a direct link longitudinally and testing the mediating effects of self-efficacy. 
Methods: The study was carried out within the elderly care sector in a Danish local governmental department. At time 1, questionnaires were distributed to 551 staff and 447 questionnaires were returned yielding a response rate of $81 \%$ for time 1 analysis. At time 2 the questionnaire was distributed to 521 staff and 274 returned the questionnaire, yielding a response rate of $53 \%$ for time 2 analysis. Surveys were sent to all employees working at the centers and thus new staff was included in the second round of the survey. A theory-driven model of the relationships between leadership, self-efficacy and psychological well-being was tested using Structural Equation Modelling.

Results: The relationships between transformational leadership, self-efficacy and psychological well-being was tested both cross-sectionally (T1 and T2) and longitudinally (T1 to T2). The results indicated that followers' self-efficacy did mediate the relationship between transformational leadership style and psychological well-being. There was only limited evidence of the existence of a direct path between leadership behaviour and employee well-being found in previous research. Only at time 1 did we find a direct relationship between transformational leadership and well-being, suggesting partial mediation at this point in time. Also a reciprocal effect was found whereby followers' self-efficacy at time 1 influenced their appraisal of the manager's transformational leadership style at time 2.

Conclusions: Our results indicate that the psychological mechanism by which managers influence their followers' well-being may be through the creation of a sense of self-efficacy. Further, it appears that followers' self-efficacy influenced managers' transformational leadership style. This may be because employees high in self-efficacy welcome the challenges offered by the transformational leader or that they simply evaluate their manager more positively.

\title{
Managers' Active Support when Implementing Teams: The Impact on Employee Well-Being
}

\author{
${ }^{1}$ Nielsen, K. \& ${ }^{2}$ Randall, R. \\ ${ }^{1} N R C W E$, Denmark; ${ }^{2}$ University of Leicester, United Kingdom
}

Background: Middle managers play an important role in communicating a clear vision and function as the link between senior management decisions and actual implementation of such decisions (Randall et al, 2005). They can also facilitate change by involving employees (Shurman \& Israel, 1995). However, it may be that the opportunities available to middle managers' to work actively towards making changes depend on their employees; it has been claimed that organisational-level interventions are more likely to succeed in organisations where the working environment already is good (Nielsen et al., 2006). Little empirical research has focused on the role of the middle manager and how employees view managers' actions during interventions. This paper examines the mediating effects of middle managers support to implement team organisation that aimed at improving employee health and well-being.

Methods: The research was carried out in a large Danish local government. Two elderly care centers participated in the study. A questionnaire survey was used to detect quantitative 
changes in a longitudinal evaluation of the implementation of teams. Immediately before the implementation of teams, questionnaires were distributed to 551 staff (81\% response rate). 18 months after team implementation the questionnaire was again distributed to 521 staff (response rate $53 \%$ ). At time 2, employees were asked to rate their manager's active support in making changes associated with the implementation of team working (Randall et al., 2009).

Results: Structural equation modeling revealed that working conditions before the implementation of teams were related to good working conditions at time 2. However, time 1 working conditions also predicted the degree to which the manager was perceived to have been actively supportive of change. This management support was, in turn, positively related to working conditions at time 2. Therefore, the impact of the intervention was partially mediated by the actions of the line manager. After controlling for baseline levels of well-being and job satisfaction, working conditions at time 2 predicted well-being and job satisfaction at time 2 .

Discussion: This study offers new knowledge on two important issues. First, this study establishes the importance of considering the role of the middle manager when implementing team structures. The study provides valuable of information of what may be required in order to the outcomes of team implementation to materialise in other settings. Second, using quantitative process evaluation may offer a cost-effective solution to manage and monitor intervention processes and may be integrated easily into existing intervention evaluation methods.

\title{
Safety Climate as Moderator on the Relationship between Risk Perception and Job-satisfaction
}

\author{
Nielsen, M.B., Mearns, K., Matthiesen, S.B., \& Eid, J. \\ University of Bergen, Norway
}

This study investigated the relationship between risk perception, safety climate, and jobsatisfaction in a random sample of Norwegian offshore workers $(N=1,017)$. It was hypothesized that high levels of risk perception is related to low job-satisfaction, and that safety climate moderates the relationship between risk perception and job-satisfaction.

The findings supported both hypotheses in that offshore workers that experienced high levels of risk perception reported lower levels of job-satisfaction, whereas this effect diminishes when workers perceived their safety climate as positive.

This study provides further evidence to support relationships between safety-related concepts and work-related outcomes indicating that organisations should not only develop and implement sound safety measures to reduce the effects of risks and hazards on workers but can also enhance other areas of organisational life through a focus on safety. 


\title{
To Work or not to Work in an Extended Working Life
}

\author{
Nilsson, $\mathrm{K}$. \\ Department of Work Science, Business Economics and Environmental Psychology. Sweden
}

Introduction: In great part of the industrial world the population is increasingly becoming older. This will probably result in changes on the labour market for a great part of the countries. In Sweden 22 per cent are going to be 65 years or older in 2020. The average pension age in Sweden for the ones who are a part of the work force at age 50 is today 63,8 years for male and 62,5 years for female (1). At the same time the remaining average duration of life for a person aged 65 is 17 years for men and 20 years for women (2). This means that the economic maintenance will increase for the society in the length of the demographic change in a close future. It will therefore be important to promote an extended working life if Sweden wants to uphold the welfare state, and it is likely that the pensionable age will be postponed. The comprehensive aim of this research projects is to examine how to make it possible to be a healthy worker and stay at work until an older age, and how to make the working life more suitable to the elderly.

Methods: The research questions have been examined by two surveys. The sample in the first study is 1.949 employees of 55 years and older in the health care sector $(3,4)$. The second study examines 905 municipal managers' attitudes towards their senior workers (5).

\section{Results:}

Study 1

The first study examines elderly workers' attitudes toward their working life $(3,4)$. The result showed that $55 \%$ could work until the age of 65 or over but only $44 \%$ wanted to do this. The main factors which affect whether the senior workers could or couldn't work until the age of 65 or above were; the physical and mental work environment, their health, the working pace and working time. The main factors that affect whether senior workers did or did not want to work until the age of 65 or above were; motivation factors like their private economic situation, stimulants and meaningfulness at work, to feel appreciation from the organisation, values of knowledge and experience and the possibility to obtain new knowledge irrespective of age.

\section{Study 2}

The second study examines managers' attitudes toward their elderly workers (5). The first study stated that it was important to the employee to feel positive appreciation and subtended by the head and the organisation if they want to work until an older age, but how were the manager's attitude towards the elderly? $41 \%$ of the managers indicated that it was important to keep the employees in the organisation until they were 65 , but only $14 \%$ indicated that it was important to keep the employees until 66 years or above. The manager's attitudes towards their elderly employees were based on some stereotypical thinking about elder's low capacity, elder's inadequate knowledge and elder's negative disposition to change. Many managers did not indicate things that will increase the senior worker's attitude in a positive direction about an extended working life. It seemed like the managers did not see their own impact and importance to motivate the employees to an extended working life.

Conclusions: For a great part of the industrial world the middle age increase which results in a demographic change. In a lot of countries this causes a situation where the community 
needs to promote an extended working life if they want to maintain the welfare state. These studies examine elderly workers' attitudes towards the working life, but also managers' attitudes towards their elder employees. The result shows that the working life needs to be adjusted to take care of the aging work force. Managers and organisations attitudes to older employees certainly affect why.

\title{
Using the Questionnaire on Experience and Assessment of Work with the Italian population
}

\author{
Pace, F., Civilleri, A., Foddai, E., Lo Cascio, V., Passalacqua, C., \& Zanca, M.A. \\ Università degli Studi di Palermo, Italy
}

Objectives: The assessment of work-related stress has been an issue that has attracted attention of Italian psychologists for a long time, but only in recent years it has been remarked in the laws protecting workers. In fact, it has just been recalled the need, in a legislative decree, to place special emphasis on psychological well-being of the worker and not just on his security.

The Italian professionals (Work, Organisational or Personnel Psychologists) who worked on the assessment of psychological risks of work-related stress, used instruments to assess the organisational climate primarily, by integrating qualitative methodologies (such as focus group) with quantitative instruments. These instruments are able only indirectly to measure the wellbeing of the subjects on the workplace. For that, they were integrated with others instruments (e.g. STAI, PANAS, GHQ etc.) built to assess the individual well-being of subjects but little adjusted to the working environments.

The strong demand for action on these issues, has recently led Avallone and Paplomatas (2005) to develop an instrument $(\mathrm{MOHQ})$ for the Italian population, able to integrate the organisational themes with the perception of stress levels and, in general, with the well-being of employees. Van Veldhoven and Meijman (1994) developed a questionnaire (QEEW, Questionnaire on Experience and Assessment of Work) which proposed a similar approach. This instrument includes a series of steps to assess some critical aspects of work organisations (Ambiguities about work, Communication, Relationships with colleagues etc.), aspects connected to the task (Pace and amount of work, Mental load, etc.. ) and issues related to a broader and articulated conception of well-being (Need for Recovery, Sleep quality, etc.).

The aim of this study is to test the possibility of Italian adapting of the QEEW, through a first survey conducted in Sicily. We are going to inquiry on internal consistency of scales and some evaluations on their concurrent validity.

Methods: The English version of the QEEW was translated and back-translated in Italian language, and a first version of the questionnaire was distributed to a pilot group of workers, as an evaluation of the content validity. We have contacted 8 companies ( 2 public/state-owned companies and 6 private companies, representing different economic sectors and productive); we will submit in these companies the QEEW Italian version with some instruments that have similar purposes, such as MOHQ and M_DOQ10 (Majer and D'Amato, 2005). 
Results: Data collection is currently in progress. We are evaluating the factorial structure and the internal consistence of the QEEW scales and subscales; we're also evaluating the concurrent validity of the measure, using other similar questionnaires.

Conclusions: The QEEW, based upon preliminary analysis of the data, seems to show a good internal consistence of the scales and a good degree of correlation with the different instruments considered conceptually overlapping.

\title{
The Effects of Job Demands, Control and Social Support on Exercise \\ Padover, D., Reeves, D., Henning, R., Faghri, P. \& Warren, N. \\ University of Connecticut, United States of America
}

Objectives: In an extension of research demonstrating a relationship between high stress job settings and health concerns, the objective of the present study is to use Karasek's model to test for relationships between job demands, job control, social support and confidence to engage in a new exercise program.

Methods: Workers from a high- tech industrial corporation were surveyed on numerous items, some of which included job characteristics, personal health practices, and social support. Based on statistical analyses, two groups of employees were identified as having high stress jobs. One group was as Karasek labeled, a High Demand/ High Control while the other was High Demand/ Low Control.

Results: A preliminary analysis of variance revealed that employees in the High Demand/ High Control group were more confident in their ability to engage in a new exercise program $(F=.038 ; 1)$. An additional qualitative analysis is planned to examine employeeresponses to open-ended questions for evidence that positive or negative social factors may be a key factor in this relationship.

Implications: It may be possible to provide various forms of social support found in the High Demand/ High Control group to employees in the High Demand/ Low Control group to improve the health status of industrial workers in these difficult jobs.

Conclusion: The present findings suggest that Karasek's Job Demand/Control model is predictive of differences in employees' confidence to complete an exercise program but further analysis of existing data is needed to determine if social support is a key factor in this relationship.

\section{Strategies of moral disengagement and attitude toward risk. Antecedents of the violation of safety rules at work}

\author{
'Palano, F., ${ }^{2}$ Cardellicchio, E., \& ${ }^{1}$ Ingusci, E. \\ ${ }^{1}$ University of Bari, Italy; ${ }^{2}$ Univerity of Verona. Italy
}

Objectives: This study investigates the combined influence of attitudes towards risk and moral 
disengagement on safety performance. Specifically, the objectives that we propose to verify with the survey is to test the influence of variables attitude towards risk and moral disengagement on the variable violation of safety rules.

Methods: The research involved a sample of 116 employees of a food company of Bari. The measure instruments used are: 1) Risk Attitude Scale, reduced and translated (Weber, Blais \& Betz, 2002); 2) an adaptation of Moral Disengagement Scale toward safety instructions (Barbaranelli \& Perna, 2004); 3) Safety Performance Scale (created ad hoc).

Results: The sample consisted predominantly of men (96\%) with different ages and years of service. The workers involved are distributed in three different places of production (pasta factory, biscuit factory, mill) and different departments. To investigate the influence of two independent variables, attitude toward risk and moral disengagement, on the dependent variable violation of safety standards, has been conducted a linear regression analysis. Are the mechanisms of moral disengagement and part of the attitude to risk to have a significant effect on the compliance behaviour toward safety rules. Cooperation with the Organisation for the protection of security appears to be inhibited by mechanisms for beneficial and comparison positive attitude towards the risk of playful nature. Compliance with the safety rules in acting business is negatively affected by the trend to the diffusion of responsibility.

Implications: The results underline the importance of contextual framework in the determination of safety behaviour. According to Barbaranelli and Perna (2004), moral disengagement is a result of the organisational behaviour toward safety. This implies that the focus of intervention for safety promotion must be the organisational context, its culture, its climate and its values.

Conclusions: This research has brought to light that are mainly moral disengagement mechanisms to predict compliance / violation behaviour of safety rules. This result emphasizes environmental responsibility in the emergence of behaviours which conflict with safety rules and the need for interventions designed not only on the basis of the worker characteristics, but also on the basis of organisational factors.

\title{
It's good to talk: exploring social support and well-being at work
}

\author{
Parkin, $\mathrm{T}$. \\ University of Edinburgh, Scotland
}

Background: Social support is a pertinent research issue as it has been found to directly influence physical health and well-being (e.g. Shumaker and Brownell, 1984). A wealth of research has been conducted on social support by academics and professionals alike, and across a range of disciplines (e.g. Karasek and Theorell, 1990; Berkman, 2000, Cullen and Whiteford, 2001; Kafetsios and Sideridis, 2006). In recent years, there has been an accumulating focus by human resource management (HRM) researchers amongst others, on the importance of social support at work as it has been found to affect employee well-being, outcomes and performance (e.g. Boselie, 2005).

Objectives: The research objectives were three-fold: 1) to explore conceptual issues surrounding social support and employee well-being. 2) to explore what factors influence experience and perception of social support and 3) to explore employee perspectives on the relative importance of social support as a factor affecting employee wellbeing. 
Methods: This was a case study of one large public sector organisation in Scotland. A 4-tier multiple methods approach was used. Firstly, all employees received an email with a brief introduction to the research and with a link attached if they wanted to access the online survey. At the end of the survey, participants could leave their contact details if they were willing to be interviewed on the same topic. A semi-structured interview was arranged for those who agreed, and held at their place of work. These participants were asked if they would be willing to complete a diary for a period of two consecutive weeks to explore the issues discussed further. Once completed, they were asked if they would be willing to attend a final interview to reflect on their participation in the research process and on their current perceptions and awareness towards social support and well-being.

Results: All four fieldwork stages have been completed. Immediate appraisal of this data indicates that there were 156 responses to the online survey. Of these 156, 31 agreed to be interviewed. Twelve of those agreed to complete the diary. All 12 diarists agreed to meet for a final interview. Analysis is due to commence imminently.

Implications: Findings from this research are of potential interest to Human Resources practitioners, Occupational Health providers and for organisational policymakers as evidence indicates that enhanced well-being at work can reduce recruitment and retention issues and improve productivity and performance (e.g. CIPD, 2007).

Conclusions: If available, preliminary results from the survey and the first interview stage will be presented at the conference.

\title{
Positive and negative organisational behaviour
}

\author{
Patlan-Perez, J. \& Zorrilla, D.M.N. \\ UNAM-UAEH, Maxico; Universidad Autonoma Del Estado De Hidalgo, Mexico
}

Objectives: The objectives of this paper are to analyze and to integrate the positive and negative organisational behaviours in order to define them, to characterize them, to generate theoretical approaches, and to develop interventions to generate positive behaviours and avoid the negative behaviours at the organisations.

Methods: The literature review include empirical researches and papers that are boarded integral or separately the positive or negative organisational behaviours. The literature review includes the papers published between 2000-2009. Also, I include previous studies that make main reference to two thematic: positive and negative organisational behaviour.

Results: The principal results obtained in this research include the conceptualization of two constructs, and the generation of the typology of positive and negative organisational behaviours. The positive organisational behaviour is the study and application of positively oriented human resource strengths and psychological capacities that can be measured, developed, and effectively managed for performance improvement in today's workplace (Luthans, 2002). In this construct its possible includes multiple positive organisational behaviours (related to positive psychology), for instance: engagement, work satisfaction, commitment, and motivation. The 
negative organisational behaviour includes the behaviours that the workers develop at the organisation with the purpose of damage to the coworkers or to the organisation (related to dark side of organisational behaviour). In this approach there are many negative behaviours, for instance: deviant behaviour, counterproductive behaviour, bad behaviour, misbehaviour, and anti-social behaviour. There are negative organisational behaviours associated to four antecedent factors: leadership, direction, and power (e.g. mobbing, discrimination, isolation, harassment, authoritarianism); organisational structure, and design (e.g. alienation, stress, work overload); work climate (e.g. hostile climate), and individual factors (e.g. violence, aggression, presenteeism, absenteeism, turnover).

Implications: The integration of two constructs (positive and negative organisational behaviours) has important practical implications at the organisations, and to development future researches. For the organisations is important to recognize the extensive potential that exists to develop the positive behaviours. Of equal forms, it's important to identify and to attend with opportunity negative behaviours that damage the productivity, the human relations, the teamwork, and the work environment.

Conclusions: The principal conclusions indicate the importance of the study, to analyze, and to design interventions at the organisations, focused: to develop positive organisational behaviours and avoid negative organisational behaviours. In both cases, the efforts should be focused to achieve the well-being at work, quality of life at work, and the equilibrium between workworker-family-company.

\title{
Which is the importance of teachers' competence development programs? The relation between burnout, depression and anxiety in Portuguese teachers
}

\author{
Patrao, I. \& Santos-Rita, J. \\ Primary Care Center, Odivelas/Lisbon and ISPA, Portugal
}

Burnout, depression and anxiety are currently major preoccupations not only on Public Health matters, but particularly on Occupational Health intervention. An increasing number of international studies report that teaching is one of the most stressful occupations (Kokkinos, 2007; Yong \& Yue, 2007). For this reason, occupational stress that teachers experience in the process of performing their professional responsibilities has been a subject of growing interest in recent years (Capelo, Pocinho, Jesus, 2009; Gomes, Silva, Mourisco, Silva, Mota \& Montenegro, 2006; Pinto, Lima \& Silva, 2003; Platsidou \& Agaliotis, 2008).

The incidence of anxiety and depression among teachers appears to be remarkably high (Aznar, RodrÃguez \& Aznar, 2006). Although there has been little research exploring these variables in relationship to burnout, given the symptoms of burnout (emotional exhaustion, depersonalisation and lack of personal accomplishment), there is a good reason to believe that burnout may well be associated with feeling that one has been placed in an unwanted subordinate position and that the stresses in one' life are so overwhelming that they have lead to a sense of defeat. Moreover, as noted by Schaufeli and Buunk (2003), burned-out individuals tend to feel helpless, hopeless and powerless, and to experience feelings of insufficiency, incompetence and poor job-related self-esteem - all experiences that suggest a subjectively low status and a sense of defeat (Glass \& McKnight, 1996; Rosse, Boss, Johnson, \& Crown, 1991). 
The current study seeks to present a part of a larger investigation, which evaluates burnout and emotional adjustment in teachers of public schools in Lisbon.

The evaluation was based on Cuestionario Burnout Profesorado - Revisado, Depression and Anxiety Scale (DAS-14, Pais-Ribeiro, Honrado \& Leal, 2004) and Demographic and Professional Questionnaire which were administered to a sample of 383 Portuguese teachers.

Our results showed that teachers with higher levels of burnout present higher levels of anxiety and depression. Related to this, teachers present special needs on professional development support, public validation and financial rewards.

It is now required a fresh and continued formation where other values rather than technical ones are taken into account. Personal, emotional and professional competence development programs are essential, because it will allow teachers to control emotions as well as their creative and innovative skills.

\title{
Causes and consequences of the utilization of work-life policies by professionals
}

\author{
${ }^{1}$ Peper, B., ${ }^{2}$ Dikkers, J., ${ }^{2}$ Vinkenburg, C., \& ${ }^{3}$ van Engen, M. \\ ${ }^{1}$ Erasmus University Rotterdam, the Netherlands; ${ }^{2}$ Free University Amsterdam, the \\ Netherlands; ${ }^{3}$ Tilburg University, the Netherlands
}

The European work place has changed. Employees increasingly ask for organisational policies that allow them to balance their work and their private life. Organisations also become aware of the work-life conflicts of their employees. In addition to these negative influences between work and home, researchers nowadays also differentiate positive influences between the two domains: work-life enrichment or facilitation. According to recent work-life studies, the utilization of work-life policies does not unequivocally lead to lower levels of conflict or to higher levels of enrichment between work and life. In this study, we examine the association of flexible (targeted at increasing employees's temporal and spatial flexibility) and care-related (aimed at assisting employees in combining work with care giving) policies with work-life enrichment. Research further suggests that managerial support is critical when it comes to the utilization and effectiveness of work-life programs (e.g. Allen, 2001; Fried, 1999; Perlow, 1997; Thompson, Beauvais, \& Lyness, 1999; Veenis, 2000; Maxwell, 2005), as it is up to managers or supervisors to communicate, implement and manage work-life policies in organisations (Lewis, 2003). In addition, research consistently shows that the level of support that employees receive from their supervisor is crucial in alleviating conflicts between work and life (e.g., Mesmer-Magnus \& Viswesvaran, 2006).

Case study research emphasizes that managerial attitudes and practices are shaped by the organisational culture in which managers operate. A manager's response to a request is influenced not only by official policy, but also by the 'unwritten rules' of an employing organisation. Consequently, this study also relates supervisor support (and work-life culture in general) to the uptake of (flexible and care-related) work-life policies and work-life enrichment. Finally, few previous studies have examined the associations of work-life integration with professionals' career progression. Objective career success in particular has rarely been related to work-life balance. Here, we study the associations of utilization of work-life policies, work-life culture and 
work-life enrichment with professionals' career advancement. Managing the work life balance by professionals is the central focus. We analyze the utilization of work-life policies in association with work-life culture, work-life enrichment, and career advancement among Dutch professionals. This analysis is based on survey research in two samples. Next, we elaborate on the role of supervisors in the (non-)utilization of policies in a Dutch financial organisation. Supervisors were interviewed on the use of work-life policies by their employees, and their attitude towards granting work-life policy requests. This analysis is based on qualitative interviews.

\title{
Quality of Life and Job Satisfaction: Multicultural Perspective
}

\author{
Pereira, J. \& Sousa, A. D. \\ Instituto Superior da Maia, Portugal
}

In the Western society, work is a central aspect in people's lives, and research has emphasized the consequences that it may have on the health, Quality of Life (QOL) and well-being of the population. The person-Work dynamic includes a broad of aspects that affect this relation. Considering the PE-Fit Model (French et al., 1972), the Six Areas of Worklife (Maslach \& Leiter, 1997;2001), the Needs and Spillover Theories (Sirgy et al., 2001; 2002; 2006; 2007), we proposed that the equilibrium person-work environment will influence the way people perceives their work, feeling it as pleasant when it allows to fulfil their needs through the existence of suitable organisational resources. On this basis, we proposed a conceptual model in order to verify and predict the relation and effect of the dynamic person-work context on the Quality of Work Life (QWL), Job Satisfaction and QOL, underlying the present cultural diversity of the Portuguese's organisations.

With regard to the proposed aim, we obtained cooperation from public and private employees. The sample is composed by Portuguese and foreign workers $(N=128)$. Using the General Population Questionnaire of The Investigation Unit in Health and Occupational Health Psychology, was collected data related to the measures of person-work congruence (workload, control, reward, community, fairness, values), Job Satisfaction and QOL (Physical, Psychological, Social Relationships, Environment). Group-specific samples were analysed using descriptive statistics, and to examine the implications, strengths and predictive effect of the proposed model, logistic regressions analysis were used.

Considering the worker's cultural particularities we verified that this imposes itself as an important variable of the perception of QWL, Job Satisfaction and QOL. In relation to the efficiently of the adopted conceptual model, it predictive validity, was confirmed, verifying that the elements of the relation work and QOL converge in an inter-dependent relation. Thus, the person-work dynamic predicts the QWL $(R 2=.880 ; p=.000)$, this the Job Satisfaction (R2 $=.403 ; \mathrm{p}=.000)$ and this last, the QOL $(R 2=.151 \mathrm{p}=.000)$. Differences between the two group samples were observed. The results show that the Portuguese workers have lower satisfaction levels in all the components of the model, comparing to the foreign workers: Congruence Person-Work, in the reward $(p=.000)$ and workload; QWL; Job Satisfaction and QOL.

The consistent association between QWL with Job Satisfaction and QOL calls for improved investments into better QWL, in particular an appropriate balance between the person and 
organisational environment. This also underlines the influence of work environment in people's general QOL.

\title{
Correlation between psychosocial factors and work accidents
}

\author{
Picchi, M.P.C. \& Erba, A.M. \\ ECoNA (Interuniversitary Centre for research on cognitive processing in Natural and Artificial \\ Systems), Italy
}

Objectives: The "human factor" has been shown as the main cause in $70-80 \%$ of labour accidents. The law asks enterprises to spend many of their resources in the evaluation and prevention of risk and they usually direct their efforts just to better employees training and individual safety devices.

Workers' mistakes or (sometimes) intentional risk-seeking behaviours are determined by their degree of knowledge about individual safety devices and, even more, by their opinions and ethical values and by the distinctive features and behaviour of their working team. We want to explore if the evaluation of some psychosocial factors as: job commitment, cohesion inside working groups, self efficacy, quality of communication, worker's personal attitude towards safety and the "direct boss" can be used to prevent labour accidents due to "the human factor".

Methods: We have tested, the above mentioned psychosocial factors with the SInPe L questionnaire (Erba. A.M., Picchi M.P.C., Olivetti Belardinelli M.) "78 items on Likert scale from 1 to $5^{\prime \prime}$ in a group of 139 workers divided into three main areas (area 1, area 2 and area 3) of the production division of an Italian industrial unit. We have also calculated the frequency of the accidents (number of accidents/number of workers) and the severity of them (number of days of absence) in each area, within a period of 20 months.

Results: We have found a fairly significant difference ( $p=, 069$ with ANOVA one way analysis) in "communication", and a very significant difference $(p=, 002)$ in "direct boss" factor. Workers in area 2 have better scores in both the factors and show lower frequency of accidents and fewer days of absence as a consequence of them.

Implications: Communication and competence of the "direct boss" are the most important means for any organisation to manage all its working groups. A working group with a high level of communication and an efficient supervision can probably operate in a safer way.

Conclusions: The evaluation of psychosocial factors can not only be used to estimate "psychosocial risks" as stress, bossing and violence at work, but can also represent an important approach to first level prevention of labor accidents and injuries. 


\title{
Confirmatory Factor Analysis of the Toronto Alexithymia Scale among a sample of nurses
}

\author{
Pisanti, R., Violani, C., \& Lazzari, D. \\ University of Rome 'Sapienza', Italy
}

Background: Both management and regulation of emotions are considered central parts of work in the nursing profession. The interaction with other health care employees, patients and supervisors demands empathy and emotional involvement which many nurses consider more and more difficult as time goes by. Sifneos (1973) applied the term alexithymia to indicate a set of cognitive-affective characteristics including a marked difficulty to verbally express their emotions, an absence of fantasy, and a concrete stimulus-bound, externally-oriented thinking style. The TAS-20 was designed to assess three dimensions (Difficulty Identifying Feelings (DIF 7 items); Difficulty Describing Feelings (DDF 5 items) and Externally Oriented Thinking (EOT 8 items) of alexithymia construct. While the TAS-20 is recognized as one of the most widely used instrument to measure alexhithymia, its structural validity is not beyond question (Caretti \& La Barbera, 2005 ). A review showed as the factorial structure and reliability of the scales 'identifying feelings' (DIF) and 'describing feelings' (DDF) could be replicated in much previous research, whereas the original dimension 'externally oriented thinking' (EOT) appeared to be unstable and unreliable (Kooiman, Spinhoven, \& Trijsburg, 2002).

Objectives: The conceptualization and psychometric properties of the 20-item Toronto Alexithymia Scale (TAS 20 Bagby, Parker, \& Taylor, 1994) were examined by this study.

Methods: The present research used confirmatory factor analysis to verify the three-factor structure for the TAS-20 in a sample of 564 Italian nurse (Female $=76.4 \%$; with a mean age of 43.7 years $(S D=7.9)$ ).

Results: A new 16 items three factor solution showed the best fitting model ( Chisquare $=365,2$ D.F. $=101 \mathrm{p}=.000 ; \quad$ Chisquare/DF $=3.62 ; \mathrm{GFI}=.92 ; \mathrm{RMSEA}=.068 ; \mathrm{RMSEA} \mathrm{Cl}=.061-$ .076). Factors corresponding to DIF and DDF scales were well defined by their original items. Analyses revealed that the scale could be improved by deleting 4 items of EOT dimension (Delta $\ddot{i} £ 2=349,8 ; \mathrm{DF}=66 ; \mathrm{P}=.0000)$. Items were removed on the basis of theoretical reasons and of their low factor loadings (.30).

Conclusions and Implications: Since all items deleted were negatively phrased, it is suggested to increase the psychometric properties of the TAS by including other positively phrased items of the EOT scale. Further research directions are discussed. The implications are discussed in terms of promotion of stress-management interventions. 


\title{
Psychometric analyses of the short-form Utrecht Work Engagement Scale (UWES 9) in Italian Health Care Workers
}

\author{
Pisanti, R., Violani, C., \& Lazzari, D. \\ University of Rome 'Sapienza', Italy
}

Background: Work engagement has been defined as "a positive, fulfilling, work-related state of mind that is characterized by vigor, dedication, and absorption" (Schaufeli et al., 2002). The construct is conceived as opposite to burnout and it is measured through 17 items of the Utrecht Work Engagement Scale (UWES). A short (9 item) version of the UWES has been published (Schaufeli \& Bakker, 2003). The psychometric testing of the both versions of UWES (17-item and 9-item versions) is still in progress and warrants further research.

Objective: The main purpose of this study was to examine the psychometric properties of the short-form of the UWES scale in Italian health care workers.

Methods: A total of 391 health care workers (Female $=72.4 \%$; mean age 43.8 years $-\mathrm{SD}=8.3$ ) participated in this study. Participants completed the brief version of the UWES and the Maslach Burnout Inventory - Human Service Survey (MBI-HSS, Maslach et al., 2001).

Confirmatory factor analysis, correlational analyses, and internal consistency were computed.

Results: The hypothesized three-factor structure of the UWES, consisting of Vigor, Dedication and Absorption is confirmed (Chi Square $=123,88$ D.F. $=24 \mathrm{p}=.000$; GFI $=.93$; CFI $=$ .93 ; RMSEA = .103; RMSEA Cl = .086-.122). The three subscales show acceptable internal consistency and significant relationships with the MBI-HSS.

Conclusion and Implications: The analyses indicate that in Italian health care workers the UWES Short Form has an adequate construct validity and reliability. Assessing of dimensionality of engagement in combination with burnout items seems to improve fit indices.

\section{A Test of influences of Work 'Family Conflict, Job Enrichment and Leader' Member Exchange on Employee Turnover Intentions}

\author{
Portoghese, I., Galletta, M., \& Battistelli, A. \\ University of Verona, Italy
}

Imbalance in the relationship between work and family can be a source of stress resulting in adverse effects on an individual's work attitudes and well-being (Frone, Russell, \& Cooper, 1992), as well as to organisations in terms of diminished employee effectiveness. Frone et al. (1992) assert that the work domain is regarded as the root cause of WFC. When examined with respect to organisational behaviours, employees with high Work-Family Conflict levels can resort to quitting organisation as a conflict managing strategy (Hammer, Bauer, and Grandey, 2003).

Since leaders can influence different characteristics of followers' jobs, including giving more autonomy at work and extra-resources (Bauer \& Green, 1996), job enrichment can be viewed as 
one of the main instrument used by leaders to feed the social exchange with followers and help them to resolve the imbalance between work and family domain. So followers who experience high levels of job enrichment should report low WFC.

The goal of this research is to test the effects of LMX and Job Enrichment on the relation between WFC and Turnover Intention.

Methods: The participants in the present study were 1047 nurses of 5 Italian Hospitals.

We tested our hypothesis by comparing models using the principles of structural equations modeling (SEM).

Results: The results show that high levels of both LMX and Job Enrichment influence negatively WFC that in turn influence positively Turnover Intention of nurses. Furthermore, LMX has a direct negative influence on turnover intention and a positive influence on Job Enrichment.

Implications and Conclusions: The results of this study may raise the level of awareness of organisations to the potential consequences of WFC into employees turnover intention. The study confirms the important role of LMX into influence the decision to quit organisation and expands the research including the role of leadership into influence the weight of job characteristics into WFC. An interesting avenue for future research would be to examine the weight of different leadership styles on WFC.

\title{
Work-related stress in the Health-Care sector. An evaluation
}

\author{
Raffaele, G., Cataldo, A., Nazzicone, F., \& Di Cecio, M. \\ Occupational Medicine IFO, Italy
}

One of the main areas of psychosocial stress that has recently attracted the attention of research is stress in the workplace. The human condition of work entails many aspects other than to simply produce 'goods'. It is a condition that involves other factors including identity, relationships and purpose. This is evident in the areas of therapeutic health and services. In fact, here work is a combination of goods/service, tasks/processes that intertwine with other more important issues, that go beyond individual characteristics, such as painful life events and uncertainty, sickness and disease and ultimately death. Deep psychological suffering and mental anguish cause patients to demand their needs being met and request the most unpleasant things subsequently stirring up a variety of profound emotional responses from their care-givers of either desiring to fulfil patients' needs or feel hostility, impatience, anxiety and behave aggressively towards them.

Despite technological advancements in the health profession, the care giver cannot exclude nor underestimate the rapport between people, that is, the one who asks for help and the one who gives help. Nor can the care-giver avoid any disturbance or kind of imbalance it may bring to the care-giver and patient relationship. It is therefore essential to train the care-giver/health worker appropriately in identifying and managing all the inevitable ambivalent emotions and situations that may emerge in the patient/care-giver dynamic. Equipping the health worker with the means to be able to be in control and not be overwhelmed by one's emotions when facing a patient in pain that can at times be frustrating. 
Stress reactions are designed to protect oneself and not harm oneself. This means that by evaluating the association between work and stress can facilitate the worker, at any level, in understanding and improving the relationship between their work situation with their work environment. Therefore, it is vital to provide tools of prevention, analysis and self-evaluation for workers and organisations that face and deal with stress in the workplace. This type of evaluation should not be underestimated nor viewed upon as a complex measure that arouses worry or anxiety. Thus, a conscious, responsible and constructive effort should be put into this approach.

In hospitals throughout Rome evaluations have been conducted to assess the association between stress and work, (risk factors, role assessments, environment, handing out questionnaires) not only with the aim to implement the rules and regulations that are currently in force (D.L.gs. $81 / 08$ and subsequent changes and additions) but to also understand and interpret situations that are at risk and as result employ adequate interventions.

\title{
Do individual coping strategies help or harm in the work-family conflict situation? Examining coping as a moderator between work-family conflict and well being
}

\author{
${ }^{1}$ Rantanen, M., ${ }^{1}$ Mauno, S., ${ }^{1}$ Rantanen, J. \& ${ }^{2}$ Kinnunen, U. \\ ${ }^{1}$ University of Jyväskylän, Finland; ${ }^{2}$ University of Tampere, Finland
}

In this study we examined the moderator role of coping strategies (problem-, emotion-, and avoidance-focused coping strategies) between work-to-family and family-to-work conflict and well-being (work engagement, job satisfaction, and family satisfaction). Furthermore, we considered coping either as a resource or risk factor in predicting work-to-family and familyto-work conflict and well-being. The study was based on the sample of 527 Finnish workers. Data were collected in spring 2007.

The results showed that emotion-focused coping was associated with higher work-to-family and family-to-work conflict. However, neither problem-focused nor avoidance-focused coping was related directly to work-family conflict. It was also found that coping strategies were directly related to the well-being outcomes. To summarize the results concerning these direct links, emotion-focused coping seemed to be a risk factor for self-rated well-being, whereas both problem-focused and avoidance-focused coping operated as resource factors, promoting employees' well-being.

Concerning moderator analyses, we found that emotion-focused coping was a vulnerability factor: emotion-focused coping was harmful for family satisfaction in a high family-to-work conflict situation. It was also found that irrespective of the level of family-to-work conflict, those who used more emotion-focused coping seemed to be less job-satisfied than the others. Emotion-focused coping might associate with rumination, or negative affectivity which, in turn, might explain its harmfulness in managing stressor or experiencing strain. In contrast to emotion-focused coping, avoidance coping was beneficial in a high family-to-work conflict situation: those who used more avoidance coping were more satisfied with their family life in such a stressful situation. No buffering effects were found for work engagement or for problem-focused coping. 
Our findings imply that employees should be offered stress management training, with a focus on their problems in maintaining a work-family balance, especially where they feel that family interferes with work. Moreover, such stress management training should emphasize a range of avoidance-focused strategies. For example, how to improve one's delegating and prioritizing skills. Employees should also be trained to avoid emotion-focused coping strategies because of their known harmful effects on well-being. Obviously, a package of different coping strategies and their flexible take-up would work best in a work-family conflict situation. Thus, employees should also be informed about the kind of coping strategies people usually use in stressful situations and their relative costs and benefits.

\title{
The Role of Sense of Coherence as a Mediator between Job Strain and Mental Health in Two Distinct Work Settings
}

\author{
Reeves, D. \& Henning, R. \\ University of Connecticut, United States of America
}

Objectives: The relationship between Job Strain, operationalized as high job demand and low decision latitude, and mental health has been well documented in the occupational health psychology literature. However, the channels by which job strain affects mental health are less clear. Sense of Coherence (SOC) is a person's global orientation toward life and has been shown to buffer relationships between stressful work characteristics and mental health. SOC has also been investigated as a mediator between job characteristics and job outcomes (e.g., job satisfaction, supervisor satisfaction), but no research to date has investigated the mediating role of SOC between job strain and mental health. Contrary to the moderating relationship that has been found in previous research, and expanding upon the mediating role of SOC, I hypothesize that SOC will mediate the relationship between job strain and mental health.

Methods: Participants were surveyed in two distinct work settings, one state agency ( $N$ $=321)$, and one large private aircraft engine manufacturing plant $(N=404)$, both in the Northeast United States. All measures used in the analysis are from previously validated and published scales. The mediation was tested using a SPSS macro developed by Preacher and Hayes (2004) which tests the indirect effect of the IV on the DV through the mediator variable, and tests the significance of this indirect effect using the sobel test of significance.

Results: The mediation analyses showed that SOC did in fact partially mediate the relationship between Job Strain and mental health in both the state agency $(p<.05)$ and the manufacturing plant $(p<.001)$.

Discussion: Although Job Strain has been shown to be related to poor mental health in countless publications, the mechanism by which it affects a person's mental health is less known. In the current study we found in two distinct organisations that a person's SOC acted as a pathway through which Job Strain negatively affected an individual's mental health. 
Implications: From these research findings we see that decreases in job control paired with increases in work demands has deleterious effects on a person's mental well-being, and that these effects occur partially through affecting one's SOC. An organisation could prevent these negative effects by increasing the control an individual has over their job and thus increasing a person's SOC and mental health. In addition SOC could be used as a key indicator of the effectiveness of interventions to increase employee control.

\title{
Culture of excellence and mental health of public service workers. A psychodynamic reading of work in Quebec and Switzerland
}

\author{
Resenterra, F. \\ Université de Lausanne, Switzerland
}

The last few decades have profoundly transformed the world in which we live. Managerial ideology has spread in very rapidly into ever larger sectors, and financial logic now reigns over all organisational systems, impregnating their modes of management with the ideas of excellence and perfection. The current of reforms of new public management aims, as elsewhere, to apply tools of management from the world of private management and business to the public sector.

In this poster presentation, I have chosen to focus on the challenge, in the public sector, of the expression of psychic suffering and the pathological manifestations which follow from it. I believe that this suffering is the consequence of new forms of work organisation and of modes of management inherent to these forms. The psychodynamic of work constitutes the approach retained for this presentation, because it is interested in the dynamic connections that permit the worker to maintain a psychological equilibrium despite the constraints encountered at his workplace. According to psychodynamic presuppositions, psychic suffering is linked to two types of transformation which threaten dignity and self-esteem: the introduction and generalisation of techniques of individual performance evaluation, and the generalisation of total quality criteria.

What is attacked in the first place, via the penetration of the culture of excellence to the centre of organisations, is the process of sense construction. Once the systems of evaluation and of recognition are disconnected from the values and the sense that the agents attribute to their work, pay will bring no satisfaction to the agent. The recognition obtained in these conditions has negative psychological repercussions for the workers, which then produce symptoms manifesting their lack of wellbeing. The introduction of management into a bureaucratic field increases the revealing signs of psychic suffering at work: stress, professional exhaustion, hyperactivity, burn-out, depression, and an increase in the consumption of psychotropic and antidepressant drugs, among others. 
This then brings me to formulate my research question in the following manner: in terms of the psychic health of public sector workers, what is the effect of the introduction of management tools from the private sector, and more particularly, of techniques of individual performance evaluation and of the generalisation of total quality criteria?

The fieldwork, properly speaking, consists in comparing two public institutions of a similar nature, but situated in different contexts, (country, culture, and so on). This will permit us to judge whether it is uniquely the organisation of work that impacts on the mental health of the workers, or whether other criteria equally play a role. It is by a qualitative approach, via focus groups, that the internal logic of the object of study will emerge. The comprehensive approach thus gives access to subjectively constructed sense of work, via the authentic speech of the participants. The psychodynamic of work, which is inspired by hermeneutics, in effect researches the workers'own understanding of the lived experiences of work. The aim being, finally, to give them in return an influence on their work situation, to the end of reducing the pathogenic effects of work and to open ways to pleasurable working.

\title{
Psychological and symptomatic stress-related disorders with Radio Electric Asymmetric Conveyer
}

\author{
Rinaldi, S., Fontani, V., Aravagli , L., Bini, S., Mannu, P., Castagna, A., \& Margotti, M.L. \\ Rinaldi Fontani Institute, Florence, Italy
}

The purpose of this study was the evaluation of the effectiveness in the improvement of the psychic and symptomatic stress-related disorders by means of radio-electric stimulation on some auricular reflex points with a device named the Radioelectric Asymmetric Conveyer. This study, was conducted on 124 subjects with psychological distress symptoms, and were assessed by the SCL-90 pre- and post-intervention. After randomization, two groups were created: 1) a group treated with effective REAC, and 2) a group that underwent the same treatment with disarmed REAC (the placebo group). There was a significant reduction in SCL-90 scores in the treated group as compared to the placebo group. The results of the psychometric tests showed that the subjects who underwent effective therapy showed a statistically significant $(\mathrm{P}<.05)$ reduction in their level of stress and psychological disorders compared to the control group. The reduction of the levels of perceived stress and the reduction of the psychic and symptomatic stress-related disorders, assessed with SCL-90 tests, obtained after one cycle of reflex auricular protocol REAC treatment, show that this treatment can be useful in the cure of psychological stress-related symptoms.

\section{Psychometric evaluation of radio electric auricular treatment for stress-related disorders: A double-blinded, placebo-controlled controlled pilot study}

\author{
Rinaldi, S., Fontani, V., Aravagli , L., Bini, S., Mannu, P., Castagna, A., \& Margotti, M.L. \\ Rinaldi Fontani Institute, Florence, Italy
}

Background: The aim of this double-blind randomized study was to test the efficacy of a radio electric stimulator device using an auricular reflex therapy protocol for stress-related psychological 
and physical symptoms. Methods The study was carried out on 200 subjects (138 females and 62 males) that voluntarily came to our Institute declaring to "feel stressed."

The participants were randomly allocated with a computerized procedure; 150 were treated with an auricular therapeutic protocol with a radio electric stimulator device (REAC) and 50 were treated with an inactivated, placebo REAC. Psychological stress was evaluated trough a self-administered questionnaire (Psychological Stress Measure [PSM]). Assessment data were collected at 2 time points (before the treatment [T0] and immediately after the therapy cycle of 18 sessions, approximately 4 weeks later [T1]). Results In the group treated with REAC, the psychometric evaluation after the therapy cycle showed a significant reduction in the PSM total scores $(107.8 \pm 23.13$ at T0 to $87.1 \pm 16.21$ at $\mathrm{T} 1 ; \mathrm{p}<0.5)$, while in the control group no significant variation in decreasing stress-related symptomatology was noted (107.86 \pm 25.80 at T0 and $106.32 \pm 25.88$ at T1; $p=$ NS). Conclusions The protocol of the auricular treatment with REAC appears to reduce the subjective perception of stress as "psychometrically" demonstrated by the significant reduction in the PSM test total score. This therapeutic procedure also provides a non-invasive, painless, and very simple innovative approach to treat the wide range of stressrelated disorders.

\title{
Evaluation of the work-stress correlation in industrial oil refining operators
}

\author{
Riso, F., De Luca, Annamaria, Barresi, G., Tanzariello, M.G., Velocci, S., Miceli, L., \& \\ Abbate, C.
}

Scuola di Specializzazione in Medicina del Lavoro,, Italy

Introduction: Vergani (2000) considers stress analogous to a job position that elicits an ensemble of mental and physical alarm responses when the job demands skills or resources that the employee does not have at his or her disposal. Gilioli (2002), instead, defines stress as a multidimensional and multifactorial reaction consisting of intrinsically negative aspects that are specifically related to work in its content, organisation and psychosocial environment. In 2004, the CEE affirmed its definition of the phenomenon as, "a condition that may be accompanied by physical, psychophysical or social disturbances or dysfunctions in response to a feeling of ineptitude in the affected individual, who often feels incapable or fulfilling what is expected of them." The objective of this study is to evaluate the neuro-psychological effects of oil refining in a group of oil plant operators in eastern Sicily.

Methods: This study was conducted on a cohort of 200 males between the ages of 28 and 55 with a mean working experience of 18 years. These subjects had all attained at least a middle school diploma and were all without a history of neuro-psychological or metabolic pathologies and of alcoholic tendencies. These oil-refining operators, responsible for a variety of different tasks within the investigated oil refinery in Syracuse, were all examined through the OSI questionnaire. The latter takes into consideration both the stress manifested at the time of the self-assessment as well as the patterns of behaviour, the attitude, the perception of control and the coping strategies displayed by the individuals. The workers were subdivided with reference to the organisation of their work schedule as either part-time or daily workers. The results were compared through the employment of statistical methodology to a group of administrative operators of the same oil refinery that were homogeneous for gender and age. The latter comprised the control group. 
Results: Among the items investigated were: 1) The satisfaction with their career, which emphasized both a difference between the oil operators and the control administrative operators, as well as a greater feeling of dissatisfaction and discontent among the part-time oil workers. 2) The satisfaction with regards to the actual work (oil refining), which highlighted a distinctly negative evaluation on behalf of the part-time oil workers who reported feeling negatively affected by the increased responsibility that they are held accountable for in the execution of their work. 3) The satisfaction with the setting and organisational structure of their work, which the experimental group failed to show a particular prevalence for, thereby suggesting that their work organisation is inflicted upon them, thus making it a suffered condition. In conclusion, this study elucidates the significant stress that oil-refining workers are subject to, and further suggests that this inherent stress is exacerbated by the perpetually altering sleep-wake rhythm inflicted upon part-time workers.

\title{
Exploring Racial and Ethnic Differences in Exposure to Work Stressors
}

\author{
Roberts, R. \\ National Institute for Occupational Safety and Health, United States of America
}

Objectives: Research indicates that racial and ethnic minority groups in the United States evidence considerably higher rates of morbidity and mortality than White Americans. For example, rates of hypertension, a major risk factor for heart disease, stroke, and adult disability, are inordinately high among African Americans/Blacks. And Type II diabetes is 2-5 times greater among Hispanics.

Scholars are increasingly speculating that these and other racial differences in health outcomes are due to greater exposure to psychosocial stressors-including stressors that are work-related. Thus, the main objective of this study was to explore whether there are racial and ethnic differences in exposure to job and workplace stressors and to clarify the nature of these differences.

Methods: In this study, analyses of the General Social Survey (GSS) were conducted to explore these questions. The GSS is a nationally representative U.S. household survey that is conducted by the National Opinion Research Center. A 76-item Quality of Work Life (QWL) module was included in the 2006 administration of the GSS. The module assessed a broad range of job and workplace stressors and was administered via face-to face interview.

Results: The sample consisted of non-institutionalized, English-speaking adult workers, age 18 and older $(\mathrm{N}=1,728)$. Roughly $73.1 \%$ of the sample was White/European-American, $12.9 \%$ Black/African-American, 8.2\% Hispanic American and 5.7\% other minorities. While preliminary analyses identified no significant differences in workload (i.e. job demands) between racial groups, minority workers reported having significantly less influence and control over their jobs and work environment (i.e. lower job control), and a greater effort-reward imbalance. Additionally, American minorities reported experiencing higher levels of racial/ethnic discrimination and harassment along with significantly lower levels of respectful treatment at work. 
Implications: These preliminary analyses suggest that racial and ethnic minorities are differentially exposed to certain stressors associated with their job and workplace which may lead them to experience higher levels of job stress than White Americans. Overexposure to generic stressors (i.e. job demands, job control) may be due to greater minority representation in blue-collar occupations while differential exposure to other types of stressors (e.g., harassment) may be directly linked to their racial and ethnic status.

Conclusions: Additional research in this area is needed. The results of this study suggest, however, that job stress models and taxonomies of job stressors are in need of considerable expansion so that they are useful in understanding occupational safety and health in racially and ethnically diverse labor forces.

\title{
Psychological well being: levels of vitality and inner resources as relevant components of eudemonic well being
}

\author{
Rodriguez-Carvajal, R., de Rivas, S., Moreno-Jiménez, B., Blanco, A., \& Alvarez, A. \\ Autonoma University of Madrid, Spain
}

Objectives: The multidimensional model of well-being, created by Carol Ryff, proposes the following dimensions to study psychological well-being (PSWB): self-acceptance, positive relations with others, autonomy, environmental mastery, purpose in life, and personal growth. One of the main criticisms labelled at this proposal is based on an excessive ethnocentrism in the operationalization of the PSWB construct linked to the contemporary individual societies. Because of this reason, the aim of this study was to extend the PSWB model with two scales: inner resources and vitality.

Methods: Four hundred and nineteen adult people representative of Madrid completed the survey, aged 18-72 years (mean $=32.4, \mathrm{SD}=13.5$ ). Through a cross-validation approach, exploratory and confirmatory factor analyses were performed.

Results: The scales of the extended version maintain and raise its internal consistency (Cronbach alpha's 0.84 to 0.70). The extended model also showed better psychometric data in validity for the scales of the extended model of PSWB. Using confirmatory factor analysis, three-, five-, six- and eight-factor models were compared. The model that fit the data best was the eight factor model with relative fit indices of $0.90(\mathrm{NNFI})$ and $0.91(\mathrm{CFI})$ (SRMR 0.06).

Implications: This study provides researchers interested in using Ryff's Scales of Psychological Wellbeing with additional information to make an informed decision on the scales and items to use.

Conclusions: The model that fit the data best was the extended version of PSWB, hereby confirming Ryff's model in a non-Anglo-Saxon culture with the extended scales of vitality and inner resources. 


\title{
Complementation and compensation effects of psychological and subjective well being on their predictive capacity of positive and negative human functioning
}

\author{
Rodriguez-Carvajal, R., Alvarez, A., Diaz, D., Moreno-Jiménez, B., Blanco, A., \& de Rivas, S. \\ Autonoma University of Madrid, Spain
}

Objectives: Research into the good life has been encouraged by the seminal work of Ryff. She developed an integrated theoretical framework of well-being and distinguished six core dimensions: Self-acceptance, Positive relations with others, Autonomy, Environmental mastery, Purpose in life, and Personal growth. Recently this model has come under discussion centered on its ethnocentrism linked to the contemporary individual societies. The main aim of this study was to analyze the predictive capacity of Psychological Well-Being (PSWB) scales extended with the validated scales of vitality and inner resources, and Subjective Well-being (SWB) scales (Positive Affect, Negative Affect and Satisfaction with Life), on positive and negative human functioning, i.e. self-esteem and depression.

Methods: Through a self-questionnaire methodology, the sample consisted of three hundred and fifty four participants living in Spain, aged 18-68 years (mean =32.2, SD=11.3), with 162 male and 192 female.

Results: Using confirmatory factor analysis, the structure of PSWB and SWB models was analyzed. The following factor analytic models were specified: (1) the original six-factor structure as described by Ryff, orthogonally related to the SWB scales; (2) the original six-factor structure as described by Ryff obliquely related to SWB scales; (3) the extended model of PSWB with vitality and inner resources scales, orthogonally related to the SWB scales; and (4) the extended model of PSWB obliquely related to the SWB scales. The model that fit the data best was the forth one with relative fit indices of $0.94(\mathrm{NNFI})$ and $0.96(\mathrm{CFI})$ (SRMR 0.04). Then, through ANCOVA analysis, the predictive capacity of extended PSWB and SWB model was tested. The data showed that different levels of both models of WB significantly predict self-esteem $(F=59.89 ; p<0.001 ; r=0.34)$, and depression $(F=46.18 ; p<0.001 ; r=0.29)$, with significant differences by paired comparisons.

Conclusions: In order to predict levels of self-esteem and depression, extended PSWB and SWB models showed complementation and compensation effects that denote their close connection and singularity.

\section{Why do some people have no back pain? Subjective characteristics of a middle- aged working population compared to a population-based cohort}

\author{
${ }^{1}$ Rolli Salathé, C., ${ }^{2}$ Melloh, M., 'Isalue, I., ${ }^{1}$ Semmer, N.K., \& ${ }^{1}$ Elfering A. \\ ${ }^{1}$ University of Bern, Switzerland; ${ }^{2}$ University of Otago, Dunedin, New Zealand
}

Objectives: evaluate characteristics of a pilot group of individuals never having experienced low back pain (LBP) in comparison to a population-based cohort. 
Methods: $\mathrm{t}$ of 200 persons aged between 52 and 68, 13 persons who never had experienced LBP before answered a structured interview assessing potential factors for never having suffered from LBP and completed a questionnaire which had been part of a nation-wide assessment about LBP. Answers were content-analysed, and questionnaires were compared to a populationbased cohort ( $n=4,213$ ) using percentile ranks (PR).

Results: Our main categories emerged for reasons why some people didn't develop LBP:

a) Sports and hobbies: specific back training, extensive endurance training, body work

b) Movements during activities of daily living and in work environment: frequent routine movements, climbing stairs

c) Disposition: absence of a family history of LBP, healthy constitution

d) Positive attitude towards life: taking responsibility for oneself, management of resources and limitations

Compared to the population-based group, the 13 individuals scored higher for the ability to achieve routine movements ( $P R=65)$. Also, they less frequently attended physicians $(P R=13)$, and had more positive attitudes towards life $(P R=87)$.

Concerning working factors, the pilot group coped better with work (PR $=61$ ), and felt treated less unfair at work $(P R=39)$. For the will to achieve something or the determination to work for success, the group was ranked with PR 65.

Implications: approximately $80 \%$ of all individuals beyond 50 years of age experiencing LBP at least once in their lifetime, little is known about those persons never having had LBP.

To minimise work absenteeism and to decrease socioeconomic costs, knowledge about persons resilient to the occurrence of LBP may help to enhance preventative behaviour in personal as well as occupational settings, such as to motivate persons to train their extensive endurance by using stairs at the office.

Conclusions: Individuals above 50 years of age never having experienced LBP are more likely to be either physically active during work and daily routine or to perform extensive endurance training.

Opinions and motivations, such as conscious and careful usage of one's bodily resources and limitations or taking good health not for granted, as well as active and proactive health behaviour and fair treatment at work possibly help preventing individuals from developing LBP.

\title{
Becoming person's values - Protection against violence
}

\author{
Romanyuk, L., \& Surkov, P. \\ National University, Ukraine
}

The problem of violence is always urgent, but especially it becomes aggravated in days of crisis periods of instability when violent psychological attacks are even more often observed. 
Often, one observes open forms of violence, and even more often hidden forms, when a subject does not perceive the reason of one's reactions (aggression, fear, depression, frustration, etc.) resulting from unconscious influence of violence. How should one survive in the environment of violence not at the level of simple physical existence, but for the sake of preservation of one's personality, dignity, and human face? This guarding and directing functions of a person are performed by person's values.

Finding-out psychological features of person's values becoming in his models of the world, and also substantiation of psychological technology of revealing compounds of this model and activization of person's values becoming as a protection method in the situation of a violent psychological attack is the objective of this research.

The technology of search for person's values by a person allows to create a certain reference point, a base of all hopes and projects, a way to decode daily experience and even behaviour. Such approach opens unperceived values and opinions that organise them and form a person's model of the world. It means advancement, movement to the future, transition from one qualitative state to another, i.e. to becoming of values.

Becoming means occurrence in the process of development. Development in the psychological and pedagogical science means changes in the private world of a human person due to external influences and person's own activity, which is based on person's values and value orientations, the activity directed at achievement of such consequence. This definition is further specified by the psychological science. The author's approach has been elaborated in practical realisation of the voluntary desire of a person to choose one's life and to be rather an actor than a passive viewer. It entails responsibility for the choice, the necessity and ability to fix one's objective in various spheres of life, both personal, and professional, the search for an often unobvious, but useful measure for an attractive idea, which in its turn refers to the issue of choice (What? Why? Depending on what? How?).

Thus, the basic material of formation of values in a model of the world of a person is the revealed list of its internal conditions, each of which is analyzed on the basis of the preliminary established matrix. It includes physiology of internal conditions, their context on the basis of the lived experience and the criterion that defines the value. The information collected in the course of use of the psychological technology serves the basis of the verbal and logical establishment of person's model of the world and establishment of values through perception of meaningful unconscious questions and statements. Mastering this technology in the system of violent influence provides that 'support point' that directs at the choice of a correct way and teaches to avoid unjustified risks, and also helps to realise values and priorities of life.

\title{
The bullying at work in the public administration
}

\author{
De Rossi, P.
}

Istituto Nazionale di Ricerca per gli Alimenti e la Nutrizione, Italy

Only in recent years has bullying been a topic of significant concern in Italy. According to the European Parliament Resolution A5-2083/2001 of 20 September 2001, approximately 12 million people - "a considerably underestimated data" - experienced bullying in 2001. Although 
this Resolution calls on Member State to equally address this issue and to elaborate relevant legislations, so far an anti bullying law has not been promulgated in Italy even if more and more attention is being paid on this phenomenon.

This results in a series of issues, such as:

- Lack of uniformity in terminology and approaches to identify the phenomenon that is, therefore, left to the discretion of the trial judge.

- The need to identify legal provisions already existing in the Italian legislation (Criminal, Civil and Administrative Law) that might be related to the phenomenon.

Consequently, to define bullying four elements should be borne in mind and they are shortened in the acronym P T H S:

P Penal: Arts. 40- 81- 572- 582- 583- 589- 590 of the Penal Code.

T Time: At least six months (EGE 6-phase Italian Model) or three months for quick bullying.

$\mathrm{H}$ Health: workers' health is affected.

S Security: occupational security is jeopardized.

According to the current elusive legislative framework, the distinctive features of bullying are:

DISHOMOGENEITY of the mobber's harassing behaviours characterized by endless series of intentional actions and omissions aimed at jeopardizing the mental and physical health of the victims;

CIRCUMSTANTIAL SPECIFICITY which enables to unmask bully actions and assess the stage reached by the phenomenon that can be:

bullying attempts;

ongoing Bullying;

false bullying.

In the light of this, Public Administration are required to comply with the various Collective Bargaining Agreements that fully acknowledged the Resolution A5-2083/2001 of 20 September 2001.

DISHOMOGENEITY characterizes bullying attempts and the bullying phenomenon in itself, whereas CIRCUMSTANTIAL SPECIFICITY is applied by who wants to clarify the matter and takes actions "a posteriori" or verifies the phenomenon.

In brief, "cultura nova propaganda est"; i.e. a new occupational culture must be popularized in Italy and all over the world, according to which no one can be victim of harassing behaviours at work for any reason and, in particular, because of or by the work the subject has to carry out. I wrote a book on this subject called "GUIDA AL FENOMENO DEL MOBBING NELLA PUBBLICA AMMINISTRAZIONE" showing an initial approach to the phenomenon of bullying with an eye to prevention, and in compliance with the existing Collective Bargaining Agreements.

Attached in the appendix of the book are the European Parliament Resolution of 20 September 2001 as well as the Italian regulations and the main sentences pronounced in the Italian context on this issue. An occupational "cultura nova" (new culture) more oriented toward the respect for the person as such will help building bridges among the various EU countries. The cultural traditions of every Member State will provide an opportunity for reflection and comparison in 
order to find a common road to go respecting the common good of all and, as a consequence, of the European Union itself.

\title{
School Burnout and Engagement during Educational Transitions
}

\author{
Salmela-Aro, K. \\ Helsinki Collegium for Advanced Studies, Finland
}

This presentation presents new concepts of school burnout and school engagement. School burnout is a school-related long lasting stress disorder including exhaustion related to school, cynical attitude towards school and inadequacy as a student, while school engagement includes vigor, dedication and absorption as a student. The aims of the presentation are to present reliable and valid measures to investigate school burnout and engagement, and examine how school burnout and engagement changes during education transitions from comprehensive school to educational tracks, during academic track and during transition to work life.

To investigate these questions, 800 students from the comprehensive school were followed five times during their educational transitions from comprehensive school to academic and vocational tracks and later on to university and work life. In addition 600 students from upper secondary high school were followed four times during their studies and transition to tertiary education. In each measurement time the students filled in school burnout and engagement scales and their life situation.

The results showed that a nine item school burnout scale including three dimensions of exhaustion towards school, cynical attitude towards school and inadequacy as a student is a reliable and valid measure. In addition, the nine item scale of study engagement, of vigor, dedication and absorption as one dimension is a reliable and valid measure. The results showed analysed by latent growth curve modeling showed that during transition from comprehensive school to academic track burnout increased particularly among girls. At the academic track $20 \%$ of girls suffered from school burnout. Moreover, path analysis showed that school burnout during academic track, during upper secondary education, decreased successful transition to tertiary education, while school engagement during upper secondary education increased successful entrance to university. School burnout and school engagement changes during critical educational transitions. School burnout can lead to delay in studies and difficulties in later career-related transitions.

\section{How do schools burn-out Portuguese teachers? The role played by age, type of contract and length of experience}

\author{
Santos-Rita, J. \& Patrao, I.
}

Primary Care Center - Odivelas/Lisbon; Escola Superior de Tecnologia da Saúde de Lisboa, Portugal

Stress is currently perceived as a cultural symbol. The World Health Organisation says that stress is a global epidemic and The United Nations suggests stress is the 21st. Century' 
disease. Occupational stress is recognized as a factor that influences not only individual health, but also organisation productivity and competition (WHO, 2004). Workers with high levels of stress are more vulnerable to illness and lack of motivation (WHO, 2004). An increasing number of international studies report that teaching is one of the most stressful occupations (Yong \& Yue, 2007). Long-term occupational stress may lead to burnout, which has negative implications on teachers'physical, mental and social health. In Portugal, some studies with teachers have concluded that levels of stress and burnout are higher within this professional group than within any other (Capelo, Pocinho, Jesus, 2009; Gomes, Silva, Mourisco, Silva, Mota \& Montenegro, 2006; Pinto, Lima \& Silva, 2003; Quirino, 2007). These facts suggest that teachers'stress and burnout are certainly a privileged area of intervention in Occupational Health Psychology.

The present study seeks to present an evaluation of levels of occupational stress and burnout in Portuguese teachers of elementary, middle and high schools. Measurement and evaluation was based on Cuestionario Burnout Profesorado - Revisado and Demographic and Professional Questionnaire (Santos-Rita \& Patrao, 2009) which were administered to a sample of 383 Portuguese teachers, in the Lisbon area.

Our results revealed high levels of burnout in $28 \%$ of teachers, which could constitute a major reason for health concerns. On the other hand, there is a statistical correlation between some demographic and professional characteristics. Age, type of contract and length of experience are directly related with higher levels of burnout. In fact, the older, fixed contract and larger length of experience teachers present higher levels of burnout.

This study suggests that the current context in educational policies in Portugal could affect individual and schools performance. Recent policy changes in areas related to teachers'careers and evaluation may be regarded as possible stress and burnout sources. The development of integrated programs could be helpful in this context, promoting individual and organisational health resources.

\title{
An evaluation of work-related stress through the integration of objective and subjective measures: the V.I.S. method and the Q-Bo test
}

\author{
${ }^{1}$ Sarto, F., ${ }^{2}$ Falco, A., ${ }^{1}$ Vianello, L., ${ }^{2}$ Corso, L.D., ' Zanella, D., ${ }^{3}$ Magosso, D., ${ }^{2}$ Marcuzzo, G., \\ ${ }^{2}$ Bartolucci, G.B., ${ }^{2}$ De Carlo, N.A. \\ 1SPISAL Prevention Department, Italy; ${ }^{2}$ University of Padova, Italy; ${ }^{3}$ Regional Reference \\ Centre for Occupational Ergonomy, Veneto Region, Italy
}

In accordance with European directives, the Authors of this study evaluated work-related stress in various organizations- within an integrated approach - using the results obtained through the Q-Bo test. Such test allows to identify the perception of the different stress sources, as well as their consequences, referring to normative statistical indices as well. The instrument was indeed standardized on a sample of about 20,000 people working in large, and small/medium-sized public and private organizations. From the general sample, several others were taken, relative to different organization categories on which further specific standardizations were conducted.

As is well known, subjective measures can be conveniently integrated, since they bring together several variables, such as, for instance, social desirability and negative affectivity, which can 
influence their validity. Moreover, their perspective is necessarily partial. In order to implement acquired and prospective knowledge, within the afore-mentioned methodologic integration, the Authors deemed it appropriate to also devise a method that might be more sensitive to "objective" dimensions. Such procedure, called V.I.S. Method - Valutazione per Indicatori di Stress (Stress Indicators Evaluation) - is based on a "multi-method" evaluation approach articulated in several phases and shorter, as it does not involve all workers. It is founded on a theoretical model in which the magnitude of work-related stress risk, measurable through focus groups and objective indicators, and consequent effects on workers' health, detected through the stakeholders' judgments, are connected to disease and symptoms assessed by the competent practitioner where present - and forewarnings. According to the value of the magnitude, the evaluation can be closed or will have to proceed with the complete stress evaluation (V.C.S. - Valutazione Completa dello Stress), obtained through the V.I.S. method and the administration of the Q-Bo test (or another instrument conceived to detect the worker's "subjective" perception).

The method is made up of 4 instruments used by company stakeholders and by the competent practitioner.

First instrument: the company focus group, composed of the employer's delegates (in particular experts of the human resource office), prevention and protection service managers, representatives of the workers' safety committee, the competent practitioner, and other professionals. Aspects are considered such as: contract typologies, quality certifications, elements of organizational management system and social responsibility, company typology, absenteeism, mobility, working hours, disputes, health data. Each indicator is compared with the standard in the literature, if present, or with the company records; and a negative or positive score is obtained. Second instrument: 40 out of the 350 items of the Q-Bo test were selected and grouped into 12 dimensions, among which work characteristics, social and organizational processes, safety management, professional growth, diversity management, and home/work interface. The answers supplied by the focus group components have to reach an agreement on the judgment. This is compared to the standard deriving from the answers to the same questions provided by the general sample of workers who were administered the Q-Bo test. Besides a final negative or positive score, such instrument allows to identify critical areas and design preventive interventions.

Third instrument: judgment of the competent practitioner, formulated for each company and department not only on the basis of objective data, but also on complaints, symptoms, related events, noted during his observation.

Fourth instrument: the competent practitioner, adequately trained, collected stress-related symptoms and psychic or psychosomatic disorders through a standardized questionnaire.

During the EAOHP Conference, this work will be extended on the theoretical level and will comprise various empirical results gained within organizations belonging to different fields. 


\title{
Influence of workload in emergency on discrimination against patients
}

\author{
Schoenenberger, S., Moulin, P., \& Brangier, E. \\ ETIC - INTERPSY, France
}

Objectives: The aim of this paper is to understand how discrimination encountered in health care services depends on the intensity of professional activities. In particular, we will examine how workload dimensions can explain organisational behaviour easily assimilated to discriminatory conducts.

\section{Theory orientation}

Studies about discriminatory behaviour against patients specified two principal dimensions:

- Refusal to provide care: direct refusal (i.e. doctors refuse to care for specific categories of patients), refusal to provide immediate care (i.e. doctors make a patient wait a long time in the waiting-room, hoping he leaves by himself), racist comments,

- Discriminatory preferential care: diagnoses correspond to the patient's presumed culture (i.e. diagnosis of an unusual pathology for a patient native to an "exotic" country), differences in care (i.e. refusing to give medicine to a patient judged a priori as non-observant). Health professionals explain those behaviours in function of their activity, in particular regarding their workload. Workload could refer to activity's intensity, communication difficulties with the patient (i.e. linguistic problems, deafness), "bad patients" (i.e. drug addict, homeless, elderly). On the other hand, patient's social characteristics (i.e. sex, age, and ethnicity) are less mentioned.

Methods: Our methodology is based on observations in situ and on verbalization from health professionals, so as to evaluate discriminatory behaviours, stereotype against different patients' characteristics, and measure workload in an emergency service.

Results: Our first results indicate that discrimination is more linked with workload than with the patient's social characteristics: for example, a foreign native patient will not be discriminated because of his native origin. Foreign origins become a factor of discrimination as soon as the patient expresses difficultly in French, complicates the interaction with the professional and increases the professional's workload. Those results corroborate a model with a link between workload and discrimination which we will discuss.

\section{Psychosocial work factors and bullying among employees in Norwegian nursing homes}

Schøning, M., Berthelsen, M., Christensen, J.O., Elka, S., Finne, L.B., Jacobsen, K., Pettersen, T., Utaaker, E., Vindsetmo, K., \& Lau, B.

National Institute of Occupational Health, Norway

Objectives: Workplace bullying is a serious problem considered to have impact on employees' health and on quality of customer service and patient care. Health care seems to be a sector facing high incidences of bullying. The scope of this study is to investigate levels of bullying in the nursing homes sector; both self-reported and observed bullying. A second scope is to test 
the work environment hypothesis on individual and on department level. That is whether poorly organized work environment may give rise to conditions resulting in bullying.

Methods: Data was collected by a web-based survey conducted in 21 Norwegian nursing homes with 2.269 employees invited to participate. The response rate was $64 \%$. The measurement instrument was based on the General Nordic Questionnaire for Psychological and Social Factors at work (QPS-Nordic), including 18 scales measuring psychosocial and organisational factors, and two single items covering self-experienced and observed bullying.

Results: $6.4 \%$ of the total sample reported self-experienced bullying, while $18.5 \%$ reported observed bullying. Conducting two-way ANOVA, preliminary results show that employees who report self-experienced bullying reported significantly higher negative scores on almost all QPSvariables than non-bullied, in particular on factors such as fair leadership, social climate, superiorsupport, and role-conflict. When the data was aggregated on department level, the results show that departments with reported bullying generally report higher quantitative demands and decision demands, less control of work pacing, less mastery of work, and less commitment to the organisation compared to departments without reported bullying. Withdrawing victims of bullying from the analyses, only quantitative demands and mastery of work remain statistical significant on department level.

Implications: The work environment hypothesis is only limited supported by our results. Our findings, however, show that individuals exposed to bullying experience their work environment inferior to non-bullied. Quantitative demands and mastery of work should be addressed with the aim of reducing bullying. It may also be worthwhile to focus upon management, conflictsolving, role-clarity and social support in order to improve work environment in general and for supporting individuals exposed to bullying.

Conclusion: Longitudinal studies and multilevel analysis are required for gaining better knowledge of the relationship between work environments and bullying on an individual and organisational level.

\title{
Job Stress Battery: Scales of Job Control and Occupational Outcome Variables
}

\author{
Sertel Berk, H.O., Ozalp Turetgen, I, Unsal, P., \& Basbug, G. \\ Istanbul University, Turkey
}

Objectives: In recent occupational health literature, job stress is treated as a multi-dimensional phenomenon to be investigated from a holistic scope considering a model of stressorsmoderators-outcomes in a sequence. However, in Turkey, there is no specific tool which measures occupational stress as a multidimensional process from this point of view.

Based on the multidimensional aspect, we embarked upon to develop the Job Stress Battery which consists of various job stress sources, personal and situational moderators and occupational and psychological outcomes. This study covers the presentation of the psychometric properties of the Job Control Scale (JCS) as one of scales of moderators, and occupational outcome scales such as Motivation (MS), Intention to Quit (IQS), Satisfaction with Performance (SPS), Organisational Commitment (OCS), and Job Satisfaction (JSS). 
Methods: Participants were a voluntary sample of employees from various sectors $(N=608$ for the occupational outcome scales; $\mathrm{N}=174$ for JCS).

The items of the moderator and outcome scales (MS-3 items, IQS-2 items, SPS-5 items, OCS6 items, JSS-4 items, and JCS-8 items) were written by the authors on the basis of job stress literature.

In order to test the construct validities of IQS, OCS, and JSS, a part of the participants $(\mathrm{N}=113)$ completed some questionnaires measuring similar constructs. The construct validity studies of JCS, MS and SPS are still proceeding.

Participants received scales in two separate weeks in random order in order to control the common variance effect.

Results: According to item analysis, items with item-total score correlations below .20 were removed from the scales. The resulting Cronbach Alpha values varied from .65 to .72.

The relationships between the developed scales (IQS, OCS, and JSS), and their counterparts were varied from .62 to $.66(p<0.01)$. The inter-correlations among the scales of the battery were significant and showed a pattern as expected $(p<0.01)$.

Implications: This preliminary attempt of developing a JSB is promising for both practical and theoretical purposes. Besides emerging as a first comprehensive measure of occupational stress in Turkey, the Job Stress Battery will serve as a tool for testing various theoretical models regarding occupational stress.

Conclusions: Results of the initial version of the moderator and outcome scales of the JSB indicate reasonable psychometric properties. Reliability and validity studies should continue across different occupations and organisations in future studies.

\title{
Job Stress Battery: The Job Stress Scale
}

\author{
Unsal, P., Ozalp Turetgen, I, Sertel Berk, H.O., \& Basbug, G. \\ Istanbul University, Turkey
}

Objectives: Considering the job stress as an interaction among the occupational context, the individual's responses to this context and the personal and situational variables became a dominant approach in the literature. This approach has crucial implications on measuring job stress. However, in Turkey, there is no specific tool which measures job stress as a multidimensional process. Based on this approach, developing the Job Stress Battery aims to investigate the sources and the moderators as well as the outcomes of job stress among Turkish employees. This study attempts to introduce the psychometric qualities of the Job Stress Scale (JSS) which is a part of the Job Stress Battery and measures stressors both in terms of the frequency and intensity.

Methods: The development of the JSS has been completed in three stages. First, a list of job stressors were determined by asking individuals the sources of stress which they experience at work $(N=79)$. Second, on the basis of the identified stressors and the review of the literature, 
an 84-item scale was formed and a pilot study was conducted $(\mathrm{N}=117)$. Finally, the JSS was administered to a sample of 589 employees. For the construct validation, the factor structure of the scale was investigated through factor analysis. Also, the correlation between the JSS and another job stress measure used in Turkey was questioned. Moreover, the relationships between JSS and job satisfaction, organisational commitment and symptoms of stress were tested. In addition, Cronbach Alpha coefficients were computed for internal consistencies.

Results: The factor analysis revealed a 7-factor structure with 49 items, explaining $46 \%$ of the variance. Cronbach Alpha Coefficients of the factors and the total scale varied from .55 to .91. The JSS was positively correlated with Stress Audit $(n=73, r=.39)$ and Symptoms of Stress Questionnaire $(n=61, r=.26)$. Also, negative correlations were found between the JSS and job satisfaction $(n=112, r=-.41)$ and organisational commitment $(n=115, r=-.34)$.

Implications: This primary attempt of developing a job stress scale as a part of the Job Stress Battery is encouraging to initiate a comprehensive evaluation of job stress in Turkey considering moderator and outcome variables.

Conclusions: The initial values of the validity and the reliability of the JSS show good psychometric qualities. However, although the sample in this study seems to be heterogeneous in terms of participants from different sectors, the factor structure should be re-validated across different occupations and organisations.

\title{
Development of Outstanding Students from the point of view the work of their teachers and companions
}

\author{
Soltero Avelar, R. \& Margarita Torres, T. \\ Universidad de Guadalajara, Mexico
}

Objectives: Define the characteristics that influence the development of outstanding student, to develop support programs for the remainder of the school population to identify factors outside the school that influence development, as family, social and cultural capital

Methods: Qualitative study, associative, Through the implementation of free listings, and focus groups, FL technique is to provide subjects with a word or term inducer and ask them to write the first words that come to mind about the word inducing, (in this case Outstanding Students). The main advantage of this procedure is that it encourages more spontaneous expression of the subjects, and therefore, is expected that the content evoked to be free about this rationalization, biases of the defense or desirability, done at the High School, between the months of June 2006 to February 2007, with student as informants, 89 semistructured interviews were applied with men and women students, and 20 protocols for characterization, key informant, was formed a focus group with 6 outstanding students in art, INSTRUMENTS; of inquiry, characterization, categorization, coding, building social graph representation.

Results: Conceptualization inductor associated with the term, "Outstanding Student", both men and women students, social representation of the concepts studied by teachers and students. 
Conclusion: Social representation graphs have 3 axis occupying the central word being studious in partnership with punctuality, be industrious and clever. With regard to the conceptualization and social representation of intelligence, effort and ability, pupils. Is directly related to the results of focus groups, of which we construct a semantic network where the main conceptualizations is associated with: the responsibility, effort, intelligence, dedication, work individually and in teams, ability, timeliness, the need to be a leader, or model.

\title{
Job insecurity, intention to quit, and psychological well-being: Analysing causal relations
}

\author{
Stiglbauer, B., Selenko, E., \& Batinic, B. \\ University of Linz, Austria
}

Job insecurity has been related to various individual and organisational consequences, such as increased psychological distress and the intention to quit one's job (Sverke, Hellgren, \& Naswall, 2002). Unknown still are the causal dynamics underlying this relation: do persons with less psychological well-being end up having less secure jobs or does perceived job insecurity have a negative effect on psychological health? Do persons with a higher intention to leave a job seek jobs with lower security or does job insecurity lead to a higher tendency to quit? In order to find an answer to these dilemmas we analysed the data from a large-scale online study, involving employed persons of various professions (nT1-T3 = 126 - 148).

The study was conducted in 2008 and 2009 in 3 waves, with six-month intervals between the waves. Across all persons, perceived job insecurity increased over time. Persons, who indicated an increase in job insecurity, also reported a decrease in psychological health. Results of multilevel modelling indicate that changes in job insecurity could explain changes in psychological well-being. Interestingly, the intention to quit rather caused changes in job insecurity than the other way around. Several moderators of the relation of job insecurity and psychological wellbeing and intention to quit were investigated. Findings will be discussed in the light of job insecurity research and related to recent economic downturns.

\section{Humour Style in Relationship to Stress and Well-being: Four Different Personalities}

\author{
Stokenberga, I. \\ University of Latvia, Latvia
}

Humour has been recognized as effective coping strategy in variety of scientific and self-help literature. Objective of the present study was to explore the role of individual differences of humour style in coping and well-being, distinguishing potentially adaptive and maladaptive humour use. Undergraduates $(\mathrm{N}=183)$ and employed adults $(\mathrm{N}=316)$ participated in this study and completed measures of daily hassles, perceived stress, humour style, several well-being scales and measure of personality. 
Results suggest four different portraits of humourous personality and their coping characteristics on the basis of the results of the present study. The first, 'soul of the company', is a highly extraverted person, quite open to experience and in a good mood. His of her ability to cope effectively depends on resources utilized from the group - social support, shared good mood and membership. The second is an emotionally stable, extraverted individual in a good mood, internally open to new, unusual experiences, and quite agreeable. This person has a 'humourous view of life' and has adequate individual cognitive resources - readiness and ability to take new perspectives and to laugh at everyday life and its absurdities. The third is 'the bad boy' who is neither agreeable, nor conscientious. He or she uses rude, hostile, and cynical jokes in the group; however using humour in order to laugh at others does not improve his or her long-term coping efficacy. The fourth character, 'The sad clown', is a stressed, depressed and anxious person, with quite a low sense of conscientiousness, overwhelmed with daily problems. He or she has difficulty coping with stress and allows and provokes others to ridicule him or her in order to gain at least some degree of attention from others.

In conclusion, it was found that different humour style dimensions have different patterns of relation to the coping with stress and well-being. Differences in the humour use should be acknowledged when promoting humour as an effective coping resource.

\title{
Life After Service Study (LASS): How are Canadian Forces Members doing after Transition to Civilian Life?
}

\author{
Sudom, K. \& Lee, J.E.C. \\ Defence Research and Development Canada \& Canadian Forces Health Services Group
}

Military to civilian transition is an extremely meaningful but poorly researched event in the life course of military Veterans. There has been surprisingly little research that tells us how many former military members have various outcomes, and how best to mitigate re-establishment problems. The research that does exist does not provide a standard approach to defining and measuring successful re-establishment. However, there seems to be consensus that there are many dimensions to re-establishment, of which employment is just one. Other dimensions include physical, mental and social health, mitigation of disabilities, determinants of health particularly income, and participation in work, family, community and leisure life to the fullest extent possible. The Life After Service Study, a collaborative effort between Veterans Affairs Canada (VAC) and the Department of National Defence (DND), was created in order to measure the health-related outcomes of released Canadian Forces members after transition to civilian life; to examine whether current programs are reaching those in need; to determine whether there are unmet needs not addressed by current programs; and to examine how outcomes compare between VAC clients and other Veterans. This study will be done in three parts aimed at measuring short and longer term health outcomes. All three parts of data collection will involve starting with a cohort of CF members that has been record-linked to VAC data on program participation. Part 1 will collect information on income changes; part 2 will collect health, determinants of health and disability measures through a telephone interview survey; and part 3 will collect information on mortality and cancer. The results will contribute to the program evaluation of the New Veterans Charter, to improved resource allocation of transition programs and services, and it will inform strategies to mitigate poor outcomes. 


\title{
Antecedents of entrepreneurial success - The role of impression management
}

\author{
Kamalanabhan, T. J. \& Reshmi, A. T. \\ IIT Madras, India
}

Impression management theory has received increasing levels of attention by organisational scholars in the last 25 years. However, most researches in the area have studied it as a part of social competence, with a special interest in social perception. This study adds to the literature as an exploratory attempt to study impression management as a precursor of entrepreneurial success. Five techniques of Bolino and Turnley were used for the study.

Objective: To study the effects of personality, social competence, entrepreneurial orientation and types of impression management techniques in entrepreneurial success.

Method: A survey of three hundred and eleven entrepreneurs was selected for the study. Closed ended questionnaire with a 5 point interval scale was used. The samples under study were entrepreneurs selected from Kerala and Tamilnadu. Convenience and snowball sampling technique was employed. The entrepreneurs were chosen based on the details available from banks. The criterion for selecting was that they should have minimum of two-year of established enterprise. The questionnaires were handed over in person to all the 311 entrepreneurs who were selected for the study.

Results: It was found that impression management techniques are indeed contributing to entrepreneurial success, along with other variables like personality, social competence and entrepreneurial orientation. Multiple regression analysis was done. The significant beta coefficients were taken in for better knowledge about the constructs. Need for affiliation, social adaptability and social perception, pro-activeness and some impression management techniques were found to have a relation with entrepreneurial success. Impression management techniques used for upward, downward and lateral relation was also verified. It was seen that entrepreneurs use supplication, self promotion more often than other tactics.

Implications: For governments, the findings can help to assist nascent entrepreneurs from the early stages of business formation, and help business development centers that screen entrepreneurs and identify patterns of psychological profiles to facilitate. Business practitioners should be aware of their own psychological profile, the social skills they possess and the type of impressions they create on others. Since the teaching of entrepreneurship has become more prevalent in Indian B-Schools, the need to focus on individual needs and skills are important. This research provides a clear understanding of the psychological perspective and the process behind entrepreneurial success. The study concludes that entrepreneurs are not people trying to self promote with superiors, but they are also fliers who supplicate even with the subordinates to get things done. 


\title{
Job-related Stress: a validation study
}

\author{
Tanucci, G., Cristina, E., Palano, F. \\ University of Bari, Italy
}

\begin{abstract}
Objectives: Stress has become one of the most relevant topics both with reference to empirical research as well as to organisational practice. Such assumption is further supported by the agreement on Stress in the Workplace (2004) recently signed by most European Countries, in order to share and improve the understanding of job related stress. Stress is referred to as a psychosocial risk for the safety of workers. Cox and Griffiths (1995) defined psychosocial risks as 'those aspects of job design and of the social and organisational context producing psychological and physical damage'. The aim of this contribution is to test the predictive validity of a tool measuring job-related stress. The tool has been developed moving from the theoretical model by Karasek. The model assumes that stress is determined by three dimensions: organisation, working relationship, individual difference. Each dimension is divided into two subareas one relative to the objective characteristics of the organisational context, the other referred to the workers' perception of the same aspects.
\end{abstract}

Methods: The study used a structured questionnaire which was given to a sample of workers selected from different organisational contexts in Italy. The questionnaire is composed by seven sections: socio-demographics variables, description of the working context, description of the work, perception of the work role, perception of the working context, self-perception, and current state of health. The data were elaborated through the software SPSS. Descriptive analyses, principal component analyses and linear regression analyses were run.

Results: The results allow highlighting the validity of the instrument for the understanding of the factors which might contribute to produce job-related stress. More specifically, results have allowed identifying both the objective and the subjective elements which might produce stress.

Implications: This study was meant as a first exploratory contribution aimed at the construction of a tool to measure job-related stress, which might account for the multiple determinants of the constructs.

Conclusions: The results emphasize the complexity of the phenomenon of job-related stress and the multi-causality linked to its onset.

\section{Coping and Quality of Life in subjects with Spinal Cord Injury}

\author{
Teixeira, A. \& Machado, F. \\ Instituto Superior da Maia, Portugal
}

The purpose of this research project is inquiring about Coping Strategies and Quality of Life (QOL) in subjects with Spinal Cord Injury $(\mathrm{SCl})$. The data collection took place in a General Hospital of Porto, Portugal, and included 44 adult subjects, $\mathrm{SCl}$ victims. Primarily, it was 
important for the objectives of this study to identify which Coping Strategies are used by SCl victims, to evaluate the presence of anxious and depressive symptoms and also to analyze how $\mathrm{SCl}$ victims perceive their QOL. Secondarily, we inferred possible correlations between these variables, highlighting the role of Coping as a central variable in the adaptation process of $\mathrm{SCl}$ victims, and because anxiety and depression symptoms, as well as the perception of QOL, are often considered good indicators of the adapting process effectiveness.

For the data gathering, we chose the following instruments: Brief COPE (Coping Orientations to Problems Experienced Scale), the HADS (Hospital Anxiety and Depression Scale) and the WHOQOL-100 (World Health Organisation Quality of Life). These instruments are validated for the Portuguese population and are largely and commonly used in other investigations in the field of Health and Occupational Health Psychology.

The results show that $\mathrm{SCl}$ victims prefer to use more the most effective Coping Strategies, particularly, Planning, Acceptance and Active Coping, and less Coping Strategies which are significantly less effective, such as Behavioural Disengagement and Substance Use. Results also show that anxiety and depression symptoms are not generalized amid SCl victims and are associated with specific Coping Strategies, namely, Denial, Expression of Emotions and Religion. Finally positive associations between Coping Strategies such as Positive Reframing, Active Coping and Humour and QOL, as well as negative associations between Coping Strategies such as Behavioural Disengagement and Denial were found.

\title{
Health care managers' boundary approaches to balance their time-commitments at work and in life
}

\author{
Tengelin, E., Wikstrom, E., Arman, R., \& Dellve, L. \\ University of Gothenburg, Sweden
}

Objectives: Complexities in health care managerial work require skills in delimiting tasks and defining boundaries in time and commitment to balance competing demands. Even though managerial challenges are described in earlier studies, processes to proactively handle them are less explored. The aim of this study was therefore to explore lower health care managers' strategies for a balanced time-distribution regarding work-commitment, work-related stress, and recovery during their everyday work and at home.

Methods: A qualitative driven mixed method design was used, including participant observations as well as qualitative interviewing of individuals and groups.

Results: The managers everyday practicing of leadership occurred in a context of timefragmentation and perceptions of boundlessness, with their time-commitment divided between their work assignment and their private, personal integrity. Overall, the approaches were enabled through acknowledging the continuous recognizing and negotiating of time-commitment. Expectations and needs could be impossible to fulfill without unreasonable amounts of work and commitment. Accordingly, strategies to limit time-commitment were used. A first step to balance time-commitments at work and in life was recognizing areas with conflicting expectations and 
inexhaustible needs. Participating in clinical practice, interaction with employees, fulfilling administrational duties, and taking active part in strategic networking, were all inevitable parts of managerial practice, which included time-consuming demands and contradictory perspectives. Goal conflicts in these areas arose from conflicting time commitments and compound role expectations due to different logics and identities related to the leadership assignment. Boundaries were handled by negotiating and manifesting the handling of managerial time-commitment and role perspectives through strategies that described the two boundary approaches of boundary setting and boundary dissolving activities.

Implications: Promoting sustainable time-use among HCMs in order to balance workcommitment, work-related stress and recovery should include (1) acknowledging boundary work as an ever-present dilemma causing a continuous negotiation process; and (2) encouraging individuals and organisations to recognize conflicting perspectives inherent in the leadership assignment, in order to decrease and better handle negotiations between them.

Conclusion: Boundaries in lower health care manager work were established through a process of individual recognizing, negotiation, and manifestation, where boundary setting as well as boundary dissolving were important approaches for achieving balance in time-commitment and worked as a form of proactive coping.

\title{
Safe workplace design: psychological risk factors
}

\author{
Tint, P., Tkatsova, L., Reinhold, K., \& Tuulik, V. \\ Tallinn University of Technology, Estonia
}

Objectives: The human body responds to stress-factors through four systems - central nervous, automatic nervous, endocrine and immune - which are constantly interacting as a complex network. The safe workplace design commonly presumes the decrease of physical overload factors like heavy weights; work in the compulsory position or monotonously, but often the other work environment hazards, like low temperatures, high noise levels etc. have to be carefully considered. The psychosocial, physical and also chemical factors which affect industrial workers are considered to be stress factors that alter the functioning of the organism and damage the peripheral and central nervous system.

Methods: The flexible risk assessment method worked out in Tallinn University of Technology was used for establishing the model that connects the psychological risk factors at workplace and the stages of developing the occupational illnesses. Occupational stress (the mobilization due to alarm of the adoption possibilities of the organism reserves) develops by three stages: 1) Hypersthenic stage (characterized with different subjective symptoms (mild asthenia, irritability, fatigue, pains in the muscles, paresthesis etc.); 2) Hyposthenic syndrome (moderate asthenization, localized pain syndromes, different objective neurological symptoms etc.); 3) The long-time exposure of the occupational stressor can cause the non-revisable changes in the organism - the development of the occupational disease. The developing of the occupational stress depends on the dose-response relationship: the length of time of the occupational stress, the specific character of the stressor and the functional state of the organism. 
The questionnaire for assessment of mental strain has been worked out for the study and introduced in different activities (garment industry, office work etc.).

Results: The results show the possibilities to improve the work environment and workers' health through good workplace design.

The health complaints at workplaces caused by the physiological stress are often very closely connected with psychological stress (not good relations between the employers and employees, stress coming from home or street with the workers to the workplace etc.). The psychological stress-factors are bad microclimate, excessive noise, insufficient lighting, monotonous work and chemicals that all together form the strain on workers' mental system. Conclusions: The number of occupational diseases is the specific indicator of influencing of existing hazards and risk factors on the worker in the work environment. The occupational diseases in Estonia are usually diagnosed in the late stage when the worker is already disabled. The mental stress at workplace has to be considered as occupational disease.

\title{
Antecedents and theory of Organisational Well-being. Variables to measure well- being at work
}

\author{
Trombetta, M. \& Franco, M. \\ Università degli Studi del Molise, Italy
}

The aim of the present research is to explain how the well-being on work place is a theme of increasing interest and how many studies analyze its functioning and diffusion. We can find many explanations to this phenomenon, first of all the strong will of lawmaker to preserve workers' health on job places. It is also possible to attribute deserts to private strategies that promote and improve wellbeing in organisations to achieve goals and high performance

In the past years the concern was only on physical health of employees, while in this last years the importance of mental health has been introduced. Today when we hear to speak about safety on job places or when we refer to TUSL (Testo Unico Sicurezza Lavoro) we consider these dual aspect of health. In Italian literature its possible to find two main different approaches on Organisational Well-Being: the first is by Avallone and its purely psychological approach; the second one is by Maggi and the field of studies is purely organisational. Today the concept of well-being in the organisations is very complex because it is composed by different variables, as Danna and Griffin (1999) suggest: job satisfaction, life satisfaction, physical and mental health. It is conditioned by many antecedents regarding work setting, personality traits or occupational stress.

The present work is based on a local survey led in an Italian district. Trough statistical analyses as correlation and ANOVA (using Bonferroni test) we want to verify the influence of several variables on well-being and to discriminate interviewees with statistically significant differences (between subjects with higher level of wellbeing). Among the variables regarding private data, such as income or level of study are not effective. Furthermore results change if we consider alternatively the three dimensions of wellbeing. Then health state derives from variables on private data and perceptions about for example environmental comfort and leadership. The influencing variables produce effect both on job satisfaction and overall wellbeing, only in a single case (social support) also on physical wellbeing. 
Then in a future research it is necessary to explore the organisational context to individuate its own characteristics because some factors must be included in a context but excluded in others and it will be possible to lead also qualitative analyses to identify the specific needs and exigencies of the reference organisation. Finally we show the limits and the other future applications for this type of work.

\title{
Verbal Aggression in Workplace Authority Structure: An Experimental Study
}

\author{
Tsang, F. T. \& Chang, Y.W. \\ National Chiao Tung University, Taiwan
}

Objectives: In the contemporary global competitive environment, accompanied with the increasing workplace stress, the observed frequency and intense of verbal aggression in workplace grows accordingly. So far the academic researchers emphasizes on investigating the causes and consequences of verbal aggression, and finding that it is highly associated with higher stress, learned helplessness experience and negative work performance, i.e. absence or in the worst case, the terminal of relationship. Especially within an unbalanced power relationship, such as the customer-employee or leader-subordinate relationship, individual in lower power position is more likely to become the target of verbal aggression. However, relevant empirical researches overemphasize on verbal samples with intensive emotions and obvious attack purposes and neglect verbal aggression in regular workplace environment. We suppose that the essential contextual factor of workplace verbal aggression, the authority structure, is very likely to add unintended aggressive meanings by the perceived unbalanced power relationship. In the other words, we predict an interaction effect between authority structure and verbal aggression.

Methods: Hereby we designed a within-subject 4-cell (superior/ subordinate- verbal aggression stimulus/ non-violent stimulus) experimental survey on 49 voluntaries to examine the influence of authority structure on perceived and cognitive stresses of verbal aggression. Respondent's biological data, the heart rate, is collected to represent their perceived stress response. We also collect respondent's cognitive stress response and intention to revenge from questionnaire survey and face-to-face interview after stimulus.

Results and Conclusions: Firstly, authority status has a direct positive influence on respondent's perceived stress response. Secondly, verbal aggression stimulus also has a direct positive influence on respondent's perceived stress response. Thirdly, the predicted interaction effect is not statically significant while we apply the linear relationship between heart rates and the level of perceived stress which is actually well-accepted in stress research field. However, if the biophysicists' U-curve relationship between the stress hormone (cf. Norepinephrine) and the heart rates suggested by is adopted, the interaction effect might become significant. Finally, the revenge behaviour is not consistent with the verbal expressed revenge intention after stimulus. Revenge behaviour occurs comparatively more frequent toward subordinates than supervisors. Implications: In the present study, we uncover this dilemma of inconsistent cognitive and physical awareness toward aggressive words by defining aggression with heart rate raise. The authority structure seems to contribute justifying the verbal aggression from supervisor in subordinate's cognitive mind, regardless of its physical impact. More considerable management policy should be taken for monitoring. 


\title{
Stress management in law enforcement: An integrative perspective
}

\author{
Turc, D. \& Turc, M. \\ Ministry of Administration and Interior, Romania
}

The theoretical and empirical researches in organisational psychology have shown a genuine occupational pathology, which circumscribes syndromes such as stress, burnout, workaholism or mobbing. From an epidemiological perspective, organisational stress stands detached through expansion area, symptoms and potential risk. Without minimizing other manifestations of organisational pathology, we can say that stress is a current issue in law enforcement institutions.

In their work, police officers have to face many complex situations. They are confronted on a daily basis with life danger, time pressure, dislike from the part of the peoples etc. Stress management in law enforcement is an essential component of the organisational management. The theoretical or practical approach of these problems is not a speculative exercise, but rather a pragmatic analysis, necessarily stemming from work complexity. The psychological assistance of employees, provided by the organisation, becomes a very important aim. It may cover, equally, skills development, stress management, crisis intervention etc.

As a prophylactic measure, periodic psychological evaluations provide active monitoring of the individual employees' functioning in terms of psychological parameters, while taking into account job specifications. The main purpose of these psychological evaluations is the early detection of dysfunctional psychological potential or psychiatric disturbance, planning, monitoring and evaluating the effectiveness of psychological intervention. Individual stress management techniques are also very important, intending to enhance individuals' ability to cope with stress. On the other hand, organisational interventions aim to change work conditions, thus reducing the stressors to witch employees are exposed. Running such activities requires a functional, regulatory and institutional framework, that establishes who should manage them, how, when and where to take action. This paper aims to present an integrative model of stress management, designed for stress relief and stress reduction in the Romanian Ministry of Administration and Interior. Issues addressed concern the legal framework governing the psychologist's profession in Romania and the internal rules that customize the activity of the psychologists in law enforcement. We also emphasize the specificity of psychological activities, referring to the main types of psychological services provided, organisational structure and regulations necessary to perform the professional activity.

\section{Job Demands-Control-Support Model and Employee Safety Performance}

\author{
${ }^{1}$ Turner, N., ${ }^{2}$ Stride, C. B., ${ }^{3}$ Carroll, A. E., ${ }^{4}$ McCaughey, D., \& ${ }^{2}$ Carter, A. J. \\ ${ }^{1}$ University of Manitoba, Canada; ${ }^{2}$ University of Sheffield, United Kingdom; ${ }^{3}$ Royal Military \\ College; ${ }^{4}$ Pennsylvania State University, United States of America
}

Objectives: Job characteristics are important correlates of employee safety performance. To date, we know little about how sets of job characteristics interact (e.g., Job Demands-Control- 
Support model; Karasek \& Theorell, 1990) to predict employee safety behaviours such as safety compliance and safety participation (Neal \& Griffin, 2006).

Methods: This paper used cross-sectional data from 289 medical staff (doctors, nurses, aides; $72 \%$ female, $\mathrm{M}$ age $=37$ years) in the emergency department of a hospital in northern England. We tested a model of the links between job demands [6-item scale; $1 \pm=.90]$, job control [6-item scale; $\left.\hat{l}_{ \pm}=.92\right]$, and social support [4-item scale; $\left.\hat{\imath} \pm=.91\right]$ on two types of employee safety behaviours (i.e., self-reported safety compliance [3-item scale; $\hat{\imath} \pm=.88$ ] and safety participation [3-item scale; $\hat{\imath} \pm=.75]$ ). We first tested the suitability of the measurement model containing all study scales, and then conducted observed variable path analyses (Kelloway, 1998) regressing safety compliance and safety participation on the 3-way, 2-way, and main effects of each of the above-mentioned job characteristics, controlling for age, sex, and occupational group.

Results: Testing alternative measurement models suggested that the hypothesized five correlated factor model was the best fit to the data $(\mathrm{CFI}=.95, \mathrm{NNFI}=.94$, RMSEA $=.06)$. The final structural model showed that job demands was related to lower safety compliance (beta $=-.12$, $\mathrm{t}=-3.07)$. Furthermore, the interaction between high job demands and high job control was related to higher safety compliance (beta $=-.12, \mathrm{t}=-2.75$ ). Greater job control was related to higher safety participation (beta $=.15, \mathrm{t}=2.45$ ). Additionally, the interaction of job control and social support was related to higher safety participation (beta $=.16, \mathrm{t}=2$.46). The 3-way interaction between job demands, job control, and social support was non-significant.

Implications: This study demonstrated that certain job characteristics alone and in combination may influence the extent to which employee work safely. Job control worked in tandem with job demands and social support to buffer and enhance employee safety performance, respectively.

Conclusions: Understanding how the psychological design of work is related to employee safety performance remains an area for research and provides ways for organisations can improve workplace safety (NIOSH, 2002).

\title{
Experimental manipulation of organisational change processes: Effects on Healthy Change Process Index, psychosocial work environment, and stress
}

\author{
Tvedt, S.D. \\ NTNU, Norway
}

Objectives: Earlier studies have found that a healthy organisational change process can improve experienced levels of control and support and stress. The level of demands, however, seems to be unaffected (see e.g. Tvedt, Saksvik, \& NytrÃ, , 2009). These findings are based on the cluster sampling of case studies. Hence, many different events in the real life of organisations threaten to bias the results in unpredictable ways. Thus, the objective of the present study was to triangulate earlier studies with an experimental approach were organisational change is simulated and subject to experimental control.

Methods: The study sample consisted of a series of experimental runs $(N=54)$, where participants were randomly assigned to either a healthy or an unhealthy simulated change process. Three main hypotheses were tested using SEM analysis: $\mathrm{H} 1$ : Exposure to healthy a change process 
increases the experience of a healthy change process (HCPI) which mediates increase in control, support and decrease in stress. H2: Control and Support will partly mediate the effect of a more healthy change process $(\mathrm{HCPI})$ on stress. $\mathrm{H} 3$ : Demands will not be affected directly by exposure to a healthy process or indirectly through a heightened experience a healthy change process (HCPI). All three hypotheses were tested by means of Structural Equation Modelling (SEM).

Results: A model incorporating $\mathrm{H} 1, \mathrm{H} 2$, and $\mathrm{H} 3$ was achieved with reasonable fit (CMIN/DF $=2.40, \mathrm{GFI}=.879, \mathrm{AGFI}=.790, \mathrm{CFI}=.959, \mathrm{RMSEA}=.067)$. The model explained $52 \%, 20 \%$, $64 \%$, and $41 \%$ of the variance in HCPI, Control, Support, and Stress respectively. H1 and H3 received full support, whereas $\mathrm{H} 2$ received no support and was rejected.

Conclusions: The experimental manipulation of organisational change process supports the causal nature of the model in the present study. The results confirm findings of earlier survey research based on clustered case samples, with increased strength of paths all around; a healthy process improves control, support and stress, but cannot improve on the increased levels of demands experienced during organisational change. Also reverberating earlier studies, meditational effects of Control and Support on Stress were not found. Being an experiment, the findings of the present study must be interpreted with caution regarding the ecological validity of the study. However, we believe that the effects we are able to produce within a 50 minutes simulation can only be expected to be more severe for real change processes with real personal implications and which are endured over months and years.

\title{
Experimentally manipulated organisational change: Importance of healthy processes and change resilience for engagement, commitment, stress, and health complaints
}

\author{
Tvedt, S.D.
}

NTNU, Norway

Objectives: Earlier studies have found that the impact of the change on the individual, and the individuals' change resilience play important and opposite roles in determining employees experience of organisational change. However, the healthiness of the change process substantially mediates the effects of resilience and impact (Saksvik \& Tvedt, 2009; Tvedt \& Saksvik, 2008). Process healthiness has also been shown to increase engagement and commitment (Tvedt \& Buvik, 2009). These findings are based on the cluster sampling of case studies. Hence, many different events in the real life of organisations threaten to bias the results in unpredictable ways. Thus, the objective of the present study was to triangulate earlier studies with an experimental approach were organisational change is simulated and subject to experimental control.

Methods: The study sample consisted of a series of experimental runs $(N=54)$, where participants were randomly assigned to either a healthy or an unhealthy simulated change process. Three main hypotheses were tested using SEM analysis: $\mathrm{H} 1$ : Exposure to healthy a change process increases the experience of a Healthy Change Process (HCPI) which mediates increase in Engagement, Commitment, and decrease in Stress and Health complaints. H2: High Resilience will increase Engagement and Commitment and decrease Stress, partly mediated by $\mathrm{HCPI}$. H3: High Change Impact will decrease Engagement and Commitment and increase Stress, and Health complaints partly mediated by $\mathrm{HCPI}$. 
Results: A model incorporating $\mathrm{H} 1, \mathrm{H} 2$, and $\mathrm{H} 3$ was achieved with reasonable fit (CMIN/ $\mathrm{DF}=1.237, \mathrm{GFI}=.845, \mathrm{AGFI}=.772, \mathrm{CFI}=.908, \mathrm{RMSEA}=.067)$. The model explained $45 \%$, $10 \%, 20 \%, 30 \%$, and $7 \%$ of the variance in HCPI, Engagement, Commitment, Stress, and Health complaints respectively. $\mathrm{H} 1$ was fully supported. $\mathrm{H} 2$ was only partly supported, only a direct effect on Stress and Health complaints was found. H3 received no support.

Conclusions: The experimental manipulation of organisational change process supports the causal nature of the model in the present study. The results confirm some findings of earlier survey research based on clustered case samples: HCPI increases Engagement and Commitment and decreases Stress and Health complaints. However, the process experience is not affected by resilience. We attribute this to the strong process manipulation in the experiment. Also, Change Impact had no explanatory power. We attribute this to a successful application of a uniform change impact in the experimental setup. This bolsters the claim from earlier studies that the Change Impact Factor (CIF) is an objective measure not affected by process nor change resilience.

\title{
Communication in emergency ambulance setting: observation coding scheme development
}

\author{
Upenieks, R. \\ University of Latvia, Latvia
}

Objectives: The importance of effective communication between physicians and patients is certain since the topic emerged in arena of health psychology. Majority of interpersonal health communication studies consider general practitioners and their patients; fewer studies are devoted to other type of medical encounters. Although there are common findings and conclusions obtained so far, there might be gray areas undiscovered yet due to specific encounters like emergency ambulance setting. On-the-scene situations, extreme levels of stress, vulnerability, and time pressure are just a few specifics of the setting. What is the content of communication between emergency ambulance staff and their patients is the question.

The objective of this poster is to present development of an original observation coding scheme for studying interpersonal communication in emergency ambulance setting.

Methods: Participant observation was employed as a method for data collecting. The author immersed himself in the study field, becoming an assistant in an emergency ambulance service. Unstructured observation was used for the first six months while developing an original coding scheme. The coding scheme was piloted on the next stage of observations.

Results: The path from unstructured observations towards structured ones is discussed. Comparison with other coding schemes is provided. An original structured coding scheme is presented.

Implications: Original observation coding scheme is developed for the field observations of emergency ambulance settings.

Conclusions: The further goal of the major study is to make systematic review and to propose practical recommendations for supporting and favouring efficient communication in emergency ambulance setting. 


\title{
Predictors of psychological discomfort and well-being in a sample of correctional officers employed in Penitentiary Sector in Italy
}

\author{
Viotti, S. \\ Università degli Studi di Torino, Italy
}

Studies carried out regrding Occupational Health Psychology amongst correctional officers highlight how this population is exposed to very high, sometimes alarming, levels of stress. A constant relationship with inmates, difficulties regarding role management (role ambiguity and role conflict), lack of autonomy, an organisational culture less oriented to the promotion of a collaborative and supportive environment both in horizontal and in vertical relationships, a high level of centralized decisions and finally, risks concerning physical health and safety (Schaufeli \& Peeters, 2000; Dowden \& Tellier, 2004) are just a few of the aspects highlighted by a literary review. If those factors are considered together, they contribute to the creation of scenarios in which considering well being and life quality improvement for this category of workers could appear clashing and paradoxical.

In the hypothesis that psychological discomfort and well-being are not, in the in the individuals' experiences, opposed to each other, and that the improvement of organisational quality's life can both reduce discomfort and promote well-being, the aim of the present study will be to explore fully, in a sample of correctional officers employed in a Italian Penitentiary Institution, the life experiences and the outcomes at an individual level on both dimension (discomfort and well-being) Moreover, in the organisational environment, factors that promote the development of both the former and the latter, will be identified.

The research (at the kick-off step during these days) is articulated in two phases. In the former, qualitative and inductive, will be privileged the 'spontaneous surfacing' of psychological discomfort/well-being experiences linked to the organisational environment in which the workers are inserted (Grounded Theory, Glaser \& Strauss, 1967). In this first phase, a small group of workers $(n=20)$ will be involved and a semi-structured interview will be administered to them. The data collected, after being analysed, together with a thorough literature review, will form the key guidelines, for constructing a questionnaire. This will be used during the second phase as a measuring tool that will be administered to the whole sample $(n=300)$, in order to determine the factors that could be explored and which tools (ready-made or tailormade) will be employed to measure them. During this phase quantitative tools of data collection and analysis (deductive way) will utilized. In particular, in order to investigate the relationship between organisational antecedents and individuals outcomes, how discomfort and well being phenomenon develop, and to discover which factors promote their onset, data collected during this second phase will be analysed using Multivariate Analysis Techniques with SPSS software.

\section{Validation Study of a Multidimensional Measure of Work-Family Conflict in Romania}

\author{
Virga, D., Sulea, C., \& Marcutoiu, L. \\ West University of Timisoara, Romania
}

Objectives: Work-family conflict is a source of stress that many individuals experience. 
Researchers have measured work-family conflict in several ways. The purposes of this study were to adapt a Romanian version of the multi-dimensional Work-Family Conflict Scale (WFCS - Carlson, Kacmar \& Williams, 2000) and to examine its reliability and validity. The scale has six dimensions of conflict which consist of time-based work interference with family (WIF), timebased family interference with work (FIW), strain-based WIF, strain-based FIW, behaviour based WIF, and behaviour-based FIW.

Methods: We have translated in Romanian the version of the WFCS scale by the following procedure: forward translation and back translation. Confirmatory factor analysis was performed on a first sample of Romanian employees $(n=275)$ and the validation analyses were performed on a second sample $(n=589)$ consisting of employees from several industries located the Western part of Romania. The average age is 35 years and $53 \%$ of the subjects are male.

Results: On a first sample, the confirmatory factor analysis (using AMOS 1.6) revealed a 6 factors structure that corresponds to original WFCS dimensions. The consistency indices were calculated for each sub-scale, varying between .70 and .80. We also calculated the inter-scale correlations and we obtained significant associations. On a second sample, we analyzed the associations between work-family conflict and antecedents (number of children, tenure, age), and outcome variables (job satisfaction, turnover, work engagement). We have identified significant associations between the WFCS dimensions and antecedent and outcome variables. These links support the predictive power of the MMWFC questionnaire.

Implications: The employees with higher tenure present higher levels on each work-family conflict dimensions. The employees with higher scores of behavioural work-family conflict tend to express lower job satisfaction.

Conclusions: The multidimensional measure of work-family conflict is considered an accurate depiction of the construct as it allows each of the six dimensions to be examined. Future use of this scale should provide a greater understanding regarding how the separate work-family conflict dimensions are associated to attitudes and behaviours relevant in the organisational environment. The results suggest that the Romanian version of the WFCS scale may be a reliable and valid instrument for assessing the conflict between work and family in Romanian workers.

\title{
Does Diversity Drive Employees Crazy? The Relationship between Organisational Demographics and Employee Mental Health
}

\author{
Waite, E., \& Avery, D.R. \\ University of Houston, United States of America
}

Objectives: Prior work has shown some degree of relationship between workplace racioethnic dissimilarity and employee mental health (Enchautegui-de-Jesus, Hughes, Johnston, \& Oh, 2006). The present study investigates this relationship using three demographic indicators of dissimilarity (i.e., racioethnicity, sex, and age) with one's coworkers. Another key extension is that we look at the effect of an organisation's structural integration (i.e., the degree to which management's demographic profile is similar to that of rank-and-file employees) on employee health, hypothesizing that higher levels of structural integration result in better employee mental health. 
Methods: The study retained data collected by the Families and Work Institute for the 2002 National Study of the Changing Workforce (total $N=3,552$; usable $N=1,391$ ) (Bond, Thompson, Galinsky, \& Prottas, 2002).

Results: We used hierarchical regression analyses to test our hypothesis. The first model, which included demographic variables as predictors, accounted for $6.7 \%$ of the variance in mental health. The main effects of sex, age, and education were significantly associated with mental health $\left(\hat{I}^{2}=-.16, .12, .09\right.$, respectively, $\left.\mathrm{p}<.01\right)$. In Step 2, we added coworker sex, age, and racioethnic dissimilarity as predictors. In this full model, coworker racioethnic dissimilarity $\left(\hat{I}^{2}=-.07, \mathrm{p}=.01\right)$ was the only significant predictor of mental health. Neither sex nor age dissimilarity reached significance. In Step 3, we added racioethnic and sex structural integration. One significant relationship emerged; that between racioethnic structural integration and mental health, $\left(\hat{I}^{2}=.08, \hat{a}^{\wedge}+R 2=.01, p<.01\right)$.

Conclusion: Racioethnic structural integration related positively to mental health, such that greater correspondence between levels, an indicator of a more favorable diversity climate, corresponded to better mental health. These results have practical implications for organisations. As the proportion of minority representatives increases in the workforce, understanding the dynamics that contribute to the well-being of employees in diverse organisations is important. Creating a work environment that considers the demographics of the organisation may have an impact on the organisation's bottom line, as previous research has linked well-being and performance (e.g. Judge, Thoresen, Bono, \& Patton, 2001; Petty, McGee, \& Cavender, 1984; Taris, 2006). Theoretically, it is noteworthy that the effects varied according to the demographic variable of interest. Specifically, racioethnic dissimilarity and structural integration seemed to be more salient than age or sex (at least with respect to employee mental health), which is consistent with the ethnic prominence model (Levin, Sinclair, Veniegas, \& Taylor, 2002).

\title{
The effect of perceived support and self efficacy on work related stress and burnout amongst intellectual disability direct care workers in Australia
}

\author{
Walker, A. \& Jackson, B. \\ Deakin University, Australia
}

Objectives: Work-related stress and burnout are considered a significant problem amongst direct care workers (DCWs) in the field of intellectual disability (ID). Organisational psychological research has found that both organisational and individual factors can significantly impact workrelated stress and burnout. However, similar research appears limited with DCWs in the field of ID. The current research aimed to investigate the relationships between two types of perceived support (peer and supervisor) and employee stress and burnout as well as the relationship between perceived self-efficacy and employee stress and burnout.

Methods: Participants were 66 DCWs from the Department of Human Services in Victoria, Australia. A self-report questionnaire measuring job stress, burnout, self-efficacy and peer and supervisor support was completed.

Results: A significant negative relationship was expected between the two types of perceived support (peer and supervisor) and work-related stress and burnout, and also between self-efficacy 
and work-related stress and burnout. It was also expected that peer support, supervisor support and self-efficacy respectively would mediate the relationship between work-related stress and burnout. Hierarchical regression analyses found significant negative relationships between peer support and work-related stress and burnout, but not between supervisor support or perceived self-efficacy and work-related stress and burnout. In addition, only peer support was found to fully mediate the relationship between work-related stress and burnout.

Conclusion and Implications: Contrary to previous organisational psychology research, only peer support had a significant relationship with work-related stress and burnout amongst DCWs within the field of ID. This might suggest that for this group of Human Service Workers at least, having peers who understand the nature and complexities of the role is more important than self-belief about capability to perform the role or receiving adequate support from one's supervisor. Implications of the findings for Human Service Workers and stress and burnout research are explored.

\title{
Workplace mistreatment and perceived support: Different perpetrators and differential relationships
}

\author{
Walsh, B. \& Magley, V. \\ University of Connecticut, United States of America
}

Interpersonal workplace mistreatment (e.g. workplace bullying, incivility) has negative effects on employee's work-related attitudes and health. Consequently, researchers have studied factors that may buffer the deleterious consequences of being mistreated such as perceived support from one's organisation or supervisor. Perceived organisational support (POS) and perceived supervisor support (PSS) refer to global evaluations employees make regarding whether their organisation and supervisor value their work contributions and are concerned for their well-being. Although the buffering role of perceived support has been studied, research has not considered whether mistreatment experiences may directly diminish PSS and/or POS. Nevertheless, because mistreatment threatens the target's welfare, there is reason to suspect such negative relationships and for considering an alternative to the buffering role of support when mistreatment is the stressor under study.

As such, we tested a model of the effects of experienced mistreatment from three sources (i.e., supervisor, coworkers, customers) on PSS and POS. Based on organisational support theory and research demonstrating that PSS is a precursor to POS, it was predicted that PSS would fully mediate the relationship between supervisor mistreatment and POS (Hypothesis 1), PSS would partially mediate the relationship between coworker mistreatment and POS (Hypothesis 2), and customer mistreatment would have a direct negative effect on POS but not PSS (Hypothesis 3).

Data were collected from 135 full-time employees who completed an online survey. Respondents completed measures pertaining to their experiences of mistreatment from their supervisor, coworkers who shared the same supervisor and customers, as well as measures of PSS and POS. Items were drawn from previously validated instruments and all measures were internally consistent. 
The hypothesized path model was tested in AMOS 5. Results provided support for Hypothesis 1 as PSS fully mediated the supervisor mistreatment - POS relationship. However, Hypothesis 2 was not supported. Coworker mistreatment was negatively associated with POS but had no direct association with PSS. Finally, Hypothesis 3 was supported as customer mistreatment was found to be unrelated to PSS and negatively related to POS. The final model accounted for $15.8 \%$ and $39.8 \%$ of the variation in PSS and POS, respectively.

Findings indicate that mistreatment has direct and indirect effects on PSS and POS and suggest the need to consider mistreatment as an antecedent to perceptions of support in future research. The results also support the value in considering source effects when studying consequences of mistreatment experiences given the differential patterns of relationships observed in the present study.

\title{
Criterion-related and incremental validity of the Civility Norms Questionnaire - Brief
}

\author{
Walsh, B. \& Magley, V. \\ University of Connecticut, United States of America
}

Recent research on workplace mistreatment (e.g., aggression, incivility, bullying) has begun to look beyond the dynamics surrounding individual experiences of such misbehaviour to understanding contextual factors that contribute to its occurrence and tolerance. Accordingly, measurement development efforts have begun examining workplace climate factors associated with respectful and disrespectful behaviour. Once such measure is the Civility Norms Questionnaire - Brief (CNQ-B), a 4-item scale designed to assess workgroup norms for mutual respect among coworkers. Although the factor structure of the CNQ-B was established and there is some evidence for its convergent and discriminant validity, there is less evidence for the criterion-related and incremental validity of the CNQ-B. Hence, the aim of the present research was to provide further evidence of the validity of the CNQ-B in predicting various work-related attitudes (i.e., intentions to quit, job satisfaction, affective commitment) beyond effects of a comparable climate measure and personal experiences of workplace incivility. We expected that the CNQ-B would be negatively associated with intentions to quit and positively associated with job satisfaction and affective commitment.

Data were collected in two waves, one year apart, from full-time employees in a department of a Northeastern state government in the United States. At Time 1 employees responded to the 4-item CNQ-B and a single item from the coworker subscale of the Perceptions of Fair Interpersonal Treatment scale (PFIT). Personal experiences of incivility, intentions to quit, job satisfaction, and affective commitment were each assessed at Time 2. All items were drawn from validated measures and all multi-item scales were internally consistent. Several hierarchical regression analyses were conducted to assess the incremental contributions of the CNQ-B. PFIT and incivility experiences were entered in the first step and the CNQ-B was entered in the second step in each analysis. As expected, the CNQ-B accounted for unique variance in each criterion and relationships were in the anticipated direction. Incremental contributions of the CNQ-B ranged from $2.6 \%$ (intentions to quit) to $3.4 \%$ (job satisfaction). Total variance-accountedfor in the models ranged from $14.7 \%$ (affective commitment) to $25.7 \%$ (job satisfaction). 
Findings demonstrate that the CNQ-B is able to predict later-assessed attitudinal criteria beyond the effects of a conceptually similar climate item and personal incivility experiences. These results provide additional evidence of the utility of the CNQ-B for understanding workgroup climate for civility and its associated consequences. However, future research is needed to understand how the CNQ-B relates to additional work attitudes and behaviours.

\title{
Authentic Leadership, Social Support and Workplace Bullying as Determinants of Worker's Mental Health
}

\author{
Warszewska-Makuch, M. \& Zolnierczyk-Zreda, D. \\ CIOP-PIB, Poland
}

Results of research in the field of workplace bullying carried out during last twenty years in European countries have shown that 50 to 80 percentage of victims of bullying indicate their superiors as perpetrators of bullying. On these basis we can suppose that workplace bullying is highly connected with types of leadership (see: Nielsen, Matthiensen, Einarsen, 2005). There is some data on relationships between workplace bullying and type of leadership. These studies have focused on destructive leadership behaviour such as laissez-faire leadership (Skogstad at al., 2007) but there is no research which would have shown a positive role of leadership in reducing pathological phenomenon such as workplace bullying in organisation. That was the reason to concentrate on one of the latest conception of leadership such as authentic leadership (Avolio \& Gardner, 2005). This conception is deeply ingrained in positive psychology and focuses mainly on individual elements of leadership which leads to its development (Luthans \& Avolio, 2003). The authentic leaders are characterized by a high level of moral hence, they are less willing to take unethical acts.

The aim of this study was to show how the authentic leadership (according to Avolio \& Gardner, 2005) is related to social support and exposure to bullying and how these variables are related to mental health. The sample consisted of 800 Polish clerical workers from different organisations and sectors. The authentic leadership was measured with the Authentic Leadership Questionnaire (Avolio, Gardner, Walumbwa, 2007). The Negative Acts Questionnaire (NAQ) (Einarsen et al., 1994; Hoel, 1999) was used to assess exposure to bullying. Whereas in assessing the state of mental health the Goldberg Health Questionnaire (Goldberg, 1978) was applied. The scale used to measure social support (from supervisor and coworkers) was derived from Job Content Questionnaire by Karasek \& Theorell (1990).

Generally, the results proved the hypothesis that all dimensions of authentic leadership (transparency, moral, balanced processing and self awareness) are positively related with social support and negatively correlated with exposure to bullying. Path modelling showed that social support mediates the effects of authentic leadership on bullying at work and that the effects of authentic leadership on mental health are mediated through exposure to bullying. These results suggest that authentic leadership may effectively prevent bullying at work and hence reduce negative effects connected with bullying on workers' mental health. This conclusion may be important for leaders in organisations who should that realize the reduction of workplace bullying begins with them and their beliefs about, and attitude towards, their staff. 


\title{
Exploring nurses' experiences of older adult care: A qualitative pilot study
}

\author{
Watts, J. \& Robertson, N. \\ University of Leicester. United Kingdom
}

Objectives: Providing support and assistance to others can impact negatively upon the health and wellbeing of care-givers as they invest their emotional resources in attending to their patient's needs. However, the exact nature of health professional distress remains unclear, with different conceptualizations offered, such as burnout and secondary trauma. Greater understanding of nurses' experiences is required in order to guide the design of future research, and to tailor workplace strategy. This pilot study aims to examine how nurses' psychological wellbeing may be affected by patient contact.

Methods: Semi-structured interviews will be conducted with staff working in residential homes $(\mathrm{N}=6)$ and hospital settings $(\mathrm{N}=6)$. Participants will be asked to comment on most enjoyable and most challenging aspects of the role, reactions to care work and feelings towards the patients or residents.

Results: Following data collection participants' responses will be typed verbatim to form a transcript. Each transcript will be reviewed to identify broad ideas and then more specific themes. Themes will be selected based on prevalence and organized using Nvivo 7 software. A list all the emerging themes will be compiled for each participant and then compared with themes from the other participants according to principles of Interpretative Phenomenological Analysis (IPA) analysis.

Implications: The findings of this pilot study will be used to inform a future qualitative investigation of nurses' experiences of working with older adults. Before this larger-scale study can be conducted it is important to find a data collection tool that participants find easy to use and that generates sufficient and meaningful data.

Conclusions: Following analysis of the interview transcripts the researchers will have gained information about the impact of patient contact upon nursing staff. The interviews will reveal nurses' reactions to their role and their appraisals of these responses. From this information a more in-depth qualitative study can be designed to investigate nurses' experiences in more detail.

\section{Positive Organisational Behaviour and Safety Science: Conceptual Issues and Future Research Questions}

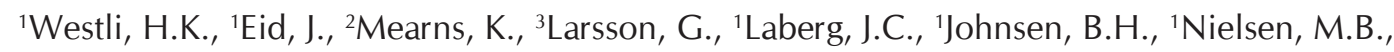
'Satrevik, B. \& ${ }^{1}$ Bergheim, K.

${ }^{1}$ University of Bergen, Norway; ${ }^{2}$ University of Aberdeen, Scotland; ${ }^{3}$ National Defense College

This theory-driven literature review examines how leadership and positive organisational behaviour (POB) may contribute to future research in safety science. According to authentic leadership theory, leader self-awareness and self-regulation processes are vital mechanisms in 
the leader - follower exchange. From emerging research on authentic leadership theory, we propose that production management values, attitudes, and behaviour are linked to safety climate and safety outcomes in safety critical organisations (SCO's) operating in hostile and environmentally sensitive environments. According to recent developments in management theory, "psychological capital" has been proposed to constitute four distinct aspects of organisational culture that can be linked to desirable organisational outcomes and sustained high quality performance in individual workers. From this we propose five hypotheses that could be explored in future research to assess how authentic leadership and psychological capital will influence safety climate and thereby safety outcomes in SCO's.

\title{
Challenge Stressors and work attitudes: Self-esteem tips the scales
}

\author{
Widmer, P., Gertsch, D., \& Semmer, N. \\ University of Bern, Switzerland
}

The challenge-hindrance model of work stress proposes two kinds of stressors: Hindrance stressors contain only stressful aspects. Challenge stressors contain both stressful and challenging aspects; hence they have positive effects on work-related outcomes, such as productivity or work attitudes (e.g., LePine et al., 2005). Previous results support these assumptions (Cavanaugh, et al., 2000; LePine et al., 2005). It has been argued that challenge stressors possess a motivational force (e.g., LePine et al., 2004) because they imply potential personal gain or growth. However, we believe that this motivational force can only occur and have positive effects if a person feels self-assured enough to use this opportunity, hence possesses a positive self-perception. More specifically, we assume that people with a high self-esteem will benefit more strongly from experiencing challenge stressors than people with a low self esteem.

We analyzed data from a convenience sample consisting of 224 individuals (52\% women). Mean age was 32.06 (SD = 11.14); all participants were white collar workers, and most of them $(85 \%)$ worked fulltime. More than half of the sample (54\%) had executive positions. Challenge stressors were measured with a German translation of the original scale by Cavanaugh et al. (2000). As dependent variables, we measured work attitudes such as affective commitment (AC; Allen \& Meyer, 1990), job satisfaction (JS; Baillod \& Semmer, 1994), and intention to quit (ITQ; Wayne, Randel, \& Stevens, 2006). Self-esteem was assessed with the job-specific measure of organisation based self-esteem (OBSE; Pierce et al., 1989).

Multiple regression analysis yielded significant interaction effects of OBSE with challenge stressors in predicting all three work attitudes. As hypothesized, challenge stressors were only related to higher JS and AC among individuals with high OBSE. For individuals low on OBSE, challenge stressors were even related to lower JS and AC. Conversely, ITQ was highest among individuals who experienced a high amount of challenge stressors but scored low on OBSE.

Results confirm earlier findings that challenge stressors may have positive effects on work attitudes. However, the study reveals that this might not be the case for all individuals. Individuals with a positive self-evaluation might benefit most strongly, whereas for people low on OBSE, experiencing challenge stressors might even be detrimental. Thus, an important theoretical contribution of our study is the moderating role of OBSE, underscoring the role of self-esteem in the challenge-experience and, more generally, pointing to the value of focusing on the self when investigating stress, challenge, and well-being (Semmer et al., 2007). 


\title{
The feeling of coherence and burnout syndrome among nurses
}

\author{
Wilczek-Ruzyczka, E. \\ The Jagiellonian University of Cracow, Poland
}

Objectives: The aim of the thesis was to measure the level of coherence and work-related emotions of the psychiatric nurses, as well as to analyse the relation between the feeling of coherence, emotions and work-related burnout syndrome experienced by nurses.

Methods: Ninety psychiatric nurses from the Psychiatrics Hospital in Krakow with an average working experience of 9,5 years took part in the survey.

The following methods were used:

- burnout: Maslach Burnout Inventory,

- emotions: Emotional State Questionnaire,

- coherence: SOC-29 Questionnaire.

Results: The results proved that the level of coherence among the nurses was slightly higher than the average level possible to have been achieved in the survey, and the feeling of resourcefulness and sense was on the highest level.

The measuring of the emotional states showed the highest intensity of favourable emotions, such as hope, satisfaction, joy and optimism.

The relation between the feeling of coherence and work-related burnout syndrome was discovered during the analysis. The higher was the level of coherence among the nurses, the lower was the level of work-related burnout syndrome.

The relation between the emotions and work-related burnout syndrome was also discovered.

Implications: The research shows the need for proper diagnosis of the job burnout and the feeling of coherence, emotions and the selection of adequate therapeutic methods.

\section{The Impact of Emotional Intelligence on Organisational Citizenship Behaviours}

\author{
Yildirim, D., Hunerli, S., Atan, O. \\ Istanbul Kultur University, Turkey
}

In the research, it is examined the effect of EQ dimensions on organisational civic behaviour by using multiple regression analysis.

In the regression analysis, Stepwise Regression, which models regression, was preferred by choosing the most effective ones among the independent variables (EQ dimensions) for the dependent variable (organisational civic behaviour dimensions). As a result of the regression analysis in order to determine the effect of EQ dimension on altruism dimension, one meaningful model was created. Depending on the research results, teachers' EQ has a correlation with their organisational civic behaviour. 
According to the regression analysis results, especially the emotional awareness dimension of the EQ dimensions is positively effective on all of the organisational civic behaviours except for conscience behaviour. Social skills dimension is only effective on alturism and sportsmanship behaviours.

As a result of the research, especially the teacher's emotional awareness and emotional management dimensions are more effective on the organisational civic behaviour. Thus, one can claim that teachers perform the organisational civic behaviours not to impress someone else, they do it for the sake of their conscience. Social awareness dimension's ineffectiveness on the organisational civic behaviour support the idea.

\title{
Workplace Violence Against Health Workers in Turkey
}

\author{
Yildiz, S., Aytac, S., \& Bayram, N. \\ Uludag University, Turkey
}

Objectives: This research was conducted as a descriptive study to determine the experience of workplace violence of workers from health sector and investigate the contributing factors. Another aim of this study was to identify the actions taken against workplace violence.

Methods: Data were collected anonymously by printed questionnaires from the subjects who participated voluntarily in the study. The questionnaire consisted of 26 questions seeking socio-demographic data, information on violence and actions undertaken. We used these instruments:

- The Workplace Violence Questionnaire developed by the International Labour Organisation (ILO) and World Health Organisation (WHO) and translated into Turkish by the authors of this study.

- The Job Satisfaction Scale developed by Blayfield and Rothe and adapted to Turkish by Bilgin (1995).

- Depression Anxiety and Stress Scale 42 (DASS-42) developed by Lovibond and Lovibond (1995) and adapted to Turkish by Uncu, Bayram, and Bilgel (2006).

The participants also filled out a 5-point Likert-type job satisfaction scale with 5 items and the DASS-42 (Depression Anxiety Stress Scales).

Results: The number of the distributed questionnaires for health sector: 1069 participants. Most of the participants were female. The mostly common type of violence was verbal abuse $(47 \%)$ followed by bullying $(37 \%)$. The most frequently reported reaction was talking with colleagues about the violent act, followed by talking with family and friends and warning the perpetrator

Implications: In the health sector, stress and violence are widespread than other sectors because they originate to a large extent from the interface between workers and customers. Several occupations, such as those of doctors, nurses and social workers, are high on the list of occupations with high stress levels. This paper deals with them as related phenomena. This correlation between emotional violence and stress is of the greatest importance not only in assessing the overall impact on the individual, but also in determining its global impact in terms of cost and efficiency for the organisations. 
Conclusion: Workplace violence at the Turkish workplaces we studied is common, especially verbal abuse and bullying. Physical assault and sexual harassment are rarely seen and committed by outsiders whereas perpetrators of bullying and verbal abuse are senior colleagues. Workplace violence in Turkish workplaces is a silent epidemic, and neither preventive measures nor social.

\title{
Work and family support, work to family spillover and job satisfaction: a model
}

\author{
Zito, M., Colombo, L., \& Ghislieri, C. \\ Università degli Studi di Torino, Italy
}

Objectives: Work-family relationship is increasingly important in organisational research, considering the growing number of women in workforce and of dual-earner couples.

This study focuses on the spillover model (Grzywacz \& Marks, 2000): the influence between work and family domain can be negative or positive and bi-directional (worktfamily, familytwork). The aim of this study is to test the effect of the family and organisational support on workât'family positive and negative spillover and on job satisfaction, but also to test the effect of the two forms of spillover on job satisfaction.

Methods: This study is based on data collected from a sample of 871 employees from two North-West Italian public organisations and it gathers information about:

- $\quad$ Family Support: emotional (3 items; $\hat{\imath} \pm$.91); instrumental (4 items; $1 \pm .91)$.

- Organisational Support: supervisors (4 items; $\hat{\imath} \pm$.95); co-workers (4 items; $\hat{\imath} \pm$.95).

- Work-Family Spillover: negative (4 items; $\hat{\imath} \pm$.77); positive (3 items; $\hat{I} \pm$.67).

- Job Satisfaction: 12 items (Î \pm .91$)$.

- Control variables: gender (64.5\% women; 35.5\% men); hours at work $(90.6 \%$ full-time, 9.4\% part-time); organisation (54.4\% Public Office for Environmental Safety, $45.6 \%$ Public Health Office).

Data analysis was performed as: means, standard deviations and alpha reliabilities (Î̃) (SPSS 15.0). Hypothesized relationships between variables were tested through a path analysis model (Lisrel 8.72, maximum likelihood).

Results: The hypothesized model fitted the data well: $\mathrm{x} 2=8.29$, df $=8, \mathrm{p} .=.40$; $\mathrm{RMSEA}=$ $.01 ; \mathrm{RMR}=.01 ; \mathrm{NNFI}=1 ; \mathrm{AGFI}=.99 ; \mathrm{GFI}=1$.

Path analysis shows the role of family and organisational supports in reducing negative spillover and increasing positive spillover and job satisfaction.

The model shows that supervisors' support influences spillover, both directly and indirectly, and job satisfaction; instrumental family support reduces negative spillover and increases job satisfaction; emotional family support enhances positive spillover; co-workers support increases job satisfaction, both directly and indirectly, through the negative spillover decrease.

T-test results show: women perceive higher level of work-family negative and positive spillover than men; part-time employees perceive higher level of work-family positive spillover than fulltime employees. 
Implications - Conclusions: Findings highlight the importance of investigating the relationship between work and family to deeper understand the dynamics of promoting well-being in organisations supporting work-life balance. The intervention areas concern: supervisors' training in order to support employees in managing their work and their 'double presence'; development of family-friendly policies to sustain work-family balance.

\title{
Flexible Working Time as a Tool to Manage Work-Related Stress and Workplace Bullying for Polish Clerical Workers
}

\author{
Zolnierczyk-Zreda, D. \& Warszewska-Makuch, M. \\ CIOP-PIB, Poland
}

According to the outcomes of the latest ESWC (2007) carried out by the European Foundation for Improving Living and Working Conditions in Dublin, Poland is the second European country, where weekly working time exceeds 48 hours. Despite the large body of evidence showing that flexible working time can effectively buffer the negative effects of long hours on worker's health and well-being, the implementation of flexible working time is still very limited in Poland. Moreover, there is some evidence that rigid working hours are related to a decreased psychological well-being and even to workplace bullying.

The aim of this study was to evaluate the role of flexible working time as a tool of managing work-related stress and bullying at work The individual variables like worker's gender and age were taken into account, since - according to the existing data - woman are a group being the most vulnerable to the negative effects of rigid working hours.

The group of 800 workers performing clerical work, having both flexible and nonflexible working hours have been diagnosed in 2008 on their mental health with GHQ -28 (Goldberg, 1980), work-related stress with Karase questionnaire (Karasek et al. 1998), and workplace bullying with NAQ (Einarsen i Raknes, 1994, 1997).

It has been shown that workers having flexible time had significantly better indices of their mental health and significantly lower scores on work-related stress. The level of bullying at work has also been significantly lower in this group, in comparison with the 'rigid time' group.

It has also been found that, the greatest costs of nonflexible working time in terms of work-related stress are woman above 50 years old. The rigid time regime was also related to significantly higher scores of bullying at work in this group comparing to the same group with flexible time. The outcome of this study clearly confirms that even in the presence of other objectively existing psychosocial risks in Poland, such as economic transition and global crisis, flexible working time can be an effective tool for work- related stress and bullying. 



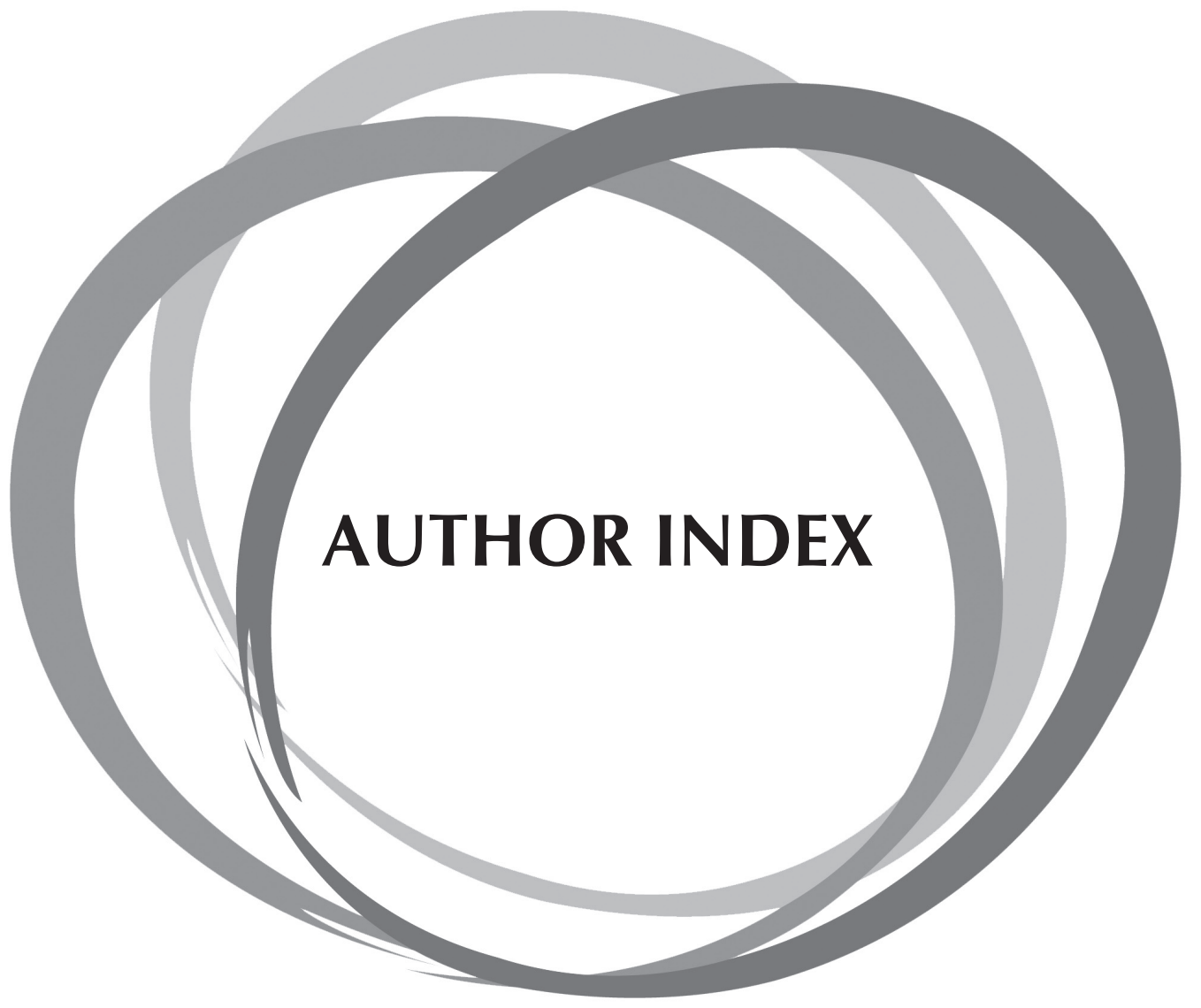



Aagestad, C. 135

Abbate, C. 422

Abjornsson, G. 209

Ahlborg, G. 281

Ahola, K. 136, 262

Aiello, A. 167

Akkermans, J. 38

Al Serkal, A. 137, 318

Ala-Laurinaho, A. 219, 251

Alarco, B. 54

Albrecht, S. 137, 232

Alessandro, A. 258

Aleyasin (Al yasin), S.A. 319

Alfoldy, S. 319

Alheiro, A.S. 320

Allen, C. 110

Alvarez, A. 424, 425

Alvarez-Bejarano, A. 268

Alves, J. 321, 388

Amponsah-Tawiah, K. 138

An, S. 322

Andreassen, C.S., 195

Angel Gonzalez, M. 342, 354

Ângelo, R. 134

Ansari Ramandi, S. 323

Antonini, E. 269

Aravagli , L. 421

Arman, R. 440

Aro, A. 108

Arsenault, A. 234

Atan, O. 456

Atzori, E. 323

Aune, M. 352

Avallone, F. 3

Avery, D.R. 306, 449

Aytac, S. 457

Baba, V.V. 298

Babic, A. 192

Bachner, Y. 215

Bagnato, C. 233

Bahamondes Pavez, C. 139

Baillien, E. 59, 67

Bakhuys-Roozeboom, M. 27

Bakk, Z. 52

Bakker, A. 168, 194

Bakker, A.B. 72, 73, 74, 195, 295, 313

Balcazar, F. 170

Baldanzi, S. 340

Balducci, C. 140
Ballottin, A. 269

Barbaranelli, C. 239, 361

Barbier, M. 141

Barkhuizen, N. 142, 143, 144, 324, 325, 326

Barnes-Farrell, J. 145

Barresi, G. 422

Bartolucci, G.B. 430

Basbug, G. 433, 434

Batinic, B. 279, 436

Battistelli, A. 416

Bauer, G. 187, 213, 336

Bauer, G.F. 30, 31, 31, 32, 33

Bauerle, T. 327, 328

Bayram, N. 457

Beata, B. 329

Beckers, D. 301

Behrendt, K. 145

Bell, E. 144

Bell, N. 87, 88

Bentivenga, R. 347, 348, 349

Berezovskaya, R. 330

Bergamaschi, A. 225, 226, 334, 391

Bergheim, K. 331, 454

Bernhard-Oettel, C. 53

Bernhardt, L. 36

Berset, M. 331

Bertana, E. 339

Berthelsen, M. 332, 432

Bini, S. 421

Biron, C. 146

Bisio, C. 339

Black C. 208

Black, Q.C. 208

Blaich, S. 147

Blanco, A. 424, 425

Blanco, L. M. 365

Bledow, R. 70

Blonk, R. 38, 148

Boccuni, F. 377, 378

Bonafede, M. 167, 347

Bonde, J.P. 311

Bonzi, F. 392

Boonprakob, M. 333

Boonprakob, P. 333

Borgogni, L. 161

Borrelli, I. 334

Bos, E. 148

Bosak, J. 149, 164 
Bosco, A. 340

Bosle, A. 335

Boudrias, J. 150

Bourguignon, D. 194

Bozeman, J. 335

Bracco, F. 152

Brangier, E. 432

Brauchli, R. 187, 336

Braun, I. 151

Bredrup, O.J. 297

Brenninkmeijer, V. 35, 38, 152

Brevik, J.I. 200

Briante, G. 241

Brooks, J. 398

Brosschot, J. 47

Bruce, M. 337

Brunet, L. 150

Bruno, A. 152

Buddeberg-Fischer, B. 213

Buech, V. 139, 274

Burba, M. 338

Buresti, G. 387

Burton, K. 398

Busch, C. 101, 145, 266

Cabianca, M. 269

Cacciola, A. 358

Caillard, J.F. 216

Callari, T.C. 153

Cambré, B. 52

Camerino, D. 154

Camicia, B. 392

Campanini, P. 154

Cardellicchio, E. 408

Carlo Picchi, M.P. 355

Carmona, I. 339

Carpentiero, L. 340

Carroll, A. E. 444

Carter, A. J. 444

Carter, L. 181

Casenave, S. 346, 380

Cassitto, M.G. 263, 340

Castagna, A. 421

Castanheira, F. 93, 94

Castellini, G. 263

Cataldo, A. 417

Catano, V. 39, 172

Catano, V.M. 364

Cecchini, F. 155

Ceja, L. 156
Ceresia, F. 157

Cetica, C. 253

Chambel, M.J. 93, 94, 134, 175

Chang, Y.W. 443

Cherng, C.F.G. 158

Cherniack, M.G. 145

Cherre, B. 158

Chiorr, C. 152

Choi, B.Y. 75, 126

Choi, H. 78, 125

Chongvisal, R. 159, 341

Choochom, O. 160

Christensen, J.O. 352, 432

Ciconte, A.R. 398

Cintar, D. 305

Cinti, M.E. 253

Ciprani, F. 225

Circenis, K. 342, 350

Civilleri, A. 407

Clarke, N. 267, 268

Clasen, J. 101

Claudino, A. 395

Clinton, M. 53

Clough, P. 173, 264

Cockburn, W 25

Coggiola, M. 178

Coll, K. 365

Colombo, L. 353, 458

Colunga Rodriguez, C. 342, 354

Consiglio, C. 161

Converso, D. 162, 343, 374, 396

Conway, P.M. 154, 263

Cooper, C.L. 5

Coronado, M.Z. 279

Corso, L.D. 430

Cortez, C. 163

Cortini, M. 315

Costa, G. 6, 154, 178, 263, 340

Costa, M. 344

Cousins, R. 164

Cowan, S. 186

Cox, T. 138, 193, 382

Cristina, E. 439

Croon, M. 302

Cropley, M. 271

Cullinane, S.J. 164

Cunha, M. J. 165, 259, 320, 344, 366, 367

Cunningham, K. 204

Cuomo, G. 225 
Curral, L. 93, 94

d'Ettorre, G. 387

D'Orsi, F. 21

Dalit Jäckel 106

Daniels, K. 243

Day, A. 39, 42, 84, 275

De Been, I. 166

de Bloom, J. 44, 48, 347

De Bruyne, E. 166

De Carlo, N.A. 430

De Cuyper, H. 52, 63

De Cuyper, N. 51, 54, 64, 66, 67

de Jong, J. 52

De Lange, A.H. 35, 37, 56, 58, 59, 61

De Lange, C. 167

De Luca, A, 422

De Luca, G. 340

de Rivas, S. 424, 425

De Rivas-Hermosilla, S. 267, 268

De Rossi, P. 427

De Tribolet, F. 173

de Weerth, C. 48

De Witte, H. 35, 51, 57, 58, 63, 64, 65, 67

Dechelotte, P. 291

Deitinger, P. 167, 347, 348, 349, 402

Deklava, L. 342

Deklavs, L. 350

Dellve, L. 281, 440

Demerouti, E. 61, 66, 69, 71, 72, 73, 74, 141, 194, 195

Demir, D. 244

den Broeck, A.V. 57, 58

Derks, D. 168, 295

Desmette, D. 169, 194

Desrumaux, P. 150

Deug Kim, H. 289

Di Cecio, M. 417

di Giannantonio, M. 334

Di Nola, C. 358

Diaz, D. 425

Diaz, L. 345

Diaz-Cabrera, D. 346, 373, 380

Diaz-Gracia, L. 345

Dikkers, J. 412

Dikkers, J.S.E. 56, 57, 58, 61

Dimpfl, S. 170

Dolan, S. 234

Dollard, M.F. 208

Dominguez Diaz, R. 354
Dominguez, J.L. 272

Dominguez-Diaz, R. 342

Donaldson-Feilder, E. 171

Donnell, C. 164

Donohoe, M 351, 364

Doosje, S. 58

Dumitru, C.Z. 288

Durepos, D. 172

Dusi, P. 351

Earle, F. 173, 264

Eid, J. 200, 331, 405, 454

Eisele, P. 352

Ekberg, K. 222, 265

Elfering, A. 173, 183, 331, 381, 425

Elka, S. 352, 432

Elovainio, M. 262

Emanuel, F. 353

Emberland, J.S. 352

Enriquez Hernandez, C. B. 354

Ensinger, K. 174, 285

Erba, A.M. 355, 414

Eriksson, A.F. 222

Eriksson, G. 356

Eriksson, T. 356

Ermakova, J. 325

Ernesto, F. 175

Ertel, M. 27, 176

Erturk, E.M. 357

Escartín, J. 156

Euwema, M.C. 82

Faghri, P. 408

Fairweather, L. 177

Falco, A. 430

Fantini, L. 19

Fattori, M. 392

Favaro, M. 358

Feldt, T. 45, 201, 375

Felfe, J. 122

Fenga, C. 358

Ferrante, P. 387

Ferrari, F. 259

Ferravante, D.G. 359

Ferreira, M.C. 359, 360

Ferreira, R.R. 359, 360

Ferrillo, M. 161

Feuerhahn, N. 177

Fiaschetti, M. 253

Fiaschi, S. 304

Fichera, G.P. 154, 178 
Fida, R. 239, 361

Figueira, C. 92, 93, 94

Figueiras, J. 395

Figueiredo-Ferraz, H. 335, 362

Finne, L.B. 363, 432

Fioravanti, M. 253

Flaspöler, E 181

Foddai, E. 407

Fontaine, A.M. 99

Fontani, V. 421

Forrier, A. 65

Fraccaroli, F. 140

Francis, L. 364

Franco, M. 442

Franke, F. 119, 122

Frascheri, C. 21

Freeman, B. 365

Galletta, M. 416

Gallo, R. 179

Gan, E. 339

Garbarino, S. 225

Garrosa, E. 339, 345, 365

Gascon, S. 344, 366, 367

Gates, D. 182

Gattai, A. 368, 389

Gatti, P. 353

Gattino, S. 343

Gertsch, D. 455

Gervais, R. 180, 181

Gervais, R.L. 89

Geser, W. 276, 277, 278

Gething, N. 112, 130

Geurts, S. 301

Geurts, S.A.E. $47,48,347$

Geving, G. 188, 369

Ghelli, M. 347, 348,349

Ghislieri, C. 353,458

Gibbes, C. 177, 224

Gilin, D. 364

Gilin-Oore, D. 84

Gillespie, G. 182

Gil-Monte, P.R. 319, 335

Gil-Monte, R. 362

Gilson, L. 396

Gilson, L.L. 371

Girard, A. 259

Gloriani, I. 359

Goian, C. 305, 370

Golay, L. 370, 371
Goncalves, S. 183, 372, 373

González-Romá, V. 85

Göransson, S. 41

Grant, L. 211

Grebner, S. 173, 183, 223

Greenberg, D. 215

Greiner, B. 184, 269

Greiner, B.A. 231, 384

Griffiths, A. 382

Grigioni, S. 291

Grimsmo, A. 188, 297

Gross, S. 185

Grzywacz, J. 205

Gudbergsson, S. 188

Guest, D. 312

Guinote, A. 194

Guise, J. 186

Gurt, J. 121

Gustavsson, J.P. 376

Gustavsson, M. 265

Gustavsson, P. 190, 270

Gustavsson, P.J. 337

Gyldholm Møller, K. 227

Hadari, I. 215

Hagen, S. 188, 369

Hakanen, J. 189

Hallsten, L. 190

Hamilton, J. 190

Hamilton, J. 191

Hämmig, O. 187, 213, 336

Han, J. 298

Hanif, R. 399

Hansez, I. 14, 192

Harris, P. 256

Hassard, J. 193, 198

Hasselhorn, H.M. 273

Hassim, N. 222

Hauke, A. 181

Henning, R. 408, 419

Henseleit, J. 331

Herman, G. 169, 194

Hernandez-Fernaud, E. 346, 373, 380

Herold, K. 254

Hershcovis, S. 335

Hertel, G. 264

Hetland, H. 194, 195

Hetland, J. 194, 195

Heurtebize, P. 216

Hillert, A. 382 
Hill-Tout, J. 110

Hindrichs, I. 162, 343, 374

Hinrichs, S. 123, 139, 196

Hoel, H. 204

Hoffmann, S. 31, 32

Holdsworth, L. 204

Holopainen, L. 197

Honkonen, T. 136

Houdmont, J. 198

Houtman, I. 27

Houtman, I.L.D. 282

$\mathrm{Hu}, \mathrm{Q} .116$

Huhtala, M. 375

Huijs, J. 148

Hultell, D. 376

Hunerli, S. 456

Hunt, N. 221, 290

Hupke, M. 199

Hystad, S.W. 200, 331

Hytter, A. 200, 376

Hyvonen, K. 201

Hyvonen, K., 375

lavicoli, I. 391

lavicoli, S 12, 25, 19, 240, 348, 377, 378, 387

Inauen, A. 33

Ingusci, E. 408

Intarakamhang, U. 202, 333

Irastorza, X. 25

Isalue, I. 425

Iskra-Golec, I. 379

Isla-Diaz, R. 380

Isometsa, E. 136

Isosomppi, M. 220

Iweins, C. 169

Jackson, B. 450

Jacobsen, K. 432

Jacobshagen, N. 202, 223, 381

Jain, A. 27, 138, 203

Jalonen, P. 47

Järvenpää, E. 250

Jennings, T. 109

Jenny, G.J. 31, 32, 33

Jin, Y.Y. 79, 126

Jochymek, S. 393

Johnsen, B.H. 454

Johnson, N.C. 145

Johnson, S. 204

Jones, C. 382
Jones, F. 205, 256

Jonsson, H. 356

Jonsson, P. 209

Jonsson, S. 235

Kadiri, S. 207

Kaelin, W. 185, 206, 223

Kajosaari, K. 219

Kajosaari, K. 251

Kallio, E. 47

Kamalanabhan, T. J. 289, 438

Kaminski, M. 207

Kang, S. 208

Kaptein, M. 375

Karlson, B. 209

Kasprzak, M. 394

Kelloway, K. 42, 364

Kelly, P. 15

Kerr, R. 210

Kim, A. 76, 124

King, N. 398

Kinman, G. 211, 212

Kinnunen, U. 45, 49, 55, 96, 98, 201, 418

Kirchner, A. 149, 164

Kivimaki, M. 262

Kivistö, S. 47

Klaghofer, R. 213

Knecht, M. 187, 213

Koch, S. 382

Kolstrup, C. 213

Kompier, M. 47, 48, 301

Kompier, M.A.J. 347

Koppes, L. 38

Koppisch, D. 181

Kortum, E. 12

Koukoulaki, T. 181

Krajewski, J. 119

Kraus, T. 217

Krause-Juettler, G. 214

Kudielka, B.M. 177

Kuehnel, J. 177

Kühnel, J. 70

Kuntz, A. 216

Kuronen-Mattila, T. 250

Kushnir, T. 215

Laberg, J.C. 454

Lacangellera, D. 340

Ladner, J. 216, 291

Ladstätter, F. 339

Lang, J. 217 
Lang, P.J.W.B. 217

Lange, A.H. 57

Larsson, B. 209

Larsson, G. 454

Larue, G. 194

Laschiner, H.K.S. 84, 218

Lau, B. 299, 332, 352, 363432

Lazzari, D. 260, 415, 416

le Blanc, P.M. 82, 85, 114, 115

Leah, C. 181

Lee, J. 76, 77, 79, 125

Lee, J.E.C. 383, 37

Lee, S.M. 75, 124

Leitão Alexandre, S. 384

Leiter, M.P. 81, 84

Leka S. 12, 27, 193, 203, 290

Lemke, P. 254

Leppänen, A. 219, 250, 251

Leskinen, J. 220

Leso, V. 391

Lewis, R. 171

Li, J. 220

Li, S. 220

Lin, P.Y. 221

Ljoså, C.H 332, 385

Llorens, S. 82

Lo Cascio, V. 407

Lo Presti, A. 386

Loera, B. 343

Lonnqvist, J. 136

Loretto, W. 255

Lotfizadeh, M. 222

Loughlin, C. 40

Lovas, J.G.L. 172

Lozar, L.G. 181

Lundqvist, D. 222

Lunt, J.A. 87

Macciocu, L. 387

Machado, F. 321, 388, 439

Machowski, S. 36

Mackay, C. 15

MacKinnon, R. 224

MacLennan, S.J. 221

Maes, S. 260

Magely, V.J. 370, 39, 451, 452

Magley, V.J. 327

Magley, V.J. 328

Magley, V.J. 371

Magnani, M. 368, 389
Magnavita, N. 225, 226, 390, 391

Magosso, D. 430

Magwentshu, S. 131, 227

Maharee-Lawler, S. 228

Maisetti, M. 392

Majer, V. 368, 389

Mäki, E. 250

Mäkikangas, A. 55

Malinowska, D. 393, 394

Malmberg, B. 209

Mamazza, L. 239, 361

Mancini, G.A. 368, 389

Mannu, P. 421

Manuti, A. 394

Manz, R. 229

Manzano, M. 216

Marcelino, D. 395

Marcutoiu, L. 448

Marcuzzo, G. 430

Margarita Torres, T. 435

Margotti, M.L. 421

Mariangela, M. 394

Maricutoiu, L.P. 288

Marini, M. 340

Marmet, M. 396

Marocci, G. 368, 389

Marowsky, I. 184

Martínez, M.B. 181

Martínez, S.V. 181

Martínez-Casariego, M.A. 181

Martinez-Jarreta, B. 366, 367

Martini, A. 240

Martini, M. 396

Masi, M. 19

Masuhr, K. 229

Mateski, M. 184

Matias, M. 99

Matthiesen, S.B. 405

Mauno, S. 55, 9596 98, 375, 418

Mauviard, E. 216

Mazzotta, M. 387

Mc Carthy, V. 184, 231

McCaughey, D. 444

McClellan, S.J. 290

McCluskey, S. 398

McHugh, M. 210

McMahon, G. 326

McNally, C. 40

Mearns, K. 405, 454 
Medhurst, A. 232

Meier, L.L. 46, 149, 164, 185

Melloh, M. 425

Mellor, S. 370

Menighini, A. 339

Messineo, A. 398

Micali, E. 233

Miceli, L. 422

Michel, A. 233

Milam, A. 399

Militello, E. 263

Millere, I 342, 350

Moccaldi, R. 261

Mohan, K.P. 202

Molan, G. 400

Molan, M. 400

Molek-Winiarska, D. 401

Moodie, S. 234

Moreno- Jiménez, B. 59, 72, 267, 268, 339, $345,424,425$

Morin, E. 183, 372, 373

Morrow, H. 39

Mottura, B. 162

Moulin, P. 432

Muehlethaler, C. 223

Muhonen, T. 235

Müller, F. 31, 32, 33

Munir, F. 403

Muratore, M. 339

Murray, P. 16, 83, 236

Musicant, O. 237

Musti, M. 178

Naegel, I. 237

Naegel, I.J. 283, 284

Nardella, C. 167, 347, 348, 349, 358, 387, 402

Naring, G. 238

Näswall, K. 41, 64, 97

Natali, E 239, 240, 361, 378

Nazzicone, F. 417

Neff, A. 107, 301

Negri, L. 241

Nelson, K. 150

Neves, J. 183, 372, 373

Nezavitina, T. 242

Niculaes, A. 403

Nielsen, K. 102, 103, 243, 403, 404

Nielsen, M.B. 299, 405, 454

Niessen, C. 107, 151, 301
Nill, A. 257

Nilsson, K. 406

Noblet, A. 147, 228, 244

Nocera, A. 339

Noehammer, E. 245, 246, 276, 277, 278, 287

Nonnis, M. 386

Notelaers, G. 35

Nyman, M. 220

O'Hara, R. 246

Oberoi, A. 170

Ochsmann, E. 217

Ohly, S. 36

Olos, L. 97, 247

Omlin, F. 331

Omne-Pontén, M. 337

Omori, M. 248

Oomens, S. 148

Osterberg, K. 209

Ouweneel, A.P.E. 115

Ozalp Turetgen, I. 433, 434

Ozkan, S. 252

Pace, F. 407

Pacelli, E. 304

Padover, D. 408

Page, K. 249

Pahkin, K. 219, 250, 251

Palano, F. 408, 439

Palaz, S. 252

Palermo, J. 252, 253

Palferman, D.J. 15

Palitti, T. 253

Pallesen, S. 195

Palombo, P. 152

Palsson, B. 209

Panfili, A. 253

Pangert, C. 254

Panunzi, M. 398

Park, Y.M. 125, 127

Parkin, T. 255, 409

Passalacqua, C. 407

Passos, A.M. 163

Pati, C. 387

Patlan-Perez, J. 410

Patrao, I. 411, 429

Paul, K. 279

Pavesi, A. 263

Pavez, C.B. 123

Payne, N. 256 
Pearce, M. 307

Peccei, R. 302

Pech, P. 27

Peeters, M.C.W. 69, 71, 74, 117

Pelizza, L. 269

Peltier, J. 257, 258

Penati, V. 259

Peper, B. 294, 412

Perbellini L. 269

Pereira, J. 259, 413

Pereira, J.P. 165 ,344, 366, 367

Perry, I.V. 231

Persechino, B. 348

Petrou, P. 71

Pettersen, T. 352, 432

Petyx, C. 378

Picchi, M.P.C. 414

Pinto, A.M. 91, 92, 93, 94

Piovanelli, S. 340

Pisanti, R. 260, 415, 416

Pizzuti, A. 402

Pontrandolfi, F. 23

Ponzini, G. 261

Pop, C. 142

Portoghese, I. 416

Pozzi, G. 334

Preciado Serrano Maria del, L. 354

Provis C. 208

Pugliese, F. 152

Puig, A. 77

Pulkki-Raback, L. 262

Punzi, S. 154, 263

Quiñones-García, C. 267, 268

Radstaak, M. 47

Raffaele, G. 417

Ramandi, M.M.A. 323

Ramos, M.E. 279

Ramos-Sapena, Y. 346, 380

Randall, R. 101, 102, 103404

Randell, K. 42

Rantanen, J 95 ,96, 98, 418

Rantanen, M. 98, 418

Rauschenbach, C. 264

Re, A. 153

Reeves, D. 408, 419

Reeves, D.W. 371

Reid, T. 173, 264

Reineholm, C. 265

Reinert, D. 181
Reinhold, K. 441

Rendon-Manjarrez, J.I. 342

Requa-Brueckner, G. 266

Resenterra, F. 420

Reshmi, A. T. 438

Rial-Gonzalez, E. 13, 25

Ribeiro do Couto, B. 362

Richter, A. 64, 97

Ricotta, S. 353

Ridley, D. 177

Riedmann, A. 27

Righini, S. 340

Rigotti, T 53

Rinaldi, S. 421

Riso, F. 422

Riva, P. 339

Roberts, R. 423

Robertson, N. 309, 454

Rodrigues, J. 259

Rodriguez- Muñoz, A. 345

Rodriguez- Muñoz, G. 184

Rodríguez-Carvajal, R. 267, 268, 365, 424, 425

Rodríguez-Muñoz, A. 59

Rodwell, J. 147, 228, 244

Roessler, R. 322

Rolli Salathé, C. 425

Rolo-Gonzalez, G. 373

Romanyuk, L. 426

Romeo, L. 269

Ronchetti, M. 240, 377, 378

Rondinone, B. 240

Rosa Pia, F. 394

Rosati, M.V. 253

Rossi, I. 387

Rosskam, E. 184, 269

Rubino, C. 306

Rudman, A. 190, 270

Russignaga, D. 178

Russo, L. 387

Rutz, J. 381

Rydstedt, L.W. 271

Sage, N. 324

Salanova, M. 82, 114

Salazar, G. 165

Salehi, B. 319

Salmela-Aro, K. 105, 197, 429

Samm, M. 213

Sancini, A. 253 
Santarém Semedo, C. 272

Santed, M.A. 366, 367

Santos-Rita, J. 411, 429

Sanz-Vergel, A.I. 59, 72, 365

Sarichlo, M.E. 323

Sarren, A. 148

Sarto, F. 430

Sartori, S. 340

Satrevik, B. 454

Savoie, A., 150

Savolainen, H. 197

Scalas, S. 340

Scatolini, E. 368, 389

Schaaf, V. 382

Schanz, K. 274

Schaufeli, W. 140, 273, 288

Schaufeli, W.B. 71, 115, 116, 117

Scheppingen, V.A. 148

Schibrowsky, J.A. 257

Schifano, M.P. 253

Schmidt, K.H. 199

Schneider, E. 181

Schoenenberger, S. 432

Schøning, M. 432

Schraub, E.M. 139, 274

Schreurs, B. 66

Schreurs, B.H. 116

Schryer LeBel, F. 275

Schuepbach, H. 123, 139, 174, 285

Schusterschitz, C. 245, 246, 276, 277, 278, 287

Schwartz, A. 111

Scimitto, L. 253

Sczesny, S. 149, 164

Seidl, J. 359, 360

Selenko, E. 279, 435

Sels, L. 65

Selvi, A. 340

Semmer, N.K. 46, 104, 183, 185, 202, 206, 223, 331, 381, 425, 455

Sertel Berk, H.O. 433, 434

Servadio, M. 351

Shabelnik, O. 330

Shin, H. 79, 127

Sieglin, V. 279

Simonsen, J.S. 280

Sinclair, R.R. 8

Sincox, A.K. 207

Sinibaldi, F. 259
Skagert, K. 281

Smulders, P.G.W. 282

Soltero Avelar, R. 435

Sonnentag, S. 47, 48, 70, 107, 151, 237, 283, 284, 301, 347

Sonntag, K. 139, 233, 274

Sossai, D. 152

Sousa, A. D. 413

Spitzmueller, C. 399

Staechele, T. 174, 285

Stanz, K. 143

Stavroula, L. 138

Steane, P. 244

Sterud, T. 18, 135, 286

Stiglbauer, B 279, 435

Stilijanow, U. 176

Stocker, D. 202

Stokenberga, I. 287, 436

Stride, C. B. 444

Stummer, H. 245, 246, 276, 277278287

Stynen, D. 65

Succop, P. 182

Sudom, K. 437

Sulea, C. 288, 399, 448

Surkov, P. 426

Sverke, M. 41, 64

Tagliaferri, C. 323

Tang, J.J. 290

Tanucci, G. 439

Tanzariello, M.G. 422

Tàpies , J. 156

Taris, T. 113, 114

Taris, T.W. 48, 116

Tavolacci, M.P. 291

Taylor-Ritzler, T. 170

Tehrani, N. 292

Teixeira, A. 388, 439

ten Brummelhuis, L.L., 293, 294

Tengelin, E. 440

ter Hoeven, C.L. 293, 294

Tetrick, L., 81

Tetrick, L.E. 73

Tham, K. 356

Thongpukdee, T. 333

Thulstrup, A.M. 311

Tims, M. 295

Tint, P. 441

Tirkkonen, M. 49

Tisato, S. 269 
Tkatsova, L. 441

Tokarz A. 393, 394

Tolyanen, A. 201

Tomao, E. 253

Tomei, F. 253

Tomei, G. 253

Torkelson, E. 296

Torp, S. 188, 297, 369

Torrente, P. 273

Tourigny, L. 298

Trombetta, M. 442

Trumpold, K. 36

Tsang, F. T. 443

Tschan, F. 185

Turc, D. 444

Turc, M. 444

Turgut, S. 233

Turner, N. 444

Tuulik, V. 441

Tvedt, S.D. 299, 300, 445, 446

Tyler, J. 184

Tynes, T. 135

Unger, D. 107, 284, 301

Unsal, P. 433, 434

Upenieks, R. 447

Utaaker, E. 432

Valenti, A. 378

van Beek, I. 113, 116

Van de Ven, B. 238

Van den Broeck, A. 35, 65, 114

van den Heuvel, M. 66

van der Doef, M.P. 260

Van der Heijden, B 54, 273

Van der Heijden, B.I.J.M. 35, 37

van der Walt, R. 325

van Dijk, F. 303

van Emmerik, H. 66, 82

van Engen, M. 412

van Hooff, M. 301

Van Rensburg, N.J. 143

van Veldhoven, M. 302

Van Vuuren, T. 54

van Wijhe, C.I. 117

Vander Elst, T. 58, 64, 65

Vansteenkiste, M. 58, 114

Varekamp, I. 303

Vasylyeva, J. 242

Vaughan, N. 87

Vecchio, G.M. 304
Vella-Brodrick, D. 249

Velocci, S. 422

Vene, D. 340

Vera, M. 82, 114

Vianello, L. 430

Vilkevicius, G. 181

Villet, H. 291

Vincent, S. 119, 120

Vindsetmo, K. 352, 432

Vinje, H. 369

Vinkenburg, C., 412

Vintila, M. 305, 370

Violani, C. 260, 415, 416

Viotti, S. 162, 343448

Virga, D. 288448

Virtanen, M. 136, 262

Visconti, S. 398

Vlerick, P. 238

Waite, E. 306, 449

Walker, A. 307, 450

Wallgren, L.G. 308

Walsh, B. 451, 452

Wang, X. 298

Warren, N. 408

Warszewska-Makuch, M. 453, 459

Watts, J. 309, 454

Wehner, T. 336

Westli, H.K. 454

Wetzstein, A. 229

Weyman, A. 89, 180, 310

Widmer, P. 455

Wieland, R. 119

Wiese, B.S. 105, 107

Wikstrom, E. 440

Wilczek-Ruzyczka, E. 329, 456

Wilde, B. 123, 139

Willert, M.V. 311

Willi, S. 331

Williamson, E. 184

Williamson, J. 89, 180

Willis, C. 170

Windemuth, D. 312

Winefield, A.H. 208

Wood, S. 302

Woodrow, C. 312

Wren, B. 109, 130

Xanthopoulou D. 295, 313

Yarker, J. 171

Yazdizadeh, M., 323 
Yermiahu, Y. 215

Yildirim, D. 456

Yildiz, S. 457

Yu, K. 78, 124, 125

Zanca, M.A. 407

Zanella, D. 430

Zapf, D. 36

Zecic, D. 168

Zhou, J. 198

Zito, M. 458

Zogran, C. 314

Zolnierczyk-Zreda, D. 453, 459

Zorrilla, D.M.N. 410

Zsoldos, L. 184

Zuffo, R.G. 315

Zwetsloot, G.I.J.M. 148 
